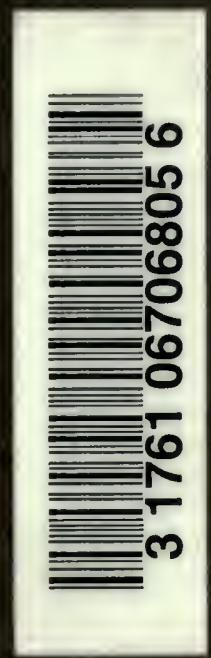



TEXT'BOOK OF ZOOLOGY 
TEXT-BOOK OF BOTANY. By J. M. Lowson, M.A., B.Sc., F.L.S., Headmaster of the Burgh Technical School, Inverness. Fifth Edition, Revised and Enlarged. 6s. 6d.

PRACTICAL BOtanY. By F. Cavers, D.Sc., F.L.S. Second Edition. 4s. 6 d.

BOTANY FOR MATRICULATION. By F. CAVER, D.Sc., F.L.S. 5s. 6d.

JUNIOR BOTANY. By F. CAVERS, I).Sc., F.L.S. 2s. $6 \mathrm{~d}$.

THE AIMS AND METHODS OF NATURE STUDY. By John Rennie, D.Sc., F.R.S.F., Lecturer in Natural History in the University of Aberdeen. 3s. 6d.

SCHOOL LESSONS IN PLANT AND ANIMAL LIFE : a companion volume to The Aims and Methods of Nature Study. By Jorn Rennie, D.Sc., F.R.S.E. 4s. 6 d. 


\section{TEXT-BOOK OF Z00LOGY}

BY

H. G. WELLS, B.Sc., F.Z.S., F.C.P.

AND

A. M. DA VIES, D.Sc.

Ninth Impression (Sixth Edition)

Revised by J. T. Cunningham, M.A. Oxon.

London: W. B. CLIVE

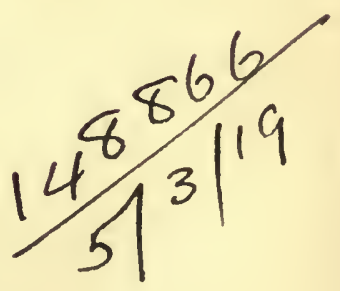

Mniversity Eutorial (Press

Hrair St., New Oxford St., W.C. 
Digitized by the Internet Archive in 2007 with funding from Microsoft Corporation 


\section{rietiale.}

IN writing this volume the authors have adopted the "type-system" as being most suitable to their purpose. The advantages of this system as an introduction to the natural history sciences are well known, though there are dangers attaching to the too exclusive restriction of the attention to isolated species; an endeavour has therefore been made throughout the writing of this work to keep both the advantages and the dangers in mind.

An experience of several years in conducting correspondence-classes in the subject has made the authors aware (to a greater extent, probably, than most ordinary teachers) of the wonderful way in which misunderstandings can lurk in the seemingly plainest sentences, and accordingly great pains have been taken to make their meaning clear at all points. Only one chapter in the book (Chapter XIV.) remains practically unaltered from the first edition, so that while the credit for the general plan of the work belongs to Mr. H. G. Wells, no responsibility attaches to him for any part of the present book.

The diagrams have been drawn specially for the book by Mrs. Davies, and it is hoped the plan adopted for labelling them will be useful to students.

Thanks are due to Mr. J. M. Lowson, M.A., B.Sc., who has read through both the manuscript and proofs, and made many valuable suggestions; also to Mr. E. Stenhouse for having called attention to several slips and errors. 


\section{NOTE TO THE SIXTH EDITION.}

THE alterations and additions which have been made in this edition are the work of Mr. J. T. Cunningham, M.A. The Supplement which he had added in the Fifth Edition has now been incorporated in the body of the work, and Part IV. Invertebrata has been rearranged so that the types follow in general the descending order in classification, beginning with the Crayfish and ending with the Protozoa:

An important addition has been made to Chapter XXII., on the Theory of Evolution, which was formerly very short and inadequate. Mr. Cunningham has summarised as far as space would allow the various facts and theories which are considered of most importance by modern biologists in relation to the problems of evolution. 


\section{CONTENTS.}

Part I - The Rabbit.

I'A $:$;

$\begin{array}{lllllll}\text { Chapter I. Introductory } & \ldots & \ldots & \ldots & \ldots & \text { l }\end{array}$

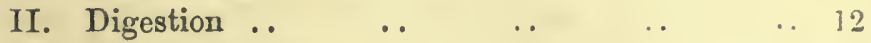

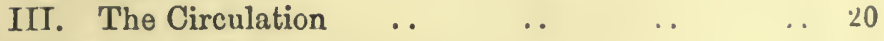

IV. Respiration $\quad$.

V. The Amoeba and the Cell

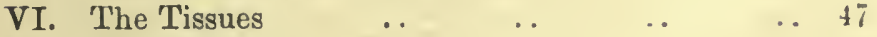

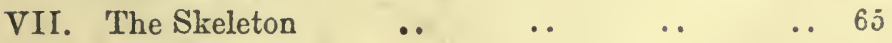

VIII. Muscle and Nerve .. $\quad$.. $\quad$.. $\quad$.. 81

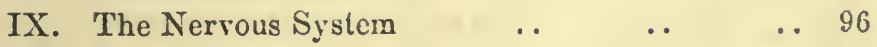

X. Sense Organs $\quad$. $\quad$.

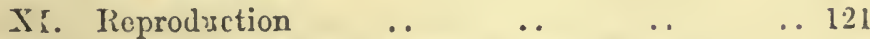

XII. The Rabbit's Place in the Animal liningdom .. 126

Questions on the Rabbit .. $\quad$. $\quad$.. 135

Pakt II.-Tne Lower Vertebrata.

XIII. The Frog .. $\quad$. $\quad$.

XIV. The Skulls of the Frog and the Dog .. $\quad$.. 159

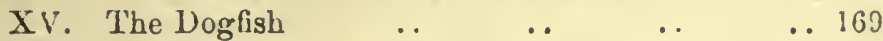

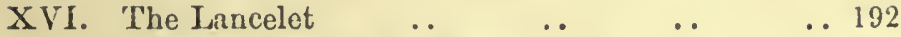

Questions on the Vertebrata _. . . 209

Part III.-The Development of Vertebrata.

„ XVII. General and Amphioxus .. $\quad$.. $\quad$.. 212

", IVIII. The Frog N

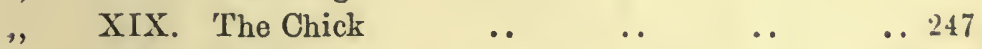

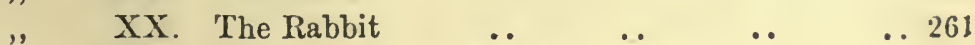

" XXI. The Systems of Organs _. $\quad$.. $\quad$.. 26;

" XXII. The Theory of Evolution .. $\quad$. . $\quad$.. 281

Questions on Vertebrate Development _. 297 


$$
\text { Part IV. - Inveriebrata. }
$$

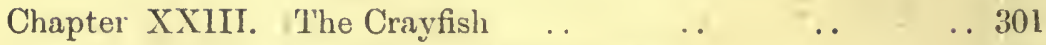

„, XXIV. The Fresh-Water Mussel $\quad . . \quad \ldots 324$

,, XXV. The Earthworm _.

,, XXVI. External Characters of Nereis .. $\quad$.. 349

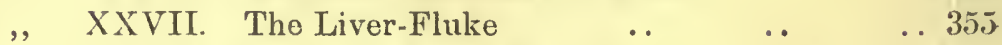

,, XXVIII. The Pork Tape-Worm . . .. $\quad$.. 366

,, XXI . The Fresh-Water Polyp .. $\quad . . \quad$.. 376

, XXX. Obelia Geniculata . . .. . . 387

,, XXXI. The Slipper-Animalcule . . $\quad$. $\quad$.. 399

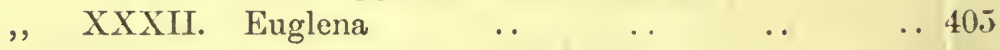

,, XXXIII. Monocystis and Coccidiun _. . . 413

„, XXXIV. The Malarial Parasite . $\quad . . \quad$.. 423

," XXXV. Diagnostic Characters of the Principal Divisions of the Animal Kingdom!.. . 428 Questions on the Invertebrata . . . . 455 


\section{PART I. - THE RABBIT。}

Chapter I.-Introductory.

§ 1. External Form. It is unnecessary to enter upon a description of the appearance of this familiar type, but it is not perhaps superfluous, as we proceed to consider its anatomy, to call attention to one or two points in its external, or externally apparent, structure. Most of our readers will already know that it belongs to that great division of the animal kingdom which is called the Vertebrata, and that the distinctive feature which places it in this division is the possession of a vertebral column or backbone; really a series of small ring-like bones, the vertebrce, strung together, as it were, on the main nerve-axis, the spinal cord (fig. 1). This vertebral column can be felt along the neck and back to the tail. This tail is small, tilted up, and conspicuously white beneath, and it serves as a "recognition mark" to guide the young when, during feeding, an alarm is given and a bolt is made for the burrows. In those more primitive (older and simpler-fashioned) veltebrata, the fishes, the tail is much larger and far more important, as compared with the rest of the body, than it is in most of the land-inhabiting vertebrates. In the former it is invariably a great muscular mass to propel the body forward; in the latter it may disappear, as in the frog, be simply a feather-bearing stump, as in the pigeon, a fly-flicker, as in the cow or horse, a fifth hand, as in some monkeys, an emotional index, as in the dog, or be otherwise reduced and modified to meet special requirements.

§ 2. Divisions of the Body. At the fore end, or, as English zoologists prefer to sily, anterior end, of the zOOL. 


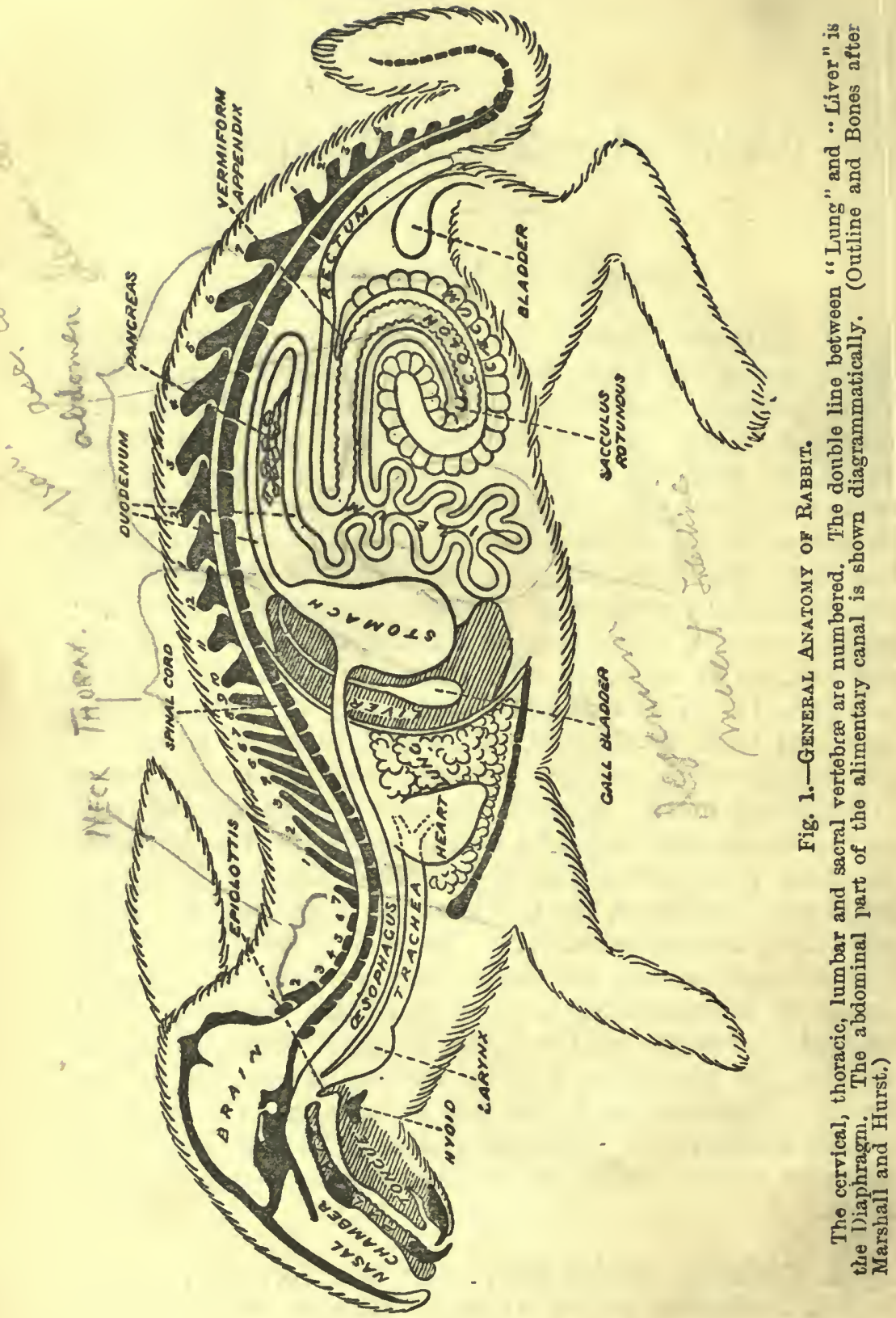


vertebral column of the rabbit, is of course the skull, containing the anterior portion of the nerve-axis, the brain (fig. 1): Between the head and what is called "the body," in the more restricted sense of the word, is the neck. The neck gives freedom of movement to the head, enables the animal to look this way and that, to turn its ears about to determine the direction of a sound, and to perform easily endless motions in connexion with biting and so forth. We may note that in types which swim through the water-in the fish and frog, for instance-there is no neck, the head simply widening out as it passes back to the body. The high resistance offered by water necessitates this tendency to a cigar outline, just as it has determined the cigar shapo of an ordinary boat or ship.

In the remainder of the trunk of the rabbit, as examined from the outside, we can make out by feeling two distinct regions, just as we might in that of a man; anteriorly a bony cage, having the ribs at the sides, a lod-like bone, the sternum (fig. 1), on its ventral surface (i.e. below in the natural position of life), and the backbone dorsally (above), and called the chest or thorax; and posteriorly a part called the abdomen, which has no bony protection over its belly, or ventral surface. These parts together with the neck constitute the trunk. Corresponding to these divisions of the body, there are four regions in the backbone: the neck, or cervical part, consisting of seven vertebræ, the thoracic part of twelve joined to ribs, the abdominal (also called the lumbar) region of seven without ribs, and the tail or caudal of about fifteen. Between the lumbar and caudal come four vertebræ, the sccral, which tend to run together into a bony mass as the animal grows old, and which form a firm attachment for the base of the hind limb.

\$ 3. The Limbs. The rabbit has two pairs of limbs. The short strong fore limbs are adapted to the burrowing habit, and have five digits; the hind limbs are very much longer, enabling the animal to progress rapidly by short leaps, and they have four toes, the one corresponding to the great toe of man being absent. In the great majority 


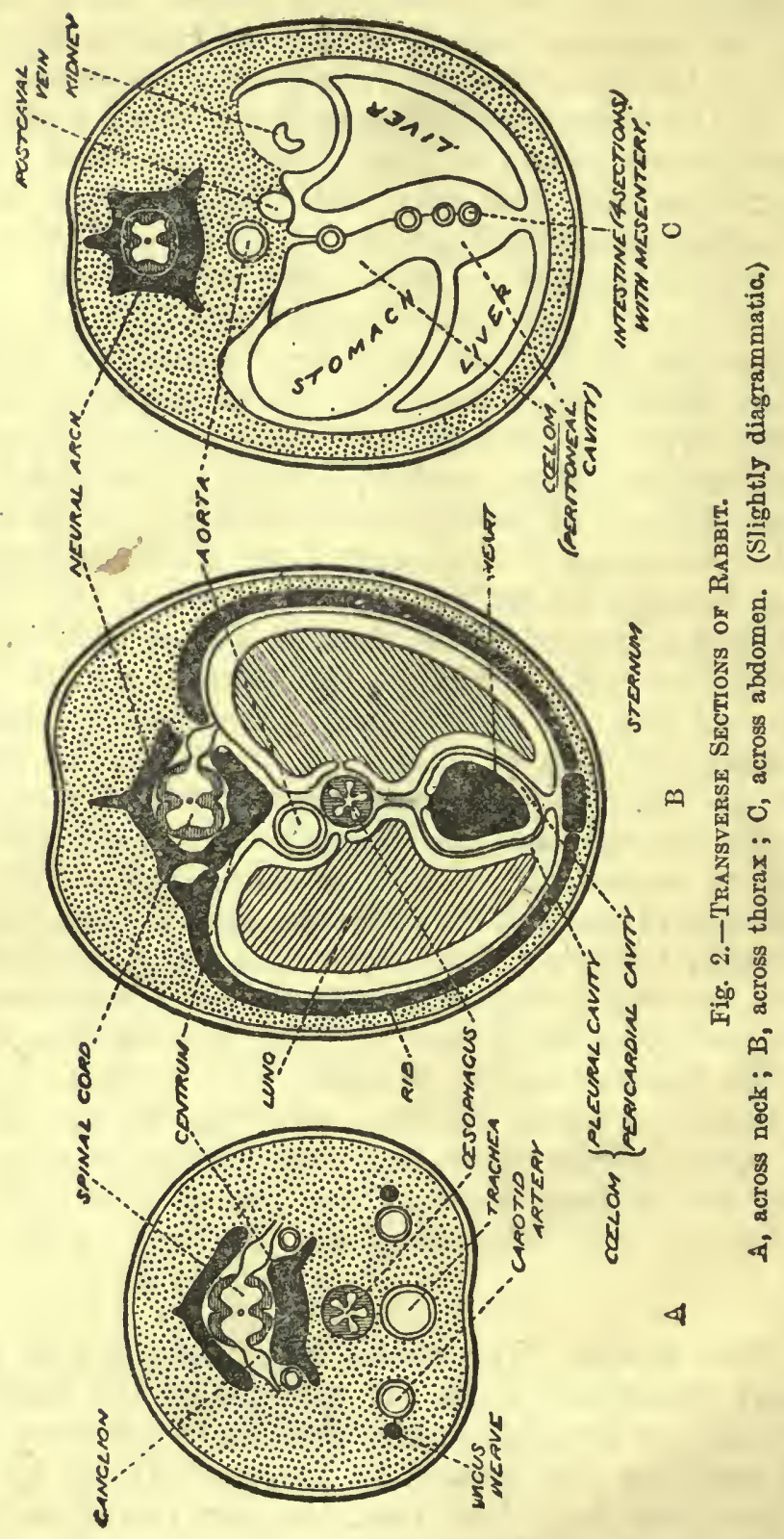


of air-breathing vertebrata, there are either five or less than five digits on both fore and hind limbs : if there are fewer than five it is always possible to determine, by comparison with other animals, which of the full set have disappeared.

§ 4. The Hair. Practically the whole surface of the body is covered by hair. This covering is distinctive of that class of the vertebrata called mammals, which includes man, most of the larger and commoner land animals, and the whales and manatee. In birds we have an equally characteristic cover in the feathers, the frog is naked, and among the fishes we find either naked or scaly skins.

§ 5. Internal Structure. In the actual examination of the rabbit's internal structure, the student will proceed by the method of dissection, or the separation of the organs from one another. To obtain a clear notion of the natural position and mutual relations of the parts, another method is useful. This is the method of taking sections across various regions of the body. Diagrammatic representations of such sections are given in fig. 2 .

$\S 6$. Section of Abdomen. Taking first the section through the abdomen, we rotice that two regions are seena smaller dorsal region, occupied by the backbone (with its contained spinal cord) and a considerable mass of muscle; and a ventral region, in which there is a large cavity-the body-cavity or colom, enclosed by a comparatively thin mass of muscle (the body-wall) and containing a greatly coiled tube, the alimentary canal or gut. The cœlom, here and elsewhere, contains a watery fluid (serous fluid). The bodywall is lined by a membrane called the peritoneum. If this is traced round from either side to the dorsal region near the middle line, it is found to be reflected ventral. wards, as a double membrane - the mesentery - which surrounds, and hence suspends, each of the coils of the gut. A little on either side of the middle line the kidneys bulge ventralwards into the cœlom, pushing the peritoneum before them. Evidently, however, the relation of the kidneys to the cœlom differs from that of the gut only in degree, 
each being separated from the actual cavity by this thin peritoneum, as though they had been pushed in from without, the gut having made its way in much farther.

$\S 7$. Section of Thorax. In the section across the thorax (fig. 2, s) we find a very similar general arrangement, but the alimentary canal (in this region called osopliagus) is a straight tube, and the greater part of the colom is occupied by the large lungs and heart. On tracing the lining membrane of the body-wall, here called pleura, we find that its median reflected portion extends right down to the ventral side, so that the right and left lungs lie in separate pleural chamber's. The heart is also surrounded by a similar but very narrow space, the pericardial chamber, lying between the outer and inner folds of a similar membrane, the pericardium* or pericardial membrane.

$\S 8$. Neck and Head. A transverse section through the neck (fig. 2, A). shows much the same arrangement in the dorsal part; but ventrally there is no colom-cesophagus and trachea are seen embedded in a solid mass of muscle and other tissues. This suppression of the colom in all parts of the body anterior to the heart is one of the most noticeable characters of the colom of ordinary vertebrates, and affords a striking contrast to the condition in many invertebrates, such as the earthworm, where the colom extends to the front end of the head.

A section through the head would also show an absence of cœlom, but here the dorsal region no longer shows vertebræ with spinal cord inside and muscles outside, but the more complex skull and brain and comparatively little muscle.

§ 9. The Diaphragm. The cavities of the thorax and abdomen are separated by a partition, the diaphragm (fig. 1). This is another of the structures distinctive of mammals, and we shall see later that it is essentially connected with the perfection of air-breathing to which this group has attained.

* The use of this term is rather ambiguous-it is sometimes applied to the membrane, sometimes to the cavity. 
§ 10. Descriptive Terms. We may note hore the meaning of certain terms we shall be constantly employing, and which are as important for our purpose as north, south, east, and west, are to the geographer. The head end of the rabbit is anterior, the tail end posterior, the backbone side of the body-the upper side in life-is dorsal, the breast and belly side, the lower side of the animal, is ventral. If we imagine the rabbit sawn asunder, as it were, by a vertical plane passing through the head and tail, that is the median plane, and parts on either side of it are lateral, and left or right according as they lie to the animal's left or right. In a limb, or in the internal organs, the part nearest the central organ, or axis, is proximal, the more remote or terminal parts are distal. For instance, the mouth is anteriorly placed, the tongue on its ventral wall ; the tongue is median, the eyes are lateral, and the fingers are distal to the elbow. The student must accustom himself to these words, and avoid, in his descriptions, the use of such terms as "above," "below," "outside," which vary with the position in which we conceive the animal placed.

A word of warning may here be given, as to the use of certain terms derived from human anatomy. The human body being conceived of as in the erect posture we find that human anatomists speak of anterior and posterior, where we should say ventral and dorsal; while superior and inferior take the place of our anterior and posterior. The human head, however, is twisted back, as it were, into the same position as in the rabbit, in spite of the erect body, and so anterior and posterior have the same meaning in both cases as applied to the head.

$\S$ 11. The Rabbit a Worker. So much for the general form: we may next note a few facts of general knorvledge in connexion with the rabbit's life-activity. In a day of a rabbit's life a considerable amount of mechanical work is done-the rabbit runs hither and thither, for instance: this means that the momentum of every part of its body is continually being altered. It scratches out earth, therein overcoming resistance. Again, its temperature is con- 
stantly kept many degrees higher than that of surrounding objects; therefore there must be a constant renewal of energy to balance that lost by radiation. Whence comes the energy which is necessary to a rabbit's existence?

$\S$ 12. Source of the Rabbit's Energy. The chemical study of the bodies of animals like the rabbbit has shown them to be more than half composed of water, while the remainder consists for the most part of the class of chemical compounds called organic, which are combinations of carbon, nitrogen, oxygen, and hydrogen, with smaller proportions of sulphur, phosphorus, and other elements. Now these organic compounds are exceedingly complex in their chemical constitution, and as the elements of which they are composed have (for the most part) less affinity for one "another than for oxygen, they are unstable. All such complex and unstable compounds, in the presence of oxygen with which their elements can combine, afford a great source of energy, and it is when this energy is transferred into other forms that work is done. Every bit of work (in the widest physical sense) done by the rabbit implies the decomposition and oxidation of a definite amount of these organic compounds. Further evidence of this is readily at hand.

$\S$ 13. Removal of Waste Material. A rabbit is constantly breathing - taking air into its lungs and expelling it again. The composition of the ingoing and outcoming air differs very definitely - the latter containing less oxygen and more carbon dioxide and water vapour than the former. At intervals, too, the rabbit expels from its body a fluid called urine. This consists of water containing in solution a compound called urea (a partially-oxidized compound of carbon, nitrogen, and hydrogen), with phosphates, sulphates, and other inorganic salts. From the skin also a certain amount of water containing inorganic salts is given off (perspiration). All these waste-materials are called excretions.

Thus the rabbit is continually taking in oxygen, and giving out compounds which are obviously the products of 
oxidation of the materials which make up its body. So far, the source of the rabbit's energy is clear; but there must evidently be some means of renewing the complex compounds, or the rabbit's life would soon come to an end. This renowal constitutes nutrition, or feeding.

$\S$ 14. The Food of Animals. A very considerable portion of the rabbit's daily labour is devoted to obtaining, nibbling up, swallowing, and treating in other less visible ways, portions of the vegetation among which it lives. Indeed, this, along with the endeavour to escape being itself eaten, and its efforts to add as many as possible to the total number of living rabbits, must pretty well exhaust the account of its life-labours. It is from this vegetation that the rabbit obtains the compounds needful for the repair of its body, and also (while growth or reproduction is going on) for the formation of new living substance. Some small portion of the food taken in is passed out again, practically unaltered, in the form of little pellets, the fuces: the greater portion, after undergoing various changes, to be described in the next chapter, enters into the formation of new living tissue. Thus the rabbit is dependent on the plant kingdom for the maintenance of its life. So, too, are all animals, directly or indirectly; for though one animal may feed on another and that in turn on another, this process cannot be carried on indefinitely: sooner or later we must come down to an animal which is a plantfeeder. In the long run all animals are dependent on plants for both the material and the energy of their bodies.

$\S$ 15. Sources of Material and Energy of Living Things. We must trace the supply one stage farther. Whence do plants obtain their material and their energy? The full answer to this will be found by the reader in his botanical text-book: here we can only point out the most general facts. The source of material is very largely the wasteproducts of animals-carbon dioxide, water, urea (or its decomposition-products), etc. But the mineral kingdom is also largely drawn upon, the decomposition of the rocks of the earth's crust furnishing a great deal of necessary 
material for plant-life. We have good reason to believe that, in spite of the enormous mutual destruction of living things, the actual quantity of living matter on the earth's surface is still increasing, on the whole, as we know it has steadily done for ages past. Thus there has been a gradual drain of material from the dead crust of the earth into the substance of living creatures, first into plants, and then through both plants and animals. But to build up from the simple compounds of the earth's crust, or from those excreted by animals, the complex compounds of plants, a constant inflow of energy is needed, and this is supplied by the radiant energy that comes to us from the sun.

$\S$ 16. Metabolism in Plant and Animal. Such is the general view which modern science takes of the relation of the animal and plant kingdoms to one another, and to the inorganic world. To express this relation briefly and clearly we need two or three terms of modern introduction. The whole of the chemical changes which go on in every living organism are collectively termed metabolism. They may bo grouped under two heads. Firstly we have synthetic processes, by which simple compounds are built up into complex ones, energy being absorbed and stored up in these complex compounds : these collectively constitute anabolism. Secondly, there are the processes by which the highly complex living matter built up by anabolism undergoes decomposition and oxidation, with transfer of energy and the performance of physical work: these constitute katabolism : the comparatively simple, oxidized compounds resulting are called katastases.

Evidently the essential difference between plant and animal is that the plant is, on the whole, passive and anabolic, the animal active and katabolic. Not that the metabolism in either case is exclusively of the one kind, but that there is a balance in the one or the other direction.

§ 17. Morphology and Physiology. In our study of the rabbit, and of all our other types, we shall constantly have to look at things from two different points of viewthat of structure and that of function. From the former 
aspect wo specially consider the form and structure, relative position and mutual relations of the various parts which collectively make up the animal, and the changes' which these undergo during development; we compare one animal with another, seek for underlying similarities among the obvious differences they present. This kind of study constitutes what is called Morphology, and it is divided into three chief branches-Anatomy, or the study of the parts (members) of an animal; Histology, or the study of the tissues or fabrics of which the members are composed; and Development, or the study of the changes by which members and tissues come to be what they are. The second point of view is that of the functions or activities of the parts of the animal, which from this point of view are organs rather than members. Here, taking anatomy and histology as our basis, we seek to learu what part all these structures play in the life of an animal. This is Physiology.

$\S 18$. Homology and Analogy. The difference of view of the morphologist and physiologist is most clearly seen when the comparison of two different animals is in question. The former looks keenly for similarities in origin and structure between organs which may serve very different functions in the two cases; the latter is more interested in seeing how similar necessities of life lead to organs of different origin and structure being adapted to the same function in the two cases. Organs of the former kind in different animals are said to be homologous, those of the latter kind are analogous. We shall, in the course of our work, come across examples of organs which are homologous with one another, but not analogous, others which are analogous, but not homologous, and many which are both.

§ 19. Distribution. Besides Morphology and Physiology there is a third branch of Biology-Distribution-which we shall barely touch. This may be called the geography and ancient history of living things. 


\section{Chapter II.-Digestion.}

$\S 1$. The Alimentary Canal is a tube, beginning at the mouth and ending at the anus, along which the food passes, in which it undergoes digestion, and from which all the available portion is absorbed into the blood, leaving the residue to pass on and out by the anus. At first sight, in dissecting, this canal appears hopelessly complicated, but in the embryo (i.e. the rudimentary rabbit before birth) it is a practically straight tube of nearly uniform diameter, running from mouth to anus. In the head and neck, where there is no cœlom, and in the thorax, where the cœlom is occupied by other organs (heart and lungs), the alimentary canal remains straight in the adult; but in the abdomen, where the colom is spacious, and has not much else in it, the alimentary canal bends and coils and varies in calibre in such a way that the maximum of digestive area is packed away in the minimum of space. The coiling is, however, on a perfectly regular plan. Just as you cannot tie knots, but only make loops on a string both whose ends are fastened, so, as the abdominal portion of the rabbit's alimentary canal increases in length during development, it can only become looped in certain ways. Indeed, it is even more confined than the string, since it is united to the dorsal middle line by the mesentery. The nature of the loops and coils of this part of the canal is diagrammatically shown (a little simplified, and very much out of proper proportions), in fig. 1 .

$\S$ 2. Food-Materials. Before proceeding to a detailed description of the canal, and of the processes to which the food is subjected while in it, we must briefly explain the various classes of substances that are found in the rabbit's food. They may be roughly classed thus, on a chemicall basis :-

(1) Proteids and allied substances, highly complex organic 
compounds, containing nitrogen and sulphur. The albumin of white-of-egg is a typical proteid, and illustrates one of the peculiar properties of proteids, that of coagulating or becoming solid and insoluble when heated. Similar proteids are the myosin of muscle (meat), gluten of wheat, and legumin of peas, etc. It is the last two and similar vegetable proteids that concern the rabbit. Allied to the proteids are such compounds as gelatin and others, characteristic of certain animal tissues.

(2) Carbohydrates are compounds of carbon, hydrogen, and oxygen, the two latter being present in exactly the proportions in which they occur in water. Examples are starch, sugar, and cellulose (the substance of cotton, and one of the main constituents of most plants).

(3) Fats are organic compounds, containing (like the carbohydrates) no nitrogen, but with less oxygen than the carbohydrates. They are compounds of glycerine with one or other of the "fatty acids."

(4) Mineral salts, such as sodium chloride, the carbonates and phosphates of calcium, potassium and sodium, etc.

(5) Water.

§ 3. The Problem of Digestion. The great bulk of the compounds in the rabbit's food, as well as of those forming its body, come under these five headings. But most of these, as they occur in the food, are either solid and insoluble in water, or if liquid do not mix with water and cannot diffuse through organic membranes. Hence the problem of digestion is to change these compounds into such forms that they can readily pass into the blood of the rabbit through the bounding membranes of the alimentary canal. This is effected by certain fluids, formed either by the walls of the alimentary canal or by certain organs called glands, which open by ducts into it; all these fluids contain small quantities of organic compounds of the class called ferments, and these are the active agents in the change. We shall discuss the structure and action of glands a little more fully in a subsequent chapter. Here we will simply say that they are organs forming each its characteristic fluid or secretion, and sending it by a conduit, the duct, to the 
point where its presence is required. The saliva, tears, and perspiration, are examples of the secretions of glands.

$\S$ 4. The Teeth. The mouth is bounded by the upper and lower jaws, bearing the teeth. In front are the chisellike incisors - a double pair above, and single pair below. 'These nibble off pieces of roots, seeds, etc., which are then crushed and ground by the cheek-teeth (three premolars and three molars above, two premolars and three molars below), which have flat crowns crossed by harder ridges. Between the incisors and cheek-teeth there is a gap : in carnivorous animals like the dog this space is filled by the biting canines, and the cheek-teeth are cutting, not grinding, structures.

The student will probably ask why the cheek-teeth, which are all similar in appearance, are divided into premolars and molars. The young rabbit has a set of milk molars-a milk dentition-which is followed by the permanent teeth, just as in man. Those cheel-teeth of the second set, which have predecessors in the first series, are called premolars; the ones posterior to these are the molars.

$\S 5$. The Saliva. When the uraterial is well ground up, it mixes with the saliva, a fluid secreted in a series of glands about the face and throat (submaxillary, sublingual, infra-orbital, and parotid glands). This fluid consists of water, with a certain amount of mucin (the slimy compound familiar to us in the.secretion of the nose), various salts in small proportions, and (most important of all) a ferment called ptyalin. This last has the effect of changing the insoluble starch into sugar, which at once begins to dissolve in the water. The mucin lubricates tho crushed-up food, and so makes swallowing easier. The saliva is of great importance in all plant-eating land-animals, as their food contains much starch. In such an animal as the dog, which has but little starch in its food, there is very little chewing (mastication) and the saliva is of less importance.

$\S 6$. The Tongue. On the floor of the mouth is the tongue, which we shall refer to again in chapter $x_{\text {., }}$ in connexion with the sense of taste. Here we may note that it 
helps in the process of mastication, by moving the food about in the mouth, and also in the process of swallowing by working it into a bolus and thrusting it back.

§ 7. Food-and Air-passages. The roof of the mouth is formed by the palate, above which lies the air passage from nose to throat. At the back of the mouth or pharynx the courses of food and air cross one another, and a small part of the throat is common to both, though the common portion is reduced to an insignificant amount. This connexion between the respiratory and alimentary systems, which is of very slight advantage to the rabbit, which does not breathe through its mouth under ordinary circumstances, is one of the most characteristic peculiarities of the vertebrate animals, and affords the first example of a feature which is inexplicable from the point of view of the particular type studied, but whose meaning will become clear when we have examined the other vertebrate types. We shall deal with respiration fully in the next chapter; at present it will be sufficient to say that throat is an opening, the glottis, into the beginning of the passage to the lungs. To prevent the passing of food down into this opening, it is protected by an epiglottis, an upgrowth which, in the rabbit, forms a complete ring round the glottis, and which during the act of swallowing is apparently thrust up above the posterior end of the palate into the air-passage. Thus the food has to go either right or left of the epiglottis* to reach the csophagus.

§ 8. The Essophagus. The food-bolus is forced down the cesophagus by the contraction of the walls of the latter. Nothing new happens to it in its course downthe separation of stomach from mouth by a long tube has no particular advantage, but is simply an anatomical necessity in an animal with a neck and thorax.

§ 9. The Stomach and Gastric Juice. In the abdomen the original tube of the alimentary canal is first expanded

* In man the process is different. 
into the large bag-like stomach, in whose walls a gustric juice is secreted. This is an acid fluid (containing a fractional percentage of hydrochloric acid), and contains a ferment called pepsin. The effects of the acid are (1) to destroy the ptyalin of the saliva and so stop any further change of starch into sugar; (2) to dissolve any such salts as calcium carbonate (in bone-eating animals, like the dog, there is a greater proportion of acid); (3) to combine with proteids; and (4) to destroy bacteria introduced with the food. The special use of the ferment pepsin is that it converts proteids into the soluble and diffusible form known as peptone.

The food remains in the stomach (the entrance to and exit from which are closed by muscular action) some considerable time, and undergoes considerable change. It becomes more liquid, both by admixture of gastric juice and solution of peptone, and also by the melting of any fats present at the higher temperature of the stomach. In this condition the food has become chyme. Probably a certain amount of the dissolved material is absorbed, i.e. diffuses into the blood in the walls of the stomach; but most of the absorption takes place farther along the canal.

$\S 10$. The Intestines. Following the stomach comes the simple U-shaped loop called duodenum, and after this a complicated series of small loops forming the ileum, which ends in an expanded bulb, the sacculus rotundus. This portion of the intestine from the end of the stomach to the sacculus rotundus is called the small intestine. The sacculus opens by a valved aperture into the colon, and this passes on into the rectum which extends to the anus. At the junction of the ileum and colon, there is given off a great blind outgrowth, the cæcum, which is large in plant-eating mammals and ends in the vermiform appendix. The ileumcolon loop and the cæcum together are coiled up in a spiral: colon, cæcum, and rectum form the large intestine.

$\$ 11$. The Bile. The duodenum is separated from the stomach by a ring-like muscular valve, the pylorus; this valve belongs to the class of muscles called sphincters, which, 
under ordinary circumstances, are closed, but which relax to open the circular central aperture. The valve at the anus, which retains the fæces, is another instance of a sphincter. When, by the relaxation of the pylorus, some of the chyme is allowed to enter the duodenum, it is met by the green bile, secreted by the liver, stored up in the gallbladder, and poured into the duodenum along the bile-duct. The bile seems to have more than one function, and the exact help which it gives to digestion is somewhat uncertain. It is alkaline, and thereby neutralizes the acidity of the chyme and puts an end to the action of pepsin. To $\operatorname{som} \theta$ extent it also emulsifies the fats (i.e. breaks them up into minute oily globules). In both these ways it may help to prepare the chyme for the next fluid it meets-the pan. creatic juice. There is no doubt, however, that a good part of the bile is merely waste material, destined to pass out with the fæces. In so far as this is the case, the bile may be put down among the excretions, though, unlike urine and sweat, it is an excretion of which some use is made. As we shall see in the next chapter, the secretion of bile is by no means the sole function of the liver. It seems also that the bile has an antiseptic action, i.e. that it checks the development of bacteria in the chyme. Its action in this way, however, is not very great, as bacteria (a fow having escaped destruction by the gastric juice) multiply and abound throughout the intestines.

\$ 12. The Pancreatic Juice. Little more than half-way along the duodenum the chyme is met by the pancreatic juice, secreted in the pancreas. This fluid is alkaline (through the presence of sodium carbonate) and has a complicated action on the chyme, as it contains several ferments. One called amylopsin acts (like ptyalin) on starch, converting it into sugar; another, trypsin, carries on the work of pepsin, from which it differs by acting only in alkaline, not acid, fluids. The alkalinity of the pancreatic juice also leads to the more complete emulsification of the fats and oils, and these, when thoroughly divided up into droplets, are acted on by a third ferment, steapsin, and each is split up (chemically) into glycerine and one of the zOOL. 
fatty acids." The latter decomposes the sodium carbonate and forms a soluble soap (i.e. a fatty salt, e.g. sodium oleate). If, however, there is any calcium or magnesium carbonate in the chyme, this will form an insoluble soap, and so a certain proportion of the fatty acid will be wasted.

$\S 13$. Absorption in the Small Intestine. The pancreatic juice is the last digestive juice of any importance that the chyme meets with. By its action the remaining foodmaterials have been changed into a soluble condition, and now absorption into the blood takes place. The absorption of the various organic compounds takes place mainly in the ileum, while in the large intestine water is absorbed and the contents gradually become more and more solid, until the characteristic appearance of fæces is produced. The large " bubbles" of gas which are frequently seen distending the rectum consist chiefly of hydrogen and methane (marshgas) produced from the cellulose in the food by the action of the abundant bacteria.

The interior of the ileum has a shaggy or velvety appearance, due to an abundance of small finger-like projections of the wall, called villi. These villi, which project into the liquid chyme, appear to be the active agents in absorption, hut the precise method of absorption is rather difficult to understand. It will be sufficient for our purpose to say that in each villus two kinds of vessels are found-ordinary blood-vessels (capillaries) and lacteals. The capillaries carry off the sugar and proteids, while the lacteals take the fat. It is a remarkable fact that, after all the trouble that is taken to change proteids into peptones and fats into glycerine and soap, the moment these have diffused into the villi they seem to be re-converted into their original forms. $\Delta t$ all events, no peptones are found anywhere in the blood, while the chyle, as the fluid in the lacteals is called, is (if there was much fat in the food) quite milky from an abundance of fat-droplets. What becomes of blood and chylo we shall see in the next chapter.

* Such as oleic acid. The adjective "fatty" means "chemically related to fats," not "fat-like." All fats are compounds of glycerine with some tatty acid. 
At intervals along the ileum are oval patches (five or six in all) called Peyer's patches. In these, and in the sacculus rotundus, the structure of the wall is modified in a way to be described later (chap. vi., § I3), and they seem to have some special function in relation to absorption.

\$ 14. Absorption in the Large Intestine. The entry from the sacculus rotundus into the colon is guarded by a valve (the ileo-colic valve: of ten called ileo-creal): this prevents the return of any of the chyme that once passes it. The chyme has now a choice of two courses-along the colon, or into the crecum and back again. The arrangement of a spiral ingrowth along the wall of the caecum and its connexion with the ileo-colic valve seem to show that normally the chyme passes into the crecum. Whether the crecum has any special function is doubtfulit is very large in the rabbit and other herbivorous animals, and small in carnivores like the dog: it has therefore been suggested that in it some special ferment is secreted for the digestion of cellulose-a compound abundant in plantmaterial, and not acted on by the other ferments. There is, however, no evidence of this, and, as we have seen, cellulose is decomposed into soluble compounds and gases by bacteria. It seems probable, therefore, that the cæcum acts merely as an additional lengthening of the canal, for more complete absorption. It ends in the blind vermiform appendix, whose walls have exactly the structure of Peyer's patches, and are therefore probably absorptive in a high degree.

The movement of the chyme along the intestine is due to a squeezing of the walls in just such a way as you might squeeze an india-rubber tube with your fingers and slide them along the tube. This is called peristaltic action.

The unabsorbed residue of the chyme becomes less and less liquid as water is absorbed, and in the rectum assumes the form of foces. These consist partly of materials taken in with the food which have remained unaltered all along, partly of the excretory compounds of the bile, in very small degree of compounds produced during digestion (such as the insoluble soaps), partly of the products of bacterial decomposition. 
Chapter iII.-The Circula'cion.

$\S 1$. The Vascular System. The next thing to consider is the distribution of the food-material absorbed through the walls of the alimentary canal to the living and active parts of the body. This is one of the functions of the series of structures-heart and blood-vessels - called the circulatory or vascular system. It is not the only function. The blood also carries the oxygen from the lungs to all parts where katabolism occurs, and it carries away the katastases to the points where they are excreted - the carbon dioxide to the lungs, urea to the kidneys, and so on.

$\S$ 2. The Blood (fig. 3) is not homogeneous; under the low power of the microscope it

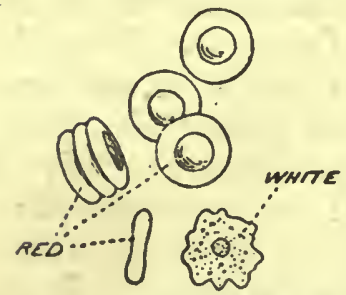

Fig. 3.-BLOOD-CoRPusures OF RABHIT. may be seen to consist of-

(i.) a clear fluid, the plasma, in which float-

(ii.) a few transparent colourless bodies of indefinite and changing shape, the white corpuscles, and

(iii.) flat round discs, which appear of a yellowish colour as seen under the microscope, but in bulk give the characteristic colour to blood, and hence are called the red corpuscles. They are vastly more numerous than the white. The two gases-oxygen and carbon diozide-are not carried in exactly the same way by the blsod. The student will know from his chemical reading that neither of these gases is very soluble, but carbon dioxide is sufficiently so in an alkaline fluid to be conveyed by the liquid plasma, as all the other materials are. The oxygen, however, is carried by the colouring matter of the red corpuscles, the hæmoglobin, with which it combines to form a bright red compound, oxy-homoglobin. This decomposes again easily 
in the presence of oxidizable compounds, to which it yields up the oxygen. The variation in the colour of the rabbit's blood is due to the relative proportions in which hæmoglobin and oxy-hæmoglobin are present.

§ 3. Capillaries. The blood must, to fulfil its functions, come into intimate contact with all the tissues of the body, and at the same time be able to travel rapidly from one tissue to another. These two neceśsities are effected by the enclosure of the blood in a series of tubes or vessels, and by the walls of certain of these vessels being so thin that the plasma of the blood can diffuse through them and bathe the tissues around. These thin-walled vessels are called capillaries: they are of microscopic dimensions and are very abundant in those tissues (such as muscles and glands) where extensive metabolism occurs. On the other hand, in tissues where metabolism is slower, especially if the tissue itself is readily permeable, capillaries are few and far between, or even absent altogether, as is the case with cartilage, which, though it may attain a thickness of perhaps a quarter of an inch, is sufficiently served by the plasma exuding from the capillaries in the surrounding tissue (perichondrium). So too the epidermis, or outer layer of the skin, is devoid of capillaries, as are all the tissues known as epithelia. (See chap. vi.)

§ 4. Arteries and Veins. The walls of the capillaries consist of the tissue known as endothelium (chap. vi., § 3), and this forms a continuous lining to the whole of the blood-vessels of the body, but in the capillaries alone is it the only layer present. The capillaries in a given portion of a tissue form a complex network through which the blood rushes rapidly, as can easily be seen in the tail of a living tadpole under the microscope. Observation soon shows that blood is brought to this capillary network in one vessel, and conveyed away through another. These two vessels frequently run side by side; usually they belong respectively to the category of arteries and veins-the artery bringing blood to the capillary network and the vein conveying it away. In certain cases both vessels are veins. 
The student has probably already heard these terms "artery" and "vein" employed, and very possibly is accustomed to associate them with a difference in the character of the blood they contain. It is well therefore to insist at once on the point that the differences between arteries and veins have nothing whatever to do with the composition of the blood they contain : they are connected solely with the different conditions of pressure in the blood.

§ 5. Arteries contain blood flowing away from the heart under the very great pressure caused by the powerful pumping of that organ. Hence they have thick and highly elastic walls, which do not allow any of the pressure to be wasted in a mere distension of the artery : though distended by the first shock of the heart's beat, the elastic recoil follows, giving rise to the phenomenon known as the pulse. Further, since the relative sectional area or calibre of the arteries determines the proportion of the blood that shall flow along them respectively, and since it is important that these proportions should vary according to varying needs, it is not surprising to find in the arterial walls a means of adjustment in the form of a muscular layer which by contracting in different degrees can alter the diameter of the tube. When the rabbit is killed, there is no longer any heart-pumping to act against the elasticity of the arterial walls, and so the calibre is at a minimum, and the arteries having thick walls and little blood remaining in them, appear white (with a bluish or pinkish tinge) with a thin reddish streak in the middle. Further, as the consequences of a cut artery in a living animal are more dangerous than those of a cut vein, we almost always find the large arteries deeper-seated than the large veins.

§ 6. Veins. In veins, on the other hand, during life, the pressure is low and variable. The blood is not pumped into them, for they are separated from the elastic-walled arteries by a sponge-like network of permeable-walled capillaries, through which pressure-variations canrot be transmitted. Hence the walls are thin, scarcely elastic, and readily distended. Through these thin walls external 
pressures of all kinds, due to the motions of the neighbouring organs, are readily transmitted, and hence there is very great variation in the blood-pressure in veins, and at times it may even bo negative, i.e. in the wrong direction, pressing the blood away from the heart instead of towards it. As a guard against these variations of pressure-direction, many veins contain valves which obstruct any flow in the wrong direction, and so bring it about that of all the varying external pressures acting on the blood, only those in the right direction shall have any effect. It follows from all this that the flow of blood in the veins is much slower than that in the arteries; and since the same amount of blood (roughly) must pass along the one sot of vessels as along the other in a given time, it is inevitable that the capacity of a vein must be greater than that of the corresponding artery. The blood-flow in a vein may be compared to the slow motion of the large body of water in a mill-stream; that in an artery to the rapid flow of the small body of water over the mill-wheel, .

$\S 7$. The Portal System. As we trace arteries away from the heart we find them branching out into smaller and smaller arteries, with thinner and thinner walls, and finally the smallest arteries pass into the capillary network, all the layers of the walls except the endothelium disappearing. In the same way we can trace the veins (in the opposite direction to the blood-flow) and find them ending similarly in the capillaries. In the other direction all arteries and most veins can be traced into larger ard larger ones, and finally to the heart.

But occasionally we find a system of veins which ends in capillaries when traced in either direction : only one such system is found in the rabbit, and it is called the portal system. This system is found in the mesentery and is therefore an approximately median one. The blood from the capillaries of the whole abdominal portion of the alimentary canal flows along a series of veins which unite into larger and larger ones, and finally into one large portal rein: these smaller veins through which the blood flows towards a larger vein may be called the factors of the portal 
vein, to distinguish them from the branches of the portal vein which convey the blood to the capillaries of the liver, and in which the blood is flowing from larger vessels to smaller. (On this nomenclature, it is evident that arteries have branches, while veins have factors, the portal vein alone having both.) The liver-capillaries also receive blood from an artery, as we shall see, and the mixed blood after undergoing certain important changes passes on into the factors of the hepatic vein and so into the general system of veins leading heartwards. The function of this portal system appears to be this-the blood coming from the capillaries of the gut contains, as we have seen (chap. ii., § 13), much material absorbed from the chyme, especially sugar and proteids. The amount of these varies, however, according to the feeding or fasting of the rabbit and the particular food it eats. Now it is important that the blood should contain its constituent compounds in fairly constant proportions: this is secured by the metabolic action of the liver-tissue, which withdraws from the blood-plasma the excess of sugar (and possibly proteids also) and stores it in the form of an insoluble carbohydrate called glycogen, and restores it to the blood again in well-regulated amounts. It acts, in fact, like a warehouse in which imported produce is stored instead of being allowed to flood the market as soon as it arrives. This, however, is a sample of the liver's functions, not a complete account.

§ 8. The Systemic Veins. The general arrangement of the systemic veins (i.e. all except the portal and pulmonary) is shown in fig. 4, the multitudinous minor factors being of course omitted. It will be seen that they are on the whole fairly symmetrical right and left, though not periectly so. Entering the dorsal side of the heart are three large vence cavae or caval veins-a right and left, precaval (vena cava superior or anterior) and a single postcaval (vena cava inferior or posterior.) The former have each as factors a small internal jugular. and large external jugular from neck and head, a subclavian from shoulder and forelimb, mammary and intercostal veins from thorax-walls, and sundry minor ones. A small azygos vein, collecting blood 
from the dorsal walls of the thorax, runs for some way in the middle line and then bends to the right to enter the precaval of that side : this is the only noticeably asymmetric feature in the anterior veins of the body. Some rabbits, but not all, possess a transverse connecting vein ventral to the thorax, uniting the $t w o$ external jugulars. This is called the jugular anastomosis, the term "anastomosis" being applied to a junction of veins of such a kind that an alternative course is thereby offered to the blood: obviously the direction in which blood flows in this vein will depend on which jugular vein has the greater blood-pressure.

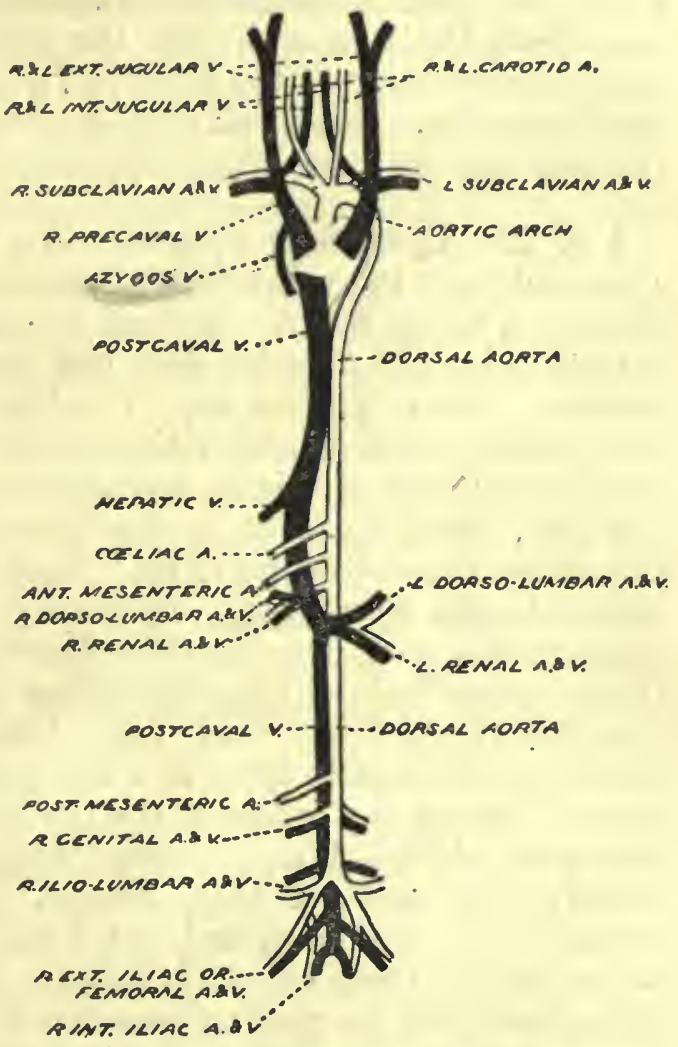

The postcaval Fig. 4.-Principal arteries and Veins of Rabit vein is not a median Heart and Portal System omitted; Median Arterics vein in the most turned to the left.

literal sense, since its anterior portion bends over to the right side, but it is median in the sense that it does not belong either to right or left side, but receives blood equally from both. Posteriorly it is formed by the union of right and left internal and external iliac veins from the hindlimbs, and ilio-lumbars from the body-wall; passing forwards its main factors are the genital * veins from the * Called spermatio in male, and oxarian in female 
reproductive organs; and the renals from the kidneys : both of these are paired (i.e. both right and left), but owing to the asymmetrical arrangement of the kidneys, the renal veins are also imperfectly symmetrical. Dorso-lumbav veins from the body-wall join the renals. Then come in the hepatic veins from the liver ( $\$ 7)$, and the phrenic from the diaphragm. Besides all these, a number of median dorsal veins enter it at intervals corresponding to the vertebræ.

§ 9. The Lymphatic System. We have now traced, in a general way, the veins from all parts of the body, except the lungs, to the heart. But all the blood-plasma carried outwards by the arteries does not travel this way back, although the corpuscles do. We have seen that some of the plasma exudes through the capillary-walls and bathes the tissues; part of this is assimilated by the tissues, but the rest, carrying with it the products of tissue-waste (katastases), travels back to the blood by a separate series of vessels, the lymphatics. These start as capillaries, quite unconnected with the blood-capillaries, and mostly blind, though some lead out from the cœlom by "lymph-stomata" (see fig. 11, p. 48). They unite into larger and larger vessels, which finally open into the precaval veins at their origin. Along the course of the lymphatics there occur at intervals whitish lumps called "lymph-glands" (though strictly they are not true glands), and in these white corpuscles are produced and added to the stream of lymph, as the fluid in these vessels is called. The lymph is forced along, partly by the general pressure of the tissues on them, and partly by contraction of the walls of the lymphatics. After death, as the blood accumulates in the veins, it may often force its way into the main lymphatics, but only under such abnormal conditions (often seen in dissecting) do the lymphatics contain red blood. The main lymphatic of the neck, with the "glands" on its course, can easily be traced in dissecting, but owing to the absence of colour the lymphatics are mostly difficult to trace.

There are three main lymphatic trunks into which the lymph from all parts eventually flows. Two of these run on the right and left sides of the neck. The third runs in 
the middle line by the side of the dorsal aorta, and brings lymph from the whole posterior region of the body and the alimentary canal. It is therefore much larger than the others, and being most conspicuous in the thorax has been called the thoracic duct. Near the anterior end of the thorax it bends round to the left, and after joining the lymphatic trunk from the left side of the neck, opens into the origin of the left precaval vein. The right lymphatic trunk of the neck opens into the corresponding vein on the right side.

The lymphatics of the alimentary canal (abdominal portion) and mesentery are specially developed and known as lactecls, because they contain (at least in animals fed on a fatty diet) a milky fluid, the chyle. This owes its milky appearance to the presence of abundant minute drops of oil, re-formed out of the soaps and glycerine absorbed in the intestine. As already mentioned (chap. ii., § 13) there is a network of lacteals in each villus of the duodenum, and there are others all along the intestine. These unite into larger and larger vessels, with "lymph-glands," some of very great size, set upon their course; they finally open into the thoracic duct.

$\S$ 10. The heart of the rabbit (see fig. 59, B) is divided by partitions into four chamber's : two anterior thin-walled ones, the auricles, and two posterior ones, the ventricles, both of which, but especially the left, have very thick, muscular walls. The right ventricle and auricle communicate, and the left ventricle and auricle, but the chambers of right and left sides are absolutely distinct. The veins open into the auricles-the three great caval veins (bringing blood from everywhere except the lungs). into the right auricle, the pulmonary veins (bringing blood from the lungs only) into the left auricle. From the auricles the blood passes into the ventricles, from which the arteries arise. The arteries do not, however, come off, as might be expected, from the posterior end of the ventricles - they come off from their anterior end, alongside the auricles, so that the posterior end (or apex) of the heart is a blind end. Each ventricle gives off a single arterial 
trunk which curves round to the left side, forming an arch; that from the left ventricle is the anterior of the two, and is called the aortic or systemic arch; the other, from the right ventricle, is the pulmonary arch. Where these arches curve back they come close to one another again, and here there occurs a connexion between them, called the ductus arteriosus. This connexion is a solid cord in the adult rabbit, and perfectly useless; in a newly-born rabbit it is much larger, and before birth it is a true artery, through which the blood flows from the pulmonary arch into the systemic. From the pulmonary arch of the adult come off two arteries only - the right and left pulmonary arteries, which take blood to the right and left lungs.

§ 11. The Double Circulation. The student will notice that the blood leaving the right side of the heart goes to the lungs only; while that leaving the left side goes to all other parts of the body. On the other hand, all the blood entering the left side comes from the lungs only, while that entering the right side comes from all other parts. Hence, since the right and left sides of the heart are completely separated internally, there is in the rabbit (and all mammals) a complete double circulation. In order that a blood-corpuscle, leaving the capillaries of a given organ, may get back to the same capillaries again, it must pass through the heart twice, or some even number of timesfirst through the right side and then through the left, and so on alternately. And each time, after passing through the right side and before it can pass through the left, it must pass through the capillaries of the lungs, and these capillaries only.

In the embryo the lungs are not in use, and there is no need for a double circulation. Thanks to the ductus arteriosus, most of the blood from the right side passes straight to the dorsal aorta without having to go through the lungs and again through the heart. Structures like the ductus arteriosus, that are useless in the adult stage, are called vestiges.

$\S$ 12. The Heart's Action. The beating of the heart is, 
of course, a succession of contractions and dilatations of its muscular wall. The contraction, or systole, commences at the bases of the venæ caræ and passes to the auricles, driving the blood before it into the ventricles, which then contract sharply and drive it on into the aorta or pulmonary artery; a pause and then a dilatation, the diastole, follows. The flow of the blood is kept in one constant direction by the various valves of the heart. No valves occur in the opening of the precavals, but an imperfect one, the Eustachian valve, protects the postcaval; the direction of the heart's contraction prevents any excessive back-flow into the veins, and the onward tendency is encouraged by the suck of the diastole of the ventricles. Between the left ventricle and auricle is a valve made up of two flaps of membrane, the mitral valve, the edges of the flaps being connected with the walls of the ventricle through the intermediation of small muscular threads, the chosdoe tendinea, which stretch across its cavity to little muscular pillars, the papillary muscles; these attachments prevent the mitral valve from flapping back into the auricle, and as the blood flows into and accumulates in the ventricle it gets behind the flaps of the valve and presses its edges together. When the systole of the ventricle occurs, the increased pressure of the blood only closes the aperture the tighter, and the current passes on into the aorta, where we find three watch-pocket valves, with the pocket turned away from the heart, which are also closed and tightened by any attempt at regurgitation (back-flow). A similar process occurs on the right side of the heart, but here, instead of a mitral valve of two flaps between auricle and ventricle, we have a tricuspid valve with three. The thickness of the muscular walls, in view of the lesser distance through which it has to force the blood, is less for the right ventricle than the left.

$\S 13$. The Systemic Arteries. From the aortic or systemic arch come off two main pairs of arteries anteriorlythe common carotids to the neck and head (each dividing into internal and external carotids as they near the head), and the subclavians to the fore-limbs. On the right side these two arteries are united for a short distance as tho 
innominate artery. Posteriorly the arch is continued into a dorsal aorta, which soon comes to lie in the middle line on the dorsal side of the cœlom. From this two kinds of arteries come off-median and paired.

The most important median ones are those which run into the mesentery to supply the abdominal portion of the alimentary canal. These are three in number, two very large ones, not far behind the diaphragm, and a third much smaller and farther behind. The first is the coeliac artery, which divides very soon into a gastro-hepatic and a lienogastric: these (as their names imply) convey blood to the capillaries of the stomach, liver, and spleen. The second is the superior or anterior mesenteric, supplying the greater portion of the intestines. The third is the inferior or posterior mesenteric, supplying the rectum.

Smaller median arteries go off dorsalwards, to the vertebral column and spinal cord, at intervals corresponding to the several vertebræ.

The paired arteries given off from the dorsal aorta correspond very closely with the veins entering the postcaval: thus there are phrenic, renal (with dorso-lumbar), genital, ilio-lumbar, and external and internal iliacs. The two lastnamed, however, start off together as common iliacs.

$\S$ 14. Comparison of Arteries and Veins. A comparison of the course of the veins and arteries in the rabbit gives some interesting results. Almost all the smaller arteries run side by side with the corresponding veins, just as the up and down lines of a railway run side by side: obviously what is the best route for the one is also the best for the other. A certain number of the larger vessels show the same arrangement-this is the case with the subclavians, renals and iliacs; it is also so with the vessels of the mesentery and intestines, except for the very largest, where the existence of a portal system makes it impossible. But when we come to the very largest vessels of all, the attempt to compare particular veins and particular arteries breaks down altogether. The portal and hepatic veins cannot be compared with the coliac and mesenteric arteries; nor the jugulars with the carotids. There are no arteries answering to the 
precaval veins, nor anything in the venous system answering to the remarkable arches of the aortic and pulmonary arteries. Why does not the simple rule, that what is the best course for artery is best for vein, hold here also? Partly it may be explained by particular necessities, as in the case of the portal system (§7). But no such explanation will hold for the neck and head vessels, nor, above all, for the remarkable arching forwards and to the left of the trunk arteries. Here in fact we find for the first time, but by no means for the last, details of structure which cannot be explained by any reference to the individual needs of the rabbit itself. How they may possibly be explained we may see later on, when we have studied other animals besides the rabbit. Meanwhile, we may just carry farther an analogy already suggested. When, in travelling on a railway, we find that the up and down lines do not run side by side, but differ in line or level, and no obvious reason exists for this, we naturally conclude that the two were not engineered at the same date, that the railway has had a past history and does not represent the carrying out of a single plan, but of several successive plans superimposed on one another. May it not be possible to explain in some similar way these anomalies in the rabbit?

$\S$ 15. Summary of Circulation. Summarizing the course of the circulation, starting from the right ventricle, we have-pulmonary artery, pulmonary capillaries, pulmonary vein, left auricle, left ventricle, aorta, arteries, and systemic capillaries. These smallest and ultimate ramifications of the circulation penetrate every living part of the animal, so that if we could isolate the vascular system we should have the complete form of the rabbit in a closely meshed network. It is in the capillaries that the exchange of material between the blood and the tissues occurs, nutritive material and oxygen passing out to the tissues and katastases in from them ; they are the essential factor in the circulatory system of the mammal-veins, arteries, and heart simply exist to remove and replace their contents.

From the systemic capillaries the blood, with less oxygen and less plasma than before, passes into the veins. From 
all the veins except those of the abdominal region of the alimentary canal the blood is gathered into the great caval veins, and so to the right auricle and right ventricle. The overflow of plasma, robbed of much of its nutritive material and loaded with katastases (carbon dioxide, urea, etc.), returns by the lymphatics to the precaval veins near the heart, receiving on its way now white blood-corpuscles from the lymph-glands, and abundance of fatty material from the lacteals.

From the capillaries of the stomach, spleen, pancreas and intestines, the blood with newly absorbed sugar and proteids is gathered into veins which unite into the great portal vein; this branches out into the capillaries of the liver, where it mingles with the blood from the hepatic artery, and finally, deficient in oxygen, but with its sugar-supply "standardized" it passes by the hepatic vein into the postcaval and thence to the right side of the heart.

In all the capillaries the blood loses nutritive materials (proteids, sugar, etc.), and, except in those of the lung, oxygen also. In the capillaries of lungs, kidneys, and skin, it most particularly loses waste-materials. It gains now nutritive material most particularly in the portal capillaries and by the inflow of lymph and chyle near the heart-receiving waste-products also in the latter case. But it is well to bear in mind the great rapidity of the circulation and the small percentage of the blood that is really affected in each capillary-journey: the variation in composition of the blood in different parts of the body is far smaller than this general statement of gains and losses suggests.

§ 16. The "Ductless Glands." Finally, we have to note a series of organs of very varied character, in whose capillaries the blood undergoes changes of an important but somewhat mysterious character. These are often collectively spoken of as the ductless glands, and several of them belong to the category of structures whose position is meaningless from the point of view of the rabbit alone, but receives surprising elucidation when a wide range of animals is studied, particularly as regards the embryonic development of these structures. Such are the thyroid gland 
(ventral to the larynx), the adrenals or supra-renals (anterior to each kidney, fig. 64) and the pineal and pituitary bodies of the brain. Removal of the two former (in surgical operations, e.g.) results in serious diseases, which can, however, be warded off to a large extent by injection into the blood of the secretions of the same glands of another animal. Hence they evidently add some necessary secretion to the blood. The spleen (lying near the stomach) and the thymus (ventral to the heart, and a large organ in young rabbits, but disappearing in adults), which are often included with the others, appear more specially concerned with the blood-corpuscles, as is also the red marrow of bones. The detailed consideration of all such organs is beyond our present purpose. 


\section{Chapier IV.-Respiration.}

$\S$ 1. Importance of Lungs. The double circulation, as we have seen, results in every blood-corpuscle passing throngh the lungs once in the course of a complete circulation. No other organs in the body are so favoured, for although the rapidity of circulation is so great, that in the course of a very short time there is ênough opportunity for a given corpuscle to try every possible route through the body in turn, yet there is no compulsion on it to do so. A corpuscle may do the whole circulation fifty times or five hundred times and never pass through the kidney, for example, but it cannot do it a single time without passing through the lung-capillaries. Evidently the lung must be an organ through which it is very needful for the blood to flow. Why this is so is obvious, when we consider the importance of a proper supply of oxygen. It is not unnatural, therefore, to take the lungs, rather than the heart, as the goal of the blood, and to apply separate terms to the blood that is travelling from the lung-capillaries to any other set of capillaries, and that travelling in the reverse direction. The former is often called arterial blood, the latter venous. These names are objectionable because they imply a wrong idea of relationship between two kinds of blood and two kinds of vessels. We have seen that the essential difference between arteries and veins is one of blood-pressure, not blood-composition. Thus the pulmonary artery contains "venous" blood, the pulmonary veins contain "arterial." To avoid these objections the terms "pure" and "impure" blood are often used, instead of arterial and venous, and the blood is said to be "purified" in the lungs. But these terms suggest too great a difference in the nature of the blood, for although "pure" blood does contain more oxygen and less carbon dioxide than "impure blood," it must not be supposed that all the carbon dioxide of blood is removed in the lungs: as a matter of fact only 
some 13 per cent. of it is removed. Besides, why should the term "purification" be restricted to one of the processes by which waste is removed? The removal of nitrogenous waste (urea, etc.) is no less needful than that of carbon dioxide, and from that point of view the purest blood in the body is that in the renal veins. The best terms to denote the difference between the blood travelling from the lungs and that travelling to the lungs are "oxygenated" for the former, and "deoxygenated" for the latter, since the taking in of oxygen to the blood is a function of the lung shared by no other organ (in the rabbit). Morcover, it is the relative amount of oxygen present which causes the difference in colour between "arterial" and "venous blood."

§ 2. Essential and Accessory Processes in Respiration. In the process of respiration we must distinguish essential and accessory processes. The essential process is the exchange of gases between the blood and the medium in which the animal lives-air in the case of the rabbit. This is a simple physical process, which takes place when the two fluids are separated by only a thin membrane. It must be clearly understood that no chemical processes go on in the lung: there is no oxidation: the oxidation by which carbon dioxide is formed goes on in the living tissues from which the blood has come.

The accessory or mechanical processes are those by which new supplies of air and of blood are brought to the respiratory organ. Part of this mechanism is of course that of the blood-circulation. The other part, concerned with the replacement of the air in the lungs, needs further description.

§ 3. Larynx and Trachea. The glottis leads into a long pipo lying ventral to the cesophagus and extending through the neck to the thorax. This is the trachea or windpipe, and the most anterior part is enlarged and modified in structure to form the lavynx, the organ of voiceof small importance in the rabbit. The wall of the trachea is strengthened and kept from collapsing by a series of cartilaginous rings, which are incomplete on the dorsal side 
where they would press on the œsophagus. In the larynx the cartilages are more complicated.

\$ 4. Structure of Lungs. On entering the thorax the trachea divides into two tubes-the right and left bronchi. These divide again - the right into four, the left into two,

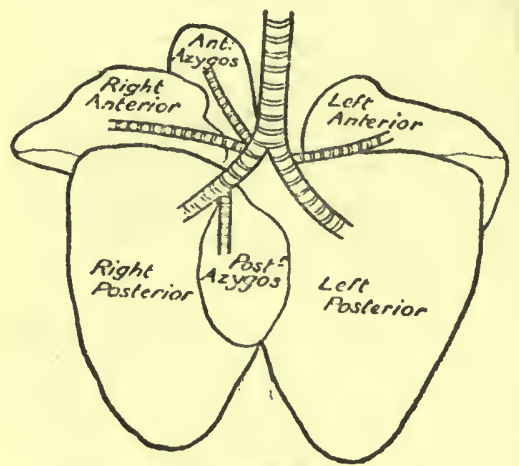

Fig. 5.-Lungs of Rabit. and on each of these six bronchi there is set one of the lobes of the lungs (fig. 5). If we could follow out each bronchus as it disappears into the lung-lobe, we should find it branching again and again into smaller and smaller branches, the bronchioles. Each final twig of this tree of tubes ends in a minute sac-an air-sac or alveolus -in whose thin walls lie the pulmonary capillaries. Here it is that blood and air, separated by only a thin membrane, exchange oxygen and carbon dioxide. The whole lung-lobe, solid as it at first looks, is little more than a mass of these air-sacs and tubes -as its extreme lightness and sponginess testify.

$\S 5$. Mechanism of Inspiration. To maintain a constant supply of oxygen to the blood the air must be continually pumped in and out of the lungs. The pumping in is called inspiration; the pumping out, expiration. In the rabbit this is effected in the following way. The walls of the thorax contain a series of ribs, and between these ribs are two series of muscles, the external and internal intercostal muscles, so arranged that when the former act,* they pull all the ribs forward (raise the ribs, in the case of man), while the latter have the opposite action. In inspiration the ribs are pulled forward : at the same time the muscular diaphragm (chap. i., § 9) pulls backwards, so that the convexity it presents to the thorax (fig. 1) is diminished. Both these changes enlarge the capacity of the thorax and so tend to

* The nature of muscular action is explained in chapter viii. 
produce a vacuum in the pleural cavities around the lungs But the walls of the air-sacs are highly elastic, and as the pressure outside them is diminished the pressure of the air inside causes them to expand, so that the lungs enlarge as rapidly as do the pleural cavities. As they do so the pressure of the air within diminishes, and if the air-passages are open, air rushes in from the outer atmosphere until the pressure is equalized. Its course is through the anterior or external nares (nostrils), through the nasal passage to the throat, and then through the glottis to larynx, trachea and lungs. The whole action of inspiration is essentially that of a suction pump.

$\S 6$. Expiration. In expiration the ribs are pulled back, and the diaphragm relaxes and is pressed forward by the abdominal viscera, which have been somewhat compressed during inspiration. Thus the pleural cavities are reduced in size and the excess of air is forced out again from the lungs by the same path as it entered. Moreover, the walls of the air-sacs are very elastic and themselves tend to return to a smaller size, as a simple experiment will show. When a rabbit dies under chloroform, its breathing always stops at the end of an inspiration. Hence its lungs remain distended. But if, after opening the abdominal cavity and exposing the posterior face of the diaphragm, you prick a hole on one side of this, air rushes at once into the pleural cavity of that side and the lung can be seen to collapse abruptly to a much smaller bulk. The lung of the other side does not collapse until that side of the diaphragm is pricked, because the two pleural chambers are perfectly separate. This is an experiment that any student can easily perform for himself. 


\section{Chapter V.-The Amoba and the Cell.}

§ 1. The Amoba. We have now seen, in a very general way, how the activities that constitute the life of the rabbit are rendered possible, and can go on to a more detailed study of the structure of the rabbit's body, and particularly of the microscopic structure, or histology of its tissues. Before doing so it will be an advantage to turn aside to another of our animal types - the simplest in structure on our list, as the rabbit is the most complex.

$A m o b a$ is the name given to a small creature that occur's in abundance in the mud at the bottom of fresh-water ponds. Like the rabbit, it is a vegetable-eater, but its mode of life is infinitely simple compared with that of the complex organism we have been studying. Examined under the microscope, the first thing that strikes one about $A$ moba is its irregularity of shape, and careful observation for a few minutes shows that the shape is continually changing - a characteristic which led the first workers at the microscope to name it "the Proteus animalcule." This is due to the fact that the substance of which it is composed - called protoplasm-is semi-fluid in nature. Careful examination shows that the protoplasm is not the same all throngh; there is an outer, clear, transparent layer called the ectoplasm and a central mass full of minute granules, the endoplasm. The constant change in shape is effected by the flowing of protoplasm towards certain points where, in consequence, projections appear, called pseudopodia. Of course the protoplasm that enters into them is withdrawn from somewhere else, and therefore if the formation of pseudopodia is mainly in one direction, the amoba must be changing its position as well as its shape: in this way locomotion is effected.

$\S 2$ Nutrition of Amœba. In the same mud in which we find amobæ, there are abundant microscopic plants 
(known as desmids and diatoms) which are almost as simplo in their own way as amœba is in its, but still distinctively plants, as amoba is distinctively an animal. When the protoplasm of an amoba comes in contact with one of these, it quickly flows round it, and completely encloses or ingests it. In a well-fed amœba, three or four or more of these plants may be seen-the recently ingested ones bright green in colour; others that have been longer enclosed are brown, and otherwise show signs of partial digestion. Not unfrequently, in watching a living amoba, we may witness the ejection of the indigestible residue of these plants from which the nutritive material has been extracted : " ejection," however, is too strong a word-we may rather say that the amoba flows off and abandons the useless residue.

§ 3. Nucleus and Contractile Vacuole. When an amoba does not contain much food in process of digestion, two other structures present in the endoplasm can be more easily seen. One of these is a spherical body, evidently firmer than the rest of the protoplasm : this is called the nucleus. The nucleus is a structure of the utmost importance to the amoeba - protoplasm from which the nucleus has been removed may remain

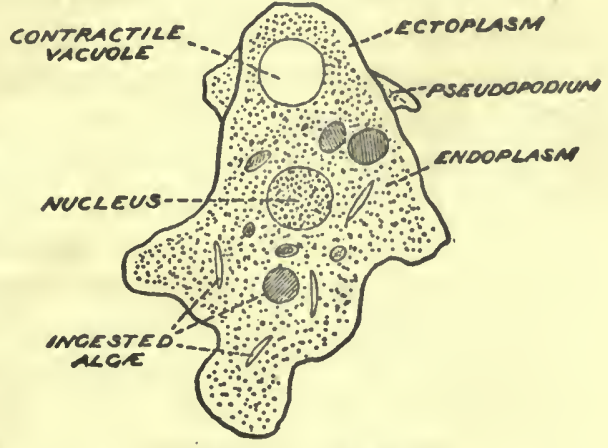

Fig. 6.-Амовв. alive for some time, but is incapable of carrying on the most essential vital functions.

The other structure seen is a spherical cavity in the protoplasm, containing some liquid. This slowly increases in size by the collection of more liquid in it, and then quite suddenly collapses with just the same appearance that is presented by a bladder full of water when it is pricked. Soon it reappears at the same spot and gradually grows 
in size as before until it again collapses. It is evident that some liquid is collected in it and periodically expelled from the protoplasm. Hence the contractile vacuole, as it is called, must be an excretory organ.

§ 4. Plant and Animal. Amœba and the desmids on which it feeds show us, in little, the whole relation of the animal and vegetable kingdoms. The desmid, too, consists of protoplasm with a nucleus, but it differs from amœba in two essential respects. Firstly, it has around it a distinct cell-wall, composed of cellulose, which gives it a definite and permanent shape, reduces its power of locomotion almost to nothing, and renders it incapable of ingesting solid food. Secondly, it has in its protoplasm a special portion which contains the compound called chlorophyll, which gives to it, as to other plants, its green colour. The first of these two features is the greatest distinction between all animals and all plants. The second distinguishes noost plants from most animals.

The pond-water in which our two organisms live contains in solution small quantities of salts, such as various nitrates, sulphates, phosphates and chlorides, and gases like carbon dioxide and oxygen. This water diffuses into the plantprotoplasm, and there the energy of sunlight, caught by means of the chlorophyll, builds up from these simple compounds complex molecules of carbo-hydrates and proteids, oxygen being set free in the process. These are finally elaborated into fresh protoplasm. Thus the plant-cell grows and multiplies. These complex products are utilized by the amœba as food: they are no doubt first broken down into somewhat simpler compounds in the process of digestion, but these are then built up into the highly complex protoplasm of the animal. At the same time every bit of work which the animal does-and the formation of a pseudopodium is work-involves the decomposition of some of the protoplasm into simpler compounds containing more oxygen. Thus the protoplasm of an amcba is constantly being increased by the anabolic process of foodassimilation, and decreased by the katabolic processes involved in all the work it performs. If the balance is on 
the side of katabolism, the amœba will dwindle and eventually die. If it is on the other side, it will grow in size.

§ 5. Reproduction of Amœba. Growth does not continue indefinitely; at some point an increase in numbers takes the place of increase in size: in other words, reproduction is effected. This process in amœba is very simple; first, the nucleus divides into two and the two move apart; then, gradually, the protoplasm becomes separated into two approximately equal parts, one around each nucleus. Thus we have two small amobre instead of one large one. Each one goes on feeding and growing and eventually divides again. The tendency of this process is to indefinitely over-populate the world with amœbæ; but it is held in check by the enormous number of accidents to which these creatures are liable. Thus the swallowing of a spoonful of pond-mud by a fish or duck might kill amœbæ by the hundred, and against such misadventures they are perfectly helpless.

§ 6. Encystment. The greatest of all dangers, however, is the drying-up of the pond. Against this danger the amœba has a slight protection; under certain conditions amobre assume a spherical shape, and cease forming pseudopodia. Presently a change appears at the surface of the protoplasm - a firm external membrane is formed, called a cyst. In this encysted condition the protoplasm is preserved from destruction by drought, for the cyst prevents evaporation of the water which forms so large a portion of protoplasm. When the pond is again filled with water, some of the encysted amœbr may still be among the dried mud : their cysts will be burst, and the protoplasm will flow out again and resume its ordinary mode of life. But while the mud is dry, the wind blowing over it will carry particles away as "dust," and among these will probably be some encysted amœbæ which may thus be blown through the air-some, doubtless, to perish, but some to reach other ponds and begin life again there. In this way among others, amœbo (like many other minute 
fresh-water animals) come to be so universally distributed. The power of encystment affords them a means of distribution.

The formation of a cyst also affords an example of the process known as secretion. The cyst is not protoplasm, but it is formed from protoplasm, and there can be no doubt that it is formed by the modification of the katabolism of the protoplasm. This is the only plain case, in amœeba, in which a product of katabolism serves a useful purpose.

$\S 7$. Cells. The body of amcela consists of a single cell. This term (which is far from self-explanatory and originated in quite a mistaken idea) is applied to any mass of protoplasm containing a nucleus (or the essential chemical substance of a nucleus). All living beings are primarily composed of cells. Some consist of one cell only, as amoba ; but all the higher animals are built up of many cells, and not of cells only, but of cell-products as well. Protoplasm must not be thought of as a definite chemical compound : it is rather to be compared to the molten mass in a furnace, into which ore, flux and fuel are being thrown while metal and slag are being withdrawn. We can, however, recognize its general constitution. Water forms more than half of it by weight; the rest consists mainly of various proteids, while other organic compounds and inorganic salts are present in smaller quantities. It must be remembered that protoplasm has always mixed in with it compounds that are in process of assimilation (anastases), and compounds resulting from its katabolism (katastases). No chemical analysis can be made of protoplasm without including these.

The nucleus is usually spherical in shape. It is less fluid than the rest of the protoplasm, containing less water and more proteid, but the chief chemical difference is the presence of a substance containing a large proportion of phosphorus. One peculiarity results from this chemical difference, which is of great advantage in microscopic study: the nucleus takes up staining reagents (carmine, hæmatoxylin, etc.) to a much greater extent than the protoplasm.

The only way in which protoplasm is known to be pro- 
duced is by the growth of pre-existing protoplasm through assimilation of food-material. The only way in which a new cell is known to be produced is by the division of a pre-existing cell, or (more rarely) the fusion of two preexisting cells into one; and in all such processes of celldivision and fusion the nucleus plays an important part. The only ways in which a new nucleus is ever known to be produced are the division of a pre-existing nucleus, and (more rarely) the fusion of two pre-existing nuclei.

§ 8. Structure of Protoplasm. All protoplasm, when examined under the highest powers of the mieroscope, is found to consist of (1) a rather less fluid portion, the spongioplasm, arranged in a complicated network; (2) a more fluid portion, the hyaloplasm, whieh oecupies the interstices of the spongioplasm just as water may fill the interstices of a common sponge; (3) granules of various kinds, in the hyaloplasm. From the faets that, in some cells the granules diminish in number when the cell is starved, while in secreting cells they appear in great abundance when the seeretion is about to be formed, it seems evident that the granules are not really part of the protoplasm, but either anastases, which are on the way to become protoplasm, or liatastases, which have been formed by the decomposition of the protoplasm.

§ 9. Structure of the Nucleus. The nueleus under ordinary conditions is surrounded by an exceedingly delicate film-the muclear membrane (fig. 7, A). The substanee within this resembles the protoplasm outside, in eonsisting of a less fluid network with more fluid substance in its interstices; hut the threads of the network are thicker, and they stain deeply when treated with earmine or other coloured reagents. Certain rounded bodies lying within the nucleus also stain deeply: these are called nucleoli. The differenee in the action of staining reagents on the several parts proves a ehemical difference between them: it is therefore convenient to speak of the meshwork and the nueleoli collectively as the chromatin of the nueleus; while the nuclear membrane and more fluid central mass is ealled the achromatin.

$\S 10$. The Centrosphere is a minute body stated to be always present in a cell just outside the nuclear membrane. It is espeeially conspicuous during the processes of division of the nucleus and eell, and there is some doubt as to whether it really exists at other times. The innermost portion of the centrosphere is distinguished as the centrosome.

$\S 11$. Karyokinesis. Division of the nucleus always precedes division of the eell: the separation from the cell of part of the protoplasm without a nucleus is not true cell-division, and the 
non-nucleated part soon dies. Sometimes the nucleus divides in a perfectly direct manner, just as a lump of putty or dough might be divided into two by nipping it round the middle with a piece of string; but this is rare, and is characteristic of degenerating cells. More usually a very complex series of changes takes place in

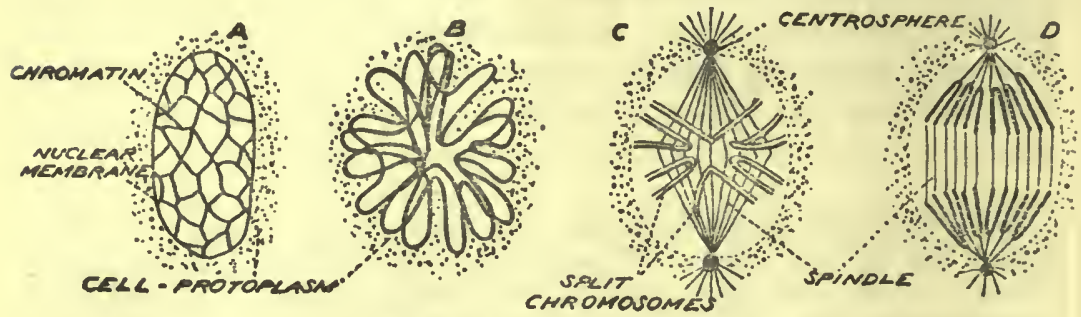

Fig. 7.-KARYOKINESIS.

the chromatin-meshwork, of which the following is a general description. The first change is said to be the livision of the centrosphere into two. The meshwork, which was irregularly tangled, takes on the form of a simple thread folded in a series of loops (the number of loops being apparently constant for all the cells of a particular animal species, but varying in different species) (fig. 7, $\mathrm{B}$ ).

The loops then become separated from one another, forming a series of U- or V-shaped structures (chromosomes), which arrange themselves in the centre of the nucleus in a radiating manner, the free double ends pointing outwards (Star or Aster stage). At this stage, the nuclear membrane disappears, the rest of the achromatin mingling with the hyaloplasm; in this mingled portion a fine series of threads (fibrils) appear, radiating out from the two centrospheres (which have moved to opposite sides of the chromatinrosette), and forming the spindile (fig. 7, c). Each chromosome now splits into two, longitudinally, and the two halves, each still $V$-shaped, separate from one another, and travel along the fibrils of the spindle towards opposite centrospheres (fig. 7, D). Thus in place of one radiating set of chromosomes we have two sets (Diaster stage). From this point onwards we really have two nuclei, and the changes undergone in each one are, in reverse order, the same that the single nucleus went through at the beginning.

It is impossible in our present state of knowledge to give any adequate explanation of this complicated series of changes. But the essential feature of the process is that nuclear division is not a rough cutting in half, but a careful division of every part of the chromatin of the nucleus into two equal parts.

§ 12. Differentiation of Cells. Animals which, like amoba, consist of one cell only (or of a few cells all equally 
self-sufficing for full life) are called protozoa. All other animals are called metazoa - the rabbit is an instance-and their bodies are built up of cells and cell-products arranged to form tissues. In such a body, each cell is not self-sufficing, but all are mutually inter-dependent. They may be compared to the different members of a human community, carrying on different trades and professions. In other words, there is a differentiation of function or physiological differentiation. And correlated with this is a differentiation of form and structure or histological differentiation.

$\S 13$. Tissue Metabolism. Every cell in every tissue performs-in a greater or less degree, according to its relatively active or passive character-the characteristic functions of assimilation, respiration and excretion, or in other words anabolism and katabolism, as does an amœba; but with certain important differences. Firstly, the food brought to it by the blood has already undergone the processes of digestion, etc., which bring it to a soluble condition, and only the final stages of anabolism take place in the individual cell. Secondly, all katabolism involves two things-a conversion of the potential energy of protoplasm into some form of kinetic energy, and the production from thesubstance of the protoplasm of simpler (and, usually, more oxidized) compounds. But in some cells it is the conversion of energy (i.e. the doing of some work) which is the primary function of the cell, and the material products are mere waste-e.g. muscle and nerve cells. In other cases it is the formation of definite products that is the main function of the cell, the energy set free taking probably the form of heat and being wasted-e.g. connective tissue-cells. Lastly, some cells seem to have a purely passive function, and their rate of metabolism must be extremely slow.

The products of katabolism that are nearest to protoplasm in composition and whose production involves the least degree of katabolism are mostly those that are retained to form part of the tissues themselves. These may be termed plastic products : when retained within the protoplasm of the cell they are termed endoplastic, when deposited outside 
and around it they are ectoplastic. A greater degree of katabolism usually accompanies the formation of those secretions (like the digestive juices) which are useful to the animal but do not form part of its tissues. The greatest amount of katabolism generally results in the production of those useless or waste compounds, the excretions (chap. i., § 13). These statements must not, however, be taken as rigid and without exception; they merely express a rough general rule, which may be put in diagram form thus:-

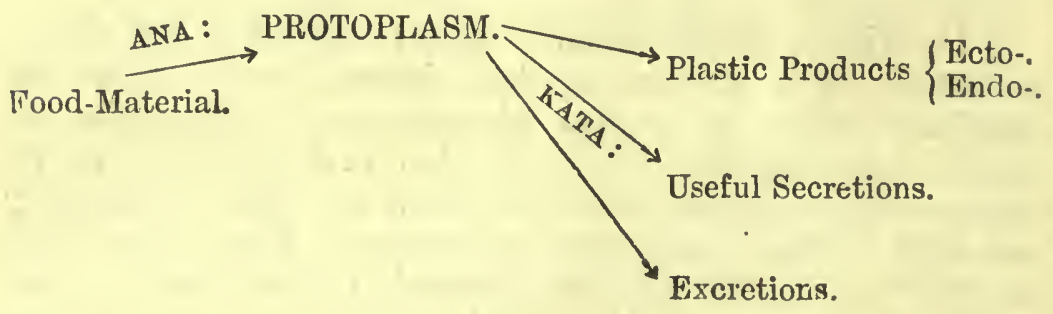




\section{Chapter VI.-The Tissues.}

\$ 1. Classification of Tissues. The tissues of the rabbit's body can be arranged in four groups-(1) Epithelial ; (2) Connective; (3) Muscular; and (4) Nervous. Of these, the last two are mainly concerned in doing work, and in them, therefore, katabolism is most active; the cells of the second are mainly concerned in forming cell-products, especially plastic products; while the epithelial

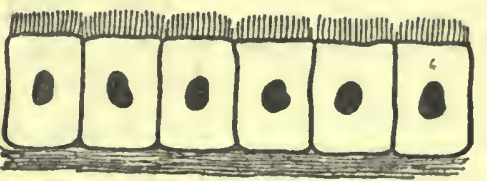

Fig. 8.-Ciliated Columak EPITHELIUM,

Section perpendicular to free surface. cells are most concerned with secretion, though they may do mechanical work (ciliated epithelium), or be purely passive (endothelium).

§ 2. Epithelial Tissues consist entirely or almost entirely of cells, a small amount of ectoplastic material comenting the cells firmly together. Epithelial tissues do not usually attain any great thickness, but form extensive thin sheets, often consisting of one layer of cells only, and abutting on some space. Thus the epidermis covering the surface of the
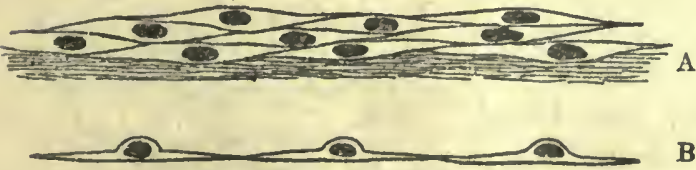

Fig. 9.-SQUamous EPITHELIUM.

A, Ordinary Overlapping; B, Pavement. Sections perpondicular to free surface. Diagrammatic. body, the lining of the alimentary canal, the lining of the body cavity, B of the blood-vessels, of all ducts and tubes-all these are epithelia.

According to the form of the constituent cells we may roughly classify epithelia into squamous and columnar. In the former the cells are flattened, their flat surfaces being parallel to the surface of the epithelium (fig. 9); in the latter the cells are lengthened in a direction 
perpendicular to the surface of the epithelium (figs. 8, 12).

We may also distinguish simple epithelia (consisting of

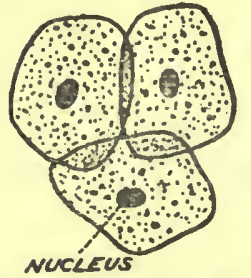

Fig. 10.-SQUAMOUS EPITHELIUM.

Surface view. one layer of cells only), and compound epithelia (consisting of many layers).

This does not by any means exhaust the varieties of epithelium, but is merely a rough provisional classification, sufficient for our present purpose.

One kind of squamous epithelium, in which the thin ends of one cell overlap those of others, can easily be obtained from the inside of the human cheek by gentle scraping with the handle of a scalpel. If the material so obtained is mounted in a drop of water and examined under the microscope, the cells can easily be seen (fig. 10). The addition of a little acetic acid makes the nucleus very plain, and carmine or magenta will stain it deeply.

$\S 3$. Endothelium. In another kind of squamous cpithelium there is only one layer of cells and they are exceedingly flat and thin, the nucleus making a little bulge ; the edges of the cells do not cverlap, but meet like the tiles in a mosaic pavement (figs. $9 \mathrm{~B}, 11$ ). Hence this kind of epithelium is often called pavement-epithelium: it is called endothelium when it lines closed cavities, as very frequently it does.

The colom and all the bloodvessels are lined by endotheliumthe walls of capillaries consist of nothing but endothelium, those of veins and arteries have, in addition,

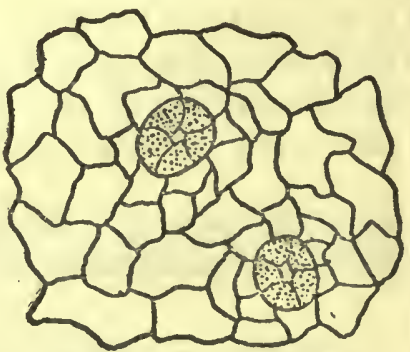

Fig. 11.-ENDOTHELIUM.

Surface view. From peritoneun. Two lymph-stomata are shown (see p. 26.) coats of connective and muscular tissue around this. The alveoli of the lungs are also lined by pavement-epithelium, but as they are not closed cavities, it is not called endothelium. 
commonly either ciliated or secretory. The protoplasm of the free surface of each cell of a ciliated epithelium is produced out into one or (more usually) many delicate processes, called cilia, which during life are constantly performing movements of a very definite and regular kind. Each cilium becomes bent and then straightens itself out again : the motion is either simultaneous for all the cilia or takes place in advancing succession, and is so rapid (under ordinary circumstances) that only the motion can bo seen, and not the cilia themselves. When the cilia are dying, or under other exceptional conditions, the motion is slow and its exact nature can be observed. The effect is that of a series of oars being pulled as in rowing: if the boat is fixed they will produce a current in the water; if not they will make the boat move. Similarly, the effect of the activity of ciliated epithelium is normally to sweep fluid in a definite direction along its surface, but an isolated ciliated cell will be whirled round and round by the action of its own cilia. Cilia, as we shall see, are of great importance among the lower animals, though of only subordinate importance in the rabbit. A cilium may be regarded as a very fine pseudopodium whose structure is such that it can perform only one movement, but can perform that with a rapidity unknown to the ordinary pseudopodium. Ciliated epithelium is found in the rabbit principally in the trachea and bronchi. Other cases will be mentioned in later chapters.

\section{$\S$ 5. Secretory Epitho-} lium. While the function of cilicted epithelium is the doing of a particular kind of mechanical work,

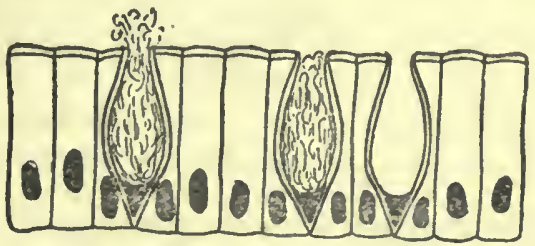

Fig. 12.-SeCretory Columarar EPITHELIUM.

Section perpendicular to free surface Three goblet-cells shown. that of secretory epithelium is the production of some definite substance, either as a result of its metabolism, or by the separation of it from the blood-plasma. It is by epithelia of this kind that all the digestive juices are secreted, and water and urea removed from the blood. zOOL. 
Sometimes the secretion is set free as quickly as it is formed ; in other cases it accumulates in the secreting cell, until at last it escapes as a large drop. A cell discharging a drop in this way is termed a "goblet-cell" (fig. 12). We shall return to this form of epithelium a little farther on ( $\$ 17)$.

§6. The Epidermis (outer portion of the skin) is a many-layered epithelium, the deepest layer consisting of columnar cells which, as they grow in size, are constantly

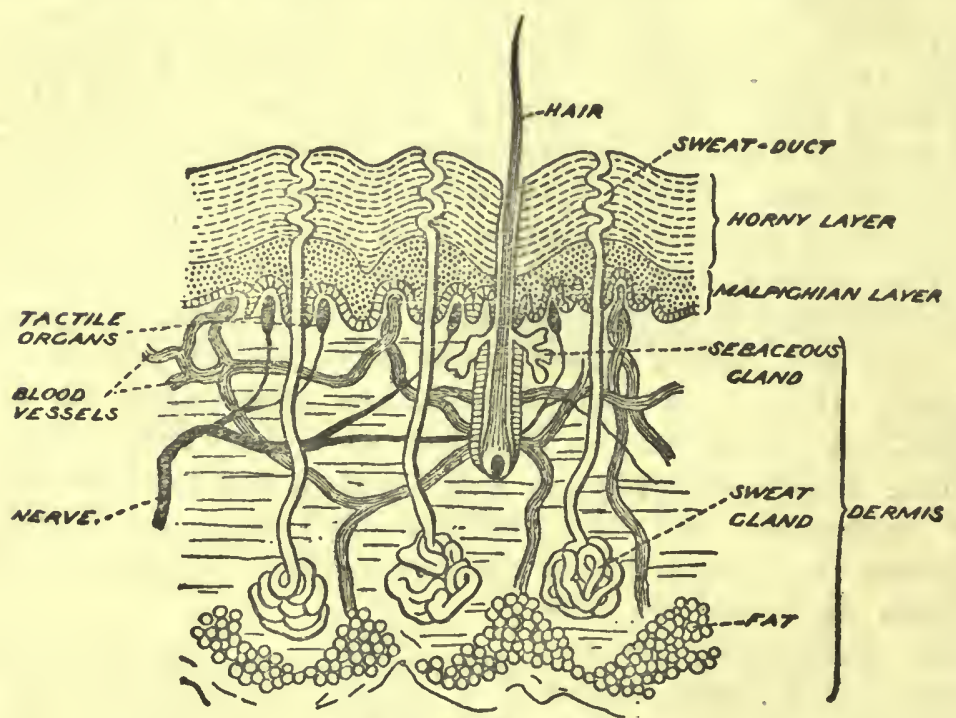

Fig. 13.-Mammalian Skin.

Diagrammatic section. (After Wiedersheim.)

undergoing division, cutting off smaller, flatter cells on their outer side, to form the superficial layers As these flatter cells become pushed nearer and nearer the surface their protoplasm undergoes change into a substance called keratin or horn, and eventually becomes rubbed off the surface. The innermost columnar layer and those above it in which the cells are still protoplasmic, constitute the Malpighian layer, or stratum mucosum: the outer ones form the horny layer or stratum corneum (fig. 13). Hairs and nails are specially developed portions of the horny layers of the epidermis. 
All epithelial tissues are characterized by the fact that no blood-capillaries occur in them. This might be expccted in tissues composed only of cells. But as the blocd must be brought near to these epithelia we always find at the base of an epithelium a layer of connective tissue containing abundant blood - capillaries. In the case of the skin, this layer is the dermis: epidermis and dermis together constitute the skin.

\section{$\S$ 7. Connective} Tissues. In the con-

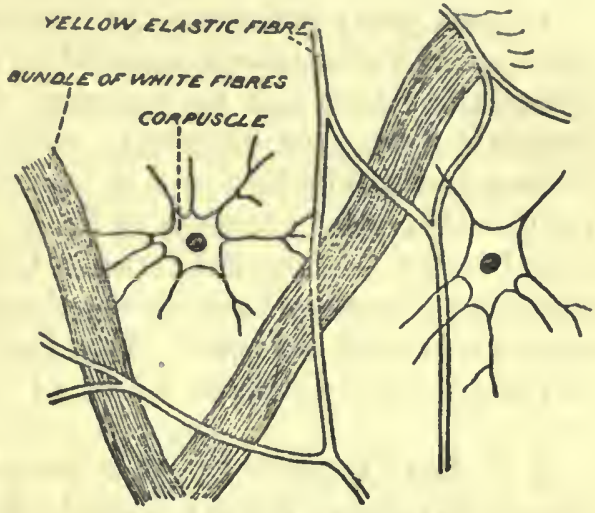

Fig. 14.-Areolar Tissue.

nective tissues, we find that both cells and cell-products are present, the latter often forming the great bulk of the tissue. Most usually the cell-products are ectoplastic. In this case it is customary to call the cells by the old name of corpuscles, and the cell-products, the matrix.

Areolar tissue (or ordinary connective tissue) consists of cells (connective tissue-corpuscles), usually of irregular shape, ${ }^{*}$ and running out into fine threads, separated from one another by a soft, jelly-like matrix, consisting mainly of the substance gelatin (a compound allied to the proteids). Running through this matrix are a quantity of fine fibres, formerly thought to be altered cells, but now known to be formed along with the matrix. These fibres are of two kinds-yellow fibres which branch and are highly elastic, and white fibres which occur in bundles, do not branch, and are inelastic. The presence of these fibres adds strength and elasticity to the delicate matrix. Areolar tissue such as we have described is found surrounding and holding together most of the organs in the body: the process of

* Hence often said to be "amœboid"; but that term is more properly used to indicate that a cell moves like an amœba, which is not the case here. 
dissection consists to a large extent in the cutting and tearing of the connective tissue that binds the organs together. A very good example of areolar tissue may be found under the skin of therabbit, especially in the region of the armpits.

In particular positions the connective tissue is found to have special characters adapted to its circumstances. Thus where the tissue has to transmit a strong pull, the white inelastic fibres are especially abundant, the tissue becomes strong and unyielding and is called a tendon. Tendons most frequently connect muscle to bone. On the other hand, if it is subjected to frequent strains, the yellow elastic fibres are abundant, and we have elastic tissue, as at many joints and in the walls of arteries. The form of the corpuscles also varies in these different kinds of connective tissue.

\$ 8. Cartilage, In this we also have cells (cartilagecorpuscles) and a matrix, but the latter contains, instead of gelatin, a substance of similar chemical composition called chondrin, which is far firmer and more elastic. Hence there are usually no fibres in cartilage, which is then called hyaline cartilage from its translucency (e.g. the cartilage at the ends of the limb-bones or that of the trachea). When

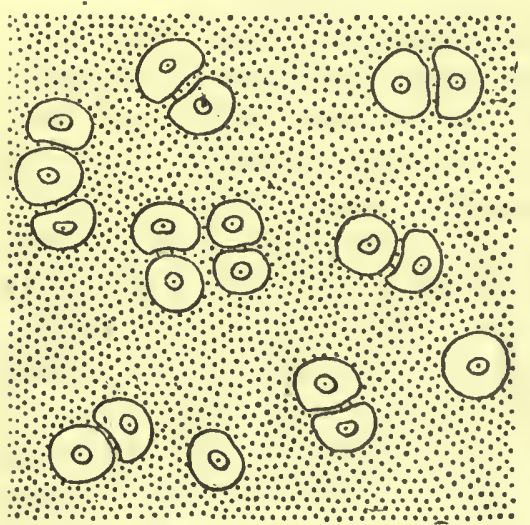

Fig 15.-Cartilage. fibres, either white or yellow, are present, we have fibro-cartilage (as e.g. in the intervertebral bodies [chap. vii., § 3] or some of the cartilages of the larynx). Another apparent peculiarity of chondrin is its ready permeability to liquids : no blood-vessels of any kind are found in the matrix of cartilage, even. when it is an inch or so thick. Yet it is obvious that the cartilagecorpuscles are not cut off from nourishment, since they are found, not only living and healthy, but often actively growing and dividing (see fig. 15). 
Around the margin of any mass of cartilage is a layer of connective-tissue called perichondrium. This contains many white fibres. Cartilage is a comparatively unimportant tissue in the full-grown rabbit. To see it in its greatest development we must go to such a type as the dogfish. Here it forms the whole skeleton, affording the comparatively rigid basis for the attachment of muscles, without which rapidity and accuracy of motion would not be possible. In the rabbit, cartilage forms the main skeleton at an early stage of its development, but it is afterwards replaced by the more efficient tissue called bone.

$\S 9$. Bone differs from cartilage primarily in the composition of the matrix-it contains not chondrin but gelatin and this is impregnated with various inorganic salts, of which calcium phosphate is the chief ; calcium carbonate and fluoride, and magnesium phosphate, being also present. 'This matrix is not readily permeable to the blood-plasma, and hence wo

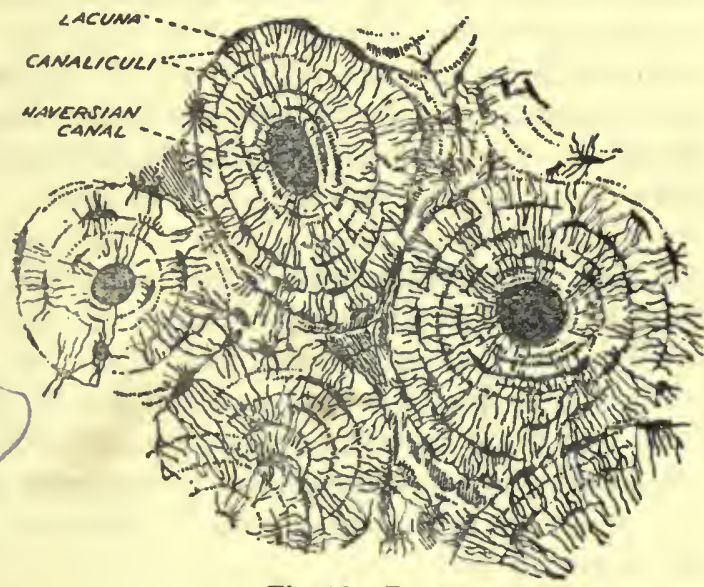

Fig. 16.-BoNe.

Transverse section.

find a complex arrangement for bringing the blood to all parts of the bone. If we examine such a bone as the femur (thigh-bone), we find it to be, roughly, a hollow cylinder-the hollowness diminishing the weight without lessening the strength. Running, for the most part longitudinally, through the bone there are a great number of canals known as Haversian canals, ench of which contains during life an artery and a vein. These are branches of a main artery and vein that pierce the shaft at one 
point. The bone-corpuscles or cells (also called osteoblasts) are arranged in concentric cylinders around each Haversian canal. Each is a much-branched cell : in sections of dead, dried bones, the places occupied by the bone-corpuscles are seen as little cavities, to which the name of lacunæ was given long before their meaning was understood. The lacunæ are connected together by very fine canais, the canaliculi, by means of which the plasma exuded from the Haversian vessels can reach all the bone-corpuscles.

Forming a close sheath round the bone is a membrane, the periosteum, the outer layers of which are of the nature of ordinary connective tissue, while the inner ones consist of closely packed cells.

The rigidity of the matrix prevents bone from growing, as cartilage does, by cell-division and intercalation of new matrix-material. Growth of a bone is partly effected on the outside by the activity of the inner layer of periosteum just mentioned. But there are more complicated arrangements at work, which will be better understood after we have described the development of bone.

$\S 10$. Membrane-bone. The simplest mode of origin of bone is found in the case of most of the flat bones of the skull. In the embryo rabbit there exists in the region where these bones will appear a kind of connective tissue with but little matrix. The corpuscles of this tissue after a time begin to secrete calcium phosphate as well as collagen, and bone is thus produced. Such a bone is called a membrane-bone.

\$ 11. Cartilage-bone. In the case of the majority of the rabbit's bones-many of those of the skull, all the vertebrae, ribs, limbs, and limb-girdles - a much more complicated process occurs. These bones are called, by distinction from membrane-bones, cartilage-bones. Each one of these bones has preceding it in the same position in the embryo a mass of cartilage of the same shape, surrounded by a "perichondrium" resembling the tissue described in the previous paragraph. After a time both the cartilage-corpuscles and the corpuscles of the perichondrium begin to deposit calcium 
phosphate in their matrix; but while the latter become true bone-corpuscles, with canaliculi to nourish them, the former by this secretion of phosphate shut themselves off gradually from nutrition. At this point we have a mass of calcified cartilage surrounded by a thin layer of true bone. Next comes a very remarkable change: a great number of very large cells from the perichondrium break through the thin layer of bone and literally eat their way into the calcified cartilage. These devouring cells are called osteoclasts ; accompanying or following them are other cells which are ordinary bone-corpuscles or osteoblasts, and these begin to form true bone among the ruins of the calcified cartilage. Blood-vessels also grow in along with the osteoblasts. Thus cartilage-bone differs from membrane-bone, in the fact that its formation is accompanied by a migration into a region previously occupied by cartilage. There is no change of cartilage into bone: the original cartilage-matrix and corpuscles-is entirely eaten up, except certain portions to be immediately mentioned, which persist as unaltered cartilage.

These persistently cartilaginous regions of a cartilagebone are. firstly, the two ends in the case of bones which are movably jointed or articulated to other bones, as are the limb-bones. Secondly, in many bones the immigration of osteoclasts and osteoblasts takes place at more than one point-usually at three, one in the middle and one at each end. These two end-regions of ossification (i.e. bone-formation) are much smaller than the main central one and are called the epiphyses of the bone. Between the epiphyses and the main ossification a narrow band of cartilage persists, and the presence of this makes possible the growth of the whole bone in length. Bone-tissue can easily grow in thickness, by additions from the cellular layers of the periosteum, but since the ends usually have a definite form of their own, growth in length cannot take place by mere additions of bone at the two ends-there must be intercalation of new material somewhere between. The actual growth in length is effected by the persistent cartilage, and in proportion as this increases its substance the osteoclasts, like rack-renting landlords, absorb the extra amount and 
so keep the cartilage small in amount in spite of its continued increase. Eventually, when no more growth in length is necessary, the whole of the cartilage is absorbed, and the epiphyses unite with the main bone.

The work of osteoclasts does not end with the disappearance of cartilage from any region of the bone. The first-

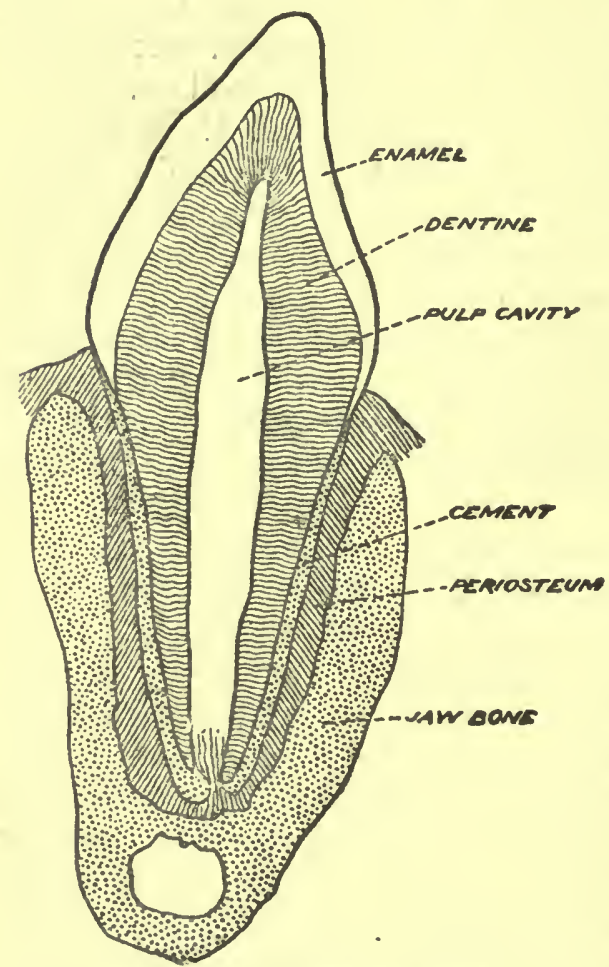

Fig. 17.-STRUcture of Tooth. (After Klein.) formed bone is in its turn absorbed to a very great extent, the marrow-cavity being thus formed. At first, too, the osteoblasts and their matrix are all arranged in one series of concentric circles (or rather cylinders); this primitive bone around the marrow-cavity is in its turn partly absorbed, and a new series of minor cavities-the Haversian canals-excavated in it, around which new osteoblasts are concentrically a ranged. In membrane-bones, also, the Haversian canals are similarly formed. Thus bones, in spite of their apparent stony character, and indeed by reason of their want of plasticity, are subject to a constant reconstruction which cartilage has no need of.

$\S 12$. The Teeth of the rabbit are of complex structure(see fig. 17) two tissues, and a secretion that is hardly a tissue, forming them. The secretion is enamel, an extremely hard substance (composed of the same compounds as bone, but 
with very little organic matter) that forms the capping of the teeth. The main portion of the tooth is formed of dentine, a tissue resembling ibone in composition, but differing from it in the fact that the active cells (odontoblasts) form a continuous layer, and deposit their secretion at their outer ends only, so that they never become embedded in their matrix as do osteoblasts. Within the dentine is a "pulpcavity," resembling the marrow-cavity of bones and containing connective tissue with abundant blood-vessels and nerve-fibres. In the incisor teeth of the rabbit this pulp-cavity persists throughout life, so that the odontoblasts continue to receive nourishment and to secrete, and the tooth goes on growing in length throughout life. The cheek-teeth, however (and the incisor teeth of such animals as the dog) have the opening of their pulp-cavity gradually constricted at the base as the tooth grows, and finally closed in, so that the odontoblasts are cut off from their foodsupply, and the tooth ceases to grow.

The third component of teeth is the "cement" at the base: this is true bone.

The development of teeth will be more conveniently considered at a later stage.

§ 13. Blood. The student will be surprised to hear blood considered among the "connective tissues." It is, however, strictly a liquid tissue, though the plasma does not agree with the ordinary matrix of a tissue in being entirely a secretion of the corpuscles. The identity of the white blood-corpuscles with connective-tissue corpuscles must have already been realized by the student. The red corpuscles, however, lack a nucleus, and are therefore not cells : in accordance with that deficiency, they have no power of growth or division, and their existence is not indefinite in length, but comes to an end after a short (but unknown) period. Old red corpuscles are constantly being destroyed and new ones formed : the spleen appears to be the region in which the dying ones are retained, and it would seem that the hæmoglobin set free from them is carried on to the liver, and there goes to form the bile-pigment bilirubin. New red corpusles are formed mainly in the red marrow 
found in the marrow-cavities of certain bones, particularly the ribs. The yellow marrow of other bones may also possibly give rise to red corpuscles, but this tissue consists very largely of $f a t$ (see below).

The white corpuscles, in spite of their power of growth and division, must also die off, for new ones are constantly being added to the blood, without causing any net increase in their numbers. These white corpuscles are mainly developed by division of the cells of those "lymphatic glands" already mentioned as occurring here and there on the course of the lymphatics. These structures are not glands at all, in the strict sense of the term : they are inasses of lymphoid or adenoid tissue, which resembles connective tissue, but has a much more fluid matrix with delicate fibres forming a network. In the interstices of the network are abundant actively-dividing corpuscles. Lymphoid tissue is the chief constituent of Peyer's patches, and the walls of the sacculus rotundus, and appendix vermiformis.

$\S 14$. Adipose Tissue. In all the tissues mentioned so far the cell-products are ectoplastic. As a common example of endoplastic products we may mention fat. Under certain conditions the corpuscles of connective-tissue secrete fat, which appears as globules in the protoplasm (fig. 18). As

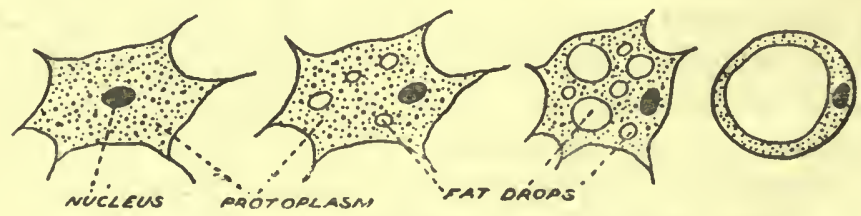

Fig. 18.-Development of a Fat-cell.

the amount secreted increases, these globules unite into larger and larger drops, until finally we have a thin bladder, as it were, of protoplasm bulged out by a huge fat-drop. Connective tissue, in which most of the cells are thus fat-laden, is called adipose tissue. This is abundant under the skin of a well-fed rabbit, and also around the kidneys.

§ 15. Notochordal Tissue. Another and more striking example is afforded by notocho?dal tissue - a tissue practically 
lost in the adult rabbit, but well developed at an early stage in the embryo. Here the bladder of protoplasm of each cell encloses as much of a watery liquid as it can hold. We all know how firm a bladder feels when fully distended with air or water-this firmness is not a property of the material of the bladder, for that is soft and flexible when the fluid inside has escaped. Exactly the same is the case with a green stem or leaf of a plant. When dry it is limp and droops, though normally it is firm and elastic. In the same way notochordal tissue, consisting entirely of cells, each of which is a bladder full of liquid, is firm and elastic, and has much the same consistency as cartilage. As we shall see later on, notochordal tissue precedes cartilage as a firm basis for certain muscles to be attached to, much in the same way that cartilage precedes bone.

$\S 16$. Tissue-Complexes. The two remaining groups of tissues-muscular and nervous-we shall deal with in future chapters. Before ending this one we must say a word on tissue-complexes.

Very fow of the simple tissues we have been describing form actual organs by themselves alone. We saw (\$6) that epithelium contains no blood-capillaries or lymphatics, but receives its nutrition by the exudation of plasma from the capillaries of the subjacent layer of connective tissue. The only exceptions are perhaps some endothelia, especially those of capillaries. The connexion between an epithelium and its underlying connective tissue is a very close one, and often they form a single anatomical structure, separable into two parts only by microscopical examination. Thus the epidermis and the underlying connective tissue, the dev"nis, together form the skin; the lining epithelium of the alimentary canal and its connective tissue form the so-called "mucous membrane"; the endothelium of the colom and the associated fibrous connective tissue form the peritoneum, mesentery, pleura and pericardium.

$\S 17$. Glands. Still more is this the case when we come to the glands: here the epithelium is secretory, and so 


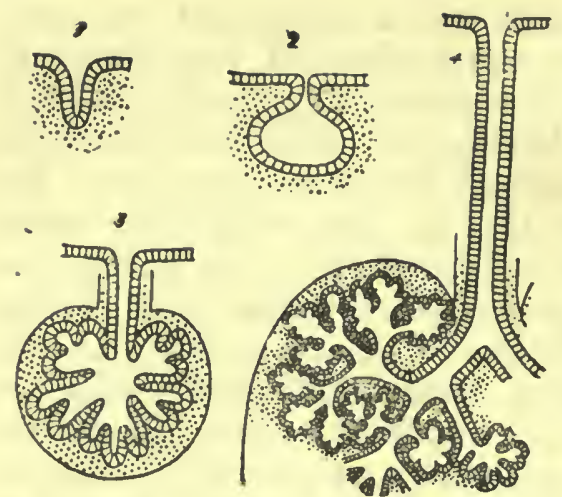

is in special noed of a continual supply of plasma, and hence the two tissues - secretory epithelium and vascular connective tissue-are in a close physiological connexion. It is often needful that the liquid secreted by a given area

Fig. 19.-Structure of Glands.

1, simple pit ; 2, flask-shaped gland with show duct; 3,4 , more complex compact glands. Vascular tissue dotted. (After Huxloy.)

of epithelium should be concentrated in a smaller area: this is effected by the double tissue being thrown into folds of some kind. It is by this process of folding and branching that the complex glands of the body are

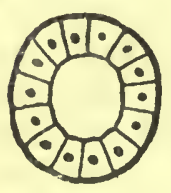

Fig. 21.

Tranguerse SECTION OF

SIMPLE

TUBULAR

GIAND. produced. Fig. 19 gives a diagrammatic representation of the successive stages of complication.

As a gland becomes more and more complexly branched, the tubular portion near the opening may become drawn out into a duct of some length, whose epithelium is no longer secretory.

The sweat-glands (fig. 13)

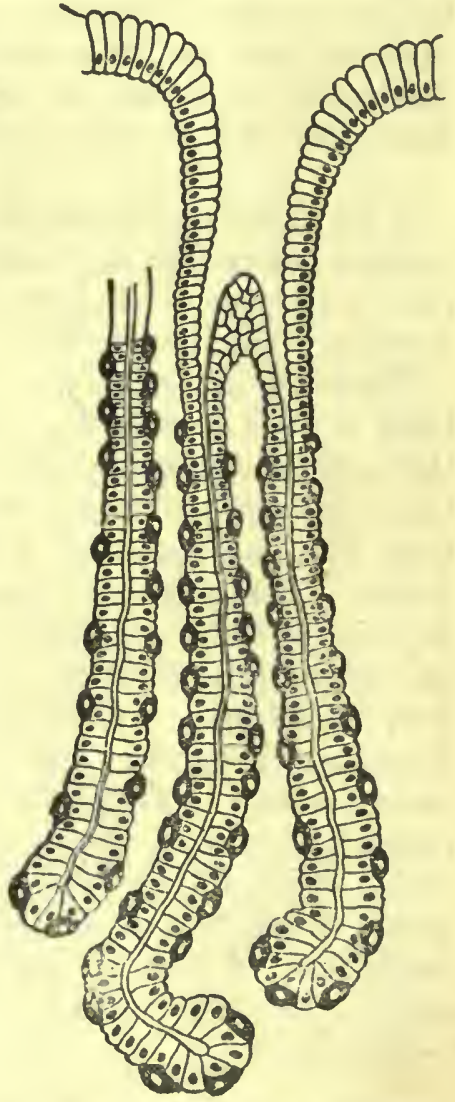

Fig. 20.-Stmple Grands in Wall of Stomich.

(After Klein.) 
are examples of a very simple type of gland; a tube, not branched, but coiled up into a little bunch at the end.

- 'The rabbit's pancreas is a good examplo of a diffuse gland, the various acini or dilated endings being scattered irregularly through the mesentery that joins the two limbs of the duodenum. 'The salivary-glands, on the other hand, are compact, that is apparently solid masses, though really consisting of just the same parts as the pancreas.

$\S$ 18. The Liver is the most compact gland in the body. It consists (fig. 22) of five lobes, from each of which a duct comes to join the others and form the bile-duct. There is also a blind outgrowth, the gallbladder, which serves as a reservoir for the bile and has its own duct, the cystic duct, joining the others. At ordinary times, bile secreted in the lobes of the liver passes down the minor ducts and then up into the gallbladder; but when food onters the duodenum the stored bile quickly flows out.

If we cut across any one of the lobes of the liver and examine its

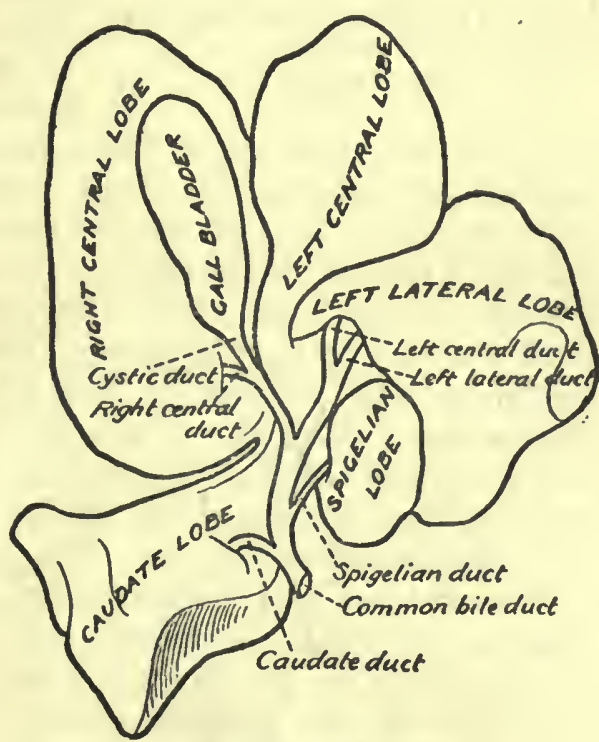

Fig. 22,-LIVER of RABBIT. cut surface, we easily see that it is made up of a vast number of small polyhedral masses, closely compacted together, about $\frac{1}{2} \delta$ of an inch in diameter. These are the lobules, and they constitute the essential part of the liver, though of course, here and there between them we see branches of the bile-duct, of the portal vein, and of the hepatic artery and factors of the hepatic vein.

If we examine a thin section of any part of the liver 
that has been suitably stained, under the microscope, we shall see that each lobule appears at first sight to bo made up of a mass of nucleated cells, the liver-cells, without any

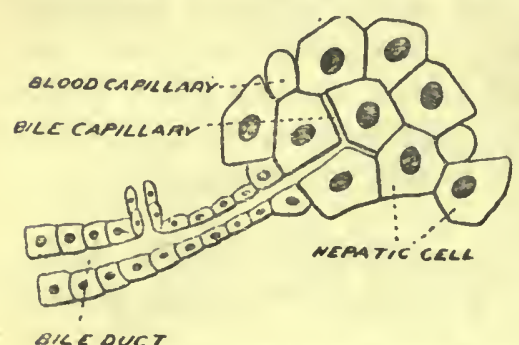

Fig. 23.-Diagram of Structure of LIVER.

(After Iuxley.)

large intercellular spaces. More careful examination shows that very small spaces occur between some of the cells-spaces much smaller than the cells themselves. It is possible to prove that these are really the ultimate bianches of the bile-ducts. This is done by the method of injection: some coloured liquid is forced by a pump into the bile-duct of a fresh liver, and on cutting thin sections the colour is found to fill these tiny spaces between the cells. In reality then the liver-cells are the epithelial cells of these narrow tubes, and we have here a remarkable case in which the epithelial cells are larger than the cavity they enclose. The change from ordinary epithelial cells in one of the bile-ducts to liver-cells is shown in fig. 23. These minute ultimate branches of the bile-ducts are called the bile-capillaries - a rather dangerous term, as they have nothing to do with blood-capillaries.

The method of injection may also be applied to trace the blood-vessels in the liver. The branches of the hepatic artery and portal vein end in small vessels which lie in the connective tissue that surrounds each lobule and separates one lobule from another : they are therefore called inter-lobular vessels. The ultimate

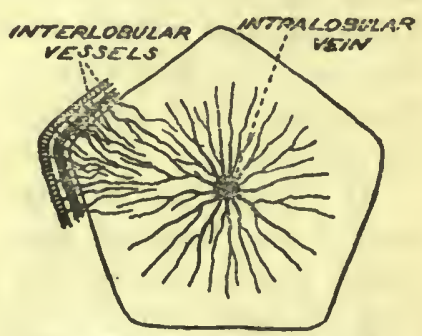

Fig. 24.-DiagraM of THE BLOOD-VESSELS OF A LOBULE OF THE LIVER.

factors of the hepatic vein, on the other hand, penetrate into the centre of every lobule: they are intra-lobular. Between the liver-cells is a network of blood-capillaries, which at the margin of the lobule are connected with the 
inter-lobular artery and vein, and at the centre open into the intra-lobular voin (fig. 24). When both this network of blood-capillaries and the system of bile-capillaries have been injected, it is found that the two are totally distinct, and that there is always at least one liver-cell between a blood-capillary and a bile-capillary. This is, of course, exactly what we should expect from the structure of simpler. glands.

The functions of the liver have already been partly stated. It secretes bile, and I'c gulates the amount of sugar in the blood. A third function is apparently this : the waste-products of katabolism, especially of muscles, cannot be directly excreted by the kidneys, but in the liver they undergo change into urea, and this can be removed from the blood by the kidneys.

$\S$ 19. The Kidneys or renal organs are a pair of compact glandular masses, from each of which a duct, the ureter, leads to the $m$ e d i a $n$ u $\mathbf{r}$ i $\mathbf{n}$ a $\mathbf{r} \mathbf{y}$ b 1 a d d e r, where the urine is stored up in the intervals between its periodic ex. pulsions from the body. If a median section of the kidney and adjoining part of the ureter is taken (fig. $25)$, it is seen

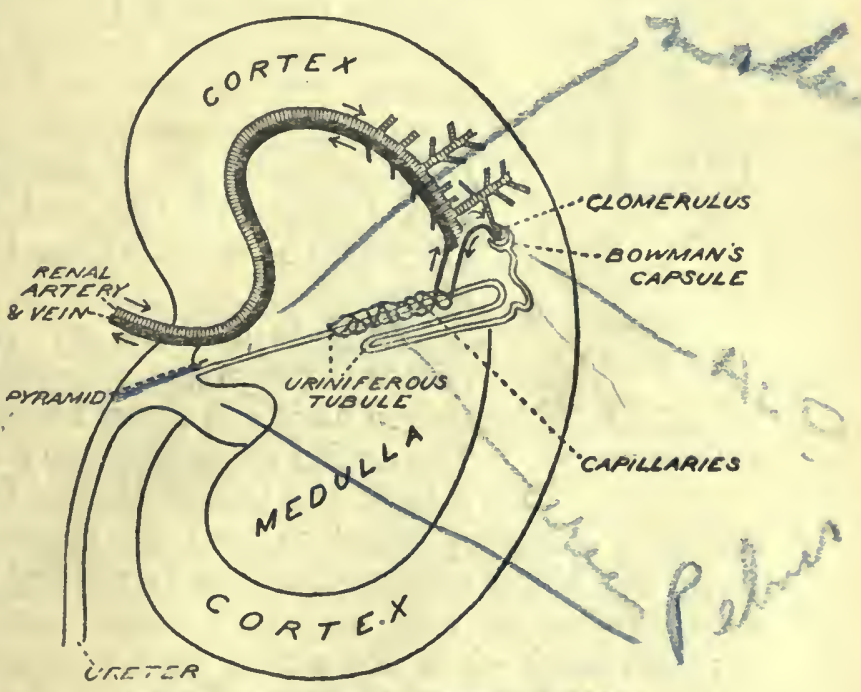

Fig. 25.-Diagram of Rabait's Kidnet.

that where the ureter meots the kidney its cavity expands into a large space-called the pelvis of the kidney. Projecting into this space is the pyramid. The main mass of the kidney is divided into an inner and outer portion, 
plainly distinguishable by the naked eye : these are called the medulla, and the cortex. The pyramid is simply a projecting portion of the medulla.

Considering now the minute structure, the whole kidney is essentially a mass of -secretory tubes - the uriniferous tubules-united together by connective tissue containing blood-ressels. Each tubule ends in one direction by opening into the pelvis, - the openings being on the surface of the pyramid - and in the other direction it terminates blindly in a structure called a malpighian body. The difference of appearance between medulla and cortex is due to the fact that while in the former the tubules have a straight course in a radial direction, as soon as they enter the cortex they curve and bend. It is also in the cortex only that malpighian bodies occur.

Blood is brought to the kidney by the renal artery: the ultimate branches of this end for the most

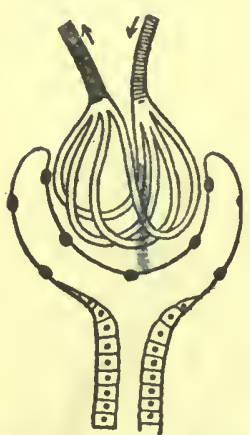

Fig. 26. - BowMAN's CAPSULE AND GromeruLus. part in a little bunch of looped capillaries called a glomerulus (fig. 26). From this the blood passes to a network of capillaries around the tubules and thence to the factors of the renal vein. A malpighian body consists of a double-walled capsule formed by the end of the tubule (Bowman's capsule) and containing a glomerulus.

Water is drained off the blood as it passes through the glomeruli; urea is removed as it circulates around the tubules. The action of an excretory epithelium like this of the kidney differs from that of an ordinary secretory gland in that its function is to select from the blood substances already present in it, and not to manufacture new materials. The selection, however, involves work-it is not a mere passive process of filtering, either in the case 'of the capsules or of the tubules. For example, if the epithelial cells of the capsules are unable to perform their work properly, the water drained off from the blood will retain the proteids dissolved in it and the disease called "albuminuria" is produced. 
Chapter VII.-The Skeleton.

§ 1. Method of Study. We are now in a position to study the rabbit's skeleton. We strongly recommend the student to do this with the actual bones at hand-they may be cleared very easily in a well-boiled rabbit. This recommendation may appear superfluous to some readers, but, as a matter of fact, the marked proclivity of the average schoolmaster for mere book-work has put such a stamp on study, that, in nine cases out of ten, a student, unless he is expressly instructed to the contrary, will go to the tortuous, and possibly inexact, descriptions of a book for a knowledge of things that lie at his very finger-tips. We have not written this chapter to give a complete knowledge of the skeleton, but simply as an aid in the actual examination of the bones.

§ 2. Physiology and Morphology of Skeleton. From the physiological point of view, the skeleton serves a double purpose-it forms the firm but movably-jointed framework without which the precision of motion, which is so necessary in an animal living under the rabbit's conditions, would be impossible ; and it affords a protective case for many of the delicate internal organs.

From the morphological point of view perhaps its most striking feature is the repetition of similar parts which it shows. We are not now referring either to the histological repetition of cells and tissues, nor to the more general repetition of such organs as veins and arteries all over the body, but to a repetition of a much more definite kind. In the first place the skeleton shows bilateral symmetry more perfectly than any other system in the rabbit; and in the second place it shows that repetition in a longitudinal series which is technically called metamerism or metameric segmentation. This is best shown by the vertebral column, which consists of a string of separate bones, which are all ZOOL. 
evidently constructed on a similar plan. The ribs afford another example; and in a reduced degree we see the same thing in the general similarity between arms (fore-limbs) and legs (hind-limbs), and the limb-grirdles (or bones within the trunk to which the limb-bones are joined).

The skull, vertebral column, ribs and sternum make up the axial skeleton, as distinguished from the appendicular or limb-skeleton (limb-girdles and limb-bones).

§ 3. Vertebral Column-Thoracic Region. We have seen (chap. i., § 2) that there are five regions in this column. It

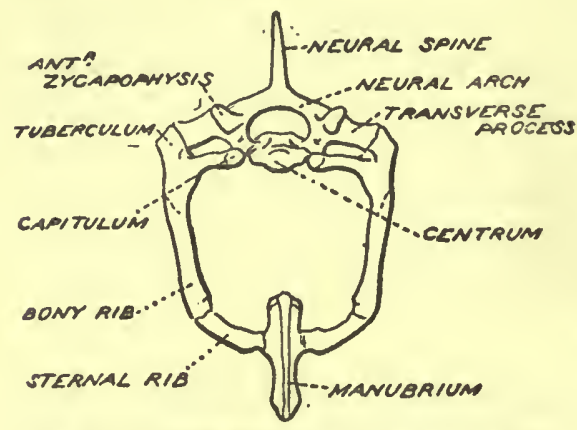

Fig. 27.-First Thoracic Vertebra and RIBS.

Front view. is convenient to start by examining a vertebra from the thoracic region. In such a vertebra (figs: 27,28 ) we see a central bony mass, the body or centrum, from which there arises dorsally an arch, the neural arch, completed by a keystone, the neural spine; and arising laterally from the arch are the transverse processes. Lookingat the vertebra sideways, we see that the arch has a notch in its posterior edge : this represents the point where a spinal nerve passes out from the spinal cord. Jointed to the thoracic vertebræ on either side are the ribs. Each rib has a process, the tuberculum, going up to articulate with the transverse process, and one, the capitulum, articulat-

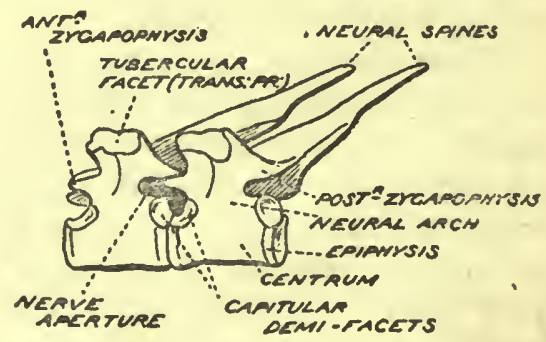

Fig. 28.-Third and Fourth Thoracic VERTEBR\&.

Side view. ing between the bodies of two contiguous vertebræ. At either end of the body of a vertebra of a young rabbit are 
the epiphyses (chap. vi., § 11). These epiphyses to the vertebral centra occur only among mammals, and are even absent in some cases within the class. In the adult rabbit they have ankylosed (fused) with the rest of the centrum. In a young rabbit's skeleton, they adhere more readily to the intervertebral pad than to the centrum, hence at the right-hand side of fig. 34 the epiphysis of the next vertebra is seen adhering.

Each thoracic vertebra is attached to its fellows in front and behind by solid masses of fibro-cartilage-the intervertebral pads, which limit to some extent the possibilities of bending of the vertebral column. A more important limitation of such motion is afforded by the zygapophyses, which are paired projections from the two ends of the neural arch, so disposed that those at the posterior end of one vertebra overlap those at the anterior end of the next. In life these contiguous zygapophyses are involved in a synovial joint, that is to say, their articulating surfaces are enclosed in a chamber containing a lubricating fluid which facilitates the sliding of one surface over the other. This allows a limited amount of lateral bending of the vertebral column, less vertical bending, and almost entirely prevents torsioni.e. the unequal rotation of different vertebræ about the longitudinal axis-as the student who sees and understands the relation of the zygapophyses will readily realize. In the macerated vertebra the articular surfaces of the zygapophyses are exposed as smooth surfaces, and afford a ready means of distinguishing the front and hind ends of an isolated vertebra: the normals to the polished surfaces of the anterior zygapophyses pointing upwards and inwards, those of the pósterior ones downwards and outwards.

There are usually twelve thoracic vertebræ in the rabbit. The first nine have long neural spines, pointing posteriorly; the tenth has a shorter spine which points vertically; the rest have short spines pointing anteriorly. In the ninth and following ones a small process is developed from the proximal end of the transverse process : this is called the metapophysis and will be better seen in the lumbar vertebræ. The distinguishing feature of the thoracic vertebræ is the presence of ribs articulated to them. 
§4. The Ribs and Sternum. The main part of each rib is bony, but their ventral ends are cartilaginous. These, in the case of the first seven pairs of ribs, articulate directly

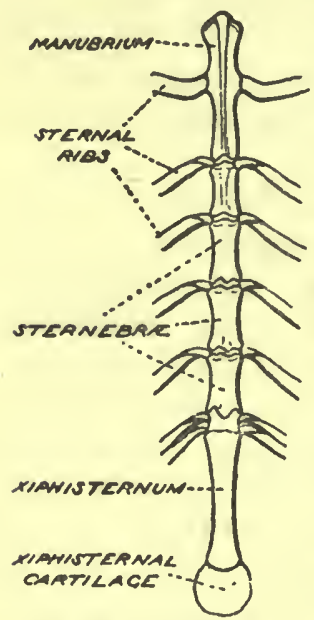

Fig. 29.-STERNUM. with the sternum; the next two pairs ("false ribs") join with the ribs in front; the remaining three ("floating ribs") ond freely ventralwards. Occasionally in the rabbit an extra pair of floating ribs is found behind, making thirteen in all: in such cases the corresponding vertebra is counted as thoracic instead of lumbar. The floating ribs have no tuberculum.

The sternum (fig. 29) is a metamerically jointed bone. Its anterior joint (manubrium) is large and projects forward; the next four are called sternebro, and the ribs articulate between these; finally there is the xiphisternum, ending in an expanded plate of cartilage.

So much for the thoracic vertebræ and related bones: it must not be supposed that they do not differ among themselves. On the contrary, the change from typical thoracic to typical lumbar is quite a gradual one. We are concerned here only with the main features, and the details must be made out by the student on the actual bones. We now pass on to the other regions of the column. Unless otherwise stated, centrum, neural arch and spine, zygapophyses, intervertebral pads and epiphyses are everywhere present.

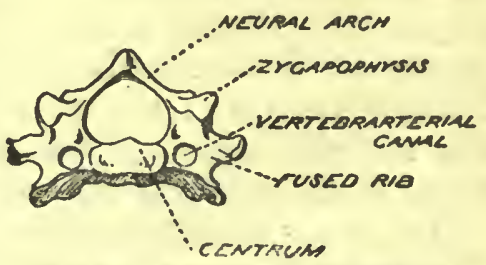

Fig. 30.-A Cervical Vertebra.

Front view.

§ 5. Cervical Vertebræ (fig. 30). These are seven in number. They seem, at first sight, to have no ribs. The transverse processes differ from those of the thoracic series in having paired longitudinal perforations, the vertebrarterial canals, so called because, in life, the vertebral 
arteries (each a branch of the subclavian of its side) are enclosed in them. A study of the development of these bones shows that the part ventro-lateral to this canal ossifies separately from the rest of the transverse process; and the form of the equivalent structures in certain peculiar lower mammals and in reptiles leaves no doubt that this part is really an abbreviated rib, fused up with the transverse process and body.

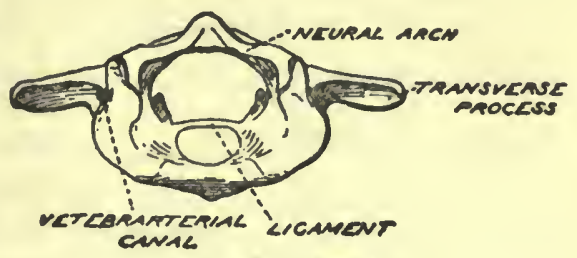

Fig. 31.-ATLas.

Front view.

The two anterior cervical vertebræ are peculiar. The first is called the atlas (fig. 31): it has great articular faces for the condyles (§15) of the skull, a deficient centrum, no zygapophyses, and very large transverse processes. The next is the axis (fig. 32), and it is distinguished by an odontoid peg, which fits into the space where the body of the atlas is deficient. In development the centrum of the axis ossifies from one centre, and the odontoid peg from another, which at that time occupies the position of centrum of the atlas.

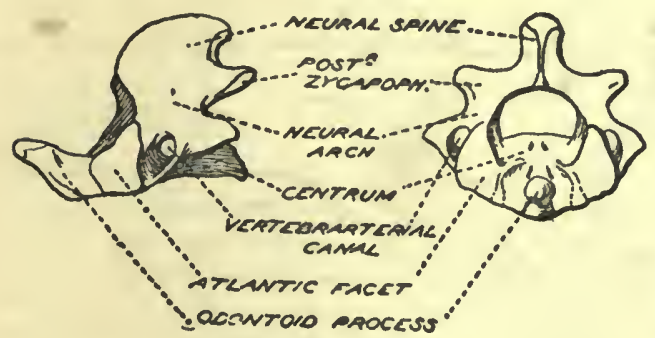

Fig. 32.-Axis.

Side and front views. So that it would seem that the atlas is a vertebra minus a centrum, and the axis is a vertebra plus a centrum, added at the expense of the atlas. The axis has a strong, ridge-like neural spine, posterior zygapophyses only, and its transverse processes are only recognizable as such through the presence of the vertebrarterial canal. The articulations between axis and atlas and between atlas and skull are synovial, intervertebral pads being wanting. This, along with the absence of zygapophyses, results in a 
much wider range of motion being possible here than elsewhere in the vertebral column. When the head is moved up and down in a vertical plane, the skull moves on the

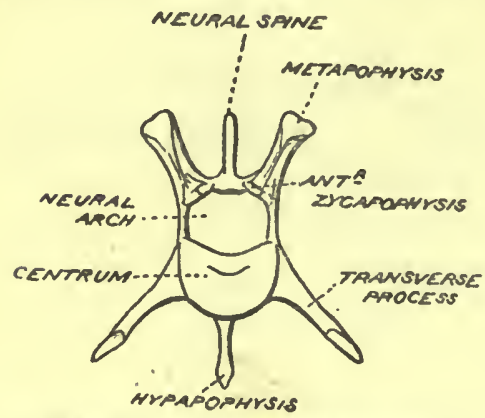

Fig. 83.-A Lumbar Vertebra.

Front view atlas ; but when oblique inclination of the head takes place, the skull and atlas together are moved on the odontoid peg. Simple side-toside motion involves bending of the neck. By combination of these three possible movements the head can be turned into a great variety of positions.

§ 6. The Lumbar Vertebræ (figs. 33 and 34) are seven in number, (except when the extra rib is present, when they are only six). They are large, and have cleft transverse processes, each giving rise to an ascending limb, the metapophysis, and a descending one. The latter (generally spoken of as the transverse proc e s s e s ) point steeply downward, and are considerably longer than those of the thoracic sories. 、

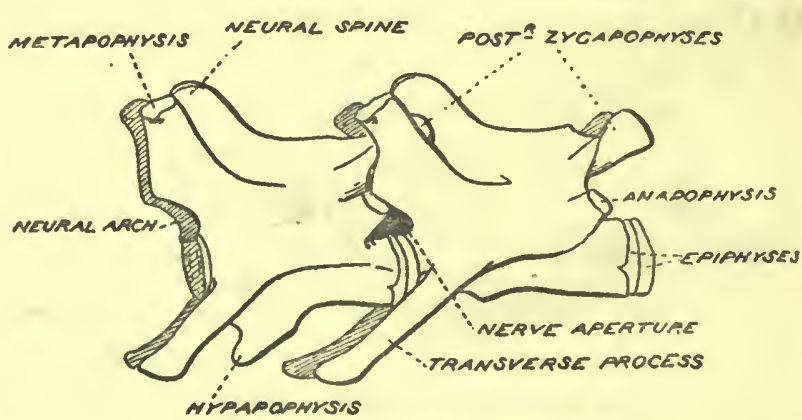

Fig. 34.-Two LUmbar Vertebra. Sido view.

$\S 7$. The Sacral Vertebræ are strictly defined as those whose transverse processes give articulation to the pelvic girdle. On this definition there are only two sacral vertebræ; but when these become fused together a variable number (two or more) of the next vertebre behind also fuse with 
them, and these are commonly counted as sacral vertebi'æ also (fig. 41, p. 75). The first true sacral has a very broad centrum, from which the greatly expanded transverse processes project, ending in a flattened surface joined im- . movably to the pelvic girdle. The remaining vertebræ narrow rapidly backwards; when fused (in the adult rabbit) their limits can be distinguished by the nervenotches (converted into holes by the fusion) which are on the ventral side.

§ 8. The Caudal Vertebræ are small, and about sixteen in number. As they are traced back they show a gradual change-zygapophyses and transverse processes disappear, then the neural arch becomes incomplete dorsally, and presently is lost altogether, the final vertebræ consisting of centrum only, and looking not unlike finger-joints.

§9. Limbs and Limb-girdles. The bones within the body which give support to the limbs are called the limbgirdles - 2 slightly misleading term, because they do not completely encirclo the body, but only partially so. Limbgirdles and limbs are constructed on one general plan throughout the air-breathing vertebrates (as distinguished from the fishes); but the plan is very variously modified to meet the requirements of running, jumping, burrowing and the multitudinous - other modes of utilizing the limbs. A diagram of this general plan is given in fig. 35 . The girdle is two-thirds of an oval hoop, and each half is divided by the point of articulation of

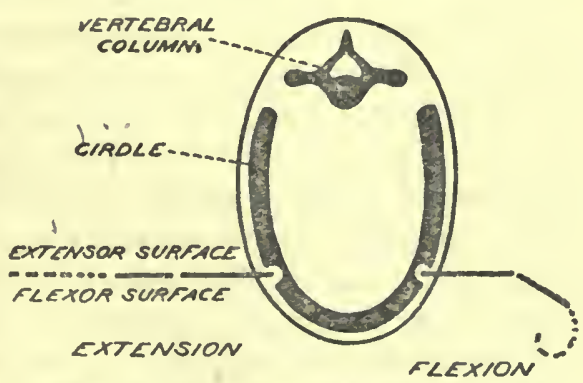

Fig. 35.-End View of Ideal GrRdue AND LIMBS. the limb into a dorsolateral and ventral portion. This ventral portion is a flat plate again divided by a large perforation (paired) into an antero-ventral and postero-ventral portion (fig. 36). To 
begin with the girdle is cartilage, but when this is ossified (i.e. replaced by cartilage-bone) each of these three portions (dorso-lateral, antero-ventral and postero-ventral) forms a

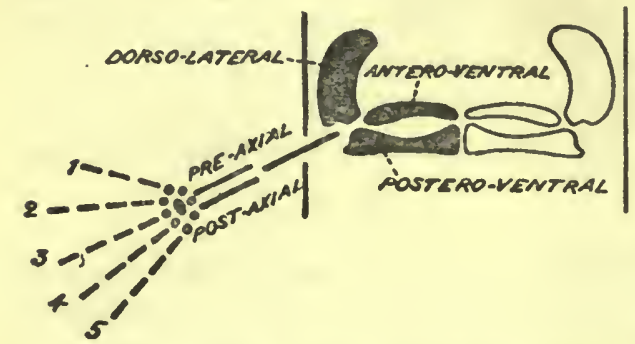

Fig. 86. - Plan of Ideal Girdle and Limb. separate bone (all paired). The limbskeleton is divided into four parts-a proximal, consisting of one long bone; the next, of two side by side; then comes a series of small bones, in three rows of three, one, and five respectively (see fig. 36); and finally five radiating rows (digits) of rod-like bones. In the primitive position, the limb points out at right angles to the body and the digits all lie in the horizontal plane: this may be imitated on the human arm, if the reader will hold it out at right angles to the side of the body with the thumb upwards (i.e. forwards) and the palm of the hand forwards (i.e. ventralwards). In this position the arm can only be bent ventralwards, so the ventral surface is called the flexor surface, and the dorsal the extensor. Looking at the $\operatorname{limb}$ from the ventral side (fig. 36) we call the forward edge preaxial, the hinder edge postaxial : these same terms can be applied to distinguish the two bones of the second segment of the limb, and also to express the relative positions of the several digits. In

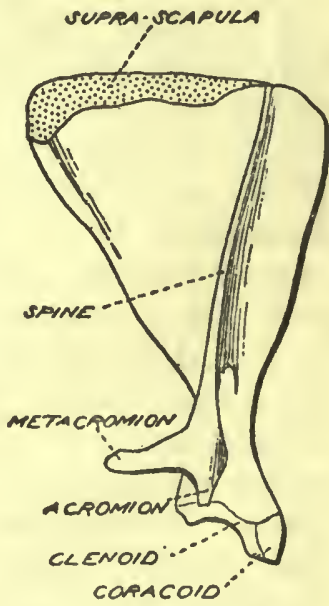

Fig. 37.-Scapula of A Young RabBit.

(The shape is slightly different in the adult.) numbering the digits we begin with the most preaxial one. So much for the general plan.

$\S 10$. The Shoulder-girdle, or Pectoral Girdle, in the rabbit is decidedly incomplete. Only the dorso-lateral bone 
is well developed-it is called the scapula. This is triangular in shape, and has on its outer surface a long ridge ending in a curious umbrella-handle-shaped free termination, the acromion and metacromion processes (fig. 37). At its ventral end it bears an articular surface for the limb, the glenoid surface. At the anterior side of this is a little curved projection which in a young animal is seen to be a separate bone : this is the coracoid or postero-ventral bone, or all that is left of it. The antero-ventral bone or clavicle (collar-bone of man) is also greatly reduced in the rabbit: it may be found in the course of a ligament that runs from the scapula to the sternum, as a small rodlike bone with a nodule of cartilage at either end.

$\S 11$. The Fore-limb in the rabbit is chiefly used for burrowing and running; to a less extent for grasping food; its range of motion is, therefore, much more limited than that of man's arm. If the student, seated before a table, will place his hands side by side on the table, palms downwards, he will have them in what is called the prone position. From this

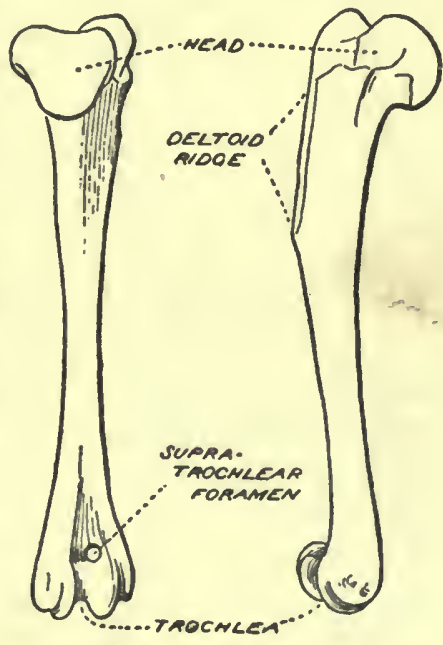

Fig. 38.-Humerus. position it is quite easy for him to turn them into the supine position, i.e. palms upwards, without moving his upper arm at all. This is because the second segment of his arm, thanks to its containing two bones, is capable of considerable rotation. But in the rabbit the limb is fixed in the prone position and cannot be supinated: this is an advantage in burrowing.

The first bone is called the humerus (fig. 38 ): the most noteworthy feature in it is the deep olecranon fossa at the distal end of its extensor surface-a deep hollow, making an actual perforation of the bone at one point: this is characteristic of mammalia, and the reason for it wo shall 
see presently. Another feature of the humerus is the prominent deltoid ridge (representing the region of attachment of an important muscle) on its preaxial border. Of the two bones in the next segment of the limb, the preaxial

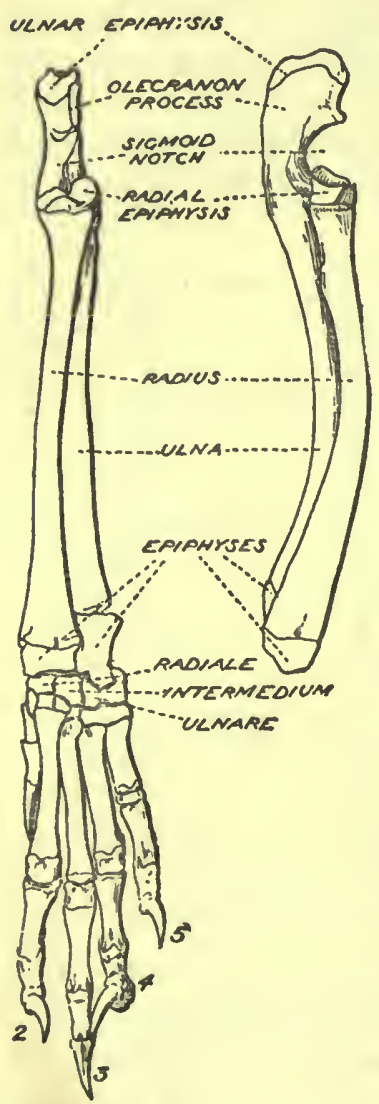

Fig. 39.-Radius and ULNa; Carpals, Metacarpals, and Phalanges.

is called the radius, the postaxial the ulna (fig. 39). The ulna has a projection, the olecranon process, beyond the articulation with the humerus (elbow): this gives leverage to the extensor muscles (i.e. muscles which straighten out the arm), whose tendons are attached to it, and it is to make room for this process when the arm is extended that the humerus has an olecranon fossa.

In the primitive position of the limb, radius and ulna lie side by side, but the partial rotation of the fore-arm necessary to bring it into the prone position causes them to be slightly crossed in the rabbit.

The small bones that follow constitute the carpus collectively-each one being a carpal. The three of the first row are called radiale, intermedium and ulnare, in reference to their position with regard to the bones of the fore-arm. The single one of the second row is called centrale; the five of the distal row are called distalia and numbered; the fifth distale is, however, fused with the fourth. Of the rod-like bones that support the fingers, it is customary to call the proximal in each finger. metacarpal, and the rest phalanges, though apart from human anatomy there is no justification for such a distinction. The preaxial digit (pollex or thumb) has two phalanges only, all the others three. 
$\S$ 12. The Pelvic or Hip-girdle (fig. 41) differs from the pectoral in practically all air-breathing vertebrates, by being articulated with the vertebral column (sacral vertebræ). The pelvic girdle is much more complete than the pectoral, but it has undergone a backward rotation from its primitive position (fig. 40), so that the dorso. lateral bone (here called ilium) which articulates with the sacrum comes to lie parallel with the ver-

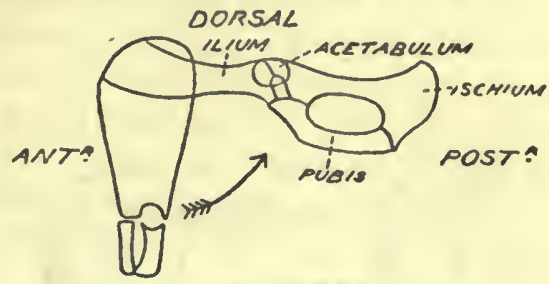

VENTRAL

Fig. 40.-Diagram of Rotation of Pelvic Girdle. tebral column instead of vertically; the antero-ventral (pubis) comes to be entirely ventral; and the postero-ventral (ischium) comes to be

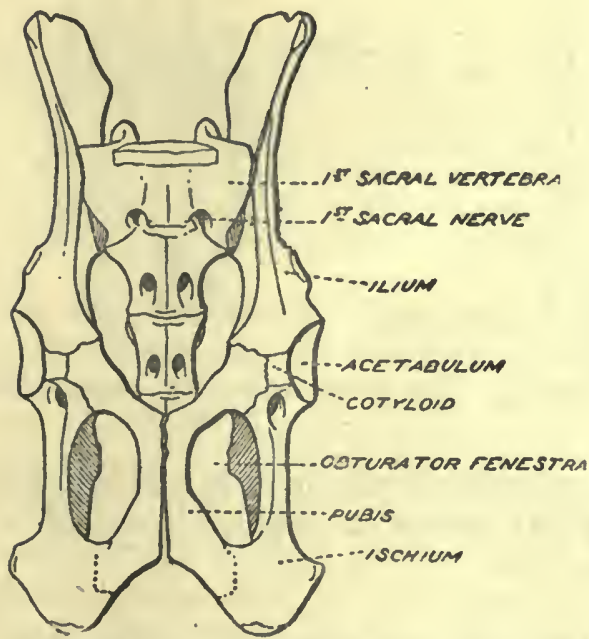

Fig. 41.-Peific Girdie and Sacral VERTEBRE. postero-dorsal, and does not meet its fellow in the middle line at all. The hollow for the articulation of the limb is called theacetabulum; at its ventral border an extra little bone is found, (possibly of the nature of an epiphysis) called the cotyloid. The large space between the pubis and ischium is called the obturator fenestra-a. fenestra meaning a portion of the skeleton consisting of membrane (connective tissue) only, as distinguished from a foramen, which is a hole through which something passes; there is an obturator foramen at one point in the fenestra-where a nerve passes through. The distinction between a foramen and a fenestra is important, but in the dried skeleton, where 
all the connective tissue is gone, the distinction cannot be recognized.

The right and left pubes are joined in the middle central line, but a narrow strip of cartilage persists between the

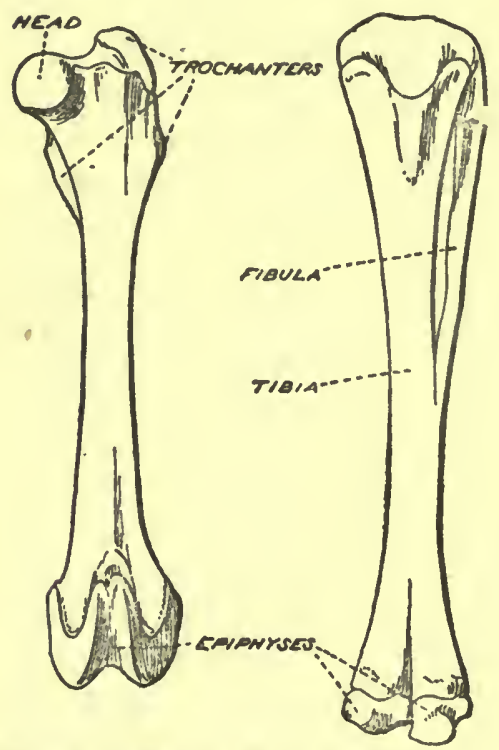

Fig. 42.-Femur, Tibia, and Fibula.

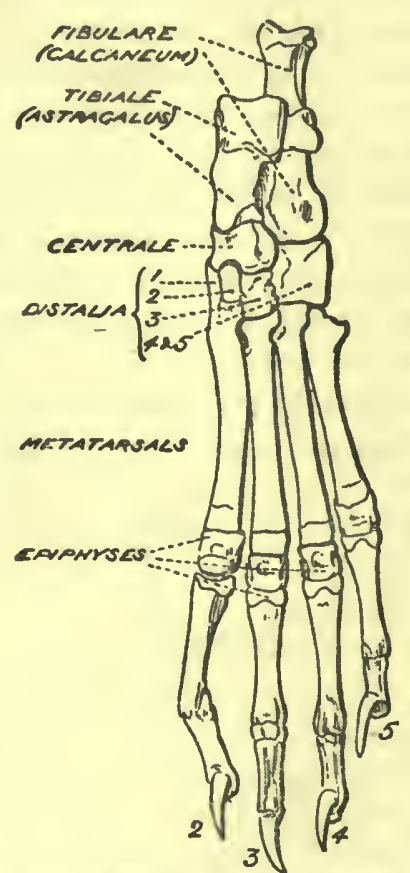

Fig. 43.-Tarsals, Metatarsals, and Phalanges.

two actual bones: such a persistent cartilage in the middle line, joining the right and left bones of a pair, is called a symphysis.

$\S$ 13. The Hind-limb (fig. 42 and fig. 43.) The first bone, or femur, differs from the humerus in the greater distinctness of its articulating head, and the greater prominence of the ridges (trochanters) which mark the points of muscular attachment. The two bones that followtibia preaxial, fibula postaxial-are fused together for their distal half; the fibula is very small and has no olecranon process. Corresponding to the carpals we have 
a series of tarsals, forming the tarsus. Of these the proximal row consists of two-the preaxial one probably represents both tibiale and intermedium fused: it is rod-like and called the astragalus. The postaxial (fibulare) is still more elongated than the astragalus and projects back into a lever-head (like the olecranon of the ulna) which forms the heel : this bone is commonly called calcaneum.*

There is a centrale, and only three distinct distalia : the preaxial distale is fused with the second metatarsal (the rest of the great toe or hallux being absent in the rabbit), and the fourth and fifth distalia are fused, just as in the carpus. Each of the four toes has a metatarsal and three phalanges. It is often convenient in comparing different animals to express the number of digits and of the phalanges in them by a brief formula. This digital formula in the case of the rabbit would be

$$
\text { 2.3.3.3.3. - 0.3.3.3.3. }
$$

$\S 14$. Sesamoid Bones. Besides the bones of the limb-skeleton already described, which are all cartilage-bones, there are a few others, which are neither cartilage-bones nor membrane-bones in the ordinary sense. These sesamoid bones are ossifications in the tendon of a muscle at a point where it has to run over a projecting surface. The best known of these is the knee-cap or patella, developed in the tendon of one of the extensor muscles of the leg.

$\S 15$. The Skull. The mammalian skull will be better understood after the study of that of some lower vertebrate. We shall describe its main features now, but their meaning will become much clearer later on. Our figures are of the dog (Canis familicaris). In section (fig. 47), we perceive a brain-case (cranium) opening behind by a large aperture, the foramen magnum. In front of this is an extensive passage, the nasal passage, which is divided from the mouth by a bony floor, the palate, and which opens into the pharynx behind at the posterior nares, and to the exterior by the anterior or external nares. Its anterior portion is

* The terms astragalus and calcaneum are taken from human anatomy ; tibiale and fibulare are the terms of comparative anatomy. Most of the other carpals and tarsals also have human-anatomy names, but it seems unnecessary to give them here. 


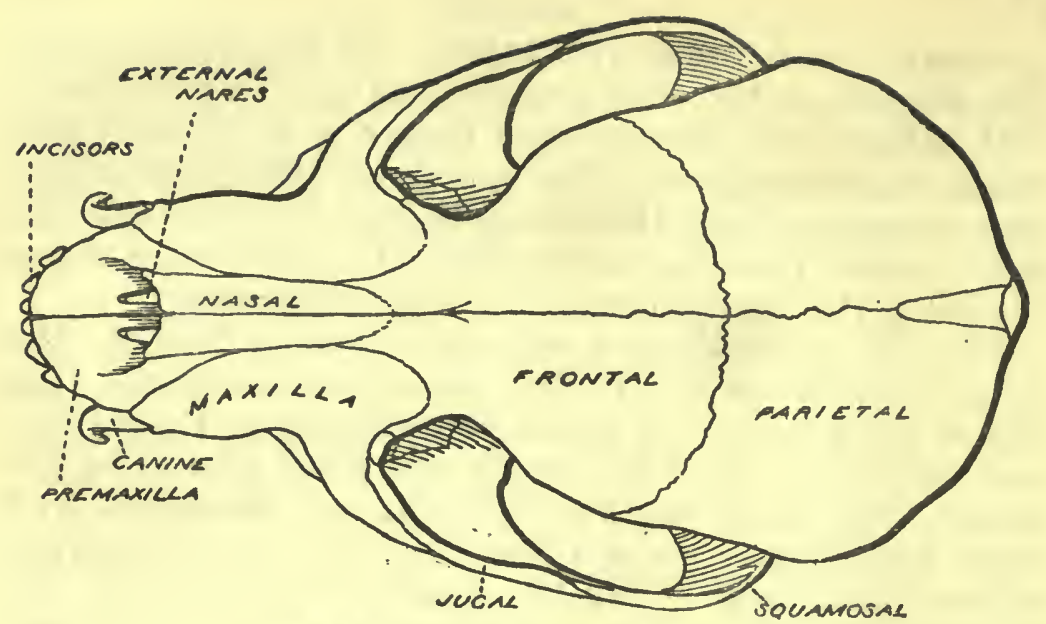

Fig. 44. - Skulc of DoG. Dorsal view.

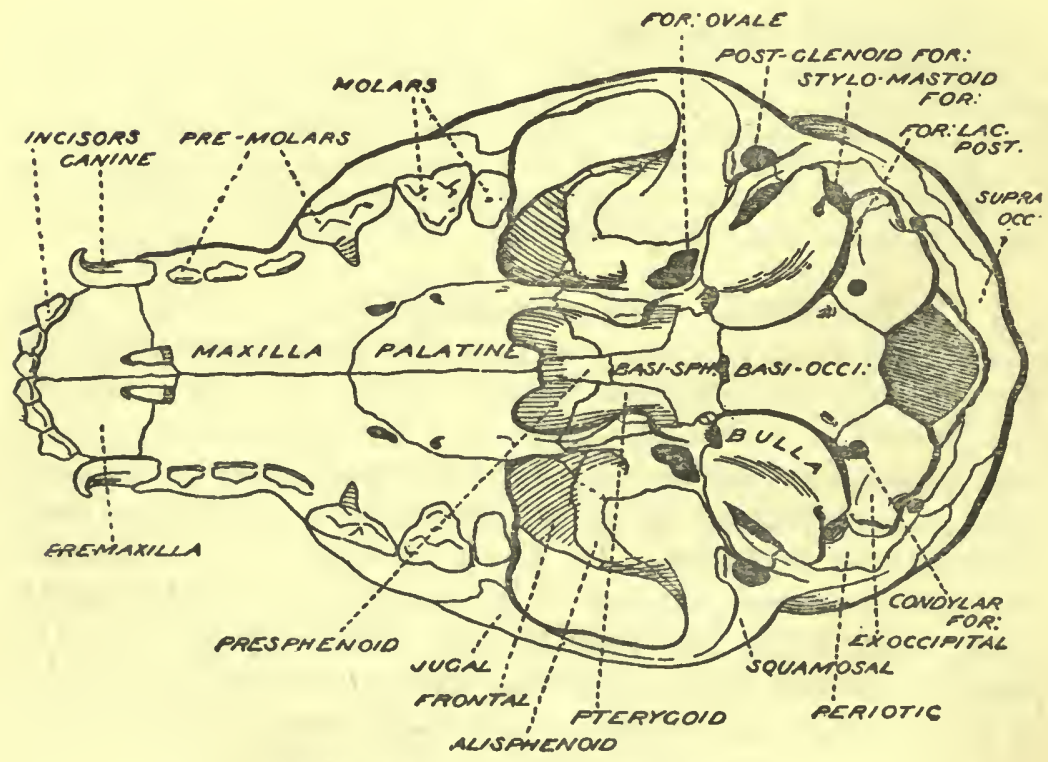

Mig. 45.-Skull of Dog. Ventral view. 

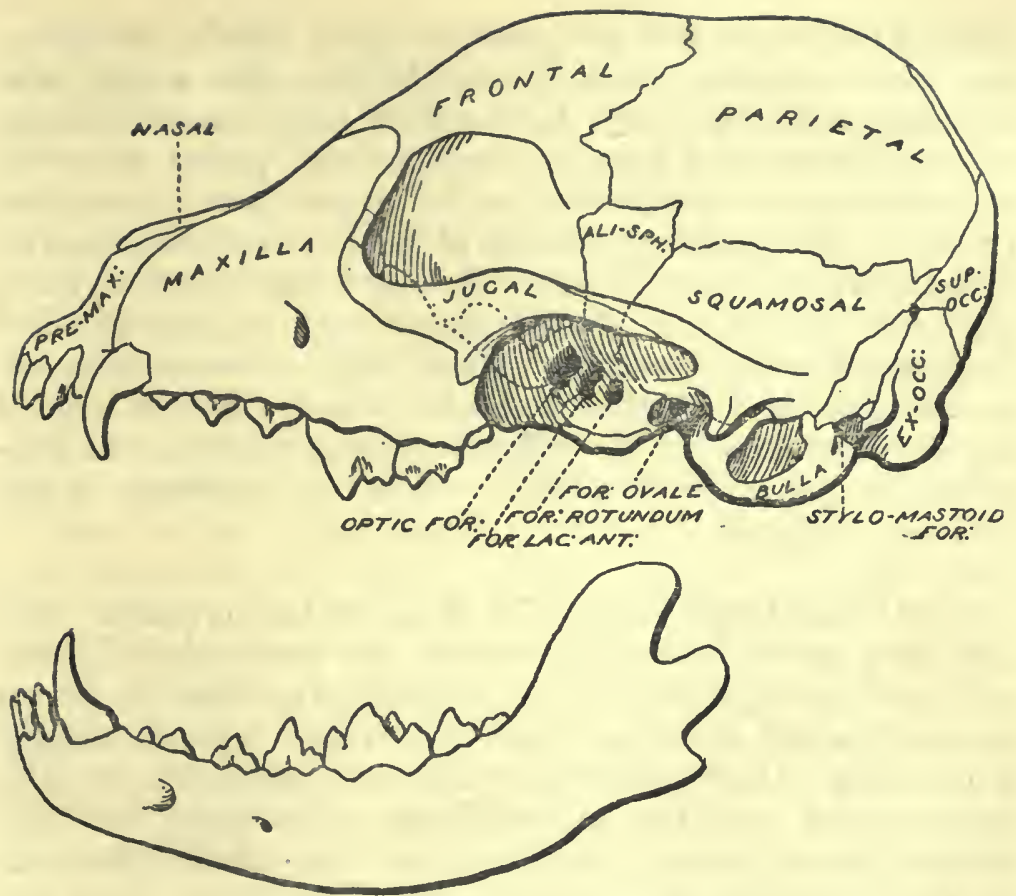

Fig. 46. - Skull of Dog. Side view.

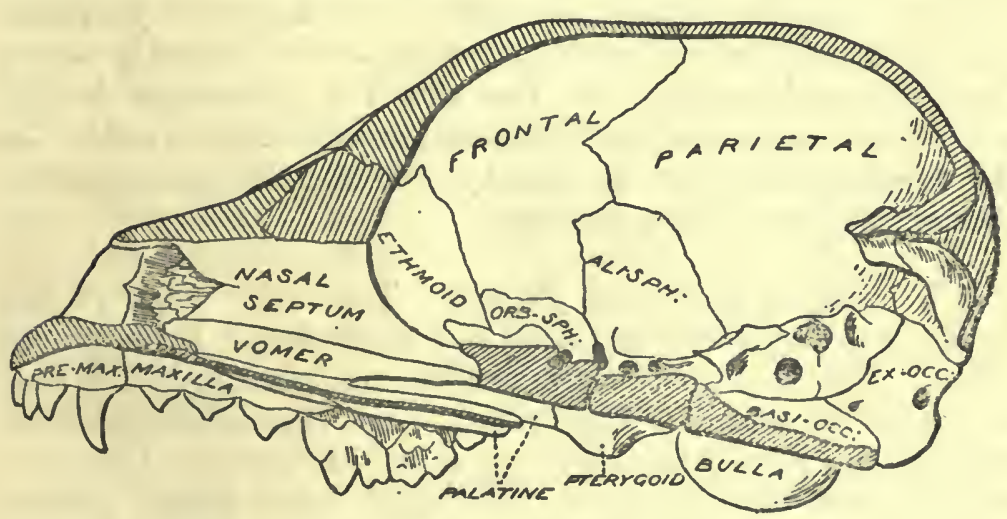

Fig. 47. -Skull of DoG. Median (sagittal) section.

79 
divided into right and left passages by a middle partition, the nasal septum. Outside the skull, on its wings, is a flask-like bone, the bulla tympani or tympanic, protecting the middle ear, and from above this there passes an arch, the cheek-bone or zygoma, to the upper jaw, forming in front the bony lower protection of the cavity containing the eye, the orbit. The cheek arch, nasal passage, and jaws, form collectively the "facial apparatus," as distinguished from the cranium, and the whole skull is sometimes referred to as the "cranio-facial apparatus." Two eminences for articulation with the atlas vertebra, the occipital condyles, lie one on each side of the lower boundary of the foramen magnum.

$\S$ 16. The Brain-case. The floor of the cranium consists of a series of cartilage-bones, the basi-occipital, basisphenoid, pre-sphenoid, and ethmoid, the last of which sends forward a median plate, the nasal septum already mentioned. Like extended wings on either side of the basi-occipital are the ex-occipitals. Similarly, the alisphenoids are wings to the basi-, and the orbito-sphenoids to the pre-sphenoid. Between the ex-occipital and alisphenoid there is wedged in a bone, the periotic, containing the internal ear. Above the foramen magnum the median supra-occipital bone completes what may be called the occipital ring, encircling the hind-brain. A pair of parietals come above the ali-sphenoids, and a pair of frontals above the orbito-sphenoids. At the side the brain-case is still incomplete, and here the squamosal enters into its wall. In the external view (fig. 46) the tympanic bulla hides most of the periotic bone from without.

$\S 17$. Upper Jaw and Palate. The outer edge of the upper jaw and the cheek arch are made up of three paired bones. First comes the premaxilla, containing, in the dog, the three incisors of either side. Then comes the maxilla, bearing the rest of the teeth-a sabre-like canine, four premolars and two molars. The jugal (also called malar) reaches over from the maxilla to meet a zygomatic process of the squamosal bone, the two forming the zygomatic arch. 
In the ventral view of the skull (fig. 45) it will be seen that the maxilla sends inwards, to meot its fellow medianly, a plate forming the front part of the hard palate. Behind, the hard palate is completed by the pair of palatine bones, which conceal much of the pre- and orbito-sphenoids, and which run back as ridges on either side of the pre- and basi-sphenoids, hiding the junction of these with the orbitoand ali-sphenoids. These ridges terminate in two small angular bones, the pterygoids, which we shall find represent much more important structures in the lower vertebrata.

The premaxillie and maxillæ bound the sides of the nasal passage, and it is completed above by a pair of membrane-bones, the nasals. Allong the floor of the nasal passage, in the middle line, lies a median membrane-bone, the vomer. This embraces in a . V-like groove the mesethmoid (nasal septum) above, and lies on the palate. The nasal passages are partially blocked by scroll-like bony ingrowths from the inner aspect of their walls, which in life are covered with mucous membrane, and increase the surface sensitive to smell. The ethmoid sends in the ethmo-turbinal; the nasal, the naso-turbinal; and the maxilla, the maxillo-turbinal. In the anterior corner of the orbit there is a small membrane-bone, the lachrymal.

\$ 18. The Lower Jaw (mandible) consists of right and left halves or rami, each of which is a single bone(fig.46). On each ramus there are three incisors which bite against the three of the upper jaw. Then come a canine, four premolars, and three molars; the first of which is blade-like (sectorial tooth), and bites against the similar sectorial tooth (last premolar) of the upper jaw. 'The third molar is small. The arrangement of teeth is indicated in the following dental formula :-

$$
\text { I. } \frac{3.3}{3.3} \text {, C. } \frac{1.1}{1.1} \text {, P.M. } \frac{4.4}{4.4} \text {, M. } \frac{2.2}{3.3}
$$

or more briefly,

$$
\frac{3.1 .4 .2}{3.1 .4 .3}
$$

In the case of the rabbit (chap. ii., $\$ 4$ ) the dental formula will be

$$
\frac{2.0 .3 .3}{1.0 .2 .3}
$$


$\S$ 19. Hyoid and Ear-bones. Attached just behind the bulla above, and passing round on either side of the throat to meet at the base of the tongue, is a peculiar series of small bones con-

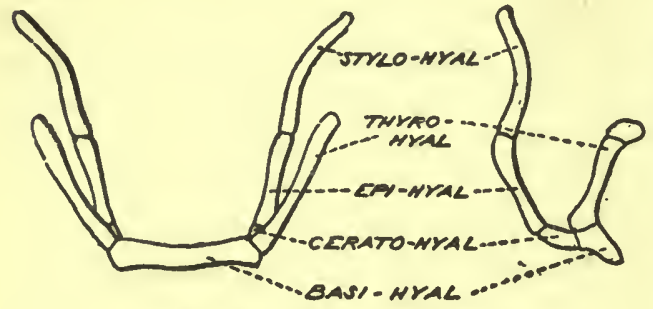

Fig. 48.-Hyord of Doa.

Posterior and side views. stituting the hyoid apparatus (fig. 48). This consists of a median bone (" body of hyoid," or basihyal), which gives attachment to the muscles of the tongue, and two pairs of projections, the horns or cornua.

Each anterior cornu consists of a row of three rod-like bones-cerato-, epi- and stylo-hyal, and a fourth smaller one, the tympano-hyal, which is fused to the periotic bone. Each posterior cornu consists of a single thyro-hyal bone, but the pair of thyroid cartilages in the walls of the larynx are really distal portions of this posterior cornu. This peculiar series of bones will be better understood after we have studied lower types of vertebrata.

Hidden by the bulla, and just external to the periotic bone, are the auditory ossicles, the malleus, incus, os orbiculare, and stapes. These will be more explicitly treated when we discuss the ear.

§ 20. General View of Skull. When we come to the study of the nerves, we shall revert to the skull, and treat of its perforations. The student should not fail, before proceeding, to copy and recopy our figures, and to make himself quite familiar with them, and he should also obtain and handle an actual skull. For all practical purposes the skull of a cat will be quite as useful as that of the dog; and even that of a sheep or rabbit will show the same bones with various differences of detail.

The following table presents these bones in something like their relative positions. A closer approximation to the state of the case will be reached if the student will 
imagine the maxilla raised up so as to overlie and hide the palatine and pre-sphenoid, the squamosal similarly overlying the periotic bone, and the jugal reaching between them. Membrane-bones are printed in small capitals, cartilagebones in italics. Median bones are named in the singular, and paired ones in the plural.

CRANIUM.

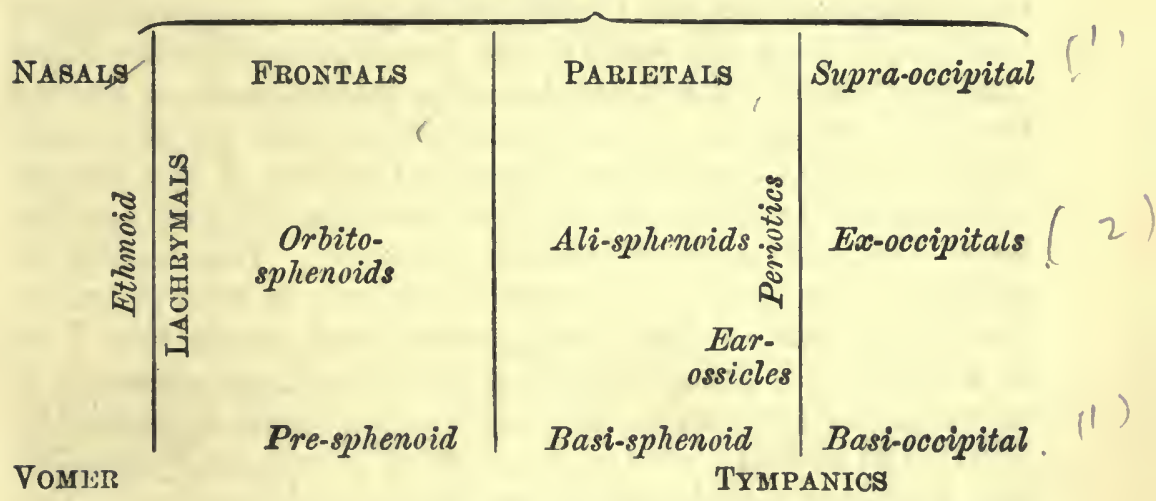

UPPER JAW.

PRE-MAXILLX Palatines

Pterygoids

MAXILLA JUGALS

SQUAMMOSALS

LOWER JAW.

HYOID. 


\section{Chapter ViII.-Muscle and Nerve.}

$\S 1$. Muscular Contraction. We have, in the skeleton, a complicated apparatus of parts hinged and movable upon one another ; the agent moving these parts is the same agent that we find in the walls of the heart propelling the blood through the circulation, in the alimentary canal squeezing the food along its course, and universally in the body where motion occurs, except only in the case of the waving cilia, and those cells which (like the white blood-corpuscles) have the power of amoeboid motion. This agent is muscle. The action of muscle is familiar to most people in the typical case of the biceps in the arm of an athlete. This is a mass of "flesh" tapering at both ends, where it is attached by tendons to the scapula and radius respectively. When the arm is bent (or flexed, as it is technically termed) this muscle is plainly seen to swell up in the centre. This change of shape in a muscle is called contraction-a somewhat unfortunate term, since in physical science "contraction" means a diminution in total volume. It must be clearly understood that muscular contraction involves no change in volume, but only change of shape--the muscle becoming shorter and thicker. As it does so, it pulls the points of its attachment towards one another (just as a stretched piece of elastic might do as it shortened itself), and so the arm is bent. It is by similar changes in shape of the appropriate muscles that all motions of the rabbit's body are effected-with the two exceptions already mentioned.

If we examine a muscle histologically we find it is a tissue-complex. It consist of a great many muscle-fibres, united together into a coherent mass by connective tissue containing blood-capillaries. Connective tissue (called the endomysium) surrounds each individual fibre, and as a muscle has abundant need of food and oxygen we find a network of capillaries set close around each fibre. The 
fibres are arranged in bundles (fasciculi), and tho connective tissue around these bundles is called the perinysium. It is the muscle-fibres which possess the power of "contracting "- the connective tissue is purely passive, though its elasticity may help in restoring the muscle to its original shape when contraction ceases. The change of shape of the whole muscle is simply the sum of the changes of shape of its constituent fibres.

It is highly probable that the change of shape of a muscle-fibre is, at bottom, the same thing as the change of shapo of an amoba's pseudopodium, only it is confined to a particular direction. The exact structure of muscle is, however, a very difficult matter to determine, and has long baffled histologists. We can only give an outline of the facts here.

§ 2. Striped and Unstriped Muscle. Two main kinds of muscle-fibre may be recognized-plain or unstriped or unstriated, and striated or striped. The former is the simpler.

An unstriated muscle-fibre (fig. 49, A) is a spindle-shaped cell (perhaps tiventy timos as long as broad), containing a single nucleus. The protoplasm shows faint striations (stripes) in the direction of its length: these are very different from the transverse striations of striped musclefibre.

A striped muscle-fibre (fig. 50) may be several hundred times as long as any unstriated one ever is-as much as an inch or more in length

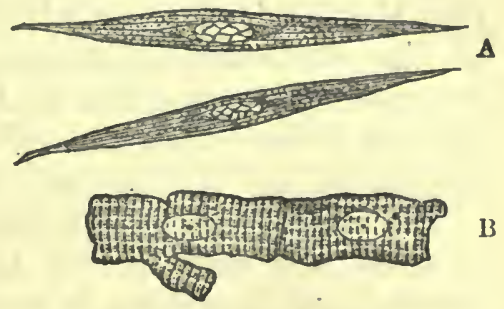

Fig. 49.-M USCI,E-Fibres.

A, Unstriped; B, Cardiac.

A large oval nucleus is seen in the centre of each. - thus better deserving the name of "fibre." Instead of one nucleus it has many. 'The outermost layer of the protoplasm forms a firm protective sheath - the sarcolemma: the nuclei lie just within this. The more liquid protoplasm within has a very definite structure, consisting apparently of alternate lighter 
and darker transverse discs, giving the transversely striped appearance. More minute examination seems to show that the darker bands are composed of many little longitudinal

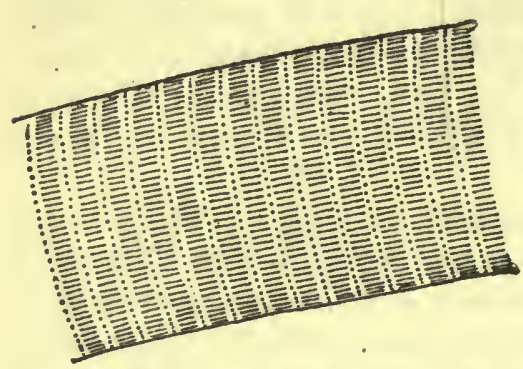

Fig. 50.-Portion of a STKIPED MUSCLE-FibRE.

The nuclei are not shown. rod-like structures (" sar"cous rods"). The precise nature of these structures is still so uncertain that we will not go into further detail.

There is no doubt that striped muscle represents a higher degree of differentiation or specialization for the work of contraction than does unstriped. This is shown, firstly, by the distribution of the two kinds in the body: all the muscles attached to bones (skeletal muscles), which therefore have hard work to do, and are concerned in the more rapid, sudden and precise movements - are composed of striated muscle-fibres. On the other hand, those which have slow, rhythmical, routine work to do (splanchnic muscles), as the muscles of the intestinal wall, which cause its squirming "peristaltic" motion, are unstriped. So, too, it is roughly true that striped muscle is voluntary, or under the control of the will, and unstriped, involuntary; while a more accurate distinction lies in the fact that unstriped musclefibres tend to contract rhythmically quite independently of any stimulus ( $\$ 10$, below), while striped fibres do not.

The most important examples of unstriped muscles are those of the wall of the alimentary canal, and those of the walls of blood-vessels, and the structure of these walls is described in the next two sections. Unstriped muscles are also found in the walls of some ducts (e.g. bile-duct and uroter) and of the urinary bladder, and in the ivis of the eye (chap. x., § 8).

§ 3. Structure of Intestinal Wall. The wall of almost the whole of the alimentary canal may be divided into three main layers, in order from within outwards-the mucous, sub-mucous and muscular coats. The layer of peritoneum on the outside may be counted as a fourth (serous) layer. 
The mucous layer consists of (1) the lining epithelinm, (2) a layer of adenoid or lymphoid tissue, containing abundance of bloodvesscls, and (3) a double muscular layer (muscularis mucosa) conconsisting of an inner layer in which unstriped fibres are arranged so as to tend to encircle the intestine (circular muscles) and an outer in which they are arranged longitudinally. The whole of the folds into which the inner surface of the intcstine is thrown, affect the mucous layer only. Where there are glands, like the peptic glands of the stomach, the epithelium is sunk down into the adenoid tissue; where there are villi, the adenoid tissue runs up into them.

The sub-mucous layer is a laycr of connective tissue containing the main blood-vessels, lymphatics and nerve-fibres from which the other layers are supplied. It is not affected by any of the folds into which the mucous layer may be thrown.

The muscular layer is just like the muscularis mucosæ, but much thicker-there is an inner circular layer, and an outer longitudinalall of unstriped fibres. It is these layers that cause the peristaltic movement of the intestine, and the analogous movements of the stomach-wall during gastric digestion. There is a layer of nervefibres between the two sets of muscle-fibres.

\$ 4. Structure of Blood-vessels. Capillaries have walls consisting of endothelium only, and this same endotbelium forms a continuous lining to the whole of the vascular system-veins and arteries, chambers of the heart, and lymphatics. In the veins and arteries there are added to this endothelium three coats-(1) a layer of connective tissue with great abundance of yellow elastic fibresthe elastic layer, (2) a layer of unstriped muscle-fibres, arranged mostly in a circular manner, (3) an outer layer of connective tissue, also with some elastic fibres. In veins the muscular layer is much thinner than in arteries; the reason for this lies in the function of the muscular layer, which is to contract or relax as may be needed, and so decrease or increase the calibre of the artery and therefore the amount of blood going to the part of the body served by that artery. In veins, where the flow of blood is from small vessels to large, there is obviously less need for the muscular layer.

§. Muscles of Heart. At the points where the walls of the large vessels merge into that of the heart, the endothelium continues unchanged, but the other layers undergo great change. The inner and outer connective-tissue coats lose their elastic character, and the muscular layer becomes enormously thickened, especially in the ventricles. At the same time, a change in the form of the musclefibres takes place; they are no longer ordinary unstriped fibres, but partake of a character intermediate between striped and unstripcd. Each fibre is still a single cell, with a single nucleus, but is rather oblong than spindle-shaped, and gives off branches which join it to adjacent fibres. Moreover, it has a distinct transverse striation, though not quite as definite as that of true striped muscle (fig. 49, B). 


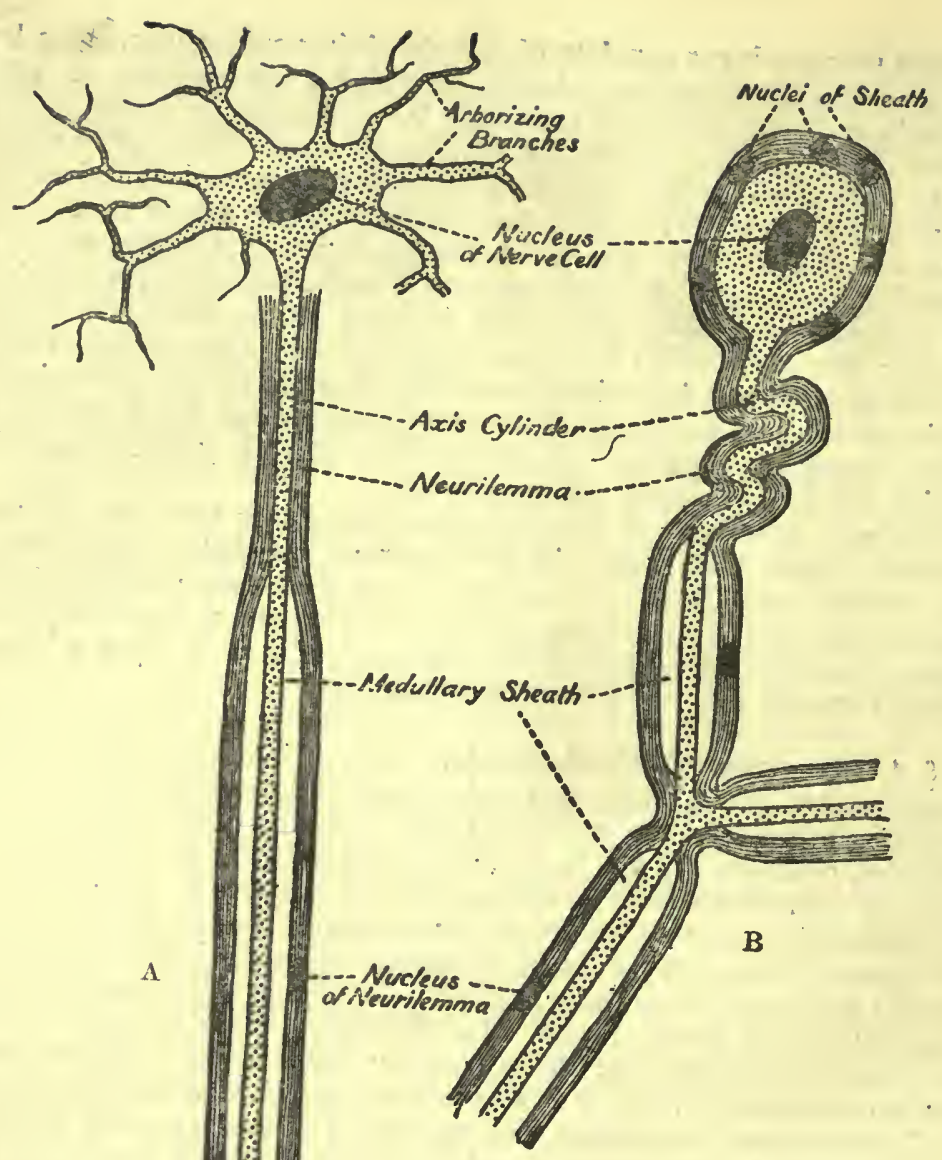

Fig. 51.-NeRve-Cells (Partly diagrammatic.)

$$
\begin{gathered}
\text { A, MUltipolir } \\
\text { (from spinal cord); } \\
\text { B, UNipolar } \\
\text { (from spinal ganglion). }
\end{gathered}
$$


$\S 6$. The Nervous System. If we watch a living rabbit, we see signs of constant muscular contraction. Eyes, lips, and snout are constantly moving, even when it seems at rest. It grasps and nibbles food, hops slowly or runs from time to time, and even when asleep the heart continues beating, and the thorax-muscles never cease pumping air in and out of the lungs. Every one of these motions in itself involves a complex series of contractions of different muscles. Obviously if the rabbit is to live for even five consecutive minutes, there must be some agent for co-ordinating all these activities, for making them work in harmony, and in such a way as is most serviceable to the whole animal. This agent is the nervous system. The nervous system may be divided into two parts - the central nervous system, which is the governing organization of the multicellular community (chap. v., § 12), and the peripheral nervous system, which forms the means of communication between the central system and the various organs of the body. In the rabbit the brain and spinal cord constitute the central nervous system, while the nerves and nerveendings (in other organs) together with certain structures called ganglia, form the peripheral system.

§ 7. Nerve-cells. The whole nervous system consists of nerve-cells and their branches-the largest and longest of the latter forming nerve-fibres-connected together by connective tissue.* Nerve-cells are usually classified according to their shape as unipolar, bipolar, and multipolar. We may take the last of these as the most typical form. Such a cell is large (fig. 51, A) and has a large and conspicuous nucleus. Its shape is irregular, as it runs out into a great many processes. The majority of these branch repeatedly, and form a fine ramification ("arborization "). Such branches are called dendrons. But one branch differing but little in appearance from them is continued on to a relatively enormous length-possibly over a foot in the rabbit, and much more in larger animals-as the axis-cylinder of a

* The connective tissue of the central nervous system is called neurnglia, and differs in certain important respects from all other connective tissues; but these are beyond our present purpose. 
nerve-fibre (see below). Bipolar cells appear to have no arborizing processes, but send off two axis-cylinders; while unipolar cells are simply bipolar ones in which the two axiscylinders are united at the base (fig. 51, B).

$\S 8$. Nerve-fibres are really complex structures, consisting of an axis-cylinder (which is a part of a nerve-cell) and a connective-tissue covering called the neurilemma. When only these two structures are present the fibre is said to be non-medullated. More usually there is between these two parts a white layer consisting chiefly of fatty material: this is called the medullary sheath, and it is always interrupted at intervals, called the nodes of Ranvier (see fig. 51). Such a fibre is a medullated fibre. As a rough rule we may say that non-medullated fibres are associated with unstriated muscle-fibres, and medullated with striated fibres and with most of the sense-organs. Nuclei are found at intervals along the course of all nerve-fibres, and these were formerly supposed to be the nuclei of cells which had united longitudinally to form the axis-cylinder. This is now known to be a mistake-the nuclei belong to the cells of the neurilemma; the only nucleus that can be said to belong to the axis-cylinder is that of the nerve-cell from which it is derived.

$\S$ 9. Functions of Nerve-fibres. Nerve-fibres are often compared to telegraph-wires, the nerve-cells answering to the telegraphic instruments in an office. The comparison is a useful means of realizing the working of the nervous system, but it cannot be carried into detail. For one thing, a nerve-fibre can only convey a message in one directionusually, but not always, from the nerve-cell to its outward termination. Nerve-fibres can be classed on this ground as afferent (conveying messages towards the central nervous system), efferent (conveying them away from it) and intracentral (conveying them from one part of the central system to another). Afferent and efferent fibres as they extend beyond the central system are bound together by connective tissue into bundles, and these into larger bundles or nerves. A nerve may consist entirely of afferent fibres, when it is 
said to be afferent or sensory; if entirely of efferent fibres, it is efferent. Most nerves contain both kinds and are called mixed.

Afferent fibres have their peripheral terminations in sense-organs-that is, in organs which are affected by external influences such as light, change of temperature, sound-waves, contact of external bodies, pressure, etc. ; and the afferent fibres convey impulses set up by these external agents to the central nervous system. Efferent fibres, on the other hand, end in connexion with muscle-fibres, gland-cells, and other cells which do work of any kind, and convey the impulses which cause them to contract, secrete, etc., or, in the case of unstriped muscle-fibres, impulses that tend to increase or to decrease their rhythmical contraction. Efferent fibres and efferent nerves are often called "motor," but this is a misleading term. Efferent fibres are (1) motor, when distributed to striped muscles, (2) accelerator, or (3) inhibitor when distributed to unstriped muscles, (4) secretory when distributed to glands. Even this does not quite exhaust the varieties of efferent nerve-fibres, but it covers most of them.

$\S 10$. Nerve-impulses. A muscle-fibre or gland-cell may be compared to the charge of explosive in a loaded gun : it contains a store of energy and is capable of doing a definite piece of work, but in order to start it to work some relatively small amount of work-the pulling of the trigger-must first be done upon it by an external agent. So a muscle (or, at least, a striped muscle) will not contract until it receives a stimulus from without, which, as it were, makes it explode. Effective stimuli of various kinds can be artificially applied to muscles, - a sharp blow on the bare muscle, a drop of acid, or an electric discharge. But under normal circumstances the stimulus is always an impulse sent from some nerve-cell along its axis-cylinder, which ends in a fine ramification over the surface of tho muscle-fibre.

What the nature of this propagation of a stimulus may be we do not know. It may perhaps be comparable to the firing of a train of gunpowder, if we could imagine only 
a small portion of the gunpowder to be burnt when the train was fired, so that the same train could be fired again. and again.

If we try to trace the origin of the stimulus farther back, we must ask what makes a given nerve-cell start an impulse in its axis-cylinder? The starting of the impulse, like a muscle-fibre's contraction, involves katabolism, and it seems that some nerve-cells (like unstriped muscle-fibres) tend to rhythmical katabolism as a result of mere healthy nutrition. But in a vast number of cases, at any rate, the katabolism is the result of a stimulus conveyed by another axis-cylinder. The axis-cylinder of one nerve-cell may end in a ramification which is entangled, as it were, with the dendrons of another nerve-cell, and in this way a stimulus be sent from the one cell to make the other send a stimulus to a muscle-fibre. In the long run the ultimate source of such stimuli is to be found in the sensory nerve-fibres. Light falls on the eye, the air or other external bodies press on the skin or alter its temperature, sound-waves beat on the ear, and so forth, and by these various external agents impulses are started along sensory nerves, whose fibres end within the central nervous system by ramifying

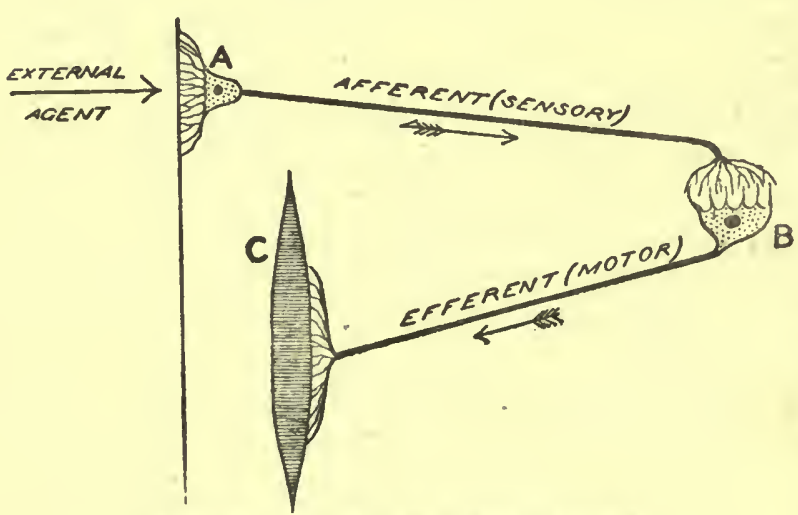

Fig. 52.-Diagram of Reflex Action. among the dendrons of nerve-cells. And thus motor impulses may be started as a direct or indirect result of a sensory impulse.

$\S 11 . \mathrm{Re}-$ flex Action.

A diagram of the simplest case imaginable is shown in fig. 52. Here external impulses directly affect the dendrons of a nerre-cell, $\mathbf{A}$. As a consequence, this cell discharges 
an impulse along its axis-cylinder, which ends in a ramification among the dendrons of a nerve-cell, $\mathrm{B}$, in the central nervous system. Through these the impulse is transmitted to the cell B itself, which in consequence sends an impulse along its axis-cylinder, which ends by ramifying over the surface of a muscle-fibre, $\mathrm{C}$, which is thus stimulated to contract. Since in this simple case the impulse sent into tho central nervous system is, in a sense, sent back or reflected at once, we call the whole process a reflex action. In one way this term suggests too great simplicity: the motor impulse is not simply the sensory impulse reflected, as a ray of light is reflected from a mirror; for at $B$, no less than at $U$ and at $A$, we have a store of enorgy that is unlocked by the impulse that arrives, and the result is in each case disproportionate to the exciting cause. We can find a bettel analogy by imagining a rifleman to dischargo a bullet at a machine which is thereby set in motion and fires a cannon.

In actual life no such simple reflex actions as we have been describing occur. For no external agent can have its effect. confined to one nerve-fibre; nor does one fibre end in a ramification about ono nerve-cell alone-it divides and influences many nerve-cells; while finally each nerve-cell in the central nervous system is surrounded by the ramifications of a number of nerve-fibres. Nevertheless, if we substitute groups of nerve-cells, nerve-fibres, and musclefibres for the solitary ones in our diagram, we shall approximate to a notion of the simplest cases of reflex action in the body of one of the higher vertebrates. Thus, if we go from the light into darkness, the pupil of our eye dilates without any knowledge or power of interference on our own part. Similarly, a sudden loud noise while I am writing will cause the muscles of my arm and hand to jerk my pen forward violently, quite against my will. Again, the sight of a body suddenly advancing towards the eye always causes the eyelids to blink. All these are reflex actions, effected in the way explained.

$\S 12$. Instinctive and Voluntary Actions. From simple reflex actions like these we pass to reflex actions of a much 
more complex nature, which constitute both what we call instincts and what we call habits. The former term covers all those which are determined by the inherited structure of the nervous system, while the latter includes those which owe their existence to structural characters in the nervous system that have been produced during the life of the animal.

Habits can only be formed as a result of those still higher modes of action which we call "rational" or "deliberate" or "voluntary"-actions which differ from all grades of reflex action by reason of their involving the association of ideas - a term under which we include all kinds of imagina. tion, memory, and reason. A young rabbit who by mistake eats some unpleasant-tasting vegetable is not likely to eat it again, because the image of the same vegetable focussed on the retina of his eye will not merely give rise in his brain to the simple sensations of colour, form, etc., as in the case of an unknown plant, but these in turn will awaken a vivid sense of the unpleasantness of taste associated with them. In this and similar ways a rabbit is educated, and all education is concerned with the association of ideas.

The distinction between deliberative actions and instinctive ones thus appears perfectly definite; but in practice the line is hard to draw, as instinctive actions may be started, or modified, or stopped in the course of deliberative ones. Moreover, deliberative actions, when often repeated, pass into habits, in which association of ideas no longer takes any part. This is strikingly shown in the case of civilized mon, in whom actions of a most complicated kind, most slowly and painfully acquired, become apparently instinctive. Writing from dictation, for example, is carried on without a thought of how a word should be spelled or how each letter should be shaped. Reading aloud from a book may be carried on without a thought of the spelling of the words, and even without a thought of the sense.

$\$ 13$. Consciousness. It is apparently only in really deliberative actions that consciousness is at all involved. It is of course impossible to know whether an animal is conscious or not, and, in arguing from analogy, we have not 
even the advantage of understanding our own consciousness with any completeness. But we know that a very large part of the activity of our own nervous system is carried on without the production of consciousness at all, or at least without any that enters into the course of that continuous consciousness which gives us our individuality. If even such elaborate and painfully learned actions as walking and writing can be carried on without consciousness entering into the details of them, we may well doubt the existence of any clear consciousness in animals with much simpler nervous systems. Without association of ideas consciousness can at best be but a series of immodiately forgotten dreams; and we shall see that even among vertebrata association of ideas is not a universal possession, while in still lower animals we can hardly find a trace of it. 


\section{Chapter IX.-The Nervous System.}

$\S 1$. Central Nervous System. We must now consider the arrangement of the nervous system in more detail. We have already said that in the rabbit the central nervous system consists of the brain and spinal cord : it is well to insist at this stage that these are really one and not two. The brain is simply the highly specialized anterior end of the spinal cord. The central nervous system is, in the earliest stages of its development, a simple tube of nerve-cells, and though it undergoes great changes in form in its development, this primitive tubular character is still shown by the presence of a central canal in the spinal cord, which expands into the cavities or ventricles of the brain.

$\S 2$. The Spinal Cord. The general structure of the spinal cord and its related nerves will be best understood from the diagrammatic transverse section of it (fig. 53). The exact outline of such a section varies with the region of the cord, but in all cases it is approximately elliptical, and is nearly cleft in two by the dorsal and ventral fissures (occupied by connective tissue). Two kinds of tissue are seen - the grey matter, $\mathrm{H}$-shaped in section, with the central canal in the middle of the cross-bar, and the white matter around this. The former consists chiefly of nerve-cells and non-medullated fibres, the latter of medullated fibres running longitudinally.

The spinal cord gives off a paired and metameric series of spinal nerves, which alternate with the metameric vertebra. These nerves are numbered according to the vertebra in front of them-thus the nerve-pair between the third and fourth lumbar vertebræ is called the third lumbar pair of nerves. An exception is made for the cervical region, because the first spinal nerve emerges between the skull and the atlas; thus there are eight pairs of cervical nerves. 
§ 3. Dorsal and Ventral Nerve-roots. Each nerve arises by two roots-a dorsal and a ventral root (often called posterior and anterior after human anatomy). The dorsal root consists entirely of afferent fibres, and is hence also called the sensory root; while the ventral contains only

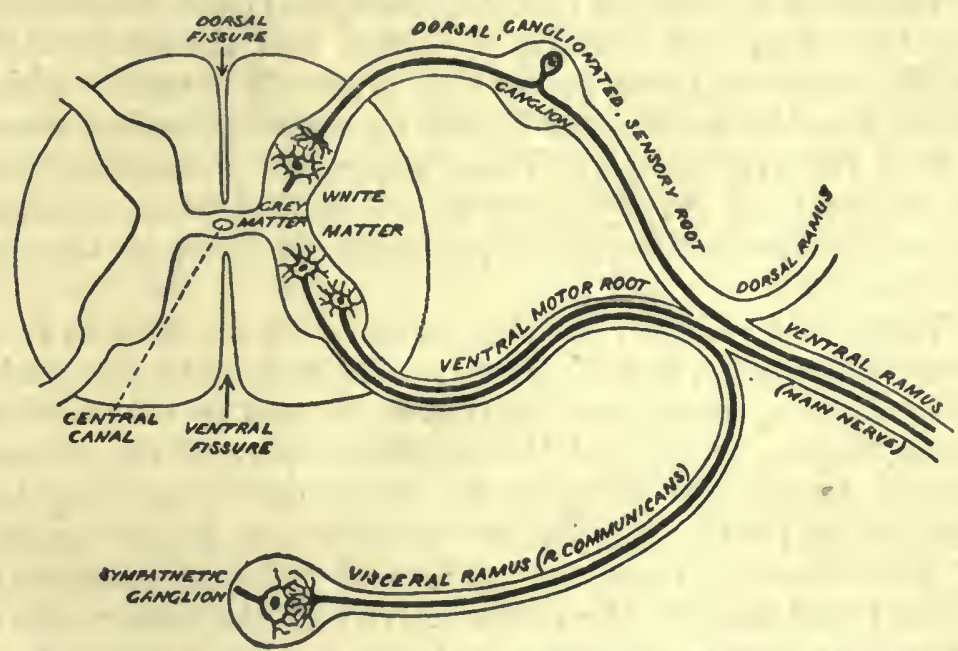

Fig. 53.-Diagram of the Spinal Cord, Dorgal and Ventral Nerve-Roots, SPINAI AND 8yMPaTHETIO Ganglia.

The thick black lines represent nerve-fibres.

efferent fibres, and is called the motor root. These roots soon unite, and the combination immediately splits up into three branches, all of which probably contain fibres of both kinds : these branches are a small dorsal branch to the region of the back, a large ventral branch to the main region of the body-wall (so much larger than the other two that it is commonly called the spinal nerve), and a visceral branch, or " ramus communicans," to which we shall return presently.

There is another difference between the two nerve-roots besides the difference in the direction in which they convey impulses. On the dorsal root there is a swelling, which on microscopic examination is found to contain unipolar nervecells: Any such collection of nerve-cells outside the central nervous system is called a ganglion*; these spinal ganglia

* This definition of ganglia applies to vertebrates only. ZOOL 
on the dorsal roots form an important distinction from the ventral roots. Of the two branches into which the axiscylinder of each nerve-cell in these ganglia divides, one passes outwards to terminate in a ramification just under the epidermis, or possibly in some special sense-organ.

The other branch, on entering the spinal cord, divides into two branches, one running forwards, and the other backwards ; each of these repeatedly gives off branches which (as well as the main threads) end by an arborization round cells in the grey matter. These latter cells are mostly those which send off axis-cylinders to the ventral root, but there are some whose axis-cylinder-processes run forwards towards the brain.

These latter fibres, as well as those which come in from every dorsal root, are all afferent with regard to the brain. They form a large part of the white matter: the whole dorsal region of it, and the superficial part of the ventrolateral region are occupied by fibres conveying impulses brainwards ; while the deeper ventro-lateral region consists of fibres coming from the brain, and bringing impulses to control and modify the reflex activity of the motor cells of the grey matter, among whose dendrons they ramify.

§ 4. Sympathetic System. The visceral branch of the spinal nerve ends in another ganglion, one of a paired metameric series called the sympathetic system, and united from end to end of the body by nerve-fibres into a continuous nerve-chain. From these ganglia nerve-fibres pass to all parts of the alimentary canal, and to the walls of all blood-vessels (including the heart) - in fact, to all the visceral (unstriated) muscles. As already hinted (chap. viii., § 8), these fibres are non-medullated. Most of them are efferent, naturally; there are no sense-organs of such importance in these internal tissues as there are on the surface of the body The function of the sympathetic ganglia seems to be to act as relays or subsidiary governing centres. The fibres from the spiral cord do not pass direct to the muscle-fibres in the wall of the intestine-they end by ramifying round cells of the sympathetic ganglia, and these, when stimulated by them, send off the impulse to the muscle-fibres. The rpinal 
cord, as it were, sends a general order for muscular contraction to be accelerated or inhibited in a particular region; and the sympathetic translates this into the necessary detailed orders. But it has no power of reflex action.

The metamerism of the sympathetic chain is imperfect in the neck-region, where only three pairs of ganglia are found -an anterior (or superior) cervical close to the head and a middle and posterior (or inferior) cervical near the subclavian artery. In the head the disposition of the sympathetic is still more obscure, but there are some four pairs of ganglia that appear to belong to it.

From several of the hinder thoracic ganglia of the sympathetic fibres come off and unite into a great splanchnic nerve on either side. These pass back through the diaphragm and across the aorta into the mesentery, between the coliac and superior mesenteric arteries. Here they pass into another set of ganglia, from which accelerator and inhibitor fibres are finally distributed to the unstriped muscles and secretory fibres to the gland-cells of the abdominal part of the alimentary canal. These ganglia have so many fibres radiating out from them as to have a stellate appearance and somewhat indefinite shape. The largest ganglion is called the coeliac ganglion, and the second the superior mesenteric, after the arteries near them. A small renal ganglion-really a portion of the cœliac-may or may not be recognizable as a distinct ganglion. The whole of these ganglia and nerves are collectively spoken of as the solar plexus. There is also a more distant inferior mesenteric ganglion near the artery of the same name.

§5. Spinal Nerves. Most of the spinal nerves are dis-tributed to the skin and muscles of the region they belong to; but a few deserve more special mention. The third cervical nerve sends off a large branch (great auricular. nerve) to the external ear. The fourth cervical has a branch which, after receiving tributaries from the fifth and sixth cervical, runs back along the precaval vein and by the side of the heart to the diaphragm-the phrenic nerve. If this nerve is injured at any point in its course, the diaphragm no longer acts, breathing ceases, and death quickly ensues. 
The last four cervical (5th to 8th) and first thoracic branch out and unite in different combination, so forming the brachial plexus, from which come the important nerves of the arm. Similarly, the last four lumbar and first two sacral give rise to a lumbo-sacral plexus, from which the main nerves to the leg are formed.

§6. The Brain of the rabbit is a highly complex structure, and the difficulties of understanding it are

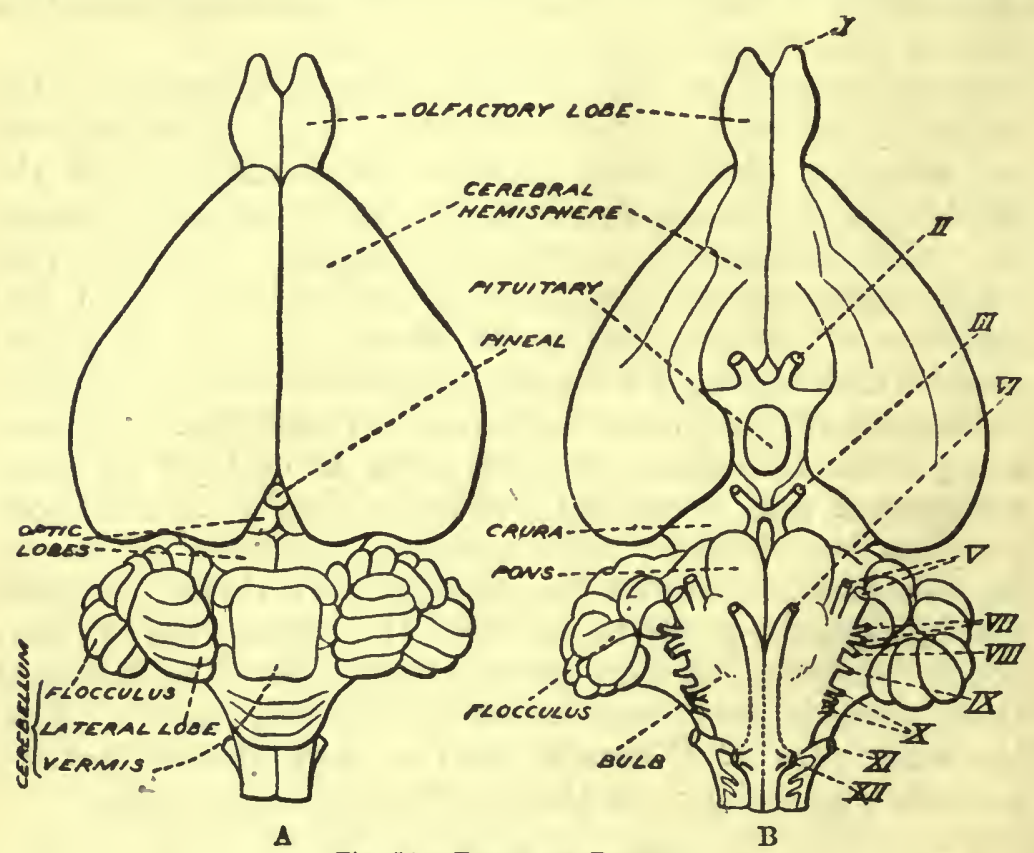

Fig. 54.-Brain of Rabit.

A, Dorsal view ; B, Ventral view. (After Wiedersheim.]

aggravated by the nomenclature of its parts, which is largely based on the very fanciful ideas of the older anatomists on the still more complex human brain. Fortunately, a new nomenclature, based on a more rational system, has grown up of late years; but the old terms have still to be learned.

As already mentioned, the central nervous system is at first a tube of nerve-tissue. The anterior end, which 
is the future brain, becomes swollen up into three hollow structures called the three cerebral vesicles (fig. 55). The first vesicle becomes the primitive fore-brain, or thalamencephalon; the second becomes the mid-brain. or mesencephalon; the third, the hind-brain, or met-encephalon. From the first vesicle are developed a pair of hollow outgrowths, the cerebral hemispheres, or prosencephalon, prolonged forward into the olfactory lobes. At first, in the embryo, the walls of these vesicles and of the spinal cord consist of a mass of simple cells, but as development proceeds these cells multiply-to a greater extent in some regions than in others. They also send out axis-cylinder processes which, pushing their way in various directions,

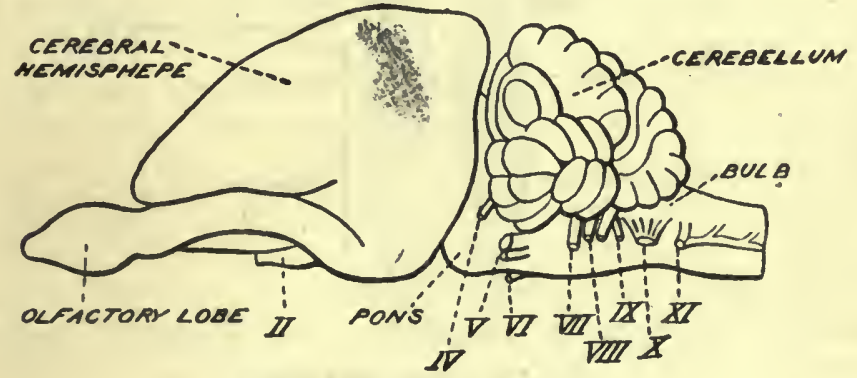

Fig. 55.-Brain of RabBit.

Sido view, (After Wiedershøim.)

give rise to the bands of white matter that we have seen in the spinal cord, and which are also found in the brain, though not so simply arranged. Thus, by growth of nerve-cells (forming grey matter) in some regions, and the convergence of numerous fibres (forming white matter) in others, the complex brain of the adult is at length built up. Diagrammatic sections of it are shown in figs. 56 and 57 ; and actual views of it in figs. 54 and 55.

The processes of growth in the brain very nearly obliterate the original cavities of the vesicles, but they can still be recognized and now receive fresh names. The cavities of the cerebral hemispheres are called the lateral (or first and second) ventricles; that of the first vesicle is the third ventricle; that of the third 
vesicle is the fourth ventricle; while that of the second vesicle, being reduced to a narrow passage, received from early anatomists the pompous title of iter a tertio

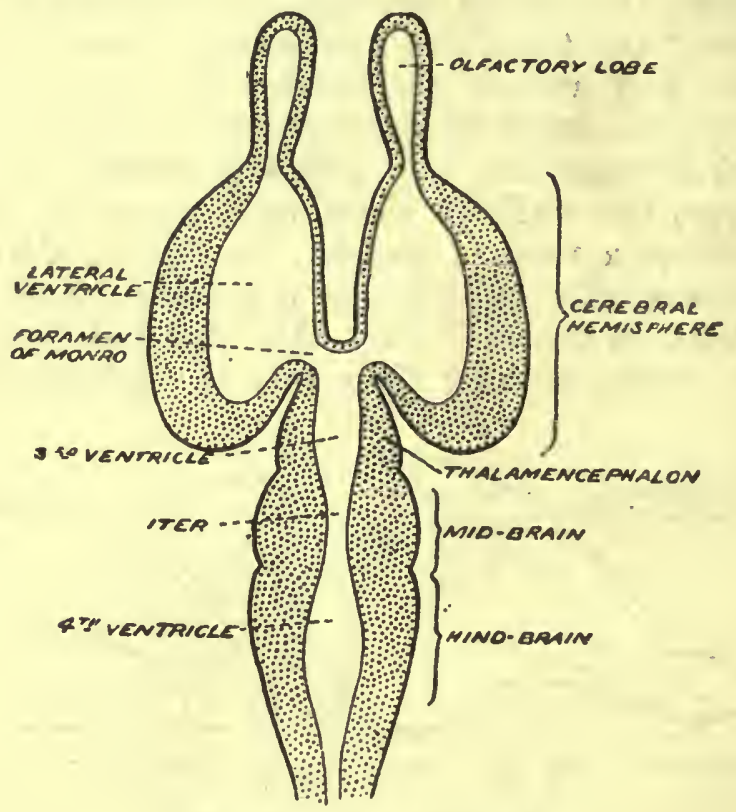

Fig. 56.-Brain of Rabit.

Diagram of a horizontal section.

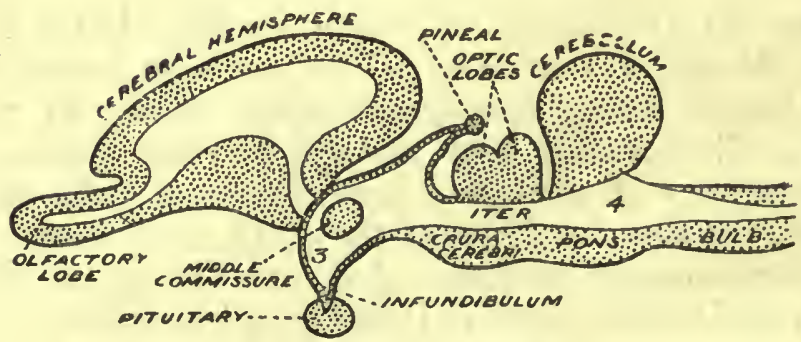

Fig. 57.-Brain of liabBit.

Diagram of a median (sagittal) section.

ad quartum ventriculum: modern biologists abbreviate this to the simple word iter. The difference in the numbering of the embryonic vesicles and the adult ventricles 
should be carefully borne in mind by the student. The openings from the two lateral ventricles into the third are known as the foramina of Monro (fig. 56).

The roof of the first vesicle is prolonged up into a conical top, at the end of which a mysterious body, the pineal body, is found; its floor is similarly prolonged down into a funnel or infundibulum, with the equally mysterious pituitary body; its side-walls become the optic thalami. The roof of the second vesicle forms the optic lobes, or corpora quadrigemina; its floor (consisting largely of nerve-fibres running towards the cerebral hemispheres) has its right and left halves, each known as a crus (plural crura) cerebri. The roof of the hind-brain has its anterior portion greatly developed into the cerebellum (consisting of five lobes-a median vermis, lateral lobes, and still more lateral flocculi), while its posterior portion, on the contrary, is extremely thin. The part of its floor beneath the cerebellum is called the pons Varolii, or more briefly, the pons; while the hinder part is the medulla oblongata, more conveniently called the bulb: this gradually passes back into the spinal cord. The cavity of the bulb (fourth ventricle) is very large, and has no roof of nerve-tissue (only connective tissue). Traced back, this cavity narrows and becomes divided into the dorsal fissure and central canal of the cord.

§ 7. White and Grey Matter in the Brain. We have seen (\$3) that the nerve-fibres to and from the brain occupy definite positions in the white matter of the spinal cord. It is a very remarkable fact that somewhere in the course of its journey every such fibre crosses from one side to the other (left to right or right to left). This crossing is called decussation: it happens to certain fibres in the spinal cord itself, but the main region of its occurrence is in the bulb.

If we took a series of transverse sections from the spinal cord forwards through the bulb, we should find the first important change in the appearance of the sections to be due to this decussation. Besides this we find that although grey matter continues to form a central mass around the central canal all the way to the front of the mid-brain, scattered patches of grey matter also appear among the white matter, and the whole appearance of the transverse section loses its simplicity. The central canal becomes more dorsal in position, its roof thins away, and, combining with the dorsal fissure, it enlarges into the fourth ventricle.

Finally in the cerebral hemisoberes. and in the cerebellum, the 
grey matter is entirely outside the white matter-exactly the opposite condition to that in the spinal cord.

Owing to the importance of the cerebral hemispheres and cerebellum as nerve-centres for the regulation of the most complex actions, the main strands of white matter in the brain are those running to and from these organs, as well as between their left and right portions. These may be briefly mentioned here. The right and left cerebral hemispheres are connected to one. another by a number of commissures, as such transverse bands of nerve-fibres are called. The largest of these in the rabbit is the great flat corpus callosum, seen on the dorsal aspect when the two hemispheres are gently pressed apart. The postero-ventral continuation of this is called the fornix. Two other smaller ones run in the anterior and posterior walls of the thalamencephalon, and are called the anterior. and posterior commissures. Lastly a large middle commissure runs right across the third ventricle, almost filling up the whole cavity (fig. 57). The crura cerebri have-already been mentioned as consisting of great bundles of fibres running to or from the hemispheres. Similar bundles running up into the cerebellum from all directions form its anterior, middle and posterior peduncles (all paired): the anterior connecting it with the hemispheres, the posterior with the spinal cord, while the middle ones connect the right and left lateral lobes with one another through the substance of the pons. The fibres of the anterior peduncles decussate in their course through the mid-brain.

§ 8. Cranial Nerves. The nerves which come off from the brain, or cranial nerves, differ from the spinal nerves, firstly in not showing such distinct metamerism, secondly in not having dorsal and ventral roots, and thirdly in that they are not all mixed nerves, some being purely motor or purely sensory. They are usually counted as forming twelve pairs, and numbered accordingly, it being customary to distinguish the cranial nerves by Roman numerals.

It is convenient to divide them into four categories.

A. Purely sensory nerves.

I. Olfactory.

II. Optic.

vili. Auditory.

\section{$B$ Purely motor-(to muscles of eye only).}

III. Oculomotor.

Iv. Pathetic or trochlear.

vi. Abducent. 
C. Mixed nerves, ganglionated at the root.

v. Trigeminal.

vII. Facial.

Ix. Glossopharyngeal.

x. Vagus or pneumogastric.

D. Purely motor nerves (to muscles of neck and tongue). xI. Spinal Accessory.

xiI. Hypoglossal.

In describing each of these cranial nerves there are three points to be noted-origin, course, and distribution.

By origin is meant the part of the brain with which the nerve is visibly connected (sensory as well as motor nerves being spoken of as running from the brain outwards). More strictly this is its superficial origin, for the individual nerve-fibres may run some way through the brain itself before they terminate in nerve-cells or in ramifications around nerve-cells. But this deep or internal origin is only determined by great refinements of investigation, and for our pürpose the superficial origin will in most cases suffice.

The course of a cranial nerve is not always straight to its destination, as might be expected. The peculiarities in the course of nerves are often quite incomprehensible from the point of view of the particular type studied, but may be explained by a comparison with other types, as we shall see in several cases.

The distribution of a nerve means the distribution of the ends of its fibres remote from the brain, whether in nervecells or in ramifications.

$\S 9$. Olfactory and Optic Nerves. These nerves differ from all other cranial and spinal nerves in that they consist of fibres whose nerve-cells, instead of being situated either in the central nervous system or in ganglia at the sides of it, are situated in the sense-organ-viz. in the olfactory membrane of the nasal passages, and in the retina of the eye, respectively. On strict morphological grounds we ought not to count these as nerves at all, but rather as tracts of white matter constituting outlying parts of the 
central nervous system. It is convenient, however, to treat them as true nerves; and we may then say that the olfactory nerve (x.) has its origin in the olfactory lobe, runs through numerous foramina. in the ethmoid bone, and is distributed to the olfactory membrane.

We may mention two interesting points: first, that the olfactory fibres are the only ones in the system which do not decussate at all; and secondly, that they are nonmedullated.

The optic nerve (II.) has its superficial origin in the floor of the thalamencephalon, just in front of the infundibulum : this region is called the optic chiasma (i.e. crossing), because here the fibres decussate. The deep origin is, however, in the optic lobes (corpora quadrigemina), whence the fibres may be traced through the side-walls of the thalamencephalon (hence called optic tracts) to the chiasma. The course of the optic nerve is forwards and slightly outwards, through an opening in the orbitosphenoid called the optic foramen (fig. 46), straight to the eye.

The auditory nerve (virr.), although we have grouped it with these two as a purely sensory nerve, does not agree with them in the peculiarities above-mentioned. We shall consider it in its proper order later on.

\$ 10. Oculomotor Series. Nerves III., IV., and vi. are solely concerned with the innervation of (i.e. the supply of nerve-fibres to) the muscles of the eye, and we shall mention their exact distribution when describing that organ. They are peculiar in their superficial origin, IIr. and VI. arising from the floor of mid- and hind-brain (crura and pons) respectively, while IV. arises from the dorsal surface, in the depression between the optic lobes and the cerebellum. Yet their deep origin is practically the same, being the tract of grey matter around the iter. If these three nerves are to be compared with spinal nerves at all, they must be regarded as ventral roots only: the ventral emergence of III. and VI. agrees with this, but the dorsal appearance of $\mathrm{IV}$. is a puzzle. All three nerves leave the skull through a foramen in the alisphenoid called the foramen lacerum anterius (fig. 46). 
§ 11. Posterior Cranial Nerves. The next series of nérves (v., viI., IX., and $\mathrm{x}_{\text {.) }}$, have much more resemblance to spinal nerves. They are mixed nerves, i.e. contain both sensory and motor fibres, though some of their branches may contain only one of the two. Their sensory fibres come from unipolar cells in a ganglion at the side of the hind-brain (bulb), and they have branches running to other ganglia which resemble those of the sympathetic. But the dorsal and ventral roots tend to run together, and are never as distinct as those of spinal nerves. These nerves, along with vIIr. (auditory), arise in order from the sides of the bulb.

$\S$ 12. Trigeminal Nerve. Nerve v. has a large ganglion (the gasserian) from which its sensory fibres come. It divides into three branches (hence its name). The first two are sensory: they emerge from the skull by foramina in the alisphenoid, called the foramen lacerum anterius and foramen rotundum respectively.* The first (v.1), called ophthalmic, runs on the dorsal side of the eye, and: is distributed to the skin of the upper part of the face. The second, or maxillary $\left(v_{\cdot 2}\right)$, runs ventral to the eye, through the maxilla, and is distributed to the skin of the snout; especially to the tactile "whiskers," or vibrissæ. The third branch, or mandibular ( $\left.v_{\cdot 3}\right)$, runs through the foramen ovale * then turns down towards the lower jaw, sends a branch to the tongue (its only sensory, gustatory branch), continues on the inner side of the mandible, eventually passes through a foramen in the mandible and is distributed to the muscles of the lower jaw, and to the teeth.

$\S 13$. Facial Nerve. Nerve vir., though included in the mixed nerves, is practically all motor in the rabbit. Its relation to the ear should be noted. It is strictly anterior to the essential parts of the ear (internal ear); but runs through the periotic bone and then bends back so as to run posterior to the tympanic chamber (chap. x., §6), and emerges

* These statements are strictly true of the dog, but in the rabbit these three foramina are united into one. These and the foramina subsequently named are all shown on figs. 45 and 46. 
from the skull behind the bulla, by the stylo-mastoid foramen. It then runs forwards close under the skin of the cheek as the facial nerve, and is distributed to the muscles of the face. Besides this main trunk, two other small branches must be noted-a palatine branch, given off before the

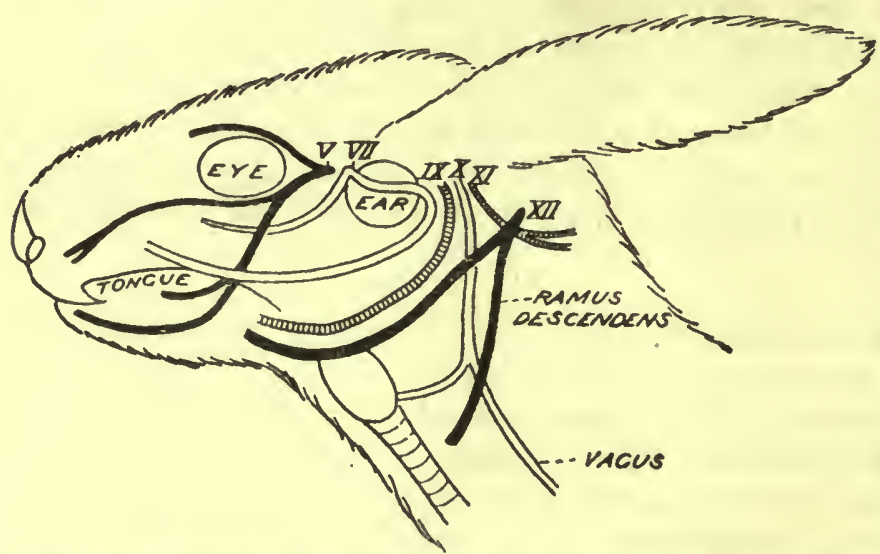

Fig. 58.-Distribution of Ciref Cranial Nerveg of Rabbit.

tympanic chamber is reached, and a chorda tympani, given off in that chamber and distributed to the salivary glands.

\$14. Auditory Nerve. Nerve virI., unlike the two in front and the two behind it, is purely sensory, yet it has no ganglion. Its fibres belong to $b i$-polar cells within the bulb itself. It enters the periotic bone, but never emerges from it to the exterior of the skull, as it is exclusively distributed to the internal ear, or essential organ of hearing, which is completely enclosed in the periotic.

$\S$ 15. Glossopharyngeal Nerve. Nerve Ix, emerges by the foramen lacerum posterius, curves round between the larynx and the posterior cornu of the hyoid, and is distributed to the sides of the throat and the posterior half of the tongue. It is mainly sensory (nerve of taste), but in part motor.

$\S 16$. Vagus Nerve. The tenth nerve has such a remarkable distribution for a cranial nerve, extending as it 
does to the heart and stomach, that it is called the vagus (wanderer), or pneumogastric. It leaves the skull along with IX., and runs down the neck and along the osophagus. Its principal branches and their distribution are as follows :-

(1) Superior laryngeal, given off opposite the larynx and running straight to its distribution in that organ.

(2) Cardiac depressor, given off at the same point as the above, running down the neck parallel to the main trunk of the vagus, but passing on the dorsal side of the great arteries while the main trunk passes ventrally. Distributed to the heart.

(3) Recurrent laryngeal. This branch is doubly interesting as a very pretty case of the determination of a nerve's course by other considerations than the needs of the animal, and as the only important instance of asymmetry in the rabbit's whole nervous system. It is therefore worth dwelling on at greater length than the other branches. The distribution of this branch is to the larynx, but instead of going direct to its destination, the fibres which compose it go by a most roundabout way. They run in the main trunk as far as the level of the subclavian artery on the right side, and still farther on the left side, namely to the level of the ductus arteriosus. At these points they separate into distinct branch-nerves and, looping under the great arteries, retrace their course along the neck to the larynx.

The explanation both of the curious course and of the asymmetry is to be found in the vascular system. When the heart is first developed in the embryo rabbit, it lies just beneath the throat, as it does permanently in all fishes. The two nerves to the larynx thus happen to run, one in front of, the other behind the great arterial arches. As the heart shifts its position into the thorax, and the neck lengthens, the first nerve (superior laryngeal) is unaffected; but the second becomes drawn out into a long loop, which is longer on the left side than on the right because the arches persist on the former but not on the latter side (fig. 59).

(4) A direct branch to the heart, given off as the main trunk passes behind the precaval vein.

(5) A direct branch to the lung. 
(6) The residue of the nerve after all these branches have been given off, runs on either side of the cesophagus, and ends in connexion with the solar plexus.

$\S 17$. Spinal Accessory Nerve. The eleventh nerve is peculiar. It arises by the union of fibres from several of the spinal nerves of the neck, as well as some from the vagus, and can only get out of the skull by first running

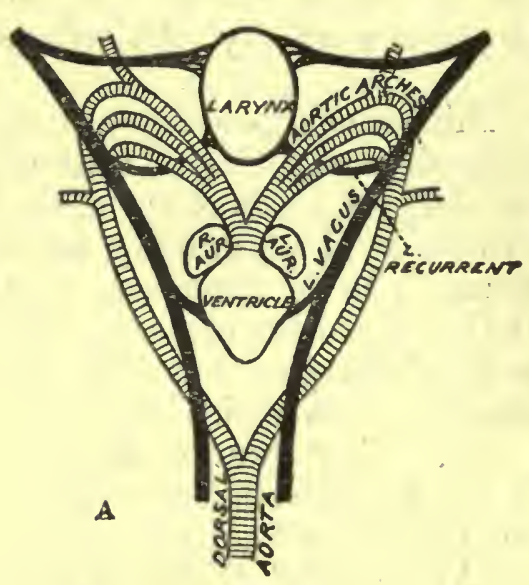

Fig. 59.-Relation of Vagus to Chief ARTERIES.

A, in embryo Rabbit and adult Frog; B, in adult Rabbit.

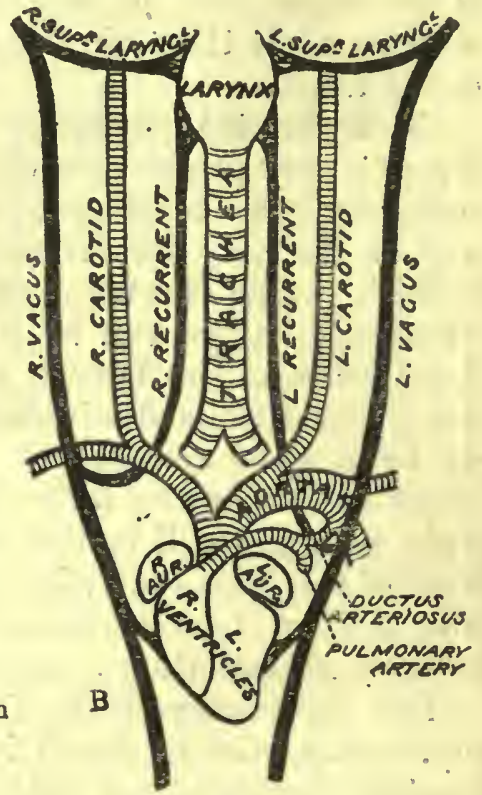

into it by the foramen magnum. It passes out by the foramen lacerum posterius, and is distributed to some of the neck muscles.

§ 18. Hypoglossal Nerve. The twelfth, hypoglossal nerve, is equivalent probably to the ventral roots only of several spinal nerves: it arises from the ventral surface of the medulla, passes through the condylar foramen, and is distributed to the muscles of the torgue, being purely motor. A small branch (ramus descendens) goes to one of the neck-muscles. 


\section{Chapter X.-Senge-Organs.}

§ 1. Sensation. We now turn to the chief sense-organs to see what their structure is, and how they are enabled to translate external forces into nerve-impulses which produce sensations in the brain. We say "translate," and it is important to fully realize that the nerve-impulse is a totally different thing from the external force which rouses it, with which it does not correspond either quantitatively or qualitatively. We have no reason to doubt that a nerve-impulse travelling along the fibres of the auditory nerve is precisely the same as a nerve-impulse travelling along the fibres of the optic nerve. The essential differences in the two cases are, firstly, in the capacity of one senseorgan to be stimulated by air-waves, and of the other to be stimulated by ethereal waves; and secondly, in the capacity of one part of the brain to produce the consciousness we call "light" when stimulated, and of the other to produce the consciousness we call "sound." If we stimulate the optic nerve-fibres in any way, the same sensation of light is produced in the brain, as the familiar experience of seeing coloured patterns when the closed eye is pressed upon shows.

$\S 2$. Simplest Sense-organs. The simplest kind of sensory nerve-ending is a simple arborization under the epidermis : only vague sensations are obtained through the help of this. For the definite sense of touch to be effected the arborizations are ensheathed in a series of layers of cellular connective tissue, the whole forming a somewhat bulbous body (see fig. 13) to which various names are applied, according to the details of structure (tactile corpuscles, end-bulbs, Pacinian corpuscles, etc.). In the other important senseorgans we find a specially modified epithelium as the organ directly affected by external agents. Around the bases of the cells of this epithelium the sensory nerve-fibres form an arborization. 
§ 3. Taste-organs are formed by portions of the epithelium of the tongue. Each consists of a number of cells with fine projecting processes, arranged in the form of a bulb (fig. 60, B). These are not found all over the surface

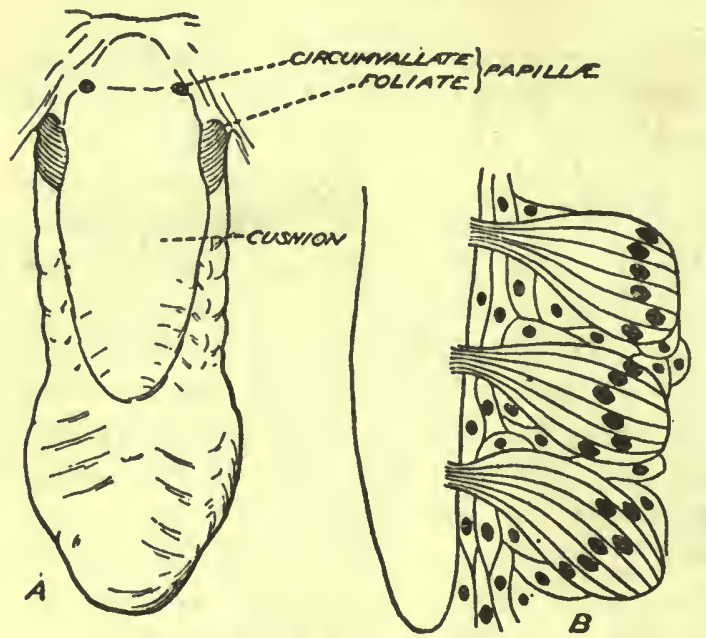

Fig. 60.-Tongur or RabBit.

A, Surface; B, Section through a fold of the Foliate Papilla, showing three taste-bulbs. (A, natural size, original; $\mathrm{B}$, greatly magnified, after Klein.) of the tongue, but are aggregated in areas called the papilloe, which are easily seen by the naked eye (fig. 60 , A). Most of these are little projections scattered around the tip; but in addition there are two pairs of special papillæ in the posterior region-the foliate papillæ are oval areas crossed by fine parallel grooves, in whose sides the tastebulbs occur; the circumvallate papillæ are rounded protuberances, each surrounded by a depression : the taste-bulbs are found round the sides of the protuberance.

§ 4. The Organ of Smell is at first, in the embryo rabbit, a simple pair of sacs each with an external opening. Later, internal openings to the throat are formed (posterior nares). The whole epithelium of the sac is sensitive to odorous vapours, and, as it develops, the epithelium with the connective tissue and cartilage that surround the sac forms a series of projections into the cavity of the sac: these become rolled up in the complicated manner that we have already noted in the skull of the dog (turbinal bones). The advantage of this complication is that without any enlargement of the olfactory sacs, an enormous increase of the sensitive surface is obtained, and so an increased keenness 
of scent. The sensitive epithelium consists partly of ordinary columnar cells, and partly of spindle-shaped cells lying between and partly below them. The latter are really nerve-cells; but in the place of branching dendrons, each one sends up one fine process to the surface, ending in fine projections (often called cilia, but that term is misleading, as they are not vibratile) which are stimulated by any vapours that come in contact with them. At the lower end each cell sends off an axis-cylinder which becomes that of one of the non-medullated fibres of the olfactory nerve. These end in arborizations around nerve-cells in the olfactory lobe.

§ 5. The Ear of the rabbit (fig. 61) is an extremely complicated organ, of which the externally visible part is the least important. The essential part (called the internal ear, or membranous labyrinth) is quite enclosed in the periotic bone. The internal ear consists of two sacs, indirectly united by a $\mathrm{Y}$-shaped tube. From one of these, the utriculus, three curved tubes, the semicircular canals, spring. The planes of the three canals are mutually at right angles; they are respectively an

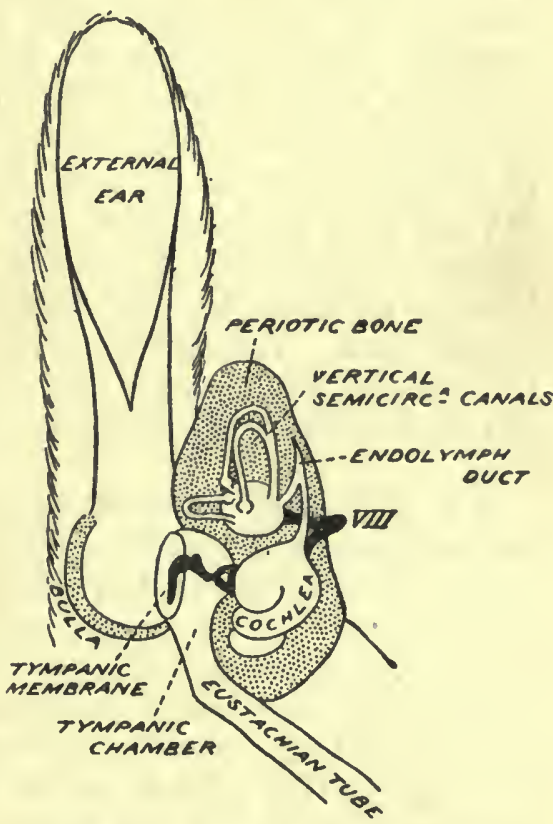

Fig. 61.-Diagram of Ear of Rabitt. anterior vertical, a posterior vertical, and a horizontal canal. There is a dilatation, called an ampulla, at the posterior base of the posterior, and similar ampullæ at the anterior ends of the anterior and horizontal canals. These ampullæ are lined by an epithelium containing cells with projecting processes zOOL. 
much like those of the olfactory cells : they are not nervecells, however; but their base is arborized around by a fibre of the eighth nerve.

The second sac, or sacculus, has a spirally twisted outgrowth resembling a snail-shell in form, the cochlea, whose epithelium resembles that of the ampullæ. This last part is distinctive of the mammalia, but the rest of the internal ear is represented in all vertebrata, except Amphioxus. The whole of the labyrinth is membranous, and contains a fluid, the endolymph; between the membranous wall of the labyrinth and the enclosing bone is a space containing the perilymph. The latter space is part of the general lymphatic system. Strange as it may appear at first, the entire lining of the internal ear is, at an early stage, continuous with the general epidermis of the animal. It grows in, just as a gland might grow in, and is finally cut off from the exterior; but a considerable relic of this former communication remains as the $\mathrm{Y}$-shaped blind tube already referred to, the ductus endolymphaticus or endolymph-duct. The eighth nerve runs from the brain case into the periotic bone, and is distributed to the several portions of this labyrinth, especially to the ampullæ and cochlea.

§ 6. Accessory Parts of the Ear. In fishes this internal ear is the sole and sufficient organ of hearing; the soundwaves transmitted by the water travel through the elastic cranium and so reach and affect the nerve-endings, just as we can hear the ticking of a watch that touches our head when our outer ears are stopped up. But in all air-frequenting vertebrates this original plan of an ear has to be added to, to fit it to the much fainter sound-vibrations of the compressible air. "A receiving apparatus" is needed, and is supplied by the ear-drum, middle ear, or tympanic cavity. In the mammal there is also a collecting ear-trumpet, the ear commonly so-called, or pinna, enclosing an external auditory meatus. A tightly stretched membrane, the tympanic membrane, separates this from the drum. A chain of small bones, the malleus, incus, os orbiculare (a very small bone), and stirrupshaped stapes, collectively called the "auditory ossicles," 
swing across the tympanum, from the tympanic membrane to the internal ear. At two points the bony investment of this last is incomplete-at the fenestr'a rotunda and at the fenestra ovalis, into which latter the ond of the stapes fits, and so communicates the sound-vibrations of the tympanic membrane to the endolymph. A passage, the Eustachian tube, communicates between the tympanic cavity and the posterior narial paissage, and serves to equalize the pressure on either side of the drum-head.

$\S 7$. The Sense of Direction. So far we have spoken of the ear as the organ of hearing simply; but the two parts of the labyrinth really have two different functions. It is only the sensory cells of the cochlea which are stimulated by the vibrations transmitted to the endolymph by the auditory ossicles. The sensory cells of the ampullæ are also stimulated by the varying pressure of the endolymph, but this varies according to the position of the three canals relative to the direction of gravitation. Hence the semicircular-canal system serves as an organ of direction, and is of the greatest importance in connexion with the maintenance of equilibrium, as we shall see presently (\$ 12). The arrangement of the canals in three planes mutually at right angles is a means by which endolymph-pressure is resolved into three components which can be separately appreciated by the brain. The giddiness which results from violently whirling oneself round is due to the rush of endolymph causing abnormal pressure on the sensory cells of the ampullæ, and so utterly disturbing the sense of direction. In birds, whose wonderful powers of flight and migration involve an extraordinary sense of direction, the canals are highly developed and very nearly circular.

$\S$ 8. The Eye (fig. 62) has a tough outer coat of white fibrous connective tissue, the sclerotic, within which is a highly vascular layer, the choroid, within which comes the essential, sensory layer, the retina. The chamber enclosed by these three layers is filled with a transparent jelly, the vitreous humour. In front, the white sclerotic passes into the transparent cornea. The epidermis is continued over 
the outer face of this as a thin, transparent epithelium, called the conjunctiva. The choroid coat is continued in front by a muscular curtain, the iris, which shows through. the cornea as the

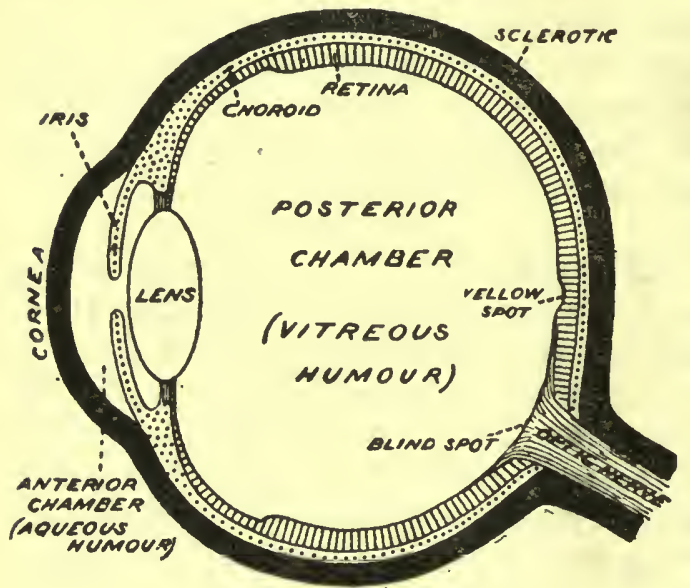

Fig. 62.-Eye of RaBbit.

Diagram of a modian section. coloured portion of the eye. This iris has a central aperture (the pupil), and as the amount of light diminishes or increases, the size of the pupil is adapted to the vàrying conditions by the greater or less contraction of the muscle-fibres of the iris: this adaptation is a reflex action. The iris serves precisely the same function as the diaphragms or "stops" used in the photographic camera or under the stage of the microscope, viz. the cutting off of oblique rays of light that tend to fog the image.

Just behind the iris is the transparent biconvex lens, the curvature of whose surfaces may be changed at will by the contraction of the ciliary muscles which pass from the sclerotic into the choroid, and pull the latter forward. By this alteration of the curvature of the lens and therefore of its focal length, the image of either a near or a distant object can be clearly focussed on the retina. The lens separates off from the main chamber of the eye containing vitreous humour, a smaller anterior chamber containing a much more liquid aqueous humour.

$\S 9$. The Retina. Fig. 63 shows in a very diagrammatic way the structure of this essential layer of the eye. The only structures which are actually sensitive to light are certain modified cells, known as the rods and cones. These 
form the layer of the retina nearest the choroid, except for a layer of deeply pigmented (i.e. black) tissue, which embeds the ends of the rods and cones. Within the rod-and-cone layer (i.e. nearer the vitreous humour) comes a layer of bipolar nerve-cells, with only short arborizing processes, and then a layer of larger nerve-cells, whose axis-cylinders become the fibres of the optic nerve. These fibres converge from all parts of the retina (forming the inner-

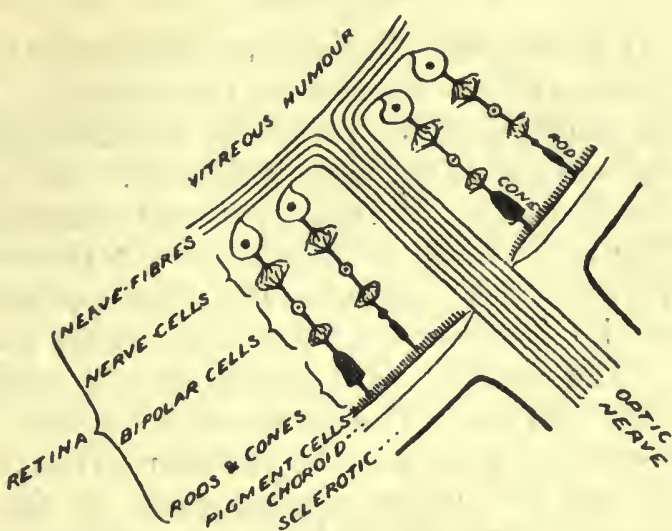

Fig 63.-Structure of Retiva.

This represents tho lower right-hand part of Fig. 62 magnified (very diagrammatic). most layer of it) to a point called the blind spot, where all the other layers of the retina are wanting: here they turn sharply out and run along the optic nerve. The structure of the retina is more complicated than the above description would imply, because besides the essential parts described there are supporting structures (neuroglia) around and among them. All the inner layers are sufficiently transparent to allow the light to pass through them, and reach the sensitive rods and cones.

\$ 10. The Blind Spot. We may pause to call the student's attention to a little point in the physiology of nerves, very happily illustrated here. The function of a nerve-fibre is conduction pure and simple; the light radiates through the fibrous layer of the retina without producing the slightest impression, and at the blind spot, where the rods and cones are absent, and the nerve-fibres are gathered together, no impression is made at all. If there is any doubt as to the existence of a blind spot 
in the retinal picture, the proof is easy. Let the reader shut his left eye and regard these two asterisks, fixing his gaze intently upon the left-hand one of them.

At a distance of three or four inches from the paper, both stars will be focussed on his retina, the left one in the centre of vision, and the right one at some spot internal to this, and he will see them both distinctly. Now, if he withdraw his head slowly, the right spot will of course appear to approach the left, and at a distance of ten or twelve inches it will, in its approach, pass over the blind spot and vanish, to reappear as he continues to move his head away from the paper.

The student will probably ask what purpose the blind spot serves. The answer is-none: its existence is a necessary result of the peculiar arrangement of the retina. In all the other sense-organs of the rabbit, the actual sensory element is always nearer the external stimulating agent than are the conducting fibres, such being the most obviously reasonable arrangement; but the rods and cones are turned away from the light. This arrangement is characteristic of the eyes of vertebrata; whereas the most highly-developed eyes of invertebrata have the sensory elements nearer the light and have consequently no blind spot. When we corne to study Amphioxus and also the development of the eye in the rabbit, we shall find the clue to this anomalous arrangement.

$\S$ 11. Eye-muscles and Glands. A series of muscles in the orbit (eye-socket) move the eye, and so enable the rabbit to vary its field of view. There is a leash of four muscles rising from a spot behind the exit of the optic nerve from the cranium, and attached to the upper, under, anterior, and posterior sides of the eyeball. These are called the "straight" muscles (recti), because each one, acting alone, causes motion of the eye in the vertical or horizontal plane. They are distinguished as superior, inferior, anterior, and posterior rectus. Running from the front of the orbit obliquely to the underside of the eyeball is the inferior oblique muscle. Corresponding to it above is a superior 
oblique. It is chiefly to these muscles that the oculomotor series of nerves is distributed. The superior oblique and posterior rectus are dignified by having a nerve each, exclusively for themselves, viz. IV. for the former and VI. for the latter. The rest, along with the iris and ciliary muscles, have to be content with nerve III. divided among them.

A lachrymal gland lies in the postero-superior angle of the orbit, and a Harderian gland in the corresponding position in front: the secretion of these has a lubricating function, enabling the eye to move easily in its orbit, and washing away all dust, etc. The excess of the secretion passes by a duct through the lachrymal bone into the nasal chamber. In addition to the upper and lower eyelids of the human subject, the rabbit has a third, the nictitating lid, in the anterior corner of the eye.

$\S$ 12. Functions of Brain. Before leaving the nervous system we must briefly allude to the functions of the greatly enlarged parts of the brain in the rabbit. The grey matter of the cerebral hemispheres is the seat of consciousness and the association of ideas, and from its nerve-cells impulses are sent out which, as it were, give orders of a general kind to subordinate parts of the central nervous system, these subordinate parts translating these general orders into the necessary detailed orders to the various active tissues. Similarly it receives only, as it were, the most important pieces of information as to what is going on in all partsonly those which are too important to be dealt with by the subordinate centres.

The chief function of the cerebellum is equilibration, or the balancing of the body. The normal position of a rabbit's body is one of unstable equilibrium, as any one will find who tries to place a dead rabbit in its normal living posture. These normal postures (whether of rest or motion) are only maintained in steadiness by constant action of appropriate muscles, just as a bicycle can only be kept from wobbling on an uneven road by constant variations in the pressure on the handles. 'The cerebellum is continually receiving impressions from the retina and from the ampullæ 
of the ear, as well as from the touch-organs of the skin and sensory nerve-endings in muscles. From all these it obtains information as to the momentary relations of the body with the external world, and is constantly sending out appropriate orders to the muscles to preserve the equilibrium of the body.

The bulb is especially the centre for the more complicated reflex actions, such as those concerned with respiration and the circulation. 


\section{Chapter Xi.-Reproduction.}

\$1. Maintenance of the Species. We have now really completed our survey of the individual animal's mechanism. But no animal that was merely complete in itself would be long sanctioned by nature. For an animal species to survive, there must evidently, also, be proper provision for the production of young and the preservation of the species as well as of the individual. Hence in an animal's physiology and psychology we meet with a vast amount of unselfish provision, and its structure and happiness are more essentially dependent on the good of its kind than on its narrow personal advantage. The mammalia probably owe their present dominant position in the animal kingdom to the exceptional sacrifices made by them for their young. Instead of laying eggs and abandoning them before or soon after hatching, the females retain the eggs within their bodies until the development of the young is complete, and thereafter associate with them for the purposes of nourishment, protection, and education. In the matter of the tail, for instance, already noted, the individual rabbit incurs the disadvantage of conspicuousness from the rear, in order to further the safety of the young.

§ 2. Essential and Accessory Organs of Reproduction. The essence of reproduction of the kind we know among the higher animals is the separation of a single nucleated cell from each of two animals, and the union of these two cells into one, which, by rapid growth, division, and differentiation of the cells formed by its division, develops into a new individual. The essential organ of reproduction is one in which special cells for this purpose are developed: such an organ is called a gonad. All other parts of the reproductive system are merely accessory to this-serving either as a duct to transmit the reproductive cells to the exterior (gonoduct), or to ensure (directly or indirectly) the 
union of the cells from the two individuals, or to provide for the nourishment of the embryo during its development.

The gonad in a female is called the ovary, the reproductive cells it produces are called ova, and/the gonoduct is an oviduct. As conveniently corresponding terms in the male we have spermary, spermatozoa, and spermiduct; but the spermary is more usually called the testis, and the spermiduct the vas deferens. We shall use the latter ter'ms in our description.

The details as to the changes that take place in these organs will be more conveniently taken in the section on embryology. Here we are concerned with the anatomical facts merely.

It is one of the peculiarities of vertebrates (not unknown among invertebrates) that there is a close connextion between the accessory reproductive organs and the organs for nitrogenous (renal) excretion, and that this connexion is usually closer in the male than in the female. The reason for this connexion of two systems of utterly different function cannot be understood from the study of the rabbit alone: we only allude to it here in order that the student may be on his guard against confusing the organs of one system with those of the other. 'The two systems are often referred to together as the Urino-genital (or Urogenital) System.

§ 3. Female Reproductive Organs. In the female we find the paired ovaries posterior and lateral to the kidneys (fig. 64). Each oviduct is a tube, open at its anterior end to the coelom, but this open end is an expanded funnel, and so near the ovary that there is every chance that an ovum when set free from the ovary will fall into the oviduct, especially as the funnel is set with ciliated epithelium which maintains a current towards the tube. Nevertheless, this imperfect arrangement for ensuring the passage of ova towards the exterior is one of the most peculiar facts in the anatomy of the higher vertebrates, especially as many less highly organised animals have their ovaries united to the oviduct, so that there is no risk of an ovum going wrong.

The oviducts are divided into two parts-an anterior 
portion with thin walls, the Fallopian tube, and a lower, stouter, and thicker-walled uterus. The two uteri cross the course of the ureters, running ventral to them, and open into a median passage, the vagina, which lies dorsal to the urinary bladder into which the ureters open. This oblique

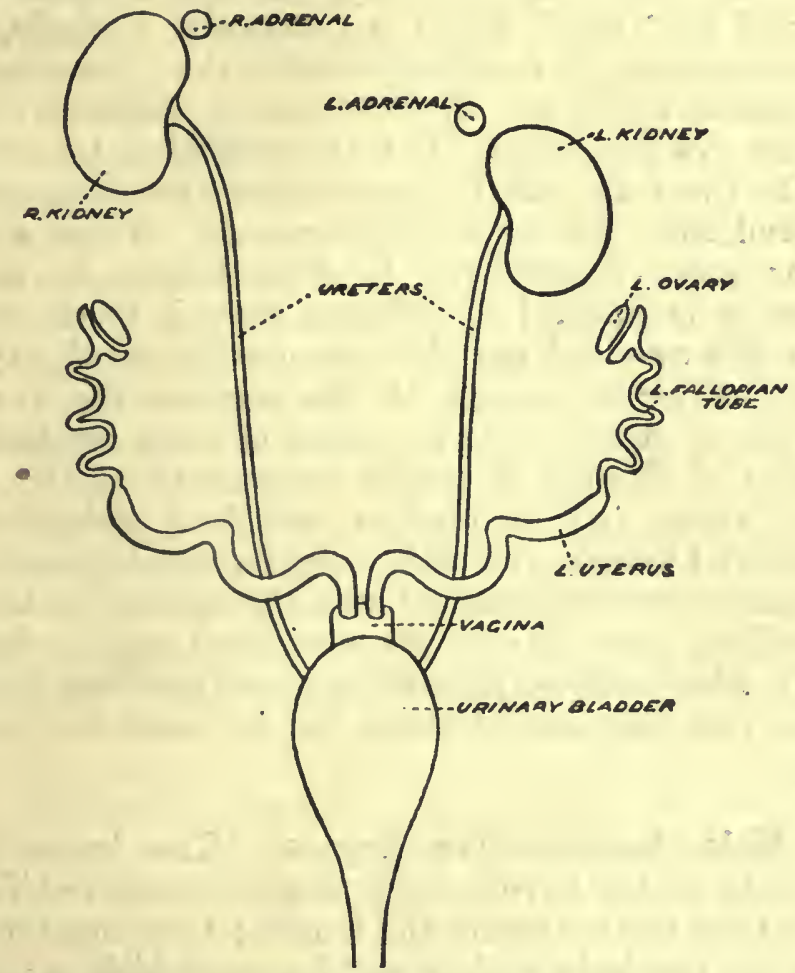

Fig. 64.-Renal and Repkoductive Organs of Female Rabbit.

Ventral view.

course of the oviducts is due to a shifting of the ovaries from their original position-nearer the middle line than the kidneys. The bladder and vagina unite farther back into a vestibule. Opening into this on its dorsal side are a pair of small glands-Cowper's glands.

\$ 4. Ova and Spermatozoa. The ova of the rabbit are microscopic nucleated cells of spherical shape (fig. 110), 
incapable of motion; this passive character is an essential feature of the female reproductive cell, and is far more strikingly shown in ova which (like those of birds) are of enormous size and stored with food-material for the embi yo. On the other hand, the male cell or spermatozoon is an active cell, capable of swimming through a liquid by the lashing of its "tail," which is practically a single, much elongated cilium. It is usually smaller than the ovum even when that is small, and the difference is still more strihing when the ova are large. But the nucleus is (at the t mo when the two cells unite) of equal size and importance in both, and since we know that animals, on the average, show as much resemblance to their fathers as to their mothers, it is natural to suppose that it is the nucleus which is the essential part in transmitting hereditary characters. Thus in respect of the nucleus the two cells are of equal importance; in respect of other matters there is a kind of division of labour between them-the ovum keeping, along with its nucleus, sufficient protoplasm and food-material to start the embryo in its development, while the spermatozoon, unburdened with these, takes on the duty of travelling about to seek the more inert ovum. Spermatozoa are also produced in vastly greater numbers than ova, and the vast majority of them fail to reach an ovum and perish.

§ 5. Male Reproductive Organs. The testes of an adult male rabbit have undergone a far greater shifting of position than the ovaries of the female; they come to lie in pouches of the body-wall-scrotal sacs-which project on either side of the urethral aperture. The vas deferens, unlike the oviduct, does not end by an opening into the cœlom-it is united to the testis through a tubulated body called the epididymis. From this it runs forwards to get over the base of the ureter and then loops sharply back to open (alongside its fellow) into a median sac called the uterus masculinus-a name which does not necessarily imply homology with the paired uterus of the female. This joins with the base of the urinary bladder to form an urethra (corresponding to the female vestibule), which 
is prolonged into an erectile projection, the penis, at the end of which is the urogenital aperture. There are a pair of Cowper's glands, like those of the female, but a much larger glandular mass lies at the dorsal and posterior side of the uterus masculinus; this is called the prostate gland.

\section{$\S 6$. Perineal Glands.} In both sexes there is present, a little behind the urogenital aperture and close to the anus, a pair of hairless depressions of the skin-the perineal pouches-into which open the ducts of the perineal glands, which secrete a fluid of powerful odour, which serves as a means of attracting the attention

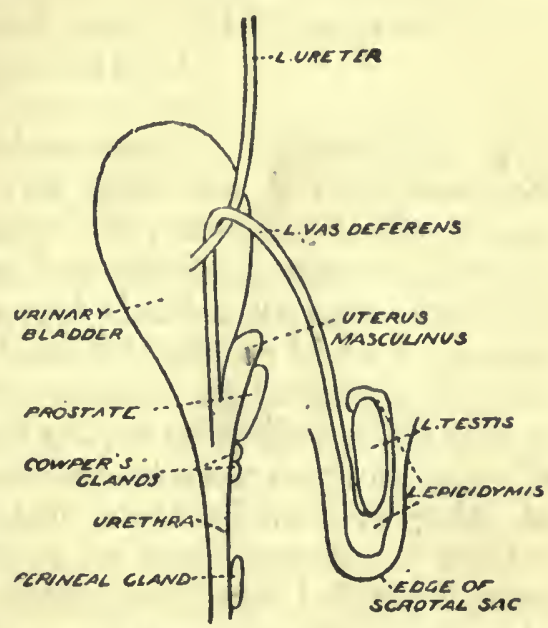

Fig. 65.-Reproductive Organs of Male RABBIT.

Side view of one rabbit to another.

Another large gland, the rectal gland, so called from its position just dorsal to the rectum, serves the same purpose.

\$ 7. Mammary Glands. In the skin of the ventral region of the female special glands are found, which undergo considerable enlargement at the time when the young are born; and secrete a nutritive fluid-milk-which supports the young until they are in a position to feed like their parents. These mammury glands appear (from their mode of development) to be highly specialized modifications of the sebaceous glands which are found in connexion with the hairs (fig. 13). They occur in a very imperfect and functionless condition in the male also 


\section{Chapter XII.-The Rabbit's Place in the Animat Kingdom.}

$\S 1$. Heredity. Innumerable wild rabbits exist in England, and if we study any one of these we find the same general structure, the same functions in the various organs, the same instincts and mode of life. Such a series of closely similar animals forms what is called an animal species; we will discuss the exact meaning of this term later on. This general resemblance of all rabbits to one another is usually explained by saying that they are all the offspring of parents which were themselves rabbits, and these in turn of other ancestralirabbits, until if we trace the history of rabbits far enough back we might conceivably find that all were descended from a single pair of rabbits. This explanation does not really explain anything: it merely brings the fact to be explained within the generalization that all new animals tend to resemble their parents. This tendency is called heredity. We have seen that the ultimate causes of heredity probably are contained in the nuclei of the reproductive cells which unite to form the fertilized ovum from which every rabbit is developed. What the nature of those causes may be we can hardly guess at present.

§ 2. Variation. We have spoken of heredity as a tendency, and no more definite term can be applied to it, for not only does it resolve itself into two partly-opposing tendencies towards resemblance of one or the other of the two parents that are necessary to produce a fertilized ovum, but there always seem to be present other tendencies towards difference from both parents. Indeed, it is probable that out of the enormous numbers of rabbits that live or have lived, no two have ever been exactly alike. Even in our description of the rabbit, which has been limited to the broadest facts of structure and function, we have had to mention that some rabbits have twelve pairs of ribs, others 
thirteen (chap. vii., $\S 4$ ); and that some have a transverse anastomosis between the right and left external jugular veins, while others have not (chap. iii., § 8). If we were to undertake the study of a series of rabbits in more minute detail, we should soon be able to add numerous other points of difference to the list.

The fact that the individuals comprised in a species differ from one another is expressed by saying that they show variation. It implies an imperfection of heredity, or tendency of offspring to differ from their parents (variability). It is worth while pointing out, however, that although a new kind of variation is in the first place due to imperfect heredity or variability, yet this variation becomes itself subject to heredity in the next generation. Hence heredity and variability are both concerned in the existence of variation; and the student should distinguish between variation and variability.

§ 3. Adaptation to Conditions of Life. Every difference in details of structure means a corresponding difference in the details of the various functions and activities in which those structures are concerned. A thirteen-ribbed rabbit does not breathe exactly in the same way as a twelve-ribbed one; and the flow of blood from the head and the variations in blood-pressure are not the same under similar circumstances in two rabbits, one of which has a jugular anastomosis while the other has not. When the variation in structure from the average is very great indeed-as when an animal is born with two heads, or without legs-the difference in functions and activities is so great that the animal is incapable of living except under very careful artificial treatment. We say that such an animal is a "monster" or "abnormal" or shows "malformation," because its variation is beyond the limits compatible with living an ordinary rabbit's life. Such extreme variations are rare, because they do not tend to be perpetuated by heredity, their possessors usually not living long enough to become parents. The variations commonly met with in any species are within the range of adaptation to the conditions of life of that species, because, if the structure 
of an animal is not adapted to the life it is born into, it must die.

§ 4. Increase or Stability of Numbers. Every wild rabbit who lives to old age has probably been concerned during its life as one of two partners in the production of not less than three,hundred young rabbits. In Australia, where rabbits have been introduced by man, and where they have found abundance of food and an absence of carnivora to feed on them, the result of this amazing fertility is a tremendous increase in the number of rabbits. But in England, where rabbits have long been established, the case is different: there is little or no room for an increase in numbers, and as a matter of fact we know that there is no perceptible increase. We must therefore conclude that out of three hundred rabbits born, only two survive to middle age, on the average : the rest being either killed and eaten by carnivorous animals, or (more rarely) dying through inability, for some reason or another, to obtain food.

§ 5. Natural Selection. Every day of its life a wild rabbit has to obtain food and escape being eaten, and failure to do either the one or the other means certain death. In a herbivorous species like the rabbit the obtaining of food is an easy matter under all ordinary circumstances, and the escaping of enemies is the great necessity of life. In the case of a carnivorous animal the relative importance of these two conditions is reversed.

Now the ability of a rabbit to escape being eaten depends, among other things, on its alertness in recognizing an enemy's presence and its swiftness and sureness in running; and though chance plays a large part in determining out of several rabbits disturbed by a dog which shall escape and which shall be eaten, still, if we consider the result, not of one such event, but of several hundreds, we may feel sure that the one rabbit out of one hundred and fifty which repeatedly escapes to a good age must have been more alert and a better runner than the average of the one hundred and forty-nine. He may not have been 
the very best-adapted of all to the necessities of a rabbit's life, but he was certainly not far short of the best. Now, what constitutes better or worse adaptation? Precisely those details of structure in which variation occurs. If, for example, the possession of a thirteenth pair of ribs enables a rabbit to breathe better during a run for life, or if a jugular anastomosis facilitates the proper distribution of blood to the organs that need it in an emergency, then we may be sure that these two features will be commoner among the rabbits that live long lives than among those that do not.* And since it is the longest-lived rabbits that are parents to the largest portion of the next generation, those features will be commoner in the next generation than in the present one.

If these same conditions determining life and death are repeated from generation to gencration-and generations overlap and succeed one another rapidly in the rabbitthen those features which are of the nature of better adaptations to external conditions will become commoner and commoner in successive generations, until at last there may be no rabbit without them, while those whose absence means better adaptation will become rarer and rarer, until no rabbit will have them.

Thus the general structure of animals (for what has been said of the rabbit might be said, mutatis mutandis, of any other species) must be continually becoming more and more perfectly adapted to their habitat and mode of life, and the special opportunities and dangers which these present. It is convenient to have a name for the process by which this adaptation is brought about; and since it is essentially a process of selecting out of the many individuals born the few that shall live to become parents, and since this selecting is done unconsciously by the multitude of external agencies which we collectively call natuve (including all other organisms than the one under consideration), the term Natural SELECTION is appropriately given to this process.

* It is not asserted that such is actually the case : it is only assumed for purposes of illustration. If the reverse is the case, then these features will be less common in the long-lived rabbits.

ZOOL, 
\$6. Rabbit and Hare. Of all the wild animals inhabiting England no one is more easily mistaken for the rabbit than is the hare. Most people probably do not know the one from the other by sight. The external differences are slight. The hare has longer ears (tipped with black) and a broader snout than the rabbit. Both limbs are longer in the hare, and the fore-limbs are not so strongly clawed. Again, in the rabbit the radius is shorter than the humerus, while in the hare the reverse is the case; and there are similar differences of proportion in other bones. If we compare the skulls of the two animals, we find that the hare's nasal bones, its nasal chambers, and its posterior narial passage are all broader than the rabbit's corresponding parts. It may also be mentioned that the young rabbit is born naked and blind, while the young hare is born hair-clad and with open eyes.

All these features are adaptations to the different mode of life of the two animals. The hare, instead of being a gregarious and burrowing animal, lives a solitary life, scraping a "form" (a slight hollow) in the ground, where it is hidden from sight by vegetation (in a cornfield, for example). Obviously a hare, living in a far more exposed position, needs a keener sense of smell than the rabbit (hence the differences in the nasal region of the skull), its limbs need to be swift for flight, not strong for burrowing, and its young ones must look after themselves from the first. Nor, in enumerating the differences between the two, must we forget the differences of instinct (involving differences of structure of the central nervous system), without which all other adaptations would be useless.

$\S 7$. Species. The rabbit and hare afford an excellent example of what is meant by the terms species and genus in biology. The two are said to be distinct species of the same genus, which means that while they agree with one another in a great many anatomical characters, they differ in certain definite respects. It is necessary, for precision of nomenclature, in biology, to give every species a double name-one being the name of its genus, or 
generic name, the other its own private specific namo. 'Thus the rabbit is called Lepus cuniculus, while the hare is Lepus timidus.

What is the exact amount of difference necessary to constitute a difference of species? 'To this question no answer can be given. Every case must be decided on its merits by the judgment of the biologist who is studying it, and there are many known cases where one investigator has made two or more species out of a set of animals which another has put in one species. There are two theories as to the origin of species, but neither affords an absolute criterion. According to the first theory, every species is descended from a pair of ancestors originally created out of not-living matter : this theory draws a perfectly sharp line between species and species, but unfortunately it makes the evidence for specific distinctness quite inaccessible, since we should never know that rabbit and hare were distinct species unless the creation of their first ancestors had been witnessed and described by a competent scientific investigator, and the pedigree carefully recorded down to the present day.

The second theory regards species as gradually differentiated by modification during successive generations, through natural selection (and perhaps other processes). We have seen that the rabbit-species is inevitably becoming more and more fully adapted to its mode of lifo; and the same might be shown of the hare. Hence every successive generation must increase the difference of average structure between hare and rabbit. Conversely, if we go back in time, every generation will show less difference between the two species, until it is conceivable that we might arrive at a common ancestral species adapted for a life intermediate in character between that of the modern rabbit and hare. Fvidently, then, it is only the disappearance of intermediate forms that makes rabbit and hare appear as distinct as they are, so that an absolute criterion again fails us.

Whichever theory of the crigin of species we adopt, then, we have to decide our species on practical grounds of convenience, For two sets of animals to be accounted distinct 
species it must be shown that there are certain constant distinctions between them, and (if possible) that these distinctions are kept up by an absence of natural interbreeding.

§ 8. Genera, Families and Sub-orders. There are about twenty species of the genus Lepus, in different parts of the world, differing from one another much in the same way as do the rabbit and hare. They all agree in possessing the same dental formula (chap. vii., § 18), in having five digits on the fore-limb and four on the hind, in having an imperfect clavicle, and in many minor points. These, then, are the generic characters.

The most nearly allied genus to Lepus is Lagomys, of which there are about a dozen living species, inhabiting mountainous districts (the picas or tailless hares). This genus is characterized by having only one or two (not three) premolars in the upper jaw, a complete clavicle extending from scapula to sternum, a second cæcum to its intestine, and no visible tail.

These differences are sufficiently great, in the opinion of systematic zoologists, to justify the placing of these genera in separate families-Leporidoe and Lagomyidoe. (Usually a number of genera constitute a family.)

Both Lepus and Lagomys agree in having two pairs of incisors in the upper jaw : this character marks them off so sharply from the most nearly allied families, that these two constitute by themselves a sub-order Lagomorpha.

If, instead of starting with the rabbit we had taken the rat or mouse, we might similarly have gone from species to genus, genera to family, families to sub-order-at each step taking in animals less and less closely like the one we started with. In this case we should get the sub-order Myomorpha. Similarly the squirrel would serve as a type for the sub-order Sciurimorpha, and the porcupine for the sub-order Hystricomorpha.

§ 9. Order and Class.-The animals of these four suborders all agree in a general gnawing habit, in having persistent-pulp incisors, in having no canine teeth, and in 
the discoidal form of the placenta.* They constitute an order of the animal kingdom, that of Rodentia.

Similarly we have the order Carnivora, typified by the cat and dog; the Insectivora, by hedgehog and mole; Ungulata, by ox and horse; Chiroptera, the bats; Cetacea, whales and dolphins; Primates, by man and monkeys; Sirenia, by the manatee; Edentata, by the armadillo; Marsupialia, by the kangaroo; and Monotremata, by the ornithorhynchus. Widely as the species in these orders differ among themselves, they agree on certain points which mark them off from all other animals. 'Therefore these orders are united as a Class, to which the name Mammalia is given. It is as a type of the Mammalia that we have been studying the rabbit in this book.

$\S$ 10. Mammalia and other Vertebrata. The rabbit agrees with all mammals, and differs from all other vertebrata (i.e., birds, reptiles, amphibia, and fishes), in having -

(a) Hair ;

(b) Mammary glands;

(c) A diaphragm;

(d) Only one aortic arch, and that on the left side of the body;

(e) Its young born alive. (But the Monotremata lay eggs, and certain fish and reptiles bear living young) ;

(f) Epiphyses to its vertebral centra;

(g) Seven cervical vertebræ (but some Edentata have more);

(h) The cerebral hemispheres covering the mid-brain ;

(i) The optic lobes transversely divided (hence corpora quadrigemina) ;

(j) A corpus callosum, uniting the two cerebral hemispheres;

(k) A spirally coiled cochlea to the internal ear;

(l) Small, non-nucleated red blood-corpuscles.

(In respect to $j$ and $k$ also, the Monotremata are scarcely mammalian.)

* This is explained in chapter $\mathbf{x x}$. 
The rabbit shares the following features not only with mammals, but with all reptiles (i.e. lizards, crocodiles, turtles, and snakes) and birds-

(a) Absence of gills (not of gill-slits), at any stage in development ;

(b) An amnion; and

(c) An allantois in development.

The meaning of $(b)$ and $(c)$ we shall explain to the student in the chapters on embryology. We simply mention them here to render our table complete.

The rabbit shares the following features with the above animals and also with the amphibia (frogs and newts), that is to say, with all the vertebrata, except the true fishes -

(a) Lungs (but many fish have a swimming bladder which answers to the lungs in its anatomical. relations, and in certain fish this has the characteristic pulmonary circulation) ;

(b) Limbs which consist of a proximal joint of one bone, an intermediate part of two, and a distal portion which has five digits, or is evidently a reduced form of the five-digit limb ;

(c) The absence of a median fin supported by fillrays.*

Finally the rabbit shares even with the true fishes (e.g. sharks, dogfish, salmon, cod, and herring) -

(a) An epidermis consisting of 'more than one layer of cells ;

(b) Skull, and vertebræ, replacing a notochord in development;

(c) A primitively three-vesicled brain, with paired eyes, paired ears, and paired olfictory organs, all of characteristic structure ;

(d) A heart ventral to the alimentary canal, and a portal system ;

(e) A large colom, which does not extend anterior to the heart, and from which a pericardial chamber is separated off:

* The frog's tadpole has a median tin, but no fin-rays. 


\section{Questions on the Rabbit.}

1. Describe the venous circulation of the rabbit (with liagrams). Comparo a vein and artery. Compare the distribution of the great venous trunks with that of the al'terial system.

2. Construct a general diagram of the circulation of the rabbit, to show especially the relation of the portal system, the lymphatics and lacteals, and the renal circulation, to the main blood-current.

3. What is a villus? Describe its epithelium, and the vessels within it. Write as explicit an account as you can of the absorbent action of a villus.

7 4. Tabulate the alimentary secretions, and their action on the food.

$>$ 5. Give an elementary account of the structure and physiology of the blood.

6. Describe cartilage and bone, and compare them with one another.

7. Give an account of the amoba, and compare it with a typical tissue cell in a metazoon (e.g. the rabbit).

8. Give a general account of connective tissue. What is tellon?

9. Compare the pectoral with the pelvic limb and girdle. What other structures of the adult rabbit display a similar repetition of similar parts?

10. What are bilateral symmetry and metameric segmentation?

711 . Describe the rabbit's brain (with diagrams).

12. Give a list of the cranial nerves of the rabbit, and note their origin in the brain.

13. Give a list of the nerve apertures of the dog's skull.

14. Describe and tigure the distribution of nerves v., vII., IX., and $\mathrm{x}$.

15. Draw and state the precise position of the hyoid bone, the clavicle, the calcaneum, and the olecranon process.

16. Describe, as accurately as possible, the position of palatine bones, pterygoids, the ethmoid bone, the pre- and basi-sphenoids, in the dog's skull. 
17. What is membrane bone? What is cartilage bone? Discuss their mutual relationship.

18. What is an excretion? What are the chief excretory products of an animal? How are they removed?

19. Describe the rabbit's liver, explaining $(a)$ its position in the body, $(b)$ its structure, gross and minute, $(c)$ its bloodsupply, $(d)$ its functions.

20. Describe the minute anatomy of the kidney, and the functions of the several parts.

21. What is ciliated epithelium? Where does it occur in the rabbit?

22. Describe the mechanism of respiration. What is the relation of respiration to the general life of the animal?

23. What are the functions of the skin? Describe its structure.

24. Name any structures that appear to you to be vestiges or rudiments, i.e. structures without adequate physiological reason, in the rabbit's anatomy. How are such structures interpreted?

25. Describe the structure of striated muscular fibre. Describe its functions, and the various means by which they may be called into activity.

26. Describe the characters and structure of the blood of the rabbit. What is the lymphatic system? Describe its relation to the blood system in a mammal.

27. Describe the structure of $(a)$ blood, (b) hyaline cartilage, $(c)$ bone, in the rabbit; $(d)$, point out the most important resemblances and differences between these tissues; (e) state what you know of the development of the same tissues.

28. Describe the lymph and the lymphatic system of a vertebrate.

29. Describe a typical animal cell, and compare any two tissue cells you may choose to select.

30. Explain the biological use of the term variation. Distinguish clearly between the different kinds of variation. Illustrate your answer by examples.

31. Describe the different kinds of skeletal tissue found in the vertobrata. 


\section{PART II.-THE LOWER VERTEBRATA.}

\section{Chapter XIII. The Frog.}

$\S 1$. External Form and Mode of Life. The common frog (Rana temporaria) has many obvious differences from the rabbit. Instead of a land-loving animal, running and burrowing, eating vegetable food, we have now a creature equally at home on land or in fresh water, leaping and swimming, and living almost exclusively on insects. Its skin is devoid of hair and nails, and so abundantly beset with glands as to be always moist. It is richly coloured, and possesses in some degree the property of varying in colour in accordance with its surroundings. The absence of a neck is characteristic of aquatic vertebrates, since a gradual passage from head to trunk offers the most suitable form for swimming (see chap. i., § 2). The absence of a tail is more surprising, since it is the organ of swimming, not only in fishes, but in newts. The frog, however, has greatly developed hind legs which replace a tail in function, while on land they enable it to progress by sudden leaps instead of the swift crawling which really constitutes the motion of a newt. The disadvantage (on land) of the neckless condition is no doubt compensated for by the remarkable way in which the eyes project up beyond the general surface of the head, instead of being deep-sunk in a boneprotected orbit, as are the rabbit's. There is no external ear in the frog, the tympanic membrane being flush with the general surface, and easily recognized as a circular patch of the skin behind the eye. The mouth-opening is huge, extending literally from ear to ear, since there are no cheeks or lips. The external nares occupy much the same position as in the rabbit. Posteriorly, instead of separate anus and urinogenital opening we have a common cloacal 
aperture, which in the absence of a tail comes to be slightly dorsal in position instead of ventral. The limbs show a general resemblance to those of the rabbit, but the fore-

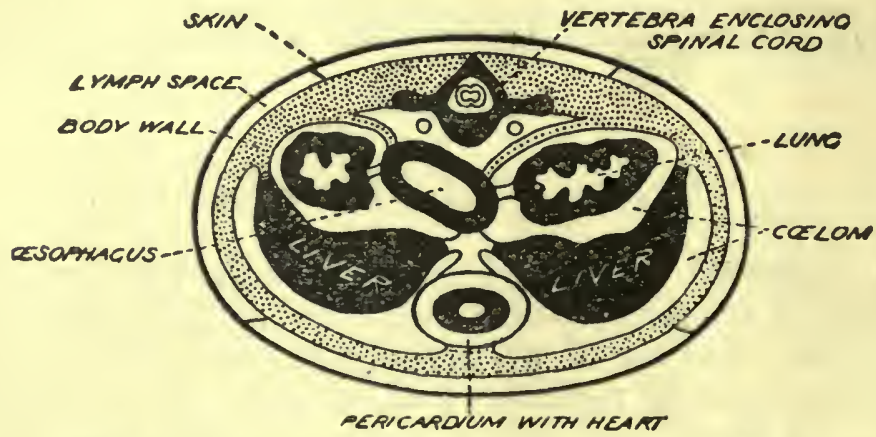

Fig. 66. - T'ransverse Sfction, anterior part of Froo.

(After Howes.)

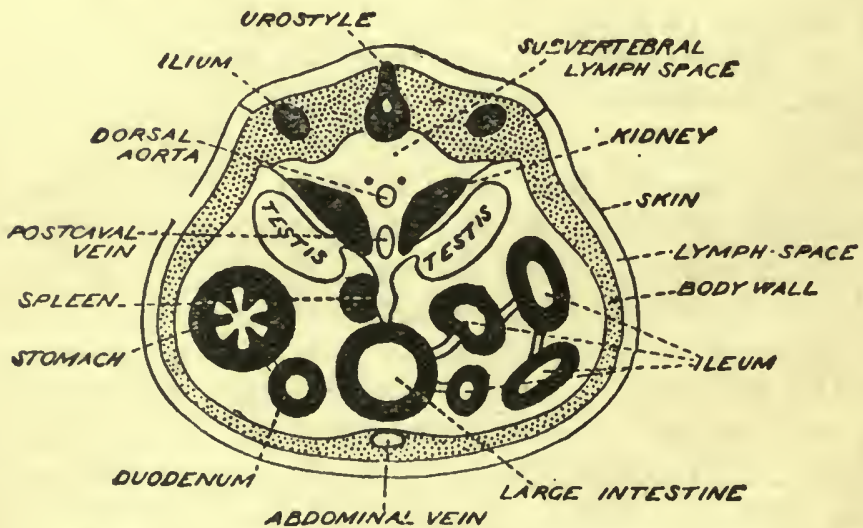

Fig. 67.-Transverse Section, Posterior Part of Froa. (Aftor Howes.)

limb has only four toes, the hind one five; the latter has an elongated tarsus and no projecting heel, and its toes are webbed.

$\S 2$. Transverse Sections. The general structure of the body, as shown in transverse sections (figs. 66 and 67), is 
the same in all fundamental respects as that of the rabbit. We have dorsally a vertebral column enclosing a spinal cord and surrounded by muscles; ventrally, a cœlom, with alimentary canal and other viscera. But there is no diaphragm, and the coolom forms an undivided space, excepting only for the narrow pericardial chamber. As in the rabbit, the colom stops short about the level of the heart and extends no farther forwards. Beneath the skin we find an almost continuous cavity, which makes the skin strikingly loose: this cavity (or series of cavities) contains lymph and connects with the lymphaties. It is called the subcutaneous lymph-space.

§3. The alimentary canal (fig. 68) is more nearly a simple tube in the frog than it is in the rabbit. Teeth are found only along the upper jaw (maxillary teeth) and in two pritches on the roof of the mouth (vomerine teeth). They are numerous, small, pointed, and slightly hooked: they cannot be classed as incisors, canines, etc., and have no masticatory function-they simply serve to prevent insects from slipping out. Instead of the two sets of teeth (milkand permanent), we find a continual replacement of wornout ones by now ones develuperl beneath. The tongue is attached to the floor of the mouth by its anterior end, and the posterior end is free and can be flicked out with great rapidity and suddenness, and aimed with precision at the small insects which form the frog's staple diet. In accordance with the absence of mastication, there are no salivary glands. The internal nares open into the mouth at its front end, just beneath the external nares: thus the narial passage is absent, and there is nothing corresponding to the rabbit's palate which separates the buccal and narial cavities. The roof of the frog's mouth answers to the roof of the rabbit's narial passage. The glottis is far removed from the nares: it is a longitudinal slit on the floor of the throat, and is completely closed wluile food is being swallowed. Near it, in a dorso-lateral position, are a pair of pits, which lead out to the inner side of the tympanic membrane: these obviously answer to Eustachian tube plus tympanum (middle car), and are called the tympano-Eustachian recesses. 
The throat gradually narrows back into the osophagus and stomach, no sharp limit being discernible between them : the stomach is simply a part of the general tube in which

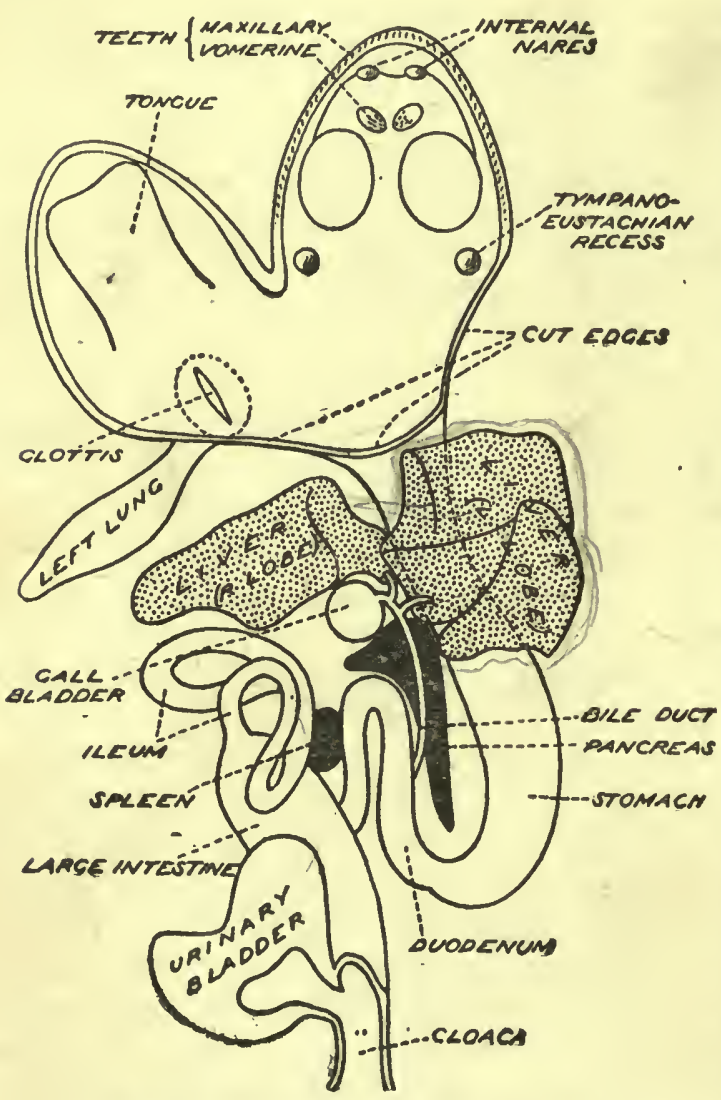

Fig 68.-Alimentary Canal of Frog.

The left side of mouth and throat has been cut through, and the floor turned over to the animal's right (left of diagram). The dotted line around the glottis is the outline of the laryngo-tracheal chamber. the peptic glands are developed. It is, however, separated from the duodenum by a sphincter muscle. The duodenum(recognizable as such by receiving the secretions of liver and pancreas) is short, and is bent back parallel to the stomach. In the mesentery between them lies the pancreas, which is a compact gland, not diffuse as in the rabbit. The liver is large, and consists of a right and left lobe, the latter partially subdivided; the gall - bladder is spherical. The pancreas surrounds the course of the bile-duct, and has no separate duct of its own, its ductlets opening into the bile-duct.

The duodenum passes on into a portion of the canal that is thrown into a series of simple folds: this is the ileum. 'There are no Peyer's patches, and it does not end in a 
sacculus rotundus, but passes abruptly into a much wider tube, the large intestine. There is no cæcum at the junction of the two. The large intestine is short, and cannot be divided into colon and rectum. Its final portion, however, receives the openings of the urinary and genital ducts on its dorsal side and that of the urinary bladder ventrally: this portion is called the cloaca.

§ 4. Respiration. The glottis opens into the larynx (or laryngo-tracheal chamber), which is a small chamber ventral to the throat. From this the lungs come off immediatelyin the absence of a neck there is neither room nor need for a trachea. The lungs are simple sacs, their internal surface somewhat increased by ingrowths from the walls, but infinitely simple in comparison with the highly complex lungs of the rabbit. The mechanism of respiration, too; is quite different, as must be evident from the absence of both diaphragm and ribs. In their stead we find the hyoid relatively much larger than the rabbit's, being a large plate of cartilage in the floor of the mouth. Muscles (sternohyoid) run from this to the shoulder-girdle, and when they contract pull the hyoid backwards and ventralwards, and so enlarge the mouth cavity. Another set (petrohyoid) runs from the hyoid upwards to the skull, and when these contract they lift the hyoid and so diminish the capacity of the mouth cavity. These two sets of muscles (in combination with minor onos) act in respiration. First the nares are opened, the floor of the mouth lowered; air, of course, rushes into the cavity. Then the nares are closed, the glettis opened, and the floor of the mouth raised. The air is, of course, forced into the lungs. Expiration takes place by the elastic recoil of the lungs and the viscera that press on them, as soon as the nares are re-opened. Thus the mechanism of respiration in the frog may be called a buccal force-pump, while that of the rabbit is a thoracic suction-pump.

The lungs are not the sole respiratory organs. There is, as we shall seo, an abundant supply of deoxygenated blood to the skin, and here also some interchange of gases between air and blood can take place. This is said to be the only means of respiration during hibernation, in winter. 
$\S 5$. Heart and Arterial Arches. The heart of the frog differs from that of the rabbit in being very imperfectly divided into right and left portions. Only the auricles in fact are paired, there being but a single ventricle into which both auriclos open. Two new structures, not recognizable in the rabbit, are also found: a sinus venosus, on the dorsal side, formed by the union of the three cavil veins and opening into the right auricle; and a truncus arteriosus, representing the united bases of both the aortic and pulmonary arches of the rabbit, and coming off from the right anterior region of the ventricle.

The arteries and veins of the frog show an almost perfect bilateral symmetry. The truncus arteriosus runs forwards and soon divides into right and left portions, and each of these soon divides into three, so that instead of two arches on the left side only, as in the rabbit, we have three arches on each side. The most anterior of these is called the carotid arch, the second is the systemic arch, and the third the pulmo-cutaneous arch. The two latter obviously answer to the two on the rabbit's left side. There is na connexion between them answering to the ductus arteriosus. Connexions of a similar kind do, however, usually occur between the carotid arch and the systemic on both sides; they are called the "ducts of Botallus." On the carotid arch is a swelling, the "carotid gland," which resembles a compacted mass of small blood-vessels.

From the carotid arches come off vessels to the whole head region. All the other systemic arteries come from the systemic arches. Each pulmo-cutaneous arch gives off an artery to the lung, and another special artery (unrepresented in the rabbit) to the skin. For the details of these vessels the student is referred to fig. 70 .

$\S 6$. Mechanism of Circulation. In spite of the undivided condition of the ventricle there is a very fair realization of a double circulation like that of the rabbit. 'I'he ventricle has its cavity greatly divided up by strands. of muscle crossing it, so that it is more like a spongy solid than a hollow chamber. IVhen the heart beats, the auricles contract together, and the right side of 
the ventricle becomes filled with deoxygenated blood from the right auricle and sinus venosus, while the left side receives oxygenated blood from the left auricle (into which the pulmonary veins open). The spongy nature of the ventricle retards the mixing of these two qualities of blood. The truncus ar-

teriosus comes off from the right side of the ventricle, so when the ventricle contracts, the deoxygenated blood of the right side is necessarily the first to be forced along the truncus; afterwards comes somewhat mixed blood, and finally the oxy-

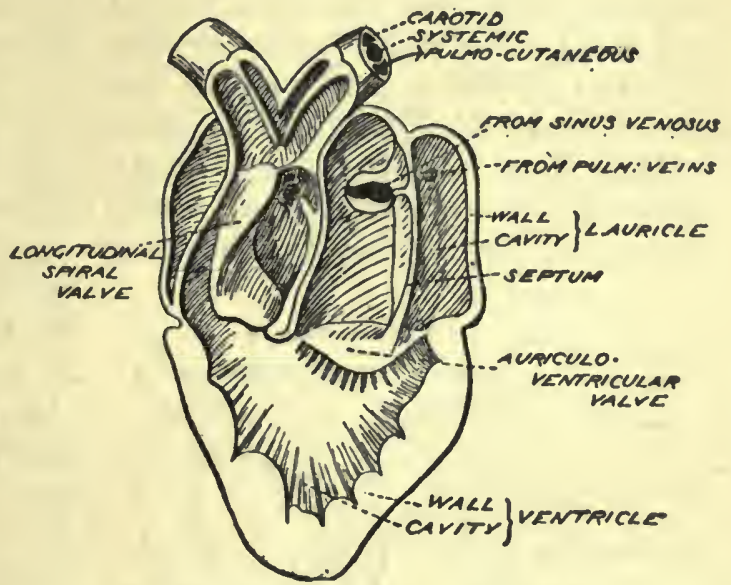

Fig. 69-Heart of Froc, Laid Open.

(After Howew) genated blood of the left side. On laying open the truncus (fig. 69) it will be found that each of the divisions that branch off from it right and left is already internally divided into three passages, and that the separation of the pulmo-cutaneous passages begins even before the division into right and left branches is visible externally. The truncus consists of two portions-a conus arteriosus or pylangium, nearer the ventricle, and a bulbus arteriosus or synangium farther from it. At each end of the former there is a series of three valves, and along its wall runs a longitudino-spiral valve. The apertures leading to the pulmo-cutaneous passages are just at the junction of the pylangium and synangium, and so placed in relation to the valves that when blood is passing to the systemic and carotid passages it tends to force the valves over and so close the pulmo-cutaneous apertures.

Now when a liquid is being forced along under pressure 
it first takes the path of least resistance : the resistance in an artery is caused by the elasticity of its walls, consequently the less the length of artery between a given point and the capillaries, the less the resistance. It follows that the resistance is least in the pulmo-cutaneous arches, and so the blood first flows along these; but as blood is pumped into these and their walls are dilatcd the resistance increases, and the blood begins to force itself along the synangium into the systemic and carotid arches. As it does so it forces over the longitudino-spiral valve and prevents further flow into the pulmo-cutaneous arches. Its next choice between systemic and carotid arches is determined in favour of the former by the extra resistance which the

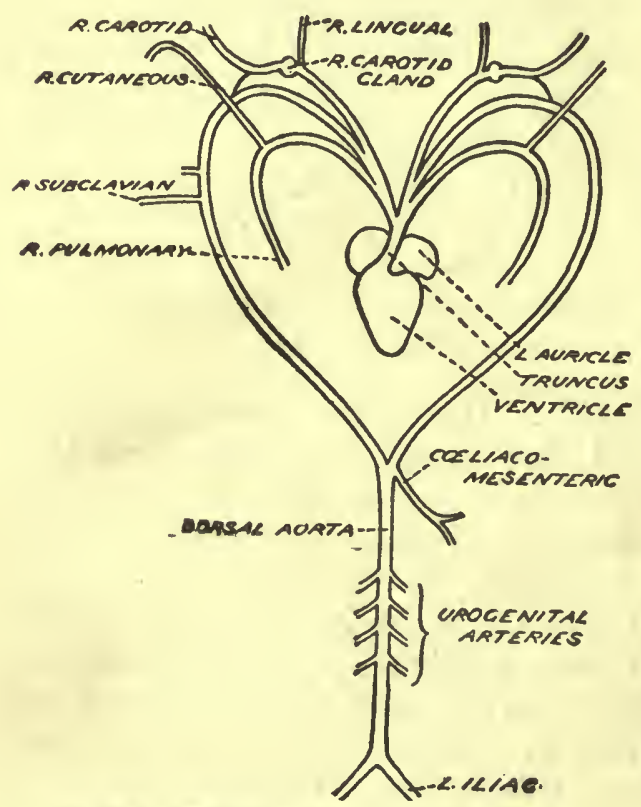

Fyg. 70.-Principal Arteries of Frog. carotid gland causes in the latter. Thus it comes about that the first and most deoxygenated blood goes to the lungs and skin, the last and most oxygenated to the head, while rather less perfectly oxygenated blood goes along the systemic arch to all other parts.

§ 7. The Arteries. Fig. 70 shows the general arrangement of the arteries. In comparison with the rabbit we mayspecially note that as there are two systemic arches, so too there are two dorsal aortæ, which eventually unite in the middle dorsal line as the median dorsal aorta. Just at the junction of the two comes off a single median vessel, the coeliaco-mesenteric, in place of the two separate coliac and anterior mesenteric 
arteries, and supplying the same organs, viz. stomach, intestines, liver and spleen. Farther along wo find a series of small modian arteries-dividing into right and left almost immediately -which answer to the renaland genital arteries of the rabbit. The subclavian arteries arise from the right and left systemic arches, as do also two occipitovertebral arteries, supplying part of the head and the region of the vertebral column. 'The iliacs are much the same as in the rabbit, but in the absence of a tail there is no caudal artery.

From the carotid

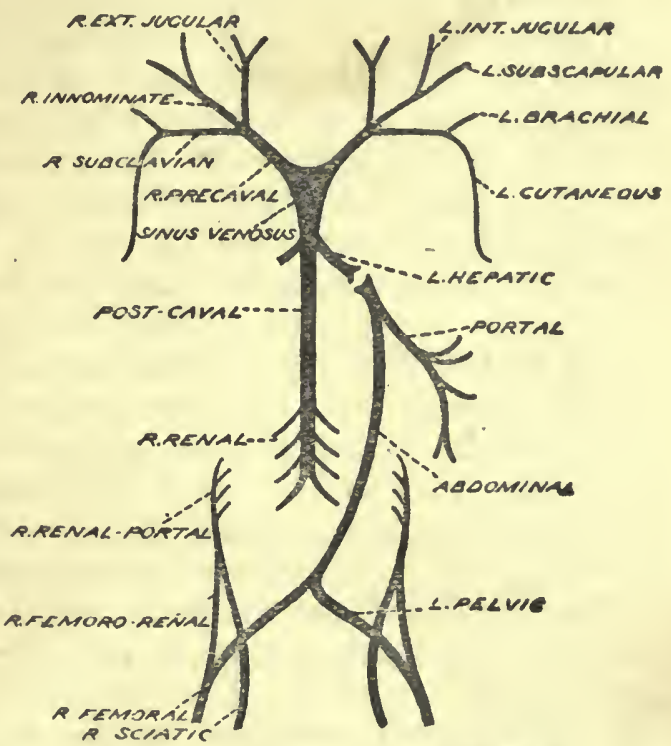

Fig. 71.-Principal Veins or Frog. arch, just before the carotid gland, there arises a lingucel artery to the tongue; beyond the gland the carotid artery soon divides into internal and external carotids which supply the head.

§ 8. The Veins. Only the pulmonary veins enter the left auricle-in spite of the fact that some oxygenation of the blood occurs through the skin, this blood returns to mix with that of the ordinary veins. As in the rabbit, there are three large caval veins, but these unite into a distinct chamber (the sinus venosus) before opening into the right auricle. As before, each precaval vein is formed by the union of external and internal jugulars and subclavian, but the internal jugular is relatively larger, more lateral in position, and its last portion, after it has been joined by an extra sulscapulır vein, is generally distinguished as the ZOOL. 
innominate. The subclavian brings blood not only from the arm (brachicl vein), but also from the skin and certain muscles by a large musculo-cutaneous vein. The azygos of the rabbit is not found in the frog.

The postcaval, as in the rabbit, receives paired hepatic, paired genital, and paired renal veins, but that is all : no blood from the hind limbs or hind part of the body passes directly into the postcaval. The explanation of this is that the frog has two portal systems-an hepatic-portal, resembling in all essentials that of the rabbit, and a paired renal-portal in which the kidneys play the part taken by the liver in the former. Blood from-each hind-limb is conveyed by two main veins-femoral and sciatic. 'The former' divides into pelvic and femoro-renal. The right and left pelvics unite into a median abdominal vein,* which flows along the ventral body-wall and into the hepatic-porta] system. The femoro-renal unites with the sciatic of its own side into a renal-portal, which, after further receiving a dorso-lumbar from the body-wall, branches out into the kidneys. It is interesting to note that the blood from the renal arteries goes direct to the glomeruli, while that from the renal-portal vein goes to the capillary-plexus around the kidney-tubules.

Thus the whole of the blood from the hind limbs and adjacent regions must pass through a second set of capillaries (either those of the liver or those of one of the kidneys) before reaching the heart.

\$ 9. The Lymphatic System. The frog has a lymphatic system like the rabbit's, but its largest vessels take the form of large, ill-defined spaces rather than true vessels. Such spaces are known as sinuses. There is a large sub-vertebral lymph-sinus between the dorsal part of the peritoneum and the vertebral column, that is, just in the position of the thoracic duct, from which it differs in the absence of welldefined walls : into it the chyle is poured by the lacteals. There are sub-cutaneous lymph-sinuses which cause the great

* Commonly called the anterior abdominal. "Anterior" here is used in the human-anatomy sense of "ventral," and is therefore misleading. 
looseness of the skin. Evidently in such sinuses the lymphpressure must be extremely low, and so, in order to force the lymph into the veins, there are two pairs of inuscular pumping organs--the lymph-hearts. The anterior pair lies between the third and fourth vertebre and pumps lymph into the subscapular veins ; the posterior ones lie on either side of the urostyle (see § 10) and pump it into the femoral veins.

$\S 10$. The Skeleton can be recognized as consisting of the same general parts as the rabbit's-skull and vertebral column, limb-girdles and limbs. The skull will repay a fuller treatment and will be dealt with in chapter xiv. The vertebral column consists of far fewer vertebræ than the rabbit's, only nine in fact, the last one being followed by an unsegmented bony rod, the urostyle. Obviously we cannot divide such a column into cervical, thoracic, etc., regions; in fact, with the exception of the first and two last, all the vertebræ are practically identical. Each consists of a centrum, neural arch, anterior and posterior zygapophyses and transverse processes. The centrum is deeply concave at its front end, and has at its posterior end a protuberance to fit into the anterior concavity of the next one behind; such a centrum is said to be procelous.

The first vertebra or atlas has no anterior zygapophyses, no trans-

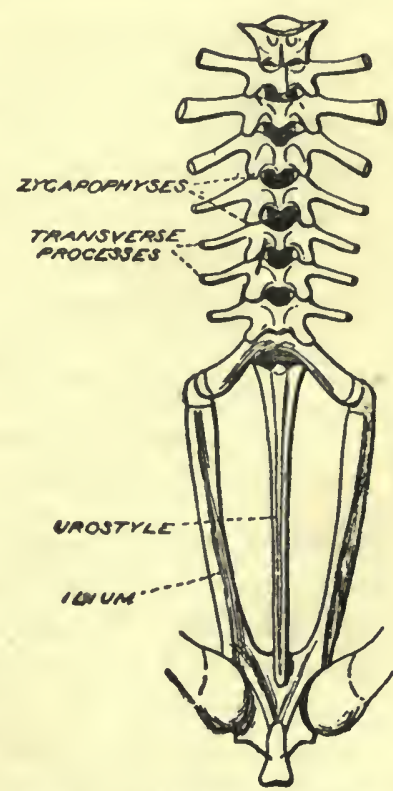

Fig. 72.-Vertebral Columa and Pelvic Girdle of Froo.

Dorsal view. (After Howes.) verse processes, an ill-defined centrum. It articulates by very small posterior zygapophyses with the second vertebra. This is not an axis vertebrathe frog cannot rotate its head as the rabbit can-and has no important pecularities. The eighth has its centrum concave at both ends (amphiccelous). The ninth has a 
single protuberance at its anterior end and a double one behind; it has no posterior zygapophyses; its tranverse processes are stout and backwardly directed and give articulation to the pelvic girdle-hence it is the sacral vertebra (cf. chap. vii., § 7). The urostyle shows signs of imperfect segmentation at its anterior end, there being a pair of foramina for the exit of a last pair of spinal nerves ; josteriorly it tapers almost to a point.

There are no ribs to any of the vertebræ. The vertebr:e are all cartilage-bones, but they have no epiphyses.

\section{§ 11. Limb-Girdles and Limbs. - The pectoral girdle (fig.}

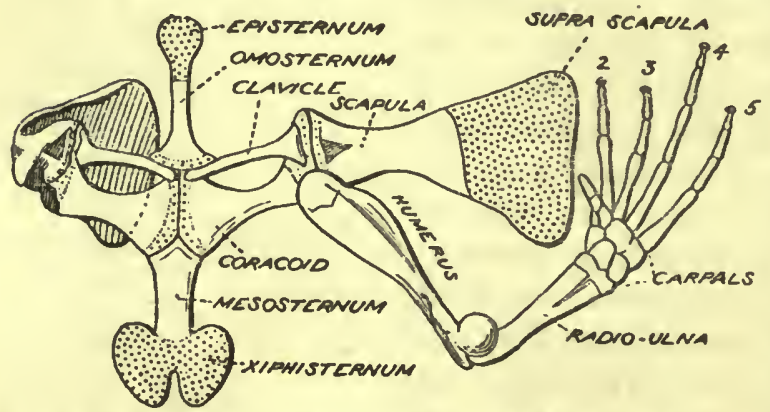

Fig. 73.-Shoulder-Girdle aNd ArM of Froa.

(After Howes.) 73) differ's but little from the typical condition (figs. 35 and 36 , p. 71). The dorso - lateral segment is partly ossified asthe scapula; the remainder is cartilage, though largely calcified, and is termed supra-scapula/ The antero-ventral portion is cartilaginous (pre-coracoid) but covered over by the clavicle - the only membrane-bone other than those of the skull. The postero-ventral portion is a stout cartilagebone, the coracoid. Between the precoracoid and coracoid comes a fenestra like the obturator fenestra of the rabbit's pelvic girdle, and similarly perforated by a passage for a nerve of the brachial plexus. In the median ventral line cartilage persists as a symphysis, but it is extended anteriorly and posteriorly into what is commonly called the sternum, though in the absence of ribs its homology with the rabbit's sternum is very doubtful. The anterior prolongation consists of episternum (cartilage) and omosternum (cartilagebone); the posterior, of mesosternum (cartilage-bone) and xiphisternum (cartilage). 
The arm has the same general bony structure as the rabbit's. The humerus is short and thick, with a strong deltoid ridge, but no olecranon fossa. Radius and ulna aro short and fused together, though there is clear indication of the double origin at the distal end; there is a slight olecranon process. In the carpus there are two rows of three small bones each. Only four digits are well-developed, the thumb, or pollex, consisting of a small metacarpal only, witlout phalanges. The digital formula is 02233 .

The pelvic girdle is much less easily understood than the pectoral; it is, in fact, in a highly specialized condition, i.e. it departs widely from the general plan of pelvic girdles to be found through most of the air-breathing vertebrates. This specialized con. dition is connected with the adaptation of the hind-legs to the leaping habit. The ilia are very long, and lie nearly parallel to one another; their posterior ends meet in a median symphysis; the ischia are small and also meet in a median symphysis; while the pubic region is cartilaginous and also median. Hence the girdle altogether loses its character of a bony case protecting some of the abdominal viscera, and serves Sido view (after Howes.) The dotted portion solely for the attach- between ilium and ischium represents the ment of the powerful

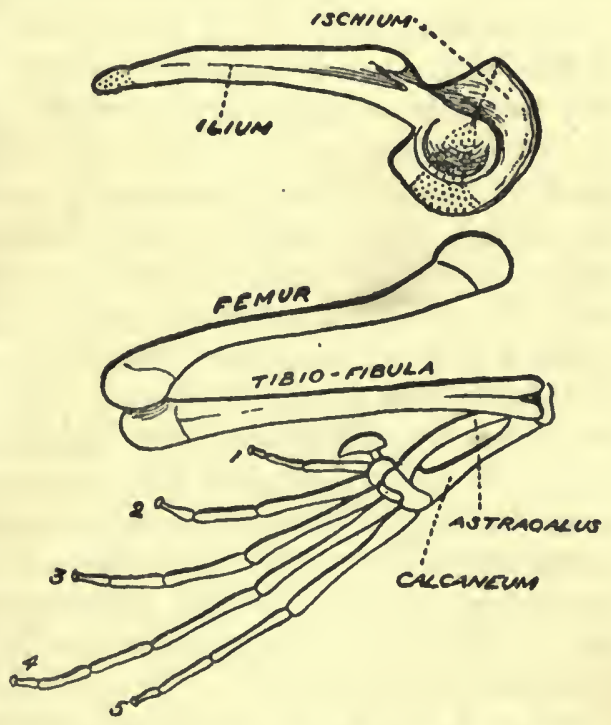

Fig. 74.-Pelvic Girdee and Hind-Leo OF

leg-muscles. Ilium, ischium, and the pubic cartilage all take part in bounding the acetabulum.

The hind-limb can be readily compared with that of the rabbit, but all its bones show a great elongation. The 
femur is long, thin and has a double bend; the tibia and fibula are fused together; the astragalus and calcaneum are remarkably lengthened, united at either end, but quite distinct between; the calcaneum has no projecting heel. These are followed by two other small tarsals. There are five digits, each with a metatarsal and phalanges, the formula being 22343. In addition there occurs on the pre-axial side of the hallux what appears to be a rudimentary extra digit-the calcar; it consists of a metatarsal, with occasionally a phalanx or two as well.

$\S$ 12. Nervous System. The central and peripheral nervous systems are built on exactly the same plan as in the rabbit, but show a general simplification.

The spinal cord has a general similarity to that of the rabbit, but is very short, and the spinal nerves number only ten pairs altogether, the first nine coming out behind the corresponding vertebræ, and the tenth through the apertures in the urostyle already mentioned. Thus, unlike the rabbit, there is no nerve emerging between skull and atlas. The first spinal nerve corresponds in distribution with the rabbit's hypoglossal nerve, a point we shall refer to again when we speak of the skull. The second and third constitute the brachial plexus. The seventh, eighth, and ninth form the sciatic plexus going to the hind limb. Evidently we cannot regard these nerves as corresponding to those of the rabbit, each to each in order from in front backwards : if we did so we should be forced to the ridiculous conclusion that the frog's hind-legs were homologous with the rabbit's fore-legs, since both are supplied by similarly numbered spinal nerves. We must recognize that we may have two animals showing metameric segmentation, and yet that the homolugous regions may be divided into a very different number of metameres in the two cases.

\$ 13. The Brain (figs. 75 and 76) shows all the same essential parts as the rabbit's, and the ten pairs of cranial nerves correspond exactly in origin, and very closely in distribution, with the first ten of the rabbit. But the general arrangement of parts is simpler; for the thalam. 
encephalon and mid-brain are fully exposed in dorsal view instead of being hidden by the cerebral hemispheres and cerebellum. The hemispheres (or cerebral lobes, as they

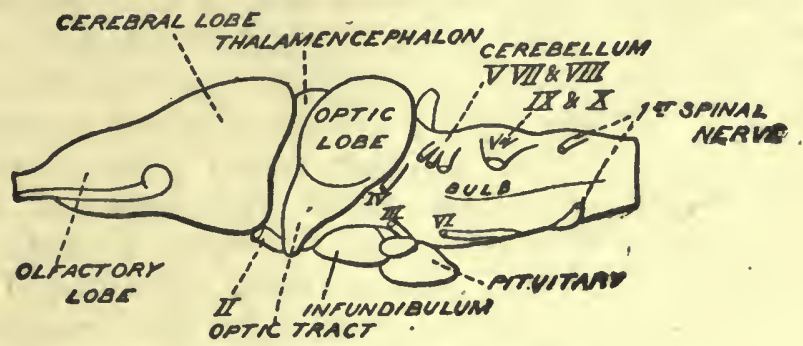

Fig. 75. - Brain or Froce.

Side view. (After Wiedershelm.)

are usually called) are comparatively small, and have large ventricles; their commissures are far fewer than in the

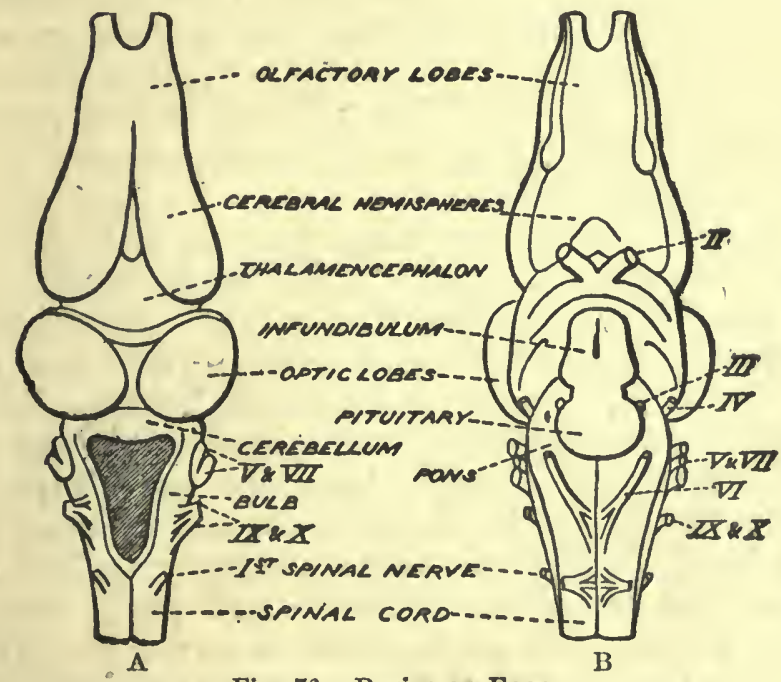

Fig. 76.-Britin of Froc.

A, Dorsal, B, Ventral view. (After Wiedersheim.)

rabbit, for there is no corpus callosum nor middle commissure, though the anterior and posterior commissures are present. The olfactory lobes are united in the median line. 
The thalamencephalon is similar to the rabbit's ; its sides forming the optic tracts, its floor depressed into an infundibulum with the pituitary at its end and the roof giving rise to a pineal stalk. But the pineal itself is disconnected with the brain, and lies close under the skin of the dorsal side of the head, having been nipped off by the growth of the bones of the skull. In the most primitive of the Amphibia (the class in which the frog is placed), long since extinct and only known by their fossil bones, the skull had a large round opening at this point, and there can be little doubt that in them the pineal was a functional organ of vision, and its stalk an optic nerve emerging by this foramen.

The optic lobes are large, and they are only one pair (hence sometimes called corpora bigemina, in contrast with the rabbit's quadrigemina), and each contains a large cavity. The floor and sides of the mid-brain do not slow any marked differentiation from those of the pons and bulb, nor these again from one another or from the spinal cord. On the roof of the hind brain comes the cerebellum, which is a very small upgrowth, quite without the complexity of structure of the rabbit's. The thin roof of the rest of the hind-brain is fully exposed, and gradually narrows back into the dorsal fissure of the cord.

The small size of the cerebellum is easily understood when we consider how much broader and less high a frog's body is than a rabbit's, and hence that a frog's nurmal position is one of stable equilibrium and needs no elaborate co-ordination of muscular action to keep it balanced. We see a result of this feeble cerebellar development in the way a frog alights from a leap: its leaps are mere leaps in the dark. Instead of coming down on its feet as a dog does it really falls down anylow, and is as ready to leap over a precipice as an flat ground. This is also partly due to the imperfect development of its cerebral cortex, the structure of which is far simpler than in the rabbit, and quite inadequate to the forming of accurate judgments of distance or to easy education by experience.

§ 14. Cranial Nerves. Of these, I., II., III., IV., VI., and vist. are so exactly like the same in the rabbit that 
they need not be further mentioned. v. also has the samo three branches, all emerging by a single aperture from the skull, and with a similar distribution to those of the rabbit. vii. differs in detail only from the rabbit's : the frog, not being a biting or chewing animal, has a much sinaller amount of jaw- and face-muscle, so the branch which is so greatly developed as to be counted the main facial nerve in the rabbit, is here no larger than the others. It is called the hyomandibular branch, and, originating anterior to the internal ear, it curves back to run on the posterior side of the tympano-Eustachian recess, on its way to the jaw muscles. This part of its course in the rabbit is obscured by the small size of the middle ear, and covered in by the bulla : in the frog it is easily made out, but its meaning will be better appreciated after we have studied the dogtish. The other branches of viI. are the vidian or palatine on the roof of the mouth, and a small pre-tympanic that runs on the anterior side of the tympano-Eustachian recess. Thus viI. is said to fork over this recess, which separates two parallel branches of it.

IX. and $\mathbf{X}$. leave the skull behind the internal ear. IX. sends a short branch to join the hyomandibular of vir.: the rest of it goes to tongue and pharynx just as in the rabbit. $x$. also has much the same distribution (see fig. 59, B.) as in the rabbit, when allowance is made for the absence of a neck. It has no superior laryngeal branch (although one has becn inserted in the diagram for comparison), but has a reçurrent laryngeal, and branches to heart, lungs, stomach, and solar plexus. A small dorsal branch of $\mathbf{x}$. to the muscles at the posterior end of the head may represent the rabbit's XI. ; otherwise it is wanting. There is a hypoglossal nerve, but it is not XII., but the first spinal.

$\S 15$. The Sympathetic System consists of a paired chain of ganglia, one to each spinal nerve and united to it by a ramus communicans. Posteriorly it is closoly connected to the median dorsal aorta: anteriorly, the right and left halves diverge to follow the paired aortre, and so on to the head where there are rami communicantes to $\mathbf{x}$., IX., vıI.. 
and $\nabla$. There is also a solar plexus in the mesentery, connected by splanchnic nerves with the main chain.

$\S 16$. The Sense-organs of the frog resemble those of the rabbit in all essentials, but differ in details. The olfactory organs are a simple pair of sacs, opening to the exterior by external nares, and to the mouth by internal nares. We can thus see that the nasal passage of the rabbit includes two things - the pair of olfactory sacs plus a portion of the mouth-cavity cut off by the formation of a palate.

The eye has almost exactly the structure of the rabbit's, but the lens is nearly spherical ; it has the same six muscles, but its lubricating glands are less developed.

In the internal ear the utriculus and sacculus communicate by a wide aperture, and the endolymph-duct is unsplit. The endolymph contains a mass of calcareous matter, known as the otolith. In the place of the four auditory ossicles of mammalia there is only one, consisting partly of cartilage, partly of cartilage-bone. This is certainly homologous with stapes plus orbiculare: whether it also represents the other two is very doubtful, but we shall return to this in the next chapter, on the skull. 'This one ossicle is called the columella auris, and of course extends from the tympanic membrane to the cartilage that encloses the internal ear. As already mentioned $(\S 1)$ the tympanic membrane is flush with the general skin, and there is no outer ear.

§ 17. Spleen, Thymus, Thyroid and Adrenals. The spleen is a spherical red body attached to the mesentery between stomach and rectum.

The thymus is a paired yellow body, and lies just beneath the skin, behind the angle of the jaw. This is a very different position from that which it occupies in the rabbit, but the identity of the organs is proved by their development, during which they shift in both cases from their original position.

The thyroid is also paired, and lies just ventral to the cxternal jugular veins, on either side of the larynx.

The adrenals are not as clearly defined as in the rabbit, 
but appear simply as a yellowish patch on the ventral surface of each kidney (figs. 77 and 78).

§ 18. Uro-genital Organs: Female (fig. 77). In the iemale, the ovaries, instead of being the tiny bodies seen in the rabbit occupy an enormous space in the colom: this is due partly to the far greater number of eggs laid, and partly to the much greater size of each egg-a rabbit's ovum being only $\frac{1}{250}$ inch, while a frog's is $\frac{1}{14}$ inch in diameter. The ovaries occupy their primitive position, nearer the middle line than the kidneys. The oviducts are relatively much longer than in the rabbit, being very much coiled and extending forwards to the lungs, close to which they open into the cœlom. The first part of the oviduct is thin and narrow; the longest, middle part is much coiled, thick - walled, and secretes an albu-

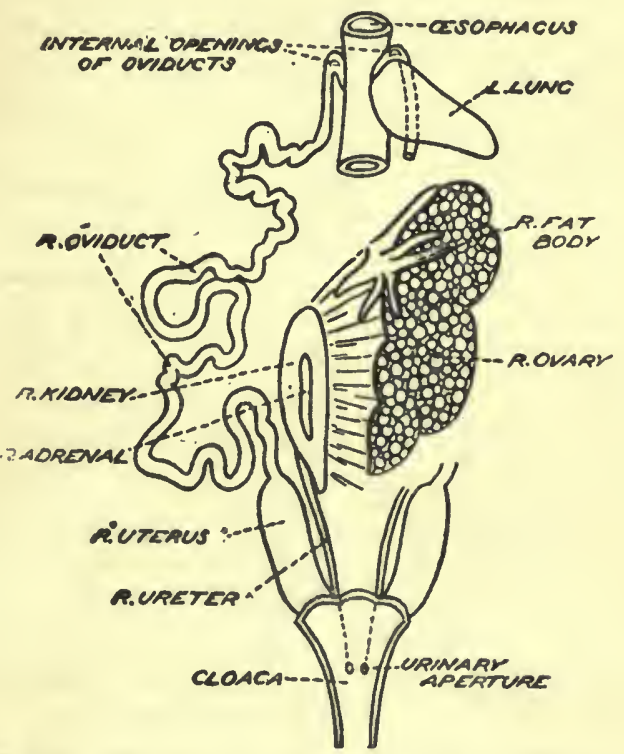

Fig. 77.-Urogenital, Organs of Frmale Frog. The left ovary, kidney, etc., and most of tho left oviduct, are omitted. (After Howes, altered.) minous substance with which each egg is coated as it passes down, and which has the property of absorbingr water when placed in it and so swelling up enormously; the final portion is broad and thin-walled, and opens, on either side, into the cloaca.

The kidneys of the female are reddish-brown organs that do not project into the colom, but lie in the subvertebral lymph-space, being covered by peritoneum on their ventral surface only. From the outer border of each comes off a 
tube, the ureter, the pair of which open separately into the cloaca.

§ 19. Uro-genital Organs: Male (fig. 78). The condition in the male is more difficult to compare with the rabbit's

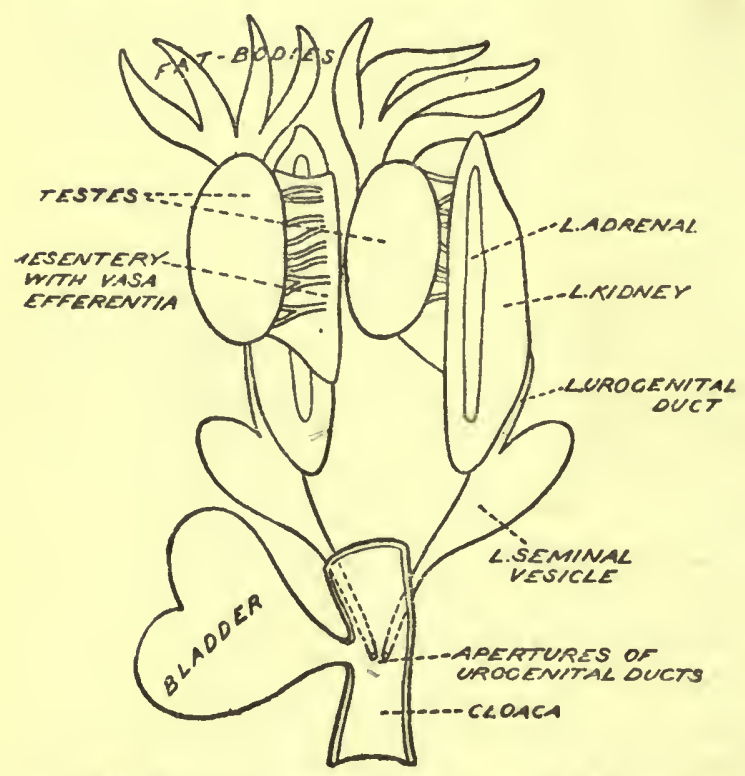

Fig. 78.-Urogenitat Organs of Male Frod. (After Howes, altered.)

at first sight. The testes occupy their primitive position in the body cavity, and are white bodies, usually dappled with black pigment. Vasa efferentia run from them to the internal border of the anterior part of the kidney, which answers, therefore, to the rabbit's opididymis. Thus the kidney (which is similar to that of the female) is not simply a renalexcretory organ, but also serves to transmit the spermatozoa. Similarly its duct is not merely an ureter, but rather a uro-genital duct; into it a seminal vesicle, which is especially large in early spring, opens. This, the permanent condition of the frog, corresponds, as we shal see when we come to study development, to temporary embryonic stages of the rabbit. In front of the gonad in both sexes is a fat-body (corpus adiposum), which acts as a fat store against the time of hibernation. There is no penis in the male, fertilisation of the ova occurring as they are squeezed out of the female by the embracing fore limbs of the male. The male has a pad, black in early spring, 
which is closely pressed against the ventral surface of the female in copulation, and which serves as a ready means of distinguishing the sex.

§ 20. Histology.-In all fundamental respects, the tissues of the frog resemble those of the rabbit, but there are certain differences of detail which it will be well to mention here. The epidermis consists of two cell-layer's only -an inner living layer and an outer horny one : the latter is shed as a whole poriodically, instead of bit by bit and constantly, as is the rabbit's. From the inner layer are developed abundant large, flask-shaped, simple glands (cf. fig. 19[2]), which are imbedded in the dermis. The frog's bones have very little development of Haversian systems-the osteoblasts being arranged for the most part in only one series of concentric rings. In the striated muscle we find the nuclei not confined to the superficial region, but at all depths. In the blood (fig. 79) the red corpuscles are much larger than those of the rabbit, and oval in shape, the central portion being thicker than the margin, and they have a nucleus; the white corpuscles are much the same as in the rabbit. The nerve-cells in the

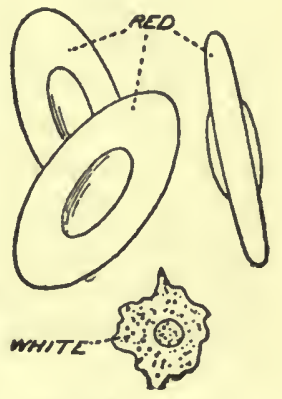

Figr. 79.-BLuUD. Corpuscles of Frog. Drawn to same scale as fig. 3. frog's sympathetic ganglia have long been described as a peculiar form of bipolar cell, which gives off two axiscylinders, one of which winds spirally round the other. It has now been proved that they are unipolar, and that the spiral axis-cylinder is efferent from a nerve-cell in the spinal cord. This close winding of its termination round the sympathetic nerve-cell makes up for the absence of dendrons in the latter.

§21. Classification.-Frogs and their allies are sharply marked off from reptiles and mammals on the one hand, and from fishes on the other, by the fact that they breathe by gills in the larval stage of their life, and by lungs in the adult stage. With this distinction go various others that 
need not here be enumerated, which characterize the important class, Amphibia. (See chap. xii., § 10.) Again, among common Amphibia we can easily recognize two clearly separated orders-the Urodela, represented by the newt and salamander, which retain a long tail and have numerous vertebræ and small limbs; and the Anura, including frogs and toads, which lose their tail, have few vertebræe, and powerful limbs. Two other orders, one confined to tropical regions, the other entirely extinct and known only as fossils in Carboniferous and Permian strata, need only be mentioned here.

Among Anura we can distinguish two sub-orders-one represented by the toad, in which (among other things) the ventral ends of the coracoids do not meet in a symphysis but end freely and overlap (Arcifera); the other, to which the frog belongs (Firmisternia). In most respects, however, a toad will show practically the same characters as a frog, in dissection. Finer distinctions mark off the family Ranidse from other families of the Firmisternia, such as the Hylidæ (tree frogs), and still finer ones, the genus Rana from other genera of the family. 
Chapter XIV.-The Skulls of the Frog and the Dog.

$\S 1$. The Chondro-Craniurn. We have already given a description of the mammalian skull, and we have stated where the origin of the several bones was in membrane, and where in cartilage; but a more complete comprehension of the nammalian skull becomes possible with the handling of a lower type. We propose now, first to give some short account of the development and structure of the skull of the frog, and then to show briefly how its development and adult arrangement demonstrate the mammalian skull to be a fundimentally similar structure, complicated and disguised by further development and readjustment:

Fig. 80, A, shows a dorsal view of a young tadpole's cranium; the brain has been removed, and it is seen that it was supported simply upon two cartilaginous rods, the trabeculæ cranii. Behind these trabeculæ comes the notochord (the fore-runner of the vertebral column), and around its anterior extremity are paired tracts of cartilage, the parachordals. These structures, underlying the brain, are all that appears at first of the brain-caser In front, and separate from the cranium, are the olfactory sacs; the eyes lie laterally to the trabecula, and laterally to the parachordals are two tracts of cartilage, the otic or auditory capsules, enclosing the internal ear.

Fig. 80 , B, shows a more advanced phase of the same structures. The trabeculæ have met in front and sent forward a median and lateral parts to support the olfactory sacs, forming the rudiments of a pair of olfactory capsules. They have also flattened out very considerably, and have sent up walls on either side of the brain to meet above it and form an incomplete roof over it. The parachordals have similarly grown up round the hind-brain and formed a complete ring. Further, the otic capsules are fusing with the brain-case. With certain differences of form these elements-the trabeculæ, the parachordals, and the otic 

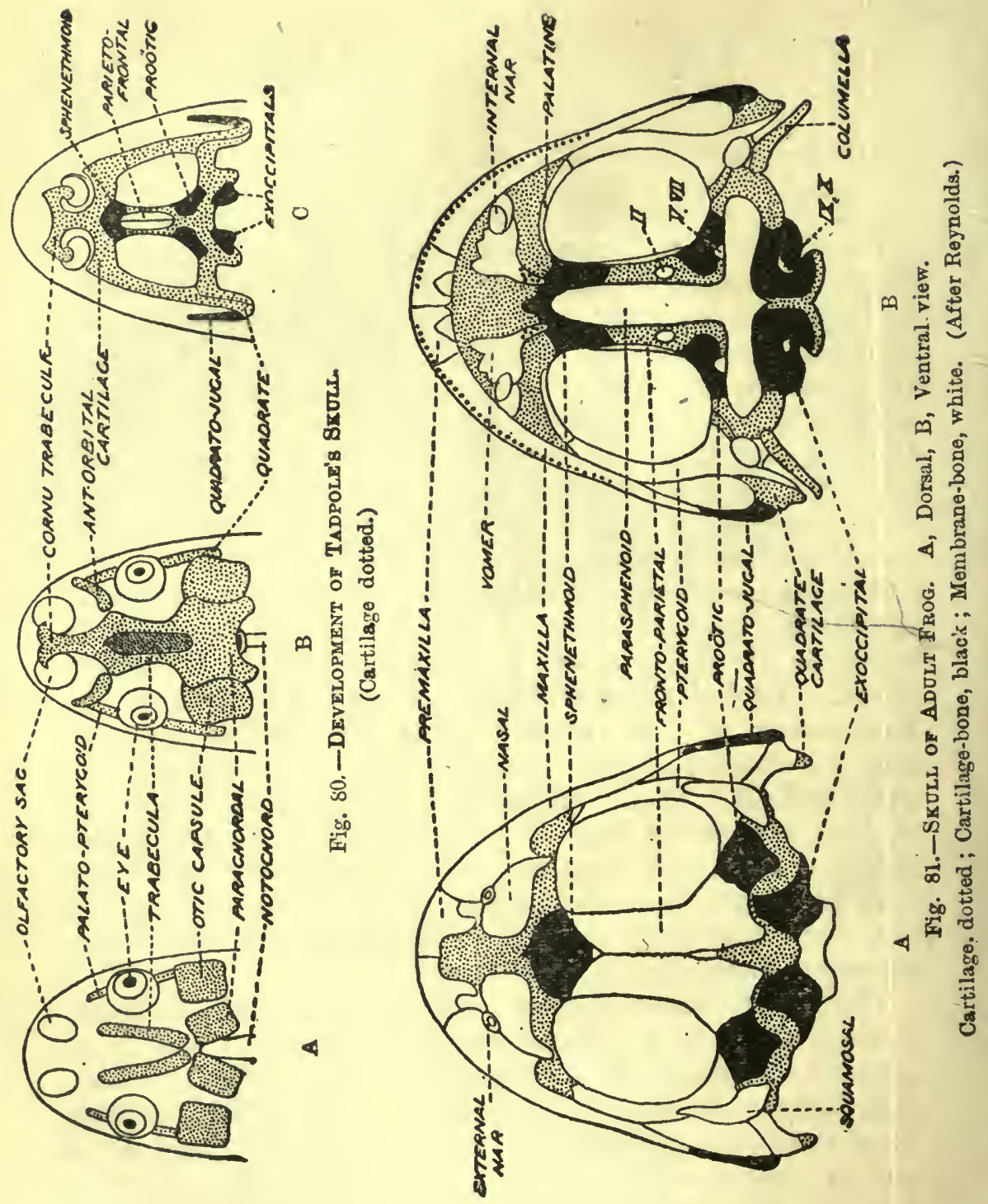
capsules, are also the first-formed structures of the mammelian cranium. The cartilaginous skull that results from their union is called the chondro-cranium.

§ 2. The Jaws. In fig. $80, \mathbf{A}$ and $\mathbf{B}$, there appears beneath the eye a bar of cartilage, the palato-pterygoid cartilage, which is also to be seen from the side in fig. 82. It will be learnt from the latter that this bar is joined in front to the cranium bohind the olfactory capsule, and behind to the otic capsule. The cartilaginous bar from the palato-pterygoid to the otic capsule is called the quadrate, and at the point of junction, at the postero-ventral angle of the palato-pterygoid, articulates with the cartilaginous bar which is destined to form the skeleton of the lower jaw-Meckel's cartilage.

§ 3. Cartilage-bones. Fig. 80 , c, shows a dorsal viow of these structures in a young frog. The parts corresponding to those in $\mathbf{A}$ and $\mathbf{B}$ will be easily made out, but now ossification (after the manner explained in chap. vi., § 11) has set in at various points of this cartilaginous cranium. In front of the otic capsule is the paired pro-otic bone; behind it at the sides of the parachordal ring is the paired ex-occipital ; in front of the brain-case, and behind the olfactory capsules, is a ring of bone, the sphenethmoid. These are all the cartilage-bones that appear in the cranium and upper jaw of the frog, so that, even in the adult, the skull consists in no small part of cartilage.

§ 4. Membrane-bones. But another series of bones, developed first chiefly in dermal connective tissue, and coming to plate over the chondro-cranium, are not shown in fig. 80, c. They are, however, in figs. 81 and 83. These membrane-bones are: on the dorsal side, the parieto-frontals, originally two pairs of bones which fuse in development, and the nasals. Round the edge of the jaw, and bearing the teeth, are premaxillæ and maxillæ, and overlying the quadrate cartilage and lateral to the otic capsules are the 'T-shaped squamosal bones. A quadrato-jugal appears at the angle of articulation with the lower jaw. In the ventral ZOOL. 
view of the skull (fig. 81, B) we see a pair of vomers bearing teeth, a pair of palatines, and a pair of pterygoids (which palatines and pterygoids, we may note, unlike those of the

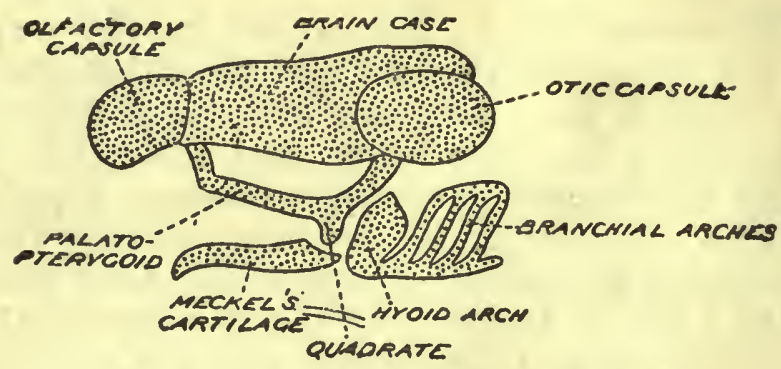

Fig. 82.-Side View of Skull of Tadpol.

Abont the same stage as Fig. 80, B.

rabbit, are membiane-bones), and a great median daggershaped parasphenoid. These two figures, and fig. 83, which shows the same bones in side view, should be carefully mastered before the student proceeds with this chapter.

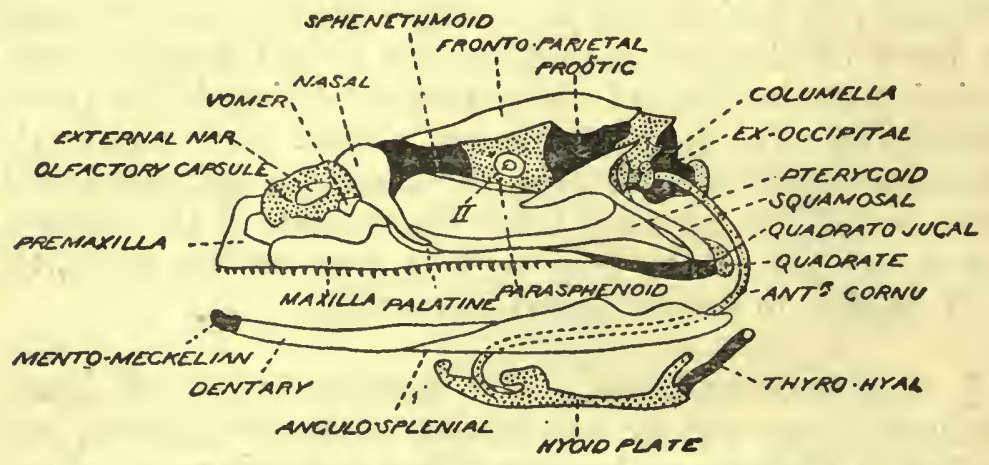

Fig. 83. - Side View of Skull of Adult Froa.

Shading as in fig. 81. (After Howes.)

The cartilage and cartilage-bones are distinguished from membrane-bones by shading (but the quadrato-jugal has been wrongly shaded throughout).

$\S$ 5. Visceral Arches. Turning now to fig. 82, we have a side view of a tadpole's skull. On the ventral side 
of the head is a series of vertical cartilaginous bars, the visceral arches, supporting the walls of the tadpole's gillslits. The mandibular arch (= Meckel's cartilage + the palato-pterygoid) is counted as tho first of these; the second is called the hyoid arch, and the four following, the first, second, third, and fourth branchial arches. Altogether there are four gill-slits, and between the hyoid arch and the mandibular arch there is an imperforate slit (i.e. a thinning of the sides of the throat from within, which nover becomes an actual perforation), which becomes the tympano-Eustachian recess. The frog no longer breathes by gills. but by lungs, and the gills are lost, the gill-slits closed, and the branchial arches consequently much reduced. The hyoid arch becomes attached to the otic capsule, and its median ventral plate, including also vestiges of the first, second, third and fourth branchial arches, is called the "hyoid apparatus." In fig. 84 the apparatus is seen from the side; the hyoid arch is now called the "anterior cornu of the hyoid." The function of the hyoid apparatus in the frog is to furnish a basis of attachment to the tongue muscles and muscles of respiration; it remains cartilaginous with two exceptions-the relic of one branchial arch, which ossifies as the thyro-hyal;

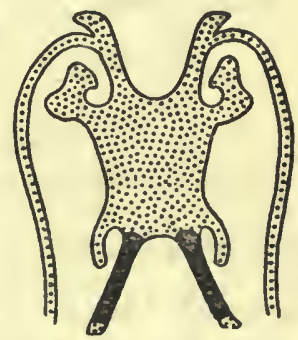

Fig. 84.-HYold or AduLT Froa.

Thyro-hyals (posterior cornua) black. and (though this is not altogether certain) the top of the hyoid arch, which is said to become the columella auris. It should be noted that, as development proceeds, the angle of the jaw swings backward, and the hyoid apparatus shifts relatively forward.

Before proceeding to the comparison of the mammalian skull with this, we would strongly recommend the student thoroughly to master this portion of the work; and in no way can he do this more thoroughly and quickly than by taking a parboiled frog, picking off the skin, muscle, and connective tissue from its skull, and making out the various bones and cartilaginous regions with the help of our diagrams. 
§ 6. The Mammalian Skull. Figs. 85 and 86 represent, in the most diagrammatic way, the main changes in form of
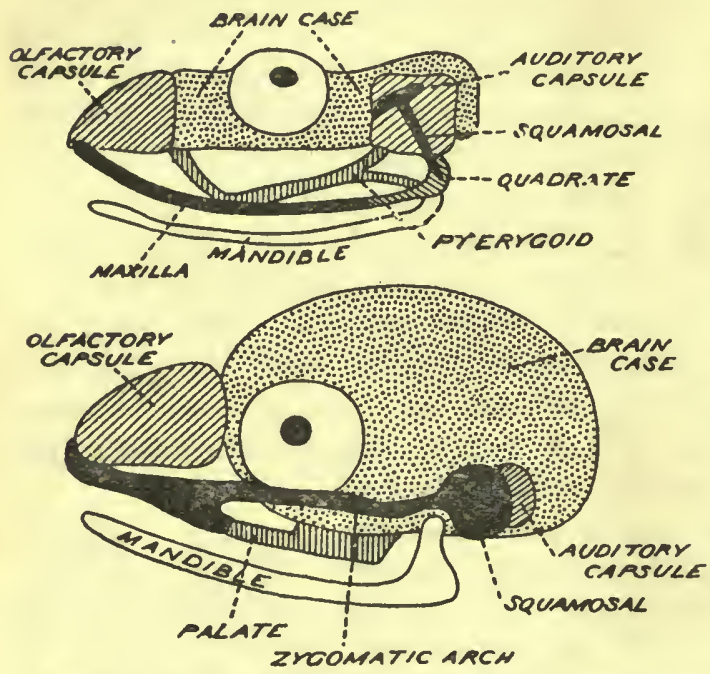

Fig. 85.-Skulls of Frog and Dog: Diagrammatic side views. the essential constituents of tho cranio-facial apparatus, as we pass from the amphibian to the mammalian skull. The student should compare with the more accurate figures of both skulls, and convince himself that he appreciates the diagrammatic rendering of these parts. Now all the distinctive The shading does not mean the same as in figs. 81 and 83. differences in form of the dog's skull from the frog's are reducible. to two primary causes-

(1) The brain is enormously larger, and the brain-case is vastly inflated, so that-

(a) the otic capsule becomes embedded in the brain-case wall ;

(b) the palato-pterygoid rod lies completely underneath the brain-case instead of laterally to it;

(c) the squamusal tilts down and in, instead of down and out, and the lower jaw articulates with its outer surface instead of below its inner, and, moreover, with the enormous distention of the brain-case it comes about that the squamosal is incorporated in its wall.

(2) The maxilla anteriorly and the palatine posteriorly send down palatine plates that grow in to form the 
bony palate, cutting off a nasal passage from the mouth cavity, and carrying the posterior nares from the front part of the mouth, as they are in the frog, to the pharynx. Hence the vomer of the dog lies not in the roof of the mouth, but in the floor of this nasal passage.

$\S$ 7. The Auditory 0ssicles. The quadrate cartilage of the frog is superseded by the squamosal as the suspensorium of the lower jaw. It is greatly reduced, therefore; but it is not entirely absent. In the young mammal, a quadrate cartilage can be traced, connected with the palatopterygoid cartilage, and articulating with Meckel's cartilage. Its position is, of course, beneath - the squamosal, and just outside the otic capsule. As development procéeds̀, the increase in size of the quadrate does not keep pace with that of the rest of the skull structures. It loses its connexion with the palatopterygoid, and apparently ossifies as a small ossicle-the incus of the middle ear. A
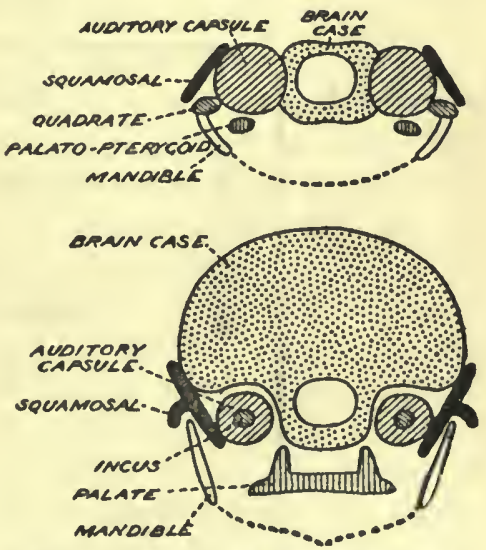

Fig. 86. - Skulls of Froc aNd Dua: Diagrammatic end views.

Shading as in fig. 85. small nodule of cartilage, cut off from the proximal end of Meckel's cartilage, becomes the malleus. Hence these small bones seem to be the relics of the discarded jaw suspensorium of the frog utilized in a new function. Considerable doubt, however, attaches to this interpretationdoubt that, if anything, is gaining ground. The stapes would appear to be derived from the hyoid arch and to answer to the frog's columella.

§ 8. Comparison of Frog's Skull and Dog's. Besides these great differences in form, there are important differences in the amount and distribution of centres of ossifica- 
tion of the skull of frog and mammal. There is no parasphenoid in the dog; and, instead, a complete series of ossifications, the median basi- and pre-sphenoids, and the lateral ali- and orbito-sphenoids occur; so that, in place of the largely cartilaginous skull of the frog, we have a completely bony skull. The points can be rendered much more luminously in a diagram than in the text, and we would counsel the student to compare this of the frog very carefully with that of the dog (p. 83).

\section{CRANIUIM.}

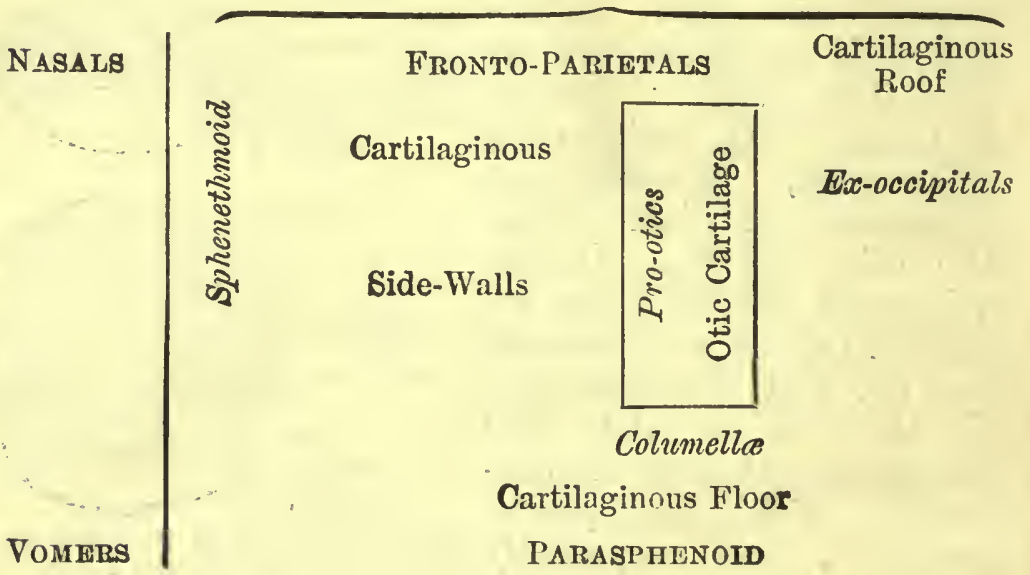

\section{UPPER JAW.}

SQUAMOSALS

PRE-MAXILLA

\begin{tabular}{|c|c|}
\hline PALATINES & PTERYGoIDS \\
\hline
\end{tabular}

\section{LOWER JAW.}

Mento-meckelians

Meckel's Cartilage

Dentaries

ANGULO-SPLENIALS 
Additional points to be noticed are:

(1) The otic capsule (= periotic bone) of the dog ossifies from three centres, one of which is equivalent to the frog's pro-otic.

(2) The several constituents of the lower jaw are not to be distinguished in the adult mammal, which consists of a single membrane-bone (on either side), Meckel's cartilage altogether disappearing during the course of development.

(3) The frog has no lachrymal or tympanic bones.

(4) The zygomatic arch of the dog is not represented in the frog.

$\S$ 9. Metamerism of Skull. We are now in a position to notice, without any danger of misconception, what is called the segmental theory of the skull. Older anatomists, working from adult structuro only, conceived the idea that the brain-caso of the mammal represented three inflated vertebræ. The most anterior had the pre-sphenoid for its centrum, the orbito-sphenoids for the sides of its neural arch, which was completed above by the frontals (frontal segment). Similarly, tho basi-sphenoids, ali-sphenoids, and parietals formed a second vertebra (parietal segment), and the basi-, ex-, and supra-occipitals a third (occipital segment). If this were correct, in the frog, which is a more primitive rendering of the vertebrate plan, we should find the vertebral characters more distinct. But, as a matter of fact, as the student will perceive, frontal segment, parietal segment, and occipital segment can no longer be traced; and the mode of origin from trabeculæ and parachordals shows very clearly the falsity of this view. The vertebrate cranium is entirely different in nature from vertebræ. The origin of the parietals and frontals as paired bones in membrane reinforces this conclusion.

But as certainly as we have no such metameric segmentation as this older view implies, in the brain-case of the frog, so quite as certainly is metameric segmentation evident in its branchial arches. We have the four gill-slits of the tadpole and their bars repeating one another; the hyoid bar in front of these is evidently of a similar nature; and tho view that the ear-drum is derived from an imperforate 
gill-slit is supported by the presence of an open slit (the spiracle) in the dogfish in an entirely equivalent position. But this matter will be more intelligible when the student has worked over the dogfish, and need not detain us any further now.

Moreover, the fact that the hypoglossal nerve emerges behind the first vertebra in the frog, and through the exoccipital in the dog, seems to show that in the latter the skull has encroached, as it were, on a region originally belonging to the vertebral column. 


\section{Chapter XV.-Tine Dogfish.}

\section{(Scyllium caricula.)}

§ 1. Form and Mode of Life. In the dogfish we have a strictly aquatic type of vertebrate, a member of an order of fishes found only in the sea. Like all the more highly organized marine animals, it is carnivorous, and it is not at all restricted in its diet, caring only for quantity. It is really a small shark, differing from other sharks in little save size; and its structure differs in many respects from that of the more familiar bony fishes, such as cod, salmon, herring, or perch.

The general build of the body is eminently adapted to an aquatic life. The whole body is laterally compressed,

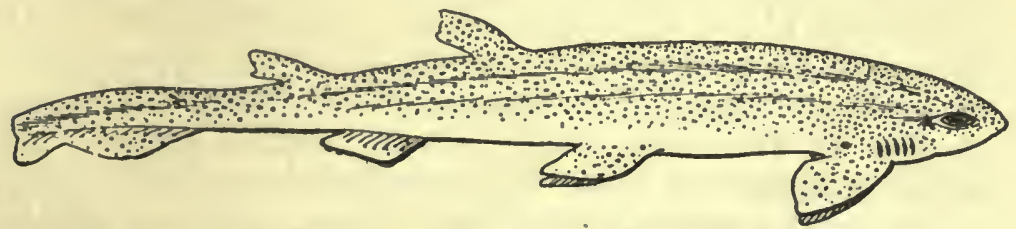

Fig. 87.-Tue Doafish (Scyllium canicula, after Day's Brilzs/t Fishes).

and smooth in outline. Head passes into trunk and trunk into tail without any sudden change. The tail is about as long as the trunk, and tapers gradually behind, finally curving upwards, the outline of the end being formed by an unequally-lobed caudal fin. Two median dorsal fins project from the back, and there is also a median ventral (or anal) fin. Farther forward ventrally, in the position where the limbs would be found in a land-rertebrate, we find paired fins, pectoral and pelvic. Though obviously answering to limbs, these have none of the complex articulations we have previously met: they are simply flexible flattened expansions, and instead of directly performing locomotion merely co-operate with the median fins in 
steering-the propelling organ being the muscular tail and, in a less degree, the trunk.

The mouth is not terminal, as in the rabbit, frog, and bony fishes -it is ventral. The cloacal opening lies between the pelvic fins. In front of the mouth are the nares-the wide apertures of the olfactory organs. These have nothing to do with respiration as in our other types, but are solely concerned with smell. There are no internal nares, but an external groove, partly covered in by a fold of skin, connects each olfactory chamber with the mouth. The eyes occupy the ordinary position, but have no movable lids. Behind them, just where we might expect to find the tympanic membrane, there is an opening on either side-the spiracle; and at each side of the throat are five slit-like openings, the gill-slits. These are concerned in respiration.

§2. Dermal Denticles. The skin of the dogfish is closely set with pointed tooth-like spines, the dermal denticles (formerly called placoid scales), and these are continued over the lips into the mouth as teeth. Each scale consists of (1) a horizontal basal plate embedded in the dermis, (2) a pointed spine arising from the upper side of the plate and protruding through the epidermis. The plate consists of true bone, the spine of dentive capped with enamel, and there is a hole in the centre of the plate through which blood-ressels pass into the cellular pulp-cavity in the axis of the spine-exactly the structure of a mammalian tooth in all essentials (chap. vi., §12). The bases of these scales are almost the only real bone-tissue in the dogfish; and perhaps, in addition to the teeth other relics of the placoid scales are to be found, in the higher vertebrata, in the membrane-bones. How placoid scales may have given rise to these structures will be understood by considering such a bone as the vomer of the frog. This bone lies on the roof of the frog's mouth, and bears a number of little teeth, and altogether there is a very strong resemblance in it to a number of placoid scales the bony bases of which have become confluent. In the salamander, behind the teeth-bearing vomers comes a similar toothed parasphenoid bone. The same bone occurs in a corresponding position in 
the frog, but without tecth. In some urodele amphibians the vomers and the splenials of the lower jaw are known to arise by the fusion of small denticles. These facts seem to point to stages in the fusion of placoid bases, and their withdrawal from the surface to become incorporated with the cranial apparatus as membrane bones, a process entirely completed in the mammalian type.

$\S 3$. Sensory Tubules. Scattered over the snout, especially on its ventral surface, are numerous fine pores. These are the openings of long tubes, filled with a jelly. "Internally they end in dilatations which resemble the ampullæ of the ear, and whose minute structure and nerve-supply show them to be sense-organs: they evidently are concerned with a sense possible only to aquatic animals, for similar tubes occur in the tadpole and are lost in the adult frog. The most reasonable suggestion is that they are organs of a pressive-sense, which must be needful in animals that swim in fish-like fashion. In most fishes similar but shorter tubes are arranged in a row on either side, forming the lateral line which is so conspicuous in the salmon, for example. But in the dogfish the lateral line is very feebly developed, though it can be made out in transverse sections.

$\S 4$. Transverse Sections. In transverse sections (figs. 88 and 89 ), the most obvious difference from frog and rabbit is the great development of the body-muscles, due to the swimming habit. These muscles, if dissected from the side, are seen to be metamerically segmented to a much more complete extent than in frog and rabbit. Each musclesegment is called a myomere or myotome, is shaped like a $\mathrm{V}$, the point directed forwards, and the ventral limb longer than the dorsal. Owing to this shape, parts of several myomeres are seen in a transverse section. At their dorsal ends the myomeres alternate with the vertebræ, i.e. one myomere is attached partly to one vertebra and partly to the next, while each vertebra is pulled by parts of two myomeres on each side. In this way the sinuous movement characteristic of swimming is rendered possible.

§ 5. The Cœlom extends farther forward than in the frog. 


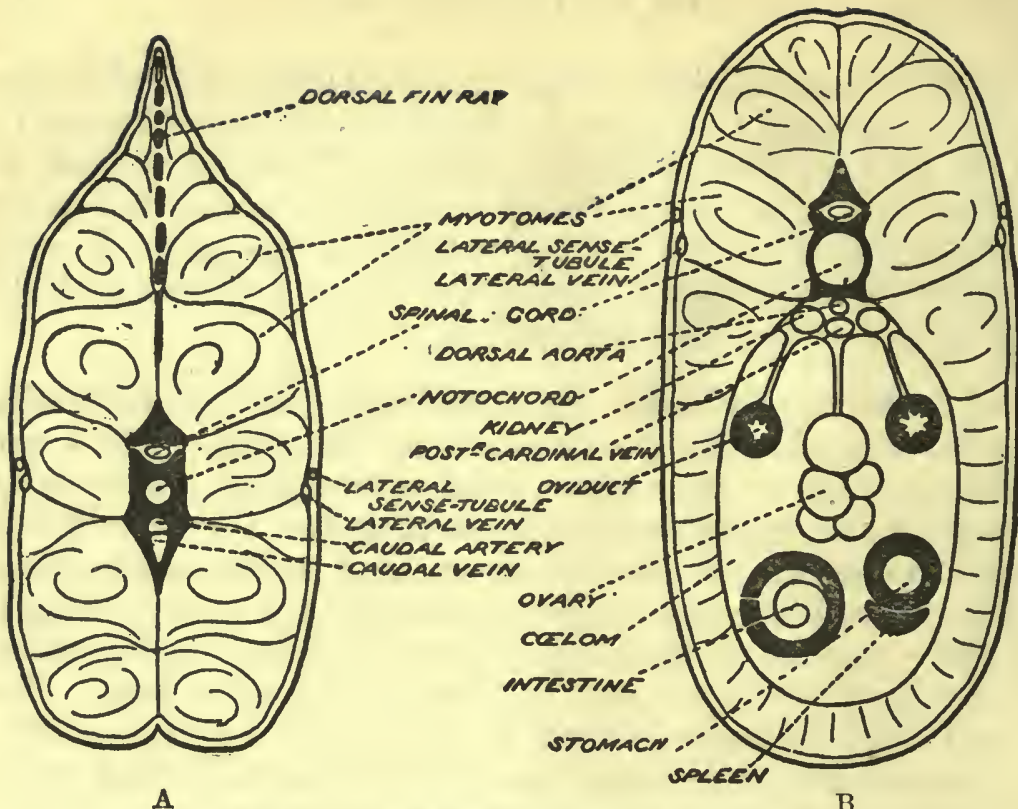

Fig. 88.-Transyerse Shctions of Dogfish.

A, through tail ; $B$, through abdominal region (partly diagrammatic).

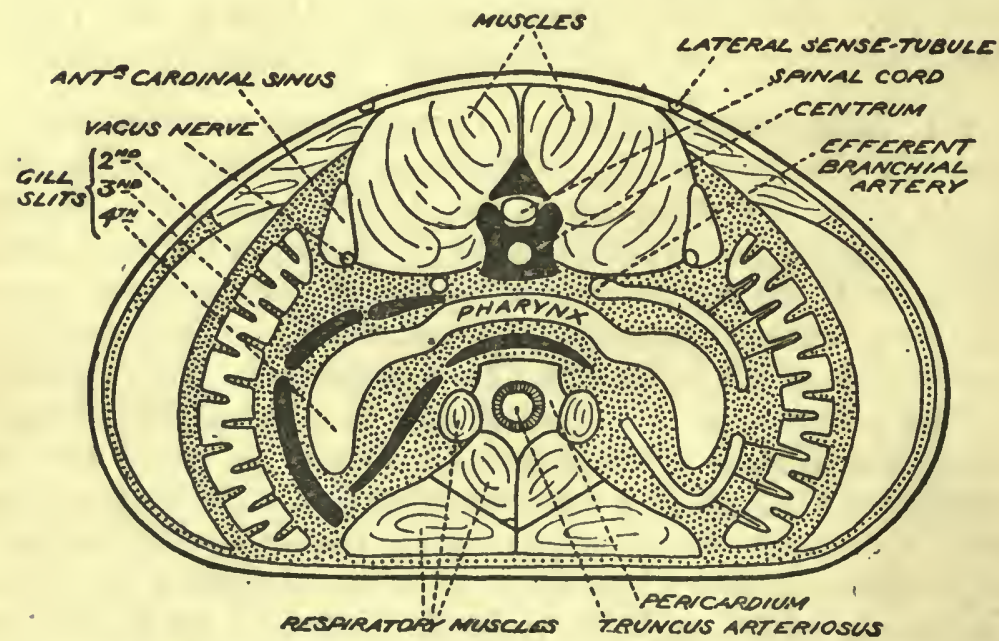

Fig. 89.-Transverse Section of Dogfish through Pharynx.

Owing to the obliquity of the gill-slits, three are cut through on each side. On the left the branchial cartilages (black) and on the right the arteries (white) ale shown diagraumatically. 
The pericardial cavity is ventral to the pharnyx (fig. 89), and its outer membrane no longer hangs like a bag in the cœlom: it forms a vertical partition between pericardial and peritoneal cavities. This partition is apt to be mistaken for a diaphragm like the rabbit's. This it is not, for the cavity anterior to it contains the heart alone, and is wholly ventral to the cesophagus. At its dorsal end this partition is imperfect, and a pericardio-peritoneal passage unites pericardium to cœlom. At its posterior end the cœlom communicates directly with the exterior by a pair of abdominal pores, one on each

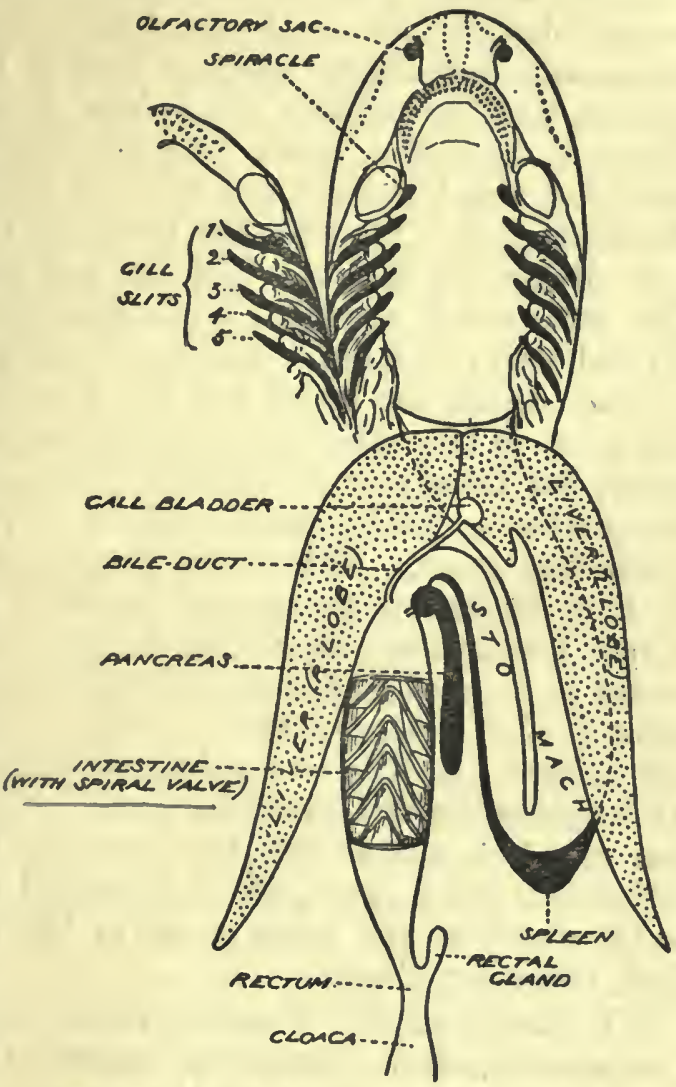

Fig. 90.-Almentary Canal of Dogrish. side of the cloaca. The mouth and pharynx have been cut open (as in Their function is not very obvious :

fig. 68). The curved rows of dots in front of the mouth represent the pores of the sensory tubules.

possibly by allowing some of the colomic fluid to escape when the external pressure on the body-wall increases (as when the dogfish descends to some depth in the sea) they may prevent the increased pressure being felt by the viscera.

§ 6. The Alimentary Canal is simpler than in the frog. It is a simple tube with one fold, giving it an $\mathrm{N}$ shape (fig. 90). 
The mouth is rough with denticles, and has a small, immovable tongue on its floor. The next part of the canal, containing spiracles and gill-slits in its walls, is called the pharynx: we will describe this in the next paragraph. It is followed by a wide oesophagus passing into a U-shaped stomach, having, like the rabbit's, the spleen on its outer curvature. There is no coiling small intestine, but the short portion receiving the bile-duct and duct of the pancreas is called the duodenum. The liver has large left and right lobes, and a small median lobe in which the gall-bladder is imbedded. The pancreas is compact, as in the frog, and is partially divided into two lobes. The next segment of the intestine is often called the colon, but more probably is the ileum: the colon in the rabbit is the chief region of absorption of water, but fishes excrete no water-vapour as the hot-blooded air-inhabiting rabbit does, and so have little need of a specialized colon. This ileum contains a spiral valve, the shelf of which points steeply forward; this acts just like a spiral staircase, the chyme having to travel round and round in its passage along the intestine. It is consequently exposed for a longer time and to a larger surface for absorption. In frog and rabbit the same result is attained by lengthening the intestine itself. The final portion of the canal is short and has connected with it a thick cylindrical rectal gland, which has no particular resemblance to the gland of the same name in the rabbit. As in the frog, there is a cloaca.

$\S 7$. Respiration. The respiratory apparatus is adapted to water-breathing, the gases concerned being dissolved in the water, and consists of the mouth, pharynx, and gillslits. The latter bear on their anterior and posterior walls the actual gills-highly vascular filamentous outgrowths serving to expose the blood to the oxygen-containing water. There is no palate, narial passage, glottis, larynx, or lungs.

Seen from the inside (fig. 90), the gill-slits are very large openings, occupying almost the whole of the sides of the pharynx, their external apertures being greatly contracted. It is easy to see, too, that the spiracle is another gill-slit whose ventral portion has been obliterated. The portions of the lateral walls of the pharynx between the slits are 
called the gill-arches (or branchial arches). They are strengthened internally by bars of cartilage, and from them arise the actual gill-processes in which the exchange of gases between blood and water is effected. These processes are very soft folds of the mucous membrane of the pharynx, covered with a thin epithelium, and containing a core of highly vascular connective tissue. The whole series of gillprocesses on one side of a gill-arch is called a demibranch (i.e. half-gill). There are on each side of the pharnyx nine functional demibranchs, and a vestigial one on the front face of the spiracle. The last branchial arch (the one posterior to the last gill-cleft) bears no gill-processes.

The aeration of the blood in the gill-processes is effected by the suction of water into the pharynx and its expulsion through the gill-slits. The muscular mechanism is roughly similar to that seen in the frog; alternate enlargement and diminution of the capacity of the pharynx are caused by the contraction of two sets of muscles attached to ventral plates of cartilage (basi-hyal and basi-branchial) which answer to the frog's hyoid plate. In inspiration the mouth opens, the pharynx fills with water, and the mouth closes. In expiration the water is forced out through the gill-slits and over the gill-processes, the branchial arches separate widely by contraction of their muscles, and the oesophagus closes by contraction of its muscular wall. The water passing over the gills is separated from the blood by a thin membrane, through which an interchange of dissolved gases takes place-oxygen from water to blood, carbon dioxide from blood to water.

$\S 8$. The Heart. The circulation is entirely devoid of that double character which is rather imperfectly attained in the adult frog, and perfectly in the rabbit. There being no lungs, there are no pulmonary veins, and no division even of the auricle into two, still less of the ventricle. All the blood that passes through the heart is deoxygenated and has but one possible course - through sinus venosus, auricle, ventricle and truncus, arteriosus. In fig. 91, a comparison of the heart of dogfish and frog is given. It will be seen from it that the condition in the frog is arrived 
at by a more complete doubling over of the heart on itself, the dogfish being obviously nearer the primitive condition of a straight tubular heart such as is found in embryonic stages of all vertebrates.

The truncus arteriosus consists, as in the frog, of two

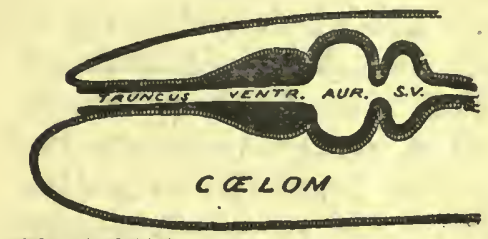

PRIMITIVE

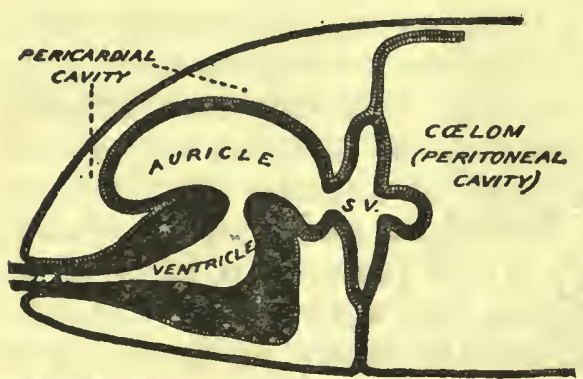

DOCF/SH

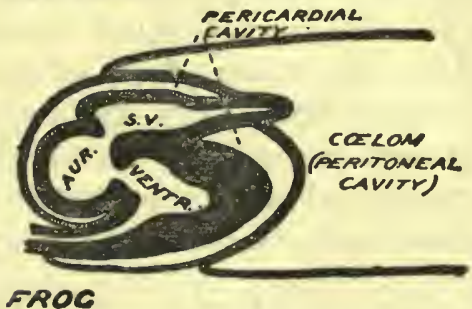

Fig. 91.-Development of the Heart of VERTEBRata.

parts: the first, the conus or pylangium, muscular, contractile, and containing a series of valves; the second, the bulbus or synangium, without valves. The bulbus is often conveniently called the ventral aorta.

§ 9. The Arteries. From the bulbus there branch, on either side, four arterial trunks, the first of which forks, so that altogether there are five afferent branchial arteries taking blood to be oxygenated in the gills. The first of these runs in the hyoid arch (i.e. the arch between spiracle and first gillcleft), the others in the first four branchial arches. Each sends 'off branch arteries into all the gill-filaments, and finally dies out near the dorsal end of the gill-arch.

The blood from the gill-filaments is gathered into nine vessels, one to each demibranch, or two to each afferent artery except the first. These unite into four efferent branchial arteries, as shown in fig. 92, and it will be seen that the two demibranchs supplied by one of the afferent 
vessels have their blood returned into different efferent vessels.

From the point where the first two of these nine vessels join, a carotid artery is given off to the head. The four efferent arteries unite into a median dorsal aorta, from which arise paired subclavian arteries (to the pectoral fins), no less than four median arteries to the alimentary canal (coeliac, anterior mesenteric, lienogastric and posterior mesenteric), a paired metameric series of small arteries to the body - wall; rencils, to the kidneys; and a small pair of iliacs to the

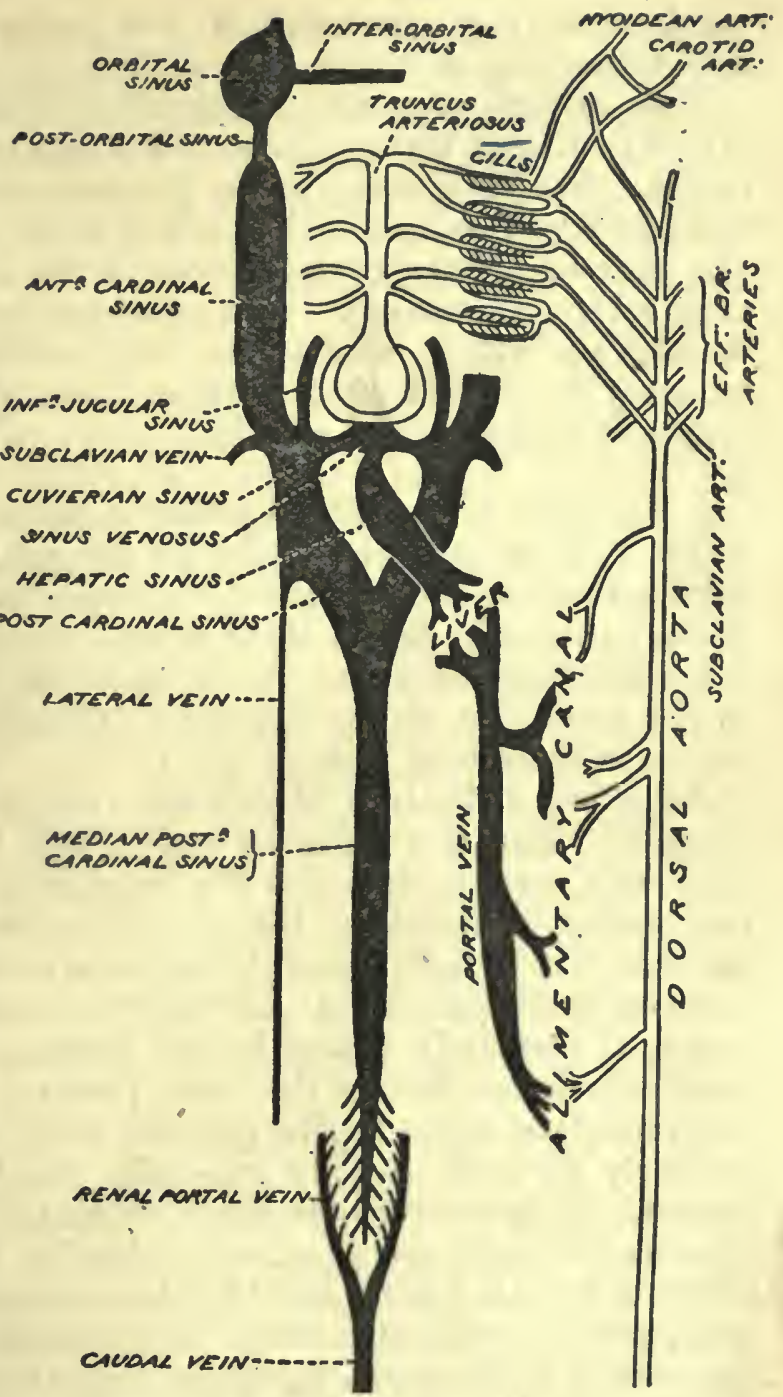

Fig. 92.-Circulation of Dogrish.

To bring the arteries into their proper position the right side of the diagram should be folded over, so that the dorsal aorta comes into the same line as the median posterior cardinal sinus.

\section{zoor.}


pelvic fins: the remainder of the aorta behind this is called the caudal artery.

Now, at last, we can see the meaning of the great arterial arches that come between heart and dorsal aorta in frog and rabbit. Evidently they are the last traces of the arrangement by which, in fishes, the blood is made to pass through the gills to be oxygenated before it is distributed to the capillaries of the tissues. This arrangement is not wanted in animals which breathe air, but although there is a tendency to get rid of it; even in the highest animals a couple of arterial arches remain.

$\S 10$. Venous System. Instead of being narrow tubes, many of the veins in the dogfish are irregular baggy sinuses, enlarged to such an extent that some structures (particularly nerves) appear to lie in them, though between the actual nerve and the blood exactly the same layers intervene as if the nerve ran alongside a vein. (Compare the case of organs in the cœlom, chap., i. § 6.)

As in frog and rabbit, three main trunks enter the sinus venosus. But the posterior one brings blood from the liver only, and so is called the hepatic sinus. It may be regarded as equivalent to the hepatic veins plus the most anterior part of the postcaval, but its actual opening to the sinus venosus is paired. At any rate, the main portion of the postcaval is entirely absent in the dogfish. Owing to the more anterior position of the heart (ventral to the throat) the precavals come to lie quite transversely instead of obliquely forward, and are generally called the Cuvierian sinuses. They receive near their confluence with the sinus venosus the inferior (= external) jugular vein. At their dorsal origin they are formed by the meeting of the anterior and posterior cardinal sinuses. The anterior cardinal sinus (answering to the internal jugular vein of frog and rabbit) lies dorsal to the gill-slits, and receives an orbital sinus from around the eye. The posterior cardinal sinus receives a subclavian and a lateral vein on either side and unites posteriorly with its fellow in the middle line. This median portion receives blood from the kidneys: it must not be confused with the postcaval-along with the right posterior 
cardinal it represents the rabbit's azygos vein. A caudal vein, bringing blood back from the tail, splits behind the kidneys, and forms the paired renal portal veins, breaking up into a capillary system in the renal organ. A portal vein brings blood from the alimentary canal to the liver.

The blood of the dogfish contains red and white corpuscles, both of which are almost identical, in size and form, with those of the frog.

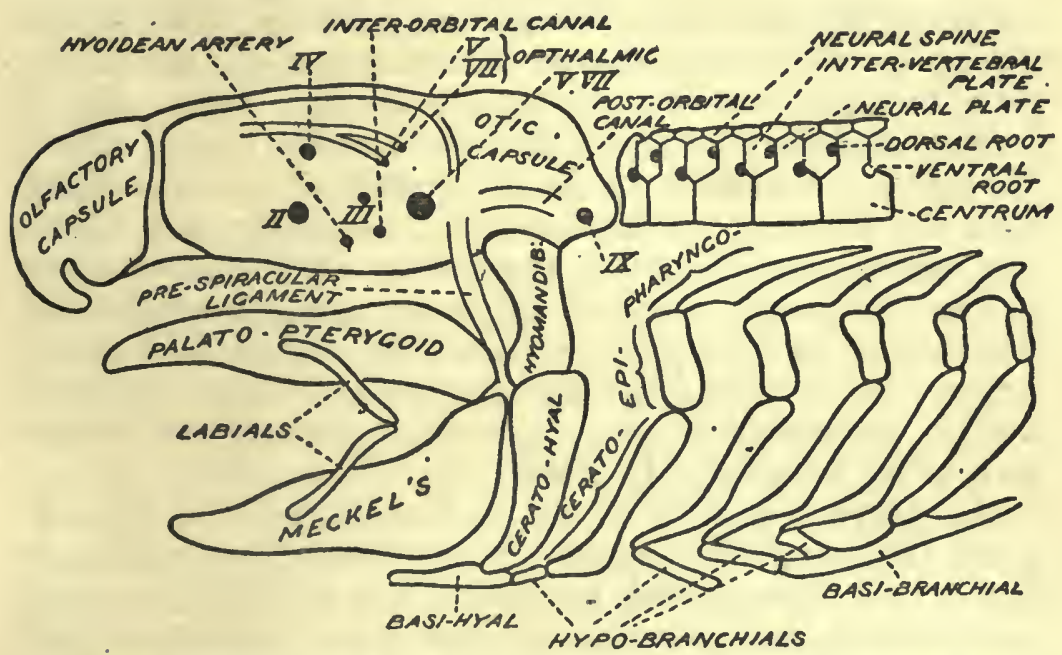

Fig. 83. - Skule, Vibceral Arches, and Part of Vertebral Colums OF DoGFish.

(Modified from Marshall and Hurst.)

$\S 11$. The Skeleton (excluding the placoid scales) is almost entirely cartilaginous, though some of the parts are calcified. Hence only those parts which are cartilage or cartilage-bone in frog and rabbit are represented here.

The vertebral column (fig. 93), consists of a very large number of vertebræ, divisible into two sets only-trunkand tail-vertebræ. No sacral vertebræ can be recognized, as the pelvic girdle does not meet the vertebral column. In a typical trunk vertebra we have a centrum, roughly cylindrical in shape, and with a deep conical hollow at each end-the two hollows meeting in the middle. Thus a 
cavity, alternately large and small, runs through the wholv, vertebral column from end to end, and this is occupied by a soft, gelatinous tissue-the persistent remains of that important embryonic structure the Notochord (chap. VI, $\S 15)$. The variation in diameter of the notochord is shown in figs. 88, a and 89 (cut through the middle of a centrum) and 88, B (through the end of one). This cavity must not be confused with the neural cavity-containing the spinal $\operatorname{cor}^{\circ} d$, which here (as in all cases) is altogether dorsal to the centra of the vertebræ. From each centrum there extend up a pair of neural plates to form the sides of the neural arch, but these are shorter than the centra, and a series of intervertebral neural plates, distinct from the centra, fill up the gaps. A double series of neural spines, alternating with the plates, completes the arch dorsally (fig. 93). From the centra there come off ventro-laterally short transverse processes, to which short cartilaginous ribs are articulated. There are no zygapophyses, and the centra and neural plates are connected by fibrous tissue, so that but little motion is possible at any single point, though the column as a whole is sufficiently flexible.

As we pass back from trunk to tail, we find the ribs cease, the transverse processes turn ventralwards, and finally unite in a homal arch (enclosing the caudal artery and vein), which may be distinguished from the neural arch above by the gaps between the successive processes (fig. 94). Ventrally it is completed by a

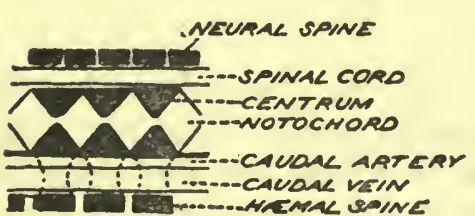

Fig. 94.-MEDran Lonartudinat Section of Three Caudal Vertebrat OF DOGFISH. series of hrmal spines. The only other difference of the tail-from the trunk-vertebræ, is that the neural spines are less regular, there being often three, instead of four, of them to two vertebræ.

Where the median fins come, there are cartilaginous fin-rays developed in continuation of the neural spines; and beyond these, in the fin itself, horny rays.

$\S 12$. The skull (fig. 93) consists of a cartilaginous 
cranium, and of jaws and visceral arches. The cranium persists throughout life, in what closely resembles a transitory embryonic condition of the higher types. There is a pair of olfactory capsules in front, a brain case proper, and lateral otic (auditory) capsules containing the internal ear. This should be compared with the tadpole's skull (fig. 80). The upper jaw has a great bar of cartilage, the palato-pterygoid, as its sole support; a similar bar (Meckel's cartilage) supports the lower jaw. In the frog these bars of cartilage are connected directly to the otic capsule by a quadrate cartilage, but this is only doubtfully represented in the dogfish by a thin plate of cartilage just in front of the spiracle. Instead, we find the jaws articulated with the skull by a large cartilage behind the spiracle-the hyomandibular, the uppermost division of the hyoid arch. A skull with this arrangement is termed hyostylic, as distinguished from the autostylic skull of the frog. The rest of this hyoidean arch on either side consists of a cerato-hyal, joined ventrally to a median basi-hyal.

Five branchial arches * follow, supporting the walls of the pharynx between the gill-slits. An ideal branchial arch would be a complete hoop of cartilage, encircling the pharynx, and divided on each side into divisions, named from the dorsal side downwards pharyngo-, epi-, cerato-, and hypo-branchials, with a median basi-branchial in the floor of the pharynx (cf. fig. 89, left side). But only in the first two do the pharyngo-branchials of each side meet dorsally to form a complete hoop; and the two last pharyngobranchials are fused together on each side. The fifth arch has no hypo-branchial. As to the basi-branchials, the first one is fused up with the basi-hyal; the other four are united into a single plate, the basi-branchial. Obviously the "hyoid plate" of the frog, and "body of the hyoid" of the rabbit or dog represent basi-hyal plus basi-branchial. The dog's "anterior cornu" is the main part of the hyoid arch, and its "posterior cornu" is the first branchial arch. (The frog's posterior cornu appears to be the fourth branchial

* There is some confusion in the use of the term "arch" : it is sometimes applied to the cartilages alone, at others to the whole sidewall of the pharynx between two gill-slits. 
arch.) But what has become of the hyomandibular in higher types? Considering the articulation of the hyomandibular with the otic capsule, and its position immediately behind the spiracle (=ear-drum), it is natural to suppose it may be represented by the columella of the frog and stapes of the rabbit. But the development of the hyoid arch in these higher types is not yet made clear enough to definitely establish the homology.

It is important to realize that the cartilaginous branchial arches lie internal to (i.e. nearer the cavity of the pharynx than) the afferent branchial arteries: the efferent ones lie alongside them. The basi-branchial similarly lies nearer the pharynx-cavity than the truncus arteriosus; it even extends back into the region of the heart and lies on the dorsal side of the pericardial cavity. This is an important point, for it enables us to plainly distinguish visceral arches from such cartilages (or bones) as ribs and limb-girdles. The latter are part of the body-wall: they are formed from somatic mesoblast, as we shall learn to call it shortly. But the visceral arches belong to the wall of the alimentary canal (splanchnic mesoblast). Owing to the obliteration of the cœlom (which separates body-wall from alimentary canal) in the throat region, this difference is easily overlooked, and at one time the limb-girdles were regarded as modified branchial arches.

Besides the main cartilages already mentioned, there are, in the head and throat, certain other much more superficial ones. Outside the olfactory capsule there are two pairs of rod-like rostral cartilages; outside the jaw-cartilages are two pairs of labials (fig. 93); and outside the ceratobranchials a series of extra-branchials. It should also be mentioned that the hyomandibular and cerato-hyal, and the first four epi- and cerato-branchials, carry cartilaginous gill-rays to support the gill-processes.

$\S$ 13. Fins and Fin-girdles (figs. 95 and 96). The paired fins and their girdles differ considerably from the limbs and limb-girdles of frog and rabbit, yet there is enough resemblance to prove their homology. At the same time there is a remarkable resemblance between the skeleton 
of the paired and that of the median fins-an interesting fact.

Each girdle is a bent bar of cartilage, divisible into dorso - lateral and ventral regions (cf. fig. 36), but there is no fenestra dividing up the latter, as in higher types. The pelvic girdle has no connexion with the ver-

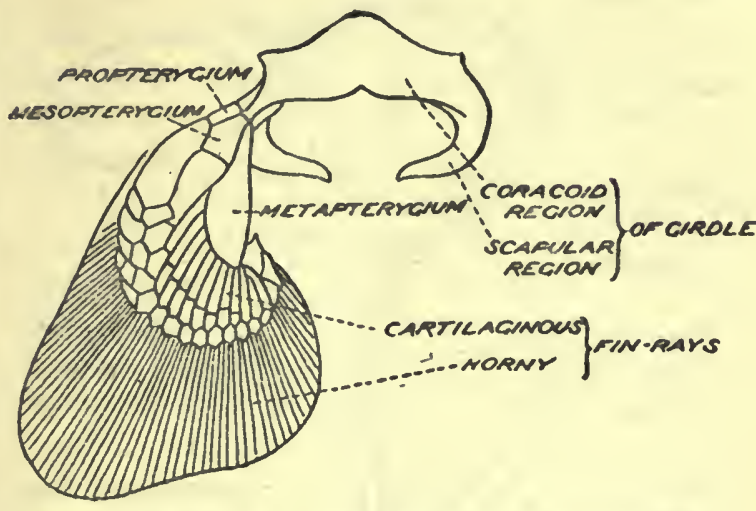

Fig. 95.-Pectoral Girdue and Right Pectoral Fin of DoGFish.

(After Marshall and Hurst.) tebral column.

The paired fins are supported proximally by a series of cartilaginous rays, and distally by a series of horny raysmuch as are the median fins.

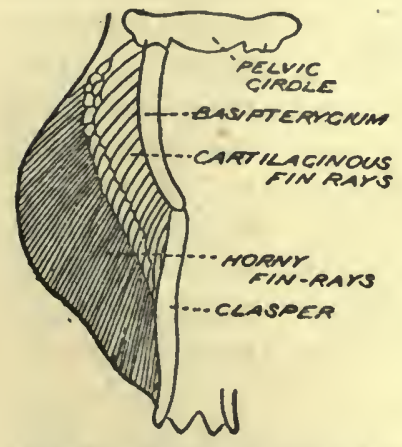

Fig. 96.-Pelvic Girdue and Right Pelvic Fin of Doofish. (After Marshall and Iurst.)

The cartilaginous rays vaguely suggest a multitude of fingers, but we cannot get nearer in our resemblance to the familiar limbs Instead of a humerus, we find in the pectoral fin three cartilages articulating directly with the girdle. These are called pro-, meso-, metapterygium - the order being from pre- to post-axial, and also from smallest to largest. In the pelvic fin, the first two are not recognizable, but the corresponding finrays articulate directly with the girdle: the meta- is here called basi-pterygium. In the male, but not in the female, the pelvic fins are united behind the cloaca, and there are two stiff grooved copulatory organs, the claspers, which have a 
cartilaginous support. These claspers form the readiest

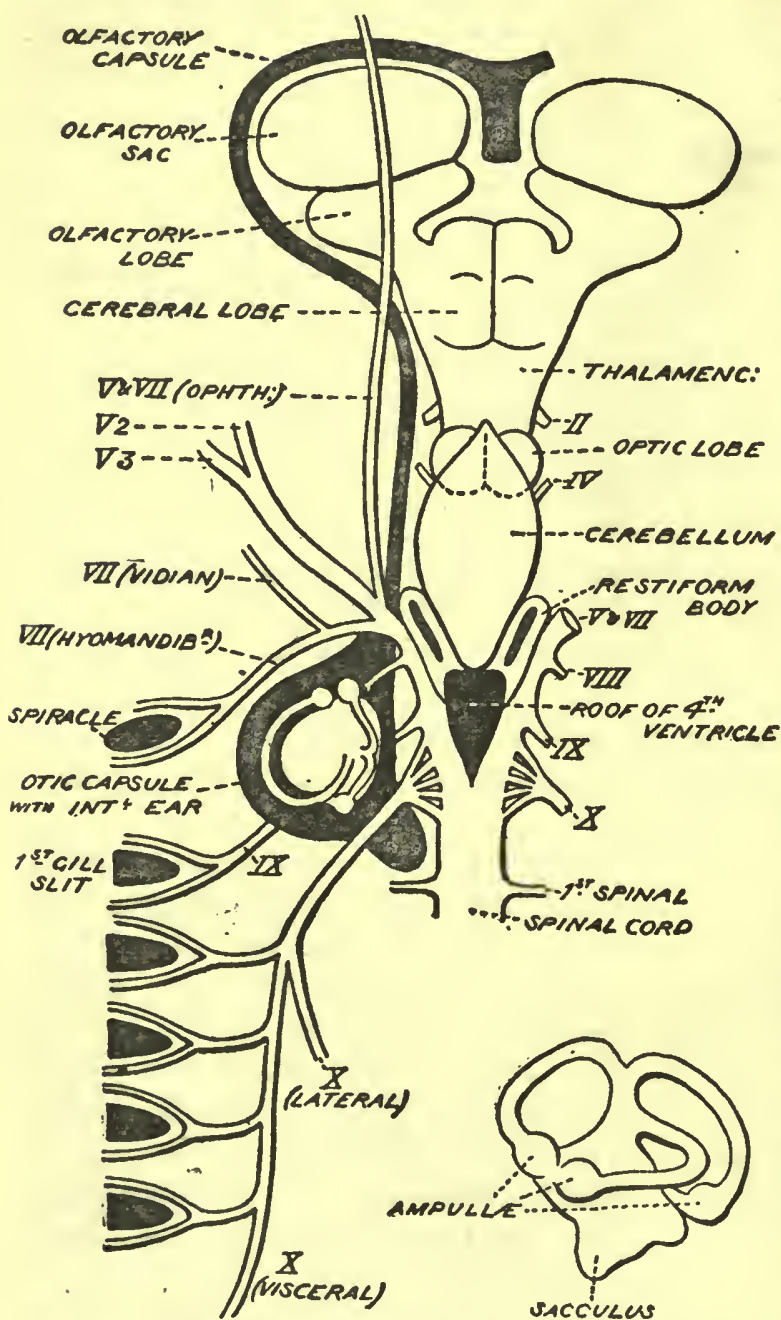

Fig. 97.-Brain, Cranial Nerves and Auditory Organ OF DogFISH. means of determining the sex of a specimen before dissection.

\section{§ 14. Ner-} vous System. The spinal cord is very like that of previous types. The spinal nerves are as numerous as the vertebræ. Their dorsal and ventral roots arise alternately, not in the same vertical plano as in previous types. They pass through the vertebral column by separate openings (fig. 93), and only unite outside it.

$\S$ 15. The Brain shows Dorsal view; cut edge of skull in black. (Partly after Wieder- the s a me $\mathrm{m} \theta$
sheim.) The separate small figure is a side view of the essential parts
internal ear. internal ear.

but the cavities are all very large, and the olfactory as in the frog,
the olfactory lobes and cerebellum, as well as the optic lobes, contain 
large cavities. The olfactory lobes lie well to the side and are joined to the small cerebral lobes by a very wide stalk. The thalamencephalon has a thin, flat roof, from which a long stalk runs forward to the pineal, which lies near the skin, as in the frog. Ventrally, it forms an infundibulum whose sides are thickened and called the lobi inferiores; while at its end is the pituitary body, with a pair of thin-walled sacs (sacci vasculosi), developed at its sides. The optic lobes resemble those of the frog, but the cerebellum is very large and laps over them. Behind it comes the usual thin roof to the fourth ventricle; but at the sides of this are a peculiar pair of hollow projections, called the "restiform bodies" (a term applied in human anatomy to the posterior peduncles of the cerebellum, which are not homologous with these structures of the dogfish), On the ventral surface, crura, pons and bulb are all much alike and pass back with little change into the spinal cord.

The small size of the cerebral lobes betokens a low grade of intelligence, and still more striking than any matter of size is the apparent entire absence of the characteristic pyramidal cells of the cortex of higher types-cells specially concerned with the association of ideas. We may therefore expect that memory and the capacity for education are either quite absent or in a most rudimentary state. The habits of the dogfish are not such as to make easy the study of its psychology, but what is known of other fishes confirms the deductions from brain-structure. On the other hand, the large size of the cerebellum, as contrasted with its small size in the frog, is readily understood.

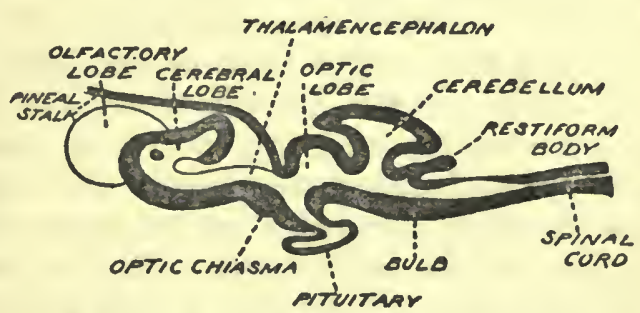

Fig. 98.-Median Longitudinal Section OF Brain OF DOGFISH.

Compare their respective shapes in transverse section (e.g. figs. 66 and 88), and it will be seen that the laterally compressed and swimming dogfish has great use for the 
co-ordinating centre of muscular action in keeping its equilibrium.

$\S 16$. The Cranial Nerves are exactly like those of the frog when allowance is made for the differences in the other systems of organs. $I$. and $I I$. are exactly the same in origin and in distribution; so also are $I I I$., $I V$., and $V I$. : but it may be mentioned that $I I I$. and $I V$. pass through the cranial wall by foramina of their own (see fig. 93).

$V$. has its usual three branches. $V_{\cdot 1}$ emerges by an aperture of its own, runs along the upper side of the orbit, and is joined by a parallel branch of VII. The joint nerve thus formed is called the ophthalmic, and is distributed to the sensory tubes (see $\S 3$ ) of the snout. The other two branches are mixed nerves, and pass to upper and lower jaws respectively.

The branch of VII. mentioned above is not apparently found in higher types, but the other branches are familiar to us-a vidian or palatine to the roof of the mouth; and a hyomandibular branch which forks over the spiracle, which is thus proved homologous with the frog's and rabbit's tympanum. These are all apparently mixed nerves, and supply the skin and muscles of the regions they run to.

$V I I I$. is the auditory nerve, as usual.

$I X$. passes through the otic capsule, ventral to the internal ear, and emerges apparently into the anterior cardinal sinus (see $\S 10)$. It soon forks over the first gill-slit, and its branches (mixed) are distributed to the same regions of pharynx and tongue as in higher types.

$X$. arises from a number of roots as though it represented several nerves run together. It runs through the otic capsule and along the floor of the anterior cardinal sinus. Its branches are (a) a lateral nerve (sensory) to the senseorgans of the lateral line; (b) four branchial nerves, each forking over a gill-slit, as $I X$. does over the first; $(c)$ a visceral nerve to heart and stomach. Of these, the first is unrepresented in frog and rabbit, as no lateral-line-organs exist in them; the branchial branches may be represented by the laryngeal nerves which supply somewhat the same region of the throat; while the visceral is of course familiar to us. 
The resemblance in distribution of $V ., V I I ., I X$. , and $X$. is singular. Each has a ventral division which forks over mouth, spiracle, or gill-slit, and each (except IX.) has a dorsal sensory branch to the peculiar sense-tubules. Evidently $X$. is really at least four metameric nerves partially united: does its extension to the stomach mean that more gill-slits once existed in that direction? And does the relation of $V$. to the mouth mean that that opening is a pair of anterior gill-slits united together? To discuss such questions will be beyond the scope of this work, but we may say that the tendency of morphologists to-day is to answer the second question in the negative.

$\S 17$. Sense-organs. The olfactory organs are a pair of spheroidal sacs, open ventrally, and having a great number of vertical laminæ projecting into the cavity from the dorsal side. These serve the same object as the turbinal foldings in the rabbit (chap. x., § 4), viz. increase of sensitive area. The olfactory sacs in fishes have no connexion with respiration, as they have in land-vertebrates.

The eye differs in no essentials from that of the rabbit, but only in details. For example, the lens is globular, and nearly fills the posterior chamber: a condition adapted to seeing through water. The eye-muscles are the same as before, but there are no glands, such not being needed in an animal whose eyes are always in contact with water.

The ear (fig. 97) is similar to that of the frog. It consists of internal ear only-utriculus and sacculus are feebly indrcated by a slight constriction of the general sac, and a little process at the posterior end is all the trace there is of a cochlea. The endolymph contains "otoliths," as in the frog. The semicircular canals are well developed.

The sensory tubules of the head and lateral line have already been mentioned. The resemblance of the semicircular canals to them suggests that the former may be specialized members of the latter series, and that the function of the tubules may be the appreciation of water-pressure. But speculations on the nature of a sense we do not ourselves possess are not very profitable without experimental evidence. 
$\S 18$. Reproductive Organs. The female is peculiar in having only one ovary, apparently median, but really the right, that of the left side disappearing during development. The oviducts occupy a similar position to those of the frog,

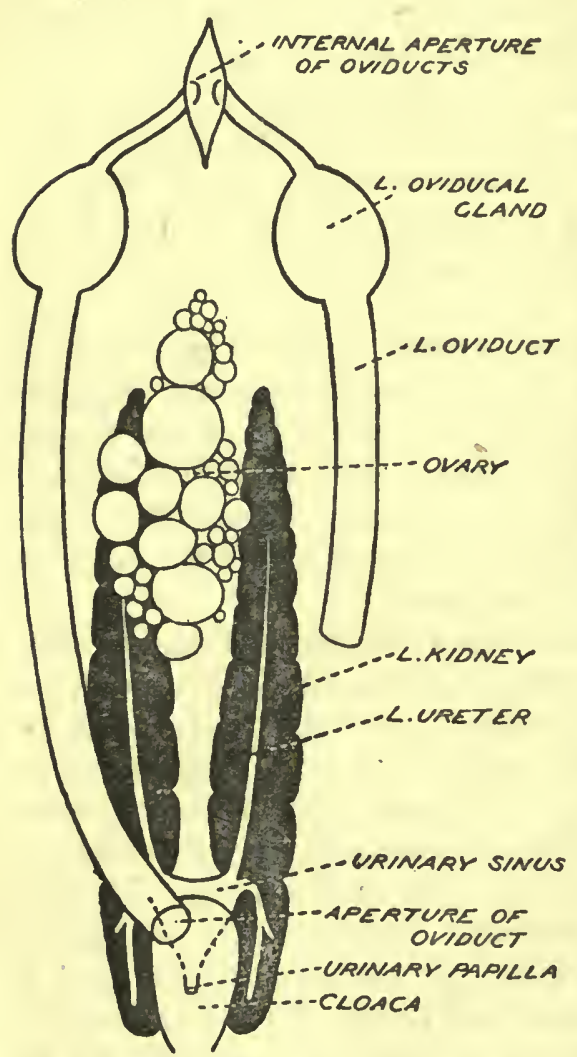

Fig. 99.-Female Reproductive Organs OF DOGFISH.

(Slightly diagrammatic.)

but owing to the absence of lungs and forward position of the heart, their anterior ends (with the internal openings) come together in the middle line between the pericardium and the liver, and there is a large funnel - shaped united opening. Each oviduct is a nearly straight tube, bearing an oviducal gland a little way from the front end, and opening posteriorly into the cloaca. The eggs are very large -about half an inch in diameter when ripe-. and only two are laid at a time, one by each oviduct. As they pass through the oviducal gland, an egg-case (fig. $100)$ is secreted around them, the curled elastic threads of which entangle in sea-weed. In this case the embr'yo safely undergoes its development. In respect of its reproduction the dogfish follows a higher method than the frog-making sure that the few eggs laid will reach the adolescent state, instead of trusting to the chance of a few being lucky out of many eggs badly provided for.

In the male we have a pair of large long testes, which are 
partly confluent at the posterior end. From the anterior ends of these, a series of vasa efferentia run to a body which obviously is the anterior portion of the kidney, as in the frog. But this has its own separate duct-the vas deferens, quite distinct from the ureter. This vas deferens is dilated posteriorly into a large cylindrical tube, which is called the seminal vesicle (though obviously not quite the same thing as the frog's seminal vesicle), and this opens into a median urogenital sinus, which opens by a single aperture into the cloaca. From the urogenital sinus one or more pairs of blind sacs, the sperm-sacs, come off. Externally the male is distinguished by the claspers.( $\$ 13)$, by which he grasps the female while injecting the seminal fluid into the cloaca and oviducts. Fertilization of the eggs must take place high up in the oviduct, before the egg enters the oviducal gland.

§ 19. Excretory System. The kidneys in the male are a pair of lobulated bodies which extend from a little behind the pericardium to beyond the cloaca posteriorly; but as the anterior part is specially connected with the reproductive system and has a duct of its own, it is advisable to apply separate

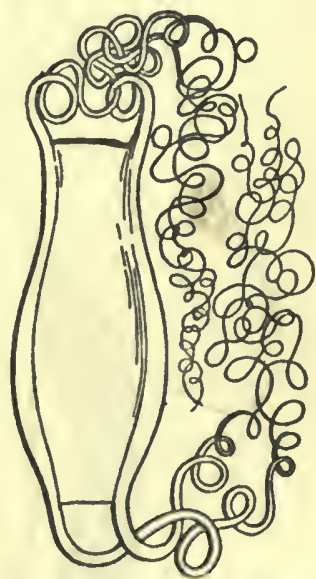

Fig. 100.-EgG-CAsE or Dogrisir. names to them. We may use the same terms as in the rabbit and call the anterior part epididymis and the posterior part kidney; or we may use terms that will be explained more conveniently in the chapters on Development, and call the anterior part mesonephros, the posterior, metanephros. In the latter case we call the vas deferens the mesonephric duct and the ureter the metanephric duct.

In the female the mesonephros is in a vestigial condition and has no separate duct. The metanephros or true kidney is like that of the male.

In both sexes the metanephric ducts open into a median sinus, and this opens by a median aperture on a small 
papilla, into the dorsal side of the cloaca. In the male this is the urogenital sinus already mentioned: in the female, it is called the urinary sinus. There is no trace of the ventral urinary bladder of frog and rabbit.

$\S 20$. The "Ductless Glands." The spleen consists of a

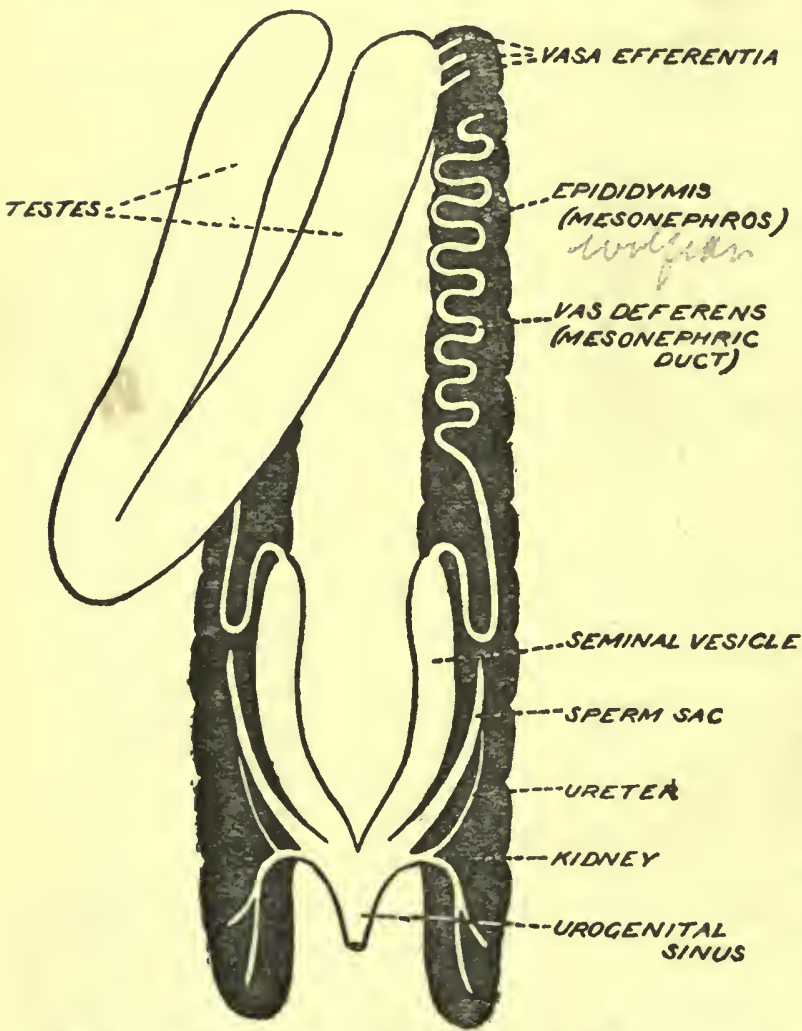

Fig. 101.-male Reproductive Organg of Dogfish. (Slightly diagrammatic.) large portion attached to the apex of the stomach, and of a long, narrow strip along the distal limb of the stomach.

The thyroid lies just ventral to the basi hyal, and laps under the front end of the truncus arteriosus. It forms a good landmark in dissection for the front pair of afferent branchial vessels.

The thymus lies on the dorsal side of the gill-arches, near the anterior cardinal sinus.

There is a pair of adrenals, and a median inter-renal body which are together homologous with the adrenals of the rabbit.

§ 21. Classiflcation. Several species of Scyllium are 
found around the British coasts, $S$. canicula, the spotted dogfish, being the commonest. The group of sharks to which it belongs is distinguished by the small size of the spiracles, the small pectoral fins and the pattern of the calcification in the vertebral centra, from the group to which some other sharks, and the torpedoes, skates and rays, belong. These two groups together make up the order Elasmobranchii, characterized by the presence of placoid scales and absence of ordinary bones, the separate openings of the gill-slits and absence of a gill-cover, the detailed structure of heart and truncus arteriosus, the large size and small number of the ova, the absence of a swim-bladder (homologous with lungs), and certain other points, from the much commoner and more familiar fishes, such as cod, salmon and herring, which form a separate order. Much as these two orders differ from one another, they agree in possessing a hyostylic arrangement of the skull ( $\$ 12)$, and so are sharply distinguished from certain rarer groups of fishes, like the mud-fishes of the rivers of the southern hemisphere, which have an autostylic skull, and which in this and other respects are much nearer theland-inhabiting vertebrates. 
Chapter XVI.-The Lancelet.

\section{(Branchiostoma lanceolatum or Amphioxus lanceolatus.)}

$\S 1$. External Characters. The Amphioxus is a small fish-like animal found in moderately deep water round the sea-coasts in many parts of the world. It is usually about two inches in length, sharply pointed at both ends, and in the living state is almost transparent. Myomeres, $V$-shaped as in the dogfish and other fishes, about sixty-two in number, show through the thin skin and emphasize the Vertebrate relationship of the animal; but these extend forwards right to the anterior end of the body, and there is no well-defined head as in all higher Vertebrates. With

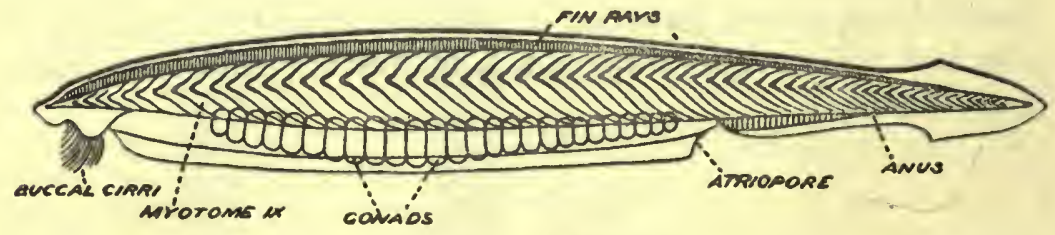

Fig. 102. - АM PHIOXU8.

(After Lankester.) For "Myotome" read (preferably) "Myomere."

the absence of a head goes the absence of all elaborate sense-organs-eyes, ears, olfactory sacs. Gill-slits are present, and very numerous, but hidden from view, as we shall presently see. Instead of a series of median fins, we find a continuous median fin extending along the dorsal edge (for the Amphioxus is even more compressed laterally than the dogfish) from snout to tail, slightly expanded at the hind end into a caudal fin, and then continuing forwards on the ventral side beyond the anus, which is shifted by it slightly to the left. Such a continuous median fin, and such displacement of the anus, are also found in some of the true fishes. This median fin extends forwards to the thirty-sixth myomere (counting from before backwards) and 
here there is a median ventral aperture called the atriopore (or atrial pore), which cannot be paralleled with any structure in our other types. In front of this pore the ventral surface no longer comes to a sharp edge, but is flattened (compare figs. 105 and 104), and instead of a median fin, a pair of metapleural folds are found. There is no trace of paired fins (limbs), but it may be that these metapleural folds represent in a continuous form the paired fins of the true fishes, just as the continuous median fin represents the separate median fins of the dogfish: this is uncertain, however.

Through the transparent skin of this flattened ventral surface a series (twenty-six pairs) of closely-set squarish bodies can be seen, one to each myomere from the tenth to the thirty-fifth. These are the gonads -whether ovaries or testes can only be told by microscopic examination, and there is no other means of distinguishing the sexes. This metameric repetition of the gonads is a feature quite new to us, and one of considerable interest. Accompanying it is a peculiar modification of bilateral symmetry-it is easily seen that the gonads of one side alternate with those of the other. This peculiarity is not confined to the gonads ; careful inspection shows that the myomeres also alternate in the same way, and so do all the metcmeric structures in the body.

The metapleural folds extend forward to the mouth - region, where they form a præ-oral hood and run in front into the median fin again. "This præ-oral hood encloses a space (præ-oral chamber) at the posterior end of which appears the true mouth - a circular aperture devoid of jaws. From the sides of the hood come off a large number of finger-like processes-the cirri.

§ 2. Mode of Life. Such are the general external characters of Amphioxus : we must now say a few words about its mode of life. It may be wondered how it can ever obtain food without eyes, ears or nose to perceive it or jaws to seize it. The explanation leads us to one of the most interesting facts of, marine life. Vegetable organisms, as we have seen, are in the long run the source of all animals' food; but such plants as afford food zOOL. 
to the rabbit, or any other of the vegetarian land-animals, are entirely wanting in the sea. But in the sea (and to a less extent in fresh water) we have conditions eminently suited to the rapid growth and multiplication of microscopic unicellular plants, and these swarm at and near the surface of the ocean. It is on such plants, and the smallest animals that prey upon them, that Amphioxus feeds. Its mouthregion is richly ciliated, and the cilia vibrate in such a way as to direct a constant current of sea-water into the mouth. Carried along helplessly in this current are

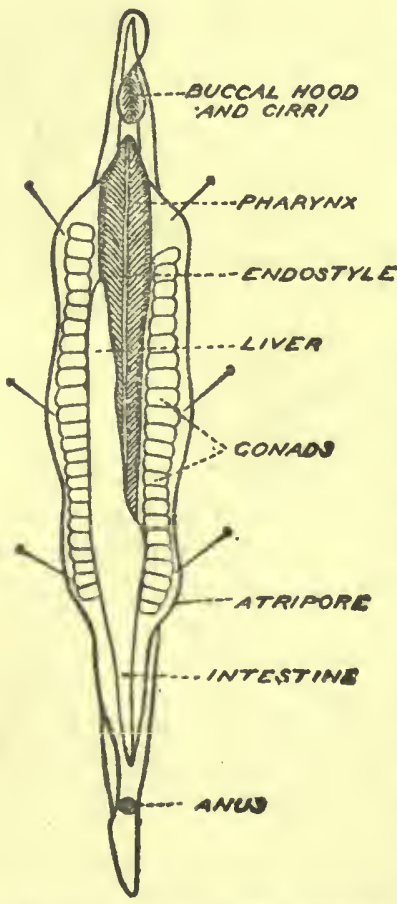

Fig. 103.-AMPhioxus DisseCted by OpEniNG THE ATRIUM.

(From Willey, after Rathke.) the minute organisms that form its food. Evidently it has little need to wander in search of such food, and so it is not surprising that Amphixous should spend much of its life buried in the sand, the mouth-region alone projecting. It does, however, also swim about actively at times, and by means of its pointed anterior end it can burrow into the sand with great rapidity.

This mode of feeding, on the minute organisms that can be wafted to the mouth by a ciliary current, is a widespread one throughout the animal kingdom. It is characteristic of the simplest members in almost all the great groups of animals. Thanks to it, many animals, such as the corals, are able to live fixed to the seabottom and to grow and branch like plants. So enormously abundant is this food supply, that it really is the ultimate support of almost all the animal life in the ocean, where all the larger animals are carnivorous.

§3. The Atrium. If we proceed to dissect an Amphioxus, 
laying it open from the ventral side in the region in front of the atriopore (fig. 103), we find a cavity which at

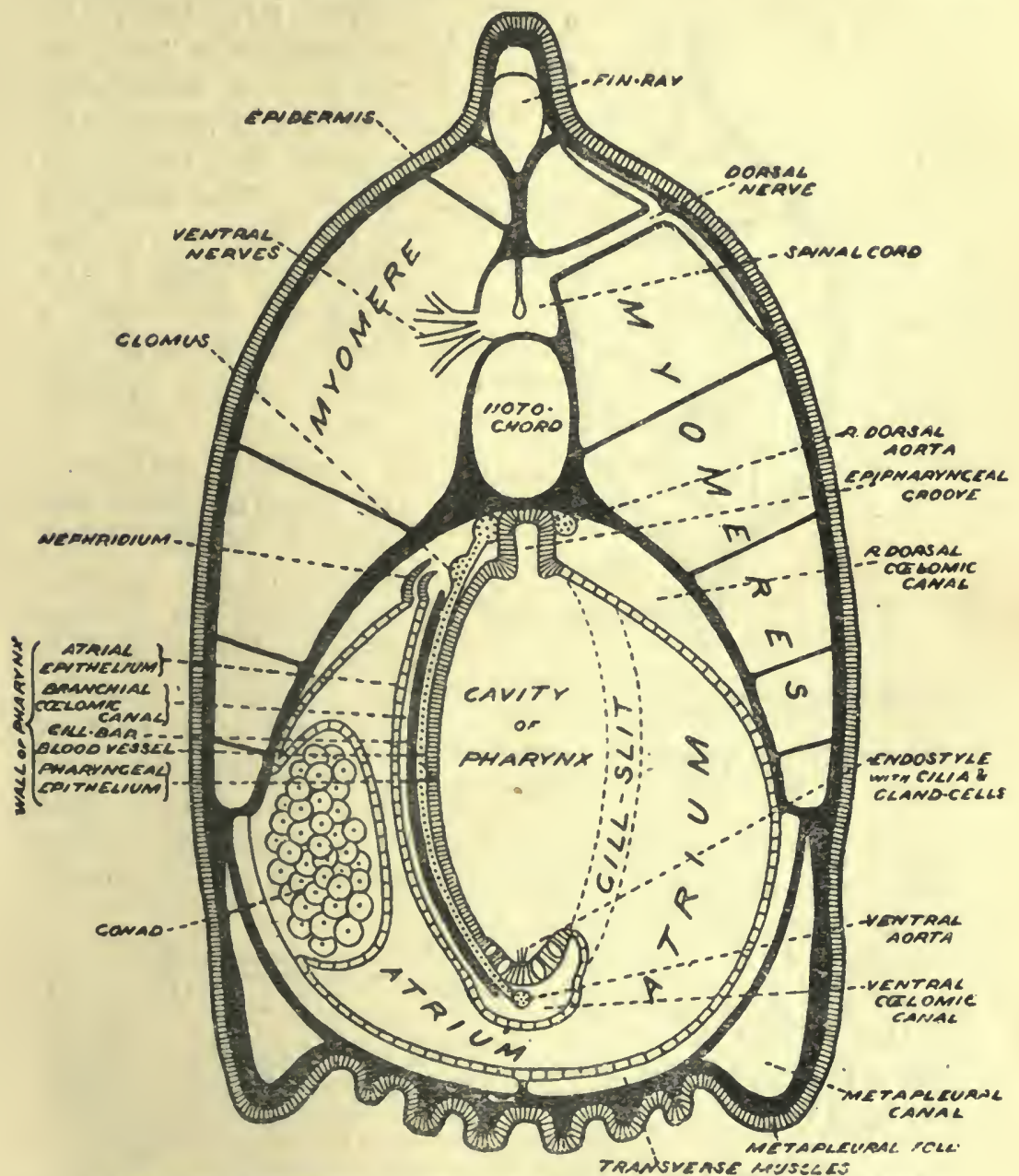

Mg. 104.-Transverse Section of Amphioxds in Phartigar Regiox.

(Diagrammatic ; combined from figures by Boveri and others.) On the left side a gill-arch, nephridium, gonad, and rentral nerve; on the right a gill-slit and dorsal nerve.

first sight may be taken for the coelom. But in this cavity lies the pharynx, with its gill-slits. Now gill-slits do 
not open into the celom - they are communications between the cavity of the alimentary canal (pharynx) and the ex-

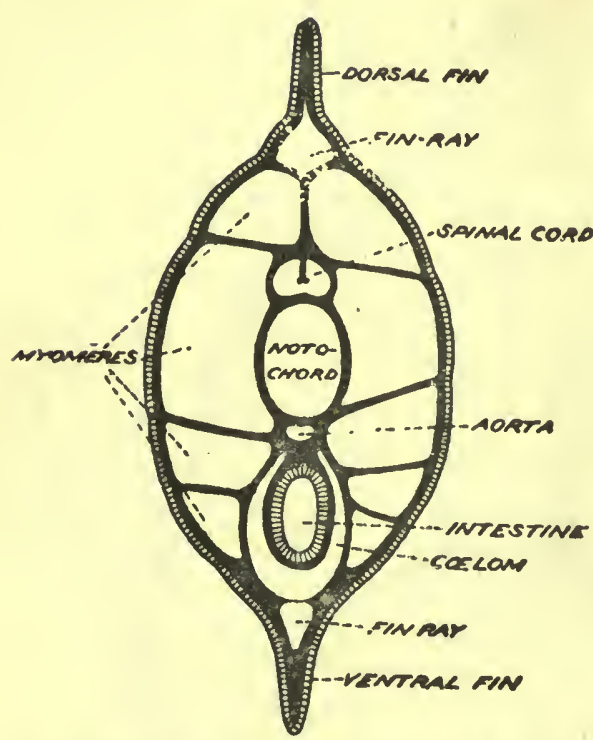

Fig. 105.-Transverse Section of Amphioxug in Intestinal Region.

'The backward extension of the atrium on the left side is not shown. terior. This apparent body-cavity, then, in Amphioxus is not the coelom, but something new, not found in the dogfish : it is called the atrium. The atriopore is its opening to the exterior. Behind the atriopore there is a blind extension of the atrium on the left side only.

The outer surface of the pharynx must correspond to the outer surface of the dogfish's body where the gill-slits open. If the student will imagine that in the dogfish a pair of longitudinal ridges of skin and muscle were formed just above the gill-slits, and that from these there grew down flaps which met in the ventral middle line so as to hide the external openings of the gill-slits, he will get a rough notion of the formation of the atrium in Amphioxus. (See also fig. 116.)

\section{§ 4. The Colom. The} true colom may be easily

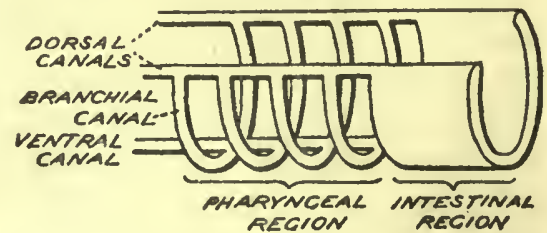

Fig. 106.-Diagram of Internal Cast of PART OF CaELOM OF AMPHIOXUS. found in a transverse section taken behind the atriopore (fig. 105). It occupies its usual position, but owing to the intestine being quite free from any coiling, the colom is a very narrow space. 
Traced forward, it is found not to stop short, as in all our previous types, but to extend right forward to the mouth-region. But obviously it cannot form a continuous longitudinal space where there are gill slits, and so we find that it is only above and below the gill-slits that longitudinally-continuous cœlomic spaces can exist. These are the paired dorsal and median ventral cœlomic canals, which are joined by lateral branchial canals in the gill-arches. These conditions may be best understood from fig. 106, which represents an internal cast of the cœlom, such as we might get by filling it with some solid material and then destroying all the soft parts around. (See also the colom in figs. 104 and 107.) Thus instead of a continuous cœlomic space we have a series of cœlomic channels. These cannot serve the function we have attributed to the cœlom hitherto: what other function they may have we may see presently.

§ 5. Mouth and Pharynx. The alimentary canal is a straight tube, divided into two portions about equal in length - a respiratory portion or pharynx, and a digestive portion or intestine. The mouth is merely a round hole in the anterior end-wall (velum) of the pharynx, and is surrounded by a sphincter-muscle, from which twelve little relar tentacles (not to be confused with the præ-oral cirri) project inwards. The pharynx is high and narrow ; its walls are perforated by an enormous number of long and narrow gill-slits with narrow gill-arches between them. They are also divided by horizontal bars which run across from one arch to the next, so that the whole side-walls of the pharynx (which are very thin) become little more than a trellis-work or sieve. We can now understand the advantage of the atrium: it enables an exceedingly delicate sieve-like pharynx to co-exist in the same region of the body with fully developed swimming muscles. In the dogfish there is no need of an atrium, because the pharynx is relatively short, and there is plenty of room for muscles in its floor and between the gill-slits.

$\S 6$. The gill-slits of Amphioxus are exceedingly numerous, 
but examination of their structure (and still more of their development) shows that, in order to compare them with the dogfish's, the number must first be halved. For each gillslit originally developed-

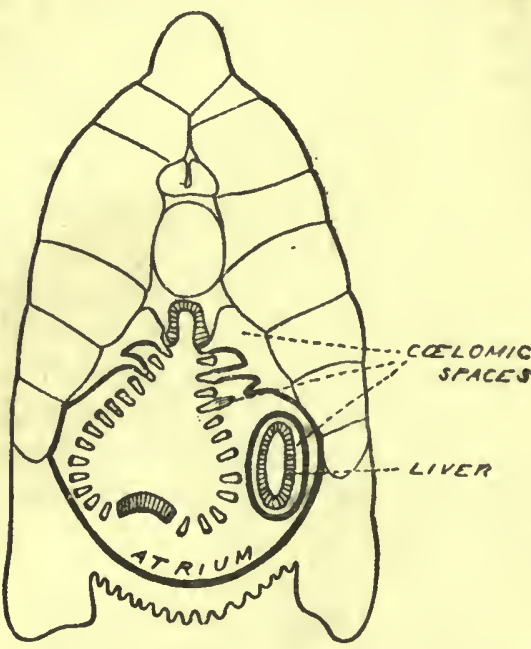

Fig. 107.-Transverse Section or Ampiloxus in Pharyngal Region.

(Altered from Marshall and Hurst.) each primary gill-glitbecomes divided into two secondary slits by a vertical downgrowth. 'These downgrowths are called the secondary gill-arches and differ in several respects from the primary gill-arches that alternate with them. For example, only the primary arches contain a branchial colomic canal. (For other differences, see § 10.)

Even when we only count the primary slits we find them greatly in excess of the myomeres in the corresponding region: the metamerism of each of these two sets of structures is independent. Like the myomeres, however, the gill-slits alternate on the two sides, a right primary bar being opposite a left secondary bar.

From fig. 108 it will be seen that the gill-slits are slightly inclined out of the vertical. It often happens that at death the pharynx is thrown into a distorted shape, the gill-slits coming to lie very obliquely while the endostyle ( $\$ 7$ below) comes to be convex upwards instead of concave. Hence in prepared transverse sections of the pharyngeal region (as fig. 107), a large number of gill-arches and slits are seen, and the whole pharynx-section appears somewhat heart-shaped instead of narrow-elliptical.

$\S 7$. Endostyle. The lattice-work of gill-arches forms the whole surface of the pharynx except for the narrow dorsal and ventral areas, which form continuous groovesdorsally the hyperpharyngeal groove, ventrally the endostyle. Anteriorly, in the velum, these two grooves are united by a 
pair of grooves, curving round right and left of the mouth. These grooves are lined by an epithelium that is partly ciliated, partly glandular. In the endostyle there are four longitudinal rows of gland-cells, separated by rows of ciliated cells.

The gland-cells secrete a slimy fluid which the cilia drive along the groove. To this slime most of the solid particles brought in by the water-current will adhere, and while the main part of the water passes out through the gill-slits into the atrium, whence it is expelled by the atriopore, the solid particles (mostly food) are carried on into the digestive region. Thus the water-current is at once nutritive and respiratory. In Amphioxus we find for the first time a reason for that utilization of the anterior part of the alimentary canal for respiration which is so marked a characteristic of the Vertebrata. As we ascend to the dogfish, frog and rabbit, we find the respiratory portion of the canal becoming smaller and smaller, until in the rabbit the courses of the air and of the food are almost completely separated.

§ 8. Intestine and Liver. Behind the last gill-slits the canal becomes more nearly circular in section, and at first there is a dilatation with glandular walls which secrete a general digestive fluid: this is therefore often called the "stomach." But into this opens a large cæcum, the nature of whose blood supply $(\S 9)$ shows it to answer to the liver. The "stomach" into which it opens is, therefore, morphologically the duodenum. The liver (hepatic cæcum) is a simple glandular sac, bearing much the same relation in structure to that of higher vertebrates that the lung of the frog does to the lung of the rabbit. It opens on the ventral side of the "stomach," but as it passes forward turns to the right side, and appears in dissection lying alongside the pharynx in the atrium. That the liver lies in the atrium adds to the resemblance of that space to a ceelom, but really what is seen is not merely the liver, but the liver surrounded by a narrow colomic space and a thin layer of connective tissue and epidermis-really the body-wall, which it has, as it were, pushed out before it.

No pancreas occurs in Amphioxus, though it may be represented by the glandular walls of the "stomach." The 
rest of the intestine is quite straight, and cannot be divided into ileum and rectum, nor does it end in a cloaca.

$\S 9$. The Circulation is very simple, there being no distinction of arteries from veins, and very little between these and capillaries. Yet its identity of fundamental plan with the dogfish's is plain when we allow for the absence of the cardinal veins. The ventral or cardiac aorta lies in the floor of the pharynx, under the endostyle, and sends branches up along the primary gill-arches. There is no heart, but the whole of the cardiac aorta is contractile, and at the bases of the aortic arches that run up the gill arches there are contractile dilatations that assist in the propulsion of the blood. Branches from the vessels in the primary arches run across the transverse bars into the secondary arches and up these. Just above the gill-slits these vessels open into a network of small vessels (glomus) which retards the flow of blood through this region: the object of this we shall see presently (§13). All these branchial vessels end dorsally in a pair of dorsal aortæ, lying on either side of the hyperpharyngeal groove. Where this groove ends, at the beginning of the "stomach," these aortæ unite into a median dorsal aorta from which branches are distributed over the intestine walls, to unite on the ventral side again into a sub-intestinal vessel, which corresponds to a portal vein, as it takes the blood back from the intestine to the liver. Thence hepatic veins take it to the ventral aorta, and so the circulation is complete.

It will be noticed that there is apparently no blood-supply to the myotomes. These, however, and other parts of the body are plentifully supplied with lymph-spaces. Of these, the metapleural canals in the metapleural folds are most conspicuous. Little is known as to the course of the lymph or its relation to the blood. The blood is colourless and contains colourless corpuscles only.

$\S 10$. The Skeleton of Amphioxus is far simpler than those of our other types. There are no vertebræ and no skull. Instead, we have a longitudinal rod, the notochord, which has already been mentioned (chap. vi., § 15), as an 
instance of a purely endoplastic tissue. Instead of being soft as its remains are in the dogfish it is at least as firm as cartilage, and even more flexible and elastic, so that it forms an excellent basis for the myotomes to act upon in swimming. Its anterior end extends right up to the tip of the snout, and thus makes it easy for the animal to burrow in the sand (§2). A notochord occurs in the embryos of all higher vertebrata, but it never extends in front of the midbrain, and it is usually replaced by. vertebræ as development goes on.

The præ-oral hood and cirri are supported on a jointed framework, composed of notochordal tissue. Possibly the labial cartilages of the dogfish represent this pro-oral skeleton.

The gill-arches are supported by a series of bars, which are of quite different chemical composition from any other Vertebrate skeleton. Their material is insoluble in hot caustic potash solution, and must therefore be very similar to the chitin which for'ms the exoskeleton of insects and many other Invertebrates. This branchial skeleton consists of a series of primary bars, lying in the primary arches, and forking below ; of tonguebars, lying in the secondary arches,

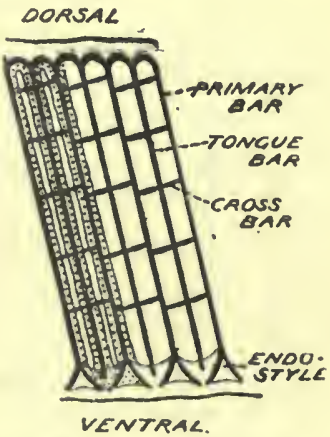

Fig. 108.-PART OF Skeleton of Pharynix of Amphioxus.

(From Willey.) The dotted regions represent the soft tissues. and not forking; and of cross-bars lying in the connexions which unite one primary arch to another and cut up the slits (fig. 108).

The median fin is supported by a series of gelatinous finrays, more numerous than the myomeres. Ventrally, between atriopore and anus these are double, indicating that the fin represents two folds united in the middle line. The metapleural folds have no fin-rays.

Lastly the connective tissue which surrounds and separates all the organs is very strong and plays the part of a skeleton both in the way of protection and in that of affording a firm basis for muscular contraction. 
$\S 11$. Nervous System. The central nervous system of Amphioxus occupies an exactly corresponding position to that of our other Vertebrate types. It consists of a spinal cord whose anterior end is but feebly dilated into a brain which shows no sign of the three primary vesicles or other familiar structures. The spinal cord has as usual a central canal, but there is no ventral fissure, and the dorsal fissure apparently opens into the central

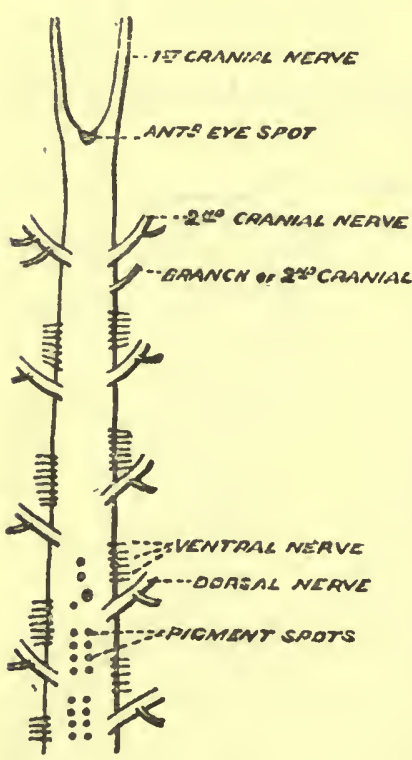
canal. Although both nerve-cells and fibres are found in the cord there is no clear segregation to form white and grey matter. Although there are both dorsal sensory and ventral motor roots to the spinal nerves, these never unite to form mixed nerves-the dorsal run straight out to the skin between the myomeres, while the ventral run into the myomeres (figs. 104 and 109). Thus they alternate in position as do those of the dogfish. But in addition to this they are affected by the alternation of the myomeres; and so a dorsal nerve on the right side comes off opposite a ventral one on the left, and vice ver'sâ. Again the Fig. 109.-ANTERIor Portion of dorsal nerves have noganglia, their SPINAL CoRd OF AMphioxus. Dorsal view. (From Willey.) fibres belonging to cells within the spinal cord; and the ventral nerves are divided into bunches of fibres.

From the brain two pairs of sensory nerves come off : these cannot be compared very well with any of the cranial nerves of the dogfish, nor can any of the latter be clearly recognized in the spinal nerves of Amphioxus. There are no sympathetic ganglia..

§12. Sense Organs. The skin, especially in the mouthregion, is abundantly furnished with sensory cells - epitlermal cells with a stiff projection after the fashion of the rabbit's 
olfactory epithelium. These cells are probably sensitive to various kinds of stimuli, but principally to touch. On the left side is a funnel, richly ciliated, leading down to the front end of the brain; this is very possibly an organ of "smell" or " taste," i.e. one which recognizes any unpleasant material that there may be in the water, and sends a stimulus into the brain which sends out a reflex motor impulse to the sphincter of the mouth, to close the aperture until the objectionable substance is gone.

Though it has no eyes and cannot see distinct objects, Amphioxus can distinguish between light and darkness, and doubtless has an instinct to burrow if a large shadow falls on it-a warning of some predaceous fish. This sensitiveness to light appears to reside in the epithelium of the central canal of the spinal cord, for irregularly shaped masses of pigment occur in it at close and regular intervals. A similar mass occurs in the epithelium at the very front end of the brain, and is usually called the "eye-spot"-a term equally applicable to the others. The existence of a sensitive epithelium inside the spinal cord is very remarkable, as it is usually the external surface which is sensitive. We shall return to this point in dealing with the development of the vertebrate eye. Of course, such a condition is only possible in a translucent animal like Amphioxus.

The simplicity of the central nervous system can be easily correlated with the other conditions in Amphioxus. There being on the one hand no elaborate sense-organs continually sending sensory impulses in, and on the other hand no elaborate muscular system for breathing, eating, the more complex kinds of locomotion, etc., there is need only of a simple co-ordinating system.

$\S 13$. The Excretory System of Amphioxus differs considerably from that of our other types. There is no compact kidney. Instead we have a metameric series of quite separate tubules-one to each primary gill-slit. Each of these tubules is bent on itself nearly at right angles, and opens at one end into the atrium.

These excretory tubules were formerly supposed to have several openings into the dorsal coelomic canal and to be 
homologous with the tubules of the urinary organs in vertebrate embryos, to correspond, in fact, to the pronephros. The original discoverers of these organs described the internal openings as surrounded by numerous long protoplasmic processes ending in little knobs; these were called thread-cells. Mr. Goodrich, having carefully reinvestigated these organs, found that there were no colomic openings, and that the thread-cells were in reality solenocytes, the thread being a very fine tubular structure and the knob a nucleated cell. Within each tube is a long vibratile flagellum which extends into the cavity of the organ. The structure is represented diagrammatically in figs. $109 a$ and $109 b$. Fig. $109 a$ shows the entire excretory tubule, only moderately magnified; fig. $109 b$ shows the structure of the solenocytes connected with the blind extremity of one of the branches of the tubule. Although it is not yet known whether these excretory organs are developed from epiblast, the presence of the solenocytes is strong evidence that they are true nephridia. The renal tubules of vertebrates, on the other hand, are not nephridia, but cœlomic ducts which have taken on excretory functions and have retained to a great degree their original function as generative ducts. The excretory organs of Amphioxus, therefore, are not homologous with those of vertebrates.

On the other side of the narrow cœlom, just opposite each nephridium, is a little network of blood-vessels, on the course of the branchial vessels just before they enter the dorsal aortæ (fig. 104). These little networks have some resemblance to the glomeruli found in the kidney of higher Vertebrates, and are often called by that name; but as they do not correspond exactly with those structures, it is better to adopt a different name for them, and the term glomus is a convenient variation of glomerulus for this purpose. Apparently water is filtered off from each glomus into the cœlom, and excreted by the nephridia. Whether urea is also filtered off from the blood at the same time is difficult to say; but seeing that the muscles-the most active tissues in the body-are not supplied with blood but only with lymph, and that this and the colomic fluirt 

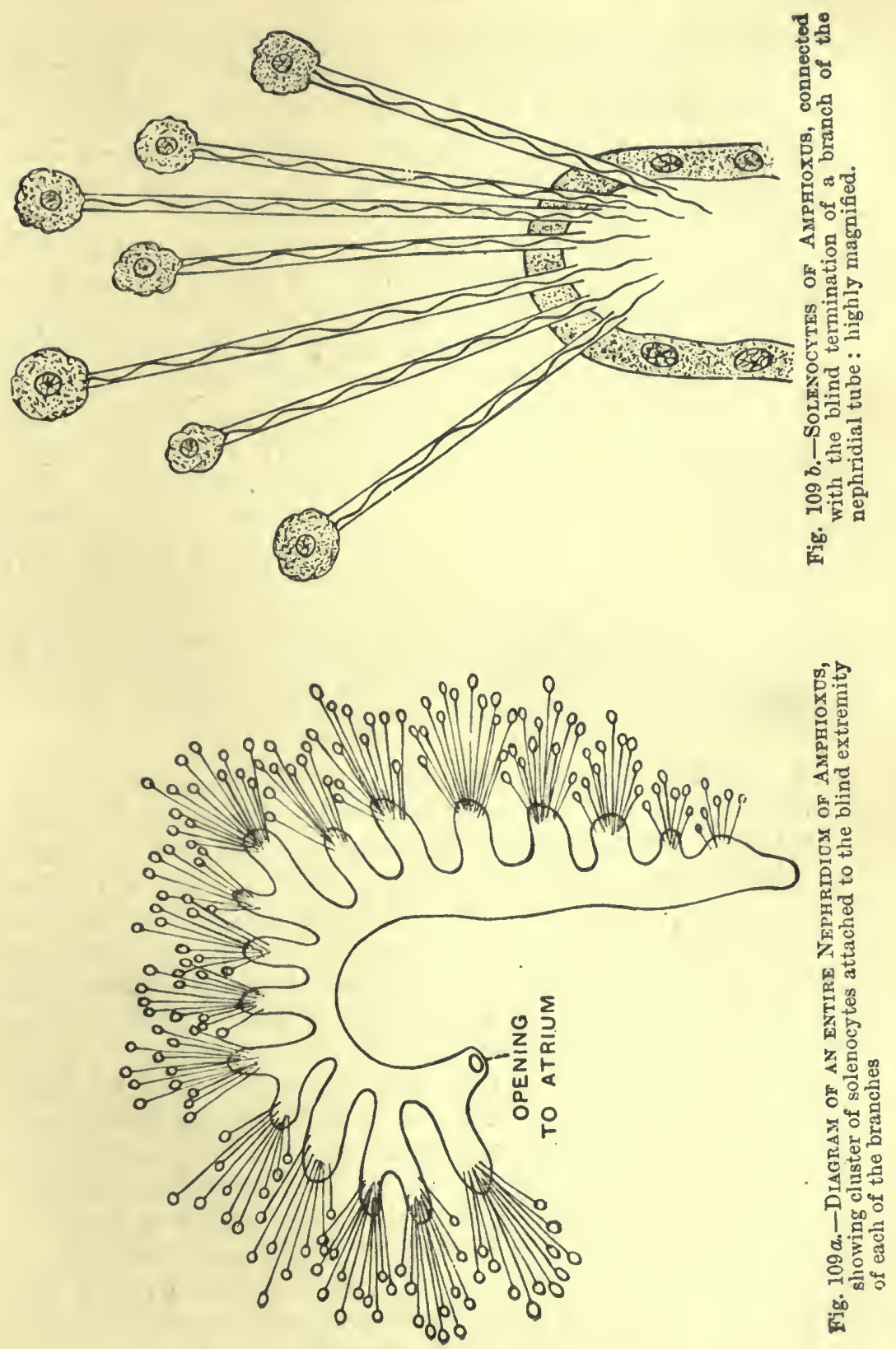
seem to be identical, it seems probable that the coelomic fluid may be the carrier of katastases to the nephridia. Thus the function of the cœlom in Amphioxus would seem to be that of a sort of auxiliary vascular system-a function perhaps still retained, but in ever lessening degree, as we pass up to higher and higher Vertebrates.

We have seen that the cœlom is to be regarded as primarily the generative cavity, but as to its mode of origin in evolution we can only speculate. It may have been a portion of the original enteric cavity which occurs in colenterates, separated off, and this view is supported by its mode of origin in Amphioxus and certain other cases. The other possibility is that it was a new cavity developed in the mass of mesoblastic cells which were developed between ectoderm and endoderm. The question is whether the schizocoel or the enterocoel is the more primitive. The latter alternative seems more probable.

Besides the nephridia, the whole atrial epithelium appears to be excretory in function, and there are a pair of "brown funnels" in the roof of the atrium, just by the posterior end of the pharynx, that are supposed, but have not been proved, to be excretory.

$\S 14$. Reproductive System. This is extremely simple, consisting only of the gonads, already mentioned ( $\$ 1$ and fig. 104). These appear to be developed on the side-walls of the atrium, into which they project; hence a further apparent resemblance of the atrium to a cœlom. But the resemblance is just as illusory as in the case of the liver. Really the gonads are in cœlomic cavities in the atrial wall (gonadic pouches), on the lining of whose walls ova or spermatozoa are developed and fill up the cavity.

If the atrium were a colom the gonads would be developed as solid outgrowths from its wall into its cavity, and the ova and spermatozoa would escape at the surface of these solid outgrowths, as in higher vertebrates. In the adult Amphioxus this appears to be the case, but study of development shows that the myocelomic pouches send off prolongations into the atrial wall, and that the ovaries and testes arise in the walls of these prolongations.

There are no gonoducts, and when the ova or spermatozoa, 
are ripe, they simply burst through the wall of the pouch into the atrium, whence they escape by the atriopore. What then becomes of them we shall see shortly.

$\S 15$. The Principle of Change of Function. The student Is now in a good position to realize how in different types the same structures are turned to different purposes. The gill-slits and arches in Amphioxus serve primarily to strain off the water that brings in the food, and as a secondary function they support respiration. In the dogfish the first function has gone, and the main one is that of respiration, but the basal parts of the cartilages serve for the attachment of respiratory muscles, while it seems probable that the jaw-apparatus is really the first of the cartilaginous arches turned to a new use. In the frog, the respiratory use of the slits is lost, and they themselves are gone, except the first, which is turned to a new purpose in connexion with the ear, forming the tympanum; the basal cartilage remains usef ul for the respiratory muscles, but it also serves a new function - the support of the tongue-muscles. Finally, in the rabbit the remains of the branchial arches (" hyoid apparatus") serve for the tongue-muscles and the larynx only, and have nothing to do with respiration.

With this example of gradual change of function in view (and it has not been put exhaustively), the student will be less surprised than he would otherwise be when he is told that the thyroid of higher Vertebrates represents the endostyle of Amphioxus. The thyroid, in fact, starts its development as a groove in the floor of the pharynx, but this groove (not needed in an animal with jaws) becomes converted into a canal and separated off from the pharynx. Its varying position in the higher types is dependent upon adaptation to the varying growth of other organs. Whether the endostyle of Amphioxus secretes the same internal secretion as the thyroid of higher types is not known; but it is reasonable to suppose that if we had a series of organisms forming as complete a chain between Amphioxus and the fishes as we have between fishes and mammals, we should find a gradual decrease in the importance of the primary function of the endostyle accompanying a 
gradual increase in the importance of the secondary function.

Equally remarkable is the fact that the thymus begins its development as a series of projections on the dorsal side of the gill-slits, closely resembling the first appearance of tongue-bars in Amphioxus. Here again we have to do doubtless, with a case of an organ of changed function.

$\S 16$. Classification. It is evident that between Amphioxus and the dogfish there is a great gap-at least as great as would be the gap between dogfish and rabbit, were no intermediate types known. Some few lowly fish, as the lamprey, come a little way nearer Amphioxus than does Scyllium, but it is only a little way. On the other side the gap between Amphioxus and the nearest Invertebrates is almost as great. The peculiar group of the Tunicates does, it is true, show considerable affinities to Amphioxus, but after that there comes a very great gap indeed. Hence modern zoologists unite the Tunicates, A mphioxus, and typical Vertebrates into one great phylum or division of the animal kingdom, to which the name Vertebrata is given. Other zoologists prefer to term this group CHoRdata, and restrict the term "Vertebrata" to those forms higher than Amphioxus. Whichever name we adopt, the following are the essential characters that mark off this great phylum from all other phyla of the animal kingdom :-

(1) At some period of existence there is a Notochord.

(2) The central nervous system is dorsal in position, is a continuous mass of nerve-tissue, and is tubular (i.e. has a central canal).

(3) The anterior portion of the alimentary canal gives rise to the respiratory organs (aquatic or aërial), and at some period of existence the pharynx is perforated by gill-slits.

(4) There is a general metamerism of the body-muscles (myomeres).

If we exclude the Tunicata we may add a fifth point.

(5) The circulation shows primitively a hepatic-portal syste $n$, and a median ventral trunk sending off' branchial resse'; between the gill-slits, which unite in a dorsal aorta. 
Next, what are the Inain points which separate off Amphioxus from the higher types? The latter all possess (with odd exceptions here and there) :-

(I) An epidermis of more than one layer.

(2) Skull and vertebrex.

(3) Two paired fins or limbs.

(4) Paired eyes, with the sensory elements of the retina turued away from the light.

(5) Paired ears, with semicircular canals.

(6) Paired olfactory organs.

(7) Sympathetic ganglia.

(8) A heart.

(9) A spleen.

(10) A compact liver.

(11) Compact kidneys.

None of these are found in Amphioxus. On the other hand, Amphioxus has

(1) Very numerous gill-slits.

(2) An atrium.

(3) Metameric gonads.

(4) Numerous metameric nephridia.

Thus we must divide the Vertebrata (Chordata) into two * great groups (or rather one great and one small) -

A. Acrania, including the Amphioxus only.

$B$. Craniata (Vertebrata of some authors), which includes fishes, amphibia, reptiles, birds and mammals.

*- The Tunicata form a third, and certain other forms may be put into a fourth.

$\dagger$ An astonishing number of other names hare been bestowed on this group by various authors: the only one in sufficiently common use to be worth mentioning is that of Cephalochorda, referring to the forward extension of the notochord.

\section{Questions on the Vertebrata.}

[Most of these questions wero set at London University Examinations.]

1. Give an account, with illustrative sketches, of the digestive organs of the common frog, specifying particularly the different forms of epithelium met with in the several regions thereof.

zOOL. 
2. Compare the coelom of Scyllium, Rana and Lepus.

3. Compare with one another the breathing organs and the mechanism of respiration in a frog and in a rabbit. Give figures showing the condition of the heart and great arteries in these animals, and indicate in each case the nature of the blood in the several cavities of the heart:

4. Draw diagrams, with the parts named, illustrating the arrangement of the chief arteries of $(a)$ the frog, $(b)$ the rabbit. (c) Compare briefly the arrangements thus described. (d) In what important respects does the vascular mechanism of the frog differ from that of the fish, in correlation with the presence of lungs?

5. Describe, with diagrams, the nervous system of Amphioxus, and compare its nervous axis, in detail, with that of a Vertebrate (Craniate).

6. Give a brief account of the physiology of respiration. Describe fully the means by which respiration is effected in the following animals :-frog, Amphioxus, rabbit, and dogfish.

7. Describe the minute structure of the blood of the rabbit, frog, and Amphioxus.

8. Give an account of the structure (including histology) of the spinal cord and spinal nerves of the frog.

9. Compare the vertebral column of the dogfish and rabbit.

10. Compare the circulation in the kidney of dogfish and rabbit.

11. Compare the skull of the frog with that of the dogfish. To what structures in the dogfish do membrane bones correspond?

12. Describe the form and divisions of the central nervous system in Amphioxus, the dogfish, the frog, and the rabbit.

13. Describe, with diagrams, the arrangement of the urinary and generative organs in the male of $(a)$ the rabbit, (b) the dogfish, and (c) the frog; (d) point out the most important differences between them.

14. Compare, one by one, the cranial nerves of the dogfish with those of any higher Vertebrate, as regards their origin and their distribution. 
15. Describe the auditory organ of the dogfish. What parts are added to this in the higher types?

16. (a) Give an account, with diagrams, of the brain of the frog; (b) point out the most important differences betweon it and the brain of the rabbit. (c) Describe the superficial origin and the distribution of the third, $(d)$ of the fifth, $(e)$ of the seventh, $(f)$ of the ninth, and $(g)$ of the tenth cranial nerves of the frog.

17. Draw diagrams, with the parts named, of the alimentary canal of (a) Amphioxus, (b) any Craniate; (c) indicate very shortly the principal structural differences between the two.

18. Describe, with a diagram, the circulation of Amphioxus. Compare it with that of the Craniata.

19. Compare the skull of the dog and the frog; especially in regard to the attachment of the jaw apparatus to the cranium, and other points which distinctly characterize the higher as contrasted with the lower Vertebrata.

20. Describe the skeleton of the upper and lower jaw (a) in the frog, (b) in the dog. Point out exactly what parts corresponds with one another in the two animals compared. (c) What bone in the dog is generally regarded as corresponding to the quadrate cartilage of the frog?

21. Give an account of the limb skeleton of Scyllium, Rana, and Lepus.

22. Describe a transverse section across the pharyngeal region of Amphioxus.

23. Describe, with the aid of diagrams, the skull and visceral arches of the dogfish. What are the essential features of difference shown in the skull of the adult frog?

24. Describe and compare the aortic-arch system in Lepus, Rana, and Scyllium.

25. What are the leading characters in which Amphioxus resombles and differs from Scyllium?

26. Give an account of the vertebral columns of Lepus and Scyllium, pointing out in what respects the two resemble and differ from each other. 


\section{PART III.-THE DEVELOPMENT OF VERTEBRA'TA.}

\section{Chapter XVII.-General and Amphioxus.}

§ 1. The 0vum. The ripe egg or ovum of any animal, as found in the ovary, is a single nucleated cell (fig. 110). In Vertebrates it is always surrounded by a follicle, which in the simplest case is a single layer of small cells; and within this is a vitelline

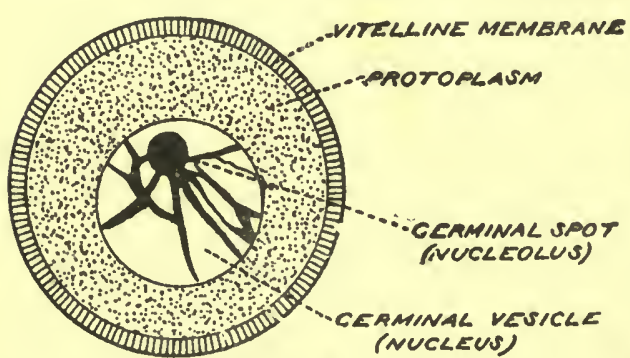

Fig. 110.-AN OvUM. membrane, which is a secretion, probably of the ovum itself. Although it varies in size enormously in different animals (from $\frac{1}{250}$ of an inch in diameter in the case of Amphioxus to over an inch in the case of the fowl), its nucleus (or germinal vesicle, as it is customary to call it) does not vary proportionately, but is always microscopic (though relatively large). The differences in size are, in fact, almost entirely due to the varying quantity of food-material or yolk which is stored within the protoplasm of the cell. This yolk is an endoplastic product of the ovum, destined in the course of development to be used up in the formation of new protoplasm. Small ova are ova with little or no yolk-alecithal they are technically called. In such cases we usually find that the developing individual becomes active and capable of feeding in regular animal fashion at a comparatively early stage in its development, while it as yet differs greatly from its parent: it is then called a larva. On the other hand, the young individual 
developed out of a large, yolk-laden egg, goes through so much of its development within the egg-membrane, that when it at last escapes it differs from its parents in only minor characters. Such a developing individual within the vitelline membrane is called an embryo.

$\S 2$. The Spermatozoon is almost always smallor than tho ovum, even when that is quito small, and as it never contains yolk the disproportion between its size and that of the ovum may be enormous. The spermatozoon (fig. 158[5]) is usually little more than a nucleus (male pronucleus) with a long vibratile flagellum (resembling a long cilium) attached, by means of which it swims actively about until it comes near an ovum.

§ 3. Maturation of the 0vum. Before fertilization can take place, certain changes happen in the germinal vesicle (nucleus of the ovum). Its bounding membrane disappears, much of its substance appears to mingle with the general protoplasm, and the chromatin that remains (far smaller in bulk than the original nucleus) divides by karyokinesis into two nuclei: one of these travels to the surface and becomes separated from the ovum, taking a little protoplasm with it. Evidently we have here a peculiar case of cell-division, in which the two cells are extremely unequal in size, though their nuclei are equal. The small cell thus cut off is called the first polar body. The residual nucleus of the ovum again divides in the same way, and a second polar body is extruded. What remains of the original germinal vesicle is now only equal in bulk to the male pronucleus of the spermatozoon: it is called the female pronucleus. 'These changes constitute the maturcttion of the ovum.

\$ 4. Fertilization. Before the ovum thus matured can develop into a new individual, it is necessary that new nuclear material should be introduced into it. This is effected by the union of a spermatozoon with the ovum. The male pronucleus enters the ovum, and unites with the female pronucleus in a very intimate manner to form a new 
nucleus possessing capacities of development not existing in either of the pronuclei alone. This union of pronuclei is the essence of fertilisation, and the resulting nucleus is called the first segmentation-nucleus. Every nucleus in the whole body of an adult rabbit or any other animal has been produced by division from one original nucleus - the first segmentation-nucleus of a fertilised ovum, and the division takes place in such a way that material derived from both pronuclei is found in every resultant nucleus.

The most important discovery of recent years in connection with this subject is that the number of chromosomes for any particular species is constant, is the same in all the somatic cells of that species whenever they divide, and that the number in the gametes is half of the number in the somatic cells. Thus in the process of fertilisation male chromosomes do not unite with the female, each with each, so that the total number in the fertilised ovum or zygote remains the same, but each gamete contributes half the specific number, and thus the whole specific number is restored in every generation. The chief result therefore of the formation of polar bodies or maturation divisions is the reduction of the number of chromosomes in the ovum to half the number in the somatic cells. In some cases it has been proved that the spermatozoon also contains half the number of chromosomes found in the somatic cells, and that this result is brought about by two divisions corresponding to the maturation divisions of the ovum. But in most cases the male cells are too small to permit of the chromosomes being counted.

In reference to this reduction in the number of chromosomes, the two divisions which lead to the formation of the mature gametes are called reduction divisions. In the case of the ovum the two divisions produce one ovum and three polar cells, for the first polar cell divides into two after its separation. These three polar cells are regarded as degenerate ova, so that the two reduction divisions produce four female gametes, of which only one is capable of conjugation and development. Similarly in the development of the male gametes or spermatozoa it has been shown in some cases that two reduction divisions take place, but the 
result is not the formation of one perfect sperm and three degenerate ones, but of four perfect sperms. As to the mode in which the reduction takes place there is still some difference of opinion, and the nuclear structures seen are not exactly the same in different species that have been investigated. According to one view the number of chromosomes in the first division is unchanged, and each of these chromosomes divides into two as in ordinary mitosis; but in the second division no division of individual chromosomes takes place, but half the number pass into one daughter nucleus, half into the other, and thus the reduction is effecter. According to the other view the chromosomes appear in half the original number in the division spindle of the first division, and then each divides into four in the two divisions. Thus the reduction of number has already taken place in the formation of the spindle of the first division.

Whatever the interpretation may be, it is now known that in a large number of cases the spindle of the first reduction division contains half the number of chromosomes, which are characteristic of the species, and that each of these chromosomes is composed of four distinct and equal parts ; these chromosomes are therefore called tetrads. In Ascaris megalocephala, a worm of the Phylum Nematoda or Thread-worms, which is a parasite of the intestine of the horse, the somatic cells have only four chromosomes. In the first reduction division of the ovum two tetrads are formed (fig. 110a), and each of these consists of four simple rounded granules; a half of each tetrad passes into the first polar body, and thus two dyads, or chromosomes, consisting each of two granules, are left in the ovum and two in the polar body. The second spindle is immediately formed without the intervention of a resting stage, and the second division takes place, half of each dyad passing into the second polar body. The first polar body divides into two in the same way, and thus there are three polar bodies and the ovum, each containing two chromosomes of one granule each. Similar divisions of two tetrads occur in the spermatocytes of the Ascaris, each spermatocyte forming four spermatozoa (fig. 110b). The spermatozoa of Ascaris are not of the usual form, 
with a long vibratile tail, but are conical and, like those of the Arthropoda, motionless; the broader end of the cone contains the nucleus. The tetrad does not always consist of four simple granules. In some cases, e.g. the spermatogenesis of Salamander and of Helix and the pollen formation of the Iily, after a long period of preparation or
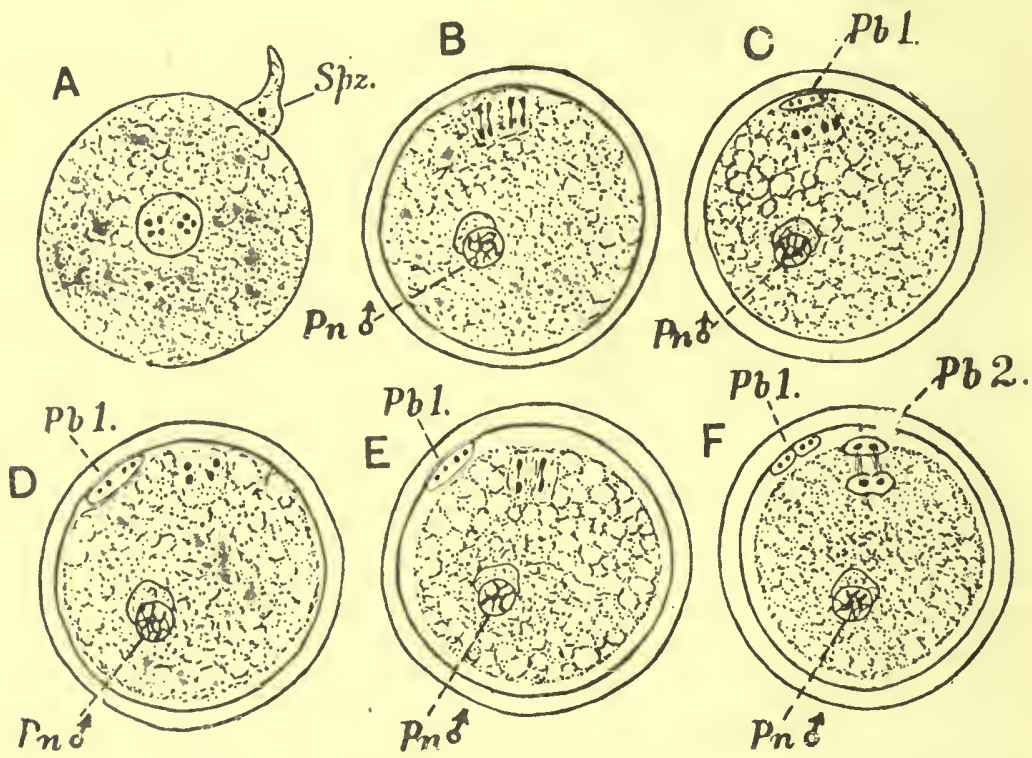

Fig. 110a.-Reduction divisions (maturation) of the ovum in Ascaris megnlo. eephula, variety with four chromosomes in somatic cells. $A$, the ovum with the spermatozoon, Spz., entering it; the nucleus of the ovum has two chromosomes, each in the form of a tetrad. $B$, the nucleus of the ovum las travelled to the sur. face and the tetrads are dividing. $C$, the division is complete, and the first polar body $P b 1$ is formed, it contains, like the ovum, two dyads. $D$, the dyads in the ovum preparing for a second division. $E$, the dyads dividing. $F$, division complete, second polar body $P b 2$ formed, first polar body divided into two. $P$ n $\delta$, male pronucleus.

prophase the convoluted chromatin thread becomes divided into lengths forming the chromosomes, and each of these splits longitudinally, the two halves separating in the middle and remaining connected by their ends, so forming a ring without any other division; it has been suggested that the two halves of the ring are really separate, chromo- 
somes in pairs, so that the number is not actually reduced until the reduction divisions have taken place. In other cases the longitudinal halves are separate and are transversely divided so that there is a tetrad of four rods. The first reduction division has been called heterotype because it differs from the type of somatic division, and the second homotype because it is less different; but there seems more similarity between the two reduction divisions, and this term itself best describes them. On the view that the
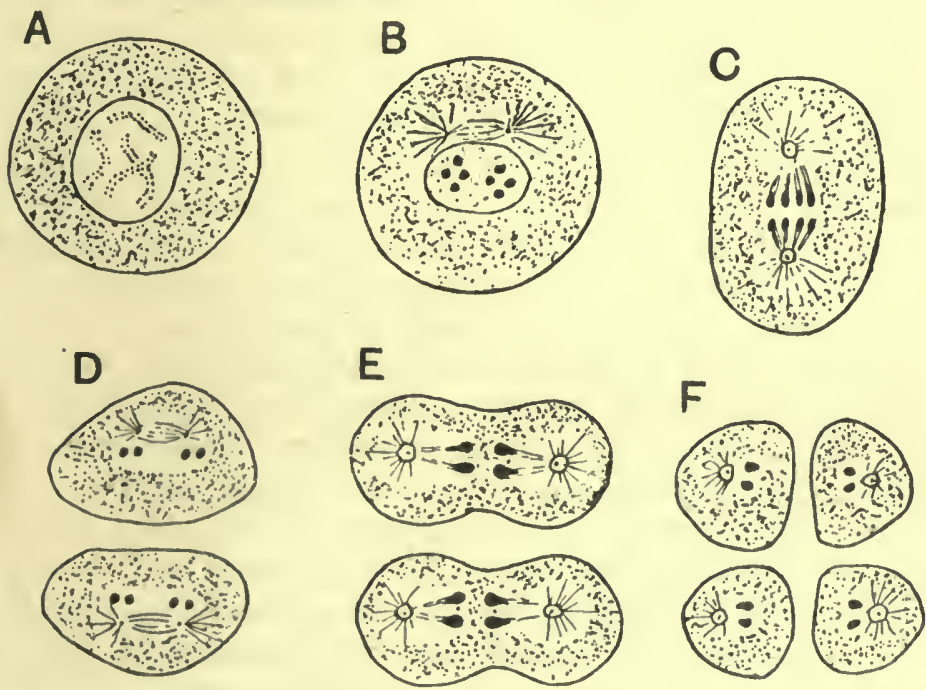

Fig. 110b. - Spermatogenesis in Ascaris megalocephala, variety with four chromo. somes in somatic cells. $A$, a cell of the reproductive organ with four thread-like chromosones. $B$, a sperniatocyte with two chromosomes each in the form of a tetrad. $C$, the nucleus dividing. $D$, two spermatocytes formed, each with two dyads. $E$, second reduction division. $F$, four sperniatids formed, each contrining two simple chromosomes or monads.

tetrad really consists of two somatic chromosomes united but not mixed together, it is supposed that one of the divisions consists in the separation of whole chromosomes, but it is not certain whether this takes place in the first or second division; the fact that in parthenogenetic animals eggs may develop without fertilisation when only one division occurs, but not after the second, seems to show that the reduction really takes place in the second division. 
$\S 5$. Reduction Divisions and Heredity. The behaviour of the spermatozoon in fertilisation, the fact that the chromatin of the male nucleus alone seems to be the essential element, together with the fact that the offspring inherits as much from the male parent as from the female, lead to the conclusion that the chromosomes are the carriers of heredity. It was discovered by Mendel in 1865 that when two individuals of different varieties, differing in some one definite character, were bred together, the character of one parent appeared in all the offspring. For example, if a Dorking fowl, which has an additional toe, five instead of the normal number four, is crossed with a fowl of another breed having only four toes, all the cross-bred chicks have the extra toe. But when these cross-bred offspring are bred together some of the chicks of the second generation have the extra toe and some have the normal number; that is to say, some resemble one grandparent and some the other. The character which appears in all the individuals of the ffrst generation is said to be dominant and the other recessive; and in the second generation the proportions of the individuals possessing the dominant character to those without it is three to one. Further, the recessives if bred together breed true, and the dominant character does not reappear; they behave as though they were pure-bred and had never been crossed Of the dominants, on the other hand, one-third breed true or are pure dominants, and twothirds produce dominants and recessives again. Now these curious facts would be explained if the gametes behaved in certain ways; or in other words we may say that from these facts certain conclusions may be drawn concerning the history of the gametes in the process of reproduction in such a Mendelian experiment. In the first cross each fertilised ovum or zygote consists of two gametes, one from each parent, and in development the dominant character conquers the other in every individual. But when the gametes of these compound or mixed individuals are formed, the characters of the two parents separate, or, as it is technically termed, segregate, so that half the gametes carry the dominant character and half only the recessive. When two individuals of this cross-bred generation breed 
together a dominant gamete may unite with a dominant or with a recessive; in the first case the offspring will be a pure dominant, in the second a mixed individual. Similarly a recessive gamete may unite with a recessive or with a dominant; in the first case the result will be a pure recessive, in the second a mixed zygote. The mixed zygotes and the pure dominant zygotes develop into adults or chicks, all showing the dominant character, and therefore the dominants will be to the recessives as three to one, as they are found to be by experiment. The gametes of the crossbred individuals may be represented by the symbols $\mathrm{D}$ and $R$, and they must be supposed to occur in the gonad in equal numbers $n \mathrm{D}+n \mathrm{R}$. The fertilisations will be represented by the symbols $\mathrm{DD}, \mathrm{RR}, \mathrm{DR}$. If we take the $\mathrm{Ds}$ in one individual, considering that they have an equal chance of uniting with a $\mathrm{D}$ or an $\mathrm{R}$ in the other individual of opposite sex, they will form an equal number of zygotes of forms DD and DR. Similarly the Rs will form equal numbers of zygotes of forms $R R$ and $R D$, the latter of which is the same as DR. Thus the total number of offspring will be $n \mathrm{DD}+2 n \mathrm{DR}+n \mathrm{RR}$, and since $\mathrm{DD}$ and $\mathrm{DR}$ resemble each other in their visible character, the proportion of dominants to recessives will be three to one.

There is a very interesting connection between this Mendelian theory of gametic segregation and the reduction of the number of chromosomes which is known to take place in the maturation of gametes. There is reason to believe that the halving of the number of chromosomes is due to the separation of the somatic chromosomes at one of the reduction divisions into two groups, each containing half the number. The reduction is thus due to segregation of undivided chromosomes. If the chromosomes are the carriers of-heredity, the character of the male parent in a cross must have been contained in the male chromosomes. In the development of the offspring each somatic cell contains both male and female chromosomes, but when the gametes are formed separation of the chromosomes takes place, so that an ovum may contain the character of one of the parents or the other, but does not contain both; and thus on the average half of the gametes contain the domi- 
nant character and the other half the recessive. It must not be supposed that in the reduction divisions the whole of the chromosomes received from the male parent are separated from those received from the female, for if this were the case the different individuals in the second generation of a cross would resemble one or other of the original crossed grandparents in all characters, and this does not happen. What has been said above of a Mendelian cross only applies to one pair of characters; when two or more pairs are considered the matter becomes much more complicated. It follows from the Mendelian theory that we must suppose segregation to take place in ordinary reproduction, but in this case the same characters occur in both parents and in both of a pair of segregated gametes. Thus we may represent a Mendelian experiment as follows :-

Crossed parents DD and $R R$.

Gametes in these $\mathrm{D}+\mathrm{D}, \mathrm{R}+\mathrm{R}$, which conjugate.

Zygotes $\mathrm{DR}$, the individuals of first generation.

Gametes of these $\mathrm{D}+\mathrm{R}$ and $\mathrm{D}+\mathrm{R}$, which again conjugate.

Zygotes $\mathrm{DD}+2 \mathrm{DR}+\mathrm{RR}$, individuals of second generation.

The individuals developed from unlike gametes are called hetero-zygotes, those from like gametes homozygotes. But an individual is not necessarily heterozygous or homozygous for all characters. Each pair of characters behaves as a rule independently.

In parthenogenesis when the egg develops without fertilisation there is probably no reduction of chromosomes, and therefore no segregation : but this is not certain.

$\S 6$. Segmentation. Usually after a very short interval the fertilized ovum begins to divide, and the resulting cells divide again and again. In these and in all cases of celldivision the nucleus divides first. At first the cells produced are all much alike, and the term segmentation is applied to this continued cell-division during the earliest stages, before the differentiation of cells begins to become so important that the mere cell-divisions are not noted. But 
from the first stages of segmentation our types begin to differ in development. We must therefore take them each in turn.

\$7. Segmentation in Amphioxus. The ovum contains scarcely any yolk, and its follicle remains around it during early development. Fertilization is said to occur always about seven o'clock in the evening, and segmentation to begin an hour later. Although the ovum is a sphore it is possible to define certain directions in it on the analogy of the naming of the earth-the polar body marks what is called the animal pole. This is one end of what may be called the axis : at the other is the vegetative pole.

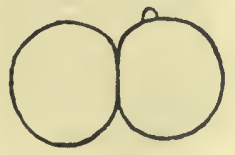

$\Delta$

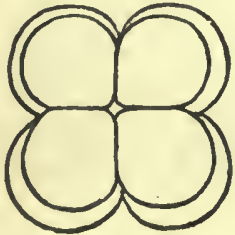

B

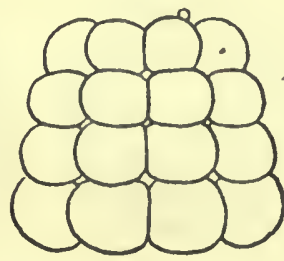

o

Fig. 111.-Sfgmentation of Ovum of Amphoxus.

(From Willey, after Hatschek). A, 2-celled stage, side view ; B, 8-celled stage, from animal pole; C, 32 -celled stage, side view.

The plane of separation of the first two cells is a meridional plane (fig. 111, A); so also is the second, which is at right angles to the first. The third is an equatorial plane. By this time there are eight cells, which remain in contact with one another (fig. 111, B). Two simultaneous meridional planes diagonal to the first ones follow, and then two simultaneous horizontal planes, and thus we have thirtytwo cells (fig. 111, c). Now, at each division the cells tend to round themselves off into spheres and so to fill up more space. The follicle stretches to allow this; but as the number of cells goes on increasing they become flattened where they are in contact, but at the same time they get pushed away from the centre. The result is that this mass of cells tends to take the shape of a hollow sphere; and this form becomes more definite as the number of the cells 
increases to sixty-four, one hundred and twenty-eight, and two hundred and fifty-six. The resulting hollow sphere is called a blastosphere, and the central cavity the segmentation cavity. The blastosphere stage is taken as the end of segmentation pure and simple.

Technically, the segmentation of the ovum of Amphioxus is said to be holoblastic and nearly equal. The former term means that the whole substance of the ovum divides up into cells (in the fowl's egg, as we shall see, it does not). The latter means that up to the end of segmentation all the cells produced are nearly equal in size. Really, those in the vegetative hemisphere are a little larger, but compared

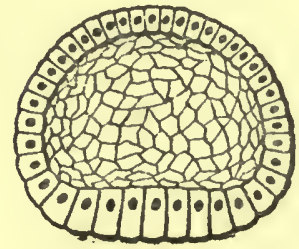

A

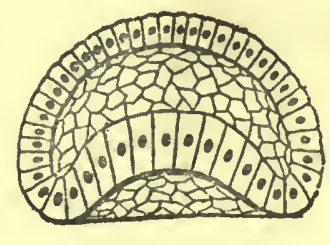

B

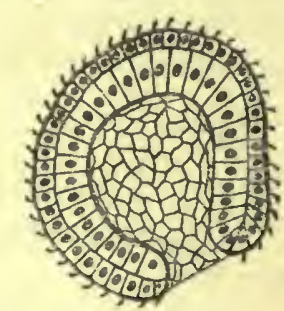

0

Fig. 112.-Sections of Eubryo Amprioxus.

A, Blastosphere; B, early gastrula; C, later gastrula, with ciliuted epiblast.

with the striking inequality of the segmentation in some types this is insignificant.

§ 8. The Gastrula. We next notice (fig. 112, A), that the vegetative end of the sphere is decidedly flattened. This flattened area becomes concave (fig. 112, B), and finally seems to bo tucked-in until it touches the animal hemisphere, and the segmentation cavity is obliterated. As cell-division still goes on, the embryo again comes to a nearly spherical shape (fig. 112, c), but now the sphere consists of two layers of cells, and the central cavity has an opening." In these stages the embryo is called a gastrula : this stage is one of the greatest interest, as such a great variety of animals go through it: in fact it is characteristic of the development of the simpler members of most of the great animal groups. Certain new 
terms are needed to dcscribe it: the outer layer of cells is called the epiblast; the inner layer, primitive hypoblast; the central cavity is the archenteron, or primitive alimentary canal ; its opening is the blastopore.

§ 9. First Vertebrate Features. The gastrula of Amphioxus is never quite spherical-in fig. 112 , $\mathrm{c}$, it is seen that one side is flattened-this is the future dorsal surface. The blastopore, originally marking the future posterior end, soon turns towards the dorsal side. This dorsal position of the blastopore is very characteristic of Vertebrates. If now we examine a transverse section of the embryo (fig. 113, A),

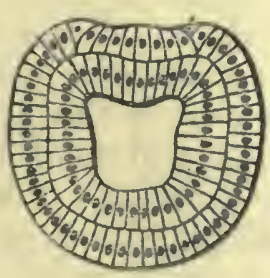

$\Delta$

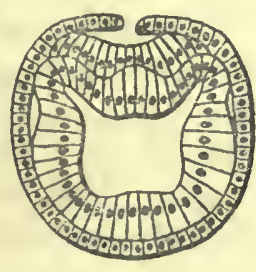

B

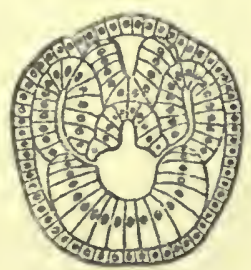

o

Fig. 113. - Trangverse Sections of Early Larta oy amphioxus. (From Willey, after Hatschek.)

In $A$, the onter laser is epiblast (with neural plate just differentiated on the dorsal side); the inner, primitive hypoblast. In $\mathrm{B}$, the neural folds are seen ; under the neural plate, the first sign of the notochord is seen, and on either side of it a myocolomic pouch. In $C$, the neural plate has begun to roll up, the neural folds have closed over it, and the myocolomic pouches are more distinct.

we see that in the dorsal flattened region a plate of epiblastcells is beginning to differentiate from the rest: this is the neural plate, another Vertebrate feature. Soon the gastrula begins to become more elongated along its antero-posterior axis. A cilium is developed from every epiblast cell (fig. $112, \mathrm{c})$, the follicle is broken through, and the gastrula escapes to swim about near the surface of the sea by tho constant motion of its cilia. Hitherto its increase in size has been due simply to change from a solid sphere to a hollow body : there has really been a diminution in material, for all cell-division is work and involves some katabolism, and there has only bcen the small quantity of yolk to serve as food to the protoplasm. From now onwards the larva 
has to feed for itself on the floating diatoms and the like in the sea-water. This escape from the egg-membrane occurs about eight hours after the beginning of segmentation.

Ciliated larvæ are common in almost all groups of marine Invertebrata; but the Amphioxus is the only Vertebrate that passes through this stage of free-

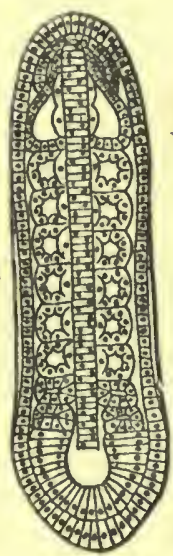

Fig. 114.- - HORIzONTAL SECTION THROUGH A LARVA OF AMPHIOXUS. swimming by means of cilia.

§ 10. Notochord and Mesoblast. Tho next changes must be briefly described. They are indicated in figs. 113, B and c, which show a series of transverse sections at different stages. 'The ordinary epiblast at the sides of the neural plate begins to grow in towards the middle line so as to cover in the neural plate. Posteriorly these neural folds close in over the blastopore, forming there a tunnel called the neurenteric canal, which continues the archenteron dorsalwards and forwards to over the surface of the neural plate. Meanwhile the median dorsal portion of the primitive hypoblast becomes thickened longitudinally, and from this thickening a In the middle is the
notochord, with longitudinal rod of cells is pinched off: this eightmyocelomic is the future notochord. Right and left of
pouches on either is side of it. (From the notochord a metameric series of hollow Willey, after
Hatschek.)

push their way in between epiblast and hypoblast, as they develop. These are the myocœlomic pouches, the cells of whose walls constitute the mesoblast (fig. 114).

$\S 11$. The Three Germinal Layers. The remainder of the primitive hypoblast, after the mesoblast has been separated from it, is called simply hypoblast, and the cavity it encloses (which is the archenteron minus the myocœlomic cavities) is called the mesenteron. Epiblast, mesoblast, and hypoblast are known as the three germinal layers. At this stage, evidently, they are all simple epithelia : it is not till 
later that connective, muscular, and nervous tissues come to be formed from them.

From Epiblast are developed the epidermis (not the dermis) and the whole of the nervous system, including the essential sensory part of all sense-organs.

From Mesoblast are developed all the connective tissues (including dermis), all the muscles, blood-vessels and blood, excretory organs and gonads-in fact, the main mass of the body.

The Hypoblast gives rise to the epithelium of the alimentary canal and of all glands that open into it; but not to any other part of the walls of the canal. The notochord is also usually counted as derived from hypoblast, although it appears before mesoblast and hypoblast are clearly differentiated.

These statements are true not only for Amphioxus, but also for all Vertebrates, and indeed (with slight modifications) for the higher Invertebrates-for all animals, in fact, in which the three germinal layers are developed. It will be convenient to indicate these three layers by distinct shading in our diagrams, and therefore in many of them * epiblast will be made black, mesoblast dotted, and hypoblast lined.

$\$ 12$. Later Development of Amphioxus. The neural plate soon begins to roll itself up into a longitudinal tube, and this becomes the spinal cord. The myocolomic pouches grow in size and push their way, as it were, between the other layers until they have oxtended as far as shown in fig. 115, A. They now become divided into a dorsal and ventral portion, the fate of which is differentthe dorsal portions retain their metamerism, their cavity becomes obliterated by the growth of the walls, and they give rise to the myomeres of the body. The ventral portions lose their metamerism, their cavities becoming connected into a single cavity - the cœlom - from end to end of the body, and the cavities of the right and left sides also become joined ventral to the alimentary canal (fig. 115, B).

Thus the mesoblast may bo said to be divided into three

$$
\text { * Figs 116, 120, 121, 123, and } 130 \text { to } 137 .
$$


purts-the dorsal part, consisting of mesoblastic somites; a continuous layer in contact with the hypoblast-the splanchnic mesoblast; and a layer in contact with the ventral half of the epiblast-the somatic mesoblast. By this time a transverse section shows us all the broad features of a section across the intestinal region of the adult (compare figs. 115, B, and 105.)

Meanwhile, the larva has been lengthening greatly, new myocœlomic pouches being added behind as this growth continues.

Mouth and anus arise as pits of epiblast (called respec-

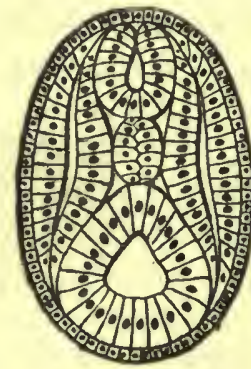

A

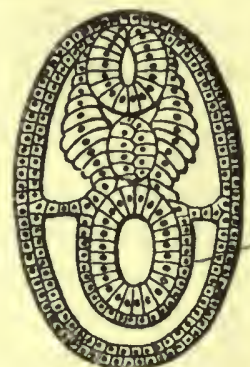

B

Flg. 115.-Dinarammatic Trangverse Sections of a linga OF AMPHIOXUS.

In $A$, the neural plate has completely rolled up to form the spinal cord; beneath it the notochord is distinct; beneath this is the mesenteron. The myocœlomic pouches are pushing their way between these structures and the epiblast, on either side.

tively stomodæum and proctodæum), that first abut against the blind ends of the mesenteron, and then open into it (compare fig. 121, showing the same in the frog). The other communications between alimentary canal and exterior, viz. the gill-slits, arise in a different way, as pouch-like outgrowths of the mesenteron (fig. 116, в). The walls of these pouches consist, of course, of hypoblast and splanchnic mesoblast. They grow out into the cœlom until they touch its opposite wall, i.e. the body wall, consisting of epiblast and somatic mesoblast. In doing so they of course obliterate the colom locally, and so this continuous space becomes divided up into dorsal, lateral, and ventral canals, as previously explained (chap. xvi., § 4). 
Then the pouches open to the exterior and become gillslits. The slits increase in number by now ones being formed in turn behind (as is the case with the other meta. meric structures).

The metapleural folds and atrium are formed when there are only a few gill-slits, and the diagram (fig. 116) indi-

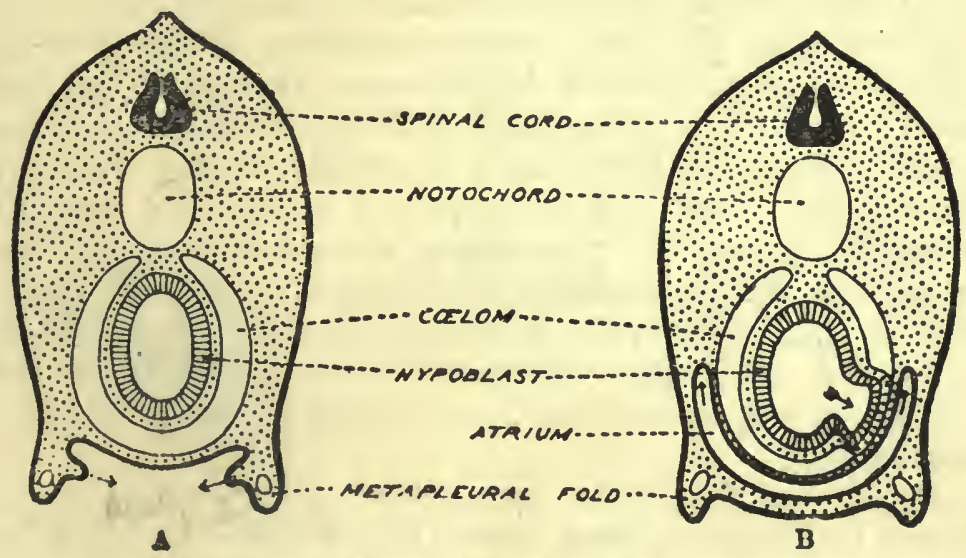

Fig. 116. - Oriain of Atridm and Gill-slits in Amphioxus.

(After Lankester and Willey, altered.) Epiblast black, mesoblast dotted, hypoblast lined. In B, the barbed arrow shows the formation of a gill-slit, the unbarbed arrows show the extension of the atrium.

cates their mode of origin. First the metapleural folds aro formed, as longitudinal ridges of epiblast and somatic mesoblast. Then ingrowths towards the middle line arise from these and meet, enclosing a space lined by epiblastthis is the atrium. It is at first small but soon grows, pushing its way dorsally on either side of the alimentary canal, as shown by the arrows in fig. 116, B. The mode of origin farther forwards, where the slits are already formed, is the same in principle; but the metapleural folds first appear above the gill-slits, and grow down to enclose them. 


\section{Chapter XVIII.-Develophent of the Frog}

§ 1. Maturation and Fertilization. The ripe ova of the frog are about $\frac{1}{14}$ of an inch in diameter, or five thousand times as large as those of Amphioxus. This relatively large size is due to the enormously greater amount of yolk contained within the protoplasm, especially of the vegetative hemisphere. The nucleus (germinal vesicle) lies near the animal pole, and the protoplasm around it contains little or no yolk, but it contains much black pigment. Thanks to this black colour, the animal hemisphere is readily distinguished from the vegetative. Such an ovum as this, having its yolk concentrated towards one pole, is said to be telolecithal.

About the time that maturation is beginning, the ova escape in large numbers from their follicles and fall into the cœlom, each surrounded only by its vitelline membrane. They travel forwards (how, is not fully understood) and enter one or other of the internal apertures of the oviducts. As each ovum passes down the oviduct, it becomes surrounded by a layer of albuminous substance secreted by the oviduct walls. During this time the process of maturation begins, and when the eggs are laid one polar body has been formed.

As the eggs are laid they are fertilized by the male, who has been waiting, attached to the female, for days beforehand. It is a curious point that the second polar body is not actually extruded until after the male pronucleus has entered the ovum.

The eggs are laid in ponds or backwaters of streams about March. On contact with the water the albuminous covering swells up and serves both to float the eggs and to protect them from the many enemies that would otherwise soon devour them in their exposed situation.

§2. Segmentation begins after the same fashion as in 
Amphioxus, but is far slower, owing to the fact that the great amount of yolk present impedes the activity of the protoplasm. For the same reason differences soon show themselves between the behaviour of the animal and regetative hemispheres, the latter of which contains so unuch more yolk than the former. The first two divisions occur along meridional planes, as in Amphioxus, and the third in a plane at right angles to both these; but instead of being equatorial, it is decidedly nearer the animal pole (fig. 117, $A, B)$. From this point onwards two kinds of cells can be distinguished-smaller black cells in the animal hemisphere, and larger yolk-laden cells forming the vegetative hemisphere. These correspond approximately to the epiblast

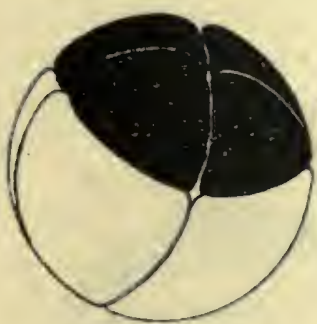

A

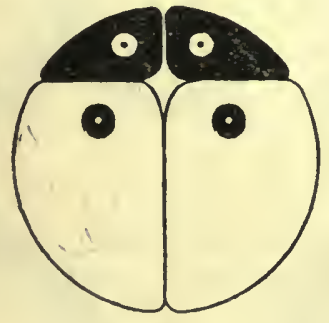

B

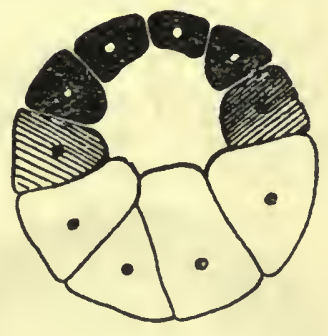

o

Fig. 117.-Segmentation of Ovum of Frog.

(After Ilowes and Marshall.) A, eight-celled stage, side view ; $B$, the same in rertical section; C, blastosphere, vertical section.

and primitive hypoblast cells of Amphioxus, respectively: we shall call them the primitive epiblast and the yolk-cells, respectively. At the blastosphere stage (fig. 117, c) we find that, owing to the larger size of the yolk-cells, the segmentation cavity lies nearer the animal pole.

§ 3. The Gastrula of the Frog. The next stage-early gastrula-in Amphioxus (fig. 113, B) showed us the primitivehypoblast cells partially invaginated. The yolk-cells of the frog are too bulky for this to take place, and so, as they continue to divide, they become a somerwhat irregular mass (fig. 118, A). This is much the same as what,we should get in Amphioxus if the primitive hypoblast cells were to greatly enlarge in size and fill up the archenteron cavity. And we 
find that later on in the frog's development the archenteron (or rather mesenteron) is formed in this irregular mass of yolk-cells by a process of splitting, or separation of the cells from one another. So far, then, the differences in development in our two types are all directly explicable by reference to the increased amount of yolk in the frog.

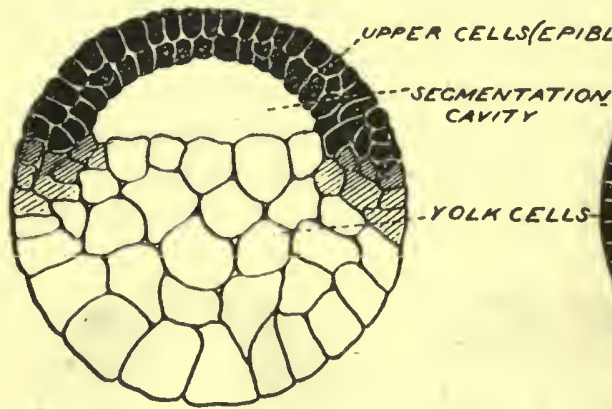

A

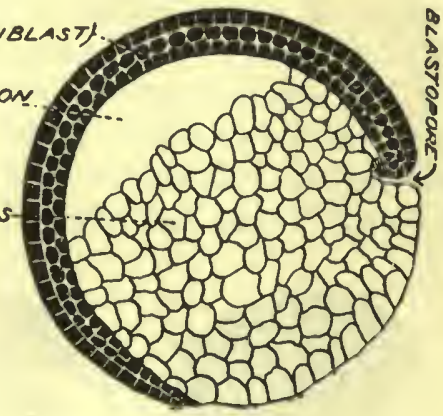

B

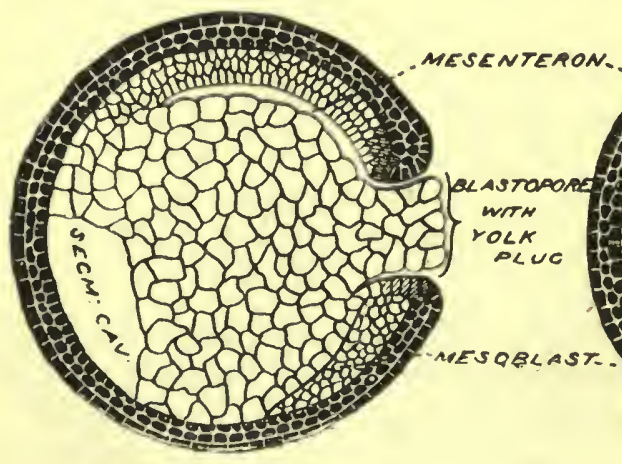

C

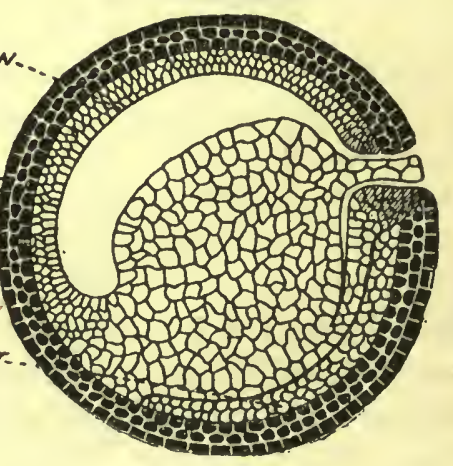

D

Fig. 118.-Development of Embryo Froa. (After Marshall.)

The next stages in Amphioxus (fig. 113, c) showed the obliteration of the segmentation cavity by more complete invagination, and the restriction of the orifice of invagination to a small opening, the blastopore-the epiblast coming to form the whole outer surface except at the blastopore itself. In the frog the primitive epiblast cells are not 
enough to form the whole outer surface over the big mass of yolk-cells, so supplementary epiblast cells are added to them by cell-divisions in the outermost yolk-cells. This process begins in the yolk-cells nearest the equator, which adjoin the edge of the primitive epiblast, and gradually extends to yolk-cells nearer and nearer tho vegetative pole (fig. 118, B, c). As the supplementary epiblast-cells at once secrete pigment like that of the primitive epiblast-cells, and as they resemble these also in size, it is easy to imagine that the primitive epiblast is really growing and pushing its way down towards the vegetative pole over the surface of the yolk-cells. This, in fact, was formerly described in many text-books as actually happening; but the process is now known to be what has been described-an extension of the epiblast, not by growth, but by addition of new cells formed from the yolk-cells.

This extension of the epiblast does not occur, however, along quite the whole of its original edge. At ono point,

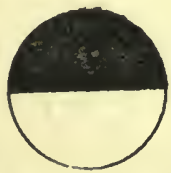

A

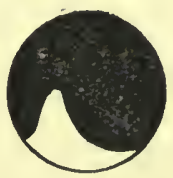

$\mathrm{B}$

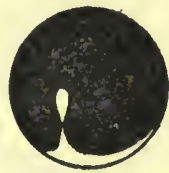

0

Fig. 119.-Formation of Blastotore in Frog. Diagrammatic posterior view. Epiblast black, yolk-cells white. The neural folds are not shown, although they would be seen in stage $C$.

which marks the future posterior end of the embryo, the edge of the epiblast remains where it was. The downgrowing edges on either side of this point approach one another and meet (fig. 119), so that by the time epiblast has come to cover over the yolk-cells right down to the vegetative pole, there still remains a little circular area at the posterior end where yolk-cells show at the surface. This is the blastopore: it corresponds, not to the original wide aperture of the archenteron in Amphioxus (fig. 112, B) but to the small, posterior blastopore of the late gastrula stage (fig. 112, c).

$\S 4$. The Epiblast of the Frog. Meanwhile the epiblast has divided into two layers. This division occurs very early in the primitive epiblast (fig. 118, $\mathrm{A}$ ), and the new epiblast formed from the yolk-cells soon follows suit. This is a special peculiarity in the development of the frogs and toads 
(Anura): it does not occur, for example, in the newts (Urodela) whose ova have quite as much yolk. This is not, therefore, a result of the yolk-laden condition of the ovum. We have seen that the epiblast gives rise to epidermis and nerve-tissue : in the frog a sort of precocious separation of these two takes place, the outer layer of epiblast being the epidermal layer, the inner the nervous layer.

\section{§ 5. Differences in Early Development of Frog and} Amphioxus. Thus there are three kinds of differences between the early stages of development in frog and Amphioxus-(1) a difference which is peculiar to the Anura (precocious differentiation in the epiblast); (2) differences which are mechanical results of the presence of yolk (e.g. the absence of an archenteron cavity); (3) those which are adaptations to the yolk-laden condition, e.g. the formation of supplementary epiblast from yolk-cells. The distinction between (2) and (3) may not be clear to the student at first. We may explain it by saying that if we could, by a delicate surgical operation, remove the yolk from a frog's ovum, the mechanical effects of the yolk would disappear and the ovum would, so far, tend to segment in Amphioxus fashion; but the adaptational characters reside in the nucleus and protoplasm and would not be changed by the withdrawal of the yolk.

§ 6. Origin of Mesenteron. The mesenteron appears as a cleft in the mass of yolk-cells, and starts from the region of the blastopore, at the time when the blastopore is only beginning to be marked out. As the epiblast comes to close more and more completely round the blastopore the split curves round it in the same way, so that it finally comes to be a circular chink, and the yolk-cells that still show at the surface are marked off by this chink as a cylindrical plug (yolk-plug) in the centre of the blastopore (fig. 118, c). The chink around this plug is the beginning of the mesenteron. As it extends farther into the mass of yolkcells, the segmentation-cavity shifts round before it and is eventually obliterated. The extension of the mesenteron can be best understood by the study of fig. 118 . 
It was formerly thought that an actual invagination took place at the blastopore, to form the walls of the mesenteron. This is a mistake, and would be strange if it were true, since we are by this time obviously past the stage at which invagination occurred in Amphioxus. The cells immediately around the blastopore do, however, show characters intermediate between the epiblast and yolk-cells, and it seems that during the next stages of growth they give rise to cells of all three layers (epi-, meso-, and hypo-blast): the same is probably true of the cells around the blasstopore of Amphioxus.

Why have we called the cavity formed in the mass of yolk-cells mesenteron and not archenteron? In Amphioxus we had an archenteron, whose walls were primitive hypoblast : the cavity became divided into mesenteron and myocelomic cavities, while the primitive hypoblast differentiated into hypoblast, notochord and mesoblast. In the frog, the yolk-cells, after the supplementary epiblast has been separated off from them, correspond precisely with the primitive hypoblast, and, like that, differentiate into hypoblast, notochord and mesoblast. But the mesenteron and myocolomic cavities both appear by splitting in a solid mass of cells, and are never connected with one another, so that there is never any actual archenteron-cavity in the frog.

$\S 7$. The Origin of the Three Germinal Layers in the frog may now be stated.

The epiblast is formed partly from the primitive epiblast cells of the animal hemisphere, and partly from supplementary epiblast-cells divided off the yolk-cells.

The hypoblast is formed of the remainder of the yolk-cells after (1) the supplementary epiblast and (2) the mesoblast, have been differentiated from them.

The mesoblast is formed from yolk-cells. Its differentiation begins near the dorsal surface, and gradually extends to the ventral side.

$\S$ 8. Neural Folds and Notochord. In Amphioxus the gastrula stage was followed by one in which the neural plate 
and folds, the myocelomic pouches, and the notochord, were formed simultaneously. The corresponding stage is found in the frog, allowing for the absence of actual myocolomic pouches.

The neural plate resembles that of Amphioxus, except that from the first it is broader in front (the future brain) than behind. Moreover, as the neural folds grow up, the edges of the plate rise with them, so that the plate becomes a neural tube as soon as the folds close in. (See figs. 120 and 121, and contrast with figs. 113 and 115.) The enlarged front end of the neural tube becomes very early constricted into three cerebral vesicles. The hind ends of the folds do not enclose the blastopore for some time, but do so at last, forming a neurenteric canal as in Amphioxus.

The notochord is formed from the hypoblast of the dorsal middle line, just as in Amphioxus ; but it never extends in front of the middle of the second cerebral vesicle (fig. 121.)

§ 9. Divisions of the Mesoblast. The mesoblast, at first

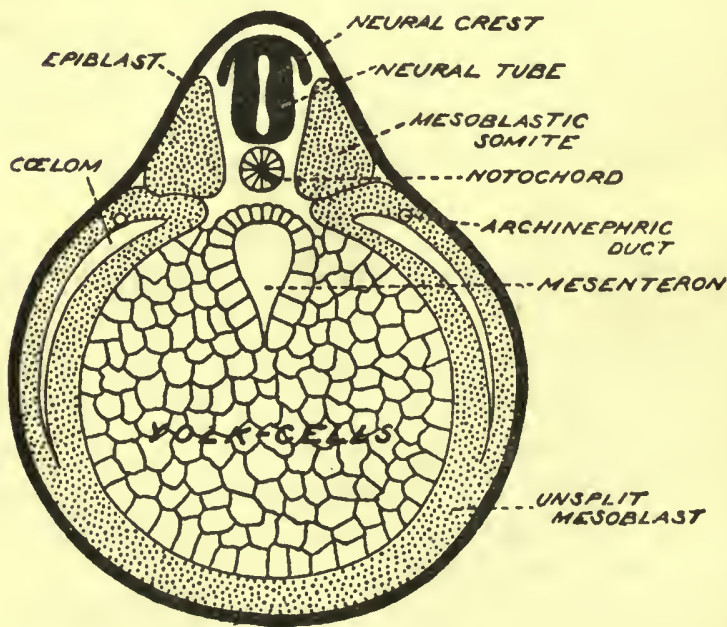

Fig. 120.-T'ransyerse Section of Embryo of Froo. (After Marshall.) solid, soon splits on each side into two layers (fig. 120), with a myocolomic cavity between them. In Amphioxus we saw that a paired $\mathrm{met} a \mathrm{mer} i \mathrm{c}$ series of such cavitios was present from the first appearance of the mesoblast; that each pouch became afterwards divided into dorsal and ventral portions: that the dorsal portions retained their metamerism but lost their cavities, becoming 
the myomeres, while the ventral portions retained their cavities, but lost their metameric character, a continuous cœlom being formed by end-to-end and right-and-left union of their cavities. In the frog a similar condition is arrived at, but by different stages. The mesoblast of each side first splits as a whole; then it becomes divided into a dorsal portion called the vertebral plate, and a more ventral portion the lateral plate. The lateral plate retains its cavity, which presently unites with its fellow of the other side, and is never metamerically segmented; while the vertebral plate becomes metamerically segmented into a series of cubical masses, whose cavities soon disappear. These masses were formerly called protovertebræ, but are now generally known as mesoblastic somites. Neither name satisfactorily expresses their nature- " protovertebræ" is misleading, because, although the vertebræ are formed from them, a great deal besides is also formed from them; while "mesoblastic somite" suggests that the whole of the mesoblast is divided into somites, whereas only the vertebral plate is so divided.

It must be added that in the head-region (i.e. corresponding to the first two cerebral vesicles) the mesoblast shows no metamerism, and its splitting is much less complete than behind.

$\S 10$. Further Development of Mesenteron. The embryo now begins to lose its spherical shape, and to elongate at its posterior end, though, being still within the vitelline membrane, it has to double on itself as it does so. This elongation takes place by growth and division of cells, of course at the expense of the yolk-the yolk-cells continually dwindling in size. The most obvious sign of growth, proving that it takes place at the posterior end, is the formation of additional mesoblastic somites behind those first formed; but of course all the other layers must be increasing equally, though, not being metameric, their growth is less obvious.

'The mesenteron, as it extends forwards, becomes wider and deeper in the anterior region than it is behind, where the yolk-cells remain accumulated on its floor (fig. 121) 'Thus it is soon divided into two regions-an anterior 
pharyngeal and a posterior intestinal. The growth in length at this stage affects the latter only. Soon little median pits of epiblast appear at the front and hind ends-the stomodæum and proctodæum (fig. 121) - and presently the proctodæum opens into the bind part of the mesenteron, forming the cloacal aperture, anterior and ventral to the blastopore. The stomodæum remains closed for some time.

In the side-ivalls of the pharyngeal region six pairs of vertical thickened ridges appear - the visceral arches-and the mesoblast-cells in the centre of each begin to secrete a cartilage-matrix. The first of these visceral arches is the - mandibular arch, which later becomes bent and divided into

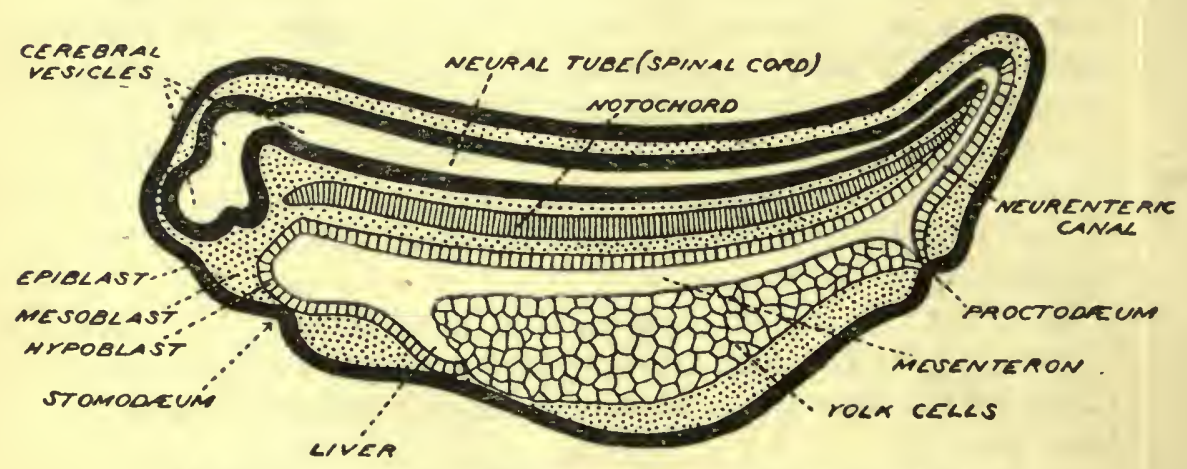

Fig. 121.-Miedian Lonaitudinal Section of Embrto of Froo (After Howes,)

palato-pterygoid and Meckel's cartilage; the second is tlo hyoid arch, the rest are the four branchial arches.

The intestinal region is a straight tube, its lateral and ventral walls extremely thick from the amount of yolk they contain. From the front end of this intestinal region a backwardly directed ventral outgrowth is formed, burrowing, as it were, into the mass of yolk. This is the rudimert of the liver.

Behind, the blastopore has closed, the yolk-plug having been absorbed; and the region of the mesenteron posterior to the proctodrum, which is continuous with the neurs? tube, becomes drawn out by the growth of the tail (fig. 121). 
$\S 11$. First Blood-vessels. The first necessity for a vascular system arises from the fact that all parts of the embryo need the food that is stored up in the hypoblast of the intestinal region. Hence the first vessels are formed in the splanchnic mesoblast of this region (vitelline veins), and extend forward as a median sub-intestinal vein, which in the pharyngeal region becomes the heart. This is at first a straight tube, giving off from its anterior end two pairs of vessels which run a little way up the third and fourth visceral (i.e. first and second branchial) arches: these are two of the future afferent branchial arteries. In the dorsal part of all the six arches the rudiments of efferent arteries appear, and connect with a pair of dorsal aortre on either side the notochord, which farther back unite into a median aorta, ventral to the notochord.

So far we have a general resemblance to the vascular system of Amphioxus, allowing for thesmall number of visceral arches. But in the frog-embryo the aorta gives off branches to the mesoblastic somites and somatic mesoblast as well as to the splanchnic, and the paired aortre in front are continued forwards as carotid arteries to the head. To return the blood to the heart from head and somatic mesoblast paired anterior and posterior cardinal veins, like those of the dogfish, are formed. 'These meet on either side just behind the last visceral arch, and a pair of $C$ Cuvierian veins run transversely from their junction to the heart. Thus even at this early stage the frog-embryo is nearer the dogfish than Amphioxus, as regards its vascular system.

$\S$ 12. The Pronephros and its Duct. In Amphioxus we saw that, though at their first development all metameric members had the same metamerism-each gill-slit corresponding to a myomere, and so on-in the adult the metamerism of the gill-slits and nephridia, on the one hand, was quite independent of the metamerism of the myomeres, nerves, and gonads, on the other, although these were all present in the same (pharyngeal) region of the body. In the embryo frog we find the metamerism of the myomeres (i.e. the somites) restricted to the post-pharyngeal region, while that of the gill-slits and visceral arches, 
and consequently of the arterial arches, is restricted to the pharyngeal region. We have next to see that the embryo frog has excretory tubules very different from those of Amphioxus: they lie altogether behind the pharyngeal region, and their metamerism corresponds to that of the mesoblastic somites.

The first rudiment of an excretory system consists in the formation of a pair of longitudinal tubes in the somatic mesoblast, just ventral to the mesoblastic somites (figs. 124 and 127). This tube is called the archinephric duct (or segmental duct). At its anterior end three tubules are formed in the somatic mesoblast, under the second, third and fourth somites respectively; these open into the colom at one end (nephrostome) and into the archinephric duct at the other. They are called the pronephric tubules, or collectively the pronephros or head-kidney (figs. 127 and 128). On each dorsal aorta, just opposite the nephrostomes, a bunch of little blood-vessels is formed. Separated as this is from the nephrostomes by the narrow coelom of this region, the resemblance to the glomus of Amphioxus is striking, and it may be called the glomus and regarded as three glomi united in one (fig. 128). The archinephric duct and the three pronephric tubules in the embryo frog have the characters which belong to cœlomic ducts : they are not intracellular but lined with an epithelium, they have an opening into the coelom, and they are developed in the mesoblast from within outwards. They cannot therefore be homologous, as was formerly supposed, with the nephridia of Amphioxus. The Müllerian duct, which becomes the oviduct is developed in the frog independently, but there is evidence in the development of the dog-fish that it was originally derived by splitting from the archinephric duct, and as we know the posterior part of the archinephric duct through the medium of the mesonephric tubules becomes connected with the testis and acts as vas deferens. The excretory tubules of the frog therefore, and of all vertebrata, have a close connection with the genital ducts, and were probably evolved as modifications of the genital ducts.

$\S 13$. Hatching of Tadpole. We have now described the 
most important structures which are developed before the liberation of the embryo from the egg as a tadpole. This takes place about a fortnight after fertilization, a long period in comparison with the eight hours of Amphioxus. One other temporary organ now developed must be mentioned; this is a pair of suckers on the ventral surface, a little behind the mouth (fig. 122 [1]). By means of these the tadpole attaches itself to water-weeds (fig. 122 [2]) for

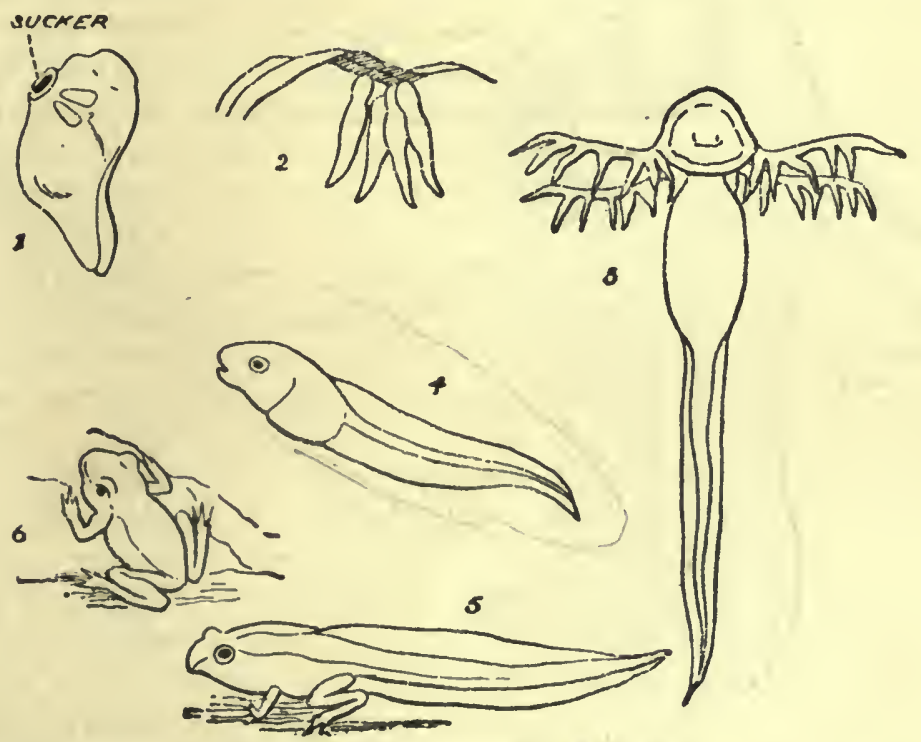

Fig. 122,-DE Elotyent of Froo.

(1) Shortly before hatching. (2) Newly-hatched tadpoles. (3) Tadpole with external gills. (4) Tadpole with internal gills and operculum. (5) During the metamorphosis. (G) Young frog. N.B. - The scale of the six figures differ's greatly.

a few days, until the stomodæum is perforated and horny jaws developed, when for the first time it begins to feed.

The tadpole just hatched is at a far higher level of development than the larva of Amphioxus when it begins its free existence. The possibility of its attaining this level while still within the vitelline membrane is due to the relatively great amount of yolk stored up in the ovum. Even at the time of hatching this is not all absorbed, though it practically all is by the time active feeding has begun. 
§ 14. History of the Gills and Related Structures. At the time of hatching the first two branchial arches show at the sides of the head as slight vertical ridges (fig. 122 [1]). From these there soon grow out long branching processes, the external gills. These are, of course, covered by epiblast, and have a core of mesoblast, containing a series of looping

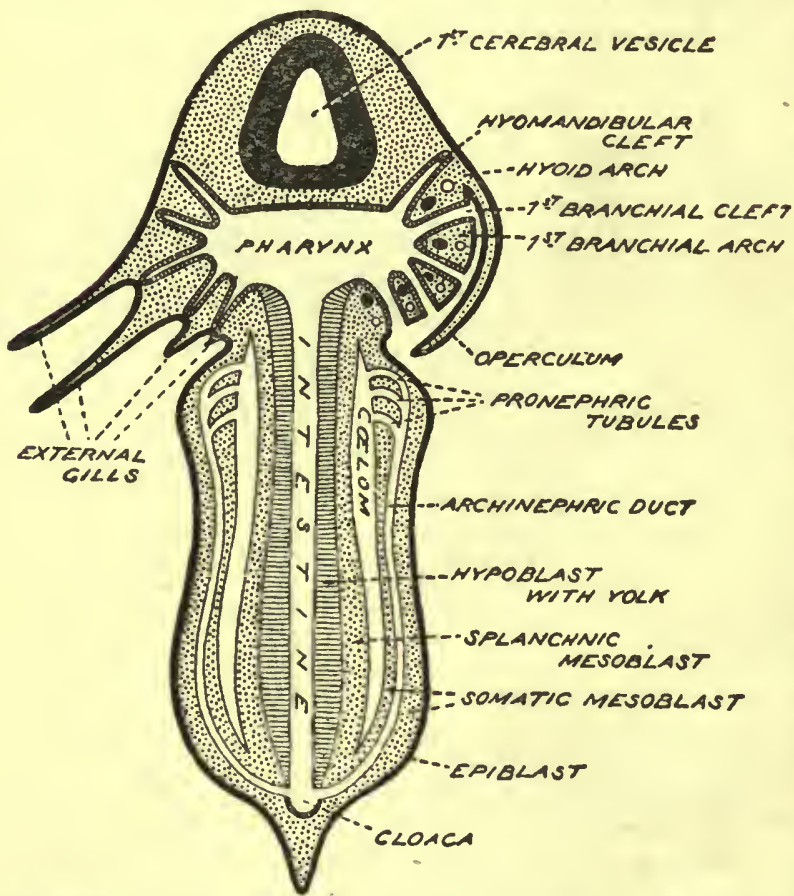

Fig. 129.-Diagramatic Horizontal Section of Tadpole, blood - vessels which connects in each case the afferent and efferent arteries of the respective arch. Presently a similar external gill grows out from the third branchial arch of either side (fig. 123, left side).

II e a n while, the hypoblast of the pharynx has been pushing its way towards black spots in the arches represent cartilage, and the white circles, arterics. (Partly after Marshall.) the surface between all the visceral arches; and these outpushings of hypoblast at length unite with the epiblast, and the gill-slits are formed. These are four pairs, for the one between the mandibular and hyoid arches (answering to the dogfish's spiracle) never becomes perforated; and there is neither slit nor arch answering to the fifth of the dogfish. These gill-slits are perforated about the same time as the mouth, so that feeding and throat- 
respiration begin together. The external gills begin to shrivel and disappear as the internal gills-vascular outgrowths from the slits, covered by hypoblast-are developed.

The vessels in the mandibular and hyoidean arches which were never more than rudiments soon disappear, but both afferent and efferent arteries are now fully developed in the other four arches. It should be noticed that the arrangement of these arteries is decidedly simpler than that in the dogfish ; there is simply one afferent and one efferent vessel in each arch (contrast fig. 92), and these are connected by a series of loops in the gill-filaments.

Presently a new structure appears, not represented in any of our other types, but existing in most ordinary fishes. This is the opercular fold, an external fold developed from the hyoid arch and extending back to cover in the gills (fig. 123, right side). Presently the edge of this fold fuses with the body-wall and only a small aperture is left, on the left side, so that the gill-slits open, not directly to the exterior, but into a chamber quite analogous (but probably not homologous) to the atrium of Amphioxus. The pore by which the water-current passes out evidently is analogous with the atrial pore of Amphioxus.*

\$ 15. Cœlom and Pericardium. About the time of hatching, two regions may be distinguished in the coelom, corresponding to the two regions of the mesenteron. In the intestinal region, owing to the great thickness of the yolkbearing mesenteron walls, the colom is a mere narrow chink (fig. 120), but it extends round all but the dorsal part of the mesenteron. In the pharyngeal region there is no yolk and the cœlom is larger, but confined to the ventral region because elsewhere the formation of the gill-slits is bringing epiblast and hypoblast close together.

As the heart develops it projects more and more from the floor of the pharynx into this expanded part of the coelom, which is, therefore, the pericardium. As the Cuvierian veins increase in size they form a transverse barrier

* In one of the toads (Dactylethra) there is a pair of these open. ings, right and left: this is obviously a more primitive condition; and the tadpoles of this genus are in other ways very primitive.

zOOL. 
which tends more and more completely to separate off the pericardium from the rest of the cœlom; but to the end of tadpole life a pair of pericardio-peritoneal pores are left, connecting the two regions of the cœlom. At this stage, therefore, the pericardium has considerable resemblance to that of the dogfish. The adult condition is produced by the shifting back of the heart as the lungs develop (compare fig. 91).

\$ 16. Further Development in the Tadpole. Meanwhile numerous other changes have been taking place. The whole

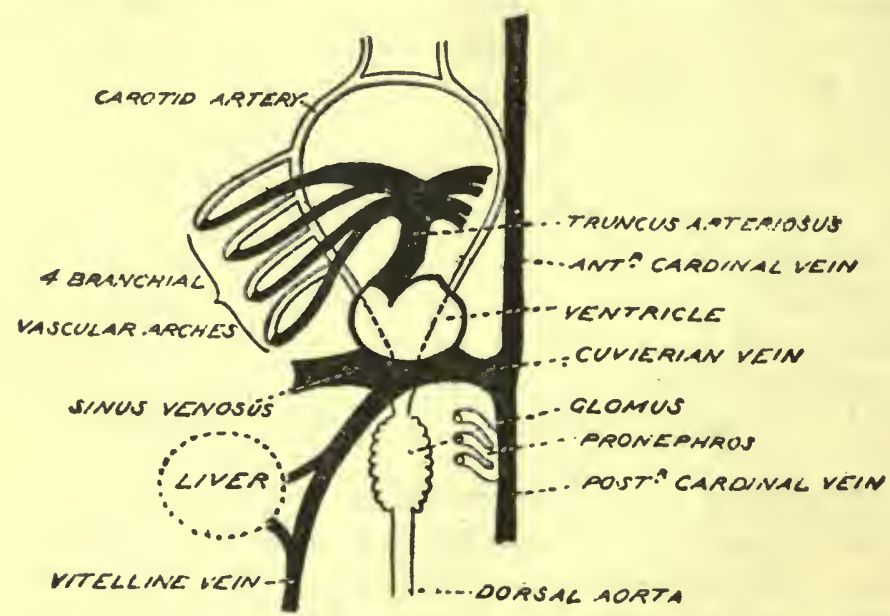

Fig. 124.-Circulation in the Tadpole.

"Vitelline Vein" is the same as "Sub-intestinal Vein."

body has grown in size, and the tail has elongated greatly and become an active swimming organ, in which fish-like myomeres are very obvious. There is a continuous median fin to the tail, along both dorsal and ventral edges, and it is interesting to note that this extends a little in front of the cloacal aperture, which consequently is shifted a little to one side, as in Amphioxus. The shifting is, however, to the right, not the left.

The intestinal region of the alimentary canal has grown much faster than the body, and forms a long coil in the cœlom. It remains of uniform diameter. All the yolk is 
absorbed. The liver has branched repeatcdly, and is beginning to assume the compact adult character; the pancreas has appeared as a pair of diverticula behind, and quite in. dependent of, the liver. The lungs, too, began to appear very early as a pair of pits in the floor of the hindmost part of the pharynx.

Blood-vessels have been developing in all parts, and joining up. 'The most important of these, at first, is the sub-intestinalvein, of which the heart is but the anterior part. This serves to carry the absorbed yolk from the mesenteron to the heart, and so to the body generally, before feeding begins. As the liver develops two series of branches grow out of this vein and form a portal system, more and more of the blood passing this way, until the original direct communication becomes closed, and the sub-intestinal rein divided into portal and hepatic veins. The chief other blood-vessels have already been mentioned in connexion with the gill-slits and pronephros. A general diagram of the circulation is given in fig. 124 .

Considerable changes have taken place in the nervous system, and nerve-fibres have grown out into all parts of the body, as must needs be the case before such active work as eating and swimming can be carried on. An interesting point in the tadpole is the development of a series of sensory tubules along the lateral line, as in fishes. Considerable histological differentiation has also taken place in all parts. But consideration of these and other details would lead us too far away from the main course of development. 'They will be better considered at a later stage.

\$17. Preparations for the Adult Stage. It is more important to note the appearance of organs that are useless to the tadpole, but needful to the frog, and which now begin to develop wherever they can without interfering with the efficiency of the larval organs themselves. The lungs have been already mentioned. As they grow in size, an artery branches off from the fourth efferent branchial of either side to supply them.

The fore limbs are developed under the opercular fold, so as not to be in the tadpole's way; but the hind ones 
arise on either side of the cloacal aperture and project back alongside the tail.

The kidney (mesonephros) of the adult frog first appears as a metameric series of tubules, called the mesonephric (or Wolfficn) tubules. These differ from the pronephric tubules in being formed from the mesoblast of the somites, and not from the somatic mesoblast below the somite. At first they are solid masses of cells, but they burrow their way, as it were, till they meet the archinephric duct. Then each acquires a lumen (central cavity), and this opens into the duct. Then the dorsal aorta sends out branches ending in little loops of blood-vessels, and these meeting the other ends of the tubules, the typical Malpighian bodies and glomeruli of an adult kidney are established. The glomerulus differs from the pronephric glomus in that it comes in actual contact with its tubule, instead of being separated from it by the width of the colom.

The mesonephric tubules acquire a nephrostome each, by growing out towards the cœlom; but this is temporary only. The nephrostomes remain, but lose their connexion with the tubules and open into the renal veins instead-a very astonishing change.

The mesonephric tubules are formed in the seventh and hinder somites; but they soon lose their metamerism, increasing greatly in number and branching, and gradually becoming compacted into a single pair of kidneys.

As the mesonephric tubules take over the functions of the pronephric, the latter begin to degenerate, and lose their connexion with the archinephric duct, which now undergoes a remarkable change, about the exact nature of which there is some dispute. It is usually stated * to become spiit longitudinally, or obliquely, into two tubes-a Muillerian duct, which includes all the anterior part, and a Wolffian or mesonephric duct, which includes all the posterior part. The Muillerian duct is said to grow back alongside the Wolffian duct and acquire an opening into the cloaca; while anteriorly it acquires an opening into the coelom, close to the position of the pronephric tubules. The Müllerian duct becomes the oviduct in the adult female,

* There is considerable doubt about this. 
and remains rudimentary in the male. The Wolffian duct remains as the duct of the kidney (mesonephros), i.e. ureter in the adult female, urogenital duct in the male.

The gonads first appear as a pair of ridges (showing no metamerism) just nearer the middle line than the mesonephric tubules. Like them, they are developed out of the mesoblastic somites, and from the first there is a close connexion between these two structures. The tubules send out branches which enter the gonad: these become the vasa efferentia in the adult male; and in the female they lose connexion with the tubules and form the cavities in the ovary itself.

§ 18. The Metamorphosis. We have now described the chief organs that appear in the tadpole in preparation for the adult stage. But when a larva differs so greatly from the adult as the water-breathing, plant-eating, tailed, swimming tadpole does from the air-breathing, insect-eating, tailless leaping frog, there must needs be a great change in the structure of many organs that are needed in both stages. The period during which these organs are changing must be one in which they are not well-adapted to either the old or the new use; it is therefore a period of helplessness, and evidently the more quickly it is got through the better. Accordingly we find in all cases where the differences between larva and adult are very marked, there is some arrangement for a comparatively abrupt change from the one to the other. Such a change is called a metamorphosis, and it may be much more abrupt than in the case of the frog.

The tadpole does use its hind legs and its lungs to some extent before the metamorphosis. After a variable time of tadpole life (usually about three months) the metamorphosis begins. The tadpole ceases feeding, but it is supplied with nourishment by the absorption of the tail. Phagocytes (cells resembling the ordinary colourless blood-corpuscles) attack the tissues of the tail just as osteoclasts absorb cartilage and bone, and the digested material is carried away by the blood. Thus the tail gradually shrinks in size. Meanwhile the outer layer of skin is shed, and the horny 
jaws and suckers go with it; the mouth at the same time becomes wider; the gill-slits close and the gill-filaments are also absorbed. The fore limbs come out, the left one through the opercular opening, the right one bursting through the operculum. Many other changes take place at the same time, and when the animal recovers its appetite it prefers insects to plants. It takes to the land and gradually becomes a frog.

\$ 19. Changes in the Vascular System. We have seen that the tadpole's circulation is just that of a fish, though the branchial vessels have a much simpler arrangement than in the dogfish. When the gill-slits close, the blood begins to flow more and more through the direct communication between afferent and efferent arteries, until these become simply one arch. There are thus four pairs of arches; of these the first becomes the carotid arch, its connexion with the aorta of the same side persisting in a closed condition as the duct of Botallus (chap. xiii., § 5). The second becomes the systemic arch. The third disappears altogether. The fourth, which already had pulmonary and cutaneous branches, loses its connexion with the dorsal aorta and becomes the pulmo-cutaneous arch. About the same time the originally single auricle becomes divided into two by a septum so disposed that only the pulmonary veins enter the left auricle.

The heart has been gradually shifting back as the larynx and lungs developed, and so the wall of the pericardium projects back into the rest of the coclom and assumes its bag-like character (fig. 91). The transverse Cuvierian veins are thus made to slope back to the heart, and are now called the precaval veins. The posterior cardinals disappear (though there aro recorded cases of frogs in which they persisted). The anterior cardinals form the innominate and internal jugular veins. 
Chapter XIX.-The Development of the Chick.

$\S 1$. The Fowl's Egg. Although we have not taken a bird as one of our types in comparative anatomy, we do so in embryology for two reasons-first, because circumstances make. it the easiest of all the higher Vertebrate types to study minutely; and secondly, because without the study of the development of either a reptile or a bird, that of the rabbit would be too puzzling to be profitable.

The fowl's ovum is about as many times larger than the frog's, as the frog's is larger than that of Amphioxus. 'This enormous size is due to an excessive gorging with yolk. Thanks to this abundant food-supply, the young bird has no need to go through the dangers of a free larval stage, but can remain within the egg until it differs but little from its parent. At the same time the presence of so much yolk necessitates a much greater deviation from the simple course of early development than was the case in the frog.

The real ovum is what we commonly call the "yolk" or "yellow" of the egg. This is, at first, a single cell, with its nucleus (germinal vesicle) close to the animal pole. Around the nucleus is a small disc-shaped area of protoplasm, free from yolk-the germinal area. All the rest of the ovum consists of a meshwork of protoplasm filled up with yolk spheres. 'The whole is surrounded by the vitelline membrane, for (as with the frog) the ovum escapes from its follicle when ripe. The difficulties of observation have, as yet, prevented any one from actually seeing the extrusion of polar bodies, but there are indications that this process does take place. Fertilization occurs about the time that the egg enters the oviduct, up which the spermatozoa must make their way from the cloaca, where they are placed by the male bird. As the ovum travels down the oviduct the walls of this tube secrete coverings for it - first the albumen or "white," and, lower down, the 
shell-membrane and shell. The structure of an ordinary hen's egg is shown in fig. 125. The ovum (" yolk") is held in place by twisted strands of firmer albumen-the chalazoe. The shell-membrane divides at the broad end into two layers, between which is an air-space. Owing to its lighter character, the germinal area is always on the top of the yolk, however much the egg may be turned round-the ovum ("yolk") swinging on the chalaze.

§ 2. Meroblastic Segmentation. The first segmentationnucleus, of course, lies in the germinal area, as did the germinal vesicle to start with. Segmentation begins as usual by division of the nucleus into two and four, and meridional

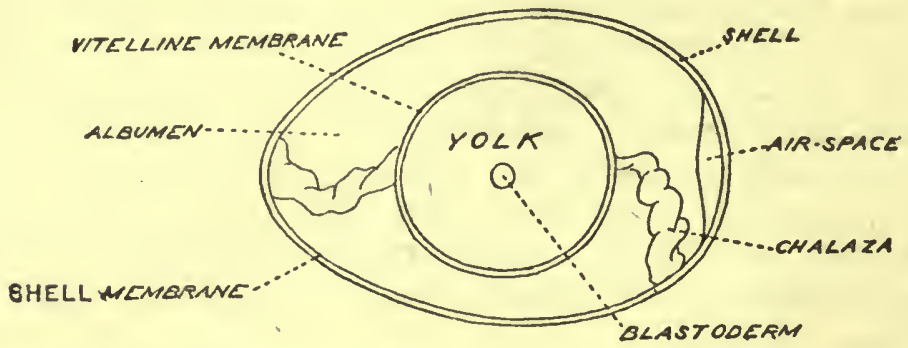

Fig. 125.-Diagras of Fowl's Ego at Tise of lariso.

planes of division appear in the protoplasm of the germinal area. But the whole ovum is not divided into two, four, etc., cells; the meridional planes die off as they pass into the main yolk-laden mass. For some time only meridional divisional planes are formed, but presently (after a corresponding division of the nuclei) a horizontal divisional plane appear's in the protoplasm of the germinal area, and cuts off an upper layer of complete cells from the main mass of the ovum, which contains, immediately under this upper layer, a "lower layer" of nuclei, surrounded by imperfectly defined cells which merge downwards into the undivided yolkladen mass below. Between the two layers a narrow space appears - the segmentation-cavity. Obviously we have here a modified blastosphere stage: the upper layer answers to the frog's primitive epiblast, and the rest of the ovum 
(by far the greater part of it) to the frog's yolk-cells. But this latter part is not divided into cells, because the enormous abundance of yolk prevents the protoplasm from dividing completely. This is expressed by saying that the segmentation of the fowl's egg is meroblastic (in contrast to the holoblastic segmentation of Amphioxus and Rana).

§ 3. The Blastoderm. Presently, however, the protoplasm around the lower layer of nuclei becomes completely marked off into cells, leaving below it the main mass of the ovum, without any cell-structure, though probably with some nuclei still present in it. This undivided mass may from this time be conveniently called the yolk. The actual cells form a small circular cap on the upper surface of the yolk : this cap is called the blastoderm.

The changes so far described take place while the ovum is in the oviduct, and though the exact stage reached by the time of laying varies, the blastoderm is always well established. It may often * bo seen as a circular patch ( $\frac{1}{8}$ inch diameter) on the surface of the yolk when an ordinary egg is broken into a dish.

The fall of temperature that occurs when the egg is laid and exposed to the air stops further development until incubation begins. This is normally effected by the warmth of the hen's body as it "sits," but artificial means are quite as successful.

$\S$ 4. Epiblast and Primitive Hypoblast. As seen from the surface, the blastoderm very soon shows division into two areas-a central area pellucida and an outer area opaca (fig. 127), whose names express their difference of appearance. In a section (fig. 126) we find the explanation of the differenco to lie in the thickness of the lower layer of cells, which is several cells thick near the margin, but only one cell thick in the area pellucida. In the latter region it is also more distinctly separated from the yolk, by the formation of a sub-germinal cavity (which must not, be confused with the segmentation cavity between the upper and lower

* It must be borne in mind that under domesticated conditions hens mas lay unfertilized eggs. 
layors). Throughout almost the whole blastoderm the upper and lower layers are quite distinct in character, but at one point in the margin there is a gradual passage between them: this point marks the future posterior end of the embryo (right-hand of fig. 126). To explain its position in relation to the whole egg we may mention that the future dorsal region of the chick is uppermost, and the broad end of the shell to its left side.

A comparison of this stage with the blastosphere and gastrula stages of the frog shows us resemblances modified by the excess of yolk. The upper layer cells obviously correspond to the primitive epiblast cells of the frog, and may most conveniently be called epiblast. The lower layer obviously answers to primitive hypoblast. Between the two

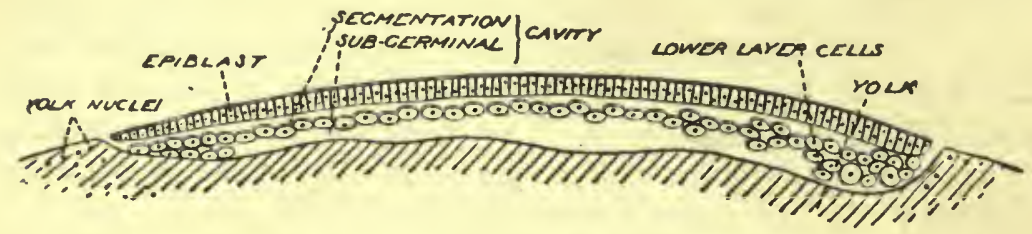

Fig. 126.-Median Vertical Section of Blastoders.

(After Marshall.)

comes the segmentation cavity, while the sub-germinal cavity answers to the mesenteron. The difference between this stage and that of the frog shown in fig. 118, c, D, is that the mesenteron has a non-cellular mass of yolk for its floor instead of a mass of yolk-laden cells. As this yolk is destined to be absorbed we may fairly say that the mesenteron has no floor, though it has a distinct roof (the layer of primitive hypoblast). In fact, we may compare this stage with the gastrula of Amphioxus (fig. 112, B), if we imagine that (by reason of the enormous yolk) the two layers of cells, instead of being hemispherical, are spread out almost flat, forming a small part of the surface of an enormously larger sphere.

$\S 5$. Growth of the Blastoderm. The most striking feature during the early stages of incubation is the extension. 
of the blastoderm over the surface of the yolk. This is effected by growth and division of the cells, which probably now begin to absorb and assimilate some of the yolk. In a day's time it has grown to throe-quarters of an inch in diameter; in two days it covers half the surface of the yolk; and erentually (though much less rapidly) it extends over the whole of the yolk. Mernwhile important changes are taking place in it.

We have already mentioned the division of the blastoderm (as seen in surface view) into area pellucida and area opaca. It is the latter which more especially increases in surface extent and surrounds the yolk; but it is from the former only (and not from the whole of that) that the embryo is developed. This statement may seem strange at first, until the reader grasps the notion that the whole ovum does not become the embryo in the case of the chick, but that certain important structures (consisting of living tissues continuous with those of the embryo) are formed outside the embryo proper. These may be called extra-embryonic structures, and are a feature not shown in the case of Amphioxus and frog.

The explanation of the existence of extra-embryonic structures again lies in the enormous quantity of yolk. All the frog's yolk can be kept in the cells of the mesenteronfloor, and this enclosed by the body-wall. But with five thousand times the bulk of yolk, enclosure within the body of the embryo would involve impossible distortions of shape ; hence most of the chick's yolk is outside its body in the obvious anatomical sense. Morphologically, however, its position is strictly the same as in the frog-embryo-the extra-embryonic structures which enclose it being really portions of the mesenteron-wall and of the body-wall extended beyond the limits of the future chick to avoid the otherwise necessary distortions of form. These points will become clearer as we proceed.

§ 6. Primitive Streak. As the blastoderm grows, the area pellucida, at first circular, becomes pear-shaped, its broad end marking the future anterior end. At the posterior end, as already mentioned, there is a point where epiblast 
and primitive hypoblast are connected by cells of intermediate character. This point becomes the seat of very active cell-division, and as the blastoderm extends the point becomes drawn out into a line,

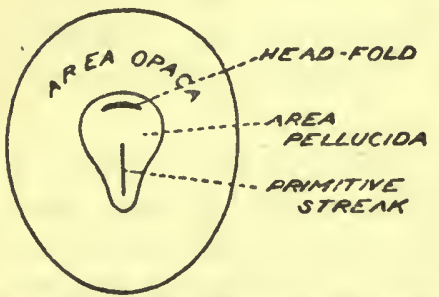

Fig. 127.-Surface View of BlastoDERa, FI DST DAY of Incubation. which shows in surface view as a streak - the primitive streak -owing to the greater thickness of the blastoderm along it (figs. 127 and 128).

Comparison with the frog shows a striking series of resemblances between thisprimitive streak and the blastopore. Both are median and posterior; both mark the point where epiblast and primitive hypoblast are connected by cells of intermediate character; both are regions of active growth; and (at a later stage) both are enclosed by the neural folds, while traces of a neurenteric canal are found in the primitive streak of the chick. We may therefore regard the primitive streak as representing the coalesced lips of the blastopore, whose actual cavity is obliterated by the compression of the blastoderm by the huge mass of yolk.

§ 7. Hypoblast and Mesoblast. These two layers are formed by cell-division and differentiation in the primitive hypoblast. A portion of the mesoblast also seems to be directly formed from the primitive streak (fig. 128), but this is in the extraembryonic region only. A great deal is formed indirectly from the primitive

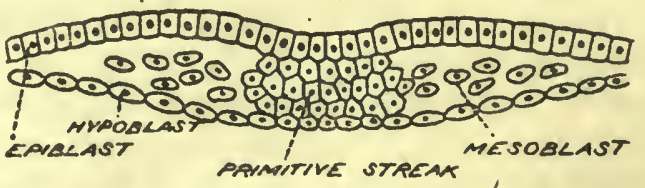

Fig. 128.-Transverse Section turovgu Primitive Streak. streak, since this latter gives rise to additional primitive hypoblast, as well as additional epiblast.

§ 8. Neural Folds, Notochord, Cœlom and Somites. In front of the primitive streak the epiblast of the middle 
line becomes differentiated into a neural plate. Along the sides of this the neural folds arise exactly as in the frog. They extend behind alongside the anterior part of the primitive streak.

The notochord is formed just as in the frog.

The mesoblast of either side, soon after its formation splits (as in the frog) to form the right and left portions of the cœlom: the union of these right and left portions is necessarily delayed for a long time by the presence of the huge mass of yolk. As the area opaca gradually spreads over the yolk, the differentiation of the three germinal layers spreads with it, and the splitting of Fí. 129.-SURFACE VIEW or the mesoblast follows close behind.

The splitting is soon followed by the division into vertebral and lateral

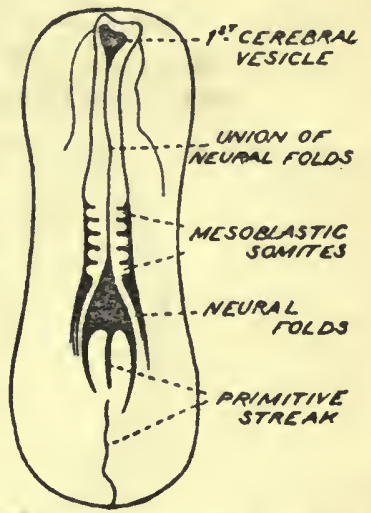
Blastoderm, Sf.cond Day OF INCUBATION.

(After Balfour.) plates, and the former soon begin to divide transversely into somites (protovertebræ), beginning in the region just

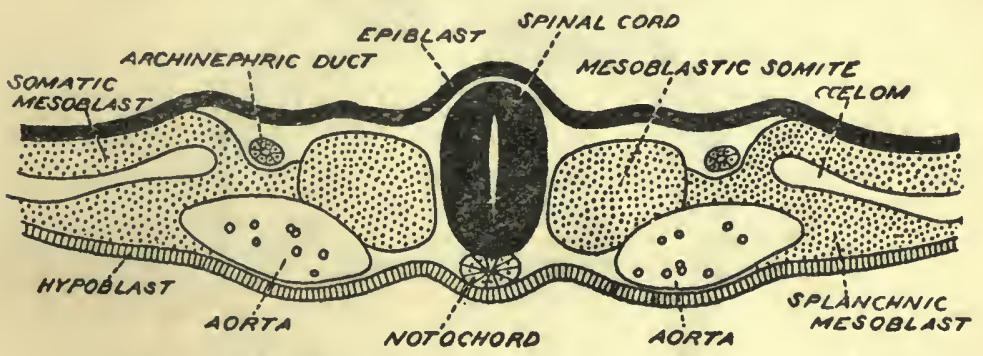

Fig 130.-Trangverse Section of Blastodram of Culck, SECond Day of Incubation.

(After Balfour.)

behind the head and gradually increasing by the cuttingoff of additional ones behind.

These changes, up to the formation of the first two or three pairs of somites, represent the progress of the first twenty-four hours of incubation. By this time, as already 
stated, the blastoderm has increased to about three-quarters of an inch in diameter. A surface-view is shown in fig.

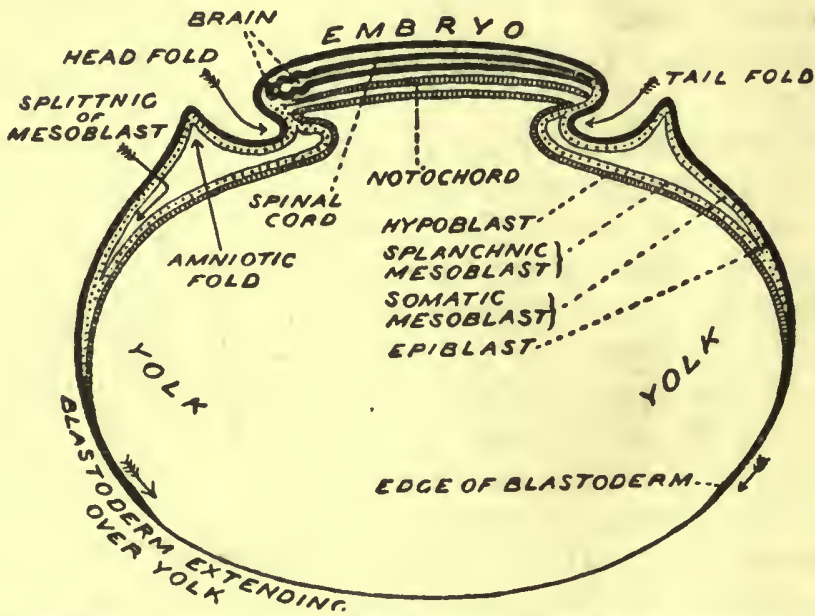

Fig. 131.-Diagramatic Median .Section of Embryo Chire

(First Stage.)

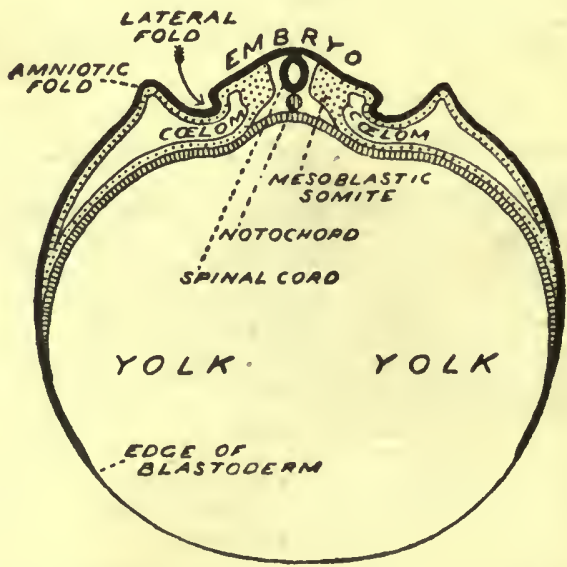

Fig. 132.-Diagrammatic Transverse Section of Embryo Cuick. (First Stage.)

129 , and a transverse section through one of the somites in fig. 130. Comparing this with fig. 120, the corresponding stage in the frog, we can see that if in the latter we slit 
the body wall and mesenteron open along the median ventral line, and spread them out flat, we should get

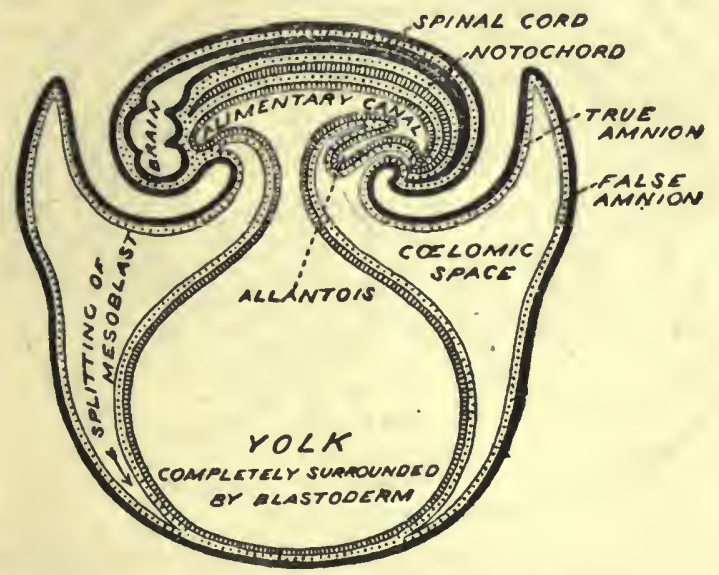

Fig. 133.-Diagramatic Median Section of Embryo Cilcz.

(Second Stage.)

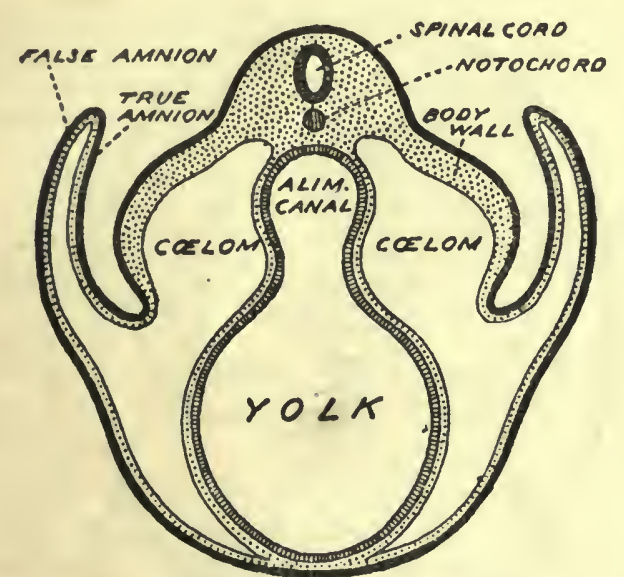

Fig. 134.-Diagrammatic Trangverse Section of Emeryo Chicr. (Second Stage.)

practically the condition in the chick. The difference is again obviously necessary in view of the difference in the amount of yolk. 


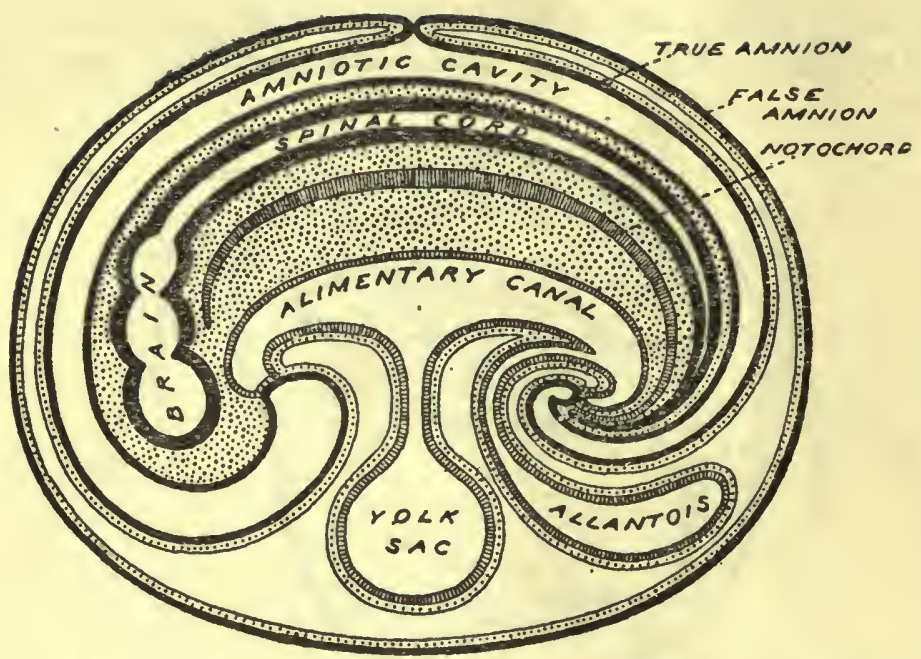

Fig. 135.-Diagrajuatio Median Section of Embryo Otick (Third Stage.)

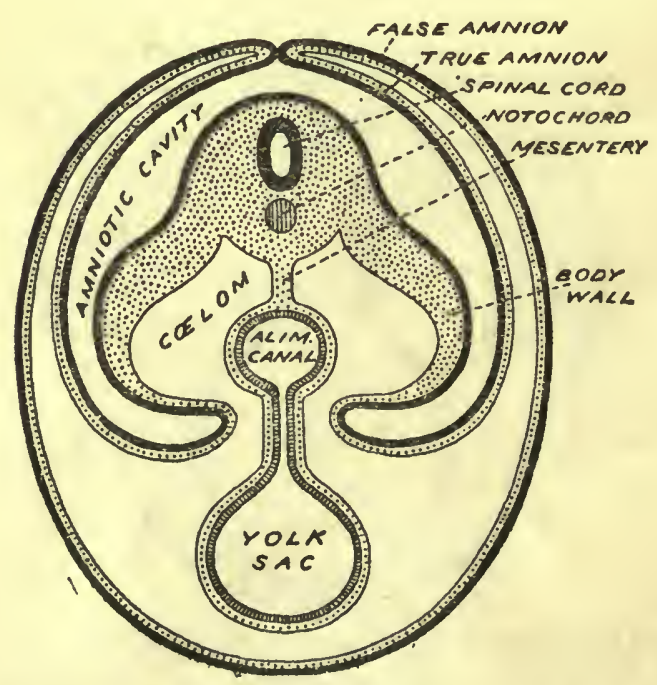

Fig. 136. -Diagramatatio Transverse Sectios ot Embryo Cinck. (Third Stage.) 
§ 9. Embryo and Yolk-sac. From about the ond of the first day of incubation we may trace the gradual progress of a process by which the embryo becomes more and more sharply delimited from the extra-embryonic structures, and at the same time assumes more and more its proper shape instead of the flattened-out condition, devoid of any ventral surface, with which it starts. This is essentially a process of pinching-o.ff of the embryo from the yolk. To get a first notion of it imagine a balloon with a rope passed round its middle : let the rope be drawn tighter and tighter, so as to gradually nip the balloon into two portions, separated by a narrow neck. The upper portion answers to the embryo, the lower to the yolk-sac, and the narrow neck to the stalk of the yolk-sac.

This first crude notion must be modified in several ways before a correct idea is obtained. In the first place, the embryo, when first its margin is pinched in, is vastly smaller than the yolk, and while the pinching-in is proceeding it is constantly growing larger; and as it gets most of its foodmaterial from the yolk (some coming from the albumen), in proportion as the embryo grows larger the yolk-sac gets smaller. This change in relative size must therefore be borne in mind when the pinching process is imaged mentally.

A much more important divergence from the simple process of pinching is the simultaneous formation of the amnion-a protective covering to the embryo. In figs. 131 to 136 an attempt has been made to show by diagrammatic transverse and longitudinal sections, the gradual change in the relation of embryo and extra-embryonic structures. These diagrams represent the embryo as far larger proportionately to the yolk than it really is at any of these stages. They show the gradual extension of the blastoderm (area opaca) around the yolk, and how the splitting of the mesoblast follows round, until the yolk becomes completely enclosed in a yolk-sac consisting of hypoblast and splanchnic mesoblast. They also show the gradual pinchingin by which the mesenteron becomes more and more completely separated from the yolk-sac, until a well-defined stalk alone unites them. They further show how the epiblast and somatic mesoblast are also tucked in ventrally to form a ZOOL. 
more and more complete body-wall. And they show the development of the amnion and of the allantois, features quite unknown in our two previous types, and which we must now describe.

\$ 10. The Amnion. The first sign of the tucking-in around the embryo appears towards the end of the first day as a curved wrinkle in the blastoderm in front of the embryo: this is called the head-fold (fig. 127). Parallel to it, farther forward, is another wrinkle, the beginning of the amniotic fold. Later on, wrinkles similar to the head-fold appear behind the embryo (tail-fold) and on either side of it (lateral folds), and each of these has a parallel amniotic fold beyond it. These wrinkles all extend and eventually unite, so that the embryo becomes surrounded by a pair of elliptical wrinkles. The inner of these may be compared to a moat, as it is essentially a depression of the blastoderm, the outer (amniotic fold) to a rampart, as it is an elevation. But whereas the depression concerns both body-wall (i.e. epiblast plus somatic mesoblast) and mesenteron (i.e. hypoblast plus splanchnic mesoblast), the elevation consists of the former only. (See fig. 134, and, for a more exact representation of the same stage, fig. 137). As the moat is dug deeper, it also begins to curve inwards so as to undermine the embryo, and separate it from the yolk-sac. As the rampart grows in height it curves inwards also, so as to form a dome covering over the back of the embryo.

The amniotic fold is from the beginning hollow, containing a space which is continuous with the general colomic space produced by the splitting of the mesoblast. Hence the dome which it begins to form over the back of the embryo is a double dome. The inner of the two layers, in which the epiblast is turned towards the embryo and the mesoblast towards the exterior, is called the true amnion ; the outer layer, in which this arrangement is reversed, is called the false amnion. When the dome is finally completed the true amnion becomes a covering for the embryo, enclosing an amniotic cavity (lined entirely by epiblast) which contains a secreted fluid and acts as a sort of watercushion to protect the embryo from shocks. The false 
amnion eventually becomes completely separated from the true amnion, by absorption of the connexion between them, and by the time that the splitting of the mesoblast has extended right round the ventral side of the yolk-sac, it forms a thin membrane completely separated from everything else, and coming to lie close under the egg-membrane when the albumen has all been absorbed. The portion of it which does not come from the amniotic fold but is split off the yolk-sac is called the serous membrane, but that and the false amnion form a single membrane in the end. It now forms a sort of extra egg-membrane, and may be disregarded after this stage.

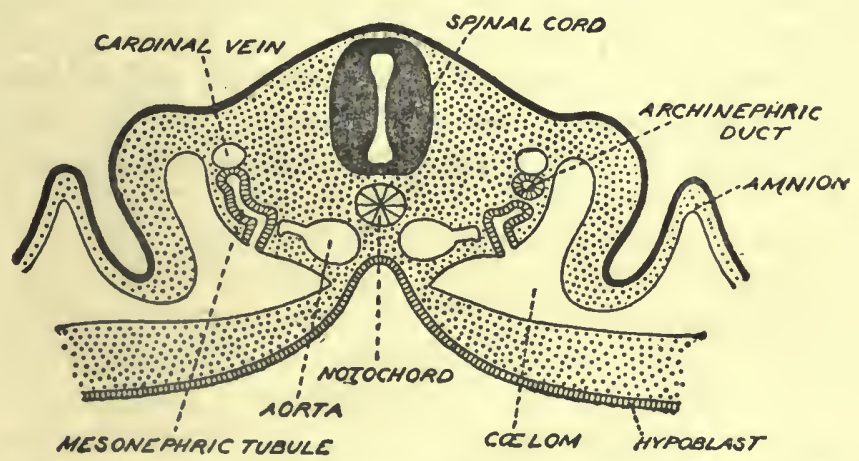

Fig. 137.-Transverse Section of Chick, End of Second Day of Incubation.

$\S 11$. The Allantois. The necessity for the nutrition of the growing embryo is met by a very early development of blood-vessels in the area opaca as it spreads over the yolk. The relations of these with the vascular system in the embryo.itself will be described later. But respiration soon becomes as great a necessity as nutrition. The tadpole's internal and external gills would be useless to the chick, which does not live in the water. Some other means of respiration is needed, and this is provided by an outgrowth from the posterior end (cloaca) of the mesenteron, called the allantois (figs. 133 and 135). This is nothing else than the frog's urinary bladder, enlarged and adapted to a new function. It consists, of course, of hypoblast and splanchnic mesoblast, the latter layer containing abundant 
blood-vessels. It grows out into the cœlom and thence into the space between the true and false amnion-a space continuous with the coelom-and then spreads out close under the false amnion or serous membrane. In this way only a series of thin membranes and the porous shell are interposed between the air and the blood, and aëration of the latter is readily effected.

We have now described the essential features of the development of the chick as a whole, and the extraembryonic structures attached to it. The detailed development of the several systems will be treated separately a little later. All we need now add is that, when the time for hatching draws near, the yolk-sac, now a tiny remnant, is withdrawn within the body-wall ; the latter closes over and nips across the stalk of the allantois, which dries up and is cast away, and the chick bursts through the true amnion just as it does through the outer membranes and shell. 
Chapter XX.-Development of the Rabbit.

§ 1 . The ovum of the rabbit is just about the same size as that of Amphioxus, and is practically alecithal. We might therefore not unreasonably expect to find segmentation, invagination, and formation of the germinal layers in the simplest way, and an embryo set free from the egg at about the gastrula stage. Yet instead of this we find that after the first stages the general development resembles in all essentials that of the chick, a yolk-sac (without any yolk), an amnion and an allantois being formed; while the embryo is retained, not merely within the egg, but within the oviduct, up to a stage of development quite equal to that of the chick when hatched. Evidently to make this long development from an alecithal egg possible, there must be some other source of food replacing the absent yolk. The yolkless condition of the rabbit's ovum is not primitive, as is that of Amphioxus; it is a secondary modification from a yolk-laden condition like that of the bird. In this fact we have a clue to the chief peculiarities in the rabbit's development.

§ 2. The Graafian Follicle. The ovarian ovum differs from that of our previous types in the much more complex character of the follicle. The gradual development of this follicle is indicated in fig. 138. The cells from which both ovum and follicle are developed are, to begin with, a specialized portion of the general coelomic (peritoneal) epithelium (germinal epithelium). Little groups of these cells sink into the underlying connective tissue, and soon we have an ovum surroundèd by a single layer of follicular cells. So far, all our Vertebrate types are practically alike. But now, in the rabbit, the follicular cells multiply, and presently a split appears in them, and grows into a large cavity, filled with liquid. The cells on the outer side of this cavity constitute the "granulosa," those around the 
ovum itself the "discus proligerus"; but these terms are not expressive, and scarcely necessary.

The vitelline membrane in the rabbit is thick and

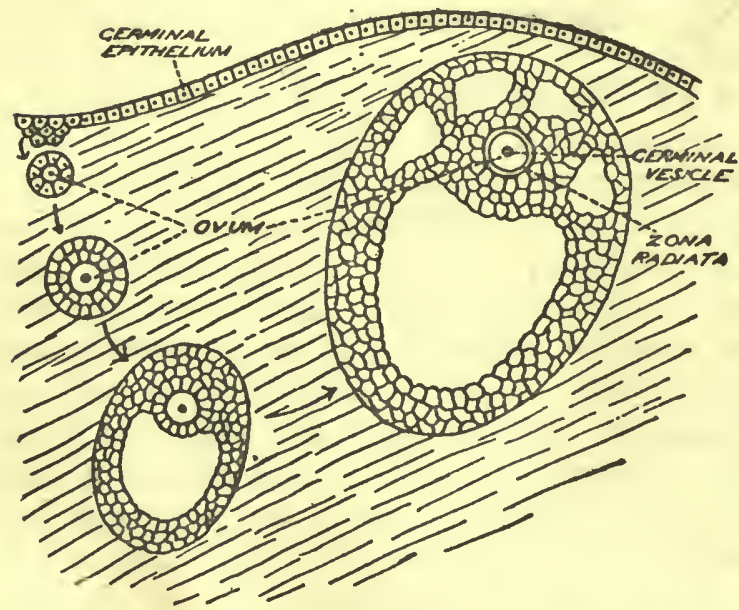

Fig. 138.-Diagram of Development of Graafian FOLIICLE OF RABBIT.

The arrows lead to successive stages. (Altered from Marshall.) marked by radiating lines (cf. fig. 110). It is therefore commonly referred to as the zona radiata.

\$ 3. Matura-
tion, Fertiliza-
tion, and Seg-
mentation. The ovum escapes from the follicle and enters the fallopian tube. It gives off two polar bodies in the usual way, and is fertilized high up in the fallopian tube. Its segmentation is holoblastic, and fairly equal, with a partial resemblance to that of Amphioxus, which later development shows to be apparent rather than real. The fertilized ovum divides into two, four, and eight cells; but at this point the segmentation cavity that was beginning to form becomes filled up by one of the cells passing into the centre. The three sister-cells of this one (i.e. the three formed from the same one of the two first-formed cells) also show a tendency to pass into the centre, and as segmentation proceeds the cells derived from them do so more and more, until at last we have a solid mass of cells (morula), consisting of two layers - an inner layer of cells, all derived from one of the two cells into which the ovum first divided, and an outer layer of rather smaller cells, all derived from the other of the two. The outer layer does not completely surround the inner-at one point the inner appears at the surface (fig. $139[1]$ ). 
$\S 4$. Explanation of the Morula. Now the explanation which naturally suggests itself is, that we have here a gastrula, and that invagination has been hurried forward, as it were, so that the blastosphere stage has been skipped. On this view (adopted by Professor Van Beneden, who first described the segmentation of the rabbit's ovum) the outer layer is epiblast, the inner primitive hypoblast, and the point where the latter is exposed is the blastopore. Unfortunately for this view, later development absolutely contradicts it. The outer layer takes no part in the formation of the embryo, but is purely extra-embryonic. The whole embryo (epi-, meso-, and hypoblast) is formed from the inner cells; and Van Beneden's blastopore, as it is still called; in no way corresponds with the blastopore of Amphioxus or frog.

Thus direct comparison with Amphioxus leads us astray; but comparison with the fowl is more hopeful. Imagine a series of eggs intermediate in character between that of the bird and that of the mammal. As the ova got successively smaller, we should find the blastoderm encircling the yolk more and more quickly; while as yolk was withdrawn from the centre, the central part of the blastoderm would sink into the hollow thus produced, By the time we reached an ovum with practically no yolk, we should find the area pellucida sunk into the centre of the ovum, and the area opaca closely surrounding it, just as the outer layer of cells does actually surround the inner layer in the rabbit's segmented ovum.

Whether this is precisely the true explanation or not is uncertain, because the difficulties of investigation have so far prevented the tracing of the fate of the outer layer fully enough. Possibly it may be an entirely new structure, not represented in the chick.

§5. The Blastodermic Vesicle. The next stage (fig. 139 [2]) shows the appearance of a space between the two layers, away from Van Beneden's blastopore. This space becomes filled with secreted fluid, and grows larger and larger, as the outer layer becomes thinner and more extensive, until at last we find the inner layer cells forming 
a little capping to a large hollow bladder (blastodermic vesicle). This capping represents the blastoderm proper, and from it alone is the embryo developed. The general

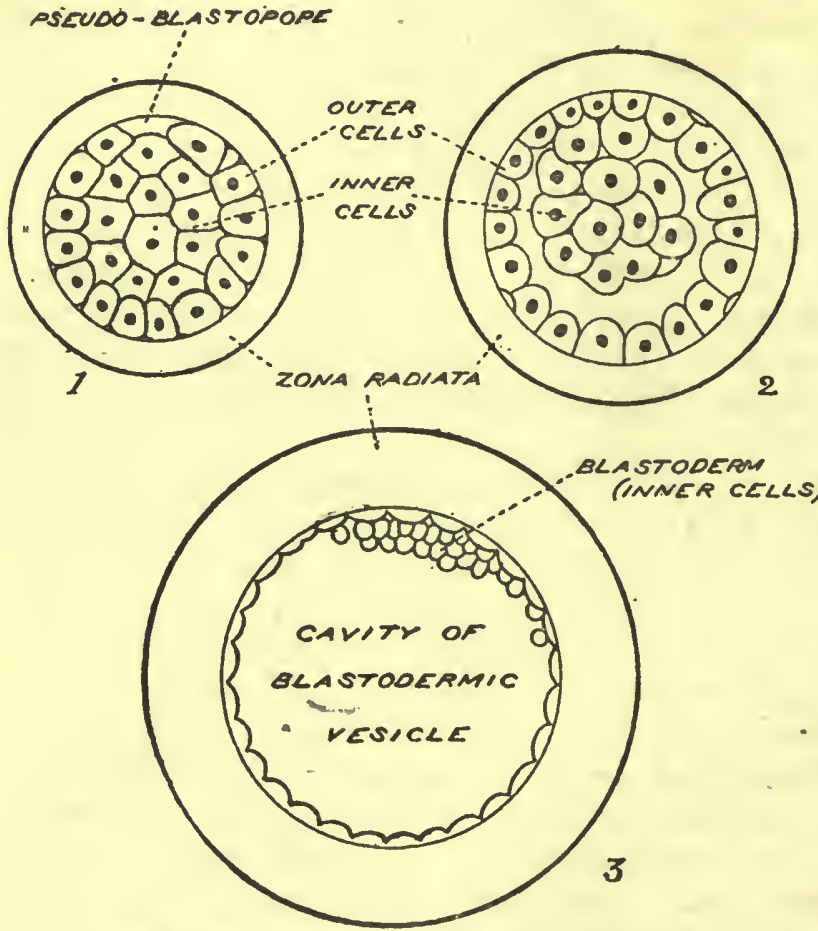

Fig. 139.-Segmentation of Rarbit's Ovum (From Balfour, after Van Beneden.) $\mathbf{r} \boldsymbol{\theta} \mathbf{s} \boldsymbol{\mathrm { m }}$ blance of this stage to the early stage of the fowl's ovum is striking, if weregard the secreted fluid as replacing the yolk.

From this point onward, the development follows that of the chick in all broad gen era l features. Primitive streak, neural folds, notochord,

mesoblastic somites, amnion, etc., all appear in practically the same way as in the chick. The only important differences concern the extra-embryonic structures.

§ 6. Yolk-Sac Placenta. The yolk-sac of the rabbit (often called the umbilical vesicle) never contains yolk, but at a very early stage of development, when the ovum has passed along the fallopian tube and entered the uterus, the yolk-sac grows out towards the wall of the uterus. It is of course separated from the actual wall of the uterus, first by the false amnion or serous membrane (cf. fig. 135), and 
secondly by the zona radiata, in which all structures derived from the ovum are enclosed. The wall of the yolk-sac, however, becomes fused with the portions of these membranes with which it comes into contact, and for the membrane formed by this fusion a distinct name is desirable: it is called the false chorion (fig. 140). 'The false chorion comes into contact with the wall of the uterus and unites closely with it, forming an attachment of the embryo to the uterus, which may be called the temporaryor yolk-sac placenta. By means of this connexion it seems probable that nutritive materials are conveyed

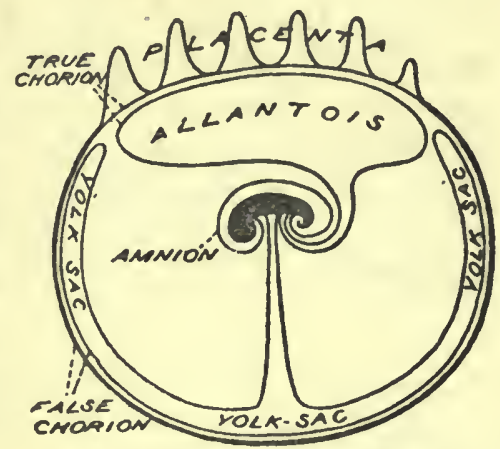

Fig. 140.-ExTra-EMBRYonio STructures of RabBit.

(After Balfour.) for a time from the blood-vessels of the mother into those of the yolk-sac, and so to the embryo.

$\S 7$. Allantoic Placenta. But when the allantois grows out, it also fuses with the remainder of the outer membranes, forming the true chorion. This comes in contact with the wall of the uterus and forms a far more important and permanent.connexion-the allantoic placenta (or placenta simply). The true chorion is a circular disc, hence the rabbit is said to have a discoidal placenta (chap. xii., § 9). From the true chorion numerous projections called villi (resembling the villi of the intestine in shape) grow out and become closely interlocked with similar processes of the wall of the uterus. It is to the compound structure thus formed (partly maternal) that the name placenta is given. Thus a very close connexion is established, and by diffusion the blood-plasma in the vessels of the uterus passes into those of the allantois. Here, then, we finally have the clue to the absence of yolk. A better method of nutrition has taken its place--a method that has the advantage of protecting the embryo up to the time of birth.

So close is the connexion of chorion and uterus that 
when the young rabbits are born part of the uterine wall is actually torn away with them. This is expressed by saying that the rabbit's placenta is deciduate. Before birth the body-wall is, of course, incomplete ventrally, as in the chick, at the point where it is continuous with the true amnion and where the stalks of the yolk-sac and allantois pass out. The scar of closure at this point persists, even in the adult, as the umbilicus or navel.

The stalk of the allantois persists as the urinary bladder. When born, the young rabbits are still enclosed in the true amnion. 


\section{Chapter XX1.-Development of the Sys'rems of Organs.*}

$\S 1$. The Alimentary Canal-Stomodæum. In all Vertebrata the beginning and end of the alimentary canal are lined by epiblast instead of hypoblast, and originate independently of and later than the main, hypoblast-lined portion, or mesenteron. The most interesting structures derived from the stomodæum are the pituitary and the teeth.

Of the former we need only say that it arises as a mass of epiblast cells in the dorsal region of the stomodæum, and at once becomes connected with the floor of the primitive fore-brain. As mesoblastic tissues increase in amount in this neighbourhood they cut off its connexion with the stomodxum. Too little is understood of the meaning of this peculiar development to make it worth while to say more.

The first sign of the development of teeth is a series of downgrowths of the epiblast into the mesoblast beneath, called enamel-organs (fig. 141). Each of these becomes greatly enlarged and nearly separated from the epiblast. A very vascular mass of mesoblast

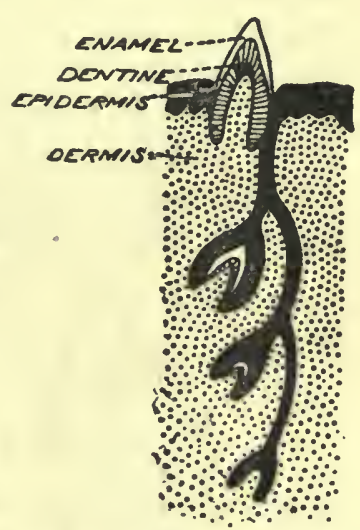

Fig. 141.-Developarent or TEETH.

(After Gegenbaur.) This represents the case of a Vertebrate with a constant succession of teeth (e.g. dogfish), three enamelorgans with rudimentary teeth being seen, besides the functional tooth. then indents it, and gives rise to the dentine; the enamel being secreted from the enamel-organ. In the case of those teeth of the rabbit which are repre-

* The development of all the essential parts of the great systems is so similar in frog, chick, and rabbit, that it is convenient to describe it in general terms, mentioning the special types only where important differences occur between them. 
sented in both milk- and permanent sets, a second enamelorgan is budded off from the first to form the permanent tooth.

§ 2. Respiratory Organs. The mesenteron is not as sharply divided into respiratory (pharyngeal) and digestive (intestinal) regions in chick and rabbit as in the tadpole or Amphioxus; but the two regions can be recognized. From the former arise the gill-slits, with arches between them, much as in the tadpole, though no gills are ever developed from them. So also do the thyroid, the thymus, and the lungs. The origin of the two former has been mentioned under Amphioxus (chap. xvi., \$ 15), and need not be further described. The lungs arise in the chick during the third day of incubation (and at a corresponding stage in the rabbit) as a median ventral diverticulum of the mesenteron, just behind the gill-slits. Its distal portion almost immediately becomes paired, giving rise by growth and repeated branching to the bronchi, bronchioles and alveoli;

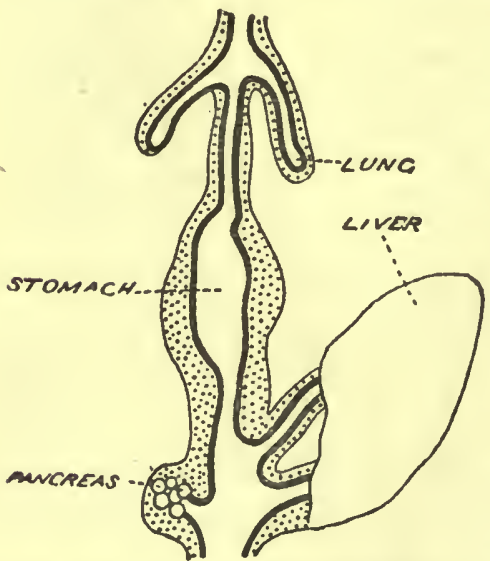

Fig. 142,-PART of MEsenteron of Embryo Chick.

while the proximal median part elongates into trachea and larynx. Thus the whole of these structures are lined by hypoblast. The cartilages, etc., in the walls of the trachea and larynx are, of course, formed from splanchnic mesoblast (fig. 142).

\section{§ 3. The Liver and Pancreas} are formed at about the same period as the lungs, by outgrowths of the digestive portion of the mesenteron (fig. 142). The liver arises as a (From Balfour, after Gottë.) Hypoblast pair of ventral outgrowths
black, sylanchnic mesoblast dotted. which later (in the rabbit) become united into one (in the frog there is only one at the first). These grow out and branch in a most complicated manner, the branching of what will be the actual liver 
being difficult to follow because, from the first, the hypoblast cells are large, and the cavities which they line, very minute. The gall-bladder arises rather late as an outgrowth of one of the main branches. The pancreas arises as a dorsal outgrowth, close behind the point of origin of the liver, and branches in a manner that leads to the diffuse condition in the rabbit, but to a compact gland in frog and chick. In the frog, at a later stage, unequal growth of the parts causes the pancreatic opening to shift its position and open into the bile-duct, as it does in the adult frog. In the rabbit, growth of the duodenum between the two points causes the considerable separation between the two apertures seen in the adult.

\$ 4. Cloaca. In all three types the hind part of the mesenteron, together with the proctodæum (which is far less extensive than the stomodæum) forms a cloaca. This persists in frog and chick: how it disappears in the rabbit we shall see in connexion with the urogenital system ( $\$ 13$ below). The urinary bladder of the frog, which becomes the allantois in the chick and rabbit, arises from this cloaca ventrally; the allantois is entirely lost in the adult fowl, but the base of it forms the urinary bladder in the rabbit.

§ 5. The Early Circulation. Fig. 143, A, shows the primitive condition of the main blood-vessels that obtains (with slight modifications) in all three types. It corresponds in a general way with the arrangement in Amphioxus, with the addition of anterior and posterior cardinal and Cuvierian veins and of arteries to the corresponding regions. One striking difference seen in chick and rabbit, though not in the frog, is due to the presence of a yolk-sac and the imperfect closing-in of the ventral side of the mesenteron and body-wall. This renders a median sub-intestinal vein at first impossible, and instead we have a pair of such veins as near on either side the middle line as possible, and which unite into one as soon as the pinching of the embryo off the yolk enables them to do so. In the rabbit, even the heart has to begin as a paired structure (the forward continuation of this pair of vessels), but these unite in the 
middle line as soon as they can. The adult division into right and left auricles and ventricles is of much later origin, and has nothing to do with this temporary arrangemont, which is simply a mechanical result of the slitting open of the floor of the pharynx, due to the spreading out of the embryo over the surface of the blastodermic vesicle.

The most important vessels at first in the chick are those of the yolk-sac, since they are essential to the nourishment of the embryo. They begin to appear in the area opaca during the first day of incubation. Mesoblast cells elongate and fuse with one another into a kind of network: vacuoles (i.e. cavities containing liquid) are formed in their protoplasm, and increase until they form continuous channels, with the remainder of the protoplasm for their walls. These are the earliest capillaries. The nuclei of the cells that form them divide, and small cells are budded off into the cavities: these are the first blood-corpuscles. The heart and the larger vessels are formed in a different way, from bundles of cells-the cavities being formed between the cells, not in their protoplasm. All the early vessels are formed in splanchnic mesoblast: the cardinal veins are the first important ones in somatic mesoblast.

Taking the chick as the most convenient type, the main course of the circulation at first (during the second day of incubation) is as follows. From the yolk-sac, by the two vitelline veins (whose proximal part represents the split sub-intestinal vein) to the heart; thence by the aortic arches (lying in the branchial arches) to the dorsal aortæ and back to the median aorta; thence by vitelline arteries to the yolk-sac again.

§ 6. Later Embryonic Circulation. On the third day of incubation we have, in addition to the above, the anterior and posterior cardinals (fig. 143, A); and on the fourth, with the great development of the mesonephros and the allantois, we find the median postcaval and the allantoic veins appearing as important factors of the original subintestinal (fig. 143, B).

While these changes are going on, changes have also been taking place in the aortic arches. In the chick, six pairs of complete arches are formed in all-in the mandibular, hyoid, and four branchial arches. (In the frog, it will be remembered, the first two of these are incomplete, 
and disappear soon.) But these six pairs are not all in existence at any one time. The first appears early on the second day, the sixth not until the fourth day, by which time the first has vanished and the second is about to follow. The fifth is so short-lived that for long it was overlooked, and the sixth is called the fifth in older textbooks.

With the great growth of lungs and liver after the fourth day, other important changes occur. 'Thesixth aortic A arch (fourth branchial), as in the frog, sends* arteries to the lungs; and veins from the lungs run $B$ direct to the heart. Tho sub-intestinal vein sends* two series of branches into the mesoblast of the liverafferent and ef ferent-so that a portal circulation is established.
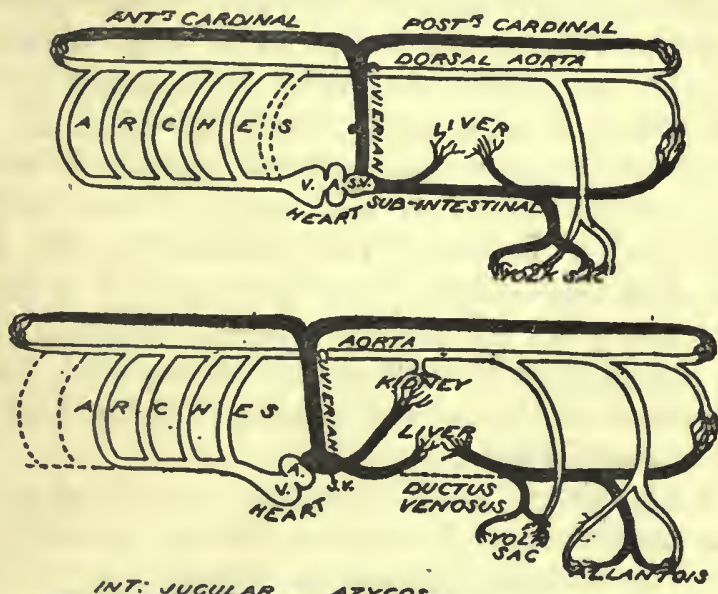

As this develops, the original main trunk of the sub intestinal vein between these vessels fails to keep pace with the growth of the rest of the vein; and thus the original sub-intestinal vein becomes separable into the

* This convenient mode of expression is figurative. What actually happens is that, as an orgar grows, parts of its mesoblast are differentiated into blood-vessels, and these become continuous with pre-existing vessels. 
following parts, in order from behind forwards :-(1) a portal vein receiving the vitelline and allantoic veins, as " ell as those from all parts of the rapidly growing digestive region of the mesenteron; (2) a ductus venosus, the original main line, largely abandoned in favour of the portal circulation through the liver; (3) a hepatic vein, the portion between the efferent vessels of the liver and the post-caval; and (4) the part nearest the heart, which is now spoken of as part of the postcaval.

Eventually the ductus venosus is obliterated.

Later stages of development begin to show the special features of the adult bird's circulation. As these do not concern us, we shall instead indicate the relations between the circulation at this stage (found also in the embryo rabbit) with that of the adult rabbit (fig. 143, c).

The third pair of aortic arches (first branchial), as in all types, give off the carotids to the head: they lose their connexion with the dorsal aorta, and, as the neck grows, become drawn out straight, forming the posterior part of the carotid arteries.

The fourth arch disappears on the right side and persists as the aortic arch of the adult on the left.

The fifth disappears very early. The sixth also disappears on the right, and persists on the left as the pulmonary arch: its original connexion with the dorsal aorta forms the ductus arteriosus, which persists till birth (chap. iii., $\S 10)$.

The posterior cardinal vein disappears on the left side, persisting on the right as the azygos (chap. iii., § 8).

The postcaval extends back beyond the kidneys so as to intercept the blood from the hind limbs, etc., which originally flowed into the cardinals.

The development of the heart is indicated in fig. 143, and hardly needs a verbal explanation.

$\S 7$. The Skeleton. The cells of the notochord become vacuolated and take on their characteristic appearance in the chick during the fourth day. About the same time the mesoblastic somites give rise to a tube of cells completely surrounding the notochord. (This tube is formed in the 
tadpole at about the time of appearance of the hind legs.) Gradually a matrix is secreted by these cells, and the tube takes on more and more the appearance of cartilage. An extension dorsalwards from the tube now takes place; on either side of the spinal cord a paired metameric series of cartilages is formed, alternating with the mesoblastic somites. The undivided tube around the notochord will give rise to the centra of the vertebræ; the metameric cartilages, to the neural arches. The tube around the notochord presently becomes thicker, at intervals which correspond to the somites in frog and chick, but alternate with them in the rabbit. In this way the notochord becomes constricted at metameric intervals, and eventually cut up into separate masses. Next, the cartilaginous tube becomes metamerically divided by transverse rifts into a series of cartilaginous centra which (like the neural arches) alternate with the somites. Consequently the isolated remnants of the notochord persist for a time within the centra of frog and chick, but between the centra of the rabbit (as in the dogfish). In the frog the hinder portion of the tube is never metamerically segmented, but forms the urostyle.

Later still, ossification occurs (chap. vi., § 11), and the cartilaginous vertebræ are replaced by vertebræ of cartilage-bone-epiphyses being formed only in the case of the rabbit. In this process, too, the final remnants of the notochord are absorbed, except that in the rabbit a little persists in the middle of the fibro-cartilaginous intervertebral discs which are unossified parts of the original tube.

The development of the skull has been fully treated of in chapter xiv., and of the rest of the skeleton little need be said. It is worth noting, however, that the cartilages of the branchial arches arise (like those of the larynx and trachea), in splanchnic mesoblast; whereas the limb-girdles and limb-skeletons are formed in somatic mesoblast. This fact effectually disposes of a theory, ably advocated at one time, that the limb-girdles were homologous with gill-arches.

§ 8. The Nervous System. The general facts of the development of the brain and spinal cord from the primitive condition of a cellular tube have been indicated in chapter ix. 
Here we need only mention the development of the dorsal and ventral fissures of the spinal cord. The dorsal fissure appears to represent, as in Amphioxus, the dorsal portion of the original central canal that becomes obliterated partly by mere opposition of its side-walls, and partly by the growth across of decussating nerve-fibres. Fig. 137 shows how large the central canal is at first, and how the sides of its dorsal portion tend to come in contact. The way the dorsal fissure seems to open out into the fourth ventricle is also in accordance with this view of its origin. The ventral fissure, on the other hand, is the space left between the right and left masses of white matter that are formed ventral to the original neural tube.

§ 9. Ganglia. The origin of the ganglia is very interesting. About the time the neural folds are closing in, in the chick, a ridge of epiblast-cells appears alongside the dorsal end of each fold. When the folds close these ridges unite as the neural crest (fig. 120 : it should have been shown also in figs. 130 and 137, in a similar position). It is from the neural crest that all the nerve-cells in the gangliaspinal and sympathetic-are formed. The neural crest soon leaves its position on the dorsal side of the neural tube, and moves to the side, being at the same time metamerically segmented-each segment corresponding to a mesoblastic somite. Next, each of these masses of nerve-cells divides into two-one remaining where it was, to become a spinal ganglion; the other moving to a more ventral and more median position, where it becomes a sympathetic ganglion. The cells of the former ganglion become bipolar, and send out one axis-cylinder-process into the spinal cord, the other in the opposite direction-thus the dorsal root is established. The two axis-cylinders at first arise from opposite ends of the bipolar cell, but eventually they join together and the characteristic unipolar cell is produced. The motor roots of the spinal nerves grow out from the spinal cord at a later stage.

In the brain-region, the origin of the nerves is less clear, owing to the obscured metamerism of this region. It seems certain, however, that all the ganglia in the head are also derived from the neural crest, and from these ganglia are derived all the sensory fibres of mixed nerves and sensory nerves, except the optic-the olfactory being doubtful, and the auditory ganglion being incorporated in the brain. The relation of the cranial nerves to the obscured metamerism of the head, and to the nerves of Amphioxus where the metamerism is not obscured, are subjects of very great interest, but beyond the scope of this work.

It should be noted that the neurilemma of all nerve-fibres is derived from mesoblast. 
$\S 10$. Sense-Organs. All, except the eye, and those connected with the muscular and other internal senses, are directly derived from the surface-epiblast. The most important are the olfactory and auditory organs. Each of these starts as a simple pair of sacs of epiblast. The olfactory sacs remain sacs in the dogfish, though their walls undergo repeated folding. But in other types they gain an internal opening into the mouth-cavity, and are given a second function in connexion with respiration. The internal ear is also a simple sac, and undergoes complications in shape which lead to the formation of the semicircular canals, etc. The endolymph duct persists as the last trace of the original connexion with the epiblast. It is a curious point that, in the frog, where the epiblast is so early divided into epidermal and nervous layers, only the latter sinks in to form the auditory sac, so that this is never actually open at the surface, as in all other types.

The first visceral cleft is never actually perforate, but it forms the middle ear (tympano-Eustachian recess); and part of the hyoid arch (in frog and fowl) or of both hyoid and mandibular arches (in rabbit) are pressed into the service of the ear as auditory ossicles. (See chap. xiv., § 7.)

$\S 11$. The Eye. The development of the eye in Vertebrates is one of the most remarkable and at the same time most instructive examples of a complex development. At an early stago (second day in chick), while the cerebral vesicles are still large thin-walled bulbs, a pair of hollow outgrowths arise from the sides of the first vesicle and extend towards the skin of each side of the head. These are the rudiments of the retina, and their cavities may be called the primary optic vesicles. They expand largely in size, except close to the cerebral vesicle, their narrow connexion with which may be called the optic stalk. Their outer ends lie against the epiblast and become first flattened and then greatly indented, so that the primary optic vesicle becomes almost obliterated, and the whole structure assumes something like the form of a goblet, but with its stem on one side, and with a slit down that side of the bowl and 
along the stem. The whole structure is now called the optic cup, and the hollow of the cup is the secondary optic

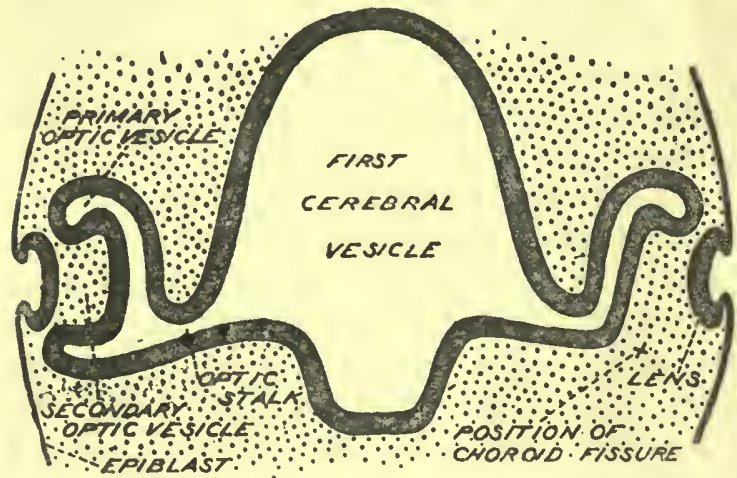

Fig. 144.-Transverse Section of Hrad of Embryo CHICK, TO SHOW Developing Eye.

On the right side the section passes through the choroid fissure.

vesicle. The stem is the optic stalk; and the slit is called the choroid fissure. The structure of the optic cup can be better understood from figs. 144 and 145 , than from any verbal description.

The lens is formed from the epiblast of the surface of the head, simultaneously with the formation of the optic cup. 'There is first formed a pit with thickened floor; the edges curve over and close in the pit, which becomes the lens-vesicle. The lens then separates from the epidermis (fig. 146), and its vesicle becomes obliterated in time.

The lens and retina thus formed, the remainder of the eye is formed from mesoblast-cells, excepting only the conjunctiva, which is part of the epidermis (epiblast). The retina at

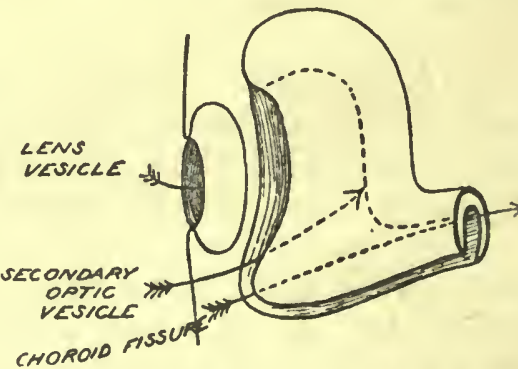

Fig. 145.-Side VIEW of LEFT EYE OF Fra. 144.

(Diagrammatic.) this stage consists of two layers, with a narrow chink (the remains of the primary optic vesicle) between them. The outer of these layers becomes the pigment-layer of the retina (often counted as part of the choroid). The primary optic vesicle becomes completely obliterated. The inner layer is divided into 
several layers-of which the outermost, i.e. nearest the primary vesicle, gives rise to the rods and cones; the inner ones to the nerve-cells and supporting elements. The choroid fissure and the groove that continues it along the optic stalk (fig. 145) become the way by which all the nervefibres growing out from the nerve-cells make their way to the first cerebral vesicle, over whose surface they extend to form the white

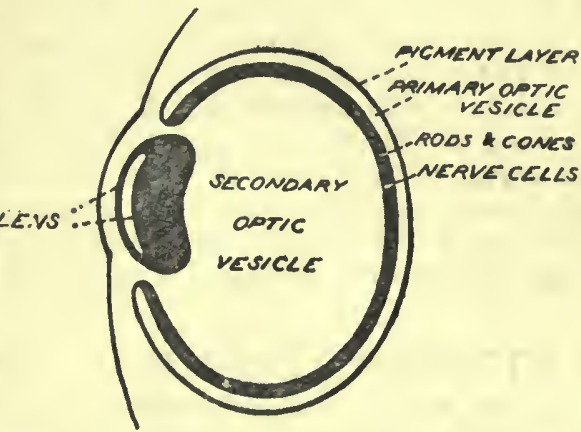

Fig. 146.-Horizontal Section throvgh Eyk at Later Stage. matter of the optic tracts. The cells of the optic stalk itself seem to degenerate and disappear after the fibres of the optic nerve are formed. The choroid fissure eventually closes up as far as it can, but its edges obviously cannot completely fuse, because of the nerve fibres running out to the optic nerve. The portion of the choroid fissure which cannot be obliterated for this reason becomes the blind spot.

$\S 12$. History of Vertebrate Eye. The study of the development of the Vertebrate eye (and it is in all essentials the same from the lamprey to man) thus shows us how the two remarkable peculiarities of the retina come about, viz. the position of the rods and cones, more remote from the light than the nerve-cells; and the presence of a blind spot. It is worth while to pause for a moment and ask why these peculiarities should be so. A moment's thought will show that the particular layer which forms the rods and cones, being next to the cavity of the primary vesicle, is really part of the lining epithelium of the tubular central nervous system, and this is shown by development to be part of the surface epiblast folded in. Thus the rods and cones agree with the sensory cells of other senseorgans in being part of the surface epiblast, but a part which has reached its final position by a complicated series of changes.

Many lowly animals, such as the sea-urchins, have the whole epidermis sensitive to light, and have their nervous system largely diffused beneath the epidermis. Starting from such a stage, if we imagine both the nerve-cells and the sensitiveness of the epidermis to be concentrated in the mid-dorsal region, and to be sunk first into a canal and then formed into a tube-we should arrive at the 
condition of Amplioxus, whose tubular spinal cord has its lining epithelium pigmented and sensitive to light for practically its whole length. Such a condition is only possible in a translucent animal. If we next imagine a series of forms intermediate between Amphioxus and the dog-fish, they will be successively more and more opaque, and the only way in which the usefulness of the sensitive lining epithelium can be retained is by its extending outwards to the surface, as the primary optic vesicle actually does in development. In a retina developed in such a way, the Vertebrate peculiarities are inevitable. We may therefore reasonably regard those pecu. liarities, not as meaningless caprices, but as indications of tbe stages by which the eye of Vertehrates has come to be what it is.

$\S 13$. Urinogenital System. We have seen how, in the tadpole, a series of pronephric tubules, in many ways comparable to the nephridia of Amphioxus, and actively functional in excretion, are replaced in later larval life by another set of excretory tubules, forming the mesonephros. In the chick the pronephros appears, but in a most rudimentary condition, and disappears almost at once ; while in the rabbit it is doubtful if it appears at all. In these types, then, the history of the urogenital system begins with the archinephric duct and mesonephros. The first appearance of the duct is shown in fig. 130. It appears close under the epiblast, and it has even been suggested that it originates from the epiblast (an origin that would meet the difficulty mentioned in chap. xviii., $\$ 12$ ), but the evidence is against this origin. It lies just on the outer side of the mesoblastic somites, and gradually sinks through the somatic mesoblast till it lies close to the coelom. Mean while, the mesonephric tubules have been formed, as in the frog, from the mesoblast of a number of somites. Their structure and relations are practically the same as in the frog, except that the nephrostomes last longer and do not become connected with the veins. A germinal ridge forms, as in the frog, nearer the median line than the mesonephros.

The mesonephros does not, however, become the permanent kidney. Just as notochord is replaced by cartilage and cartilage by bone, so pronephros is replaced by mesonephros and mesonephros by metanephros. The metanephros is, however, not so distinct from the meronephros as that is from the pronephros. 
THE DEVELOPMENT OF VERTEBRATA.

\begin{tabular}{|c|c|c|c|c|}
\hline 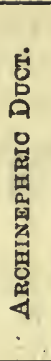 & 站 & 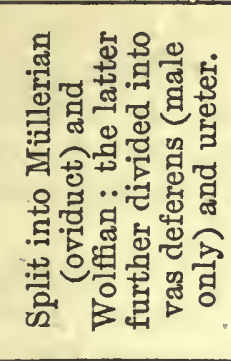 & 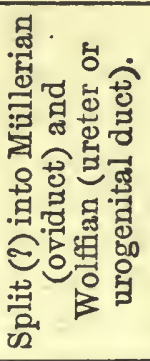 & 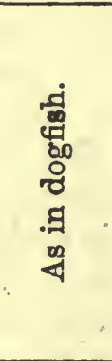 \\
\hline 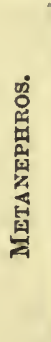 & 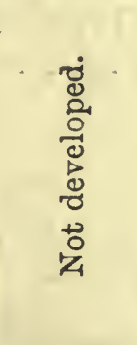 & 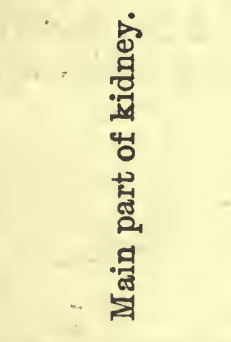 & 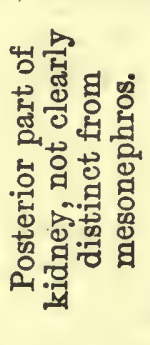 & 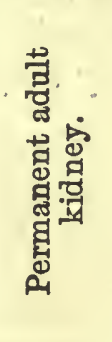 \\
\hline 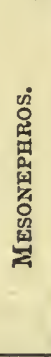 & a. & 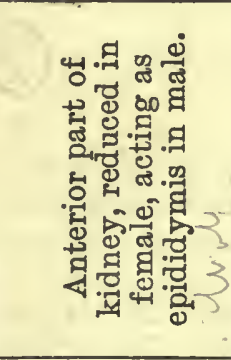 & 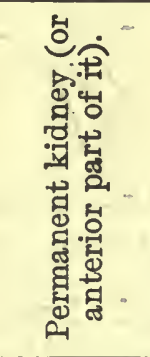 & 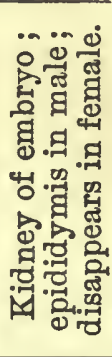 \\
\hline 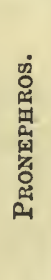 & 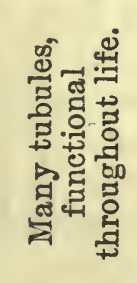 & 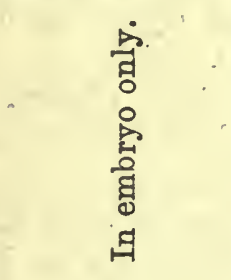 & 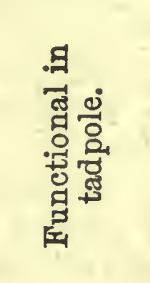 & 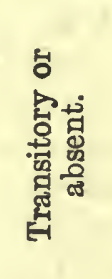 \\
\hline 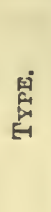 & 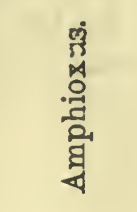 & $\begin{array}{l}\text { 데 } \\
\stackrel{0}{0} \\
80 \\
\circ \\
0\end{array}$ & $\sum_{0}^{\circ 0}$ & 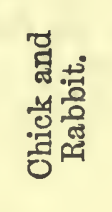 \\
\hline
\end{tabular}


Its exact origin, and that of its duct (the ureter) have never been fully traced out, but they appear to be derived from the posterior part of the mesonephros and its duct. As the metanephros develops, the mesonephros aborts in the female, but in the male it becomes connected with the testis and forms the epididymis.

It will be useful at this point to tabulate the facts about the excretory system of our. Vertebrate types (see p. 271). 279

In the rabbit only, an important change takes place in the position of the apertures of the ducts (fig. 147). Primitively they open into the cloaca on its dorsal side, as they
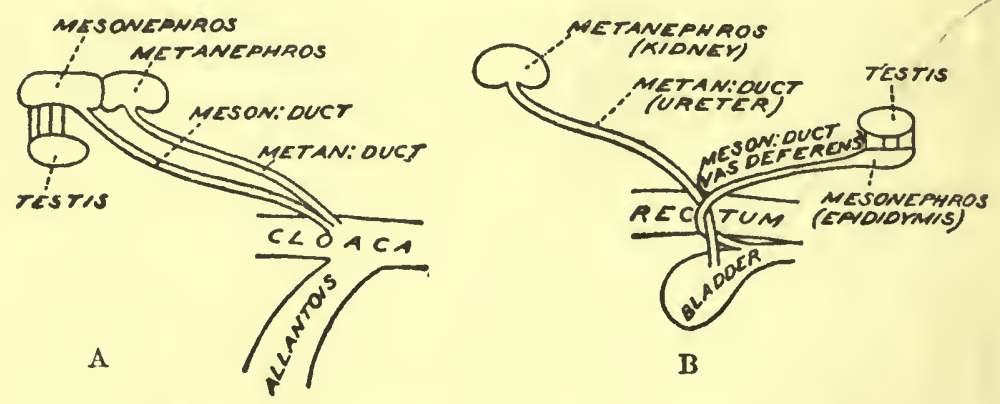

Fig. 147.-Urogenital Oranis of Male Rabits.

A, embryo. B, adult, showing shifting of parts.

do permanently in lower types. Afterwards they shift their positions round the sides of the cloaca, and the metanephric duct shifts further still and opens into the base of the allantois (or urinary bladder).

The ventral portion of the cloaca, into which the allantois and (at this stage) the Wolffian and Müllerian ducts open, becomes separated off as the urethra. A further complication is caused by the shifting of the gonads from being more median than the kidneys to being more lateral. This shifting involves the Müllerian duct (oviduct) in the female, and the Wolffian duct (vas deferens) in the male: the shifting is much greater in the male, where the testes pass into the scrotal sacs. 
Chapter XXII. - The Theory of Evolution.

$\S 1$. Relationship between Types. We have now considered our Vertebrate types, both from the standpoint of adult anatomy and from embryological data; and we have seen through the series a common structure underlying wide diversity in external appearance and detailed anatomy. We have seen a certain intermediateness of structure in the frog, as compared with the rabbit and dogfish, notably in the skull and skeleton, in the circulation, in the ear, and in the reduced myomeres; and we have seen that the rabbit passes in these respects, and in others, through dogfish-and frog-like stages in its development, and this alone would be quite sufficient to suggest that the similarities of structure are due to other causes than a primordial adaptation to certain conditions of life.

$\S 2$. Theory of Common Ancestry. The explanation of these resemblances according to the theory of evolution is that they are family likenesses-in other words, the marks of a common ancestry. The theory attributes the actual structure of organisms at the present day to heredity with modification. We have no scientific evidence of any other origin for individual organisms than by the process of reproduction from pre-existing individuals. We must conclude that all Vertebrates are descended from the same primitive type.

It must not be imagined for a moment that rabbits are supposed to be descended from frogs, frogs from dogfish, or dogfish from lancelets. Adopting the customary likening of genealogy to a tree, we may say that these and other animal species now existing do not represent parts of the trunk and branches of the trees, but only the terminal leaves. Nevertheless their structure gives us clear evidence of the approximate point where the branches which bear them diverged from one another or from the trunk. 


\section{§ 3. Amphioxus-Primitive and Specialized Characters.} Thus while in the broadest facts of structure Amphioxus remains at a stage left behind by very distant ancestors of the other types, it has not retained all the characters of those ancestral forms unchanged, but has developed, in the course of its descent from them, special peculiarities of its own that adapt it more and more fully to the conditions of its life. This is expressed by saying that Amphioxus, though belonging to a very primitive group of Vertebrata (all the other members of which are extinct), is specialized on lines of its own. Instances of this specialization are the forward extension of the notochord, the large number of gill-slits, and the great development of the atrium. We are so much in the dark, however, as to the nature of the common ancestor of Amphioxus and the Craniates, that it is difficult to say of many of the peculiarities of Amphioxus whether they are primitive or specializations.

\section{$\S 4$. Specialization of Dogfish and Frog. Of the other} types we may speak with greater certainty. The dogfish, though retaining many characters that must have been possessed by the common ancestor of the Craniates, has specialized in the direction of greater care of its younglaying, as it does, but two eggs at a time, well-supplied with yolk and protected by a horny case, instead of the enormous number of ill-cared-for eggs that the bony fishes and the frog continue to produce. The hyostylic condition of the skull, the detailed structure of its paired fins, its pouched gill-slits, and the elaborate arrangement of afferent and efferent vessels, are also specializations which cannot be regarded as intermediate between the conditions of the homologous parts in Amphioxus and the frog.

The frog again shows great specialization in the reduced number of its vertebræ and complete loss of its tail ; in the structure of its pelvic girdle and lind-limbs, and many other characters which could not possibly have existed in ancestors common to it and the rabbit.

§ 5. Palæontological Evidence. Fortunately, we are not condemned to mere speculation as to the extinct common 
ancestors of existing animals. The geologist has shown us that many of the rocks that form the surface of the earth beneath the soil were in immeasurably remote times formed as sediments on the floor of the sea or lakes or elsewhere; and he has shown how we can determine the relative ago of these sedimentary rocks and recognize a series of periods in the Earth's history. Now embedded in these rocks aro the fossil remains of extinct animals, and though the knowledge so obtained of the life of these successive periods is of necessity very incomplete, it goes far to confirm the conclusions of Comparative Anatomy and Embryology. Although at all periods there were abundant forms of lifo which were, as it were, only leaves on the lower branches of the tree of life, and in no way ancestral to the forms of later periods and the present day, yet many ancestral or approximately ancestral forms can be recognized.

In the Vertebrate series, palæontology (the study of fossils) begins only with the fishes, for only organisms with hard, durable skeletons can as a rule be fossilized. In the Silurian and Devonian periods, there were abundant fishes -many of them specialized in ways of their own, but some of which may very well have been ancestral both to the dogfish and to the land-Vertebrates. In the succeeding Carboniferous period, the earliest Amphibia are found, far more primitive and fish-like than any existing Amphibia. In the Permian period come forms which link the Amphibia to the Reptiles; in the Triassic period, Reptiles which have many striking resemblances to Mammals; and at the end of that period true Mammals were in existence. Birds, which are nothing but highly specialized Reptiles, did not appear till a later stage. Later still we can trace the evolution of most of the great orders of Mammalia from simpler members of that group.

$\S 6$. Investigation of the Causes of Evolution. When this great fact of the descent of all Vertebrates-and, by extension of the idea, though on less complete evidence, of all other animals-from some common ancestor is admitted, the question arises: How have these wonderful changes of form, structure, liabit, and instinct been 
brought about? This is a question which has engaged the attention of many of the ablest biologists from the time of Darwin to the present day, and the problems which it involves are so complicated that only a brief outline of the present state of our knowledge of the subject can be given here.

We may point out in the first place that Darwin's theory of Natural Selection, though proposed as an explanation of the origin of species, was in reality an explanation of the origin of adaptations. It was assumed by Darwin that species differed from one another because they were adapted to different modes of life. The marvellous adaptations that occur in animals and plants are obvious to every naturalist: we may take the structure of a bird, or of a whale, or of a flat-fish as conspicuous examples. On the other hand systematists spend the greater part of their lives in studying and describing the minute differences which distinguish allied species from one another, and in the great majority of cases no evidence has been discovered that these differences correspond to differences in mode of life, or have any function or utility which could bring them within the scope of the theory of Natural Selection.

There are then two distinct problems to be considered, the origin of species and the origin of adaptations-in other words, the causes by which organisms are so modified as to be fitted for their modes of life, and the causes by which organisms living under similar conditions are divided into distinct species. It may be in some cases, as in that of the rabbit and hare, that the differences of species correspond to differences of habit, but it is certain that this is not the case with a vast number of specific characters, and these have to be explained.

§ 7. Gametes and Heredity. Weissman by his theory of the independence of the germ-plasm directed the attention of biologists to the reproductive cells as the only connecting links between parents and offspring. Without entering into the theoretical question of the existence of a special germ-plasm distinct from the ordinary proto- 
plasm of the body, we recognise that the reproductive cells are distinct from the rest of the body, and that when they are separated from the parent they give rise to new individuals. In most cases two germ cells unite in fertilisation before they are capable of development, and therefore they are called gametes.

In these gametes, and, as we have seen, probably especially in the chromosomes of their nuclei, are properties which give rise to the peculiarities or "characters" of the individuals developed from them. The individuals may to some extent be modified by the conditions of life and by functional exercise, but we can usually distinguish many characters which are congenital, or due only to the properties of the gametes. When such characters are carried on from generation to generation they are said to be inherited, or developed by heredity, but when a new character appears it is a variation.

§ 8. Distinction between Soma and Gametocytes. It is admitted by all biologists that the gametes in a given adult organism are derived by a continuous succession of cell divisions from the fertilised ovum or zygote from which the organism was itself developed. In the early stages of development the embryo consists of a number of embryonic cells of which none can be distinguished as reproductive cells. At a certain stage of development some of the cells become distinct as reproductive cells, the rest forming the soma or body.

\section{$\S 9$. Special Characters of Reproductive Cells or} Gametocytes. It may perhaps be urged that the reproductive organs are differentiated in the same way as other organs, such as the brain or the liver, and that the cells of the other organs are capable of multiplication and growth as well as the reproductive cells. In the lower animals and in plants the organs are not so definitely differentiated, and continuous growth takes place in various parts of the body, producing shoots, buds, etc.; also parts other than reproductive cells separated from the body may produce new individuals. 
All this is perfectly true, and must be included in a complete general view of reproduction and heredity; but at the same time the reproductive cells, especially in the higher animals, have this peculiarity-that they are set free from the parent before they develop into new individuals or at any rate they begin to develop as single cells not as pieces of tissue, that they retain the primitive undifferentiated condition of embryonic cells and are not formed from the latter by special changes, and that they are the only cells in the body which are capable of the rejurenating process known as conjugation or fertilisation.

It is very important to have a clear conception of this process of development in relation to the problems of reproduction and evolution. Development is in all cases the formation of a large mass of cells by the division or "segmentation" of the original ovum. The organs and tissues of the body are formed by the differentiation of groups of these cells. How this differentiation is brought about, how one group is caused to form the brain, another the muscles, and so on, is a much debated question which we have not space to discuss here. The important point for our present purpose is that all the cells are not used in the formation of the body, but a certain group of cells remain undifferentiated, retaining all the properties of the original ovum from which they are descended: these are the reproductive cells or gametocytes, which multiply by cell division but always remain undifferentiated primitive cells, and ultimately give rise to the gametes which produce the next generation. We have thus the important modern conception of the distinction between soma composed of somatic cells on the one hand, and the reproductive cells or gametocytes contained in the reproductive organs on the other.

The gametocytes then are not produced by the soma but have only a fraternal relation with the somatic cells, and the developmental properties of the ovum of a frog, for example, are not derived from that frog, but are due to the persistence or continuation of the properties of the ovum from which both the soma and the gametocytes of the frog are descended. The relation of the individuals 
of successive generations may be compared to that of successive leaves on a growing shoot. Each leaf is not produced by the preceding leaf, but owes its resemblance to the latter to the fact that it is produced by the same shoot. The gametocytes are continued by cell division from generation to generation, the individuals are outgrowths from the line of gametocytes.

$\S 10$. Blastogenic Origin of Variations. It was formerly assumed that modifications of the soma by external conditions and by use and disuse were inherited in some slight degree, so that in each generation the amount of modification was increased, and in this way adaptations might be produced. Since the time of Weissman, as the above distinction between soma and gametocytes has been more thoroughly realised, the majority of biologists have adopted the view that changes in the soma do not affect the gametocytes, in other words are not inherited, and that all variations arise in the gametocytes, or are blastogenic.

$\S 11$. Minute "Continuous" Variations. That blastogenic variations frequently occur is certain, but the study of variation presents many difficulties and has been attempted from different points of view. A distinction must be made between the minute differences which always exist between a number of individuals of the same species, and even between the offspring of the same parents, and those definite and conspicuous variations which are not connected with the normal type by intermediate degrees. For the latter the term mutation, introduced by the Dutch naturalist de Vries, has been adopted. The minute individual differences were those on which Darwin chiefly relied for his theory of Natural Selection, and they have been investigated statistically by Galton, Weldon, and Karl Pearson, who maintain Darwin's original view.

Many objections have been raised to this view: one is that the differences are too small to determine death or survival; another that it is not certain that such differences are inherited, as many of them may be due to external 
conditions, and therefore not hereditary; and a third that differences of environment which are supposed to give rise to selection are slight or "continuous," while species are separated by distinct jumps or are "discontinuous." To all these objections may be added that already mentioned, namely, that specific differences are not always or in the majority of cases differences of adaptation.

$\S 12$. Mutations. The Mendelians on the other hand conclude from the results of experimental breeding (see p. 218) that characters are transmitted as "units" or "factors," which are not subdivided but are either present or absent. Mutations consist in the addition or omission of one of these units. The cause of the mutation is not known with certainty, but it is blastogenic, and possibly arises from unequal distribution of the factors in the cell divisions of the gametocytes within the parent. There can be little doubt that the differences between species in nature are of the same kind as those which distinguish breeds and varieties in cultivated animals and plants, and that many of these characters have arisen suddenly and not by a gradual process of selection.

$\S$ 13. Mutations and Metamorphosis. On the other hand there are many zoological phenomena which the doctrine of mutation and blastogenic variation does not explain or agree with, and which appear more in accordance with so-called Lamarckian principles, i.e. the heredity of modifications due to conditions. One of these phenoinena, which is not considered by the mutationists, is that of metamorphosis. In the well-known case of the frog and other Amphibia, the development of the lungs and disappearance of the gills take place gradually as the individual changes from an aquatic to a terrestrial existence, and this is only a type of numerous similar cases, such as the flat-fishes, in which the change of structure proceeds gradually and continuously in correspondence with a gradual change of habit.

Recapitulation, i.e. the repetition of ancestral stages in development. is not universal, but it is verv freccuent, in 
cases of adaptation, and it is a continuous process which has no resemblance to discontinuous variation and is not satisfactorily explained by any theory except that of the heredity of modifications due to external conditions. There is no evidence of variations which could possibly lead to lungs, except in animals that breathe air. Similarly there is no evidence that steps in the changes which take place in the eyes of flat-fishes have ever occurred except in those fishes which lie on one side. It is natural to conclude, therefore, that the habits and conditions produced the adaptations. There is plenty of evidence that such changes of structure as are required on this theory are produced by external conditions in the individual.

$\S 14$. The Metamorphosis of Flat-fishes. The flounder, when first hatched, is a minute larva not quite one-eighth of an inch in length. The right and left sides are perfectly similar to one another, and it swims vertically in the water. But the larva has no fin-rays and no bones: a continuous fin-membrane passes along the edge of the back round the end of the tail. The conversion of this larval form into the fully developed flounder takes place when it is from two to three months old, and about half an inch long. When the bones and fin-rays begin to develop, the left eye rises first to the edge of the head and then passes completely over to the right side. At the same time the little fish begins to lie on its side on the ground, and loses the power of sustaining itself in the water. With slight differences in details, the development and metamorphosis of other species of flat-fishes are similar. The early condition of the flat-fish, therefore, is not that of any fully-developed fish at all, but of a fish-larva without bones or fin-rays. It is in all its principal features similar to the larvae of other marine fishes; for instance to that of the mackerel, or that of the cod. When the bones begin to develop the eye begins to become asymmetrical, and we have not the ancestor but the flat-fish. We do not know at present whether the elongated fins along the dorsal and ventral edges had the same form in the ancestor; we have reason to believe they 
had not so great an extent, yet they are developed directly, not by gradual increase. The true reading of the matter therefore is not that the ancestral condition is repeated, but that the larval condition of the ancestor is retained; because the larva is still hatched and still lives in the same way; but the structure after metamorphosis is different, because the fish has acquired different habits. On the theory of mutations we must suppose, not that individuals were hatched which had asymmetrical eyes, but that symmetrical individuals in consequence of mutation became asymmetrical, and there is no evidence of such a mutation having occurred in any fishes which do not lie oll the ground on one side.

$\S$ 15. Secondary Sexual Characters. Another class of zoological facts which cannot be explained on the mutation theory, nor on any selection theory, is that class which concerns what are called secondary sexual characters. It is well known that in a vast number of cases the male possesses structures or colours which are not present in the female: the male and female are different, and the sexes constitute two "forms" in the same species; hence this condition is called "sexual dimorphism," which means sexual two-formedness. Examples of this are seen in nearly all divisions of the animal kingdom, the cock and hen of the common fowl, the stag and the doe, and the black horny excrescences on the fore-foot of the frog afford familiar examples. Darwin explained such facts by “ sexual selection," the males having such variations most developed being either preferred by the female or conquering other males in combat. It is certain that the selection of the male is no explanation, because the variation would be inherited by the female unless it was only capable of inheritance by the same sex, and this must therefore be an original peculiarity of the variation.

Mutationists explain these characters by supposing that certain mutations are "coupled" with one sex, so that they are only inherited by individuals of that sex. But there is abundant evidence to prove that the characters are really inherited by the female sex, but in a latent con. 
dition. It may be true in certain cases that the characters are coupled with the sex character, but it is difficult to believe that mutations could ever occur, and occur so frequently, which consisted of structures which not only were adapted to a particular function, e.g. the excrescences of the frog's fore-foot for holding the female, but actually developed in the breeding season when the function was required and disappeared afterwards. Such a close correspondence between the structure, and the season of its development, and its function seems to point very strongly to the conclusion that the function itself, which is confined to the male, must be the cause of the structure.

$\S 16$. Growth and Shedding of Antlers. The antlers of stags are in many respects the most remarkable of secondary sexual characters: there is no other case in which so large a mass of bony tissue is annually produced and annually shed. The growth begins in spring soon after the old antlers have dropped off. The new bone is covered with skin and hair, the skin being very vascular and the lair short, whence the covering is called the velvet. The growth of the great branched structures is complete by the end of August or beginning of September, and then the animal voluntarily scrapes off the velvet by rubbing the antlers against the trunks of trees or palings. The velvet at this time is not actually dead, but full of blood, yet the process seems to give the animal no pain. At the base of the antler, where the skin joins the bare bone, is produced a projecting ring of bone called the "burr." With the bare antlers the stags fight with one another during the breeding season, which lasts from September to Christmas. Then at some time in the beginning of the following year the bone below the burr is absorbed, the antler drops off, and the whole process is repeated. The most important fact about this history of the antler is that the processes which take place in the stag by heredity are essentially the same as those which occur occasionally in mammals in general in consequence of injury or disease. It is a well-known fact that bone cannot live after the death of the periosteum that surrounds 
it, and also that dead bone is absorbed by the adjacent living tissue. Therefore the stripping of the velvet is the real cause of the shedding of the antler. It was formerly supposed that the formation of the burr caused the death 'of the antler by constricting and closing the arteries that entered from the bone below; but the bone within the antler is alive and blood circulates in it after the burr is formed: it dies gradually. When it is dead the living bone below the burr absorbs the layer of bone next to it and the antler necessarily drops off, the separation taking place below the burr, not above it.

It is true that the growth of the antler and the stripping of the velvet do not actually follow, but precede the fighting of the stags, but on the other hand it is difficult to believe that processes so similar to natural physiological processes could have arisen by "mutations," which were not caused by the fighting of the stags. If the mutation was independent of the habits of the animal it is impossible to understand how the habits and the development of the antlers should correspond so closely. If the fighting of the stags caused the original growth, and then the stripping of the skin, which was followed by the shedding of the antler, we could understand that in course of ages the development might gradually take place earlier in the year until the present state of things was brought about.

$\S 17$. Effects of Castration. It is held by many, however, that there is no evidence of the heredity of such modifications, and until recently it was maintained that there was no means by which a change in a part of the soma could affect the gametocytes. It is true that mutilations are not inherited even when repeated for countless generations, as in the docking of lambs' tails, but this does not prove that physiological changes such as increased growth or "hypertrophy" may not be inherited, and an infinitesimal inheritance in one generation may become complete inheritance in time.

The view that the development of all parts of the body is due to the properties of the ovum alone is contradicted by the recently discovered facts concerning internal secre- 
tions or hormones. It was contradicted from the beginning by the long-known facts of the effects of castration on secondary sexual characters. Darwin explained such characters by the selection of the males by the females, or by the victory of the males which were strongest and provided with the best weapons. But such selection among the males would not explain the absence of the characters, e.g. antlers of deer, in the females. The selection of a male with antlers to mate with the female without any would not cause the heredity of the antlers to be confined to male progeny. The limitation of the heredity must have preceded the selection. In fact the antlers are inherited by both sexes but only developed in the males, and this development does not take place if the gonads are removed.

The development of the antlers is therefore due not entirely to the properties of the ovum from which the animal is developed, but partly to the presence of the testes, and it is now known that the influence of the testes is a chemical one; something absorbed from the testes acts as a chemical stimulus on the skull which causes the inherited tendency to produce antlers to become active. The absence of antlers in the female, therefore, is to a great extent, if not entirely, due to the fact that the internal secretion of the testes is absent in the female, and an internal secretion from the ovaries is present which stimulates the development of female characters.

Development, therefore, is not always a mere differentiation of the mass of cells derived from the ovum, depending on properties already contained in the ovum before segmentation, but the degree of development in one part of the body may depend on chemical compounds derived from another part. This is a conception of development quite different from that of Weissman and his successors, who regard development as the predestined course of heredity.

$\S 18$. Internal Secretions or Hormones. Many hormones are known besides those derived from the sexual organs. It has been proved that the resniration centre is stimu- 
lated, not by afferent nerve-impulses, but by the carbon dioxide contained in the blood. When the proportion of this gas in the blood increases, as during muscular exertion or partial suffocation, the respiration centre is excited and the respiratory movements increased. If the tension of carbon dioxide in the blood is reduced, as by rapid breathing for a short time or by an excess of oxygen in the air breathed, the respiratory centre is not stimulated and respiration ceases for a time, and we have the condition called apnoea. The carbon dioxide produced in the body is a hormone which stimulates the respiratory centre.

Starling and Bayliss have shown that the secretory activity of the pancreas is due to a substance called secretin, which is formed in the mucous membrane of the duodenum by the action of the acid chyme, and which is carried to the pancreas by the blood.

The thyroid gland affords another example. Although this is not a gland in the ordinary sense, with a duct through which its secretion is delivered, it secretes specific substances which when absorbed into the blood are necessary for the normal development of the organs of the young animal or child. Cretins are children whose development is arrested in consequence of disease of the thyroid gland : administration of the thyroid of calves or of thyroid extract causes renewed growth and development so that the child grows taller and more intelligent.

The adrenal bodies produce a substance, adrenalin, which has the same effect on the involuntary muscles as stimulation of the sympathetic nerves.

$\S$ 19. The Hormone Theory of Heredity. These facts, especially those concerning the growth of special parts of the body such as secondary sexual characters, or the whole development as in the case of the thyroid gland, give as before stated a new conception of the process of develop. ment, and dispose finally of the contention that no process is known to physiology by which modifications of the development of the body could affect the properties of the ovum.

Darwin, in his theory of Pangenesis, suggested that all 
parts of the body gave off living particles, which he called gemmules, which passed into the reproductive cells and in the next generation developed into organs and structures similar to those from which they had been derived in the parents.

These gemmules were purely imaginary, and there is no evidence in plysiology of any living substance passing from the somatic cells or tissues into the reproductive cells. It is, however, now certain that special chemical compounds pass in certain cases from one organ and profoundly affect the functions or development of other parts. It is also certain that all parts of the body give off their waste products to the blood. It is possible that these waste products include not only carbon dioxide and compounds formed in all living tissues by oxidation, but also specific chemical compounds peculiar to each organ or tissue.

Since the reproductive cell or gametocyte contains something corresponding to each structure and character of the individual developing from it, and therefore to each part of the soma of the parent in which it is contained, it is a reasonable hypothesis that each part of the soma gives off a hormone which, passing into the blood, acts upon the reproductive cell and affects the corresponding part in it, the corresponding determinant we may say. Thus, although it is true that the "determinants" in the gametes are derived directly from those of the gametes or of the fertilised ovum of the previous generation, it may be also true that these determinants are to some degree modified by the hormones of the soma in which the gametes are contained.

Moreover, we have reason to believe that hormones are specially produced by actively growing tissues, and by functional activity of all kinds, since growth and functional activity necessarily involve the production of more waste products. Hence external stimulation, whether of the nature of mechanical irritation producing growth or of nervous stimulation producing functional activity of muscles, bones, sense-organs, etc., may increase the hormones of the parts affected, and so stimulate the "determinants" in the gametes; and cause the modification of 
the same organs or parts in the offspring. Such a process, at present hypothetical, would afford a better explanation of the evolution of adaptations than any other theory hitherto put forward.

$\S 20$. The Two Chief Factors of Evolution. In the above brief outline we have endeavoured to show that there is some valid basis for the conclusion that evolution has been the result not of this single process or the other, but of the interaction of two main factors, one the occurrence of blastogenic mutations which do not directly correspond to external conditions and which have given rise to species and often to genera and to non-adaptive characters generally (metamerism for instance), and the other the direct action of habits and conditions, by mechanical and functional stimulation, producing adaptive modifications of structure. Every type and every species of animal is the combined result of these two factors: it is neither all adaptation nor all spontaneous mutation, but a combination of the two.

In some cases, as we have already suggested, a special adaptation may be confined to a single species, but in the majority of cases it is easy to perceive that allied species present differences in adaptive characters which canmot be of importance to the animals in which they occur. For example, if we assume that the function of the antlers of stags is to serve as weapons in fighting, we find that the species of deer are distinguished by differences in the size and branching of the antlers, and in the colour of the coat. It is easy to distinguish the red-deer, the fallow deer, the roe-deer, and the moose or elk. In all these the antlers are different in form and branching, but it is impossible to contend that in each species the special character of the antlers is useful or adapted to the special mode of fighting of that species. In fact Darwin himself was obliged to conclude that the branched antler was more for ornament than use, because a single sharp spike would be a more effective weapon: but there is no adequate basis for the view that the females have any opportunity for selecting branched antlers for their beauty. 
The more reasonable conclusion is that the mechanical stimulation caused by fighting set up the growth and branching of antlers, and that spontaneous variation or mutations gave rise to a number of types in different species. There is everywhere spontaneous tendency to the formation of species, just as there is to the formation of varieties in domesticated animals. Similarly in flatfishes, all the species show the chief modifications adapted to the exceptional position of the fish, and again, differences of the jaws and teeth are adapted in different flatfishes to the different kinds of food. But it is impossible to perceive any relation between the character of the scales and the colour markings by which the species are distinguished and differences in the mode of life. Some flat-fishes like the sole have spiny scales, others small smooth scales like the plaice, and others like the flounder and turbot no true scales at all.

\section{Questions on Vertebrate Development.}

1. Describe the changes in the egg-cell which precede fertilization; describe the process of fertilization and the formation of the primary cell-layers, as exhibited in three of the animal types known to you. What is the notochord, and how is it developed in the frog?

2. (a) Explain what is meant by the term "central nervous system." (b) Describe the tissue elements which enter into its composition. (c) Explain, as far as you can, the function of each structure described. (d) How is the central nervous system developed in the frog, and $(e)$ in the rabbit? $(f)$ What conclusions may be drawn from the facts stated as to the origin of the central nervous system in evolution?

3. Describe the structure and cleavage of the ovum (a) of the frog, (b) of the fowl, and (c) of the rabbit. (d) Explain as far as possible the differences in the cleavage of these three eggs. (e) Point out how the embryo is nourished in each case, and $(f)$ describe the constitution of the placenta in the rabbit. 
4. (a) What are the protovertebro? (b) How does the notochord originate in the frog? (c) How are the vertebræe laid down in the tadpole? (d) Describe the vertebral solumn of the adult frog. (e) In what important respects do the centra of the vertebræo of the frog, the dogfish, and the rabbit, differ from one another?

5. What temporary organs are developed in the embryo frog which are absent from the embryo bird and mammal, and what in the two latter which are absent from the former?

6. Describe fully the coelom of Amphioxus, and compare it with that of the frog in regard to $(a)$ development, $(b)$ ite relation to other organs in the adult.

7. How do protozoa differ from higher animals (metazoa) as regards $(a)$ structure, $(b)$ reproduction? Compare the process of fission in an amoba with the segmentation of the ovum in Amphioxus, pointing out the resemblances and differences between the two cases.

8. Compare the auditory organs in Scyllium, Rana, and Lepus, especially with regard to the homologies of the structures connected with the tympanic cavity, namely the auditory ossicles, the columella, and the Eustachian tube. Describe the course of the 7th cranial nerve in each case.

9. 'Trace the history of the post-oral gill-slits and their accompanying cartilaginous bars and vascular arches in the frog, fowl, and rabbit.

10. Give a short account, with illustrative figures, of the mode of formation of the primary germinal layers in Amphioxus and in the frog. What explanation can you give of the differences between the two cases?

11. Give a short account, with diagrammatic figures, of the principal changes which occur in the circulatory and respiratory organs during the metamorphosis of the tadpole into the frog.

12. Describe the mode of development, and adult structure of the vertebrate eye.

Explain the position of the retinai layers, illustrating your answer by a careful comparison with the eye of the sravfish. 
13. Give a brief explanation of each of the following terms:-(1) primitive streak; (2) Graafian follicle; (3) blastopore; (4) protovertebræ; (5) female pro-nucleus.

14. Give an account of the developmental origin and the structure of the notochord and (in the last three) of its relations to the vertebral centra in Amphioxus, the dogfish, the frog, and the rabbit.

15. Describe the egg, the process of fertilization, and the formation of the germ-layers in the rabbit, fowl, frog, and Amphioxus.

16. Give an account of the first appearance and development of the amnion and of the allantois. In what animals do they occur? What appear to be their functions?

17. Describe and compare the egg of the fowl and rabbit, and give an account of their mode of fertilization and early developmental changes. 



\title{
PART IV.-INVERTEBRATA.
}

\author{
Chapter XXIII.-The Crayfish.
}

\section{(Astacus fluviatilis.)}

\$ 1. Mode of Life. The crayfish is found in England in those rivers which, flowing over chalk or limestone, contain a considerable amount of calcium carbonate in their water. Compared with its companion in the same streams-the fresh-water mussel-it is a highly-developed animal. In-

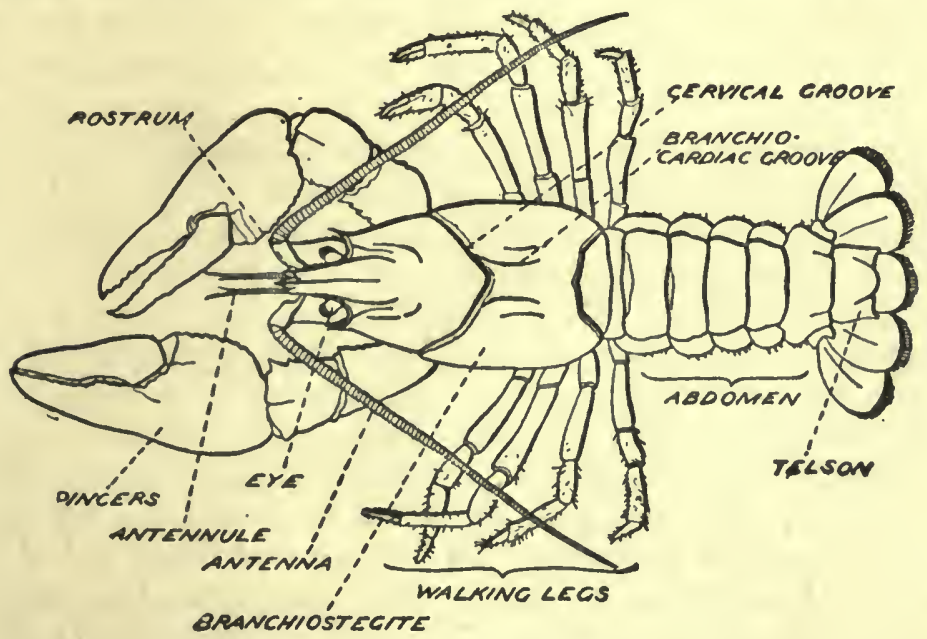

Fig. 148.-The Crayfish.

Dorsal view. (After Huxley.)

sterd of passively feeding on diatoms and the like, it is active and carnivorous (or rather omnivorous), burrowing holes in the river-banks, in which it lies in wait for prey. At night it sallies forth to crawl over the bottom. It has well developed eyes and olfactory organs by which to recognize prey or enemies ; its large pincers serve as efficient weapons of attack or defence; and it can jerk itself backwards through the water by the sudden flexion of the "tail" 
(abdomen). It cannot, however, swim like a fish, the body being flexible only in the vortical and not in the horizontal plane.

$\S 2$. Exoskeleton. This limited flexibility is due to the high development of the exoskeleton. As in the earthworm and mussel this is a cuticle secreted by the epidermis, but instead of being equally thin throughout as in the former, or merely thickened and calcified in tro valves which cover in the whole animal as in the latter, we havo an alternation of thickened and calcified regions (sclerites) with thin uncalcified flexible areas. In this way the exoskeleton combines rigidity with movableness.

Hair-like setce are abundant on the cuticle of various parts: unlike those of the earthworm, these are not developed in sacs of the epidermis, but the epidermis extends up for some way into them.

The front half of the body is encased in a single unjointed sclerite called the carapace. The region covered by this is called the cephalo-thorax, and vaguely divided into "head" and "thorax," the remainder of the body being called "abdomen" (fig. 148). The head so far resembles the Vertebrate head that it bears the chief sense-organs, but otherwise no comparison can be made between these three divisions of the body and the similarly named divisions of a rabbit: the organs they respectively contain differ altogether. The carapace runs out in front into a pointed median rostrum projecting beyond the real front end of the - body. An oblique groove in the carapace-the cervical groove-marks off the "head" from the "thorax." In front of this groove the carapace forms a simple circle round the body; but behind the groove it is extended down on either side as a gill-cover or branchiostegite, and ends apparently in a free edge. Really the branchiostegite is double - there being an inner thin uncalcified layer on its inner face (see fig. 157). The line of origin of the branchiostegites is indicated on the dorsal surface by the pair of branchio-cardiac grooves.

§ 3. Structure of Abdominal Region. The abdomen is 
obviously metamerically segmented, seven divisions being present. The last of these is much flattened, and is called the telson. The other six are alike, each showing the same rounded - triangular

form in cross section (fig. 149). The exoskeleton here eonsists of two partsa larger dorsolateral tergum and a smaller ventral sternum. A part of the tergum which projects laterally is called the pleuron, and a small portion that forms part of

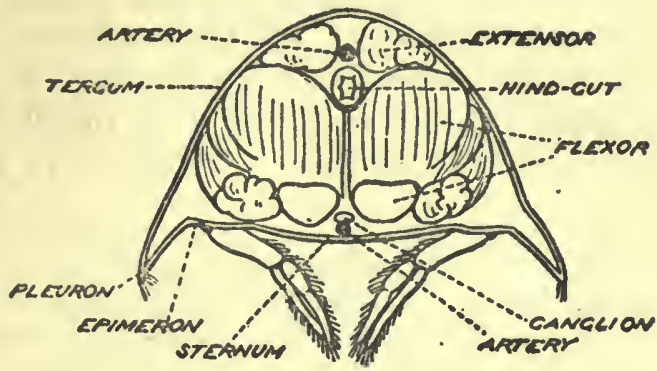

Fig. 149.-Transverse Section or Abdomen of Crayfish.

(After Huxley.) the ventral surface is the epimeron. Between the latter and the sternum the appendages or limbs are attached. These are covered by a cuticle continuous with the general exoskeleton, and jointed as that is. There is one pair of these jointed appendinges to each of the six abdominal somites. As the telson has no appendages, it is not usually considered to be a somite, but rather an unsegmented terminal portion of the body (just as the urostyle is the terminal unsegmented portion of the frog's vertebral column).

§ 4. Cephalo-thorax. The fact that thirteen pairs of more or less similar jointed appendages are attached to the cephalo-thorax is regarded as an indication that in that region there are really thirteen somites fused together; the crayfish thus being a metameric animal in whose anterior region there has been a coalescence of somites masking the metamerism, much as is the case with the head of Craniate Vertebrates.

§ 5. Appendages. Although the nineteen pairs of appendages show great variety of form, because they aro adapted to a variety of functions, they can all be described by 
reference to a common plan, from which each one diverges in its own way. In this typical form (cf. fig. 150 [5]) we have a proximal portion attached to the body; the protopodite. Distally this divides into two parallel branches-an endopodite towards the median line, and an exopodite more lateral in position. All three of these are divided into few or many articulating segments. A fourth portion, the epipodite, which comes off from the protopodite, is unjointed, turns up dorsalwards, and may carry a gill.

The protopodite is usually divicled into two joints, of which the proximal is called coxopodite, the distal, basipodite. The endo- and. exopodites may have an indefinite number of divisions, but in several cases the former has five : these are then termed, in order from proximal to distal-ischio-, mero-, carpo-, pro-, and dactylu-podite .

The nineteen pairs of appendages are as follows. The first two pairs are at the anterior end of the body, and modified to serve as "feelers" or antennæ; the next three are set close round the mouth to serve as jaws, the third being called mandibles, the fourth and fifth maxillæ. The next three (6, 7, and 8) are intermediate in character between these jaws and the walking-legs behind, and are therefore called maxillipedes (fig. 150). Next comes the biggest of all the appendages, the strong pincers; followed by four pairs of walking-legs (fig. 148). Lastly we have the abdominal appendages, the first of which is vestigial in the female, and forms a stout style in the male; the next four (fig. 149) are swimmerets (though the first of them in the male is also modified); and the last form the lateral portions of the tail-fin (fig. 148), the central part of which is formed by the telson

§ 6. Head Appendages. The general form and appearance of these various appendages is shown in figs. 148, 150, and 158, and the following notes on their peculiarities are only intended as an assistance to the practical examination of them. All are, of course, paired, although spoken of in the singular.

The antennule has a protopodite of three joints: both endo- and exopodite are many-jointed and whip-like.

The antenna bears on its coxopodite the excretory aperture; its exopodite is a flat, sharp-pointed plate, called the squame; its endopodite is long, many-jointed, and whip-like. 
The mandible consists of a very stout, hard, unjointed protopodite, serving as a biting organ; and a small three-jointed endopodite, serving as a feeler or palp. The reason for calling this endopodite and not exopodite will be found by comparing it with the next two or three appendages behind.

The first maxilla is a small, thin appendage, consisting of three curved chitinous plates: these are shown, by comparison with the next two, to be the two joints of the protopodite and the unjointed endopodite, respectively.

The second maxilla consists of a portion resembling the first

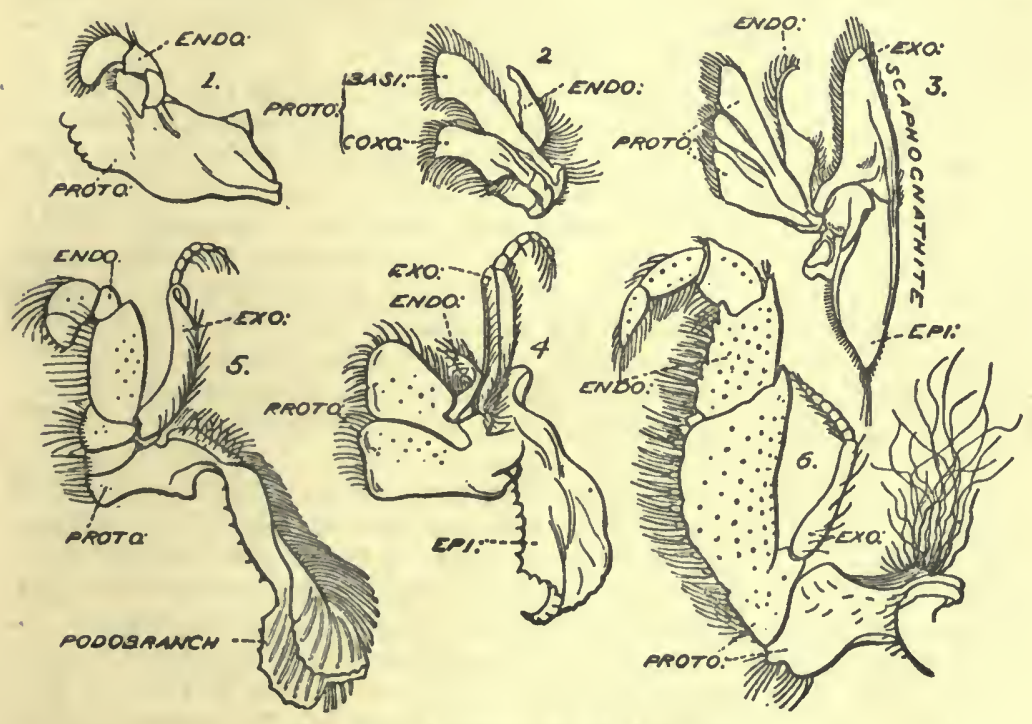

Fig. 150.-APPENDAGES or Astacus.

(After Huxley.) 1, Mandible ; 2, first maxilla ; 3, second maxilla ; 45 and 6, first, second, and third maxillipedes. All are drawn in the inverted position (dorsal part downwards.)

maxilla, but larger, and a more lateral portion called the scaphognathite. 'The two joints of the protopodite are each cleft nearly into two; the endopodite is unjointed but rather long. The scaphognathite is a large flat, flexible plate: it has a special function in connexion with respiration $(\$ 20)$, and is regarded as representing exopodite and epipodite united.

§ 7. Thoracic Appendages. The first maxillipede shows the same flattened, expanded condition of the two protopodite-joints as the appendages in front; the endopodite is small; the exopodite is large and consists of a stout, unjointed proximal half and a flexible, jointed distal portion; while the epipodite is a thin ovate plate 
resembling the dorsal part of the scaphognathite, but quite free from the exopodite.

In the second and third maxillipedes the two divisions of the protopodito lose their expanded flat character; the endopodite becomes larger and larger, consisting of the definite five joints; the exopodite becomes relatively smaller; and the epipodite takes the form of a small oval plate bearing a gill. (F'or description of gills, see $\S 20$ ).

In the pincers and four walking-legs the exopodite is wanting; the epipodite with its gill is similar to that of the two hinder maxillipedes (except in the fourth walking leg, which has none). The main portion of the limb consiste of the two joints of the proto. podite and the five of the endopodite.

In the pincers, and often in the first walking-leg, the basipodite and ischiopodite are fused together-or, more accurately, the cuticle is continuously calcified over both, and no motion can occur at their junction, which is recognizable by a slight surface-marking only.

In the pincers and first two walking-legs the propodite has a distal prolongation, as long as the dactylopodite, and the latter bites against it, forming an excellent nipping mechanism. This is called a chela, and these limbs are said to be chelate. It is a curious thing that the process of the propodite is developed on the postaxial'side in the pincers and on the opposite side in the other two.

The third and fourth walking-limbs are not chelate.

§ 8. Abdominal Appendages. The swinmerets consist each of a two-jointed protopodite, and an exo- and an endo-podite which are very similar and many-jointed. Most of them are rather larger in the female (where they serve for the attachment of the eggs between the times of laying and of hatching) than in the male.

The first pair, however, is quite vestigial in the female; whereas in the male it is specially enlarged, the exopodite is absent, and the endopodite is hard, unjointed, and grooved. The second in the male is large, has a small exopodite, and the basal joint of its endopodite resembles the appendage in front. These grooved appendages serve to transmit the seminal fluid from the male genital apertures to the ventral surface of the female.

The last pair of abdominal appendages has small protopodites, and broad flattened exo- and endopodites, the former of which alone are two-jointed.

§ 9. Articulations and Tendons. It will now be well to get a clear idea of the structure of the articulations between the various movable sclerites that make up this skeleton. Any one of the articulations of the walking-legs or pincers (fig. 151) will do to illustrate this. If we examine such a one we find that the area of soft cuticle between the two sclerites is divided into two by the approximation of the sclerites to one another at two opposite points, where one has a rounded peg-like projection which fits into a socket of the other. 
By the presence of these two peg-and-socket articulations, motion of the two sclerites relative to one another ean only take place about one axis-that which passes through both articulations. Thus the range of motion at any one articulation is much less than at one of the articula. tions of the human arm; but this is made up for by the greater number of artioulations, so that after all a erizyish can grasp its food with its pineers quite as quickly and certainly as a human being. If we examine the two areas of thin cuticle we shall see in the centre of each a narrow transverse groove: this marks the point where an invagination of the cuticle (and the epidermis with it) oecurs, for the purpose of giving

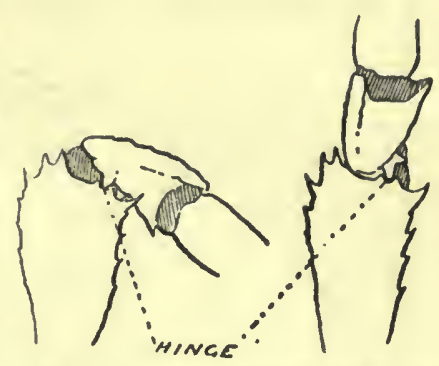

Fig. 151,-An Articulation in THF: Cray Fish.

The soft cuticle is shaded. Flexion, left; Extension, right. a larger surface of attachment to the museles. Ingrowths of the exoskeleton for museular attacliment are characteristic of the elass Arthropoda, to which our crayfish belongs. When, as in this ease, they are thin, flat, flexible plates they are called tendons (they are of course analogous to, but not in the least homologous witb, the tendons of a Vertebrate, which are mesoblastic connective tisssue, not epiblastic cuticle). There are thus two tendons at each articulation (fig. 152). If one is pulled by the contraction of the muscles attached to it the corresponding soft area is wrinkled up and the two sclerites are brought nearer one another on that side. When the other tendon is pulled, they are brought nearer on the other side. The articulations are so arranged that bending (flexion) can take place on one side only: one set oi muscles

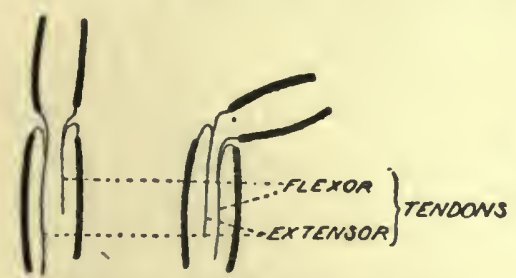

Fig. 152.-SECTION THROUGH AN ARticulation.

Flexion, right ; Extension, left. (flexcors) acting on their tendon cause the flexion; while the contraction of the other set (extensors) pulls the limb straight again (extension). In the chelate joints (see $\S 6$ ) the extensors beeome adductors (bringing the movable claw into contact with the fixed one), and the flexors, abductors.

The motions of the abdomen are of just the same kind, only here the terga overlap or telescope when extension occurs, while the sterna are brought nearer together and the soft areas between them wrinkled, when flexion takes place and there are no tendons. The arrangement of the extensor and flexor muscles is shown in fig. 153, and a 
comparison of that figure with fig. 154 will show the nature of the flexion and extension of the abdomen.

$\S$ 10. Endophragmal System. A further development of the plan of

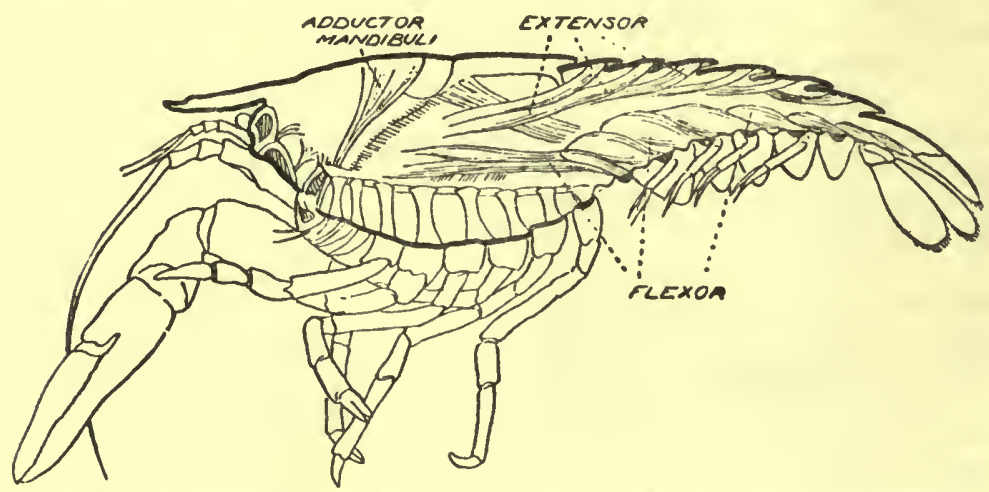

Fig. 153,- Median Section of Crayfish, witu Abdomen Exiended

To show flexor and extensor muscles.

exoskeletal ingrowths for muscular attachment is seen in the thorax, where a complicated series of hard arches is formed in this manner, constituting what is known as the endophragmal system (fig. 154). This is arranged as follows.

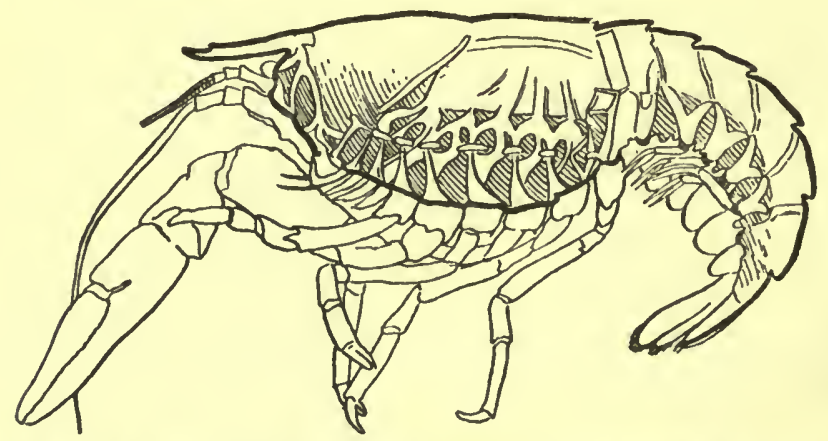

Fig. 154.-Median Sfction of Crayfish, with Abdomen Flexed.

To show endophragmal system.

From the side-wall of the thorax a paired metameric series of ingrowths (endopleurites) passes inwards towards the middle line, forking in a $Y$-shaped manner, the ends of each $Y$ being in contact with corresponding ends of the next $Y$ in front and behind. At the same point of contact they are joined by the top of one of 
another paired metameric serics of ingrowths (endosternites) which arise nearly vertically from the sterna. Each endosternite further sends in a process to meet one from its fellow in the middle line, a series of arches being thus formed, which cover in the thoracic part of the central nervous system.

\$11. Ecdysis. The whole of the exoskeleton, including tendons and endophragmal system, being a cuticular product, containing no cells, and bcing largely calcified, is incapable of growth, and hence a periodic moulting or ecdysis takes place-once a year, during the late summer, for adult crayfish, but more frequently in young ones. Not only is the whole outer shell, the tendons and endophragmal system shed, but even a large part of the alimentary canal has a cuticular lining, which goes also. The process is a very hard and exhausting one, and leaves the crayfish for some time in a very weak and defenceless condition, until such time as be has a new cuticle secreted and partly thickened. During this time crayfish hide in their burrows.

$\$$ 12. Alimentary Canal. The mouth of the crayfish is a rounded aperture; it is not defined by distinct lips, and the jaws are simply modified external appendages. A short œesophagus leads vertically dorsalwards into a large spheroidal sac, commonly called the stomach. As it is essentially a crushing organ, it is better spoken of as the gizzard or gastric mill. The whole of the esophagus and gizzard is lined by a cuticle continuous with that of the outer surface. In fact, they really represent a portion of the outer surface which has been tucked in during development. We have seen that a stomodoum, lined by epiblast, forms the first part of the alimentary canal in Vertebrates, and a similar proctodacum forms the anal portion. But whereas in Vertebrata these epiblast-lined portions are quite insignificant in comparison with the main hypoblastlined part of the gut (mesenteron), in the crayfish they together form at least nine-tenths of the length of the gut, and the mesenteron is quite small.

$\S 13$. The Gizzard. The cuticle of the gizzard is mostly thin, but eight areas of it are thickened and calcified: these are known as the "ossicles," and specially thick projections from them into the cavity of the gizzard are known as "teeth." All theso aro cuticular structures, and have no 
homology whatever with the like-named structures in Vertebrates. The eight ossicles form a sort of framework, capable of definite movements by which the three main teeth are brought forcibly together at one point, thereby effectively chewing up the food. The ossicles have been given names-the four median ones are, in order from in front back, the cardiac, uro-cardiac, pre-pyloric, and pyloric. Of these the pre-pyloric is doubled back on the

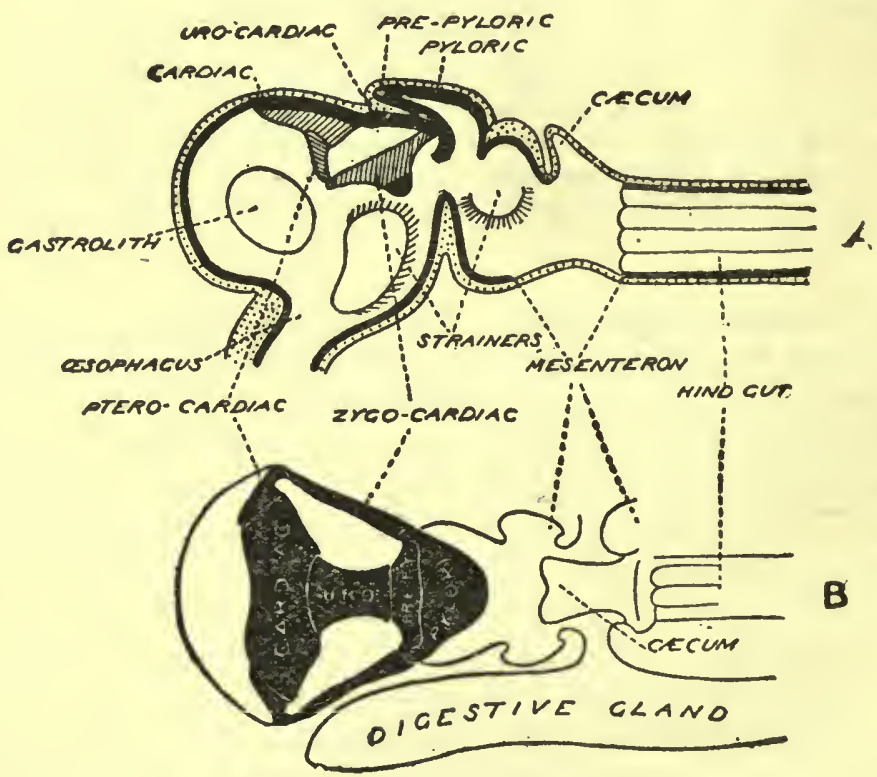

Fig. 155.-Gizzard of Crayfish.

A, median aection; $B$, dorsal view.

uro-cardiac, and from the acute angle between them projects the median tooth. The paired ossicles are the small ptero-cardiacs at either end of the cardiac, and the zygo-cardiacs stretching from these to the pyloric and bearing the lateral teeth. The exact arrangement ard working of this framework can only be fully understood by a study of the actual thing, though fig. 155 may help the understanding of them a little. As usual, two sets of muscles act in opposite ways on this mechanism : intrinsic muscles, in the wall of the gizzard itself, pull the cardiac 
and pyloric ossicles nearer one another, and so cause the teeth to diverge. More powerful anterior and posterior extrinsic muscles, running from cardiac and pyloric ossicles respectively to the tergum of the carapace, pull those ossicles away from one another and cause the three teeth to converge.

The main division of the gizzard, in which these ossicles occur, is called the cardiac* chamber. The hinder division is called the pyloric chamber, but it is hardly a chamber, as the walls are thickened and bulge in, reducing its cavity to a tri-radiate slit. The front end of this slit is fringed by a series of long setæ, which form a lattice-work strainer. preventing any insufficiently comminuted food from passing into the mesenteron.

It has already been mentioned that the whole of the cuticle lining the gizzard (ossicles and teeth included) is got rid of at every moult. As the moulting-time approaches, there are developed in the side-walls of the gizzard a pair of white, button-shaped masses of calcium carbonate called the gastroliths. These have nothing to do with the grindingup of food, but are simply a reserve-storage of calcareous material. Just before the moult they are set free into the gizzard and ground up, and their material is absorbed into the blood, to be presently used in the secretion of the calcified parts of the now cuticle.

§ 14. Mesenteron and Digestive G]ands. The mesenteron is a small chamber, with a median cæcum projecting up dorsally (fig. 155). Laterally there arise from it a pair of much-branched tubes, constituting the "liver" or digestive gland. These are the largest organs in the body, occupying an astonishing amount of space, and at first sight, in dissecting, look compact; but they are soon found to be merely masses of tubules loosely held together. They not only

* The application of the term "cardiac" to this organ is a reductio ad absurdum of the plan of naming parts of Invertebrate animals after the vaguely or fancifully corresponding parts of human anatomy. The "cardiac" part of the human stomach is so called because it is the part nearer the heart; in the crayfish the "cardiac" region is away from the heart. 
secrete the main digestive fluid, which has a similar universal digesting power to the rabbit's pancreatic juice; they also appear to absorb the digested food, and store up both oil and glycogen as reserves agamst the starving period that follows a moult.

\$ 15. The Proctodæum (hind-gut, or intestine) is long and straight. If it be true that all absorption takes place in the mesenteron or digestive gland, then the proctodæum can scarcely be said to have any function. It is lined by a

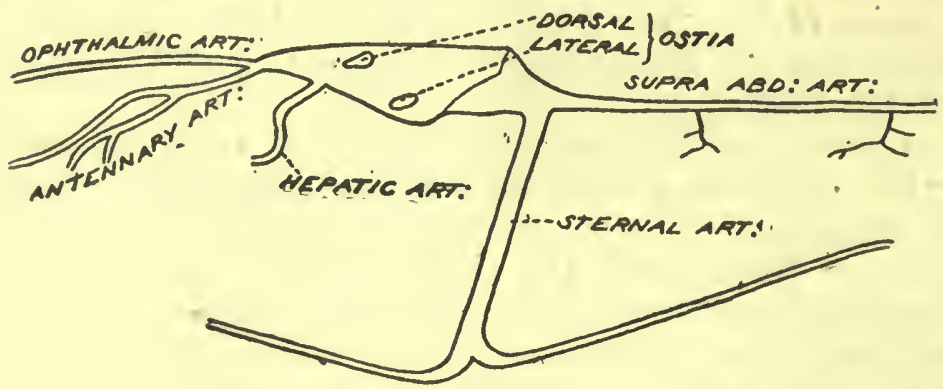

Fig. 156.-Heart and Arteries of Astaous.

Side view, (After Howes.)

cuticle thrown into longitudinal ridges which run in a very slow spiral.

§16. Vascular System-Heart. The crayfish has a well-defined heart, which, like that of the mussel, is systemic. In its structure, however, it differs from that of any other of our types. It lies near the dorsal side of the thorax, above and behind the mesenteron. It consists of a single muscular chamber, the walls of which are perforated by three pairs (dorsal, lateral, and ventral) of openings-the ostia (figs. 156 and 157). The cavity in which the heart lies is not a coelom, as might easily be supposed from dissection: it is a blood-sinus. When the heart dilates (i.e. at its diastole) blood rushes in from this pericardial sinus through the ostia; but these are valved so that when the heart contracts (systole) the blood cannot return, but is 
forced along the arteries. This "ostiate" condition of the heart is characteristic of the class Arthropoda: we may imagine its origin by supposing the enlargement of the main veins entering the heart until they come in contact with one another all around the heart. Then let their party-walls be absorbed, and an ostiate heart surrounded by a blood-sinus is the result. On this view the six ostia represent six veins entering the heart, and the last remnant

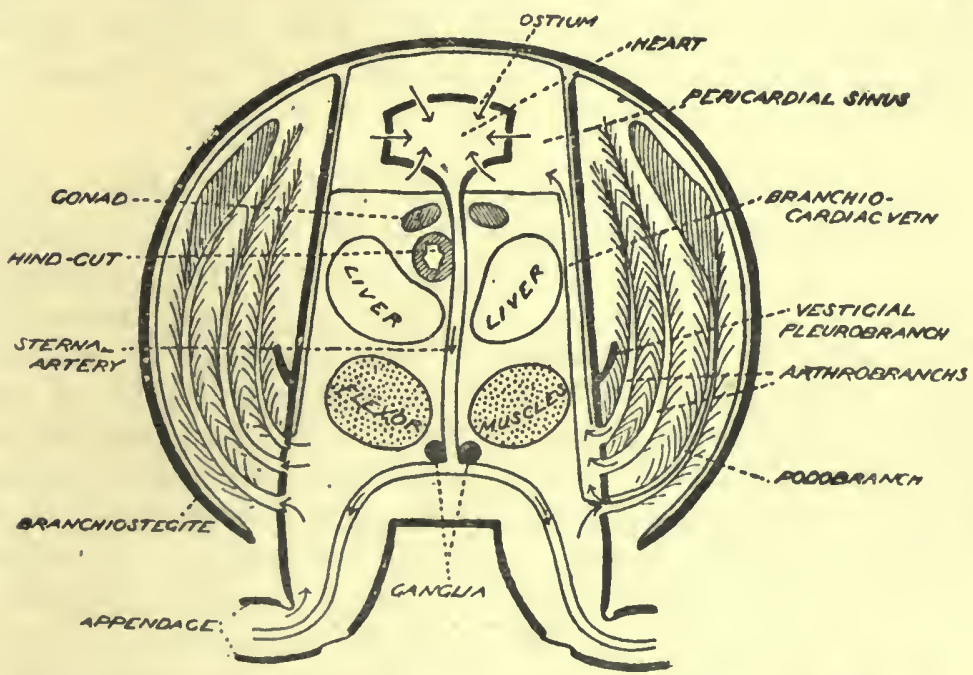

Fig. 157.-Diagramuatic Transverse Segtion of Trorax of Astacos.

The arrows show the course of the blood. (After Huxley.)

of their walls is seen in the strands of connective tissue which connect the heart to the sides of the sinus.

$\S 17$. Arteries and Sinuses. The arteries leaving the heart (fig. 176) are:-

(1) A median ophthalmic artery running forward to supply the front part of the gizzard, and adjoining regions.

(2) A pair of antennary arteries, supplying not only the antennæe, but almost the whole anterior portion of the body.

(3) A pair of small hepatic arteries, to the digestivo gland. 
(4) A median sternal artery, running vertically ventralwards and dividing into an anterior and posterior median ventral arteries supplying the ventral region of thorax and abdomen respectively.

(5) A dorsal abdominal artery, supplying the proctodxum and extensor muscles of abdomen.

From these arteries the blood passes into the capillaries of the various tissues, and thence into large venous sinuses, of which the main one lies along the ventral surface. From this sternal sinus it passes into the gills, and from thence back by a series of branchio-cardiac canals into the pericardial sinus.

\$ 18. Absence of Cœlom. The series of large venous sinuses containing deoxygenated blood, and the pericardial sinus containing oxygenated blood, constitute the anatomical "body-cavity" of the crayfish - that is to say, the cavity which serves in dissection as the limit between "alimentary canal " and "body-wall." But since it contains blood, and forms part of the vascular cavities, it is obviously a different thing from the cœlom of Vertebrates, or of the earthworm, or the pericardium of the mussel. The colom in the crayfish is very much reduced. It is represented by the cavities of the tubular gonads; the excretory organ and the genital ducts are cœlomic ducts, nephridia being absent.

$\S 19$. The blood is a colourless fluid, containing colourless amœboid corpuscles. Dissolved in the plasma is a faintly bluish compound called hæmocyanin: this has similar oxygen-carrying properties to hæmoglobin, and further resembles it in the fact that a metal-copper, not iron, however-is an essential constituent of it.

$\S 20$. Respiration. The gills (fig. 157) are vascular outgrowths of the body, on which the cuticle is very thin. Some arise from the epipodites of appendages: these are called podobranchs. Others are outgrowths from the soft articular region at the base of an appendage: these are called arthrobranchs. Others arise from the sides of the body above the articulation of an appendage: these we 
INVERTEBRATA.

pleurobranchs. The following table shows those appendages from or beside which gills are found :-

\begin{tabular}{|c|c|c|c|}
\hline APPENDAGE. & Podobraxchis. & ARTIIROBRANCIIS. & Pligurobrancis. \\
\hline $\begin{array}{l}\text { 2nd Maxilla } \\
\text { 1st Maxilliperle } \\
\text { 2nd " } \\
\text { 3rd " } \\
\text { Pincers ". } \\
\text { 1st Walking I,eg } \\
\text { 2nd " " " } \\
\text { 5rd " } \\
\text { 4th " }\end{array}$ & $\begin{array}{l}e p . \\
e p . \\
1 \\
1 \\
1 \\
1 \\
1 \\
1 \\
0\end{array}$ & $\begin{array}{l}0 \\
0 \\
1 \\
2 \\
2 \\
2 \\
2 \\
2 \\
0\end{array}$ & $\begin{array}{l}0 \\
0 \\
0 \\
0 \\
0 \\
0 \text { or } v . \\
v . \\
v . \\
1\end{array}$ \\
\hline Total . & 6 & 11 & $1+2$ or 3 \\
\hline
\end{tabular}

ep. signifies an epipodite without a podobranch.

v. aigmifies a vestigial pleurobranch.

Thus there are in all eighteen functional gills and two or three vestigial ones on each side.

In structure the arthrobranchs and pleurobranchs are similar and simpler than the podobranchs. Each consists of a vertical axis from which a number of flaments come off all round, so that the whole gill looks like a test-tubebrush. Oxygenation of the blood takes place in the filaments. There are separate afferent and efferent channels in the axis.

The vestigial pleurobranchs consist of a short axis only, without filaments.*

The podobranchs arise from oral plate-like epipodites. Each has an axis like the other gills, and filaments come off from the anterior face of this axis; but posteriorly there comes off a double membrane, the two folds of which diverge to embrace the front of the next podobranch behind (figs. 150 and 157).

The whole series of gills, as well as the epipodite of the first maxillipede and the scaphognathite of the second maxilla,

* In the lobster, which closely resembles the crayfish in all respects, all four pleurobranchs on eacls side are functional. 
are enclosed in a sort of gill-chamber by the branchiostegite. During life the scaphognathite is continually working backwards and forwards in a rapid and vigorous manner, and so producing a current of water forwards through the gill-chamber. Thus fresh oxygen is continually being brought in solution, and the carbon dioxide carried away:

§ 21. Renal Excretion-Green Glands. The renal-excretory organs occupy a position not seen in any of our other types, viz. in front of the mouth. There is a single

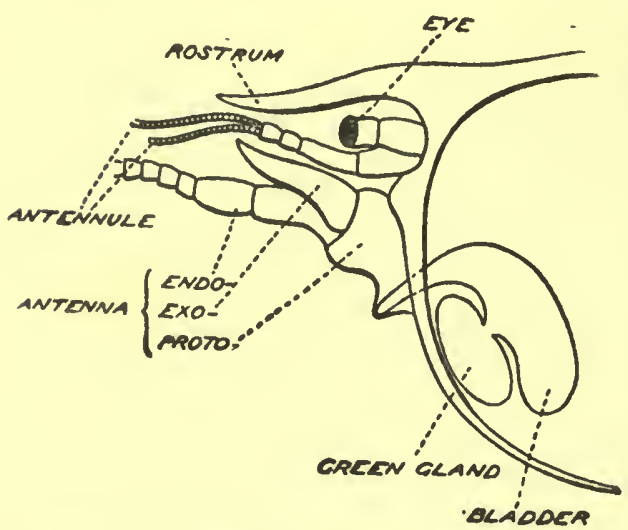

Fig. 158.- Green Gland and Neighbourina Parts of Astacus.

The protopodite of the antennule should have three oints, not four.

pair, each consisting of a gland, green in colour (when fresh), from which a thinwalled, distensible, bladder - like tube leads to the external opening on the "papilla of the green gland" on the basal joint of the antenna (fig. 158).

\section{$\S 22$. The Nervous} System (fig. 159) resembles that of the earthworm more nearly than any other we have seen. It consists of a pair of cerebral or supra-œsophageal ganglia in an antero-dorsal position, a metameric series of ganglia along the ventral side of the thorax and abdomen, the first of which is united to the cerebral ganglia by a pair of circumosophageal commissures like those of Lumbricus. But the ventral part is not a continuous nerve-cord as in Lumbricusit is a chain of separate ganglia, joined by longitudinal commissures. It shows (as does that of Lumbricus in its development) a distinctly paired structure, as though two lateral series of ganglia had fused in the middle line. This is most clearly seen in the hind part of the 
thorax, where the sternal artery is; this artery has to get to the ventral side of the nerve-chain, before it divides into anterior and posterior branches, and it is enabled to do so by the nerve-commissures diverging slightly on either side of the middle line to which the artery keeps.

The metamerism of the ner'vechain is less perfect than that of the appendages, owing to the fusion together of what we may suppose to have originally been distinct pairs of ganglia. 'Thus while there are nimeteen pairs of appendages, there are only fourteen pairs of ganglia. Of these, the celebral send motor fibres to the muscles of antennules and antennæ, and must therefore include representatives of the ganglia of the first two segments, which have shifted forwards in front of the mouth. Behind the mouth is a subœsophageal ganglion, from which motor fibres pass to the muscles of the mandibles, first and second

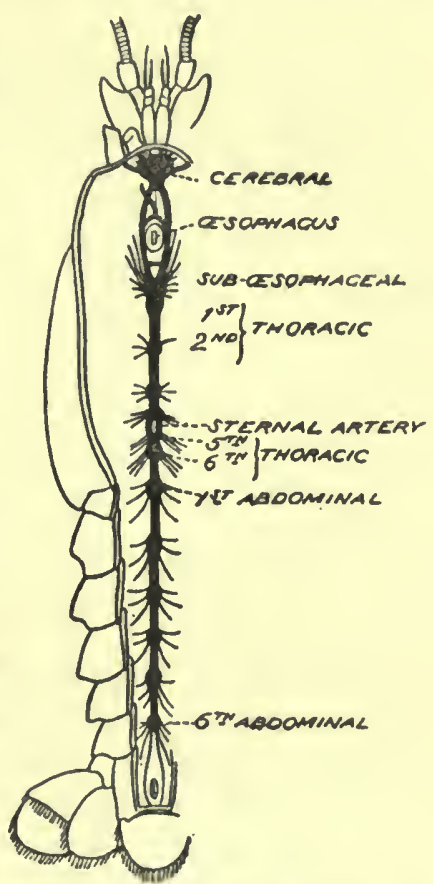

Fig. 159.-Nervous BYstem of Astacus.

(After Huxley.) maxillæ, and first and second maxillipedes. Here then we apparently have five pairs of ganglia fused together. Behind this there is one ganglion-pair to each segmentsix thoracic and six abdominal. In the thorax the ganglia are protected by the endophragmal arches, but in the abdomen they lie just ventral to the flexor muscles.

We see here a phenomenon similar to what we have seen already in Vertebrata-the tendency for the anterior somites of a metameric borly to become integrated or united in a compound structure in which the metamerism is traceable only with difficulty (compare the head of a Craniate Vertebrate with that of Amphioxus). The study of a few other arthropod types would make this point clearer to tho 
student. We may just mention here that in the crab this concentration of ganglia has gone farther, and we have only two ganglionic masses - the cerebral and a large thoracic mass representing all the rest fused together.

The ganglia contain unipolar cells almost exclusively. From the single process of the cell comes off both axis-cylinder and a series of dendrons. The axis-cylinders convey efferent impulses only (including those from one ganglion to another as well as to muscles). It would seem that the fibres distributed to muscles include not only motor fibres, but also inhibitory fibres, as is the case only with the splanchnic muscles in Vertebrates. The sensory fibres seem to originate, as in the earthworm, from cells in the sensory epithelium itself.

In function, the cerebral ganglia so far resemble the Vertebrate brain that they control the reflex action of the other ganglia (as has been proved by the actions of crayfish in which the cerebral ganglia have been destroyed). Undoubtedly in its nervous system the crayfish shows a great advance on any of our other Invertebrate types-an advance necessary to its more active existence. But there is no satisfactory evidence that it has any such powers as memory, association of ideas, etc., or any other of those mental powers without which we can scarcely speak of consciousness as existing.

\section{$\S 23$. Sense Organs.-The Eyes are borne on short} movable stalks, and lie between the antennules and the rostrum. In structure they differ from the Vertebrate eye as completely as any two organs fulfilling the same function very well could. In the first place the eye is a practically solid organ, devoid of the large "chambers" of a Vertebrate eye. Then it is compound, i.e. composed of a number of elements each physiologically com-

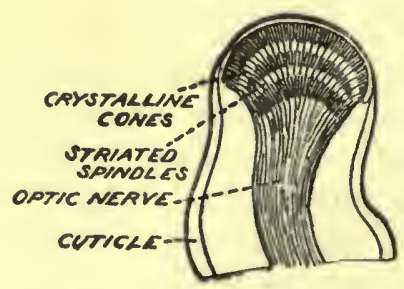

Fig. 160.-Median Section THROUaH EYE OF CRAYFisH.

(After Huxley.) plete in itself; and lastly, its sensory. cells (rods and cones) are nearer the light than the nervefibres.

The "elements" of the eye are arranged in a radiating manner, as shown in fig. 160. Each one (fig. 161) consists of (1) a corneal facet, sometimes called a lens, though its refractive power is doubtful: this is simply a polygonal area of the ordinary cuticle, slightly thickened.

(2) A crystalline cone, formed by the secretion of a clear substance by four adjacent 
cells, whose nuclei lio close under the corneal facet. These cells are simply ordinary epidermal cells modified. Next comes a group of cells called a retinula, surrounding a central rhabdom, which is spindle-shaped, and shows peculiar transverse striations (hence called striated spindle). This rhabdom is really made of four rod-like bodies closely apposed. The base of each rhabdom is surrounded by a process from a nerve-cell. These nerve-cells form a mass at the base of the eye (optic ganglion) and send their axis.cylinders into the cerebral ganglion.

Each nervo-element is separated from its neighbours by tissue full of dense pigment which effectively screens off all

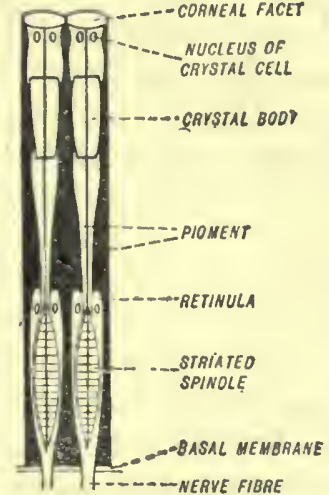

Fig. 161.-Two Elements of the Crayfish's Eye,

(After Huxley.)

light other than that falling directly normal to the surface of each particular corneal facet. Each element thus subtends a particular sector of the field of vision, and an imago of each such fraction may be said to be separately focussed by each particular crystalline cone on to each retinula. But the "image" so projected might as well be a mere spot, for" it can give rise to only a simple sensation of varying intensity. But the whole series of such spots put together would make an image differing from the image on a Vertebrate retina much as the familiar stippled "halftone" photo-engraving differs from a real photograph. Moreover, the compound image would be erect, not inverted as is that on the Vertebrate retina. In practice the crayfish's eye is quite as good as he needs, for it guides him in seizing an object with the greatest precision.

$\S 24$. Other Sense Organs. On the dorsal surface of the basal joint of each antennule is the opening of a little sac, lined by a continuation of the external cuticle. The cavity of this sac contains a gelatinous fluid into which project setæ, 
furnished each with a nerve-ending. In the gelatinous fluid are solid particles-grains of sand and other foreign particles presumably wilfully placed there by the crayfish itself. The analogy of this structure to the Vertebrate ear in a simplitied condition is striking, - that also originates as an open sac, it contains sensory "hairs," and otoliths in a gelatinous fluid. Hence this organ has been universally termed the auditory organ of the crayfish. But when we remember that the Vertebrate ear has the function of equilibration as well as that of hearing, we must allow the possibility that this sac in the crayfish may have the former function as well as, or even without the latter.

Many setæ on the appendages, especially the antennules and antennx and the mouth-appendages, are tactile; and there are certain peculiarly shaped setæe on the antennules which are supposed to be sensitive to odours.

§ 25. Reproduction. As we have already seen, the sexes are distinct, and have externally-visible differences. The gonads of both are similar in shape and position. It is

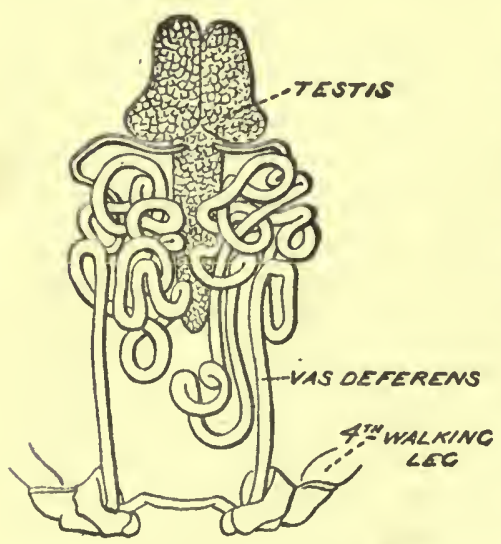

Fig. 162.-Rephoductive Organs of male Crayfisir.

(After Huxley.) hard to say if they should be called median or paired, each being a hollow sac, paired in front and median behind (figs. 162 and 163). It lies between the hear't and the proctodæum. 'The ducts are paired and quite continuous with the gonads, from which they come off on either side at the junction of the median and paired portions. The oviduct is thinwalled, short and straight, running down to its opening on the basal joint of the second walking-leg. 'I'he vas deferens is long and coiled, and opens at a similar point on the fourth walking-leg.

The spermatozoa are unique among those of our types in 
having no moving flagellum. Instead there is \& radiating series of stiff spokes (fig. 164). The ova are large (about

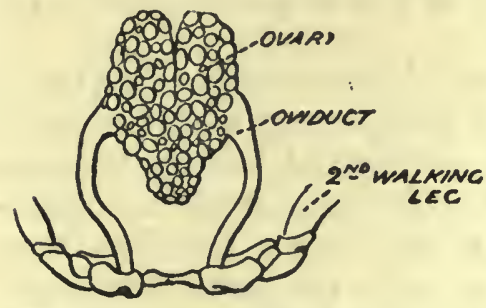

Fig. 163.-Reproductive Organs of Female Crayfish.

(After Huxley.) $\frac{1}{8}$ inch diameter), containing much yolk: they are red in colour when ripe, but vary from white and yellow to purple during development; so that the ovary is usually a striking object in dissection. The seminal fluid, largely secreted by the walls of the vas deferens, is white and - sticky, and is smeared by the male's first abdominal appendages over the abdominal sterna of the female. When the ova are laid they are attached to the swimmerets, and now fertilization is effected.

Segmentation is centrolecithal, i.e. the yolk forms a central mass, around which the nuclei derived by division of the first segmentation-nucleus are arranged. Hypoblast is formed by an invagination on what will be the ventral surface, and on this surface the main part of the embryo develops (contrast the dorsal development in the chick). There is no free larval stage, although in most marine Crustacea such stages occur. Details of the development, without comparison with other Crustacea, would be of little interest. The young when hatched are very like the parent: they cling to the mother's swimmerets persistently, until their first moult,

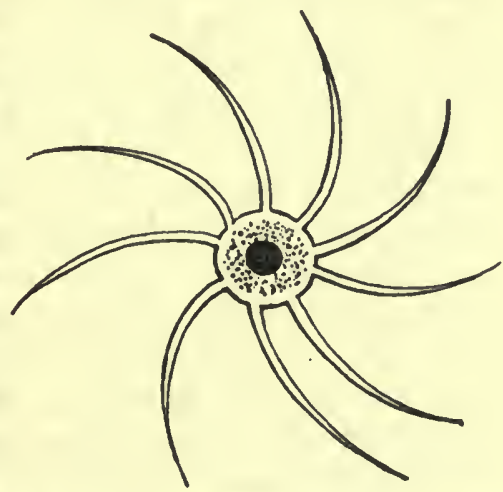
after which they do so only Fig, 164,-Spermatozoon oy Craytish. when alarmed. During the first year, when growth is rapid, moulting recurs two or three times.

$\S 26$. Histology. Two correlated points in the histology ZOOL. 
of the crayfish are of great interest, One is the complete absence of ciliated epithelium; the other is the high development of the muscular tissue, all of which is striated.

$\S 27$. Classification. The crayfish is obviously allied to the various marine lobsters, shrimps, and prawns, and less closely to the crabs. These constitute the highest order of the class Crustacea, called Decapoda from their possessing ten conspicuous limbs, viz. the pair of pincers and four pairs of walkinglegs. The Decapoda, together with the wood-lice, sandhoppers, and less familiar forms, form a division of Crustacea with a fixed number of segments, viz. 19; whereas the humbler water-fleas, barnacles, etc., have no such constant number. All these forms constitute the class Crustacea. Its nearest allies are probably the king-crabs, scorpions and spiders constituting the Arachnida; and less nearly, the Centipedes and Insects. The whole of these constitute the phylum Arthropoda, characterized by the jointed appendages, peculiar ostiate heart, absence of ciliated epithelium, and certain other points.

Comparing the crayfish with our other Invertebrate types, we at once think of the similarity in the metamerism and nervous system to the earthworm. But the wide differences in the cœlom and vascular system, excretory and reprodućtive organs show how great a gap there is between them.

Looking back on our Invertebrate types, the student will realize that they do not form a homogeneous assemblage, united by close relationship, as do the Vertebrates. In fact, the historical division of the Animal Kingdom into Vertebrata and Invertebrata can no longer be upheld as a natural one. As we have seen, the primary division of Animals must be into Protozoa and Metazoa. The Metazoa again must first be divided into animals with two germinal layers, or Colenterata, and those with three germinal layers, termed Cœlomata (not a very happy term, since not all of them possess a cœlom). After this no such sharp divisions in classification can be made. While among Colomata at least five well-defined and important phyla can be recognized-viz. Echinodermata, Mollusca, Chrtopoda, Arthropoda, and Vertebrata-no one of these, taken as a whole can 
safely be said to represent a lower grado of development than any other, or to stand towards it in anything liko the position in which, for example, one class of Vertebrata does to another. Moreover, these five great phyla are far from exhausting the list of Colomate animals. Numerous other groups-some represented by a wide and varied series of forms, others by very few or even by a solitary speciesremain to puzzle the morphologist. If we accept the doctrine of a common descent for all animals, we must admit that only the most fragmentary and uncertain evidence exists as to the way in which the ancestors of the great groups diverged from one another. 
Chapter XXIV. -The Fresh-water Mussel.

\section{(Anodonta cygnaea.)}

\$1. Mode of Life. There are several species of fresnwater mussel, that known as the Swan Mussel (Anodonta cygncea) being the commonest. In its mode of life it shows a very close resemblance to Amphioxus, for it lies habitually buried in the mud, with only a small portion protruding. At this protruding end are two openings close together (figs. 165 and 166); if a little colouring-matter is

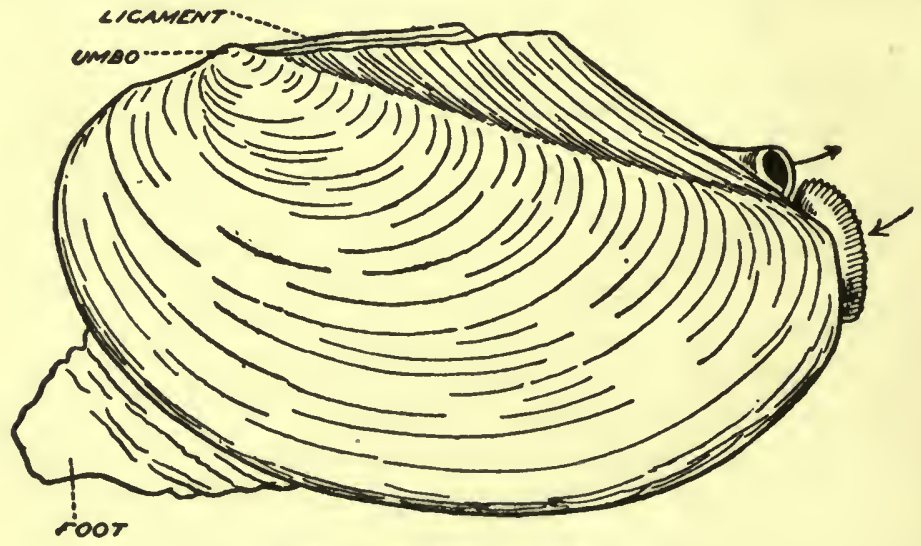

Fig. 165.-Side VIEW OF ANOdONTA.

(After Howes.)

put in the water near by, it can be seen to flow in at one of these openings (inhalent); from the other an outward current flows (exhalent opening). These constant currents are maintained by ciliary action as in Amphioxus, the inward current taking in both food and oxygen, the outward taking out all waste products. Here again we shall find a filtering apparatus by which the excess of water is allowed to escape and oxygenate the blood as it does $\mathrm{so}_{2}$ while the 
solid particles are carried on to be digested. But closer examination will show, beneath this superficial resemblance, very deep divergences of structure. Resemblances of this kind, due to adaptation of primarily

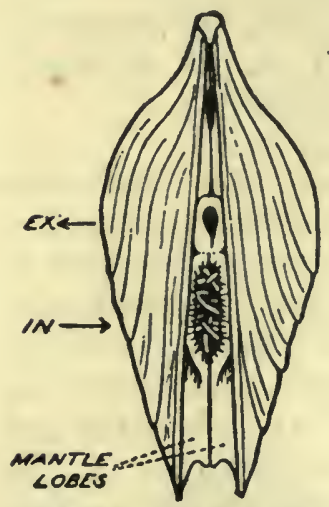

Fig. 166. -ViEw of Pos. TERIOR END OF ANODONTA. (After Howes.) distinct organisations to similar modes of. life, are said to show homoplasy, in contradistinction to resemblances such as we have found between the dogfish and frog, for example, where primarily similar organs have been adapted to different modes of life. Homoplasy may be defined as the resemblance between two animals due to a similar combination of analogous but not homologous structures.

§ 2. Shell and Mantle. The body of Anodonta is always for the most part hidden, and can be entirely hidden, in a shell. This shell represents a portion of the cuticle which the epidermis (as in the earthworm) everywhere secretes-a portion which has been greatly thickened and rendered rigid by impregnation with calcium carbonate. The shell consists of two valves, occupying symmetrical right and left positions, ending along the anterior, posterior, and ventral edges in an apparently free margin (though really continued over the rest of the epidermal surface as a delicate cuticle) and united along the dorsal margin by a leathery, elastic portion of the cuticle (called the elastic ligament), which tends, by its elasticity, to make the valves diverge from one another (fig. 165). While the animal is alive this tendency is counteracted, partly or fully, by the contraction of two adductor muscles which run from one valve to the other and keep the ligament in a state of tension. After death, when these muscles relax, the valves gape apart.

The shell is secreted for the most part by the epidermis of a pair of muscular folds constituting the mantle, which grow down from the dorso-lateral region of the body and cover in the sides and ventral region of the animal, some- 
what after the manner of the atrial folds in Amphioxus, though unlike those they do not fuse in the ventral middle line. Thus there is enclosed by these folds a mantlechamber, and it is into and from this that the watercurrents already mentioned flow. It is a comparatively large chamber, so that the actual body of the animal is much smaller than one might at first imagine.

§ 3. General Structure of Body. Fig. 168 (transverse sections) will give an idea of the size of the mantle-chamber and of the true body; while fig. 167 shows a side-view of the body and indicates the chief regions into which it may be divided. Anteriorly we have a region composed entirely of muscle-the anterior adductor. Behind this comes the great visceral mass in which most of the digestive and reproductive systems are contained, the cavities of the latter being part of the cœlom. From the ventral surface of the visceral mass there is developed a median muscular mass - the foot-the only locomotor organ of Anodonta. Postero-dorsally lies the pericardial region, also containing a cœlomic cavity. In this region we have part of the alimentary canal, the heart and related blood-vessels, and the excretory organs. Finally, at the extreme posterior end is another muscle-the posterior adductor.

§ 4. Gills and Labial Palps. Certain outgrowths from the body-wall occupy a good part of the mantle-chamber. The disposition of these structures may be understood from a careful comparison of fig. 161, which is a diagrammatic side-view, with the series of transverse sections given in fig. 162.

There are four * gills in all, distinguished as inner and outer of the right and left side respectively. Each gill is further composed of two plates or lamellæ, united along their ventral border and at both ends, so that they form a pouch whose opening is directed dorsalwards. The dorsal

* Strictly, there is a single pair of ctenidia, each ctenidium having an axis and two plates, which latter are doubled back on themselves. But in the absence of any other Molluscan type to compare with, the terms given in the text are sufficient. 


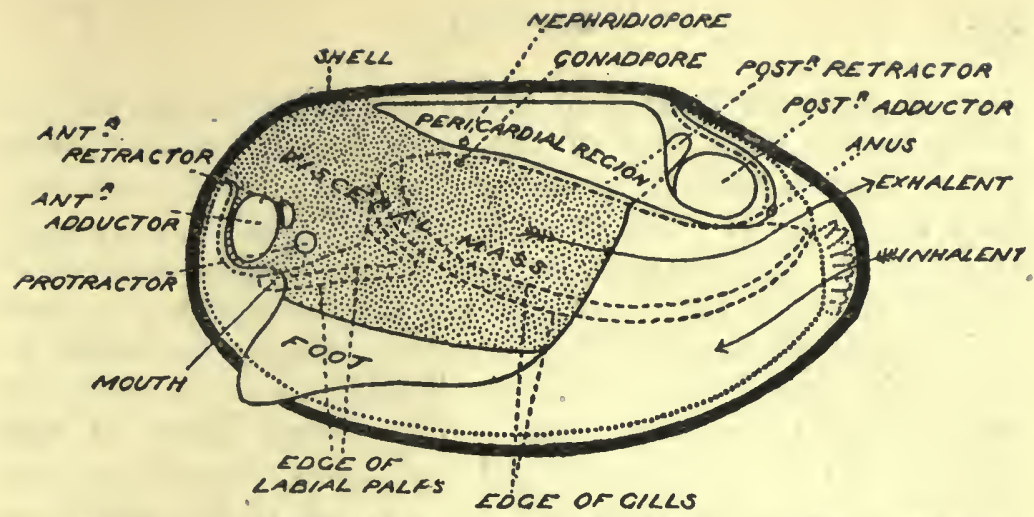

Fig. 167.-Side View of Avodonta After Removal of Letr Valve.

Diagrammatic. The dotted line is the free edge of the mantle, the dot-and-dash line its ine of attachment to the body.

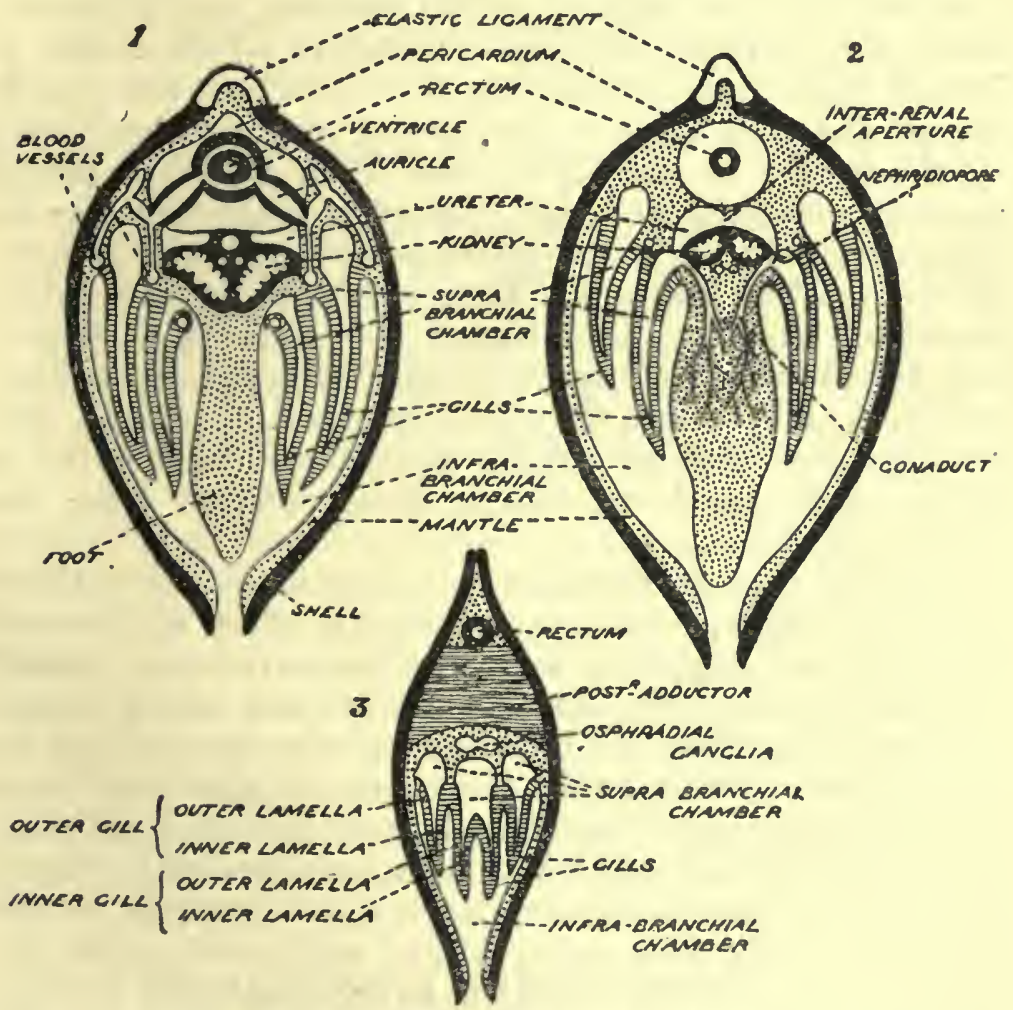

Fig. 168.-Transverse Sections of Anodonta.

(1) Through centre of pericardium. (2) Throngh anterior end of pericardium

(3) 'Ihrough posterior adductor. (After Howes.) 
edge of the inner lamella of each outer gill is united for its whole length with that of the outer lamella of the inner gill, so that in transverse section the two gills of each side show a W-shape. The outer lamella of each outer gill is attached to the mantle for its whole length, while the inner lamellæ of the inner gills are united together in the middle line posteriorly, but separate farther forwards to run right and left of the animal's body, to which they are attached at their front ends. The united edges of the inner lamella of the outer gill and outer lamella of the inner gill are also attached to the side of the body in front. By these various unions of the gill-lamella, the mantle-chamber becomes incompletely divided into two9. larger ventral or infra-branchial chamber, and a smaller, more dorsal supra-branchial chamber, which latter is further imperfectly divided into four chambers, corresponding to the four gills. The former may be open freely by the gaping of the valves, and when they are drawn together communicates with the outer water by the inhalent aperture; the latter communicates with the exterior by the exhalent aperture. The mouth lies in the infrabranchial chamber, just behind the anterior adductor muscle: the anus, the excretory apertures, and the genital apertures all open into the supra-branchial chamber. The posterior, undivided part of the supra-branchial chamber is often called the cloaca, though quite different from the Vertebrate cloaca.

We must next realize that each lamella is not really a continuous plate, but is a numerous series of vertical filaments, each supported by two chitinous rods and covered by ciliated epithelium. These filaments are united to one another at very frequent intervals, so as to form a sieve or lattice-work. Besides this, there are connexions between the two constituent lamelle of each gill, and in these inter-lamellar connexions run blood-vessels (fig. 169). These inter-lamellar connexions are much more abundant in the Inner gills than in the outer, so that while each outer gill contains a large cavity (in which, in the female, the developing embryos are protected), the cavities of the inner gills are reduced to a spongy condition. 
We can now more fully realize the method of life of Anodonta. The cilia which abound on the gills and inner surface of the mantle produce a constant current inwards through the inhalent aperture. This aperture is fringed with sensory tentacles, so that if there is any obnoxious substance in the water the shell can be tightly closed and no water allowed to enter. Normally, however, the water brings in oxygen and food (microscopic plants and animals). Most of the water is strained through the gills, exchanging its oxygen for the carbon dioxide of the blood as it does so, and then as it is swept outwards along the supra-branchial

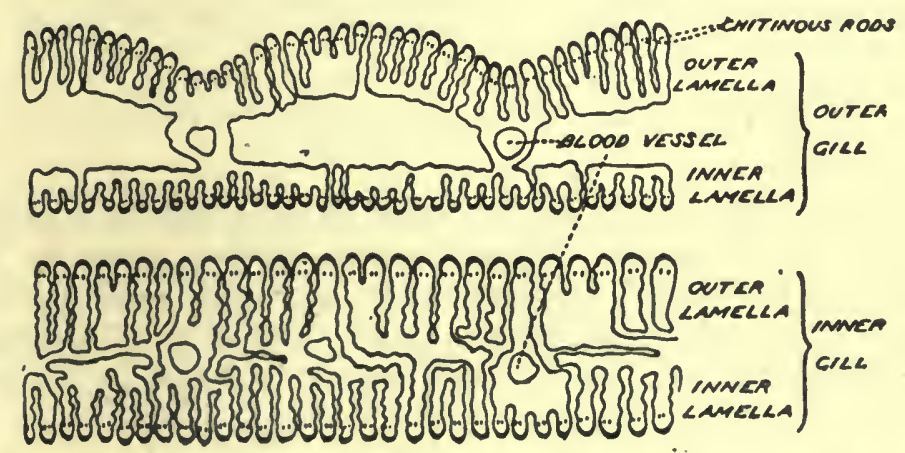

Fig. 169.-Section in a IIorizontal Plane across thr Two Gills of ONe SIDE or ANOdONTA.

chambers it carries off to the exhalent aperture the products of excretion, frces, and (in the mature male) the spermatozoa. Meanwhile the rest of the water, with the solid particles that could not pass through the gills, is carried forwards to the mouth. 'This is effected by the cilia on the labial palps. These are two pairs of lamellæ, not unlike the gills, but smaller and simpler in character. They lie in front of the gills on either side, and the corresponding right and left ones unite anteriorly, the mouth lying between the two.

$\S$ 5. Alimentary Canal (fig. 170). The mouth is a simple opening, without lips or jaws. It leads by a short œsophagus to an expanded stomach. Into this there open, 
right and left, the ducts of the liver, as the sole digestive gland is called; this is a semi-compact gland which closely surrounds the stomach. From the ventral side of the stomach comes off the in. testine, which, after several coils in the visceral mass, turns dorsalwards and runs nearly straight(hence it is here called the rectum) in the middle line through

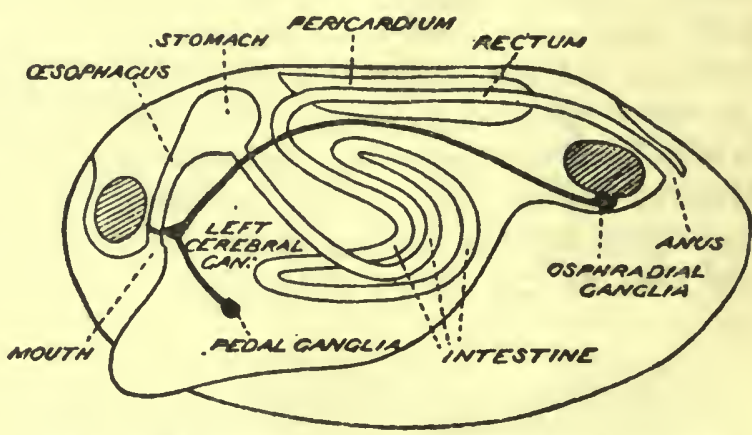

Fig. 170.-Almentary Canal and Nervous Sx8tem OF ANODONTA.

the pericardium (cœlom) and dorsal to the posterior adductor muscle to the anus. These various names given to the parts of the canal indicate only a vague analogy, not homology, with the similarly named parts of a Vertebrate. The whole of the alimentary canal is lined by ciliated epithelium.

§ 6. Circulation. The heart of Anodonta consists of a ventricle and two auricles: verbally, the same is true of the frog, but there is no real resemblance between the two. Both auricles here are alike - the presence of two is merely due to the bilateral symmetry of the body. The ventricle wraps round the rectum-a somewhat surprising position, until we remember that even in Vertebrates the heart arises as part of the wall of the alimentary canal. From the anterior end of the ventricle comes off an "anterior aorta," which runs on the dorsal side of the rectum and distributes blood to all the anterior part of the body. A "posterior aorta" similarly comes off from the posterior end of the ventricle and runs on the ventral side of the rectum. From the ultimate branches of these arteries the blood passes, not into proper capillaries, but into irregular spaces, whence it eventually finds its way for the 
most part into a largo longitudinal rein ("vena cava") just ventral to the pericardial cavity. From this it passes right and left, through the excretory organs (to be presently described), to the gills. The blood, thus purified, first of nitrogenous waste and then of carbon dioxide, passes into the auricles and so to the ventricle. The gills, however, are not the sole organs of respiration: the mantle is also adapted for this function, and the blood from it returns directly to the heart without passing through nephridium or gill.

In fig. 171 we have this circulation compared with that of the dogfish. It will be seen that whereas in the latter the heart contains deoxygenated blood, which it sends to
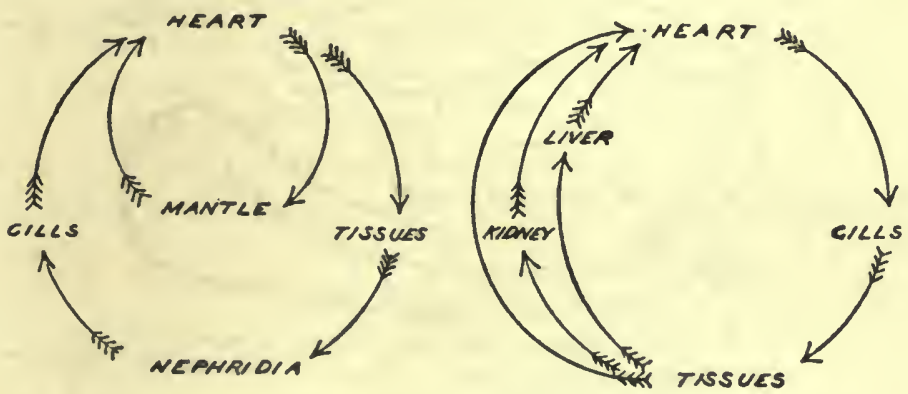

Fig. 171,-Comparison of Circulation in Anodonta (Left) and Scylliud (right).

the gills, the heart of Anodonta receives oxygenated blood from the gills. This difference is expressed by calling the heart of Scyllium a branchial heart, and that of Anodonta a systemic heart. The blood of Anodonta is colourless and contains colourless amœboid corpuscles.

$\S 7$. The Renal-Excretory Organs of Anodonta have none of the characteristics of nephridia, but are cœlomic ducts with excretory functions. They are often spoken of as the organs of Bojanus (fig. 172). They lie side by side just ventral to the pericardium, being partly separated by the vena cava, and partly by the posterior retractor muscle. Each is a tube doubled upon itself: the two limbs of the tube communicate at their posterior 
end, and at their anterior end they cross, the ventral one turning upwards to open into the pericardium, and the dorsal one turning down to open to the exterior in the supra-branchial chamber. This opening is called the nephridiopore. The ventral limb is brown, and its walls are glandular, hence it is often called the "kidney"; its nephrostome (or reno-pericardial pore) is ciliated and carries off the pericardial fluid ; as the wall of the pericardium is glandular in places (forming the so-called " organ of Keber"), it doubtless has an excretory function also. The dorsal limb of the nephridium is thin-walled and nonglandular, and often called the "ureter." Close to the

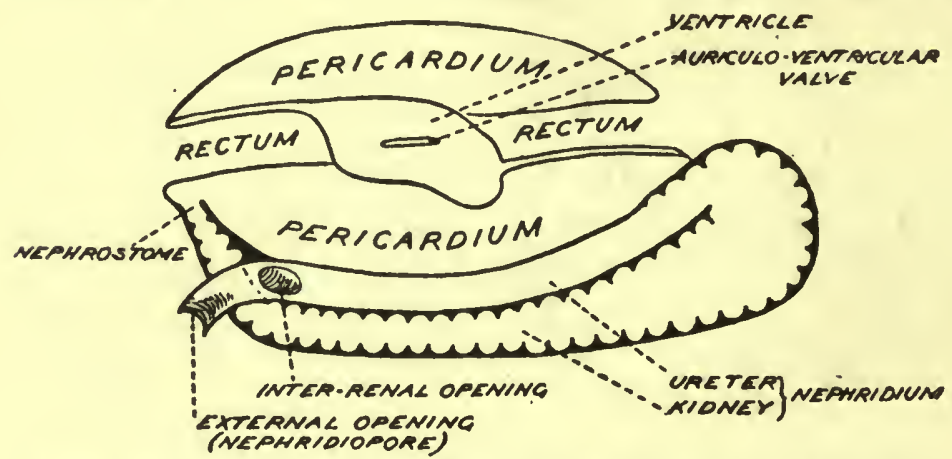

Fig. 172.-Diagrasi of Side Dissection of Nephridiom of Axodonta.

point where it opens to the exterior, it has a communication with its fellow of the opposite side ("inter-renal opening ").

$\S 8$. The Nervous System of Anodonta is very different from any we have yet seen. The central nervous system consists of three pairs of small ganglia, connected with one another by commissures consisting of nerve-fibres only (fig. 170).

One pair, commonly called cerebral, lies right and left of the mouth, the two being connected by a commissure in front of the mouth. From these ganglia motor fibres are given off to the anterior adductor and other muscles in the neighbourhood. 'They also send off commissures to the other ganglia of the same side. 
The pedal ganglia lie close together, just dorsal to the foot, to whose muscles they send motor-fibres.

The third pair, osphradial (or parieto-splanchnic) ganglia, are also close together, just ventral to the posterior adductor, which, as well as the posterior retractor and the inhalent and exhalent apertures, they innervate. The cerebroosphradial commissures run from the cerebral close under the skin until they reach the front end of the nephridium, when they approach the middle line, and run close together, diverging again to get round the posterior retractor. These commissures are shown in section in fig. 168 (1) and (2), and the osphradial ganglia in fig. 168 (3).

Sense-organs are very few, as might be expected in an animal shut up so completely in its shell. Doubtless the epidermis is generally sensitive where it does not secrete a thick shell, and the tentacles around the inhalent aperture are more especially sensitive to touch and odour. A special patch of sensory epithelium (olfactory?) occurs just over the osphradial ganglia; and in the foot there are a pair of little round sacs, containing liquid and a solid particle (otolith), which are generally assumed to be auditory organs. Their nerves run to the cerebral ganglia, in the cerebropedal commissures. There are no eyes.

It would seem that each ganglion has an independent power of reflex action; and it is very doubtful if any process in such an animal can claim to be anything more than a reflex action, simple or complex. Seeing how much simpler this nervous system is than that of the unintelligent dogfish, we cannot expect to find any trace here of memory or the capacity for education, and it is probable that the consciousness of Anodonta is of a most rudimentary description.

§ 9. Reproduction. The gonads of Anodonta resemble diffuse glands in structure, being tubular and quite continuous with their ducts. They are paired, and the ducts open on either side close to the nephridiopore, into that portion of the supra-branchial chamber which is enclosed between the two lamellæ of the inner gill (fig. 168 [2]). Ovary and testis are exactly alike until examined micro- 
scopically. They are found in separate individuals. The ova and spermatozoa when ripe pass into the suprabranchial chamber; in the case of the male, the spermatozoa pass out into the water, and by good luck some of them may be carried into the mantle-chamber of a female. Here the ova are fertilized, and here-more precisely, between the lamellæ of the outer gills - they remain until they have reached a larval stage of development known as the glochidium (fig. 173). This larva has a bivalved shell, very different in shape from that of the adult;

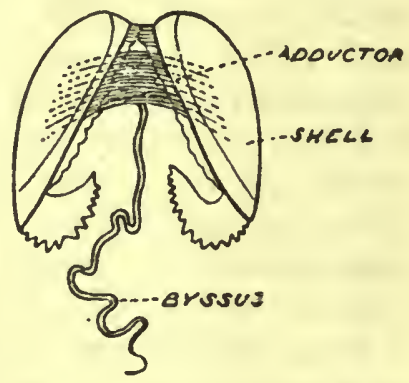

Fig. 173.-A GLOCHIdIU⿴囗 there is only one adductor muscle, and the intestine is spirally coiled. Each valve has large recurved teeth, and an epidermal gland secretes a long adhesive filament, the byssus. The glochidia are retained in the " brood pouches" (the outer gills) until a stickleback or other suitable fish approaches the mother. They are then discharged into the water, and some may attach themselves to the fish, first by the byssus and afterwards by the valve-teeth. The irritation set up leads to a growth of the skin of the fish around the glochidium, which, thus protected, goes through a metamorphosis, and when it drops out it is a miniature adult. This temporary attachment to an actively swimming fish secures the distribution of the young mussels up stream as well as down, and insures them to some extent against the danger of being washed out to sea-a danger to which all fluviatile larvæ are liable.

\$10. Classification. The mussel is a type of the phylum Mollusca, characterized among other things by the ventral muscular foot, the mantle-chamber with its gills, the dorsal systemic heart, reduced colom, ganglionic nervous system, and absence of metamerism. It belongs to the class Lamellibranchia, or Pelecypoda, which also includes the oyster, cockle, scallop, and similar forms with bivalved shells. The study of its structure would be much 
more interesting and instructive if it were compared with that of members of other Molluscan classes, such as the snail, whelk, or cuttle-fish. Here we can only consider it as illustrating a type of structure so utterly different from that of any Vertebrate that only by contrasting both with that of some form like Hydra can we find points of agreement between the two. If Mollusca and Vertebrata have had a common ancestor, it must be in the immensely distant past. And indeed we find that whereas the whole history of Vertebrate development is recorded for us in the rocks, in the earliest rocks in which fossils have been found there are Lamellibranchs closely allied to some of the most primitive Lamellibranchs of the present day. The divergence of the Lamellibranchs from the other classes of Mollusca must have taken place at a still earlier period, and that of the primitive Mollusca from the primitive forms of other groups much earlier still.

There can be little doubt that the most primitive Mollusca were much more active organisms than Anodonta, which affords us an example of a degenerate group-one in which specialization, in adaptation to a simple mode of feeding, has involved a general simplification of structure instead of a general elaboration. It is probable that Hydra is also a degenerate form. Degenerate forms can usually be distinguished from primitive forms by the fact that along with general simplicity of structure they exhibit an undue elaboration of some organs; whereas primitive forms show equal simplicity in all their parts. 


\section{Chapter XXV.-The Earthworin.}

\section{(Lumbricus terrestris.)}

\$1. Form and Mode of Life. This familiar animal (fig. 174) has a cylindrical body varying in length up to six inches or more, pointed at the anterior end, somewhat swollen for the anterior third of its length, and rather flattened dorsoventrally in the hinder two-thirds. The mouth is almost terminal, ventral, and overhung by a præ-oral lobe (an outgrowth from the first somite); the anus is quite terminal. The most striking feature is the obvious external metamerism, the whole body being divided into a very large number (about a hundred and fifty) of

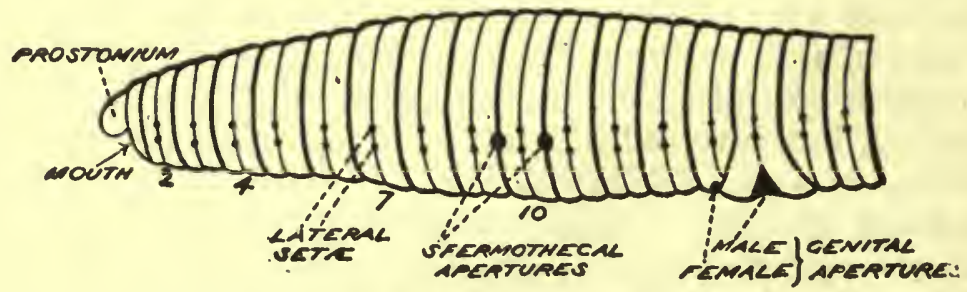

Fig. 174.-Front Portion of Earthwory.

Side View, (After Howes.)

segments (somites or metameres) all almost exactly alike. No other of our types (unless it be Amphioxus) exhibits metameric segmentation so fully and plainly. With the exception of the first thirty-five or so of the somites, where the reproductive organs and certain special parts of the alimentary canal are found, each somite is an exact repetition of any other. There is no distinct head. In a mature worm the region of somites 32 to 37 is enlarged, forming the clitellum; here the skin is very glandular (see § 2).

Though devoid of limbs, the earthworm has a highly muscular body-wall, and there project from its surface a 
series of small stiff spikes, called setæ. Of these there are eight on each somite, in four double rows, in the position shown by the transverse section in fig. 175. They can readily be felt in a dead worm by gently passing it through the fingers. By means of these the earthworm can readily make its way over and through the ground.

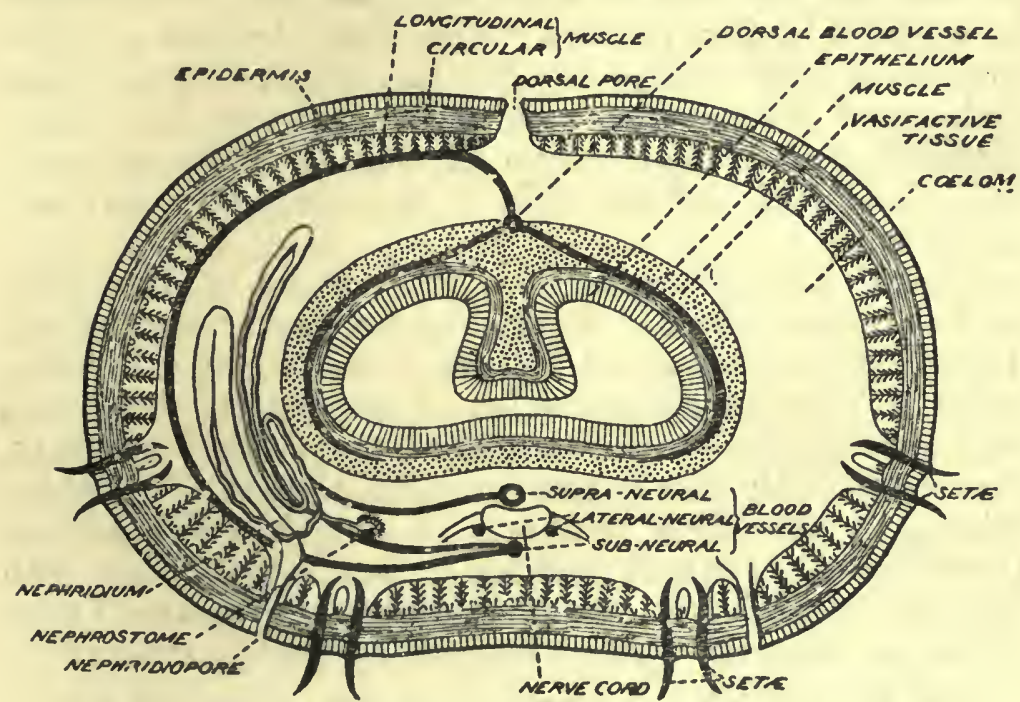

Fig. 175.-Transverse Section of Hinder Region of Eartuwork. (After Howee, altered.)

Its burrowing habits are known to all, as is probably also the fact that it very largely eats its way through the soil, deriving nourishment from the decomposing plant-remains in it. It also devours leaves.

§ 2. Skin and Body-wall. A transverse section of an earthworm in the region behind the clitellum (fig. 175) shows that there is a body-wall and an alimentary canal separated by a colomic cavity. Thus it is as typically a cœlomate animal as any of our Vertebrate types, but differs from them in many important respects.

The epidermis consists of a single layer of cells, among which are numerous glandular cells, but no multicellular glands. On its free surface is secreted a cuticle composed 
of that substance chitin, which we have already met in the gill-bars of Amphioxus. The presence of a cuticle is very common in Invertebrate Cœlomates, and contrasts strikingly with the typical condition in Vertebrates. In both cases we have a dead protective layer outside, and a living layer inside, but in the Vertebrates the outer layer is composed of dead cells, and is not a secretion. An epidermis overlain by a cuticle is often called a "hypodermis." The setæ are specialized derivatives of the cuticle, each developed from a little sac of the epidermis not unlike that seen in a Mammalian hair. In the clitellum all the cells of the epider'mis are glandular, and the skin is therefore very soft and easily torn.

Beneath the epidermis come the muscles of the bodywall-an outer circular layer, and an inner longitudinal. These layers are of about the same thickness all roundthere is no such concentration of muscles in the dorsal region as we have been accustomed to in the Vertebrata. Correlated with this is the complete absence of vertebral column or notochord, or indeed of any internal skeleton (endoskeleton): the only skeleton in fact is the cuticle with its setæ (exoskeleton). Another contrast with the Vertebrata is that the central nervous system (nerve-cord) is ventral in position.

$\S 3$. The Colom. If a longitudinal section were taken it would be found that the cœlom, which extends almost from end to end, is divided up into a metameric series of chambers by vertical partitions called septa, partly membranous, partly muscular, corresponding to the external divisions between the somites. This partitioning of the colom is not complete, however, and there is free communication from one end of the body to the other for the colomic fluid. At the front end of each somite there is a direct opening from the cœlom to the exterior, in the middle dorsal line: this opening is called the dorsal pore, and is controlled by muscles. The cœlomic fluid contains large amœboid cells, like blood-corpuscles.

§ 4. The alimentary canal is a straight tube (fig. 176). 
It begins with a small buccal chamber (in somites 1 and 2) without jaws or any other hard structures, and this is followed (in somites 3 to 5 ) by a strong muscular pharynx, and this by an osophagus (somites 6 to 12). Into this a fluid is poured from three pairs of calciferous glands (somites 10 to 12), the first of which are hollow, the other solid. The fluid contains minute crystals of calcium carbonate, and its function is to neutralize the organic acids always present in the soil which is eaten. Behind the asophagus is a dilated portion called the crop (somites 13 to 16); this is followed by a strong muscular crushing gizzard (somites 17 to 19). All the rest of the canal is intestine, not differentiated into regions. It is lined by a glandular epithelium surrounded by a layer of vascular connective tissue. These two layers are infolded on the dorsal side to form a longitudinal typhlosole (fig. 175). There is, also a thin muscular layer, and finally the cœlomic epithelium. This latter is specially modified on the exterior of the intestine, and

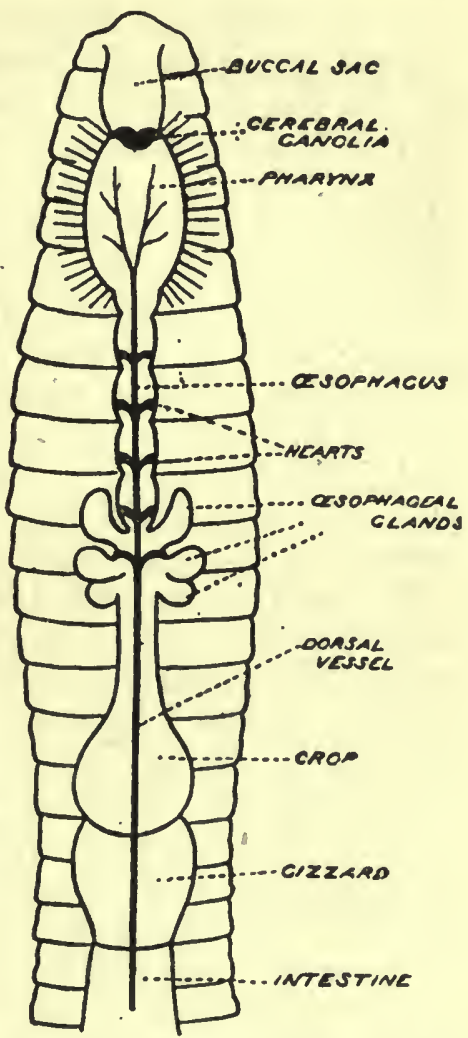

Fig. 176. -Alimentany Canal of EARTHWORM.

Dorsal view of anterior portion. (After Howes). to a less extent farther forward, giving rise to a yellow tissue, which has been called chloragogen tissue. This term appears to mean green-excreting, the term chloros in Greek meaning yellowish-green. It has been shown that this tissue contains uric acid and also glycogen, so that it would appear to perform two of the functions of the vertebrate liver. 
There is some evidence that the intestinal opithelium actually ingests food-particles : if so the process of digestion must in part be intra-cellular, as in Hydra.

§ 5. Vascular System. The colomic fluid, as in Amphioxus, probably carries on part of the functions of blood; but there is in addition a true vascular system, with a red blood circulating in definite vessels. As in the Vertebrata, the red colour of the blood is due to hæmoglobin, but here it is dissolved in the plasma and not in special corpuscles. There are small corpuscles in the blood, but they are colourless. The chief vessels are longitudinal, a dorsal one (fig. 176) above the alimentary canal, and four ventral ones below it, whose relation to the nerve-cord is expressed in their names-supra-neural, lateral-neural (paired), and infra-neural. In each somite from 6 to 11 there is a pair of contractile vessels, connecting the dorsal and supraneural vessels around the œsophagus: these are commonly called the "hearts" (fig. 176), there being no better claimant to that title in the earthworm. In each somite there is a connecting vessel between the dorsal and infra-neural, running in the body-wall, and there are branches to all the tissues from one or other of the main vessels.

In rough outline, there is a curious similarity between the circulation in the earthworm and that in Amphioxus. In both, the main vessels (indistinguishable as veins or arteries) run along the dorsal and ventral sides of the alimentary canal, and are connected towards the anterior end by a paired series of contractile vessels embracing that canal. But in Amplioxus the direction of circulation is forwards in the ventral vessel, up through the contractile vessels, and backwards in the dorsal vessel. In the earthworm the course of the blood is just the reverse. Moreover, the connecting vessels are not associated with gill-slits. Indeed, there are no special respiratory organs-the epidermis and cuticle are sufficiently thin to allow of diffusion of gases between the air and the blood in the sub-epidermal capillaries. There is no lymphatic system, but the cœlomic fluid certainly carries on the functions of lymph, and in some degree those of blood also. 
$\S 6$. The excretory system of the earthworm is of great interest. It consists of a paired metameric series of tubules, which open at one end into the colom, and at the other to the exterior. Like the excretory tubules of Amplioxus these tubules are called nephridia. A diagrammatic view of a nephridium is given in fig. 154. The internal end is a ciliated funnel (nephrostome) lying near the mid-ventral line, just in front of a septum, and with its open end facing forwards. This passes back at its narrow end into a tube formed of a single row of cells, the duct perforating the individual cells instead of being surrounded by an epithelium-in other words, it is intracellular. This tube runs through the septum into the next segment behind, where the rest of this nephridium lies. The tube is looped dorsalwards and ventralwards repeatedly, in a complicated manner, and shows three portions - a first where the duct is very fine, a second where it is larger and ciliated, and a third where it is still larger and not ciliated, the protoplasm around it being actively secretory. Finally, there is a portion where the duct is no longer intra-cellular, but lined by an epithelium, and outside this are muscle-fibres, by whose contraction the excretory liquid may be expelled through the external aperture. The secretory part of the nephridium is surrounded by a plexus of capillaries, and here doubtless urea (or some similar. compound) is removed from the blood. Through the nephrostome it, is obvious that the cœlomic fluid must be continually driven by the cilia towards the exterior, and it may be that some waste-products may be removed in this way, while it is certain that effete cells are thus removed. In this, as in many other cases, however, our knowledge of the details of physiology is far inferior in the case of Invertebrates to what it is in that of Vertebrates.

\$ 7. Nervous System (fig. 177). As has been already mentioned, the main nerve-cord runs in the mid-ventral line, internal to the body-wall. In addition to this there is a pair of ganglia in the head-region on the dorsal side of the alimentary canal. 'These ganglia, which are small pear-shaped bodies lying side by side in the depression 
between the buccal cavity and the pharynx, are known as the cerebral or supra-œsophageal * ganglia. They aro connected to the ventral nerve-cord by a pair of circumœsophageal * commissures. The

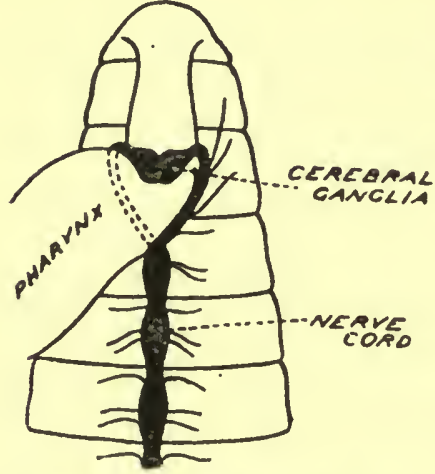

Fig. 177.-Central Nervous SYSTEM oF EarTHWORM, ANTERIOR END.

(After Howes.) nerve-cord is very slightly thicker towards the middle of each somite, and this has led to its being inaccurately described as a "chain of ganglia": but the thickenings are not ganglia, because nervecells are not confined to them. The cord is just as much a continuous cord as is the spinal cord of Vertebrates. It differs from the latter not only in its ventral position, but also in structure. It has no central canal; the nerve-cells are aggregated to the ventral side, the longitudinal fibres to the dorsal side (cf. grey and white matter). At the extreme dorsal side there are three giant fibres, resembling nerve-fibres in structure but of much greater diameter: they may be supporting structures only.

Nerves are given off metamerically-three pairs in each segment-but they have no separate roots for their sensory and motor fibres. The sensory fibres differ from those of all the nerves of Vertebrates except the olfactory, by being outgrowths of cells in the sensory epithelium (in this case the epidermis) : they resemble the sensory fibres of a Vertebrate's spinal nerves, however, in their behaviour on reaching the cord, as they divide into an ascending and descending branch, each of which ends in arborizations around a nervecell. The motor-fibres originate from nerve-cells in the cord, as in Vertebrata.

There are no specialized sense-organs-eyes, ears, or olfactory organs. Scattered through the epidermis, but

* These terms (as applied to the earthworm) are inconsistent with the sense in which the term "œsophagus" is used, as they lie in front of the "pharynx." 
especially abundant in the head-region and around the setæ, aro globular aggregations of the sensory cells already mentioned. These are probably concerned mainly in tactile sensibility, but we know that the earthworm is sensitive to light, perhaps also to sound and smell.

§ 8. Reproduction. Like Hydra, the earth worm is hermaphrodite, but unlike it, is incapable of self-fertilization, there being elaborate arrangements for mutual cross-fertilization between two worms. The gonads, as in Vertebrates, are derived from the cœlomic epithelium: in many members of the group to which Lumbricus belongs, they are metameric (as in Amphioxus), but in Lumbricus itself only a trace of this is seen, there being two pairs of testes, and one pair of ovaries. The

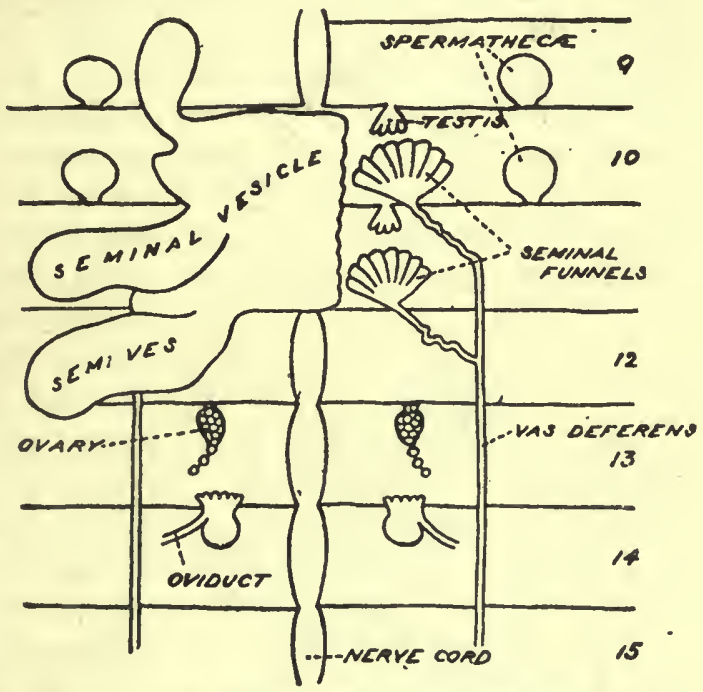

Fig. 178.- Reproductive Organs of Earthworm.

Dissection from dorsal side. (After Marshall and Hurst, altered.) For "Spermathecæ" read "Spermothecæ." former are attached to the septa at the front end of somites 10 and 11 ; the latter occupy a similar position in somite 13 (fig. 178). In Vertebrata we have seen that the oviduct has a free internal opening into the cœlom, whereas the male duct is directly continuous with the tubules of the testis. But in Lumbricus both male and female ducts open freely into the cœlom, by funnel-shaped ends lying a little behind each gonad. The two vasa deferentia of either side unite into a pair of longitudinal ducts which run back to their external openings on the ventral side of somite 15 : these apertures 
are usually very plainly visible on the surface (fig. 174). The oviducts are shorter, and only pass back into somite 14: at the base of its funnel, each oviduct bears a little pouch, the receptaculum ovorum.

So much for the essential organs : next there are certain important accessory structures. The cells from which spermatozoa develop are set free into the cœlom and there undergo further development before they pass out along the vasa deferentia. To allow for their development, the septa of the somites concerned become greatly modified, and there project out from them, into adjacent somites, large white pouches, called the seminal vesicles. It is in the cavities of these vesicles (really a portion of the cœlom) that the early development of the spermatozoa goes on. Quite distinct from these are two pairs of little sacs called spermothecæ, developed between somites 9 to 10, and 10 to 11, from ingrowths of the skin, and having no communication with any internal

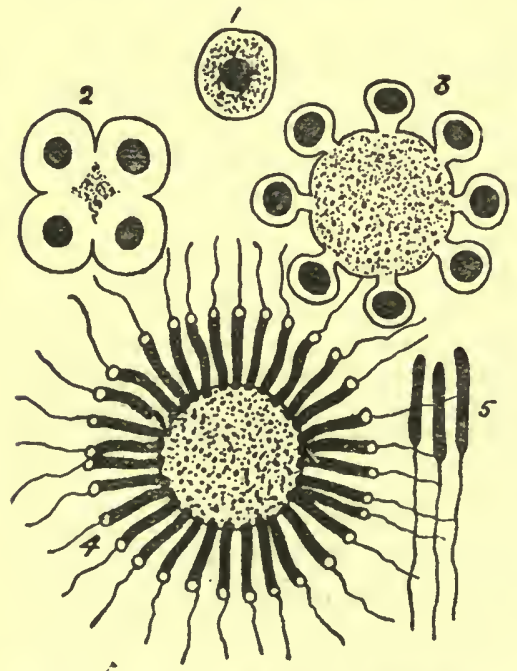

Fig. 179. -Development of Spermatozoa OF LUMBRICUS. stage of development than those in the former. The several stages in their dovelopment are shown in fig. 179. In the testes spermospores or sperm-mother-cells 
are formed by cell-division : each of these is a cell, whose nucleus soon undergoes repeated division, the nuclei arranging themselves at the surface. Corresponding to each nucleus a wart-like swelling, the spermoblast, appears on the surface, and soon the spermospore has the appearance of a blackberry. Each little protuberance eventually elongates and becomes the tail of a spermatozoon, with the nucleus for its head. The central mass of protoplasm (sperm-blastophor) is apparently wasted.

When the ova are ripe, the clitellum secretes a cylindrical cocoon, out of which the worm wriggles itself backwards. As the fourteenth segment passes through the cocoon, the ova are laid in it; and as the tenth passes through, the "foreign" spermatozoa are shed into it. The cocoon is elastic and closes when the worm has finally got out of it. In this cocoon the ova develop into young worms. It is unnecessary to detail the stages of development, for the earthworm is a specialized member of its group, and without a comparison with that of more typical marine worms its development is as uninstructive as would be that of the rabbit without a knowledge of fowl, frog, and Amphioxus. It will be sufficient to say that a gastrula stage is passed through; epiblast, mesoblast, and hypoblast are formed much as in craniate Vertebrates, and give rise to the same tissues respectively as in them; that the cœlom is formed by splitting of the mesoblast; the central nervous system by a paired thickening of the epiblast, without neural folds or groove; and that the blastopore, instead of being posterodorsal, is ventral, and between the positions of the future stomodæum and proctodæum, there being nothing resembling a neurenteric canal. These points illustrate the fact that only in the very broadest features of development does Lumbricus agree with the Vertebrates: as soon as we pass to the features which come second in importance, striking differences at once show themselves.

§ 9. Systematic Position. Comparing Lumbricus with Hydra, it is evident that we have a much higher organiza. tion. The presence of a definite, coelom (cf. figs. 175 and 195, A) of well-defined tissues other than epithelia, of elaborate 
circulatory and excretory organs, and of a central nervous system, all show this. With such a lowly Vertebrate as Amphioxus, the earthworm shows some resemblances-such as the general metamerism, and the structure of the nephridia; but the differences are many and great. There are no gill-slits, no notochord, the nerve-cord is ventral and solid, and the anterior end of the central nervous system encircles the mouth-region; the circulation is different.

$\S 10$. Former Theory of the Nephridia. The older view concerning nephridia and genital ducts was that they were homologous structures, which could be recognised in at least the majority of the phyla or sub-kingdoms of animals. According to this view, largely due to the influence of Prof. E. Ray Lankester, generative and excretory tubes were all modifications of one type, one of whose primary characteristics was that it had an opening into the coelom, or a nephrostome. The excretory tubules of Anodon and other mollusca, the pronephric and mesonephric tubules of vertebrates, the excretory tubules of Amphioxus, and the nephridia of the earthworm were all regarded as homologous structures, and this is the interpretation of them which was given in earlier editions of this book and all other zoological literature until recently.

$\S 11$. Recent Researches. This theory, however, has been much modified by the results of recent investigations, especially by those of $\mathrm{Mr}$. Goodrich. Embryologists had long ago found that the mode of development of structures regarded as nephridia was different in different cases-the tubules in some arising from epiblast, in others from mesoblast; in others partly from one layer, partly from the other. The most important factor, however, in the development of the new theory was the discovery by Mr. Goodrich that in several Polychaetes the nephridia did not open into the coelom at all, but that their internal extremities were blind, and that connected with these blind extremities were remarkable cells having a structure similar to that of the flame-cells of the Flat-worms, such as the liver-fluke. These peculiar cells are conpected with the 
nephridium by long delicate tubes, composed of a kind of cuticular substance and opening into the cavity of the nephridium. In the interior of these tubes is a long vibrating flagellum, which is attached on the one hand to the protoplasm of the cell, on the other extends into the cavity of the nephridium. These cells have been named solenocytes or tube-cells (solen, a pipe or tube; kutos, a cell). In some cases it has been found that ciliated funnels formed on the walls of the coelom, usually in the angle between body-wall and septum, grow out into ducts, and when the worm becomes sexually mature open into the cavity of the nephridium. Such funnels and ducts act as generative ducts.

$\S 12$. Characters of Nephridia and Coelomic Ducts. By the consideration of such facts as these we are led to the conclusion that nephridia and generative ducts are not modifications of one kind of organ, but organs of two distinct types, whose essential and primary characteristics are strongly contrasted. The characteristics of a primitive nephridium are :-

1. Its tube is intracellular-not a tube lined by an epithelium, but a tubule pierced through the actual substance of a scries of single cells (drainpipe cells).

2. It develops from without inwards, or centripetally from the epiblast.

3. It has no internal opening, but whether its internal extremity is simple or branched, it ends blindly in flame-cells, or cells provided with a vibratile flagellum.

The characteristics of a coelomic duct, which is primarily a generative duct, on the other hand are:-

1. Its tube is intercellular: it is a tube lined by an epithelium.

2. It develops in the mesoblast from the coelom outwards.

3. It has necessarily a coelomic opening, because this is the first part developed, and this opening is a ciliated funnel. 
In the earthworm we find nephridia which satisfy the first two of the above conditions but not the third, and it is held that a true nephridium may develop a coelomic opening, but that this is a secondary modification, not a pimitive character. The generative ducts in the earthworm are true coelomic ducts, not modified nephridia, and this explains why there are nephridia in the same segments as the oviducts and sperm ducts. 
Chapter XXVi.-External Characters of Nereis.

$\S 1$. Nereis as Type of the Polychaeta. The Polychaeta are so called because they possess large numbers of chaetae or cuticular bristles, in contrast with the Oligochaeta, of which the earthworm is a type, in which the chaetae are few. The genus Nereis, of which there are several species, is the most typical of the Polychaeta. It possesses, with the exception of branchiae, almost all the structures and characters which are found in any of the other forms, so that they are most conveniently distinguished by the absence or modification of one or more of the complete set of organs seen in Nereis. This applies more especially to the external characters. Like the earthworm, Nereis is a metamerically segmented animal, but it is more primitive in that the segments are more similar to each other,-it is homomerous. There is no clitellum, and no restriction of generative organs to certain anterior segments. The sexes are separate and the generative organs are merely tracts of the epithelium of the coelom, from which the generative cells become detached to undergo development in the coelomic cavity. There are no special generative ducts, ova and spermatozoa escaping by dehiscence of the bodywall. The only differentiation of the intestine is at the anterior end, where there is an eversible pharynx, provided with chitinous jaws and teeth, and a short oesophagus and stomach. The Polychaeta are all marine. Nereis lives between tide marks and in shallow water, and either burrows in soft ground or hides in crevices among stones and rocks.

$\S 2$. The Parapodia. The most characteristic external features of Nereis are the lateral appendages known as the parapodia. A pair of these is borne by every segment except the first and last. They are outgrowths of 
the body-wall, Hattened in an antero-posterior direction, and symmetrical not only on the two sides of the somite, but each one in itself. A parapodium consists of two similar halves, a dorsal and a ventral, the former called

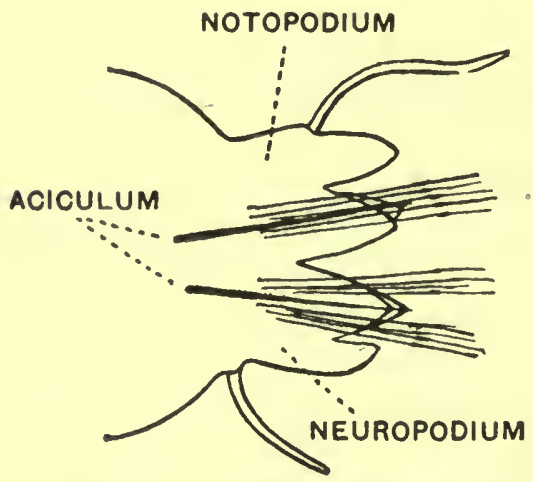

Fig. 180.-Typical Parapodium of Nereis magnified. the notopodium, the latter the neuropodium-because it is nearer to the neural surface and the ventral nerve cord (from notos, back; neuron, nerve; and podium, foot-organ). Each of these halves consists of a cirrus and two lobes, which are somewhat pointed. Thus the notopodium consists of dorsal cirrus, outer and inner lobe; the neuropodium of ventral cirrus, outer and inner lobe. Each of the inner lobes bears a fan of long chaetae, which are compound or jointed, each consisting of a shaft and a blade. The chaetae are really contained in a chaetigerous sac, which is an invagination of the skin, and extends deeply into the interior of the body. The inner lobe of each half of the parapodium is really double, forming the lips of this chaetigerous sac. In addition to the ordinary slender chaetae there is in each chaetigerous sac a much thicker and more rigid needle-like chaeta called the aciculum, which projects very slightly externally, but extends further than the other chaetae into the interior of the body and serves for the attachment of the muscles that move the whole bundle of chaetae. The two acicula thus act as an internal skeleton of the parapodium, though of course really belonging to the external cuticle. The cirri are well supplied with nerves and serve as tactile organs. The whole parapodium is an organ of locomotion, acting as a lever in the wriggling movement of the worm over the rough ground of the sea bottom, and in crevices between rocks and stones; at the same time the lobes are well supplied with blood-vessels and serve as organs of respiration. 
§ 3. Chaetae. The chaetae, as represented in Fig. 182, are of two kinds-one with a long slender blade or head as in $\mathbf{A}$, the other with a much shorter curved head. In both the head is furnished with fine teeth on the concave edge. The head is articulated in a socket at the top of the shaft, and is somewhat easily detached. The shaft shows a number of bands side by side, marked with transverse striae.

$\S 4$. The Heteronereis or Epitokous Form. Many species of Nereis exlibit remarkable and interesting differences in

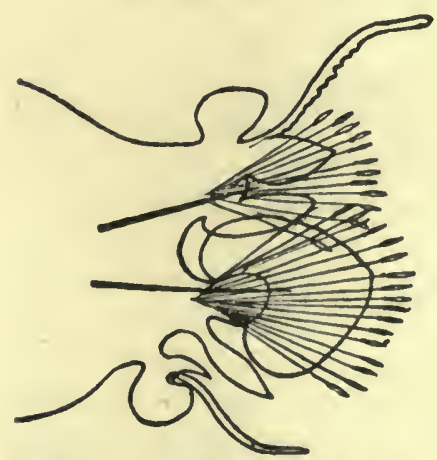

Fig. 1S1.- PARAPODIUM OP THE HETRRONKREIS OR EPITOKOUS FollM of Nrieis MaGNIFIED. the structure of the parapodia of the posterior part of the body when they become sexually mature, the appendages of the anterior third of the body remaining unchanged. The modified parapodia appear much larger and broader. The differences consist in the development of very broad flat expansions on the inner lobes of the parapodium and at the bases of the cirri, while the

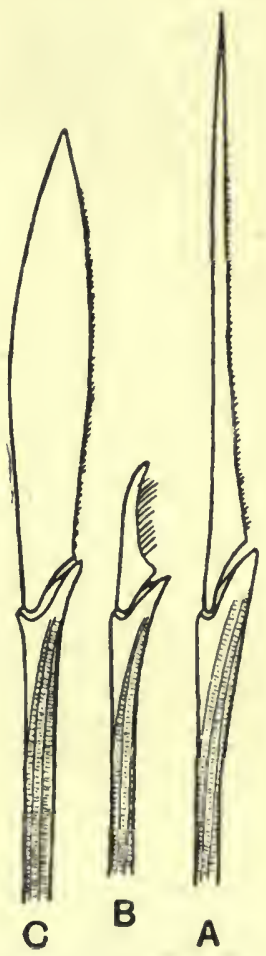

Fig. 182.-Ciraetae of NHREIS HIGHLY MAGNIFIED.

A, Chaeta of ordinary form with long blade. $B$, Ditto with short blade. C. Chae$\mathrm{ta}$ of Heteronereis or epitokous form with broad blade. outer lobes are little affected. The chaetae in the modified parapodia are more numerous and of different shape, the blades or heads being remarkably broad and flat, like the blade of an oar. All these changes make the parapodium (Fig. 193, c) much more efficient as a fin, and enable the worm to change its mode of locomotion from creeping or 
burrowing to swimming in the open water. Accordingly when the sexual products are ripe the worms of both sexes swim about actively while the eggs and sperms are discharged, and thus fertilisation takes place. A parapodium in this condition is shown in Fig. 181. Originally specimens of Nereis with such parapodia were supposed to belong to a distinct genus, which was named Heteronereis,

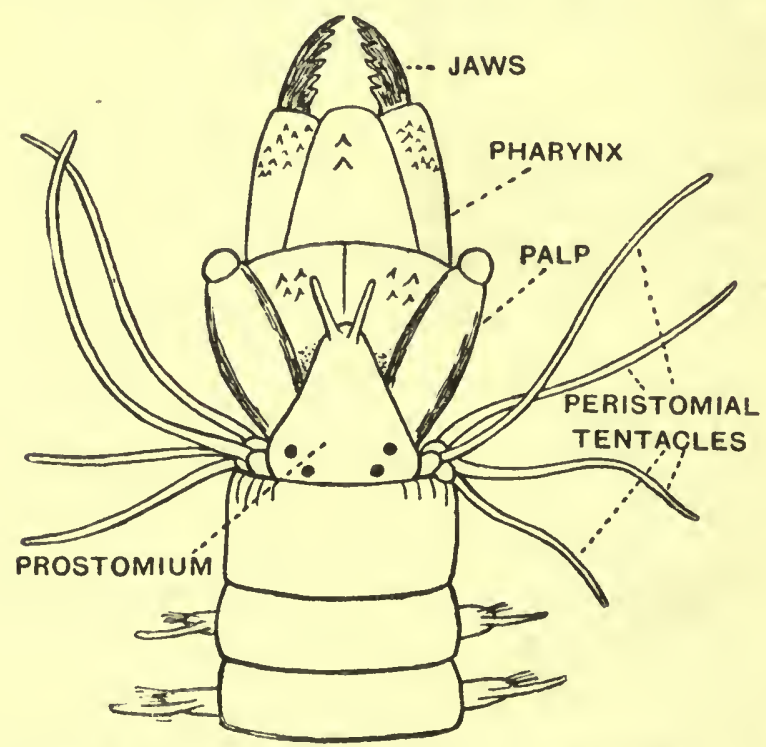

Fig. 183.-Anterior End of Nireis with Pharynx equrted, from Dorsal Side, knlarged.

and this term may be used to signify the sexual modification; the two stages have been distinguished also as the atokous and epitokous conditions.

$\S 5$. Head and Pharynx. Fig. 183 represents the anterior end of a Nereis from the dorsal side, with the pharynx everted. The pharynx is usually seen in this condition in preserved specimens, owing to the contraction of the muscles which occurs when the worms are killed. The extremity of the body is formed by the prostomium, which is of triangular outline and projects dorsally in front of the oral aperture. The prostomium bears two 
small prostomial tentacles at its anterior end, and near its base two pairs of eyes, of which the anterior pair are farther apart than the other pair. At the sides of the prostomium project obliquely two conspicuous cylindrical structures called the palps. These are also preoral, and may be regarded as outgrowths of the prostomium. At the end of each palp is a rounded papilla, which is retractile. The palps are well supplied with muscular tissue and nerves ; they are sense organs, perhaps more especially olfactory.

The first segment of the body behind the mouth and prostomium is called the peristomium. It is longer than the ordinary somites, and bears no parapodia. Attached

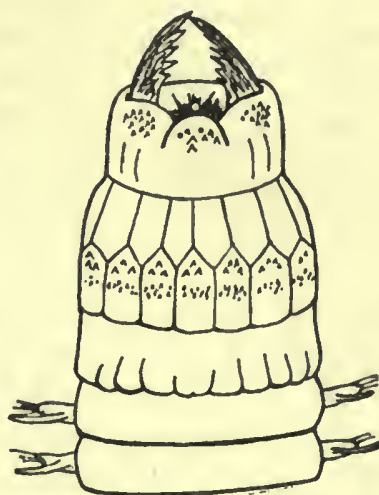

Fig. 184.-Everted Pharenx and Anterior Segmets OF NRREIS FRON VhNTRAL SIDE. to its anterior border, however, on each side are four long slender cirri, called the peristomial tentacles. They aro arranged in two sets-a dorsal and ventral anteriorly, and a dorsal and ventral posteriorly. Each cirrus is slightly enlarged at its base.

The pharynx, when protruded, consists of two layers-an external thinner layer, which bears the chitinous teeth, and an inner thick muscular tube which is continued back into the intestine. The pair of large chitinous jaws are attached to the end of the muscular part, so that when the pharynx is fully protruded they are terminal, and serve for seizing the prey, for Nereis is carnivorous and. predatory. The jaws are curved and toothed on their inner edges. The external surface of the outer layer of the pharynx is marked by grooves into distinct areas, in which are situated groups of small chitinous teeth. There is an anterior zone and a posterior zone. Dorsally the anterior zone has three areas, two large teeth in the central area, a group of small teeth in each of the lateral. The posterior zone shows two areas, with a group of rather large teeth in each. Fig. 195 shows the ventral view of the zOOL. 
protrudad pharynx, with the posterior zone divided into a number of small areas, each containing two groups of

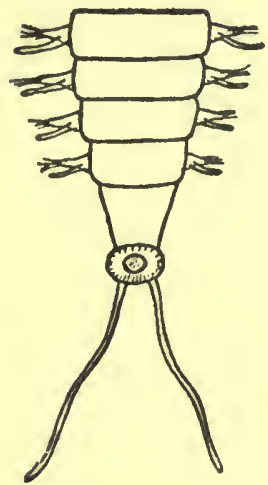

Fig. 185.-Posterior END OF BODY OF Nereis, showing ANal Segment aNd ANal Ciriri. teeth. When the pharynx is withdrawn the external layer becomes the wall of a buccal cavity, with the chitinous teeth projecting inwards: then the teeth serve to prevent the escape of the prey, and to some extent to masticate it. The jaws then lie at the junction of the buccal cavity with the muscular pharynx, and hold and tear the prey.

$\S 6$. Posterior End of Body. The segments diminish gradually in size towards the posterior end of the body, and the latter terminates in what is called the anal segment. This bears no parapodia, but the anus is situated at the end of it and is surrounded by a somewhat tumid annular border. From the ventral side of this thickening arise two long delicate cirri, called the anal cirri. The terminal segments of the body are represented in Fig. 185. 
Chapter XXVII.-The Liver-Fluke (Fasciola hepatica or Distomum hepaticum): a Parasitic Metazoan.

$\S 1$. Form and Habitat. The well-known and unfortunately abundant liver-fluke is a flat worm belonging to the class of Trematodes. In its adult state it lives chiefly in the bile-ducts of the sheep, but occurs also occasionally in other mammals and even in man. It is flat and leaf-like in shape, tapering to a point posteriorly, and having a triangular projection at its blunt anterior end. At the end of this projection is the mouth, and a short distance from this anterior end, on the ventral surface, is a sucker which is entirely superficial and has no connection with the interior of the body. The name of the group to which the animal belongs, Distomidae, is derived from the mouth and sucker, regarded as two suckers or two mouths (di, two, and stoma, mouth), the word stoma being used in the names of Trematodes in reference to the suckers which are so characteristic of their structure. The mouth in the Distomidae, however, has little claim to the title of sucker.

§ 2. Size and Structure. The liver-fluke is about one inch in length. The body consists externally of a body wall consisting of a homogeneous cuticle on the outer surface, with two layers of muscle-fibres-an outer circular layer and an inner longitudinal layer. An epidermis of the usual character is wanting, but there are numerous unicellular glands which open on the external surface. Internally the substance of the body consists of a peculiar kind of connective tissue which fills up the intervals between the organs. There is no spacious, continuous body cavity. The mouth leads into a pharynx formed of muscular walls, acting as a sucking bulb, and this leads 
by a short oesophagus into a bifurcated intestine, each branch of which extends nearly to the posterior end of the body. There is no anus or aperture to the intestinal cavity except the mouth. Each main branch of the intestine gives off numerous lateral diverticula, those on the inner side towards the middle line being short and simple, those on the outer side longer and branched. Thus the various ramifications of the intestinal cavity extend through all parts of the body.

The nervous system consists of an anterior nerve collar encircling the pharynx, having a ganglion on each side and one ventral. From each lateral ganglion there passes a ventral nerve cord to the posterior extremity of the body.

The excretory organs consist of a paired system of tubules communicating with a median duct in the posterior two-thirds of the body. This duct opens by a pore at the posterior extremity. The branches of this duct break up into numerous ramifications, which extend into all regions of the body. The ultimate tubules take their origin in cells which are enlarged and hollow, and are provided internally with one or more long cilia or flagella, the undulating motion of which suggests the flickering of a flame, whence the cells are called flame-cells. The function of these vibratile flagella is to cause a current in the liquid secreted by the flame cells and tubules towards the efferent ducts. The tubules appear to be intracellular, like that of the nephridium in the earthworm.

§ 3. Generative Organs. The animal is hermaphrodite and the generative organs are complicated. Like the other systems of organs, they extend into all parts of the body (fig. 186). The female system consists of vitelline or yolk glands, ovary, shell-gland, and uterus. The vitelline glands are very small and very numerous, and are situated in the lateral regions on each side of the body. Each gland has its own duct, and the various ducts unite together to open into a longitudinal duct on each side. From each longitudinal duct passes a transverse duct a little behind the ventral sucker, and the two transverse ducts unite together and 
open into the shell-gland, which is in front of them. The ovary is of limited extent and consists of a branched tubular structure, the blind ends of the tubes being somewhat dilated. It is situated on the right side of the body in

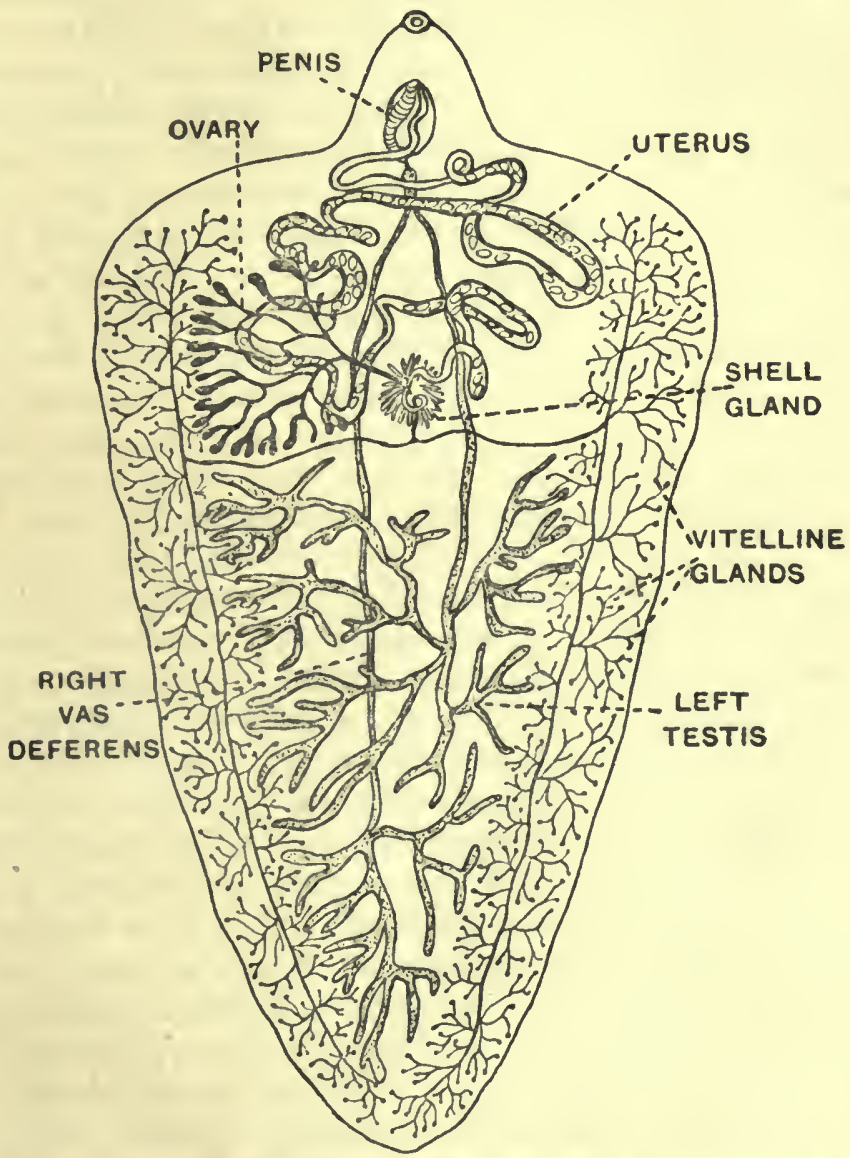

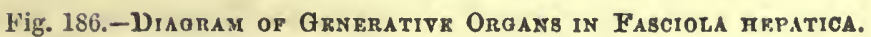

front of the transverse vitelline duct. The single terminal oviduct opens into the shell-gland. The shell-gland is a small spherical structure in the middle of the body behind the ventral sucker; it is composed of a number of unicellular glands secreting into a central cavity; the secretion forms 
the egg-shells. From the shell-gland passes forwards a much convoluted duct called the uterus, because the fully formed eggs accumulate in it; it passes forwards to open on the ventral surface between the oral and the ventral suckers. The testes are paired, but not symmetrical. They are branched tubular structures like the ovary, occupying the middle region of the posterior two-thirds of the body between the vitelline glands. Each testis gives off a vas deferens which passes longitudinally forwards; the two ducts unite into a dilatation called the vesicula seminalis. From this a fine short duct passes to the penis, a muscular tubular organ surrounded by a sheath called the cirrus-sac. In copulation the penis is everted or evaginated and protruded from the mouth of the cirrussac. The opening of the uterus lies close beside the opening of the penis. A short straight canal called the LaurerStieda canal leads from a dorsal pore to the junction of the ovarian and vitelline ducts with the shell-gland.

$\S 4$. Production of Ova. The true ova are produced in the ovary. Copulation has apparently not been observed, but probably consists in the injection of spermatozoa from another individual by the penis into the Laurer-Stieda canal. This duct is known to contain spermatozoa, and thus the ova are fertilised just as they reach the shellgland. Each ovum becomes surrounded by a large number of yolk cells produced by the vitelline glands and brought to the shell-gland by the vitelline duct. Then in the shellgland the ovum and its envelope of yolk are enclosed in a tough chitinous egg-shell, and the complete egg then passes into the uterus, finally to escape by the aperture of that duct. The number of eggs produced is very large, as in most parasites, where the probability of any single egg escaping destruction and successfully developing into a mature individual is small.

§ 5. Life-history-the 0vum. The completely formed eggs of the liver-fluke are extruded in large numbers from the uterus through its aperture, then make their way down the bile-ducts into the intestine of the sheep, where 
the resistant shell protects them from the action of the digestive secretions. Passing along the course of the intestine they are finally ejected from the body of the sheep with the faeces. The embryo within the egg-shell then begins to develop if the necessary conditions are present. These conditions are moisture and an ordinary summer temperature. Thus the multiplication of the parasite goes on in damp pastures in the summer season, but is checked in winter, in dry weather, or on dry ground. The eggs may develop in wet meadows in rainy weather, or in ditches and pools. The egg-shell contains originally a small protoplasmic ovum and a large number of spherical yolk cells, which serve as food for the developing embryo. One end of the egg-shell forms an operculum, which becomes detached when the larva is fully developed (Fig. 187).

§ 6. Larva. The newly hatched larva of the liver-fluke is of conical shape and uniformly ciliated all over its surface. By the active movements of the cilia the larva swims about in the water. The broad end is the anterior, and in the centre of this broad end is a small papilla, which can be extended till it is long and pointed, or contracted so that it is short and blunt. The most external layer of the body consists of large flat cells which bear the cilia. These cells are hexagonal in shape and are disposed in five transverse bands. Beneath the ciliated cells is a layer of nucleated protoplasm in which cell outlines are difficult to distinguish. In this layer are circular and longitudinal muscle-fibres. Anteriorly on what may be called the dorsal side are a pair of eye-spots, each consisting of a pigmented cell provided with a lens-like particle of refractive substance. These eye-spots are beneath the external ciliated cells. There are also a pair of rudimentary nephridia, each consisting of a single flame-cell. The central part of the body consists of large rounded cells, which in later stages of the life-history develop into new individuals, and are therefore reproductive cells or germ-cells. The external and internal structure of the larva are shown in the second and third of the diagrams in Fig. 187. 
§ 7. The Sporocyst. The ciliated larva swims about until it meets with a specimen of the fresh-water snail Limnaea truncatula. This snail is of small size, abundant in moist pasture lands such as those in the valley of the
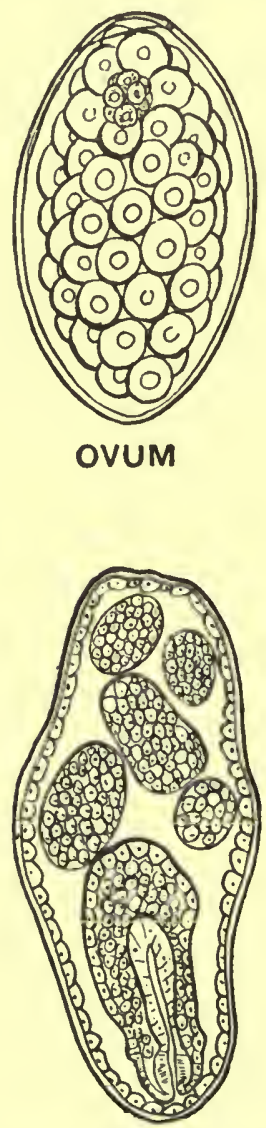

SPOROCYST
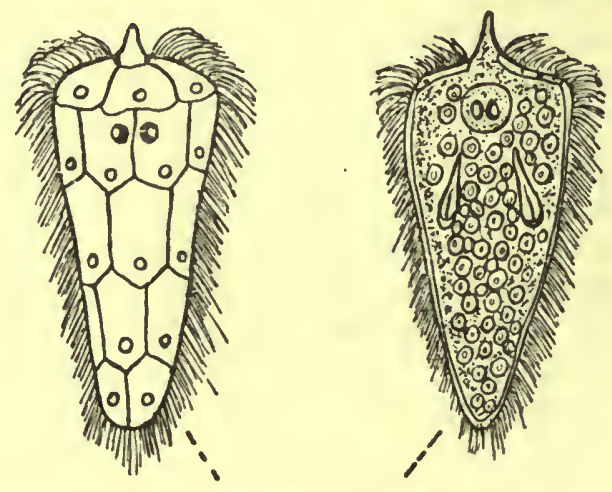

MIRACIDIUM

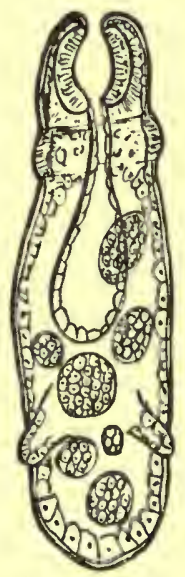

REDIA

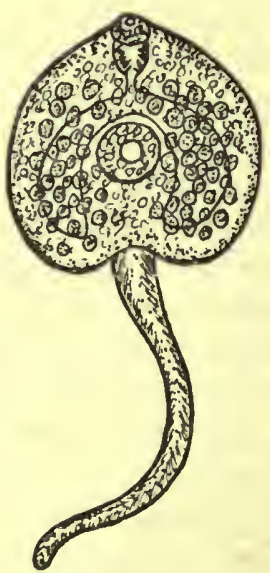

CERCARIA

Fig. 187.-Stagks in tur Lify-IIistort of Fasciota hepatica.

Thames, and amphibious in its habits; that is to say, it is scattered over the pastures during floods, and is able to live in the wet grass as well as in the ditches and pools. The fluke larva bores its way into the soft tissues of the snail, penetrating them by means of its anterior papilla 
while its body is rotated by the motion of its cilia. Inside the tissues of the snail the larva throws off its external covering of ciliated cells and develops a large cavity in its interior, lined by the germ cells. The anterior papilla disappears and the eye-spots degenerate, so that the larva is converted into a simple sac of somewhat indefinite elongated shape. In this stage the animal is called a sporocyst, which grows to a length of about $5 \mathrm{~mm}$., and occasionally may be divided to form two sporocysts.

§ 8. The Redia. Within the cavity of the sporocyst the germ-cells become free and undergo development like ova, but without fertilisation. We have, therefore, here a case of parthenogenesis (parthenos, virgin; genesis, generation). The germ-cell segments and forms a blastula, which is invaginated to form a gastrula. The invaginated layer forms an endoderm surrounding an enteric cavity, the anterior part of which is developed into a muscular pharynx. The external layer or body-wall develops a muscular ring or collar just behind the position of the pharynx, and near the posterior end of the body are developed a pair of blunt outgrowths, apparently serving as rudimentary organs of locomotion. Between the enteron and the body-wall are numbers of germ-cells like those of previous stages, and in the interior of these a cavity is again developed to serve as a generative cavity. The animal in this stage is called a redia.

In the sporocyst there is no special or permanent aperture from the generative cavity to the exterior through which the rediae can escape: they simply burst through the walls at any point, and the opening so formed contracts and closes up after the redia has passed through. But in the redia there is a definite permanent opening to the generative cavity; it is situated on one side of the body a little behind the collar, and is called the birth-opening.

Large numbers of rediae are developed in succession in a sporocyst, and as they become mature they force their way through the wall of the sporocyst and penetrate into the tissues of the host-snail, especially into the liver, feeding on the tissues by means of their mouth and muscular pharynx. 
New generations of rediae are developed in the same way in the generative cavity of a parent redia, so that a single snail becomes infested with large numbers of the parasites and is ultimately killed by them.

§ 9. The Cercaria. Eventually, usually on the approach of autumn, the germ-cells of the rediae, instead of developing into new rediae, give rise to individuals of a different form and structure, called cercariae. ("Redia" is derived from "Redi," the name of an Italian zoologist; "cercaria" from kerkos, the Greek word for "tail.") The cercaria may be described as an immature stage of the adult fluke, of cordate shape, and provided with a long muscular tail. Like the redia it consists of external body-wall and an internal digestive sac, but it already possesses many of the characters of the adult fluke. Around the mouth at the anterior end is a small oral sucker, and in the middle of the ventral surface is a large dermal sucker. The enteron bifurcates immediately behind the pharynx, and the two halves extend back, one on each side of the body, as simple tubes without branches. In the tissue of the body is an excretory system which is paired and symmetrical, as in the adult, consisting of longitudinal ducts into which open numerous branches, the ends of the branches arising from flame-cells. The generative organs of the adult are represented only by a germinal tissue between the wall of the enteron and the body-wall. On each side of the body near the surface are numerous large cells loaded with granules. These are called the cystogenous cells, because they subsequently secrete a substance which forms a spherical envelope, or "cyst," around the cercaria.

$\S 10$. Encystment. The cercaria, after escaping from the birth-aperture of the parent redia, does not remain in the tissues of the snail in which it was produced, but very shortly passes out and becomes free. It then, by means of the movements of its tail and of its suckers, for a while moves about in the water or among the wet grass; but before long, attaching itself to a blade of grass or to a water plant, ceases to move and becomes encysted-that is, 
enveloped in a spherical capsule formed by the secretion above mentioned. The substance of the cyst is gelatinous when first formed, but quickly solidifies and adheres to the grass or leaf on which it is situated. In this condition the cyst has a chance of being swallowed with the herbage by a sheep, or occasionally by some other animal: it is possible that such cysts may be attached to watercress or other vegetable, and be eaten by human beings.

§ 11. Development of Cercaria into Adult. In the stomach of the sheep the cyst is dissolved and the cercaria is set free to enter upon another phase of parasitic existence. It makes its way from the duodenum into the bileduct and its branches, and there feeds and develops into the mature Fasciola hepatica. The sheep can tolerate the existence of a small number of flukes in its liver for a long time without suffering much in health, but if the parasites are numerous the consequences to the host are serious and often fatal. The disease is known to farmers as rot or liver-rot, and large numbers of sheep are killed by it every year in districts where it prevails.

$\S 12$. Characters of Trematoda. Fasciola is a member of the phylum Platyhelminthes or Flat-worms, and among those, of the class Trematoda. The Trematoda are usually divided into two orders: I., the Digenetica, to which Fasciola belongs, in which there are several generations in each life-cycle and an alternation of hosts, the larval forms usually living in an invertebrate host and the sexually mature form in a vertebrate; $\Pi$., the Monogenetica, in which the egg of the sexually mature animal develops directly into a form like the parent, and in which the parasite lives always on one host. The majority of the Monogenetica are external parasites on fishes.

$\S 13$. Parthenogenesis and Alternation of Generations. The life-history of the liver-fluke illustrates some very important phenomena, both physiological and morphological. Physiologically, we have here an alternation of parthenogenetic and sexual reproduction. The term digenetic is 
not strictly correct, for there are not merely two generations in the life-cycle, but many. As a general rule, in Metazoa the reproductive cells or ova are unable to develop into new individuals until they have been fertilised-in other words, have conjugated with other reproductive cells in the form of spermatozoa. The germ cells in these cases are gametes, cells destined to conjugate with other gametes. But the reproductive cells of the sporocyst and redia develop directly without fertilisation, although in structure and mode of development they do not appear to differ from true ova. This case of alternation of generations is, therefore, to be distinguished from the metagenesis seen in Obelia, where the sexual individuals are produced not by generation, but by gemmation; not by the development of a single cell separated from the parent, but by the proliferation of a number of cells, of a portion of the tissues of the host, including both ectoderm and endoderm, separation occurring after development.

$\S 14$. Paedogenesis. The liver-fluke also illustrates what is called paedogenesis, or the reproduction of young individuals not fully developed. It is evident that the sporocyst and the redia have proceeded a very little way on the road of development which leads to a fully developed liver-fluke. They are even less developed than the Cercaria, which is incapable of reproduction until it has become adult. (Paedogenesis is derived from pais, child or boy, and genesis, generation.)

$\S 15$. Coelom and Nephridia. Morphologically the various stages of the liver-fluke illustrate the significance and relations of the coelom or body-cavity and the nephridia. At first sight the mature liver-fluke appears to be destitute of a coelom : it appears to consist, apart from the digestive cavity or enteron, of solid tissues in which the generative and other organs are imbedded. But it is to be noted that all the generative organs are saccular; they consist of cavities containing the generative or accessory cells, and these cavities are directly continuous with the generative ducts. Now if we consider other animals, 
vertebrates for example, or the earthworm, we find that the coelom is a cavity on the walls of which are situated the generative cells, and which is connected with the exterior by the generative ducts. In the sporocyst and redia the generative organ is one large cavity occupying most of the interior of the body with reproductive cells on its walls, but here there are no definite ducts, unless the birth-opening of the redia be regarded as such. The cavities of the generative organs in the liver-fluke, then, correspond to the coelom, and the coelom generally is to be regarded as primarily the generative cavity.

It was formerly supposed to be characteristic of the nephridia that they had an internal opening into the coelom, as in the earthworm, but the excretory tubules of the liver-fluke evidently resemble nephridia, and are now held to be homologous with them. The connection of the ultimate tubules with flame-cells is now regarded as the primitive condition of true nephridia, and similar structures have been discovered in many Polychaeta, and even in the excretory tubules of Amphioxus, which do not open into the coelom as formerly supposed. Generative ducts or gonaducts, therefore, are not modified nephridia as formerly held, but are organs of primarily distinct origin and nature. 
Chapter XXVIII.-The Pork Tape-Worm.

\section{(Taenia solium.)}

$\S 1$. Form and Mode of Life. In its adult and sexually mature condition this parasite lives in the small intestine of human beings. It consists of

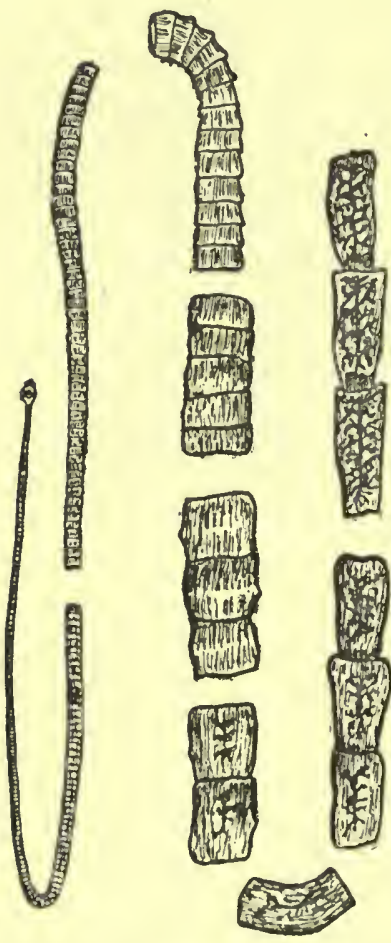

Fig 188.-Diagram of Taenia sólium AS IT APPEARS TO THE NAKED EYE, ONLY REPRESENTATIVE PORTIONS FROMT DIFFERENT REGIONS BEING SHOWN. a somewhat enlarged rounded extremity called the head, which is provided with hooks and suckers and is fixed to the mucous membrane of the intestine, and of a long body consisting of a very large number of flat oblong segments called joints or proglottides. The part behind the head is slender and is continually growing and producing new segments, so that the oldest and most mature segments are those at the posterior extremity. There is no mouth or anus, the animal being entirely destitute of intestine and all other digestive organs, obtaining its nourishment from the digested food of its host, by which it is surrounded.

The name Taenia meaning a band, like the English name tapeworm, refers to the characteristic flattened shape of the animal. When fully developed this tapeworm may reach a length of 6 to 9 feet, and the presence of such an enormous parasite in the intestine has naturally a bad effect on the health of the host: 
the length, however, is indefinite, for the posterior proglottides as they mature become successively detached, and are expelled to the exterior.

§ 2. Structure. The head (fig. 189) is quite small and somewhat globular: the central termination forms a muscular projection called the rostellum, which is surrounded by a ring of chitinous hooks. On the sides of the thickest part of the head are four muscular suckers at equal distances from each other. Behind this the head narrows to the neck. The hinder part of the neck shows slight transverse constrictions, which separate segments from each other, the anterior much less in length than in breadth, but as we pass backwards the segments become longer as well as broader, and flatten till we reach the mature proglottides at the posterior end of the body. The number of proglottides reaches 850 .

The structure of the body resembles closely that of Trematodes. In a transverse section is seen on the outer side a cuticle; there is no regular

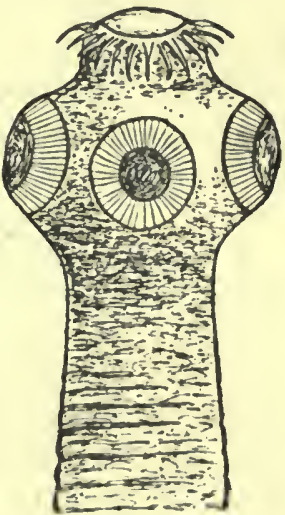

Fig. 1S9.-HEAD or Tuenia solium MAGNIFIED, SHOWING THE ROSTELLUM WITH ITS CHITINOUS IIOOKS AND THE FOUR SUCKERS. epidermis, but beneath the cuticle is a layer of spindleshaped cells with large granular gland-cells scattered in it, and longitudinal muscle fibres passing through it. Beneath the cellular layer is a layer of longitudinal muscle fibres, and internally again a parenchymatous tissue traversed by bundles of longitudinal muscles, and a layer of circular muscles : there are also, especially at the borders of the proglottides, groups of dorso-ventral fibres. All these muscle fibres give the proglottids a great power of contraction in all directions. Scattered in the parenchyma, especially in the cortical region, are calcareous corpuscles.

The other organs which are embedded in this muscular and connective tissue are the excretory tubes or nephridia, the nerve cords, and the complicated reproductive organs. 
§ 3. The Nephridia or Excretory Tubes. Running through all the segments are on each side two excretory tubules: these are united with one another in the head by a transverse annular vessel, and also in the posterior border of each segment by a straight transverse anastomosis. As in the liver-fluke, the longitudinal vessels open, in the entire tape-worm before the ripe proglottides have begun to detach themselves at the posterior end, into a caudal vesicle which opens to the exterior. In some tape-worms there are other lateral openings as well. Communicating with the longitudinal vessels are numerous ramified tubules, the extremities of which are formed by flame-cells similar in structure to those of the liver-fluke.

\section{$\S 4$. The Nervous System consists firstly of a broad} transverse band in the head behind the rostellum: this contains nerve cells chiefly aggregated in two swellings forming indistinct ganglia ; and secondly of two longitudinal nerve cords which pass backwards, one on each side, through all the proglottides. Nerves pass from the anterior band or brain to the suckers. Sensory terminations of the nerves given off from the longitudinal cords have been described in the cuticle, but no organs of the higher senses such as eyes, auditory or olfactory organs, are present: in an internal parasite living in the dark immersed in semiliquid nutriment such sense-organs are not required.

$\S 5$. Reproductive 0rgans. As in the liver-fluke, these are complicated, hermaphrodite, and highly developed. The testes are a number of minute spherical sacs, somewhat resembling in shape and arrangement the vitelline glands of the liver-fluke, scattered through the dorsal region of the proglottis, each of which has its own complete system of reproductive organs independent of those in every other proglottis. Each testis has its own fine duct, and these ducts unite together into larger and fewer branches or "factors" until all are united into a single convoluted vas deferens, which runs from the central region transversely to the right or left margin of the proglottis. The end of the vas deferens forms a long narrow 
process, the penis or cirrus, which can be protruded through the male aperture, and which is contained in a muscular sac, the cirrus sac.

The ovaries are a single pair of organs, each consisting of a number of branching tubes, which run from the lateral region in the posterior part of the proglottis to open into a central oviduct. The latter is connected posteriorly with

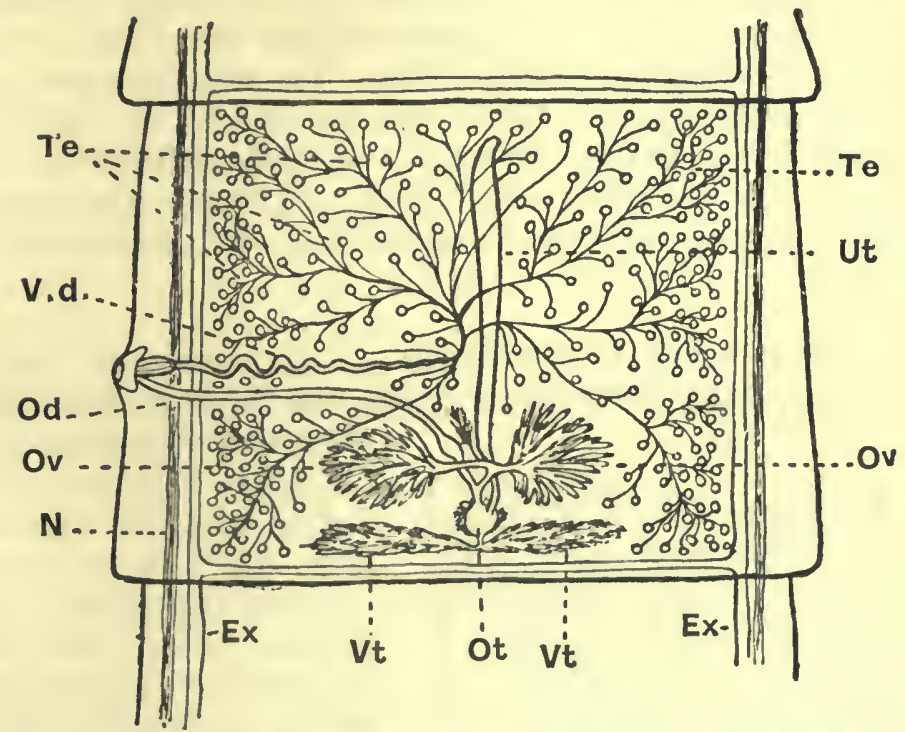

Fig. 190.-Proglottis of Taenia solium magnified to show the Generative Organs.

$T e$, testes ; V.d., vas deferens; $U t$, uterus ; Ot, oötype $V t$, vitelline glands; $N$, nerve; $E x$, excretory tube: only one pair of these is shown; Ov, ovaries; $O d$ oviduct.

a small spherical chamber called the ootype, surrounded by small glands which secrete the egg-shell, and this ootype receives posteriorly a short duct from the single vitelline gland, a transversely elongated mass of lobules near the posterior margin of the proglottis. From the ootype is given off a wide straight tube, which runs forward through the middle of the proglottis : this serves for the retention of the completely formed and fertilised eggs, and is called the uterus. From the ootype the oviduct runs parallel to the vas deferens to the female opening which adjoins the zooL. 
male opening; near the ootype the oviduct forms a small dilatation, the receptaculum seminis, in which the spermatozoa received in copulation are stored.

The genital papilla is situated on the margin of the proglottis, but not always on the same side; it is sometimes on the left side, sometimes on the right, but not in regular alternation.

The above description applies to the fully-developed genital organs, which in Taenia solium are found in the 450 th and following proglottids. In the youngest proglottids immediately following the neck no trace of genital organs can be detected: as they grow older and farther removed from the point of origin the organs begin to develop, the male organs first and afterwards the female.

$\S 6$. Process of Reproduction. The process of copulation is not completely understood, but it is believed that the penis protruded from

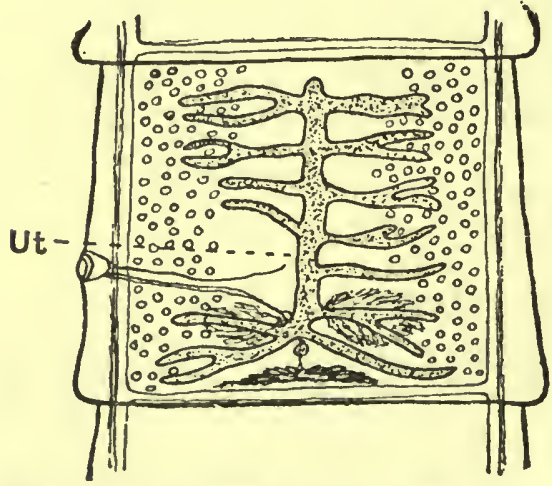

Fig. 191.-HalfF-RIPe Pronlottis of Tuenia solium, SHOWING BRANCHED UTERUS. $U t$, uterus. the male aperture is inserted into the vagina of the same or a neighbouring proglottis, and spermatozoa are thus conveyed to the receptaculum. As in Trematodes, the ova derived from the ovaries are fertilised in the ootype, surrounded by yolk cells from the vitellarium or yolk gland, and then enclosed in an egg-shell secreted by the shell-

glands.

The eggs when complete pass successively into the uterus, in which they accumulate, and as the number of perfect eggs increases the uterus enlarges and develops lateral caeca, as shown in fig. 22, until at last it occupies nearly the whole of the proglottis, and the rest of the reproductive 
system degenerates. This is the condition of the ripe proglottids at the posterior end of the worm, and these become detached singly or in groups and pass out of the host with the faeces.

§ 7. Development of Hexacanth Embryo. The segmentation and early development of the ovum take place in the uterus before the detachment of the proglottids. The embryo is formed from only a portion of the total number of segments. The first segmentation forms two blastomeres, of which one is filled with refringent granules and takes no further part in the formation of the embryo. The other continues to segment and gives rise to three megameres and numerous micromeres.

The megameres grow larger and form what is called a yolk-envelope. The micromeres form a spherical mass, and two of them at one end flatten out and divide to form a superficial envelope, which gives rise to a chitinoid coat. The remaining central mass forms the embryo proper, on the exterior of which are developed three pairs of pointed spines commonly called hooks. This hexacanth embryo consists of a solid mass of embryonic cells. This is the stage of development reached in the eggs in the ripe

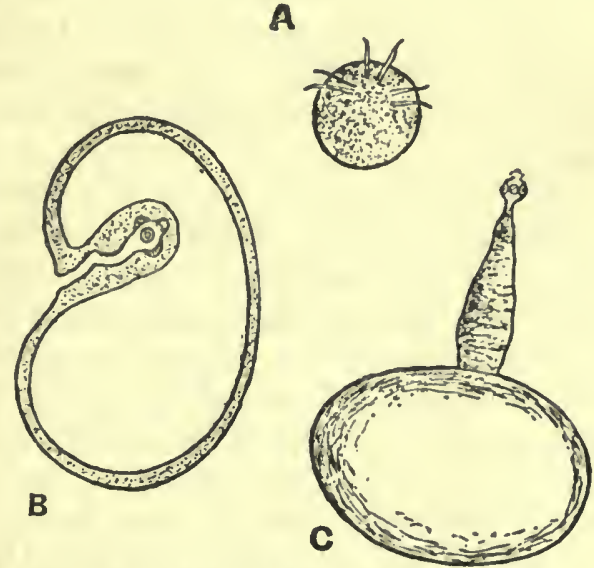

A

Fig. 192.-Developaent of Taenia solium. $A$, six-hooked embryo; $B$, cysticercus ; $C$ ditto with head overted. proglottid at the time it becomes detached and expelled from the host. The expelled proglottis either decays and sets free the eggs, or it may be swallowed by a pig in its entire condition. In either case the egg envelopes are digested in the stomach of the pig and the hexacanth embryos set free. 
$\S 8$. Development of the Cysticercus. The hexacanth embryo is incapable of further development except in the body of the first host, which in the case we are considering is usually a pig. By means of their six hooks, aided by the peristaltic movements of the host's intestine, the embryos make their way through the intestinal wall, and reach some factor of the portal vein, in which they are carried along till they reach a capillary of smaller diameter than themselves.

In the species we are considering the further development usually takes place in the muscular tissue, giving rise to what is called " measly pork."

The embryo is nourished by absorption from the host's tissues; it developes a central cavity and becomes an ellipsoidal vesicle, in the walls of which excretory tubules with flame-cells are developed. At one point the wall of the vesicle is invaginated and at the base of the invagination are developed the four suckers and double circlet of hooks which characterise the "head" of the mature tapeworm.

These structures are on the inside of the invagination, which of course corresponds to and is continuous with the external surface of the vesicle. By pressure the invagination can be everted, and then the suckers and hooks are on the exterior of the extremity of a structure similar in all respects to the head of the adult Taenia, which in fact it becomes. This head is often called the "scolex." The whole embryo at this stage is known by the English term "bladder-worm," or the technical term cysticercus, meaning "bladder-tail," because the larva is like a small tape-worm with a bladder at the tail end.

The cysticercus undergoes no further development in the body of the pig: it may live for a long time in this condition without change, but if not transferred to another host it dies and shrinks to a small mass which is finally absorbed. In the muscle tissue of the pig the cysticercus is enclosed in a "cyst" formed round it by the host's tissue in response to the irritation caused by the presence of the parasite. 
§9. Development of the Tape-worm. When measly pork is eaten by human beings the meat is digested and the cysticercus (or several of them) is set free. Thorough cooking kills the cysticerci and prevents infection. In the duodenum of the new host the scolex or head of the cysticercus is everted, and attaches itself to the mucous membrane by its suckers and hooks. The bladder is digested and disappears, and the neck begins to grow and develop proglottids until the adult stage already described is fully developed. Thus the life-cycle is completed, and we see that it requires two distinct hosts, both in this case belonging to the Mammalia.

$\S 10$. Interpretation of the Structure of Taenia. At first sight it seems obvious that the head or scolex of the tape-worm is the anterior end, and if this is correct it follows that the formation of the proglottids is not comparable to that of the segment in an Annelid such as the earthworm or Nereis. In the latter the new segment is formed between the last segment and the posterior extremity, in the tape-worm the new segment is formed between the first and the head. The proglottids might be on this view regarded as buds, comparable to the zooids which are budded off in succession from certain Annelids, each bud arising from the anterior asexual region.

There is, however, some reason for the view that the socalled head of Taenia really corresponds to the anal segment of an Annelid and is not anterior but posterior. Assuming that the six spines of the embryo are developed at the anterior end, it is certain that in the larva of Dipylidium caninum, a tape-worm of the dog, and in other species, the scolex is developed at the end opposite to the hooks, and is therefore posterior. On this view the proglottids of the tape-worm would be formed much in the same way as the new segments in the Annelid, and the structure of the tape-worm would be a true instance of metameric segmentation.

Another circumstance in favour of this interpretation is that if we compare the structure of a proglottis of Taenia with that of Distomum hepaticum, the Liver-fluke, the 
testes in the latter are posterior to the ovary, and therefore the side on which they are placed in the former, i.e. towards the scolex, should also be posterior. In many Trematodes again, though not in Distomum, the chief organ of attachment is posterior, and many of these animals are in the habit of attaching themselves by the posterior end and moving the anterior mouth-carrying end freely about: If this were the original habit of the primitive tape-worm it would be certain that the so-called head was really the posterior end.

In any case it is certain that the proglottids become detached, and these may be regarded as asexually produced individuals, which are themselves sexual, so that we have here an example of metagenesis as in Obelia.

$\S 11$. 0ther Tape-Worms. Another tape-worm which frequently occurs in man is Taeniarhynchus saginata, sometimes called Taenia mediocanellata, which differs from Taenia solium in the absence of the circlet of hooks and rostellum; it has the four suckers only. It is larger than Taenia solium, reaching a length of four metres, having 1,200 proglottids, the 600 th being the first fully developed. This is the beef tape-worm, its cysticercus inhabiting the flesh of the ox, and infection being due to eating infected beef insufficiently cooked.

Man may also serve as first host in the life-history of tape-worms, the larvae developing in his body instead of the adult. The most dangerous case of this kind is that of Echinococcus, a bladder-worm, which develops new bladders by successive internal gemmations, the bladders developing very large numbers of heads or scolices. The proliferating Echinococcus usually develops in the liver, and may grow to such an extent as to cause extensive destruction of neighbouring organs and cause the death of the host. The adult form of this larva is Echinococcifer, a very small tape-worm with four suckers and a circlet of hooks, but having only four proglottids and a total length of $5 \mathrm{~mm}$. It lives in very large numbers in the intestine of the dog, wolf, and jackal. The infection in man occurs principally in Iceland and Australia. 
Another proliferating cysticercus is Coenurus cerebralis, which occurs usually in the brain of the sheep and causes the disease known as "staggers." The adult Cystotaenia coenurus lives in the ileum of the dog and wolf: thus the shepherd's dog may be a source of infection to the flock.

The bladder-worm almost invariably found in the body cavity of the rabbit when opened for dissection is the larva of Taenia serrata, which lives in the dog or wolf. 
Chapter XXIX.-The Fresh-Water Polype.

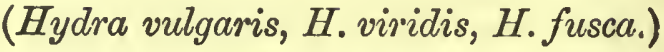

$\$ 1$. Occurrence and Mode of Life. Hydra is a common inhabitant of fresh-water ponds in which duck-weed (or other water-plants) and small crustaceans are abundant. If a glass jar be filled by dipping it into such a pond and left in a light place for an hour or so, Hydræ are likely to be found sticking to the sides of the jar in an excellent position for examination with a hand-lens. If fresh water is added every now and then to make up for loss by evaporation, the Hydræ can be kept alive for an indefinite time.

Each Hydra is a cylindrical body usually attached to water-weed (or the sides of the vessel) by one end, and having at the other end a variable number of long, fine tentacles (see fig. 193). If roughly touched, contraction takes place, resembling that of a muscle-the body becomes shorter and thicker-almost globular-and the tentacles very short and thick (fig. 193, в). Very soon, however, it regains its normal shape. Occasionally a Hydra may be seen changing

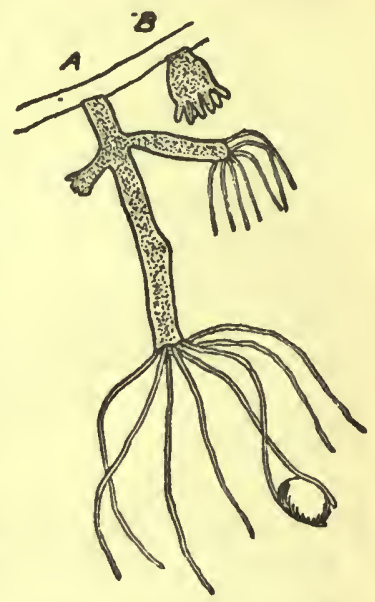

Fig. 193.-I'wo HYDRA ATTACHED TO WATER-WEED. its place, moving very slowly, after the fashion of a looping caterpillar. Its normal position, however, is the fixed one, and it affords us the only example among our types of a fixed animal. The fixed habit of life, which we usually think of as one of the most distinctive features of plants, is, however, by no means uncommon among aquatic animals, and is very often accompanied by another plantlike habit, viz. that of branching (fig. 196 and § 14. below). 
It may seem surprising that a fixed and relatively slowmoving animal such as Hydra should feed upon the active little Crustacea ("water-fleas") that swim about in pondwater, but such is the case. In its ordinary position Hydra is in fact fishing for them with its extended tentacles. As soon as a water-flea touches one of these tentacles it stops, evidently paralyzed, and remains fixed to the tentacle. (How it is stunned we shall see presently.). The Hydra gradually contracts the tentacle so as to bring the crustacean near its mouth, which lies in the centre of the circlet of tentacles, at the summit of a little cone-the hypostome. The other tentacles help in forcing the prey against the mouth, which then expands widely and seizes it. An ordinary water-flea is thicker than a Hydra, and when swallowed usually causes a prominent bulge in the cylindrical body of its captor. It is easy to see that the cavity in which the prey undergoes digestion extends for the whole length of the cylinder, but ends blindly at the attached end. There is no anus, the unassimilable parts of the food being ojected at the mouth.

$\S 2$. General Structure. Examined under the microscope, whole or in thin sections, further details of structure can be made out. Hydra is multicellular, i.e. composed of many cells, not of a single cell as are the Protozoa. These cells are arranged in two very definite layers-an outer one called the ectoderm, and an inner one, the endoderm, which forms a lining to the digestive cavity or enteron (fig. 195, A). These two layers, as well as the cavity, are continued into the tentacles, each of which is thus a miniature of the main body (see fig. 194). Between these two layers of cells, everywhere, there is a layer called the mesogloea which is a secretion, and not a cell-layer. This is highly elastic and performs the same function as the cuticle of Paramecium, causing the body to revert to its normal form as soon as contraction has ceased: it is in fact the skeleton of Hydra.

$\S 3$. The Ectoderm. The main part of the ectoderm consists of cells resembling those of columnar epithelium, tapering somewhat internally and ending in a process which 
lies against and is firmly attached to the mesogloea. These processes show a faint structure resembling that of unstriped muscle-fibre, and they are distinctly of a muscular nature, their combined contraction being the cause of the change of shape of the whole animal. Hence they are called muscle-tails, and the cells which bear them muscletail-cells (fig. $151, \mathrm{~B})$.

\section{$\S$ 4. Nema-} tocysts. Filling up the spaces between these last are $\mathrm{n} u \mathrm{~m}$ erous $\mathrm{s} \mathrm{m} \mathrm{a} 1 \mathrm{l}$ er rounded cells,

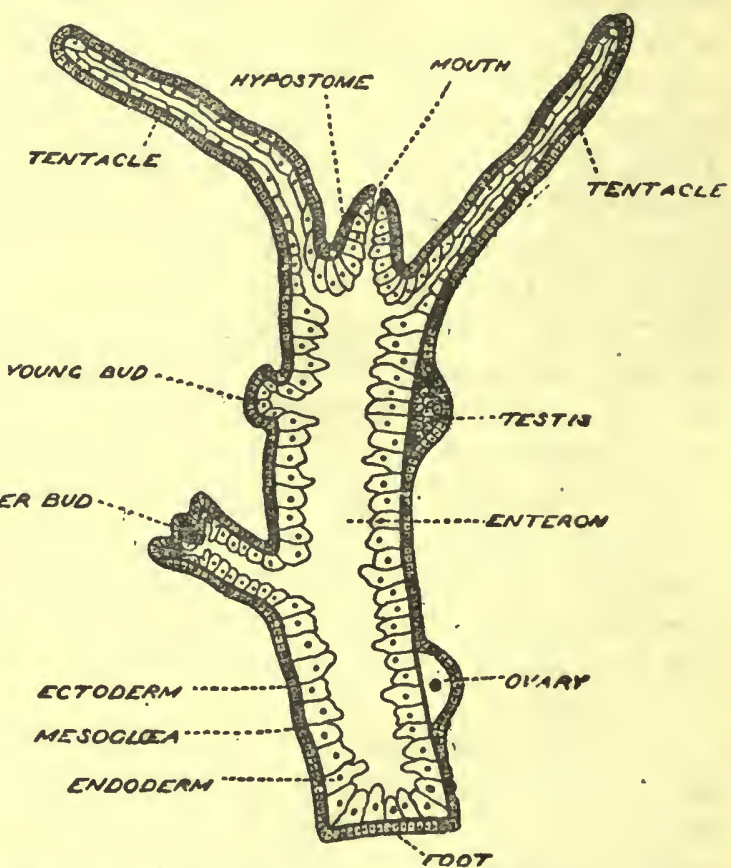

Fig. 194.-Median Longitudinat Section of Hyura.

(Rather diagrammatic.) the interstitial cells. These may be regarded as reservecells, comparatively undifferentiated and ready to take on one of several possible functions as may be necessary. Most commonly, perhaps, they develop into cnidoblasts, cells which secrete within themselves remarkable structures called nematocysts. Imagine a closed bladder drawn out at one end into a long slender hollow thread; let this thread be turned outside in and be coiled up within the main part of the bladder. Imagine further that this bladder is tensely filled with liquid - then, evidently, any pressure on the bladder will cause the hollow thread to be more or less completely everted, and a sudden and sufficient pressure would cause the thread to be shot out with considerable violence. Such 
is essentially the structure and action of a nematocyst. The cnidoblast which encloses it comes, when fully developed, to the surface of the ectoderm, and from its free surface there projects a fine stiff protoplasmic process, like one of the sensory hairs of a sensory epithelium, called the cnidocil. It would seem that when any external body touches this process, the protoplasm of the cnidoblast is stimulated to move in such a way as to press on the nematocyst, and cause the ejection of the thread (fig. 195, B, c). Apparently some poisonous substance is ejected with it, and it is this which stuns the water-flea that touches a tentacle,

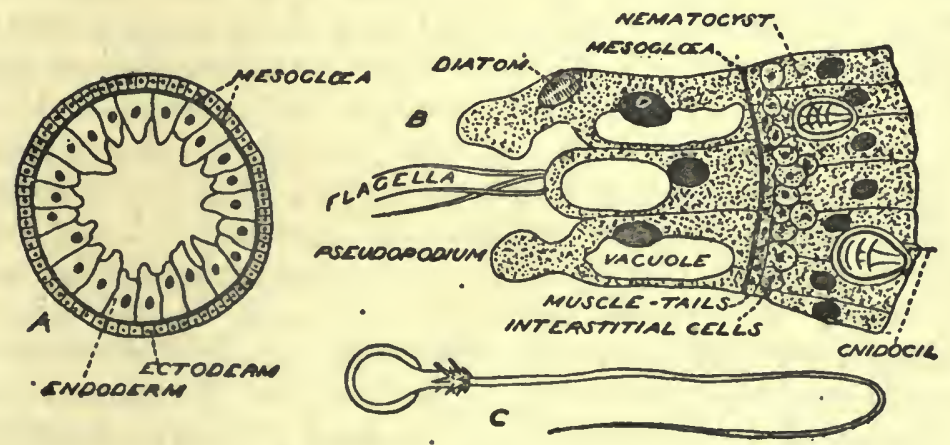

Fig. 195. - Structure of Hydra.

$\Lambda$, transverse section; $B$, small portion of the same enlarged-ectoderm to the right, endoderm left. $\mathrm{C}$, an everted nematocyst. (B and $\mathbf{C}$ after Parker.)

nematocysts being specially abundant on the tentacles, where they are grouped in "batteries." New cnidoblasts are continually being formed to replace those which have been "fired." Thus the soft-bodied Hydra, incapable of flight and seemingly quite defenceless, is not only efficiently protected against enemies, but is even enabled to prey upon animals much more active and more highly organized than itsolf.

§ 5. Nervous System. If the description given above of the action of a cnidoblast is correct, we have in it an example of a cell which combines the characters of a sensory and of a motor (muscular) cell. A stimulus of a 
definite kind (pressure on the cnidocil) unlocks a store of potential energy, and causes an amount of work to be done quite disproportionate to the pressure itself. In view of the fact that pressure on some one part of a Hydra will be followed by both muscular contraction and the firing-off of nematocysts through a much larger region-even over the whole body-it is evident that some sort of a nervous system must exist for the transmission of stimuli to a distance. And in fact we find, scattered among the other ectoderm cells, a number of multipolar nerve-cells, whose processes enter into intimate association with the sensory-motor cnidoblasts, with one another, and with the muscle-tailcells: The exact nature and extent of this association is not fully known - the processes have been described as continuous between one cell and another, but modern methods of histological research have so constantly shown such supposed continuity not to really exist, that we may well feel doubtful about the condition in Hydra. It is not even known whether the distinction between axis-cylinder and dendrons, so characteristic of nerve-cells in the higher animals, is found here. However this may be, the scattered condition of the nerve-cells, which are not compacted into any central nervous system, is a more primitive condition than we have in any of our other types which possess a nervous system.

$\S 6$. Gland-cells. The only other modification of the ectoderm cells that need be mentioned at present is the glandular character of those at the attached end, or "foot" as it is often called. These secrete a sticky substance which causes firm adhesion. In this region interstitial cells are absent.

§ 7. The Endoderm (fig. 195, B) consists for the most part of larger cells than those of the ectoderm. Each of these contains a large vacuole (i.e. a space containing fluid), which differs from the food-vacuoles and contractile vacuoles of Protozoa, in being a comparatively permanent structure in the cell. The end of the cell which is next the mesogloa is extended into a muscle-tail, less conspicuous than that of 
an ectoderm-cell. The free surface, abutting on the enteron, is amœboid, projecting either into blunt pseudopodia or into long thread-like ones called flagella. A flagellum is intermediate in character between a pseudopodium and a cilium.

The pseudopodia can, like those of Amœba, ingest small particles of food, so that part of the process of digestion takes place within the protoplasm of the endoderm cells (intra-cellular digestion); but not all. For the endoderm cells of the hypostome, which are smaller than the rest, are actively secretory. There can be little doubt that they secrete a juice something like the rabbit's pancreatic juice, containing one or more ferments, and that part of the digestion is carried on by this secretion in the enteron (enteric digestion).

The food of Hydra is not limited to organisms large enough to be grasped by the tentacles. Diatoms may also be found ingested in its endoderm cells: in the absence of true cilia, we must suppose these to have been brought in by a current of water caused by the flagella.

§ 8. Chlorophyll. There are three generally recognized common species of Hydra, distinguished principally by the difference of colour of the endoderm, which shows through the transparent ectoderm, and gives an apparent colour to the whole animal. Thus Hydra viridis is green; H. fusca, yellow-brown; $H$. vulgaris, colourless. In the first of these the green colouring-matter is none other than chlorophyll, so characteristic of the vegetable kingdom, and it occurs in specialized portions of protoplasm, which are identical in character with the chromatophores (chloroplastids) of plants.*

There can be no doubt that the nutrition of $H y d r a$ viridis is partly effected in the manner of a plant, viz. by the utilization of sunlight-energy, in building up organic compounds from simple inorganic ones (holophytic nutrition), as well as by the purely animal method (holozoic nutrition). In proof of this, we find starch present in the endodermcells. Attempts have been made to show that these chroma-

* See Text-Book of Botany, chapter ii., §§ 9 and 14. 
tophores are not parts of the Hydra, but independent organisms-unicellular Alga-living in close and mutually advantageous association (symbiosis) with it. But as the chromatophores have neither nucleus nor cell-wall, they cannot be unicellular Algæ.

In $H$. fusca, chromatophores are also found, but with another colouring-material instead of chlorophyll. It has been suggested that the two species are really the same under different conditions which cause the one or the other colouring material to appear. But there is no evidence of this : the two forms live side by side under the same conditions; and colour is not the only difference between them. $H$. fusca is always longer and slenderer than $H$. viridis, and has much longer and finer tentacles. The balance of evidence is in favour of their being distinct species, though the reader who remembers what we have said in chapter xii. will see that there is no absolute criterion.

\section{§ 9. Physiological Differentiation. Taking the two} layers as a whole, we see that the ectoderm is essentially protective, sensory, and nervous, the endoderm digestive. Both are also in some degree contractile (or muscular) and in places secretory. Both are epithelia, though in both we have, as it were, a beginning of differentiation of muscletissue, and in the ectoderm of nerve-tissue. The secreted mesoglœa evidently represents a very simple kind of endoskeleton, but there are no connective tissues of any kind. Neither is there any rudiment of a vascular system, the products of digestion and of katabolism easily diffusing from cell to cell in so simple an animal. The absence of respiratory organs is not remarkable, for in so small an animal every cell is in contact with the water, or nearly so. It is somewhat surprising, however, to find no trace of renal-excretory organs, seeing that even Amœba has a contractile vacuole for this purpose. Possibly wasteproducts may be excreted along with the useful products by the gland-cells of the endoderm. The absence of true cilia in Hydra is accounted for by its method of feeding; cilia are abundant in some allied organisms.

§ 10. Asexual Reproduction. So far we have said nothing about reproduction, for Hydra stands alone among 
our motazoan types in having no permanent organs of reproduction. It does, however, reproduce itself freely if well fed, and the common method can easily be watched. It is known as budding, and is not unlike the branching of a plant: in fact it simply differs from ordinary growth by being localized and eventually discontinuous. Both ectoderm and endoderm grow rapidly at one point, and a protuberance containing a diverticulum of the enteron.appears at the surface. This increases in length, tentacles develop one by one at its distal end, and a mouth is presently opened in the centre of them. Gradually the bud grows into the form of a Hydra, the attachment to the parent becomes constricted and the enteric cavities separated, and finally the young Hydra is detached to look after itself. Soveral buds may often be seen at once on a Hydra. In this way Hydra will multiply fifty-fold in a few months.

Again, if a Hydra be cut across, each half will soon grow so as to restore its lost parts, and two Hydræ will thus be formed.

In both these cases, it is to be noted that ectoderm-cells give rise only to ectoderm-cells, and endoderm to endoderm. No exchange of character can take place between them. One of the earliest observers of Hydra tried the experiment of turning Hydra inside out, and thought the animal could live as well as before in this new condition. This is now known to be a mistake, as a knowledge of the detailed structure of ectoderm and endoderm would indicate.

§ 11. Sexual Reproduction. Asexual reproduction by budding cannot apparently be continued indefinitely. As in the case of Paramecium, complex nuclear changes seem to be necessary (or, at least, advantageous) from time to time: these are effected by sexual reproduction. The gonads are developed from the interstitial cells of the ectoderm, testes and ovarios being formed simultaneously on the same Hydra-the testes near the hypostome, the ovary or ovaries nearer the foot. In each case there is rapid multiplication of the interstitial cells till a slight protuberance is seen on the surface; this differs from a 
bud in that the mesogloea and endoderm take no part in it. If the gonad is to become an ovary, one of the interstitial cells becomes amceboid and increases in size by feeding on the others. Yolk-spheres are stored up in it, and also (in $H$. viridis) chromatophores-a remarkable fact in a cell of ectodermic origin. Eventually the ovum, for such it is, ceases growth and becomes spherical, its nucleus divides twice, and the usual polar bodies are cut off. About this time the layer of ectoderm cells that had been covering it hitherto splits, and the ovum is exposed to the water. It is now a large spherical body, about a millimetre in diameter, often more conspicuous than the Hydra that bears it.

Meanwhile the interstitial cells in the testes have been dividing to form spermatozoa of the ordinary form. These escape and swim about, one of them eventually meeting the ovum and fusion of the pronuclei taking place.

An animal in which, as in Hydra, both ova and spermatozoa are produced on the same individual is said to be hermaphrodite. In many hermaphrodite animals there is provision of some kind for making cross-fertilization (i.e. the fusion of pronuclei derived from separate individuals) easier than self-fertilization, but no such provision is found in Hydra. The fertilized ovum segments until a hollow blastosphere is formed. The single cell-layer then becomes divided into two, and a two-layered stage is produced, differing from a gastrula in the absence of a blastopore. From this point development is simply a matter of differentiation.

§ 12. Somato-plasm and Germ-plasm. Thus, while in asexual reproduction ectoderm gives rise only to ectoderm, and endoderm only to endoderm, in sexual reproduction a cell derived entirely from ectoderm gives rise to a complete Hydra, consisting of both ectoderm and endoderm. Evidently the nuclear material of the interstitial cells of the ectoderm contains, in addition to what we may call its "ectodermal potentiality," a further "full Hydra potentiality" which becomes active as a result of the sexual process. According to the theory of Weissmann, these two potentialities belong 
to two distinct nuclear materials combined in one nucleusa somato-plasm which has only the potentiality of producing (in this case) ectoderm-cells, and a germ-plasm which has the potentiality of producing a complete Hydra.

These two nuclear materials would correspond very closely with the two which form the two very distinct nuclei of Paramecium-somato-plasm answering to meganuclear material, germ-plasm to micronuclear. Attempts have been made to distinguish between these two materials in the nuclei of Metazoon cells, but without much success. We need therefore say no more here, but would only call the reader's attention once more to the comparison of the life-histories of higher Protozoa and Metazoa on p. 283.

§ 13. Hydra and the Gastrula. The student will probably have already been struck with the general resemblance, in respect of the broadest facts of structure, between Hydra and the gastrula of Amphioxus. In each there are two layers of cells with a central enteric (or archenteric) cavity - the outer layer in each case giving rise to sensory, nervous, and protective structures; the inner, to digestive structures. Thus it may be said that Hydra remains permanently at the gastrula stage, so far as general structure goes. On the theory of evolution, the remote ancestors of Amphioxus were probably permanent gastrulæ, and Hydra may be regarded as a representative of the group of animals from which the ancestors of Amphioxus sprang. But we must be careful to avoid the easy mistake of assuming that Hydra closely resembles those hypothetical ancestors. Hydra is by no means a simple gastrula-its tentacles, nematocysts, lack of cilia, fresh-water habitat, and crustacean diet, are all features very unlikely to have been found in the primordial gastrulæ. The following words are as true now as when they were written forty years ago by Huxley, who first pointed out the similarity of Hydrozoa to Vertebrate embryos:- "All that can be justly affirmed is that the Hydrozoon travels for a certain distance along the same great highway of development as the higher animal, before it turns off to follow the road which leads to its special destination." 
§ 14. The Cœlenterata. Animals which, like Hydra, never advance beyond the gastrula stage in the direction of the development of mesoblastic tissues constitute the great phylum of the animal kingdom called Coclenterata. All animals that do advance beyond this stage are grouped as Coclomata.

If we regard the mode of origin of the colom in Amphioxus (by outgrowths from the archenteron) as the primitive method, then we may say that the primitive enteron (or archenteron) of Coelenterata has been differentiated in at least some of the Coelomata into two cavities-mesenteron and cœlom.

The Coelenterata include a great variety of organisms, almost all marine, such as sea-anemones, coral-polyps, jellyfish, and forms like Hydra, but more

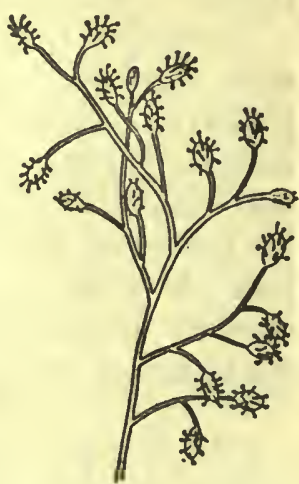

Fig. 196.-A CoMPound HYDROZOON.

(Coryne.) permanently fixed and branched. Such forms, in which buds, formed as in Hydra, remain permanently attached to the original individual, themselves budding in turn, are spoken of as colonial animals (fig. 196). 
Chapter XXX.-Obelia geniculata.

$\S 1$. Form and 0ccurrence. This organism occurs in two forms, one that of a fixed compound zoophyte, the other that of a free active medusa. The fixed form is called the hydroid stage, the free form the medusoid. The hydroid develops from a fertilised ovum, the medusa is produced by budding from the hydroid. The hydroid is a compound organism consisting of a number of members connected with one another, and produced by gemmation. I propose therefore to call it a gemmarium, a term which is more appropriate than those hitherto employed. It consists of small upright stems attached by their bases to the surface of sea-weeds, and is very common on our shores on the fronds of Fucus and Laminaria. Each stem is about an inch in length, and the members of the compound are "zooids" attached alternately to opposite sides of the stem (Fig. 201).

§ 2. Structure of Zooid. Each zooid has a structure similar to that of a Hydra. It consists of a body wall composed of ectoderm, mesogloea, and endoderm, enclosing a simple cavity. At the distal end of the zooid is an aperture, the mouth, while at the attached end the body passes into a cylindrical stalk or peduncle, which is connected with the stem, and the stem itself is tubular. The part immediately below the mouth is conical and is called the hypostome; this is about one third of the length of the zooid, and is separated from the main part of the body by a circle of tentacles, which are narrow outgrowths of the body-wall, longer than the hypostome. Each tentacle consists of ectoderm externally and endoderm internally, but it contains no cavity, the endoderm consisting of a single row of vacuolated cells. Between the ectoderm and endoderm there is a layer of mesogloea. The stem to which 387 
388

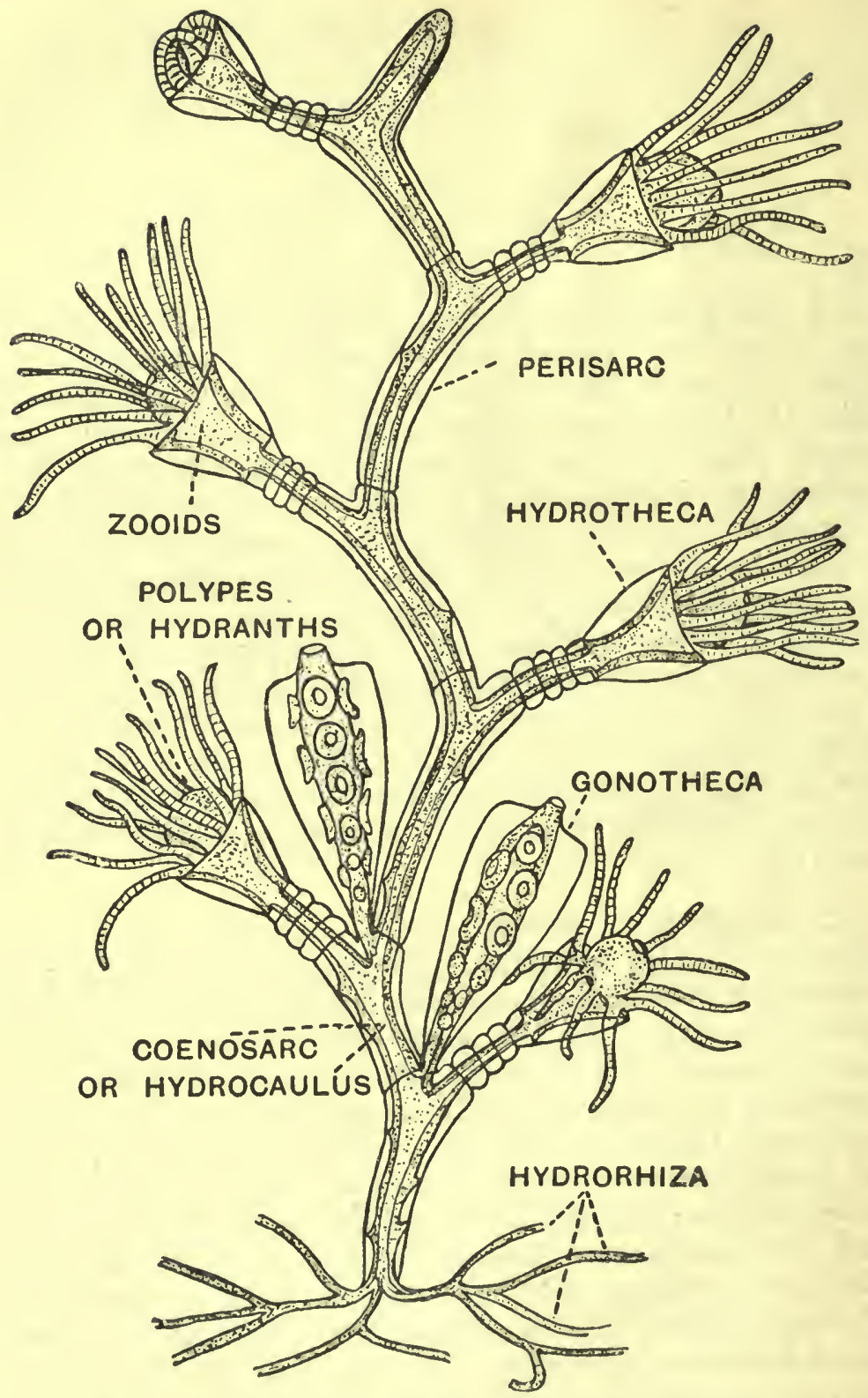

Fig. 197.-A Geminariun of Obulia genicuiata mannified. 
the zooids appear to be attached is in reality not a distinct structure, but is composed of the basal portions of the successive zooids, for the whole gemmarium consists entirely of a series of zooids each budding from its predecessor. Each zooid produces but one bud, and the buds are first on one side and then on the other alternately.

$\S 3$. The Perisarc. The whole gemmarium is surrounded by a cuticular secretion of the ectoderm, called the perisarc. 'This is not in contact with the ectoderm, but separated from it by an interval, except at occasional spots, where the ectoderm cells extend to meet it. The tubular perisarc expands at the end of each branch into a conical cup open at the end, which protects each zooid. This cup is the hydrotheca, and into it the zooid when contracted is withdrawn. Between each hydrotheca and the stem the perisarc is divided by annular constrictions into a number of rings, and there is usually one such ring in the stem itself above the origin of each zooid. In the interior of the hydrotheca near its base the perisarc forms a little annular shelf, on which rests the base of the "hydranth," by which name the enlarged terminal portion of the zooid is distinguished. This base is continued into a narrow stalk, the hydrocope, which passes into the peduncle of the zooid. The interior of the whole organism is occupied by the coelenteric cavity, everywhere continuous. The upright stems arise from a root-like branching system of filaments, which have the same structure as the stems, but are much thinner : these are attached by their whole length to the surface of a sea-weed. The details of structure of the hydroid zooid or hydranth are represented in fig. 198, as seen in a longitudinal section highly magnified. It will be seen that the structure is closely similar to that of the fresh-water Hydra. The ectoderm is thin, and consists of columnar cells and smali interstitial cells. In it there are numerous nematocysts, more especially in the tentacles, where they are arranged in annular ridges. The endoderm cells are large and granular; most of them are furnished with flagella, which are in vibratory motion during life, but the flagella may be withdrawn and pseudopodial processes protruded. 
$\S 4$. Blastostyles and Gonothecae. When an erect stem has reached its full development it produces special reproductive zooids in the axils of the peduncles of the ordinary zooids. These arise by gemmation, and have the form of
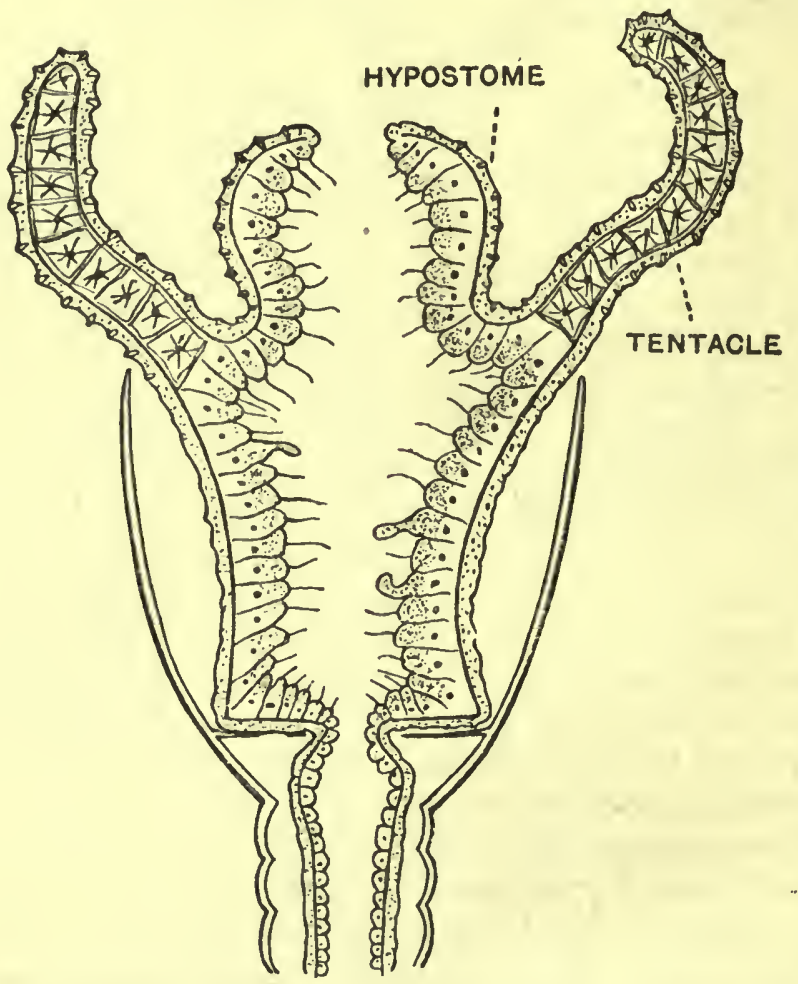

Fig. 198. -Lovaitud: 1L SfCrion or a IIroroll Zoom minly Magified.

narrow cylindrical tubes closed at the outer end. They are therefore zooids, without either mouth or tentacles. They are contained in special capsules formed of perisarc. These capsules are called gonothecae, and have an elegant shape like that of an ancient amphora, with a short marrow neck ending in an aperture. The blastostyle gives rise to a considerable number of lateral buds, which develop into medusae. Each bud at first is a simple process of the wall of the blastostyle, and the manner in which it becomes a 
medusa is best explained when the structure of the medusa has been described.

$\S 5$. The Medusa. The medusa is about $\frac{1}{4} \mathrm{in}$. in diameter when full-grown. It consists (Fig. 199) of a circular disc slightly campanulate or bell-like in shape: this is called the umbrella. From the middle of the concave surface of the umbrella hangs a short hollow process, the manubrium, at the end of which is the mouth. The cavity of the

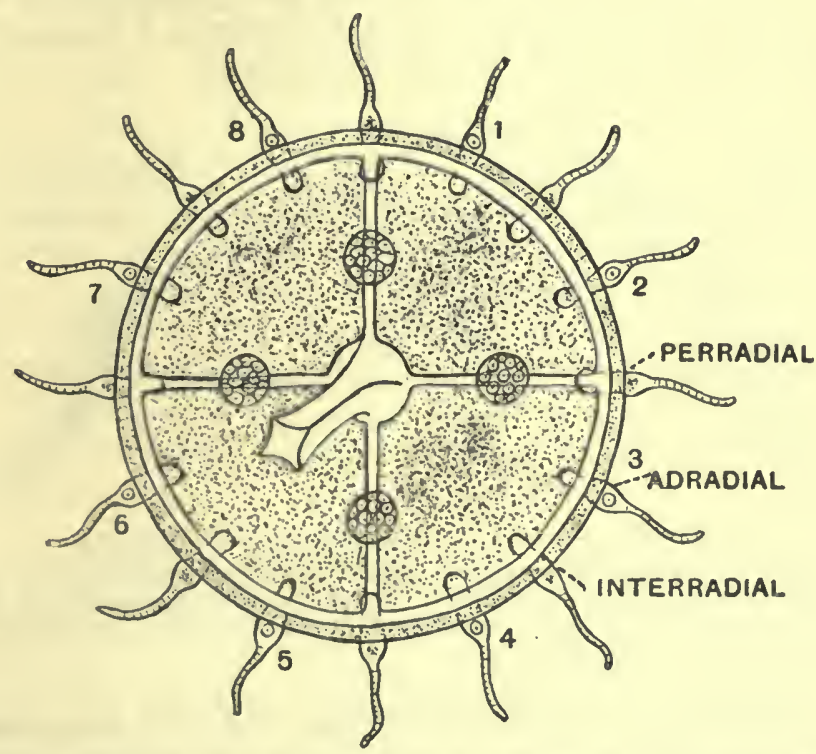

Fig. 199.-Mrduba of Obelia seen frox tilk Vremtral or Orai, Sidr.

The figures 1 to 8 are placed near the bases of the adradial tentacles containing the statocysts. The gonads are seen on the radial canals and the manubrium in the centre.

manubrium leads into a central cavity in the umbrella, and from this radiate four radial canals which open into a circular canal running near the margin of the umbrella. The cavities and canals are, of course, lined with endoderm, but the endoderm is not confined to them. In reality the umbrella is divided into two layers, a dorsal and ventral, or upper and lower, by a continuous double layer of endoderm (Fig. 200). The upper layer is called the exumbrella, the lower the subumbrella. The two layers of 
endoderm are in close contact, except in the course of the radial canals and circular canal, where the two layers separate to form these canals. Thus the umbrella is to be regarded as due to the approximation of the upper and lower walls of a flattened sac, and the mesogloea is everywhere between endoderm and ectoderm, as the general structure of coelenterates requires. The double layer of endoderm extending between the radial canals is called the endoderm lamella, and if this were not present the mesogloea of the umbrella would be between two layers of ectoderm, as it never is in coelenterates.

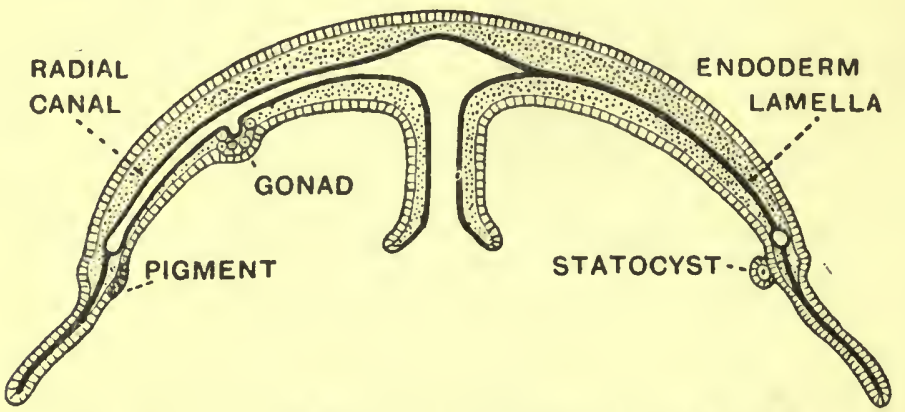

Fig. 200.-Diagram of a Vrertical Section of a Mrdusa.

The endoderm is represented by a black line, the ectoderm by a band of transverse lines, the mesogloea by dots. The section really consists of two separate halves, that on the left passing through a radial canal, that on the right through the endoderm lamella between the canals.

$\S 6$. Tentacles and Sense Organs. Around the margin of the umbrella are numerous tentacles, whose number increases with the age of the medusa. The position of these tentacles and other organs is fixed by reference to radii of the circular umbrella. The four radial canals fix the position of four principal radii, called per-radii, and at the ends of these are four per-radial tentacles. Intermediate between the per-radii are four interradii with interradial tentacles. Bisecting the angles between these are eight adradii with adradial tentacles. The tentacles are somewhat swollen at their bases and on the lower side in all of them there is a little spot where the ectoderm cells are pigmented. These pigment spots are probably sensitive to the stimulation of light, but have not the 
special structure of ocelli. The eight adradial tentacles have, in addition, projecting from the inner side of the basal enlargements, small vesicles formed from ectoderm and containing calcareous concretions. Such organs were formerly regarded as rudimentary auditory organs, but as our own semicircular canals are organs of equilibrium, so experiment indicates that equilibration is the principal function of these vesicular organs in the medusa of Obelia and others of the lower animals. It is better, therefore, to call them not otocysts, but statocysts. The presence of these statocysts is characteristic of the medusae called Leptomedusae, which are derived from hydroids with hydrothecae. In the Anthomedusae there are ocelli, but no statocysts, and the hydroids of these are not provided with hydrothecae or gonothecae. In Obelia there are never more than the eight statocysts on the eight adradial tentacles, but the number of tentacles increases considerably in older medusae, and each bears a pigment spot. The tentacles are solid, a core of vacuolated endoderm cells extending along the axis of the tentacle as in the hydroid.

$\S 7$. Development of Medusa. The mode in which the medusa is developed as a bud on the blastostyle can now be understood without difficulty (fig. 201). The first stage of the bud is a simple small diverticulum of the cavity of the blastostyle. This diverticulum grows longer and then enlarges at the extremity so that it forms a little vesicle enclosed by ectoderm, mesogloea, and endoderm, and connected with the blastostyle by a narrow stalk. The cavity of the subumbrella is now developed in a peculiar way which would not be expected. The cavity is not formed by the simple moulding of the vesicle to the shape of a medusa, but the distal ectoderm separates into two layers; the inner layer acquires a cavity, and this little sac, which may be called the bell-rudiment, enlarges and acquires the shape of the subumbrellar cavity with the manubrium in its centre. The cavity therefore, although lined throughout by ectoderm, is at first closed by a layer of ectoderm which extends from one margin of the 
umbrella to the other. This membrane is at last broken through and the remnant of it forms a circular shelf projecting inwards from the margin of the umbrella. This shelf is called the velum, and is permanent and conspicuous in most hydroid medusae, but in Obelia it diminishes

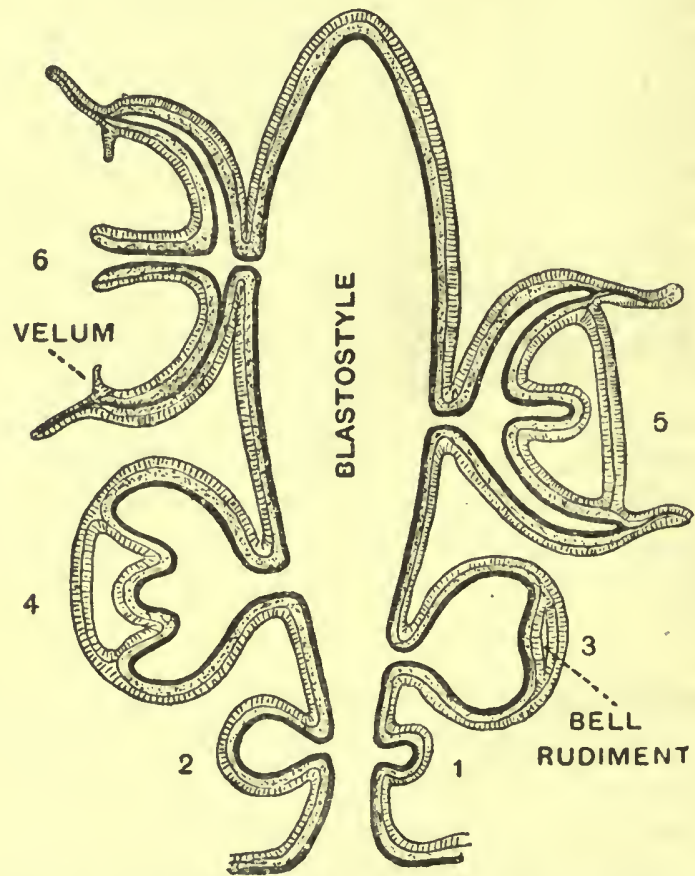

Fig. 201.-Diagra of tile Development of a Medusa from the Blastostyle.

The successive stages are numbered 1 to 6 and are represented as all connected with the same blastostyle,

and becomes quite vestigial. It is therefore not represented in our diagrams. When the medusa bud has acquired its final shape the mouth breaks through at the extremity of the manubrium and finally the stalk is broken, the aperture into the enteric cavity formed by the rupture closes up, and the little medusa becomes free and escapes from the gonotheca through the aperture at its end.

§8. Comparison of Hydroid and Medusa. By comparing the structure of the two it can be seen that the medusa is 
a modified hydroid zooid. The mouth is homologous in the two cases, and so likewise are the tentacles. The subumbrella therefore corresponds with the hypostome, the central part projecting as the manubrium. The oral and aboral layers of endoderm, instead of being separated by a continuous cavity, have come into contact to form the endoderm lamella, except where the central cavity and the radial and circular canals occur. The mesogloea is very much thickened. The original cavity is thus divided up. The hydroid is elongated along the axis of symmetry which passes through the oral and aboral poles, the medusa is shortened along this axis and expanded in the plane which passes through the bases of the tentacles. The medusa therefore is a modified zooid which separates from the gemmarium.

§9. Generative Organs or Gonads. The generative organs of the medusa are four in number, one in the middle of the course of each radial canal. They are formed by ventral diverticula of these canals, which, pushing the mesogloea and ectoderm before them, form projections from the subumbrellar surface. 'The sexes are separate. 'The germ-cells have been traced in the early development of the medusa on the blastostyle as originating in the ectoderm of the manubrium, but they afterwards migrate to the endoderm, and then to the gonads, where before maturity they leave the endoderm and lie between the ectoderm of the subumbrella and the mesogloea. When ripe they escape through the ectoderm into the sea-water, where fertilisation and development take place.

$\S 10$. Development of 0vum. The fertilised ovum segments to form a hollow blastula, which becomes solid by the delamination of cells from the blastula wall. There is no invagination and therefore no gastrula. The outer layer of cells becomes uniformly ciliated and the embryo is now called a planula. A cavity appears by splitting in the solid endoderm. The planula is easily converted into a single hydroid zooid. It attaches itself by one end to stone or weed, forms a mouth by rupture at the other, and then 
the circlet of tentacles grows out in a circle below the mouth. By gemmation the hydroid gemmarium is produced and so the life-cycle is completed and starts again.

$\S 11$. Metagenesis. It will be seen that in the lifehistory of Obelia there is a regular alternation of hydroid and medusoid. In this species and many others the medusa never gives rise to medusae, but its eggs develop into hydroids, from which in turn the medusae are produced by budding. The phenomenon was originally called an alternation of generations. This term as first used referred more to the organisms than to the mode in which they were produced, just as we speak of the members of a family as of the first, second, third, or fourth generation. The fact of the alternation is a matter of observation: the zoophyte produces medusae and the medusae produce zoophytes. Objections, however, have been raised to this use of the term generation, and if we restrict this term to the production of new organisms by fertilised germ cells or gametes it will be seen that there is only one process of generation in the life-history of Obelia, namely the sexual reproduction of the medusa. The medusa, however, is not produced directly from the egg, but is budded off from a hydroid form which develops from the egg. Generation, therefore, is, as it were, postponed, and the process has been called metagenesis (meta, after; genesis, generation). There is, therefore, an alternation of gemmation and generation or sexual reproduction. In gemmation not single cells but whole tissues grow to give rise to the new organism.

Further, we see that there are not one but several successive processes of gemmation before the medusa is produced. The medusa is not to be compared with the zoophyte, but with the zooid. The zoophyte is a compound structure, a gemmarium, consisting of several zooids. The medusa is not budded from the first zooid developed from the egg, but from a blastostyle which is itself a modified zooid, and the blastostyle is budded from a zooid, which may be the second or third in succession 
from the egg. We see, therefore, that generation does not strictly alternate with one gemmation, but with a whole series of gemmations.

$\S 12$. Individuality. The study of the gemmarium of Obelia gives rise to certain other important theoretical considerations. We are accustomed from experience of the higher animals to think of individual animals as separate from one another, and incapable of continuing to live when divided. In Obelia, however, we see a considerable number of distinct animal units each with its own organs, but all organically connected. We have seen that the medusa is an individual constructed on the same general plan as the sessile zooid, but it is separate and independent. We can scarcely, therefore, deny the claim of the zooids to be considered as individuals. At the same time, these individuals are not so independent and complete as those which are separate: the ordinary zooids have no reproductive organs, the blastostyles have no feeding organs, but are specialised for the production of medusae. There is thus an individuality, an organisation of the gemmarium, and the zooids have to a certain extent lost their independence and completeness and become subordinated to the needs of the aggregate to which they belong. In other compound coelenterates this loss of individuality has gone much further, so that the zooids have become mere organs of the gemmarium, entirely dependent for their existence on the life of the compound of which they are members. It is thus difficult to give a generally applicable definition of the individual, or organic unit. It is rather a question of degree, the degree of centralisation. When the units are separate from one another there is no difficulty, but when they are organically connected the individuals may be reduced and subordinated to a new organisation or individuality, that of the compound. And in other cases, as in metamerism, it is sometimes difficult to decide whether the similar parts are to be regarded as individuals which are losing individuality, or parts of a single individual which are acquiring it. 
$\S 13$. Polymorphism. Closely associated with this question is the phenomenon of polymorphism, which may be defined as the existence of individuals of different form and structure in the same species. This is distinctly exemplified in Obelia. The medusoid and the hydroid are both produced from the same parents, but they have a different structure and different organs. In the gemmarium, again, the blastostyle is different from the feeding zooids. In allied forms such different zooids are distinguished as trophozooids and gonozooids. Obelia, therefore, may be said to be trimorphic. In the gemmarium polymorphism is connected with the specialisation of the zooids and the development of the individuality of the gemmarium ; from the latter point of view the ordinary zooids are feeding organs, the blastostyles the reproductive organs. Thus the relation between the medusa as an independent zooid and the gemmarium as a compound consisting of many zooids is somewhat similar to that between a Protozoan consisting of one cell and a Metazoan which is a compound of many cells. In Herbert Spencer's language, if we take the cell as the unit, then an ordinary individual such as the Medusa is an aggregate of the first order, and the gemmarium an aggregate of the second order. 
Chapter XXXI.-The Slipper-Animalcul\%.

\section{(Paramecium aurelia.)}

$\S 1$. Form and Mode of Life. Paramecium is a minute organism very common in fresh water where there are decaying vegetable remains. It is just visible to the naked eye as an elongated whitish spot. Microscopic examination soon shows that its body consists of a single cell, as does Amœba; but two striking differences from the latter type are at once seen. Firstly, Paramecium has a definite and

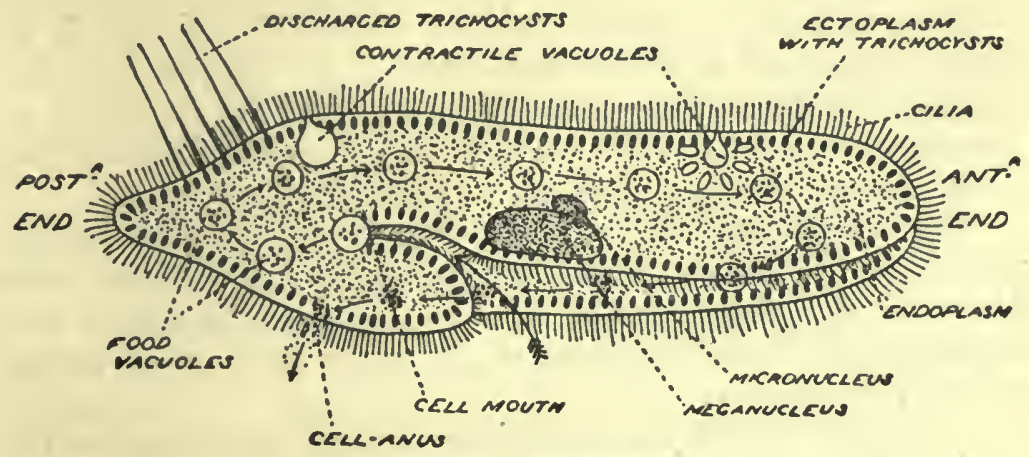

Fig. 202.-Paramecius.

(After Parker.)

constant shape; and secondly, it swims about through the water (rotating as it does so) or glides over the surface of any solid with what appears to be great rapidity as compared with the slow crawl of Amceba.

The shape of Paramecium (fig. 202) is not unlike that of the slipper which gives it its common name-that is to say, it is elongated in one direction, rounded at one end and bluntly pointed at the other, while on one of the flattened 
surfaces there is a shallow groove (answering to the hollow of the slipper). The rounded end always moves first, and is therefore anterior ; the pointed end being posterior. Here we see the same adaptation of shape to the swimming habit that we found in the dogfish. The grooved surface, being always towards the surface over which the animal glides, is ventral. These terms "anterior," "posterior," "dorsal" and "ventral," are merely used for convenience of description. As applied to an animal consisting of one cell only, they cannot imply any morphological comparison with the regions similarly named in a Vertebrate.

§ 2. Protoplasm and Cuticle. While having a definite and constant shape, Paramecium is flexible and elasticbeing able to squeeze itself through narrow operings and bend round corners. These characters are due to the enclosure of the protoplasm in a thin but firm membrane secreted by its outer surface; a membrane of this kind secreted by the outer surface either of a unicellular or multicellular animal, is called a cuticle. Since its function is to keop the animal in shape, and afford a firm support for all internal motions of the protoplasm, it may be regarded as the skeleton of the Paramecium. A skeleton forming the external surface, as this does, is called an exoskeleton.

The protoplasm within is differentiated into two layers, which are far more unlike and permanently distinct than the ecto- and endoplasm of Amœba. The terms cortex and medulla are therefore given to the inner and outer protoplasm respectively.

From the cortex there project through apertures in the cuticle into the surrounding water a great number of delicate cilia, just like those of the ciliated epithelium of a Vertebrate. It is by the vibration of these cilia that the locomotion of the animal is effected.

Embedded in the cortex at right angles to the surface are numerous little bodies called trichocysts. When pressure, or some irritant liquid, is applied to the surface of the animal, a fine long thread is ejected from each trichocyst. Owing to their small size it is difficult to say how this takes place, but it seems probable that these structures are similar to 
the larger structures which we shall meet in $H y d r a$ (chap. xxiv., $\$ 4$, nematocysts), and like them are means of attack on or defence from other small creatures, though differing from them in that a multitude are developed from a portion of the single cell, instead of each one in a separate cell.

§ 3. Cell-mouth and Cell-anus. At the bottom of the ventral groove there is a small area where cuticle and cortex are both wanting. This is the only point at which ingestion of food-particles can take place, and it may therefore be called the cell-mouth. It is obviously quite a different thing morphologically from the mouth of a multicellular animal; its function, however, is the same. The cilia in the groove sweep all solid particles along with a current of water towards this point, and here the former are taken into the medullary protoplasm along with a drop of water, as a food-vacuole. The food-vacuole travels along in the medulla and undergoes a kind of circulation through it, while the food-particle is being digested. Eventually all that remains unabsorbed is cast out at a point near the cell-mouth, called the cell-anus or-from the fact that the medulla is only exposed here at the moment of defæcation and not permanently as at the cell-mouth - the potential cell-anus.

$\S 4$. Contractile Vacuoles. Paramecium has two contractile vacuoles-one anterior and one posterior. These show a more complex structure than that of Amœba, for while the vacuole is refilling, a number of radiating tubes can be seen along which the water is plainly being conveyed into the vacuole. Otherwise the action of these is exactly like that in Amoba, and there is no doubt that they serve the same function, viz. the elimination of water containing in solution some of the waste-products of katabolism.

§5. The Two Nuclei. There are two nuclei, embedded in the medullary protoplasm; but these two nuclei have a very different significance from the two or more sometimes found in an Amoba. They differ in size and appearance, and also, as we shall see presently, in function. It is convenient to distinguish the larger as the meganucleus, and the smaller as the micronucleus. 
\$ 6. Multiplication takes place much as in Amoba. First the micronucleus and then the meganucleus divides - the former by karyokinesis, but not the latter-and the animal becomes divided into two in a transverse plane. Each half by slight changes soon comes to have the form of a perfect Paramecium.

When well fed, Paramecium will multiply in this way very rapidly, dividing once or twice in a day. Carefu! observations on the descendants of a single Paramecium, carefully isolated, have shown that this fission may continue for several months. By the end of that time, however, the character of the Paramecia was found to be changed; their vigour was diminished, their nuclei and protoplasm showed signs of decay, and eventually death became inevitable. Thus the power of continued division is not unlimited.

§ 7. Conjugation. Under normal conditions, this condition of "old age" is avoided by means of a process termed conjugation, which is of the greatest interest as aftording a means of comparison between the higher Protozoa and the Metazoa (multicellular animals). Conjugation in Paramecium consists in the temporary union of two individual Paramecia, during which certain internal changes take place. The exact details of these changes are very difficult to observe, and somewhat different accounts are given by different observers. But about the essential facts there can be no doubt, and to these we will confine ourselves.

Firstly, the internal changes concern the nuclei, and especially the micronucleus, which is very active; the meganucleus of each individual is at first unaffected, but eventually becomes disintegrated and disappears (possibly being digested by the protoplasm). The micronucleus of each individual, on the other hand, increases in size and repeatedly divides by karyokinesis; some portion of the products of division is exchanged between the two individuals, which then separate; fusion of the native and imported micronuclear material occurs in each one, and from the product a new meganucleus and new micronucleus are formed. The two Paramecia are now "rejuvenated" and at once proceed to divide in the ordinary way. 
§ 8. Reproduction in Protozoa and Metazoa. It has often been said that Protozoa are potentially immortal, while Metazoa must inevitably die. That is to say that in the former death is always the result of some external agent and may be indefinitely avoided, each generation becoming the new one, instead of being replaced by it; while in the latter old age and death inevitably follow, sooner or later, after reproduction is effected. This distinction is true as long as we consider only such Protozoa as Amceba, which appears to be capable of indefinite reproduction by fission. But in the case of Paramecium the matter is rather different. The material of its meganucleus is as inevitably doomed to death as the material of the nuclei of ordinary tissue-cells in a Metazoon; while the immortality of its micronuclear material is, like that of the nuclear material of the ovum and spermatozoon of Metazoa, entirely dependent on admixture with the similar material from another cell.

In fact, the analogy between the conjugation of Paramecium and the sexual reproduction of a Metazoon is very close, as the following comparison will show :-

PARAMECIUM.

A single "rejuvenated" individual divides repeatedly, forming a large number of cells which are all alike, and each of which is an independent individual.

Each of these cells contains two kinds of nuclear materialone (meganucleus) destined eventually to die; the other (micronucleus) having a potential immortality, which is, however, dependent on an interchange of part of its material for the sirnilar material of another individual-not derived from the same rejuvenated ancestor.

This process (conjugation) results in two rejuvenated individuals. a Metazoon.

A fertilized ovum divides repeatedly, forming a large number of cells which become differentiated, and are mutually dependent parts of a single multicellular individual [or, if asexual reproduction* also occurs, of several such individuals].

These cells are of two kinds - one (somatic cells) destined eventually to die; the otber (reproductive cells) having a potential immortality, which is, however, dependent on a removal of part of the nuclear material and its replacement by similar material from another reproductive cell not derived from the same individual.

These processes (maturation and fertilization) result in a single fertilized ovum.

* This will be explained in the next chapter. 
The wording of the right-hand column of this comparison is a little too positive : the occasional occurrence of parthenogenesis (chap. xvii., § 5), and of "self-fertilization" (i.e. the union of ovum and spermatozoon from the same parent) is sufficient proof that the ordinary "laws" of sexual reproduction in Metazoa express rather what is generally advantageous to a species than what is inevitable; and under peculiar circumstances the advantageousness may disappear. Parallel cases in the higher Protozoa are furnished by forms which are able to dispense with conjugation completely, or to a much greater extent than Paramecium. 


\section{Chapter XXXII.-Eughena.}

$\S 1$. Occurrence and Mode of Life. The organisms distinguished by the name Euglena are minute, unicellular forms of green colour, which occur commonly and often in great abundance in stagnant water containing much organic nitrogenous matter. Pools or ditches which are contaminated with the urine and excrement of farm animals almost invariably contain Euglenoids of one or more species, and one of the commonest forms is that known as Euglena viridis. The longest diameter of the organisms varies in different species from 03 to $.2 \mathrm{~mm}$., so that details of their structure are only to be made out by careful examination under high powers of the microscope. The most characteristic condition is that of active motion. So small that they cannot be separately distinguished by the naked eye, but merely give the water containing them a green colour, under the microscope they are seen as green specks moving rapidly in all directions.

The individual Euglena (fig. 203, A) is a single cell having a spindle-like shape, the end which is foremost as the organism moves being blunt and the opposite end tapering to a point. The body is surrounded by a distinct cuticle, which though firm and strong is very thin and is sufficiently flexible and elastic to permit of considerable and characteristic changes of form which the body occasionally exhibits. These changes consist of the contraction of one part of the body and the dilatation of another, and are spoken of as Euglenoid when they occur in other Protozoa. The surface of the cuticle is marked by spiral lines or striations, which appear to be delicate ridges on 
the surface. In Euglena viridis and many other species these spiral markings are very fine and close together, but in some cases, as in Euglena spirogyra (fig. 204), are
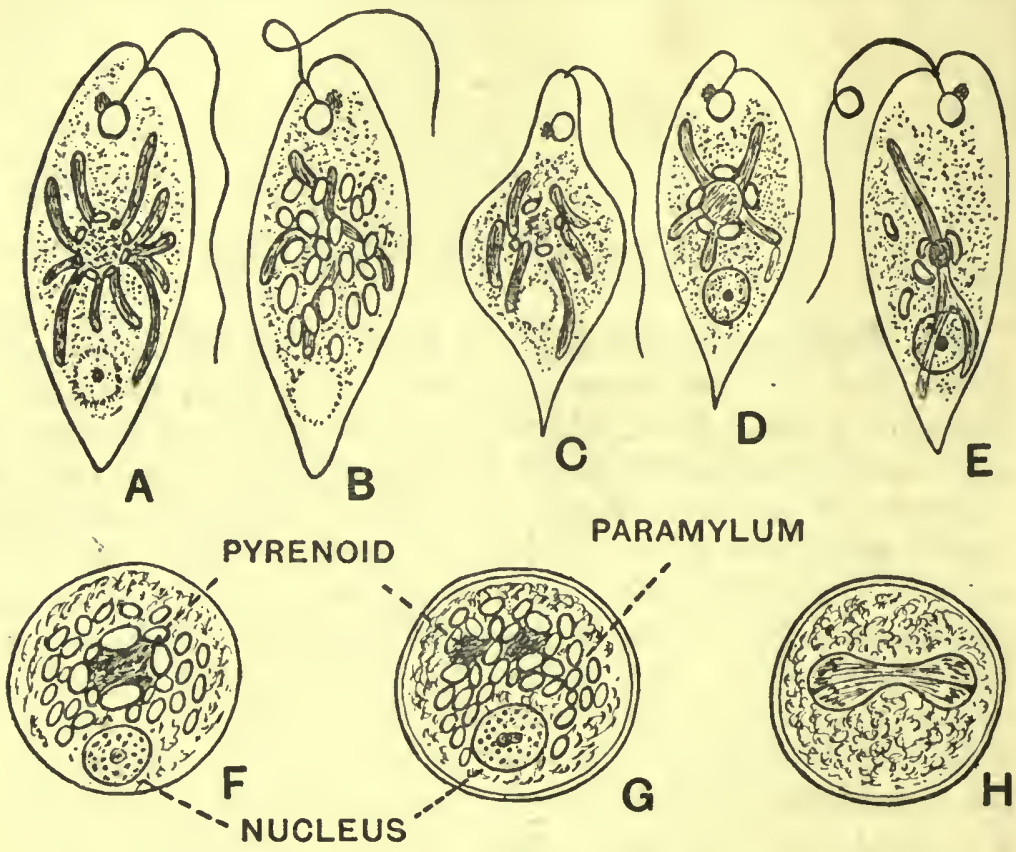

ARAMYLUM
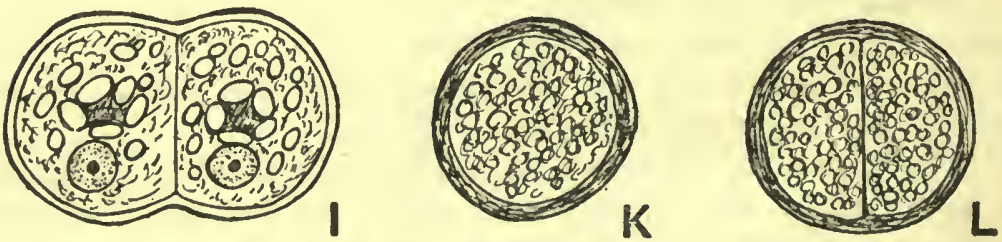

Fig. 203.-Euar,ENA viridis.

$A$, usual form with central pyrenoid and radiating elongated chloroplasts. The pyrenoid surrounded with small grains of paramylum. $B$, specimen with more numerous paramylum bodies. $C$, specimen with separate chlorophyll rods. $D$, specimen treated with alcohol and acid fuchsin to show nucleus. $E$, specimen with chloroplast in form of a single bifurcated band. $F$, specinien in sphcrical motionless condition preparing to divide. $G$, specimen with pyrenoid dividing. $\Pi$, specimen with nucleus dividing. I, division completed. $K, L$, encysted forms showing division, but not the internal details.

much coarser and far apart. In the species mentioned they are not uniform lines, but are marked with series of rounded nodules. 
§ 2. Organ of Locomotion. At the blunt anterior end of the body there is a single long delicate protoplasmic process such as is usually called a flagellum. This whip-like filament is the organ of locomotion, and to its lashing movements the motion of the organism through the water is due. As the flagellum is anterior in the movement of the Euglena, and does not propel the body from behind but draws it from the anterior end, it has been distinguished by the term tractellum. The tractellum arises from the side of a little conical depression of the surface of the body, which is sometimes called the mouth, and which somewhat resembles the cytostome of Paramecium. In the species of Euglena, however, observers are agreed that no solid particles are ever taken in as food through the mouth. The mouth is continued into the protoplasm of the cell for a short distance as a minute tube, which is for convenience termed the gullet.

§ 3. Organ of Excretion. Near the inner extremity of the gullet is a cavity of spherical shape, and this was formerly supposed to correspond to the contractile vacuole of other Protozoa. It is not, however, contractile, but permanent and almost constant in size and shape. Careful observation shows that near the surface of this cavity are smaller vacuoles which from time to time contract and disappear. The large permanent cavity, therefore, seems to serve as a reservoir into which the contractile vacuoles open. There is reason to believe that the reservoir communicates with the lumen of the gullet, and that in this way the excreted liquid is conveyed to the exterior. According to Dangeard the true contractile vacuoles increase in size and then fuse together to form a single vacuole which enlarges until it comes into contact with the reservoir, into which it then bursts, and the contents of the reservoir escape gradually through the efferent canal which leads into the gullet.

§4. Stigma and Haematochrome. In contact with what may be called the dorsal and anterior side of the reservoir is a pigment spot known as the stigma, which 
has been supposed to be an organ of vision. Taken literally, of course, this is an absurd supposition, but, on the other hand, it is possible that the pigment, by absorbing light, may cause the protoplasm to be specially stimulated by luminous rays at this point, and this function if it exists may be regarded as similar to the primary function of an organ of vision in higher animals. The stigma consists of a special portion of the cytoplasm containing a large number of minute red granules of a substance called haematochrome. This substance is, like chlorophyll, soluble in alcohol and ether. It is insoluble in ammonia and acetic acid. In the species called Euglena sanguinea haematochrome is often present not merely in the stigma, but in the general cytoplasm, in such quantity that the whole cell has a red instead of a green colour. The cell in this species appears in fact to pass regularly from a green to a red condition; it is always full of haematochrome when dividing, and it has been suggested that the haematochrome is produced by modification of the chlorophyll.

$\S 5$. Nucleus. The rest of the interior of the body of the cell consists of protoplasm containing a nucleus and certain other structures, of which the principal are the chlorophyll bodies and granules of a substance called paramylum. In Euglena viridis the nucleus is situated near the pointed posterior end of the cell, and in the living condition appears merely as a large colourless space in which no details of structure can be distinguished. (See Fig. 1, A, B, c.) It can be studied further in specimens which have been killed and fixed in absolute alcohol and stained with acid fuchsin. The nucleus is then seen as a spherical vesicular body containing a dense central nucleolus, which has been called a nucleo-centrosome, and around it about 40 chromatic (i.e. stained) granules scattered in the nucleoplasm (Fig. 203, D).

§ 6. Chloroplasts, Pyrenoids, and Paramylum. It is characteristic of Euglena viridis that the chlorophyll corpuscles are of an elongated rod-like form and that they radiate in all directions from a central mass of granules, which, according to Dangeard, constitute a large pyrenoid 
of the same nature as the pyrenoids of the lower Algae. Other authorities do not consider the central body as a pyrenoid. According to some observers, the chlorophyll rods constitute a single chloroplast containing a pyrenoid in the centre, so that the pyrenoid would be contained in the chloroplast as is the case in Algae. The pyrenoids are believed to consist of proteid substance and to be accumulations of nitrogenous food material. Around the pyrenoid in Euglena viridis, between the radiating chlorophyll rods are grains of paramylum of rounded or ellipsoidal shape. They have a homogeneous transparent appearance contrasting with the granular protoplasm. The typical relations of these various structures are represented in fig. 203 , D. Sometimes the paramylum grains increase in number to such an extent that they obscure the other structures as in fig. 203, B. Fig. 203, E, shows a specimen in which the chloroplast consists of one long rod bifurcated at its posterior extremity and evidently continuous with the pyrenoid at its centre.

§ 7. Reproduction. Euglena reproduces only by simple cell-division; no conjugation has yet been observed with certainty in any species of the genus or of the family to which it belongs. In the case of $E$. viridis division seems to occur only in the motionless condition of the cell. The cell becomes stationary and spherical, and surrounds itself with a mucilaginous envelope. The flagellum disappears. The pyrenoid is almost central, and large: it is surrounded by paramylum grains. The nucleus is situated below the pyrenoid, it is large and its chromatin granules are conspicuous. Division commences in the pyrenoid, which elongates transversely and then constricts itself in the middle and separates into two halves. Then the nucleus divides. The nucleo-centrosome elongates transversely and the chromosomes also elongating arrange themselves parallel to the nucleo-centrosome, then break in the middle and the nucleus separates into two. Division may be repeated several times in the resting condition so that a pellicle is formed consisting of numerous motionless Euglenae embedded in a mucilaginous film: such films 
may float on the surface of the water or adhere to the sides or bottom of the receptacle.

Sometimes cysts are formed in which the cell is surrounded by a thicker and more resistant envelope of a yellowish-brown colour. Division takes place also within these cysts, but the details are not distinctly visible. In other species division has been observed to occur in the motile condition, and repeated division may occur in the resting state so that a number of very small individuals are formed and ultimately set free.

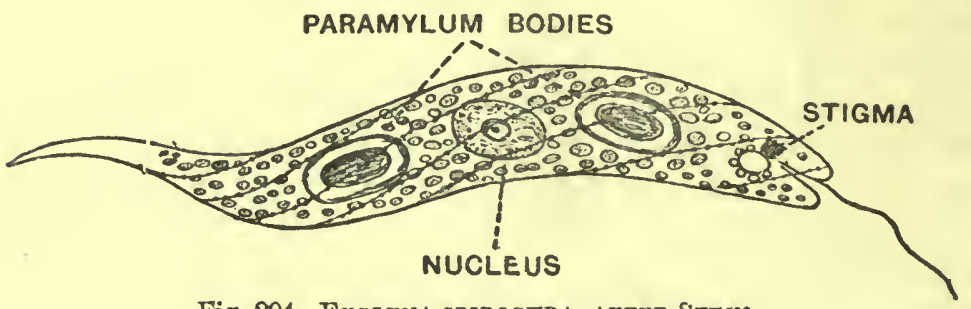

Fig. 204-Euglrina spirogtra, AFTrR StEIN.

§ 8. Specific Characters. There are a number of species resembling Euglena viridis in general characters but differing in minute details, and there is another group of species in which the chloroplasts, instead of being elongated and rod-like or stellate, are small separate discs like those of plants. To this latter group belongs the E. spirogyra shown in fig. 204. This group again can be divided into two divisions: (1) those species in which each chloroplast contains a pyrenoid, (2) those in which it does not. Where a pyrenoid is present in the chloroplast it forms a layer of paramylum outsideitself on each side, but paramylum also occurs in the cytoplasm independently of the chloroplasts. Thus in E. spirogyra and other species there are two large annular bodies of paramylum, one in front of and the other behind the nucleus. In other species again, as in $E$. deses, there are more than two paramylon bodies in the cytoplasm unconnected with the chloroplasts.

§ 9. Mode of Nutrition. We have seen that there is no evidence that Euglenae ingest solid particles of food and digest them. In other words, the nutrition is not holozoic 
or similar to that of typical animals or animal cells, as, for example, Amoeba or Paramecium. The presence of chlorophyll, and experiments showing that $\mathrm{CO}_{2}$ is absorbed and $O$ evolved by Euglena when exposed to light, indicate that holophytic nutrition occurs, or that the organisms are able to live like green plants. But experiment shows that when Euglena is cultivated with only inorganic materials in solution and exposed to light the cells do not divide rapidly and do not appear vigorous. On the other hand, when the organisms are kept in the dark in solutions containing decomposing organic compounds including carbohydrates and nitrogenous compounds in solution they thrive and multiply, but lose their chlorophyll. This shows that they can nourish themselves saprophytically, like fungi. The normal condition appears to be a combination of these two forms of nutrition, the holophytic and the saprophytic; hence the fact that in the natural state Euglena occurs chiefly in waters containing organic matter in considerable quantity. But, like fungi, they absorb organic compounds in solution and do not ingest them as solid particles.

$\S 10$. Paramylum. This substance has the same chemical composition as starch, $\mathrm{C}_{6} \mathrm{H}_{10} \mathrm{O}_{5}$, but differs from it in its reactions. It is not coloured blue by iodine; it is not affected by organic acids or by alcohol or ether. Potash in strong solution dissolves it rapidly, and sulphuric acid more slowly. Paramylum is in Euglena a store of carbohydrate food, but it appears to be formed not only in the chloroplasts, as in plants, but also in the cytoplasm independently of the chloroplasts. The latter process is due to the saprophytic mode of nutrition, the former to the holophytic.

$\S 11$. Nuclear Division. We have seen that the nucleus of Euglena in division goes through certain changes which have a distant resemblance to the mitosis of the cells of Metazoa. According to Keulen the nucleo-centrosome is surrounded by somewhat elongated rod-like chromosomes which during division arrange themselves parallel to the 
central part of the nucleo-centrosome and then split longitudinally like the chromosomes of Metazoan cells. Dangeard, on the other hand, considers that these threads are not separate chromosomes, but lengths of a continuous thread forming a skein, and that all the threads of the skein are broken transversely at the moment of division. The character of the mitosis is sufficiently indicated by fig. $203, \mathrm{H}$. This special kind of mitosis is distinguished by the term haplomitosis (haplos, simple; mitosis, weaving).

$\S 12$. Classification. Euglena is generally considered to belong to the animal kingdom in spite of the presence of chlorophyll. The occurrence of the flagellum and the active motion in themselves prove nothing, for flagellate cells occur in the life histories of many algae. But the absence of cellulose and the presence of the contractile vacuole are animal characters, and Euglena seems to be allied to many forms which have no chlorophyll and have a holozoic mode of nutrition. Such forms are placed in the class Mastigophora or Flagellata, distinguished from the Ciliata, of which Paramecium is an example, from the Rhizopoda, illustrated by Amoeba, and from the Sporozoa, which include Monocystis and Coccidium. 
Chapter XXXIII.-Monocystis and Coccidium, Examples of Sporozoa (Parasitic Protozoa).

$\S 1$. Structure of Monocystis. Various stages in the life-history of this organism are almost invariably seen when a small piece of a seminal vesicle of the earthworm is cut off, slightly spread out on a slide, and examined with the microscope. When the worm is opened, brownish or black specks are usually seen on the surface of the seminal vesicle. These are produced by the reproductive cysts of Monocystis, and in them under the microscope are seen groups of the spores. The mature organism before it enters upon the reproductive stage can usually be found in a preparation, and is a single cell of somewhat elongated cylindrical form, limited externally by a smooth cuticle (fig. 205, c). The interior substance shows a distinction into external ectoplasm and more granular, somewhat opaque endoplasm. The deepest part of the ectoplasm forms a layer of fine contractile fibres, which have been called the myocyte fibrillae. Within the endoplasm is the nucleus, which is limited by a delicate membrane, and contains a clear liquid in which are suspended denser bodies called karyosomes.

$\S 2$. Formation of the Gametes. The Monocystis in the stage we are considering is free in the contents of the seminal vesicle, and moves by contractions of the ectoplasm. The next stage is the commencement of the process of reproduction, and the organism in this stage is therefore called the gametocyte, the cell which forms the gametes. Two gametocytes come into close contact with each other, and surround themselves with a spherical cyst, the gametocyst, in which two layers can be distinguished, the epicyst and endocyst (fig. 205,D). No fusion or conjugation takes place between the gametocytes, but each proceeds to divide independently by mitosis into a number of small cells, 
which are the gametes, that is to say the cells which conjugate. In each gametocyte about to segment the nucleus undergoes the following changes. The karyosomes break up and become partially dissolved in the nuclear sap, and a number of grains or short filaments of chromatin appear, which are believed to be derived from the karyosomes. These chromosomes are collected in a group and form the segmentation nucleus. The nuclear membrane disappears, and the segmentation nucleus divides by mitosis (Fig. $3, \mathbf{E})$. The remains of the karyosomes are left free in the cytoplasm and are gradually absorbed. The numerous nuclei formed by division pass to the surface of the gametocyte and its cytoplasm divides to form cells around them. These are the gametes, and the central cytoplasm of the gametocyte is left as a cystal residuum (Fig. 205, F).

$\S 3$. Conjugation and Spore-formation. The gametes exhibit for a time active movements, and then conjugate in pairs, forming the zygotes, or sporoblasts, in which the two nuclei of the two united gametes fuse together (Fig. 3, a). The zygote becomes a spore: it assumes an oval form, and secretes a tough membrane or sporocyst.

The spores have a characteristic fusiform shape and are very conspicuous in preparations of the seminal vesicles. From their resemblance to a diatom called Navicella, they have been known as pseudo-navicellae.

$\S 4$. Formation of Sporozoites. A new process of division takes place in the spore within the sporocyst, the nucleus dividing three times, so that eight nuclei are formed. This division is amitotic. The cytoplasm segments to correspond with the nuclei, each segment being called a sporozoite, and as before a central residuum is left unused. The sporozoite has been sometimes called a falciform body.

$\S 5$. Mode of Infection. Nothing is known with certainty of the means by which the spores are transferred to other earthworms, although as a rule every earthworm examined contains the parasite. It might be supposed that the spores would be transferred from one host to another in the process of copulation, when the spermatozoa and seminal fluid are conveyed from the seminal vesicles of one 
worm to the spermathecae of another. But the spores have never been seen in the spermathecae or in the cocoons. It seems probable, therefore, that the spores are only set free when the host worm is killed and destroyed, as, for instance, when the worm is devoured by a bird. Pfeiffer has actually observed the spores in the contents of the intestines of various birds. In this case the spores would pass with the excrement of birds into the soil, and there be swallowed by other earthworms. The sporocyst is then probably dissolved by the digestive secretions of the worm, and the sporozoites set free, being actively motile, traverse the tissues and reach the seminal vesicles.

$\S 6$. Development of Sporozoite and Trophozoite. The earliest known stage in the development of the parasite is a minute nucleated body contained in the central residual protoplasm of one of the sperm morulae (fig. 205, A). The parasite is, therefore, in its earlier stages intracellular, like many other sporozoa. The sporozoite seems, therefore, to attack one of the male germ cells, and to enter it, remaining in it when it divides and develops into spermatozoa. This is called the trophic or feeding stage, and the individual is called a trophozoite; it is nourished by the protoplasm in which it lives. It grows at the expense of the residual protoplasm of the sperm morula until that protoplasm is all consumed, and then for a time the trophozoite is found surrounded with the spermatozoa of the earthworm which have developed around it, so that it might be mistaken for a ciliated organism (fig. 205, B). Ultimately, these spermatozoa are detached and the trophozoite becomes free, and thus we reach the stage with which we began the description of the life-cycle.

\$ 7. Life-history Formula. The life-history may be briefly exhibited by the following symbolic formula:-

Sporozoite $\rightarrow$ trophozoite $\rightarrow$ gametocyte $\rightarrow n$ gametes $\}$

Sporozoite $\rightarrow$ trophozoite $\rightarrow$ gametocyte $\rightarrow n$ gametes $\}$

$$
+=n \text { zygotes } \rightarrow n \text { spores } \times 8^{n} \text { sporozoites, }
$$

which is read thus: The sporozoite becomes a trophozoite which becomes a gametocyte, which segments into a 

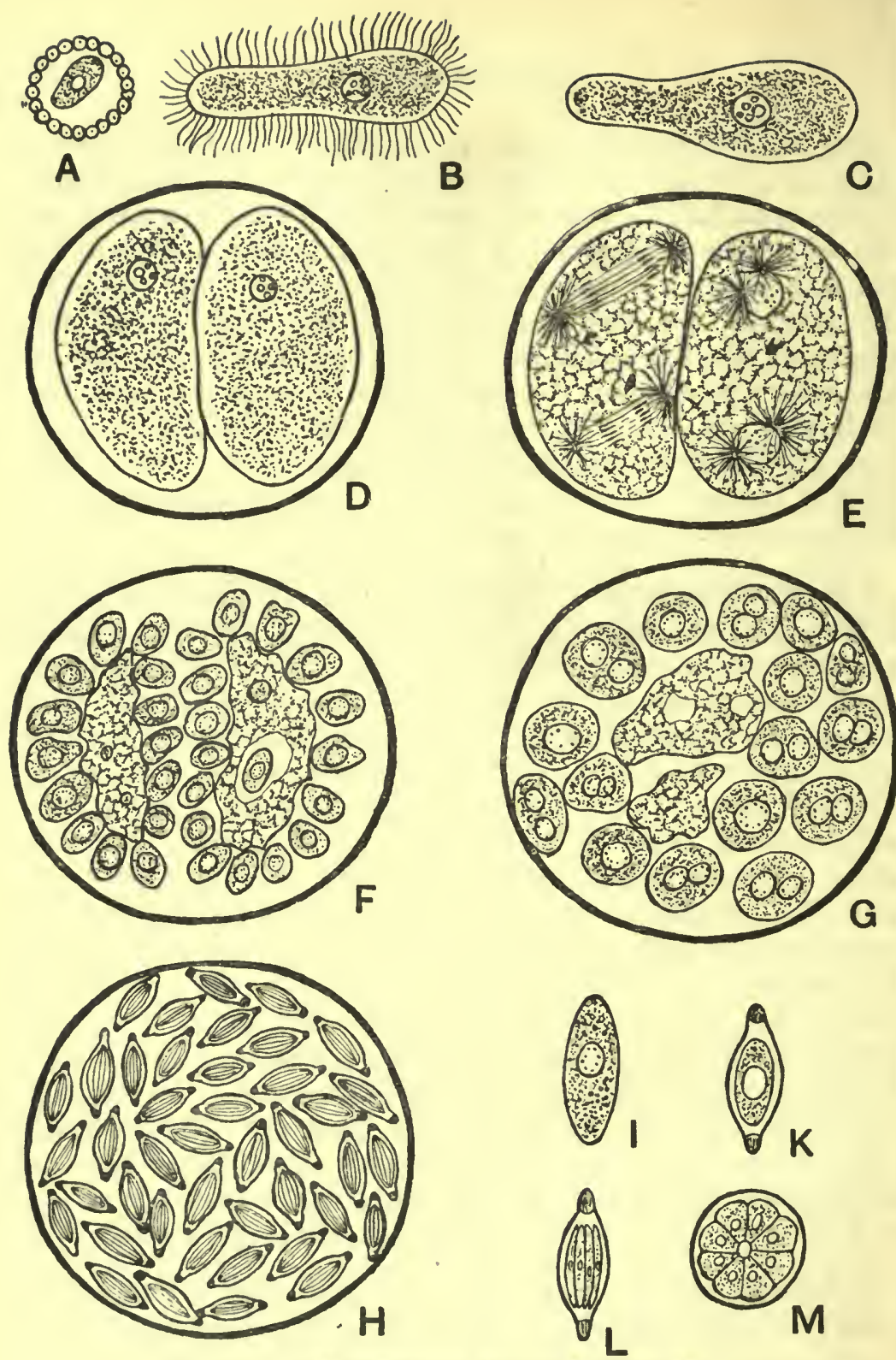

Fig. 205. - Life-mistory of Monocyerts Aarirs.

$A$, Young Trophozoite in residual protoplasm of a sperm-polyplast. $B$, Adult trophozoite surrounded by spermatozoa of earthworm. $C$, Free trophozoite. $D$, Gametocyst containing two gametocytes. $E$, Gametocytes showing nuclear division. $F$, Gametes formed from gametocytes, and residual protoplasm. $G$, Zygotes, some with two separate nuclei, some with the two fused into one. $H$, Fully developed spores (pseudo-navicellae) in gametocyst. $I$, Young stage of spore with single nucleus. $K$, Spore with sporocyst formed but nucleus undivided. $L$, Fully developed spore containing eight sporozoites. $M$, Transverse section of mature spore. 
number of gametes, which conjugate within a cyst in pairs with an equal number of other gametes to form the same number of zygotes, which form the same number of spores, each of which divides into eight sporozoites.

There is another species of Monocystis, usually found in the same worm with M. agilis. It is larger and is called M. magna. It occurs attached by one extremity of the elongated body to the epithelium of the seminal funnel, and only becomes free in the sperm sac just before encystment and conjugation.

§ 8. Characters of Coccidiidea. These are Sporozoa which are intracellular parasites living in tissue cells of Metazoa, usually in epithelial cells, not in blood cells. They are chiefly found in Arthropoda, Mollusca, and Vertebrata. The species of Coccidium are usually confined to vertebrates, attacking the cells of the kidney, liver, spleen, or testis, but never the ovary. One species attacks the epithelium of the bile-ducts in young rabbits and causes an acute disease which is often fatal. The liver becomes greatly enlarged, the secretion of bile is diminished, the blood becomes thin and watery, and the animal dies in convulsions, or may survive the acute stage of the disease and recover.

§ 9. Development of Sporozoite into Schizont. The species, however, whose life-history has been most completely investigated is not one that lives in a vertebrate host, but one that occurs in the intestinal epithelium of Lithobius forficatus, a centipede. The name of this form is Coccidium schubergi, and the name of its investigator is Schaudinn. The youngest stage of this parasite is a free sporozoite which is sickle-shaped and very minute, measuring 15 to $20 \mu$ in length and 4 to $6 \mu$ in breadth. (The symbol $\mu$ indicates the thousandth part of a millimetre, or .001 mm.) It consists of finely granular protoplasm without cuticle, and contains a single nucleus. The latter appears in the living organism as simply a clear transparent spot. When killed and stained the nucleus is seen to consist of a number of chromatin granules contained in 


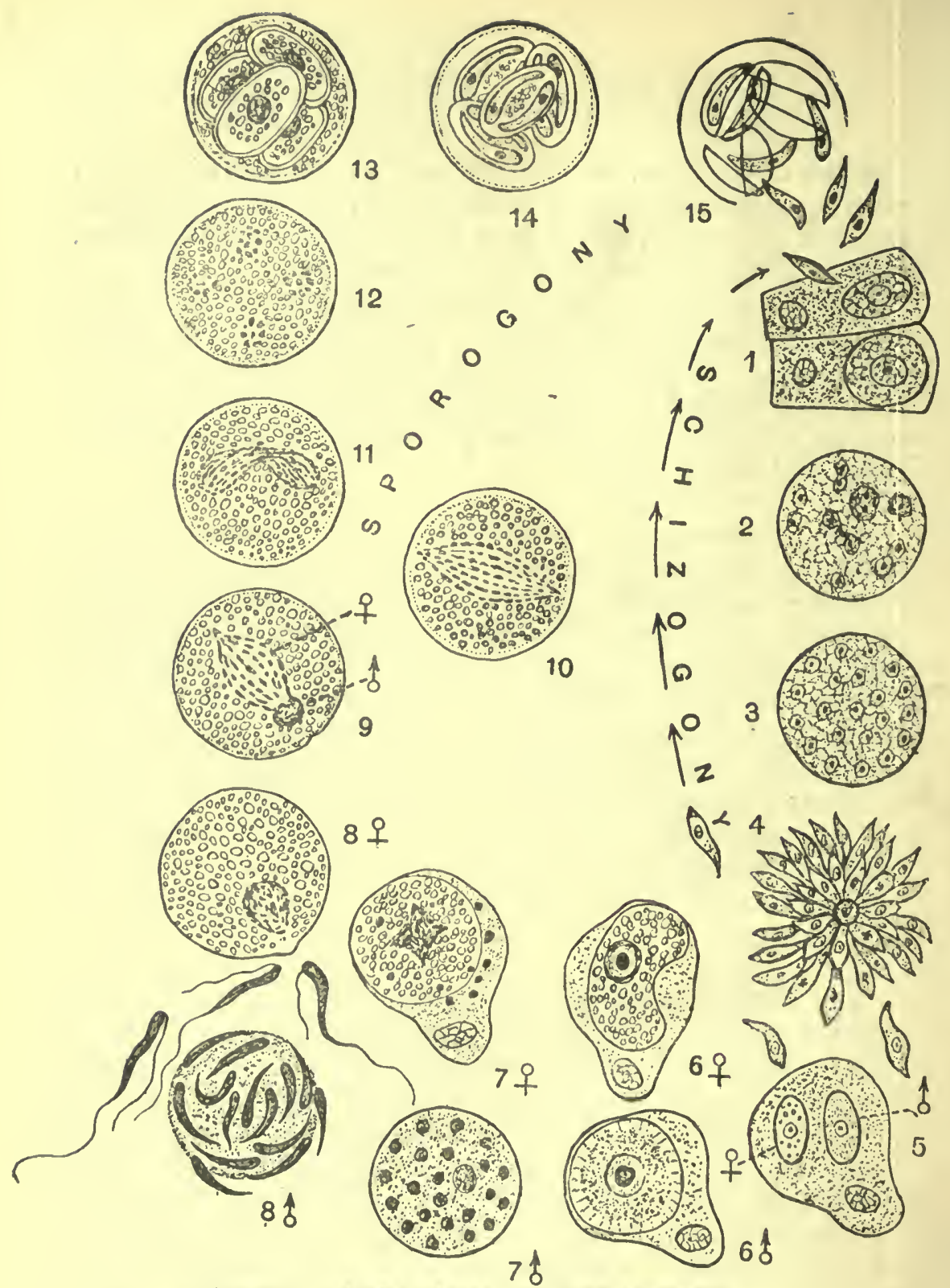

Fig. 206.-ITrN-history OF Coccidium 8ChUBRRG1.

1, Two epithelial cells containing sehizonts, with a sporozoite shown entering one of them. 2, Sehizont fully developed, with nuclei dividing. 3, Sehizont, with numerous nualei formed by division of original nueleus. 4, Group of merozoites formed by division of schizont. 5, An epithelial eell containing a male and female gametoeyte, $6 \delta$ and 6 , , Male and female gametoeytes more developed. $7 \delta$ and 7 , Gametocytes showing separation of chromatin from karyosome. 8 f, Male gametocyte dividing up into male gametes. 8 o, Female gamete, with nueleus of chromatin and projection of protoplasm attracting male gametes. 9, Zygote just after conjugation. 10, Zygote later stage, forming ooeyst. 11, Zygote, with nueleus dividing. 12, Zygote, with four nuclei produeed by division. 13, Oocyst containing four spores. 14, Each spore divided into two sporozoites, with sporal residuum. 15, Oocystand sporoeyst ruptured and sporozoites eseaping. 
an alveolar structure of unstained linin, but there is no central body similar to the structures called nucleolus or karyosome in other cases. When in a free state the sporozoite exhibits spontaneous movements, but it soon penetrates into an epithelial cell of the Lithobius and then becomes motionless and spherical. The nucleus now undergoes important changes. The chromatin granules collect gradually to the centre of the spherical cell and become fused with another substance to form a "karyosome." At this stage the parasite undergoes division without conjugation, and this phase in the life-history is called schizogony (Greek schizo, to split), the cell being called a schizont.

$\S 10$. Schizogony. In the process of schizogony the nucleus divides directly into a number of nuclei, which travel towards the surface of the schizont. The protoplasm then grows out into small projections, each taking a nucleus with it, and then each projection separates, forming what is called a merozoite. The central part of the protoplasm remains as a residuum. Each merozoite escapes from the cell where it was formed, passes into another epithelial cell and there grows into a new schizont, and several schizogonous generations succeed each other.

$\S 11$. Sporogony. After a period of schizogony lasting about five days the process of sexual reproduction begins, and this is termed sporogony, because it results in the formation of spores. The merozoites now grow into cells, which give rise to the sexual or conjugating elements, and these elements being now termed gametes, the cells which form them are called the gametocytes. The gametes are of two kinds-one small and actively motile, the microgametes; the other large and motionless, the megagametes. Obviously the former resemble spermatozoa, the latter are similar to ova, in the Metazoa, and they may be regarded as male and female. In the male gametocyte the chromatin separates from the karyosome and travels to the surface, leaving the rest of the karyosome at the centre. At the surface the chromatin forms a number of 
nuclei, which assume an elongated form and then separate from the gametocyte, each provided with a protoplasmic flagellum at each end (Fig. 206, $b \delta^{\pi}$ ). In the female gametocyte, on the other hand, it is the chromatin which remains at the centre while the achromatic part of the karyosome is expelled at the circumference in a fashion which reminds one of polar bodies, though there seems to be no real similarity between the two cases. The female gametocyte also accumulates a certain amount of food granules resembling the yolk of the Metazoan ovum.

$\S 12$. Conjugation. A microgamete enters the megagamete, and fuses with its chromatin nucleus, and the result of this process of conjugation or fertilisation is the zygote. The nucleus next divides by direct division into four, and the protoplasm also segments, forming four sporoblasts. The zygote surrounds itself with a cyst called the oocyst. Each sporoblast also becomes enclosed in a thick coat called the sporocyst, and the contents of the spore divide into two sporozoites. We thus reach the stage from which we started in following the succession of stages in the life-history.

$\S 13$. Mode of Infection. The infection of a Lithobius is due to the accidental swallowing of oocysts with the food. The parasite is intracellular in all stages except the free sporozoites and merozoites, which enter new epithelial cells and there develop. When the oocysts are developed they become free in the intestine of the host and then pass out with the faeces. When these oocysts are swallowed by another Lithobius, the cyst wall is dissolved by the digestive secretions, the four spores-are liberated, and then each spore splits lengthwise and the sporozoites are liberated and at once make their way into the epithelial cells of the intestines.

$\S 14$. Life-history Formula. The life-history of Coccidium schubergi is represented in the following formula:Sporozoite $\rightarrow$ schizont $\times$ merozoites $\rightarrow \ldots$ schizonts $\rightarrow$

$\rightarrow$ merozoites $\rightarrow$ $\rightarrow$ gametocytes $\times n$ व gametes

. . . merozoites $\rightarrow q$ gametocytes $\rightarrow q$ gametes $\}+=$ zygote $\times 4$ spores $\times 8$ sporozortes. 
$\S 15$. Comparison of Monocystis and Coccidium. It will be seen that the two chief peculiarities of Coccidium as compared with Monocystis are (1) the occurrence of schizogony, (2) the differentiation of the gametes. Monocystis does not multiply by asexual division within the same host, Coccidium does. In Monocystis the gametes are similar, there is no distinction of microgametes and megagametes. In Coccidium, on the other hand, the microgametes are much smaller than the megagametes, and are furnished with flagella.

$\S 16$. Characteristics of Parasitic Protozoa. As the Protozoa in many cases correspond to single cells of the multicellular Metazoa, it is natural that the parasitic forms should be intracellular, that they should be parasites within other cells, just as a Metazoan parasite like Distomum is parasitic within the body of the Metazoan host. The rule, however, is by no means universal. The parasite of malaria lives within the blood corpuscles, as Coccidium lives within epithelial cells, but on the other hand the ciliate Opalina lives free in the cavity of the rectum of the frog. The special characteristics of parasitic forms are to a certain degree similar in Protozoa and Metazoa. In the former the power of locomotion is much reduced; it is entirely absent in many stages, as in the schizonts of Coccidium, and is exhibited only in those stages which migrate from one host cell to another, as in the sporozoites and merozoites, and in the microgametes of Coccidium. Cilia are absent in all the intracellular parasites of the class Sporozoa, although flagella are present in certain stages, as in the microgametes. Food is never ingested in the form of solid particles, as in free Protozoa such as Amoeba and Paramecium. This is one of the most characteristic features of parasitism. The parasite living within another animal takes for its own use the food material which has been obtained and digested by its host, and loses in consequence all the special organs and activities which an independent animal employs in seeking and digesting its food. Parasitic protozoa absorb their food in liquid form at their surface, but whether they 
actually destroy and digest the protoplasm of the host cell is not certain. The formation of firm resistant cysts or envelopes is also very characteristic of parasitic Protozoa.

All parasites, both Protozoan and Metazoan, have enormous powers of multiplication, without which they would necessarily be in danger of extinction. For as they are usually carried passively into the appropriate host, and in any case depend upon chance for the opportunity of entering a host, it is necessary that there should be an enormous number of spores or eggs to ensure that some will survive. A large proportion of the spores or eggs fail to find entrance into a host of the proper kind, or are killed by unfavourable conditions. 


\section{Chapter XXXIV.-The Malartal Parasite.}

$\S 1$. Mode of Occurrence and Effects. The disease called malaria or ague is an acute or intermittent fever, formerly supposed to be due to pernicious exhalations in the atmosphere of marshy districts (malo, bad; aria, air), but now known to be due to a Protozoan parasite in the blood. This parasite lives in the red blood-corpuscles, and belongs to a group of the Sporozoa called Haemosporidia (haima, blood; sporidion, a spore-like body), because they are blood parasites. There are several forms of malarial fever, and it is uncertain how far these are due to different species of the parasite, and how many species of the latter exist. Three chief forms have been distinguished. Laverania malariae, which causes the pernicious malaria of hot countries; Plasmodium malariae, found in cases of quartan ague; and Plasmodium vivax, in tertian ague. The characters and life-histories of the three are closely similar.

$\S 2$. Schizogony. The minute sporozoites are introduced into human blood by the bite of a mosquito of the genus Anopheles. The sporozoite is slender, almost filamentous, sharp at both ends, with a thicker central portion containing the nucleus. Within the corpuscle the sporozoite becomes rounded in form and forms an amoeboid trophozoite which grows at the expense of the corpuscle until it nearly fills it. The youngest amaebulae are without pigment, but contain in fixed and stained preparations a conspicuous vacuole, giving the so-called ring-form of the parasite. At a later stage the vacuole disappears and grains of pigment termed melanin are formed round the nucleus (fig. 207, 6). 
When full-grown the trophozoite is spherical and almost as large as the corpuscle, and it proceeds to divide by schizogony. The nucleus divides, the daughter nuclei travel to the surface, and the protoplasm divides into a corresponding number of merozoites surrounding a small mass of residuary protoplasm which contains all the pigment granules (fig. 207,9). During the schizogony the corpuscle disintegrates, and it is this destruction of the corpuscles which is so harmful to the host. The merozoites are set free in the blood, and the residuary protoplasm is disintegrated, its pigment being scattered and afterwards deposited in the spleen, liver, lungs, kidneys, and brain.

§ 3. Conjugation. By repeated schizogony the number of parasites increases by geometrical progression like the Coccidia in an infected epithelium until a very large number of the corpuscles are destroyed: the only check is the action of the leucocytes, which destroy the merozoites.

When the host is becoming exhausted certain trophozoites develop into gametocytes as in Coccidium. In Laverania malariae the gametocytes are like curved sausages, exceeding in length the diameter of the corpuscle the remains of which are seen in the hollow of the gametocyte (VIII. $a$, VIII.b). These are known as crescents and are very characteristic of pernicious malaria. In the male crescent the pigment granules are scattered, in the female they are aggregated round the nucleus. The crescents appear to originate in the spleen and bone-marrow, but when fully developed they are found in the superficial blood-vessels. They afterwards become oval, then spherical, and free themselves from the remains of the blood corpuscle. They do not get beyond the spherical stage in the human blood, and may be transferred to the stomach of the mosquito as crescents, or ovals, or spheres.

The development of the gametocytes and the process of conjugation take place in the stomach of the mosquito. In the male gametocyte (fig. 211 , IX.a) the nucleus breaks up and the daughter nuclei pass to the surface of the gametocyte. Then, as in Coccidium, each of the several nuclei becomes elongated and with a small quantity of 


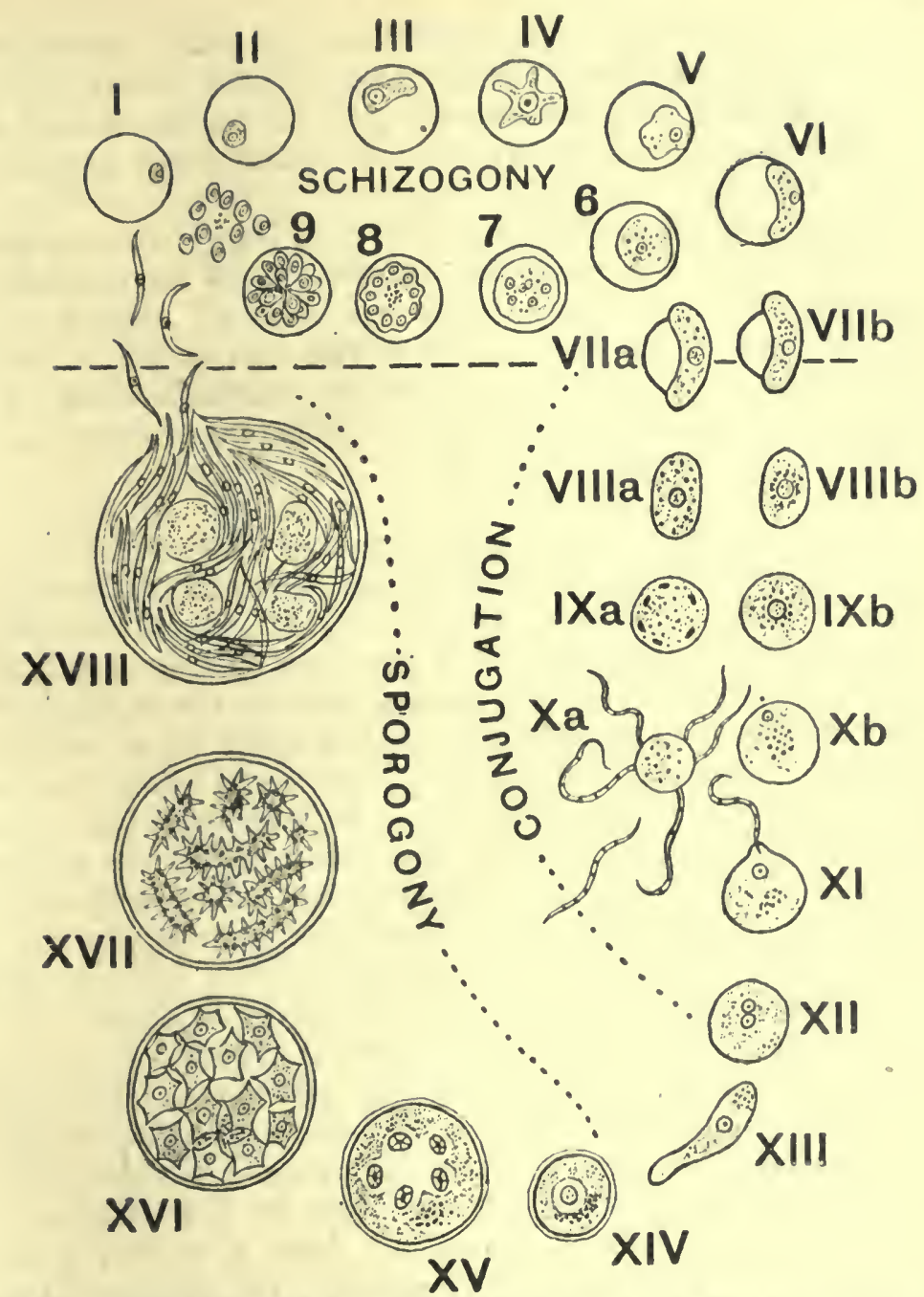

Fig,207.-Diagram Illustrativa the Ijife-history of the Malarial Parasite.

I.V. 6, successive stages in the growth of the trophozoite within a red corpuscle. 7-9, division of trophozoite into merozoites. VI., trophozoite in crescent form, becoming a gametocyte. VII. $a$, VII. $b$, male and female crescents. VIII, $a$, VIII. $b$, male and female ovals. IX. $a$, IX. $b$, male and female gametocytes; IX. $a$, the male with divided nucleus. X. $a$, formation of male gametes; X. $b$, female gamete which has ejected a particle of chromatin. XI. and XII., conjugation. XIII., the motile zygote which penetrates the epithelium of the mosquito's stomach. XIV.-XVIII., development of the sporozoites. Stages above the dotted line occur in the human host, those below in the mosquito. (After Minchin, Art. Sporozoa in Lankester's Treatise on Zoology.) 
protoplasm forms a long slender motile body, the microgamete, analogous to a spermatozoon. These microgametes are themselves filamentous and perform rapid undulatory movements; they are not provided with distinct flagella as in Coccidium.

In the megagametocyte (fig. 207, IX.b) the uucleus remains undivided, and ejects a portion of its substance. Conjugation then takes place; a cone of attraction is formed to meet the apex of one of the microgametes which enters the megagamete, and whose chromatin forms a male pronucleus within the latter. The two pronuclei now unite and the zygote is formed.

\$ 4. Sporogony. The zygote instead of secreting a cyst loses its spherical shape and becomes elongated and actively motile : it is called a vermicule or oökinete. It bores its way through the epithelium of the mosquito's stomach and becomes encysted in the tissues outside the epithelium. Here it increases greatly in size, the cysts in an infected mosquito forming small rounded projections on the external surface of the insect's stomach. In this stage the zygote may be called the oöcyte; its nucleus divides without mitosis, and then the protoplasm divides into corresponding portions. The products of this division evidently correspond to the sporoblasts of the Coccidium, and in their formation a small quantity of residual protoplasm is left containing the pigment granules of the zygote.

The sporoblasts now behave very differently from those of Coccidium or Monocystis : they form no external cyst, but continue to grow, the nucleus in each divides into a large number of nuclei; these pass to the surface of the sporoblasts which grows out into a corresponding number of spindle-shaped processes. In this way each sporoblast gives rise to a large number of slender sporozoites (Fig. 207, XVIII.) and a few masses of residual protoplasm. Each sporozoite is about $14 \mu$. long and $1 \mu$. broad, and a single oöcyst may contain several thousands of them.

When the sporozoites are completely developed the oöeyst bursts and they are set free in the haemocoel of the 
mosquito. Then they penetrate into the salivary glands of the insect. Only the female mosquito sucks blood, and when a specimen with sporozoites in its salivary glands pierces the skin of a human being it injects into the puncture a drop of saliva containing numbers of the sporozoites, and in this manner the person becomes infected with the so-called malarial disease.

$\S 5$. Other Haemosporidia. Haemosporidia are found in various other vertebrates besides man. Drepanidium ranarum lives in the corpuscles of the frog. In birds occurs a genus called Haemoproteus, which is closely similar to the malarial parasite of man. It lias been shown that this parasite of birds is transmitted by mosquitos or gnats of the genus Culex, which is the common guat in England.

If Anopheles bites an infected bird all the parasites in the blood swallowed by the insect are killed and digested, when Culex takes the bird's blood all stages are digested except the gametocytes, which go through the processes of conjugation and sporogony. Conversely, if Culex bites an infected person all stages of the malarial parasite are digested, while in Anoplieles the gametocytes survive. It is a curious fact that ague was formerly common in the fen districts of England, where it is now almost or quite extinct. Yet gnats of the genus Anopheles still occur there, and it is supposed that this race of the insect has become immune, that is the gametocytes do not survive in them, so that they no longer transmit the infection. 
Chapter XXXV.-Diagnostic Characters of the Principal Divisions of the Animal Kingdon.

The animal kingdom is first divided into a number of subdivisions which are called sub-kingdoms or Phyla. The latter term is now generally used, because it means a tribe, or clan, or stock, and implies common descent among the collection of organisms to which it is applied. The largest and most important phyla are : the Protozoa or unicellular animals ; the Porifera or sponges ; the Coelenterata, including hydroids, jelly-fishes, corals, and sea-anemones; the Platyhelmia or flat-worms; the Nemathelmia or threadworms; the Echinodermata, including star-fishes, seaurchins and their allies; the Annelida or segmented worms ; the Arthropoda or animals with jointed appendages, such as Insects and Crustacea; the Mollusca; and the Chordata, which include the vertebrate animals. In the London University Syllabus for the Intermediate Science Examination which comes into force in 1909, all these phyla are mentioned except the following :-Porifera, Nemathelmia, Echinodermata, Mollusca. The Arthropoda, however, are called the Articulata, the term used by Cuvier at the beginning of the nineteenth century for one of the four principal divisions of his classification; this division included the Annelida and the Arthropoda. It is not usual at the present time to employ the term Articulata as synonymous with Arthropoda. In the University Syllabus also the term Chordata is used as distinct from and contrasting with the Craniata, whereas it is generally understood to include the latter, the primitive forms without cranium or vertebrae. being called Prochordata. 


\section{PHYLUM PROTOZOA.}

The general characteristic of the Protozoa as distinguished from all other animals is that in them the cell is the individual or organism, while in others the individual is composed of multitudes of cells which are not capable of permanent existence apart from the compound organism to which they belong. The Protozoa, however, are not always simple cells each with a single nucleus. Many have two nuclei, like Paramecium, or more than two, as in one stage of the Foraminifera. Such forms may be considered as equivalent to several cells potentially, not actually, divided. Again, many Protozoa when they multiply by simple division do not separate but remain connected by peduncles or otherwise, forming compound structures, just as the zooids of Obelia remain connected with one another. Such forms are usually called colonial, which as explained in the case of Obelia is not an appropriate term. We cannot call the compound in this case a gemmarium, because the new individuals are not formed by gemmation but by fission; we may therefore call it a fissorium. In such cases every cell is equally capable of conjugating and reproducing the compound, whereas in Metazoa these functions are possessed by special cells of the compound organism and have been lost by the others. Again, in the compound Protozoa all the cells are similar and each performs all the necessary functions of life, and the cells are not arranged in layers. In Metazoa the cells are arranged in layers which have different functions, one layer being specialised for digestion. In the Protozon parts of the cell are differentiated to form organs, in the Metazoa organs are formed of groups of cells.

The Protozoa are divided as follows :-

Chass : Rhizopoda.

Protozor without distinct cuticle, the amoeboid form being predominant, the protoplasm capable of protrusion in the form of pseudopodia, which are not constant in form nor' permanent. This class is sometimes called Gymnomyxa. 
Order 1, Lobosa.-Rhizopoda with blunt pseudopodia which do not anastomose to form networks. Amoeba is an example of the Lobosa. Some of the species, e.g. Diflugia and Arcella, possess a chitinous cuticle or shell enclosing the greater part of the body but leaving one part uncovered, from which pseudopodia are protruded.

Order 2, Foraminifera.-Pseudopodia fine, branched, and anastomosing ; body contained in a shell which may be chitinous, calcareous, or arenaceous; shell usually compound, consisting of chambers successively formed and communicating by a terminal aperture to each cliamber or by numerous perforations. In the life-history there is an alternation of generations, one form with large initial chamber and a single large nucleus gives rise after division of the nucleus to a large number of minute biflagellate zoospores which conjugate in pairs, and each zygote thus formed becomes a microspheric form, that is a form with a small initial chamber, which by simple division without conjugation gives rise again to the megalospheric form with large initial chamber.

Order 3, Radiolaria.-Pseudopodia long and delicate, either free or anastomosing; imbedded in the protoplasm is a "central capsule" of chitin, within which is a large and complex nucleus, or sometimes several nuclei. Skeleton in many forms siliceous, either of separate spicules, or of a firm connected framework, of spherical, stellate, or discoid shape, frequently produced in radiating spinēs : in one division the skeleton consists of the chitinous substance acanthin : in some cases no skeleton is present. Usually symbiotic unicellular organisms containing chloroplasts of yellow colour are found living in the protoplasm of the Radiolaria; they are usually regarded as Algae, but by some authorities as Flagellate Protozoa. Reproduction by simple fission, which in some cases leads to the formation of compound or colonial organisms, or by the formation of flagellate zoospores; no conjugation has been observed.

\section{Crass: Sporozoa.}

Protozoa which are all parasitic, destitute of organs of locomotion in their principal stages, without contractile vacuole, enclosed by a distinct cuticle, absorbing food only 
in solution. Only a single nucleus. Reproduction by fission, conjugation occurring regularly at certain stages in the life-history, and resulting in the formation of resting spores surrounded by a firm sporocyst, within which the protoplasm divides into several minute sporozoites. Cilia never present, amoeboid stages may occur, and the microgametes are frequently flagellate.

Monocystis and Coccidium are typical examples of Sporozoa, and represent the two great divisions of the Telosporidia, namely the Gregarinida and the Coccidiidea. The essential difference between the two groups is the association of the gametocytes in Gregarinida in a common cyst; the Coccidiidea are also more permanently intracellular.

Class: Flagellata.

Protozoa which move by means of long flagella which are either single or few in number; pseudopodia either absent or transitory; a firm cuticle or cell-wall usually present; nucleus single, or if multiple not differentiated; nutrition holozoic, saprophytic, or holophytic ; reproduction by fission, sometimes alternating with conjugation, the zygote breaking within a cyst into minute spores.

The Class Flagellata is somewhat artificial, since many other Protozoa have flagellate stages, e.g. the microgametes of Coccidium, and many forms are placed in this group which by botanists are regarded as plants, e.g. the Volvocidae. The group can scarcely be said to belong exclusively to the animal kingdom or to the vegetable kingdom, as many of its members combine the characters of animals and plants, or show in allied forms a transition from an animal character to a vegetable. In mode of nutrition the same individual may be at one time holozoic, at another saprophytic, or, like Euglena, at one time holopliytic, at another saprophytic, according to the conditions of life. Even the presence of cellulose is not diagnostic, for the Dinoflagellates, which are common in the sea, have a cellulose membrane, and yet some of them lose their chromatophores and take in solid particles and digest them like an amoeba; many of them also possess a contractile 
vacuole. Volvox and some of its allies show animal characters in the possession of a contractile vacuole and an eye-spot in each cell, and in the absence of a cellulose envelope. In Volvox reproductive cells are differentiated, while the other cells cannot reproduce the compound organism, and thus this Flagellate forms a transition towards the Metazoa.

\section{Class: Infusoria.}

Order Ciliata.-Complex Protozoa of definite shape with cilia as organs of locomotion, and a cytostome; usually with undulating membranes or large rigid cilia called cirrhi; with two kinds of nuclei, a meganucleus and one or more micronuclei, the latter alone taking part in conjugation (karyogamy), and giving rise after conjugation to all the new nuclei.

The above definition applies to the Ciliata proper of which Paramecium is an example, but there is another group called Suctoria, which are allied to the Ciliata, and united with them in the Class Infusoria. The Suctoria are ciliated in the young state only; they have no mouth, but absorb food through one or more tentacles which are perforated at the apex. The Ciliata show the highest differentiation in the cell-body which is found among the Protozoa, the ectoplasm being differentiated into muscular fibrils or myonemes, and often containing trichocysts resembling the nematocysts of Coelenterata. They are to be regarded as organisms evolved from simple cells in which great complexity has been attained in the cell itself without union of cells into a compound organism as in Metazoa. At the same time they are not homologous with simple uninucleate cells, but are multinucleate with the nuclei differentiated from one another like the parts of the protoplasm. Only one micronucleus gives rise to the conjugating nuclei, and at the moment of karyogamy the cell is hermaphrodite, equivalent to two gametes, and two zygotes are formed. Hence the two conjugating individuals are not gametes, but conjugants. In Vorticella and its allies one of the conjugants is small and does not produce a zygote; this has been interpreted as a step towards the 
differentiation of ovum and spermatozoon, but is not so; it is a modification of the process peculiar to the Ciliata.

\section{METAZOA.}

All other animals are united together in contrast to the Protozoa under the name of Metazoa, and are distinguished by the fact that the organism is a compound of cells arranged in layer's differing from one another in character and functions. In all Metazoa at least three kinds of cells are present: in the first place there are reproductive cells (gametes) and somatic cells, the former alone capable of reproducing the organism, usually after conjugation. The somatic cells are differentiated at least into an internal digestive and an external sensitive layer. The gametes are always differentiated into ova and spermatozoa, megagametes and microgametes, and the fertilised ovum or zygote undergoes segmentation leading to the formation of distinct layers of cells. 'The soma or body often exhibits various kinds of asexual reproduction such as fission or gemmation, but this process is due to the growth of portions of the body tissues, not of single cells. Prof. Sollas takes the view that the Sponges or Porifera should be regarded as quite separate from the rest of the Metazoa, and that they were evolved independently from a special group of the Flagellata: he therefore proposes to give them the name Parazoa, distinct from both Protozoa and Metazoa. As generally understood, however, the term Metazoa includes Sponges.

\section{PHYLUM COELENTERATA.}

The essential peculiarity of this Phylum is that the body is saccular, containing only one cavity, which, however much it may be complicated or differentiated into canals and chambers, is continuous throughout, and has only one principal aperture, the mouth. Other small apertures may exist in some cases, but there is never a large number of small pores in the walls of the body through which a current of water passes as in sponges. The body is usually

zOOL. 
radially symmetrical with the mouth at one end of the principal axis, but minor features of bilateral symmetry are not uncommon. The body-wall consists of two primary layers of cells, the ectoderm and endoderm, the latter being digestive. These two layers are separated by a layer of gelatinous substance, cliemically allied to mucin and called the mesogloea ; in this layer many cells of various kinds may occur, and these are derived from ectoderm and endoderm. In all Coelenterata except Ctenophora are found nematocysts or thread-cells; the Ctenophores have no nematocysts, but the surface of the tentacles contains peculiar adhesive cells called colloblasts, which may be equivalent to nematocysts. Ctenophores also differ from other Coelenterata in having a distinct layer of mesoblast cells in the embryo: they are therefore separated by some zoologists as a distinct phylum. They agree, however, with Coelenterates in having no coelom, and may for the present be included in the same phylum. This phylum constitutes a distinct grade of organisation, contrasted with the Coelomata or animals possessing a coelom, a group which includes the remaining phyla.

As thus defined the Coelenterata are subdivided into Hydrozoa, Scyphozoa, Anthozoa, and Ctenophora.

\section{Class : Hrdrozoa.}

Coelenterata in wbich the coelenteron is simple and the mouth opens directly into it, not into a tube lined by ectoderm or stomodaeum: no mesenteries; reproductive cells discharged directly to the exterior.

The Hydrozoa as exemplified by Obelia exhibit two distinct forms of individual in the life-history, only one of which is sexual: these are the hydroid and the medusa. The class includes forms like Obelia, in which the zooids of the hydroid stage are supported and protected by cups of the perisarc forming hydrothecae, and other forms in which the perisarc stops short at the base of the zooids and there are no hydrothecae. The medusae of these two orders differ in structure, those, like Obelia, the Lepto-medusae, having statocysts and the gonads on the radial canals, while the Anthomedusae have no statocysts and the gonads on the 
manubrium. In many cases the medusae are never set free, but remain attached to the hydroid gemmarium and show various stages of degeneration. There are also two orders in which the medusa develops directly from the egg or with a rudimentary lyydroid stage. Another order is -that of the Siphonophora, which are free swimming pelagic gemmaria, whose various zooids are differentiated for various functions, so that the gemmarium is polymorphic.

Class : Anthozoa.

Coelenterates of cylindrical shape, attached at one end, with mouth and tentacles at the other: the mouth leading into a tubular stomodaeum, lined by ectoderm and opening into the coelenteron below: the coelenteron around the stomodaeum divided into compartments by radial partitions called mesenteries: the free edges of the mesenteries below the stomodaeum elongated and thickened, forming the mesenterial filaments. The gonads are endodermic, situated on the sides of the mesenteries, and the reproductive cells are discharged by the mouth. The majority of the species form compound gemmaria, the corals.

This class includes the sea-anemones and corals. There are two great sub-classes : the Alcyonaria, represented by the Dead Men's Fingers of our coasts and by the red coral of commerce, and the Zountharia, represented by the common sea-anemones and the corals of the tropical coral reefs and coral islands. In the Alcyonaria the number of mesenteries and tentacles is always eight, in the Zoantharia the number is usually six, twelve, or some higher multiple of six, but in certain species the circles of tentacles are in multiples of five. The moutl is always elongated, not circular, and many of the internal structures are paired; an anterior or dorsal can be distinguished from a posterior or ventral side, so that the structure is always really bilaterally symmetrical.

\section{PHYLUM PLATYHELMIA.}

The Platyhelmia or flat worms form a phylum of which the different divisions show marked differences from one another, so that it is somewhat difficult to state 
the common characters. They are elongated, bilaterally symmetrical animals, not metamerically segmented; the alimentary canal has no anus and may be absent entirely. Coelom represented by the cavities of the generative organs, which form a complicated system of hollow organs and ducts, usually hermaphrodite; usually a number of yolk glands distinct from the ovaries. Nephridia, a single pair of branched tubular organs, the branches ending internally in flame-cells. Blood-vessels absent.

The Nemarteans were formerly included in the Platyhelmia, and there are reasons for thinking that they are allied to this phylum. But they differ in the remarkable eversible proboscis, which lies above the alimentary canal, in the possession of an anus, and of blood-vessels, and in the simple character and matameric arrangement of the generative organs. Many of them are extremely long and flexible; they have no external signs of segmentation.

Apart from the Nemertinea the Platyhelmia comprise the Turbellaria, the Trematoda, and the Cestoda or tapeworms. The last group are all internal parasites, the second are either internal or external parasites, while the Turbellaria are free-living and inhabit the sea or fresh waters; a few, however, live in moist soil in the tropics.

Class : Turbeluaria.

Mostly small animals flattened dorso-ventrally and not much elongated, ciliated over the whole of the external surface; by means of these cilia they glide along at the bottom of the water or on the lower side of the surface film; they can also swim by undulations of the body. The mouth is usually on the lower surface of the body and leads into a muscular pharynx connected with a blind intestinal cavity which is either simple (Rhabdocoelida), or consists of a median anterior division and two paired posterior divisions (Tricladida), or is complexly branclied (Polycladida). In a few species the digestive cavity is absent and the endoderm is a solid mass of cells (Acoela). In the Polycladida there are numerous testes and ovaries but no separate yolk-glands, and male and female genital apertures are usually separate. In Tricladida there are 
two ovaries, numerous testes, and numerous yolk-glands ; the genital aperture is single. In Rhabdocoelida there are one or two ovaries, two or many testes and yolk glands may be present or absent. The nervous system consists of a large cerebral ganglion divided into two lobes from which pass off a pair of long posterior nerve cords and smaller nerves to the anterior end. A pair of eyes are usually present at the anterior end.

Class : Trematoda.

Either internal or external parasites provided with suckers, with mouth at anterior end, and usually bifurcate intestine. No external cilia as a rule. The reproductive system is similar to that of Turbellaria and is exemplified by that of the Liver-fluke, but a special feature is the presence of one or two canals leading from pores on the dorsal surface to the shell gland. These are called Laurer's or Laurer-Stieda canals and serve to conduct the sperms from another individual to the shell gland, where fertilisation takes place.

The Trematoda are divided into two orders: the Digenea, resembling Fasciola hepatica, which exhibit alternation of generations and pass from an invertebrate to a vertebrate host in the course of their life-history; and the Monogenea, which develop directly without alternation of generations and without distinct metamorphosis, and which are attached throughout life to one host. The Monogenea are usually external parasites, they are common on the gills and other parts of fishes; but Polystomum lives in the bladder of the frog and Aspidogaster concharum is another example living in the pericardium of Anodon. The suckers vary in number, and are situated on a special disc at the posterior end of the body. Hooks are often present as well as suckers. The Digenea mostly have two suckers only, like the Liverfluke, which belongs to the family Distomidae, while the Monogenea have numerous sucliers, and the typical forms belong to the family Polystomidae.

Class : Cestoda.

Endoparasitic Platyhelmia without cilia and without 
alimentary canal, or endoderm; consisting in the adult of a rounded head bearing suckers and hooks for adhesion, and budding off a series of segments called proglottides, each containing a complete set of hermaphrodite reproductive organs. The life-history consists of two stages passed in separate hosts, usually both Vertebrate; the larva is sexually immature, rarely reproduces itself asexually, and develops directly into the sexually mature adult.

The proglottides are to be regarded as sexual individuals produced by gemmation from an asexual individual, so that the Cestoda or Tape-worms present the phenomenon of metagenesis.

The larva is known as a Cysticercus or Cysticercoid, consisting of a more or less enlarged hollow vesicle invaginated at one point; hooks and suckers are developed on the invaginated surface, which is afterwards everted to form the head of the adult. When the larva is swallowed by the final host the head of the tape-worm becomes attached to the wall of the intestine and the proglottides are budded from the head in succession, so that the youngest are always next to the head. As the proglottides get older the generative organs begin to develop in them, the male parts first. In each proglottis is formed a complete set of these organs, which consists of (i) a large number of small rounded testes, whose ducts unite and lead finally to a single vas deferens opening at the lateral margin of the proglottis, and (ii) a pair of ovaries placed near the posterior border of the proglottis, and opening into the oviduct, whose external aperture is at the side of the male aperture. Behind the ovaries is situated a yolk gland, whose duct opens into the oviduct, and a shell gland opens into the yolk duct. Leading from the oviduct is a uterus for the storage of fully formed eggs, and beyond this is a receptaculum seminis. The end of the vas deferens forms a protrusible intromittent organ or cirrus, but it is not known whether copulation occurs between cirrus and vagina of the same or of neighbouring segments. In the oldest seginents large numbers of fertilised eggs accumulate in the uterus, while the rest of the 
generative organs disappear. Ultimately groups of ripe segments or proglottides become detached and pass to the exterior, and there the eggs are dispersed. If swallowed accidentally by the first host the eggs develop into the cysticercus. Cysticercus pisiformis in the abdomen of the rabbit becomes Taenia serrata in the intestine of the dog; Coenurus cerebralis of the brain of the sheep becomes Taenia coenurus in the dog; Echinococcus is a proliferating cysticercus in the liver of man, sheep, pig, etc., and develops into Taenia echinococcus in the dog; the larva of Taenia solium, which occurs in man, is a cysticercus in "measly" pork; while the larva of Taenia saginata, another human tape-worm, occurs in beef.

\section{PHYLUM ANNELIDA.}

Bilaterally symmetrical, metamerically segmented animals in which each segment primitively contains a large coelomic cavity, a pair of gonads, a pair of nephridia, and a pair of coelomic ducts which are functionally generative ducts; nervous system consisting of a pair of cerebral ganglia connected by perioesophageal commissures to a double ventral nerve cord, with more or less distinct ganglion enlargements in each segment; usually a closed vascular system, including a dorsal and ventral vessel. The vascular system and the metamerism distinguish the Annelida from the Platyhelmia, while the condition of the coelom and vascular system are very different from that seen in the Arthropoda. They include two principal classes, namely, the Chaetopoda and the Hirudinea or leeches, and also a number of smaller groups which for the present may be considered as classes, namely, the Archiannelida, the Echiuroidea, the Sipunculoidea, and the Priapuloidea. The last three were formerly united in the Class Gephyrea.

Class : Chantopoda.

The possession of chaetae alone does not suffice to define the Chaetopoda, for chaetae are present in the Echiuroidea, but the Chaetopoda have well marked metamerism with 
chaetae on every segment and usually borne on segmental appendages called parapodia.

\section{Sub-Class : Polychaeta.}

A typical Polychaete has already been described in Nereis. The diagnostic characters are: Chaetae numerous and large, often compound as in Nereis, and usually borne on the segmental appendages or parapodia; sexes separate, with gonads in many or all of the segments; coelomic ducts usually united with the nephridia into a single series of tubes which function as both excretory and genital ducts, except in Nereidae, in which the genital cells escape by dehiscence. They are all marine and show a great variety of forms, the parapodia being in many reduced or much modified, and branchiae may be present in addition.

\section{Sub-Class: Oligochaeta.}

In the Oligochaeta, of which the earthworm is an example, the parapodia are rudimentary and represented only by a few chaetae. They are hermaphrodite, with the gonads restricted to a few segments and genital ducts distinct from the nephridia ; the clitellum, a band of thickened epidermis which secretes the cocoon, is usually present. The head has no tentacles or other appendages as in the Polychaeta. They live in the soil or in fresh water.

\section{PHYLUM ARTHROPODA.}

Literally the term Arthropoda means animals with jointed feet, i.e. limbs (Greek arthron, joint; pous, foot). This might be considered equally applicable to the Vertebrata, but the joints or segments of the Arthropoda are segments of thicker, more rigid, often more calcified cuticle connected by parts of the cuticle which are thinner and more flexible. One pair of these limbs or appendages primitively occurs on each segment or metamere of the body. At least one pair of the anterior appendages at the sides of the mouth is adapted for use as jaws; for this reason the name Gnathopoda is sometimes preferred. In the perfection of their metamerism Arthropoda resemble 
Annelida, but they differ in the absence of chaetae, the character of the appendages, and the small number of the segments. Processes of the cuticle are common, but these setae are never imbedded in sockets like the chaetae of Chaetopoda. The internal characters are the reduction of the coelom, which is represented only by the cavities of the generative organs and the terminal sacs of the excretory organs, the absence of true nephridia, and the ostiate heart surrounded by a blood space representing the venous vessels.

The Arthropoda include the following classes: Crustacea, Arachnida, Prototracheata, Myriapoda, and Insecta.

Class: Crustacea.

Arthropoda with two pairs of antennae; at least three pairs of appendages converted into jaws, i.e. mandibles and two pairs of maxillae; these five pairs of appendages are attached to the compound head formed by the union of the corresponding segments; the rest of the body usually divisible into two regions, the thorax or pereion, and abdomen or pleon. The dorsal wall of the head is usually prolonged backwards to form a shell which covers more or less of the body, and in the higher forms fuses with some or all of the thoracic segments to form the cephalo-thorax. Respiration, with few exceptions (woodlice), aquatic, branchiae consisting of outgrowths of the body wall or of the limbs. There is a median eye which often disappears in the adult, and a pair of compound eyes often raised on peduncles. Astacus is one of the order Decapoda which is distinguished by the great enlargement of the five posterior pairs of thoracic legs and the modification of the three anterior pairs into maxillipeds. Forms like Astacus with well-developed pleon form the sub-order Macrura or long-tailed Decapods, contrasted with the crabs or Brachyura, in which the pleon is rudimentary and the cephalothorax is broad and short. The Decapoda, with other orders, such as the Arthrostraca (including wood-lice, sand-hoppers, etc.), form the subclass Malacostraca, distinguished by having with few exceptions 19 segments, while the remaining Crustacea are 
placed in the sub-class Entomostraca, the orders of which are not very closely allied.

\section{Class: Arachinida.}

Arthropoda without antennae, body divided into cephalothorax and abdomen; the cephalothorax bears two pairs of gnathopods, chelicerae and pedipalpi, and four pairs of legs; abdomen usually without appendages. The organs of respiration when present are either tracheae or booklungs, except in Limulus the king-crab, in which they have the form of book-gills attached to the last five pairs of appendages of the abdomen. Like the Crustacea the Arachnida possess a large pair of digestive glands, the so-called liver, opening into the mesenteron.

The Arachnida include the following orders :

1. Xiphosura (Limulus),

4. Araneida (Spiders),

2. Scorpionida (Scorpions),

5. Acarida (Mites),

3. Phalangida (Harvest-men),

and some others of less importance.

The species of Limulus are marine, and occur off the eastern coast of North America and in the East Indies. They are the only living representatives of the marine ancestors of the Arachnida. The cephalothorax is covered by a broad carapace convex on the dorsal side and semicircular in outline; the lower surface of the carapace is flat in front and hollow behind, and its posterior angles are produced. The carapace bears dorsally a pair of simple eyes in the median line anteriorly and a pair of lateral compound eyes; the abdomen or mesosoma is somewhat triangular in shape and consists of seven segments, the first six of which bear broad flat appendages; the first of these form the operculum, on the posterior surface of which are the genital apertures; the next five pairs bear the gill-books, and the seventh bears the anus. Behind the anus is a long spine or telson which is rigid and unjointed; it probably corresponds to the post-anal telson of the scorpion, the anal segment representing the whole of the scorpion's post-abdomen. Inside the body, pear the ventral surface, lies a plate of cartilage of meso- 
blastic origin, the endosternite, as in scorpions and some spiders.

In Scor pionida the segments and appendages correspond with those of Limulus. The prosoma or cephalothorax is much smaller and narrow; the chelate pedipalpi are large and strong; the operculum is small and bears the genital apertures; the next pair of appendages are fringed and called the pectines, whose function seems to be tactile; they are modified gill-books; the remaining four pairs of gill-books are sunk into pits in the body-wall and form the lung-books; the last segment of the mesosoma is without appendages and is succeeded by a metasoma of six narrow segments without appendages; the anus is at the posterior border of the fifth segment, and the sixth forms the characteristic pointed sting containing poison glands.

The Phalangida differ from spiders in having no constriction between prosoma and metasoma, and in the fact that the latter is distinctly segmented. The four pairs of legs are very long; they have no lungs, but breathe entirely by tracheae; they spin no web, but pursue their prey.

In the true Spiders the metasoma is unsegmented and separated from the prosoma by a very deep constriction. The chelicerae are not chelate, but the terminal joint is pointed and provided with poison glands; the pedipalpi are also non-chelate, and in the males modified to serve as copulatory organs. The genital openings are at the anterior end of the abdomen and protected by an operculum; in some there are two pairs of lungs, like those of the scorpion; in others the second pair of lungs is replaced by a pair of openings leading to tracheae. Near the posterior end of the metasoma are three pairs, or in some cases only two pairs, of spinnerets, or papillae, on which the ducts of the silk-glands open ; these papillae are modified abdominal appendages. The prosoma bears two or three pairs of simple eyes.

In the Acarida the body is entirely unsegmented and shows no distinction into prosoma and metasoma. The chelicarae are sometimes chelate, but in the majority are 
stylets for piercing and enclosed in a sheath formed by the pedipalpi. The respiratory organs are tracheae or entirely wanting.

Class : Insecta.

Air-breathing Arthropoda which possess the power of flight. The presence of wings distinguishes Insects not only from other Arthropoda, but from all other Invertebrata, since no others are adapted to aerial locomotion. There are, however, some insects in which wings are absent; in some cases this is obviously due to the disappearance of the organs, but in the order Aptera there is no evidence that wings were ever developed; wings therefore, though highly characteristic, are not absolutely diagnostic. The body is divided into three regions-head, thorax, and abdomen; the head shows no external segmentation and bears a pair of compound eyes, a single pair of antennae, a pair of mandibles, and two pairs of maxillae. The thorax consists of three segments, each bearing a pair of jointed unbranched legs, and the second and third segments each bearing a pair of wings, which are outgrowths of the terga, not appendages. The abdomen is distinctly segmented, the maximum number of segments being 11, but in many cases only 7 to 10 can be distinguished; the abdominal segments bear no fully developed appendages, but at the posterior end processes representing reduced appendages may be present. The heart is elongated, tubular, divided into eight chambers situated in the abdomen; blood-vessels are not highly developed. The organs of respiration are trachae, i.e. much ramified tubes supported internally by a spiral thread; the air enters by a number of small apertures called stigmata, situated on the sides of the thorax and abdomen. The nervous system consists of cerebral and ventral pairs of ganglia, highly developed. A liver is wanting, but salivary glands are always present; the excretory organs are a number of caecal tubes, the Malpighian tubes, opening into the intestine. The sexes are separate and the development is sometimes direct, more usually remarkable for a very complete metamorphosis. 
The wings are folds of the integument supported by thickened branched linear structures called nervures and consisting of a trachea and a blood-vessel. The class is divided into a number of orders distinguished by the structure of the wings and of the nouth-parts; the principal of these are the following:-

Order Aptera. Wings entirely absent, body covered with cuticular scales or hairs. Development direct.

Order Orthoptera. Two pairs of wings, anterior pair more or less tough and thickened. Prothorax free, development direct or with incomplete metamorphosis. This order includes Cockroaches, Grasshoppers, Locusts, Stickand Leaf-insects, and Earwigs.

Order Neuroptera. Botl pairs of wings membranous with numerous reticulated nervures, mouth parts masticatory, prothorax free. Metamorphosis complete or incomplete. Includes Dragon-flies, May-flies, Ant-lions, Caddis-flies, and Termites or White-ants.

Order Hemiptera. Proximal half of the anterior wings usually horny and rigid; mouth parts modified for piercing and sucking. Prothorax free; metamorphosis incomplete Includes Bugs, Lice, Scale-insects, Plant-lice or Aphides, and Cicadas.

Order Diptera. Posterior wings rudimentary; mouth parts adapted for piercing and sucking. Prothorax fused with the other segments of the thorax. Metamorplosis complete. Includes Flies, Gnats, Mosquitos, and Fleas.

Order Lepidoptera. Both pairs of wings large and covered with scales, not folded. Maxillae forming a spiral sucking-tube. Prothorax fused with mesothorax. Metamorphosis complete. Includes Butterflies and Moths.

Order Coleoptera. Anterior wings horny and rigid, acting as covers for the posterior pair. Prothorax free. Mouth parts adapted for biting. Metamorphosis complete. Includes true Beetles.

Order Hymenoptera. Both pairs of wings membranous, hinder pair smaller and attached by hooks to anterior pair, Mouth parts adapted for biting and licking. Prothorax united. Metamorphosis complete. Includes Ants, Bees. 
and Wasps, Gall-flies and Ichneumon-flies, whose larvae are parasitic in caterpillars.

\section{PHYLUM CHORDATA.}

Animals which possess temporarily or throughout life the following three characters: (1) a dorsal tubular nerve cord; (2) pharyngeal gill-slits; (3) a notochord. The more primitive forms are distinguished by never developing any skull or vertebrae, nor any complicated brain like that of the higher forms; they may be termed therefore the Prochordata.

Of the Prochordata two classes are included in the syllabus, the Urochorda and the Cephalochorda.

\section{Class: Urochorda.}

These are the animals commonly kuown as Ascidians or sea-squirts. The great majority of them are sessile in the adult condition. In the larval stage of all and throughout life in the Larvacea there is a notochord in the tail which does not extend into the anterior part of the body. There are at first only two gill-slits, which become enclosed in a secondary cavity or depression into which the anus opens; this cavity is the atrium, thougl not exactly homologous with the atrium of Amphioxus. The gill-slits become subdivided into numerous small openings. The atrial opening is dorsal and near the mouth. Thus the usual form of the adult is that of an irregular somewhat cylindrical sac, attached at one end, with two openings, mouth and atrial opening, at the other. The cilia of the pharynx maintain a constant current of water, entering at the mouth and passing out at the atrial aperture. A ciliated and glandular endostyle is present along the ventral median line of the pharynx as in Amphioxus, and the epipharyngeal groove is represented by a dorsal series of projections called languets. Another very characteristic feature is that the epidermis secretes a thick gelatinous "test" into which extends an outgrowth from the hinder part of the body, and from this outgrowth the test receives cells and blood-vessels; the test contains cellulose. Many 
of the Urochorda or Tunicata exhibit the process of gemmation, forming compound gemmaria as in other sessile animals. One class of the Urochorda is pelagic, that is, adapted to a free swimming life at the surface of the sea.

\section{Class : Cephalochorda.}

The characters of this class are those of Amphioxus, for the differences between the various genera and species, which are few in number, are only in small details. Cephalochorda differ from the other Prochordata in the elongated fish-like form, in the fact that the notochord extends to each extremity of the body, and in the special relations of the atrium.

\section{SUB-PHYLUM VERTEBRATA OR CRANIATA.}

Since all the members of this group possess some rudiments of vertebrae if it be only as in the Lamprey a few neural or haemal arches without centra, there seems no reason why the old name Vertebrata should not be retained. Some authorities, however, regard the skull as more characteristic and therefore prefer the name Craniata. Other common characters are the well-developed brain with its common plan of structure, the well-developed heart, the portal system and other general features of the vascular system, the urogenital system. In fact all the Vertebrata are modifications of a common fish-like ancestor.

The Lampreys (Petromyzon) and Hag-fishes (Myxinoids) form a group which has been placed in various positions in the classification of the Vertebrata. These animals have no paired fins, but have fin-rays in the median fin; they have gill-slits which take the form of pouches, communicating with the exterior and with the pharynx by tubular ducts. Accordingly they are considered by Huxley as an order of the class of fishes. The fact, however, that the mouth is suctorial and possesses no movable jaws, and that the pituitary sac opens permanently outside the mouth, with the nasal sac single and opening into the pituitary sac, differentiates these animals from all other fishes and therefore later authorities have made them a separate 
class of Vertebrata under the name Cyclostomata. The condition of the mouth and pituitary sac is so extrabordinary and so different from the usual condition which, with modifications, is common to all other Vertebrates that modern zoologists have given the Cyclostomes a still more isolated position and divided the Vertebrata into two main divisions, one, the Cyclostomata, containing only these peculiar forms, and the other, the Gnathostomata, containing the rest of the Vertebrates. Lately it has been stated as the result of new investigations that the so-called tongue of the Myxinoids is really a modified mandibular arch, and this would suggest that the Cyclostomes are modified fishes; but the position of the pituitary sac is still so exceptional that we cannot unite them with the latter, but must continue to regard them as a separate class.

\section{Class : Pisces.}

Craniata which possess gill-slits and gills throughout life, and which possess paired fins or ichthyopterygia, and usually unpaired fins also. 'This character distinguishes all fishes from Amphibia, for although the fins of fishes differ from one another in structure they never resemble the limb of the terrestrial Vertebrate consisting of a segmented axis terminating in five digits.

The fishes consist of the following orders :-

Order 1, Elasmobranohii.-Gill slits opening on surface of body, not covered by an operculum. Skeleton cartilaginous, more or less calcified, but not ossified. Exoskeleton consisting of dermal denticles. Skull hyostylic, i.e. jaws suspended by the hyomandibular cartilage. A preoral rostrum or snout. No air-bladder. Truncus arteriosus with several rows of valces. Fins with long cartilaginous endoskeletal rays, and beyond these dermal horny fibres.

Order 2, Holocephali.-Similar to Elasmobranchs, but differing in the following characters: dermal denticles absent except on the frontal clasper; bony rings surrounding the notochord; skull autostylic, i.e. the palato-quadrate is fused with the cranium and the jaws are not suspended by the hyomandibular; gill-slits covered by an operculum. 
This order contains a curious fish called Chimaera living in European seas, and a few allied forms in other oceans.

Order 3, Dipnoi.-Fishes with persistent notochord, without centra, the vertebrae represented by neural arches, bearing ribs, and haemal arches. Skull cartilaginous, with little ossification; paired fins pinnate in structure and elongated, dermal fin-rays horny. Dermal skeleton of flat, overlapping scales on the body and membrane bones over the skull. Skull autostylic, palato-quadrate fixed to it. Gills covered by an overculum. Single or paired lungs opening ventrally into the pharynx, supplied by pulmonary arteries from the fourth efferent branchial arteries, a pulmonary vein opening into a separate left auricle.

There are only three genera of Dipnoi, all living in fresh water. One, Ceratodus, lives in Australia; another, Lepidosiren, in South America; and the third, Protopterus, in West Africa. They form an approximation in many respects to the Amphibia.

Order 4, Teleostomi. - Fishes with an air-bladder homologous with lungs, but with veins returning blood into the cardinal veins, not into the auricle, which remains undivided. Skull hyostylic, that is jaws suspended by the hyomandibular bone. A well-developed operculum, the gill-septa reduced to narrow bars. Dermal skeleton of scales on the body and membrane bones on the head; along the edges of the mouth membrane bones (the maxillae, premaxillae, and dentaries) form the jaws and bear teeth. Paired fins fan-shaped with endoskeletal rays well developed in the more primitive forms, much reduced in the others. This order includes both the more primitive forms formerly distinguished as Ganoids and the numerous specialised modern fishes called Teleosteans; examples of the former are the Sturgeons and the Bony Pike (Lepidosteus) of North America; to the Teleostei belong all the more common and familiar fishes such as carp, perch, cod, herring, salmon, and flat-fishes.

\section{Class : Amphibia.}

The frog is completely distinguished from fishes by the absence of gill-slits in the adult state, but as there are zOOL. 
some Amphibia which retain gill-slits throughout life this character is not diagnostic of the whole class. A complete distinction is, however, found in the fact that no Amphibia possess dermal fin-rays like those of fishes; endoskeletal fin-rays are also wanting, the endoskeleton of the paired limbs having another form, that of the terrestrial limb or cheiropterygium; this consists of a single proximal bone, then two parallel bones, then several small bones, the limb terminating in five digits which alone have some resemblance to fin-rays. The median fin-membrane when present has no fin-rays at all. The skin is usually soft, moist, and glandular, and serves as an accessory organ of respiration, having a large vascular supply, and dermal scales are usually absent; but this is not a diagnostic character, as dermal scales are present in the snake-like Amphibia of the tropics called the Gymnophiona and in some Anura there are bony plates in the skin of the back. The membrane bones of the skull represent the scales of the head in fishes. The heart is three-chambered, the auricle being divided into two, but not the ventricle. A post-caval vein is present, but this occurs also in Dipnoi. The posterior cardinal veins disappear in the adult. In the vertebrae bony centra are well-developed, and the neural arches possess zygapophyses which are not found in any fishes.

The Amphibia include four orders: the Urodela, which retain the tail throughout life and contain some forms in which one or more gill-slits persist in the adult, and others, like the common newts, in which the gill-slits are entirely closed in the adult; the Anura, of which the frog is an example; the Gymnophiona, which are elongated snake-like burrowing forms destitute of both limbs and tail ; and the Stegocephalia or Labyrinthodonts, extinct tailed Amphibia of large size with well-developed membrane bones on the skull, and dermal exoskeleton.

Class: Reptilia.

In Reptilia, Birds, and Mammals, the fish-like larval stage of the life-history is wanting, or rather it is passed in the egg or in the oviduct. The embryonic development 
requires a special organ of respiration, which is supplied by the allantois, and the embryo is also enclosed in the amnion. These three classes are therefore grouped together as the Amniota, contrasted with the Fishes and Amphibia which form the Anamniota. Reptiles are coldblooded, that is to say the temperature of the blood is not much above that of the surrounding medium, and varies with that of the latter, while in birds and mammals it is not only high but constant. In Reptiles two aortic arches are present, as in the frog, and there are two auricles and one ventricle, except in Crocodilia, in which the ventricle is completely divided. The skin is almost entirely destitute of glands, and the outer layers of the epidermis are thickened and cornified in distinct areas, either meeting edge to edge (scutes) or overlapping (scales). The scales of Reptiles are therefore very different from those of fishes, which are dermal structures; many Reptiles, however, have also dermal ossifications, for example, the crocodiles and turtles and many lizards, but these do not in all cases correspond to the epidermic scales. The skull articulates with the vertebral column by a single condyle, and has more cartilage bones than in the Amphibia; some of the anterior vertebrae are included in the skull, so that the hypoglossal is a cranial nerve. The conus arteriosus is merged in the heart. The metanephros is entirely separated from the mesonephros, the latter forming the epididymis in the male and being vestigial in the female; this is true also of all the Amniota. In Reptiles there is a renal portal system of veins. There are four orders of living Reptiles: the Rhyncocephalia, containing only a single genus, Hatteria, confined to New Zealand, which has many primitive features; the Squamata, in which the quadrate bone is moveable, and which include the lizards and snakes; the Chelonia, or tortoises and turtles, distinguished by the com. plete bony investment formed by the fusion of dermal bony plates with the endoskeleton; and the Crocodilia, which have bony dermal plates covered with sculptured horny plates of corresponding shape on the dorsal surface, and in some cases on the ventral surface also; the ventricle of the heart is completely divided, but the oxygenated and 
deoxygenated blood is not completely separated. In Hatteria and many lizards the pineal gland has the structure of a distinct eye, known as the pineal or parietal eye, situated in the parietal foramen of the skull and covered by a transparent scale.

\section{Class : Aves.}

In the case of Birds one character is sufficient to distinguish them from all other kinds of animals, for they are the only animals which possess feathers, and no bird is without feathers. These structures are peculiar developments of the epidermis, composed of the middle layers of the latter, the outer layers being shed as the feather grows. The feather is developed like a tubular scale or quill, and the tube splits down the posterior side; the barbs are portions of the tube separated by oblique divisions and attached to a longitudinal, undivided thicker portion on the anterior side, which forms the stem or rachis. The lower part of the feather remains tubular and does not shed its outer layers; this forms the quill, and is sunk in a socket or invagination of the skin. The structure of the wing and that of the leg are peculiar and diagnostic in birds. That of the former is specially adapted for flight, the requisite extent of surface being supplied not by the limb itself but by the feathers. The radius and ulna are parallel to each other, not crossed, the radius being anterior and smaller than the ulna. The carpal bones are reduced to two, there are three metacarpals united together, of which the first is very short and bears a single phalanx, the second bears two phalanges, the third one. The peculiarity of the leg is that the tarsus is entirely ankylosed with the neighbouring bones, and the ankle joint, instead of being between the tibia and the tarsus, is between the proximal and distal rows of tarsals; the proximal row is united with the tibia, and the distal with the second, third, and fourth metatarsals, which are united together. The first metatarsal is free, but the fifth is absent. The fibula is vestigial. The segments of the leg are therefore the femur, the tibio-tarsus, the tarso-metatarsus, and lastly the digits. There are no teeth, the jaws being enclosed in a 
horny beak. The skull is globular with indistinct sutures, and the lower jaw is articulated to a quadrate bone as in reptiles. The pectoral girdle consists of scapula, strong coracoid articulating with the sternum, and a clavicle united with that of the other side. The sternum is broad and long and produced ventrally, except in ostriches and their allies, into a deep keel. The sacrum consists of about twelve vertebrae united together, the ilium is long and parallel to the sacrum, neither pubis nor ischium meet in a ventral symphysis. 'There is only one aortic arch, the right, and the oxygenated blood and deoxygenated blood are completely separated. In spite of these peculiarities birds are in many respects, as in the possession of a quadrate, of large coracoids, of an undivided cloaca, similar to reptiles, and therefore the two classes are often grouped together under the name Sauropsida.

\section{Class: Mammalia.}

A list of the mammalian characters is given on p. 133 of the original text-book, but the list requires the following additions and explanations. The chief external peculiarities are the hair, the mammary glands, and the external ear. A hair is a cylindrical rod of epidermic cells produced from the surface of a papilla which lies at the bottom of a follicle sunk in the skin; the dermal vascular tissue of the papilla does not extend up the centre of the hair as in the case of a feather. Mammary glands are special enlargements of the sebaceous glands which open into the hair follicles. The external ear is an outgrowth of the skin around the external auditory meatus supported by cartilage and supplied with muscles. The diaphragm dividing the thoracic cavity from the abdominal and the presence of only one aortic arch, viz. the left, are the special features of the circulatory and respiratory systems.

Of the skeletal characters, epiphyses to the vertebral centra are wanting in Monotremata, some of the Sloths have eight or nine cervical vertebrae, and one has only six ; the Manatee also has only six; all other Mammals have seven. Coracoids are absent as separate bones, and only represented by the coracoid processes of the scapulae; in 
this also the Monotremata are reptilian, having complete coracoids. In the skull the articulation of the mandible with the squamosal, and the conversion of the articular and quadrate bones into the malleus and incus of the tympanic cavity, are peculiarities found in all Mammals and in no other Vertebrates. The backward development of the cerebral hemispheres, the corpus callosum, the corpora quadrigemina, and the spirally coiled cochlea, are characters of the brain and sense organs. In the reproductive system the cloaca is divided into a dorsal passage terminating the rectum, and a ventral connected with the bladder, and forming the urinogenital duct; the Monotremata, however, have an undivided cloaca like the Sauropsida. In the latter also the eggs are rather large, provided with yolk, and are laid and hatched afterwards : in other Mammals the egg develops in the uterus, and the young are born alive; the diagnostic character is, however, not the latter fact but the formation of the placenta by the union of the allantois with the uterine wall. The true allantoic placenta is only fully developed in the higher Mammals ; in Marsupials, with the exception of one, Perameles, the yolk sac alone adheres to the walls of the uterus and forms what is called an umbilical placenta. In accordance with these important differences Mammals are divided into three subclasses : the Prototheria or Monotremata, containing only Ornithirhynchus and Echidna; the Metatheria or Marsupials; and the Eutheria, including the rest of the class. 


\section{EXAMINATION QUESTIONS.}

\section{Questions on Invertebrata and General Zoology.}

1. Describe the minute structure of the respiratory organs of the earthworm, crayfish, Anodonta, and Amphioxus.

2. Give a short account, with examples, of the essential features in (a) asexual, (b) sexual, (c) partheno-genetic reproduction.

3. Give as complete an account as you can of the structure of the ectoderm and of the endoderm of Hydra. Describe the structure of the chlorophyll-corpuscles of Hydra, and their mode of occurrence and origin, and discuss their nature and significance.

4. What is a nephridium? Describe those of the animals named in the University Schedule* which possess such organs.

5. Give an account of the structure and reproduction of the Protozoa enumerated in the schedule, and point out the differences between Protozoa and Metazoa.

6. Describe the naked eye structure of the alimentary canal and the microscopic character of its walls in the rablit, the earthworm and Hydra. Give some account of the process of digestion in each of these.

7. Describe the blood, blood-vessels, and circulatory

* The schedule of types is: Lepus, Rana, Scyllium, Amphioxus, Astacus, Lambricus, Anodonta, Hydra, Paramecium, Amœba. 
organs of the dogfish, the earthworm, and the pond mussel. Give a brief account of the chemical constituents, structure, and functions of the blood in each case.

8. Give an account (with diagrams) of the lowest and highest degrees of structure known to you in the Protozoa. How do unicellular animals differ from unicellular plants?

9. (a) Describe from without inwards the structures visible in a transverse section of a Hydra; (b) describe similarly a transverse section of an earthworm taken about the fortieth segment. In both cases describe the tissues present and the characters of the cells which form them. (c) State what is the most important structural difference between Hydra and the earthworm as shown by these two sections.

10. In what essential respects does the structure of Hydra differ from that of Paramecium? Describe the methods of reproduction in each of these animals.

11. Describe the reproductive processes and the development of the egg in Hydra, and contrast therewith the modes of reproduction observed in Paramecium and Amœba.

12. What substance is excreted by the renal organ of a frog, and what relation does this substance bear to the general life of the organism? Describe the parts by which similar excretion is believed to be effected in Amœba, Hydra, earthworm, mussel, and crayfish.

13. Describe fully $(a)$ the central nervous system, $(b)$ the notochord and its sheath, of Amphioxus. (c) Compare the central nervous system of Amphioxus with the central nervous system of Lumbricus.

14. What is a gastrula? With what lower type has the gastrula been compared? Discuss the comparison.

15. What is meant by the term serial homology? Illustrate your answer by reference to the appendages of Astacus.

16. Draw diagrams, with the parts named, of the central nervous system and renal organs of (a) Lumbricus, (b) Astacus, and (c) Anodon. (d) Compare Lumbricus and Anodon, in respect of their nervous systems; and $(e)$ Astacus and Anodon, in respect of their renal organs.

17. Describe the renal excretory and the reproductive organs of the mussel, the earthworm, and the frog. 
18. Describe in detail the gill of a mussel. What are the functions of the gills?

19. Give an account of the body cavity of the mussel, and compare it with that of any vertebrated type (say which you take).

20. Describe fully the circulatory and respiratory organs of a crayfish, and compare them with those of a mussel.

21. Describe the urinary and generative organs in $(a)$ Astacus, (b) Lumbricus, (c) Anodon. (d) In what respect do the urinary organs of Lumbricus and Anodon resemble one another and differ from those of Astucus? (e) What is the function of urinary organs?

22. Draw diagrams of the blood vascular system of $(a)$ Lumbricus, (b) Astacus, (c) Scyllium. Describe the course of the circulation of the blood in each of these animals.

23. Describe the digestive organs of Astacus, including the mouth appendages.

24. Describe the heart of the frog and compare it with that of the crayfish.

25. What is meant by the term coelom? Give a short account of the coelom in Lumbricus, Rana, and Isepus, including (1) development, (2) relation to the gonads and gonaducts.

26. What is the nature of the renal excretion? Describe the structure and exact position of the excretory organs in the earthworm, mussel, crayfish, and frog.

27. Describe the microscopical characters of the circulatory fluids met with in the rabbit, frog, Astacus, and earthworm.

28. Describe and compare with one another by means of diagrammatic figures the structure of the eye in (1) Astacus, (2) Rana or Lepus. Explain briefly the mode of action of the compound eye.

29. In the series of organisms (1) Amoeba, Paramecium, Hydra, Earthworm, Frog; (2) Spirogyra, Fucus, Pteris, Vicia (Bean Plant); show that the structures characteristic of the animals as such, whether high or low on the one hand, and of the plants as such on the other, are the expression of a fundamental difference in the mode of nutrition of the members of the two series. 
30. Describe and compare the excretory apparatus of Iepus or Rana, Astacus, Lumbricus, and Anodon.

31. Describe the heart of Astacus, Scyllium, Rana, and Lepus, with especial reference to the relationship of each to the great vessels and vascular channels.

32. What are the phenomena characteristic of sexual reproduction? Describe any case with which you are familiar, and contrast one of asexual reproduction.

33. Describe and compare the nervous system of Anodonta, Lumbricus, and Astacus.

34. How is respiration effected in Lumbricus, Amphioxus, and Scyllium? Describe the organs concerned in each case.

35. Describe the excretory apparatus of Lumbricus and compare with it that of Astacus and of Anodon.

36. Describe the integument of Lepus, Scyllium, and Astacus.

37. Give a general account of the structure and lifehistory of Paramecium.

38. Explain the meaning of the following terms:metabolism, assimilation, secretion, coolom, metamere, gamete. 


\section{APPENDIX.}

\section{GENERAL ADVICE TO THE STUDENT.}

We would at the outset impress upon the student-especially the isolated worker, for whom these notes are more particularly written-the importance of some preliminary reading before dissection is undertaken. No one would dream of attempting to explore a deserted city without some previous study of maps and guide-books, but we find again and again students undertaking to explore the complicated anatomy of a vertebrated animal without the slightest, or only the slightest, preparatory reading. This is entirely a mistake. A student should be familiar with the nomenclature of the structures he contemplates examining, he should have some idea of their mutual relations and functions, or his attention will inevitably be diverted by the difficulty of new names and physiological questionings to the neglect of his dissection, and that careful observation of form and mutual position which is the essential object of dissection. On the other hand, it is equally necessaryperhaps more so-to warn students against the bookish fallacy, and to assure them of the absolute impossibility of realizing biological facts from reading alone. Practical work can alone confirm and complete the knowledge to which the text-book is the guide. In scientific teacbing it may sometimes be conveniont for the thought to precede the thing, but until the thing has been dealt with the knowledge gained is an unsatisfactory and unstable possession.

\section{How to Read.}

Our first caution to the reader is to avoid the common and easy delusion that he really understunds some state- 
ment, because he can remember the words of it. He should always test his understanding of difficult points by trying to express them in words of his own. Many statements, especially descriptive ones, are often difficult to follow unless the accompanying diagrams are studied. These should not be merely looked at, but copied: only thus can you be sure of seeing every detail. Moreover, careful copying is a good training in that accurate observation without which dissec. tion loses much of its value. In copying diagrams, a set of coloured pencils is absolutely indispensable. Although the diagrams have been made as clear as possible in black and white, their clearness can in many cases be increased, and at the same time the trouble of drawing them diminished, by the use of different colours in place of different modes of shading. The "Blaisdell " coloured pencils are recommended, as more readily sharpened and less easily broken than the ordinary cedar-wood pencils; but they can only be had in four colours, and greater variety than this is often wanted. Both kinds can be obtained through any shop where artists' materials or school appliances are sold.

The abundance of unfamiliar technical terms is a difficulty with many beginners. These terms fall into two categories. There are ancient terms whose derivation is often so obscure that they are practically meaningless : these terms have to be learnt as a new-comer in any neighbourhood learns the names of the streets-by constant familiarity impressing them on the memory. On the other hand, most modern terms are self-explanatory, if once the meanings attached to a number of Greek and Latin roots are understood. For this purpose the following list of some of the commonest roots will, we hope, prove useful. We strongly recommend the student to take note of all such roots, as he comes across them; it will save him more trouble later on.

\section{Greik Roots.}

ana, up.

archi, ancient, primitive.

blast, develop. cephal, bead. chloro, green. chromo, colour. coel, cavity. derm, skin. ecto, outside. endo, inside. entero, intestine. epi, upon. gastro, stomach. haemo, blood. 
GREEK Roots (continued).

hepato, liver. hyper, above. hypo, below. karyo, kernel, nucleus.

kata, down.

lieno, spleen.

macio, large.

mega, large.

mere, division, part.

meso, middle.

meta, behind.

micro, small.
myo, muscle.
nephro, kidney.
neuro, nerve.
noto, back, dorsal.
odonto, tooth.
osteo, bone.
-oid, resembling.
peri, around.
plasm, matter, sub-
stance.
pleuro, side, lung.

podo, foot.

$p^{*} \mathrm{o}$, in front of.

procto, anus.

proto, first, primor-

dial.

pseudo, false.

soma, body.

splanchno, viscera.

stoma, month.

tom, cut, divide.

zygo, unite.

\section{LAATIN Roots.}

branch, gill.

circum, around.

infra, beneath.

inter, between.

intra, within.

nuulti, many. or-, mouth.

post, behind.

pree, in front.

pulino, lung.

reno, kidney.

supra, above.

The student should notice that both Latin and English forms of some terms are in use : e.g., appendix vermiformis, vesicula seminalis, tuberculum, ductus endolymphaticus, etc., are absolutely synonymous with vermiform appendix, seminal vesicle, tubercle, endolymph-duct, etc. As a rule the English form has been used in such cases in this book.

If any statement is found to be still obscure after diagrams and list of roots have been consulted, the student should mark it for future reference. It may be that dissection will show him its meaning, or that the wider knowledge that comes imperceptibly as his study extends will afterwards make it clear. But whatever he does, he should never delude himself into believing that he does understand it if he doesn't.

\section{Comparisons.}

When the student has read through and dissected the rabbit, and goes on to other types, he will find the making of comparisons a most valuable means of testing the reality of his knowledge and showing him its weak places. In the 
book, the various systems of any type are necessarily described in comparison with the same in types previously described. The student should, in the case of the Vertebrate types, reverse this, and first describe the lower of two types, and then contrast the higher one with it. The questions set at the end of the chapters (most of this nature being taken from London University Examinations) will give the student a good opportunity for testing himself. In all cases he should illustrate his answers with diagrams drawn from memory -if only for the sake of practice in drawing. As a guide we append two examples of comparisons.

1. Compare the vertebræ of dogfish, rabbit, and frog.

The vertebræ of the dogfish are of cartilage; those of the frog, of cartilage-bone; those of the rabbit also of cartilage-bone, with epiphyses to the centra.

The centra of the dogfish are amphicolous (i.e. hollow at either end); the centra of the rabbit are flat-faced, and separated from one another by fibro-cartilaginous intervertebral bodies. The centra of the frog are mostly procœlous (hollow in front).

The vestiges of the notochord persist between the centra in the dogfish and rabbit, within the centra in the frog.

The transverse processes of the rabbit typically bear ribs. Short ribs occur in the dogfish, but their homology with those of the rabbit is doubtful. The frog has no ribs.

The intervertebral neural plates are peculiar to the dogfish in this comparison.

The dogfish has no zygapophyses, such as are found in frog and rabbit. There are trunk-and tail-vertebræ only in the dogfish; trunk-and sacral vertebræ only in the frog; cervical, thoracic, lumbar, sacral, and caudal in the rabbit.

2. Compare the skull of the dog with that of the frog. The Brain Case-

Of the frog is a cylindrical box, from which the otic capsules project conspicuously on either side. It contains only three ossifications in its cartilaginous substance (the median sphen-ethmoid and the paired ex-occipital), 
being protected by the membrane bones, the parietofrontals above and the parasphenoid below.

In the dog it is enormously inflated, and the otic capsules are imbedded in its wall. There are supra- and basi- as well as ex-occipital bones; the parasphenoid is absent, and its place is taken by the basi-and pre-sphenoids, and the lateral walls contain fresh paired ossifications, the ali-and orbito-sphenoids - all cartilage-bones. Thesphenethmoid is perhaps represented in part by the ethmoid. As a result of the inflation of the brain-case, the squamosal, which slopes out from above downward in the frog, and overlies the cartilaginous suspensorium (quadrate cartilage), has become a constituent of the brain-case wall, and slopes in from above downward. Jaw Suspension.

The point of attachment of the jaw has shifted outward, and the original suspensorial cartilage (the quadrate) has, in the dog, taken on a new function as the incus of the middle ear-the squamosal superseding it as the suspensory part.

Lower Jaw.

Meckel's cartilage, with one cartilage-bone (mentoMeckelian) and two membrane-bones (dentary and angulo-splenial, on either side, in the frog. A single membrane-bone on either side in the dog.

Otic Capsule.

Position as specified. One centre of ossification in the frog, forming pro-otic ; several fuse together and form periotic of the dog.

There is no bulla in the frog.

Palate.

In the frog the posterior nares open into the front of the mouth. In the dog the maxillæ and palatines send plates down and in (the palatine plates) to cut off a nasal passage from the rest of the buccal chamber, and carry the posterior nares back to the pharynx, thus cutting the vomer off from the roof of the mouth.

'The pterygoids in the dog are much reduced, and do not reach back to the suspensorium.

The frog has no lachrymal nor jugal. 


\section{Practical Work.}

Two excellent works cover the practical work requiredviz. Marshall's The Frog (David Nutt, 4s.), and Marshall \& Hurst's Practical Zoology (Smith, Elder \& Co., 10s. 6d.). Useful instructions are also given in Parker \& Parker's Practical Zoology (Macmillan, 10s. 6d.), and the figures in Howes' Atlas of Biology (Macmillan, 14s.) are of great help to a student working alone. The latter does not, however, include rabbit, dogfish, or amphioxus among its types.

The student who cannot attend a practical class regularly is strongly advised to get some practical work done as early as possible under the guidance of a teacher: he will then be in a far better position to continue by himself.

We confine ourselves here to such general advice as is needed by a student working alone. The following instruments are indispensable for practical work :-

(a) Two or three scalpels of various sizes.

(b) Scissors, which must taper gradually, have straight blades, and be pointed at the ends, and which must bite right up to the tips (or they are uselcss). 'Two pairs, small and large, are advisable.

(c) 'Two pairs of forceps, large and fine. Both should have the inner surface of the tips roughened, and the points should meet accurately. The fine ones may preferably be curved at the tips.

(d) Two needles set in wooden handles.

(e) A seeker, i.e. a blunt piece of wire, slightly bent near the tip, and preferably mounted in a wooden handle.

$(f)$ Some sort of lens (magnifying glass). A watchmaker's glass is very good, but the student is not advised to attempt learning how to keep it in his eye by contracting the muscles of the orbit,- he may think he has learnt the trick, but it will only be to find that at the critical point of a delicate dissection, his attention being concentrated on his work, the glass will fall from his eye and spoil his work. An elastic band made to fit the head will hold the glass much more conveniently.

(g) Pins of at least three sizes: ordinary ones, about an inch long, for such types as the frog and crayfish; "baby" pins, half an inch long and very fine, for earthworm and Amphioxus; and stout laundry pins or nails, one and a half inch long, for rabbit and dogfish (blanket pins are too long and too easily bent: you want something you can hammer on).

(h) A board (an old drawing-board, or a packing-box lid) on to which to pin the rabbit and dogfish. Failing this an old table will do.

(i) A dissecting dish with either paraffin or cork to pin smaller types on. A pie-dish will do very well, but something with vertical sides is better. If you use paraffin it must be melted in the dish, 
lamp-black mixed with it till it is quite black, and while it is cooling small shot or other small pieces of metal dropped into it. You thus get a smooth black surface as a background for dissection, and one which will not float up when water is poured in. The thickness of paraffin should be enough to allow plenty of room for pins to be stuck into it, while leaving enough depth in the dish for the animal to be entirely covered with water. The surface of the paraffin will soon be damaged by the pins, but can be restored by passing a Bunsen-flame over it a few times.

If cork be used, a flat piece is needed, not less than $6 \mathrm{in.} \times 4$ in., and $\frac{1}{8}$ in. thick. This must be firmly attached to a flat piece of lead of the same size.

(j) A pickle- or other wide-mouthed jar to keep specimens in.

(k) A microscope, with low- and high-power objectives (Leitz's nos. 3 and 7 ).

(l) Glass slips ( 3 in. $\times 1$ in.) and medium cover-glasses ( $\frac{7}{8}$ in. diameter or square).

$(m)$ Bristles are useful as very fine seekers. They may very conveniently be cut off the rabbit's " whiskers."

\section{The Animal Types.}

Rabbits can be got anywhere: the tame varieties will do quite as well as the wild. They are most conveniently killed by chloroform : take a small box, just large enough to hold the rabbit, pour a spoonful of chloroform on a rag and spread this out in the box so that it may evaporate quickly, keeping the lid down; after a minute or two, place the rabbit in the box, put a weight on the lid, and wait two or three minutes.

Frogs may be found in spring and summer in abundance in marshy places (brickfields, for example), or town-dwellers can get them by post from Mr. T. Bolton, 25, Balsall Heath Road, Birmingham, or from dealers in other large towns who generally advertize in such periodicals as Nature, Knowledge, Natural Science, Science Gossip, etc. Failing frogs, toads will do quite well, except as regards the shouldergirdle (see chap. xiii., § 21). Frogs may be killed in the same way as rabbits, using a correspondingly smaller box and quantity of chloroform.

The Marine Biological Laboratory, Citadel Hill, Piymouth, supply preserved Dogfish, Obelia, Nereis, and other marine types; " a list can be obtained from the Director." 
Amoeba, Paramecium and IIydra may be obtained by post from Mr. Bolton. The two latter can easily be studied under the microscope (Hydra with the naked eye also), but we do not advise any beginner to attempt to find amœba for himself-the mud in which it lives is always full of an abundance of other strange creatures, much more conspicuous than the one he is in search of.

Earthworms can be dug up in any garden or field. They are killed by immersion in methylated spirit. Mussels occur in most English rivers, and crayfish in many: these types can also be had through the post from dealers. They are killed by immersion for a minute or so in water as hot as the hand can just bear.

For preserving animals that cannot be completely dissected on one day, common methylated spirit answers very well. It both preserves and hardens, but has no great penetrating power, so that if large animals are placed in it care should be taken to open them up as nuch as possible to let the spirit have access to all parts. Formalin has lately come into very general use as a preservative; it has several advantages over spirit, particularly its penetrating power, but its vapour is very irritating to the nose and eyes. Formalin is really a standard solution of formic aldehyde in water. For preserving animals or parts of them, it may be very considerably diluted (4 to 6 parts of formalin to 100 of water). In its full strength it acts as an excellent hardening re-agent for animals of which sections are to be cut-e.g. the mussel (removed from shell), brain and spinal cord of rabbit or dogfish, rabbit-embryos and the earthworm.

\section{Suggested Order of Practical Work.}

By the time the student has read the first three chapters, lo may begin dissecting the rabbit; but if he is quite without both help and experience, he would do better to wait until he begins chapter vii. (The Skeleton), when he can take a dead rabbit (as sold for food) and boil it whole until he finds that the muscles (flesh) can be removed quite easily from the bones. $\mathrm{He}$ can then proceed to clean the skeleton completely, taking care to keep all the bones in 
their proper relative positions. The comparison of bones with the descriptions in his practical text-book will be an excellent training in biological observation, free from the uupleasantness that accompanies the first unskilled dissection of a fresh rabbit. But he must see that every point described is clearly verified on the specimen; the habit of shirking difficulties is easy to acquire and difficult to get rid of. Drawings should be made. of the bones, if only for practice.

If a dog's head can be got (preferably that of a young animal) it can be treated in the same way, the brain being cleaned out by alternately wriggling a bent wire through it and washing out the débris by a strong stream from a tap. Failing a dog's, a cat's skull will do very well. The rabbit's has a very narrow palate as its chief peculiarity: it should be kept for guidance in dissecting the head.

All bones should be thoroughly dried after being cleaned. If they smell unpleasantly, soaking them in water to which a little chloride of lime has been added will cure this, but too strong a solution will rot the bones. The best preventative of smell is thorough cleaning. Soap and a nailbrush are very useful in the final stages.

Having mastered the skeleton, the student will be in a better position to carry on ordinary dissection. If, however, he finds this very unpleasant to him, our advice would be to leave the rabbit, and start on the frog, returning to the rabbit when he has gained experience in dissection on the smaller animal.

A good deal of bleeding is sure to occur in the earlier dissections of frog and rabbit by a beginner. The application of ferric chloride solution to the cut vessel will make the blood clot rapidly and stop further bleeding.

For detailed instructions in dissection we must again refer to the books already recommended. For the study of the rabbit's brain, which is especially difficult, we offer the following suggestions: Take a fresh rabbit (cut off its head if you like), and skin the dorsal side of head and neck; remove also the muscles running from the occipital region to the neck as far as you can. During the rest of the operation keep a dried rabbit's skull by you for comparison 
before trying to remove any part of the bone. Then, using a bone-forceps if you have one, or, if not, a strong knife, start cutting through the bone at the conspicuous raised pentagonal area where the inter-parietal and supra-occipital meet: the bone is spongy here, and the corebellum lies underneath. Starting from here, go on to expose the cerebral hemispheres in front and the bulb and spinal cord behind. In the rest of the dissection do not try to remove the brain from the head, but cut away the rest of the head from the brain. Cut away nose and jaws, remove the eyes. and gradually break away the bone all round the brain and beginning of the spinal cord, leaving bits of bone attached at any point where you do not feel safe in removing them. Then place in pure formalin, in a wide-mouthed bottle, with a lump of cotton-wool at the bottom for the brain to rest on. After a few hours the brain will be harder: take it out and wash it (have plenty of water at hand to keep the formalin off your fingers), and remove as much more of the bone as you can. Also remove very carefully the tough membrane that surrounds it-don't tear it, but cut it away' in pieces with scissors. Put the brain back in formalin for a few days. When well hardened keep in methylated spirit. It will be in a very good state either for dissection or for cutting sections.

The frog and dogfish are so well described in Marshall's books that we need say little here about their dissection. 'The student should not fail to cut a series of transverse sections of the dogfish. Before such can be made of the frog it must be soaked in chromic acid to soften the bones. The frog's skeleton may be prepared in the same way as the rabbit's, but great care must be taken not to lose the small bones. The dogfish's may be prepared similarly, but must not be boiled too much, as it consists of cartilage, not bone.

The dissection which usually gives most trouble in the dogfish is that of the afferent branchial arteries. A very neat dissection of them can be made by the following method. Remove the skin from the ventral region between the pectoral girdle and the mouth. Slice away the median ventral part of the pectoral girdle and adjoining 
muscles so as to fully expose the pericardial cavity. Dissect away the superficial layer of muscles from the region between the pericardial cavity and the lower jaws, so as to expose a pair of longitudinal muscles running forward side by side from the pericardial region to the basi-hyal cartilage. On slightly separating these the thyroid gland can be seen in the middle line: it is a useful land-rnark, as it lies just ventral to the anterior T-shaped end of the truncus arteriosus (fig 92, p. 177). Before going further, it is necessary to stretch the floor of the pharynx by pushing something down the throat: a bone-forceps does very well, or a carrot may be cut to a suitable shape and pushed in at the mouth. You next proceed to carefully dissect away the two longitudinal muscle-bands above mentioned, taking great care not to cut the arteries that lie just beneath them. These arteries (first and second afferent branchial) are thus exposed, and the first can be traced very easily along the hyoid arch, its branches to the gill-filaments of the first demibranch being well shown. The truncus arteriosus should be traced from its anterior end back to the pericardium, not vice versa. When traced half-way it may be left until the other afferent branchials have been found, as they can easily be by dissecting just anterior to the inner ventral corner of earh of the three posterior gill-pouches. Each artery at this point lies within a small cavity (probably homologous with the branchial cœlomic canals of Amphioxus) and so is less likely to be cut than if searched for at its origin from the truncus. When once the level at which the truncus and afferent arteries lie is clearly realized, all muscles lying ventral to that level may at once be sliced away. The rest of the dissection consists in tracing out and cleaning the various arteries. The ventral portions of the gills may be cut away.

Another dissection that should not be omitted is that of the internal ear or membranous labyrinth of the dogfish. The surface of the auditory capsule should first be scraped thoroughly clean, and then the cartilage cut away piecemeal so as to leave uninjured the semicircular canals, etc., which are embedded in it.

The odour of the dogfish is unpleasant and clinging. 
The most satisfactory way to get rid of it is to bathe the hands in a solution of permanganate ("Condy's fluid"), diluted to a light pink colour, and afterwards with soap and water.

Formalin-preserved dogfish are much less objectionable than others.

Amphioxus affords good practice for those who enjoy fine dissection, but is distinctly difficult for the ordinary student. We advise the following as the best procedure: Use as shallow a dissecting-dish as possible, and work in a good light. Rest your wrists on some solid support while dissecting (this advice applies to all fine work). Holding the lancelet in one hand insert the point of a fine pair of scissors into the atrial pore, and cut forward along the middle line of the floor of the atrium, taking great care not to push the point of the scissors farther into the cavity than is absolutely necessary, or you may cut open the pharynx also. Stop cutting just short of the mouth-region. 'Then lay hold of both cut edges simultaneously by means of two pairs of forceps, and gently draw them apart, until you can clearly see both pharynx and liver, and that they are both separated from the atrial wall. If this is not done the liver at least is sure to adhere to the atrial wall, and will be badly damaged in the subsequent dissection. You can now pin the animal down on its left side (dissect from the right side in order to display the liver). Three pins (the smallest size obtainable) will be enough to transfix it: two through the notochord (whose position is marked on the surface by the V-shaped bend in the myomeres) and the other through the left atrial wall. The right atrial wall, with the right gonads, may now be cut away with scissors, and then the rest of the muscles of the body gradually dissected away. It is advisable, while this is being done to turn the dissection round so that the dorsal side is towards you; you are then less likely to injure the pharynx. In this way all the essential structures can be exposed in a single side dissection. If the dissection is properly done, the sphincter oris muscle in the velum will be plainly visible just behind the præoral cirri and their supporting hoop: the two may be removed together. 'The sphincter with its tentacles if the 
mouth is wide open looks not unlike a wheel, the tentacles being the spokes; but if, as is often the case, the sphincter contracted at death, it will lie on its side, appearing as a cone with the tentacles at the apex. In removing the dorsal portions of the myotomes, the yellow notochord will be exposed. Dorsal to this lies the spinal cord, which needs some scraping to be fully exposed : it shows as a very thin white line; with a lens the row of pigment-spots is plainly seen. The dorsal fin-rays are also very noticeable. 'The dissection of the spinal corr may be made much easier if the whole animal is soaked for a day or two in 20 per cent. nitric acid and washed. It is possible then to completely isolate the spinal cord and mount it - the dorsal and ventral nerves and pigmentspots are well shown. To see the skeleton of the pharynx a piece should be cut out, laid open on a glass slide and mounted in caustic potash solution, with a cover-glass.

Young specimens of Amphioxus can be had mountel whole for microscopic examination. These differ from the adults in that the gill-slits are few and simple, and the gonads undeveloped.

In the earthworm the finding of the ovary gives much trouble to beginners. The following method is recommended. Cut across the beginning of the intestine; grip the gizzard with forceps (it is the only part of the alimentary canal that will stand handling), lift it up and cut the septa beneath it. Work forwards in this way, carefully cutting each septum in turn with a sharp scalpel on both sides of the middle line. Cut them also between the oesophagus and the seminal vesicles, or you are likely to tear away the latter. In this way you can remove a considerable part of the alimentary canal. It is also well to pin out the posterior pair of seminal vesicles, or they may lap back and hide the ovaries. The ovaries should now be visible to the naked eye in Segment 13, a little on either side of the nerve-cord.

For the remaining types, we do not think any special advice is needed. We may, however, mention some of the most conspicuous and common internal parasites, which are likely to puzzle the solitary worker. 'The rabbit frequently has clear spherical bodies, 
$\frac{1}{8}$ ill. or so in diameter, in its liver and mesenteries : these are encysted tapeworms ('Tænia), unable to continue their development until introduced into the stomach of a carnivorous animal. The frog may have its lungs full of black and white worms (Ascaris), and one or two six-suckered worms (Polystomum) in its urinary bladder. I.ong white threadworms infest all parts of the dogfish. A l'aramecium-like Protozoon is generally found running over the surface of Hydra. The earthworm, thanks to its mode of life, is specially given to unintended hospitality: its nephridia are never free from minute wriggling threadworms, and the fluid in its seminal vesicles contains spore-forming Protozoa, mistaken by the unwary for spermnspores: other stages of the same Protozoon may be seen attached to the funnels of the vasa deferentia. The gills of Anodonta harbour a little spiller-like creature which the uninitiated mistake for a glochidium. On the hind-gut of Astacus, egg-shaped orange bodies may be found (encysted Echinorhynchus).

For the re-agents used in staining and mounting animal tissues we must refer the student to the works before mentioned. For the rough examination of fresh tissues, normal salt solution ( $\frac{3}{4}$ per cent., or $7 \cdot 5$ grammes common salt to a litre of water) is used. If the object appears too opaque in this fluid, it can be mounted in weak glycerine (not less than half water). In all cases a cover-glass should be carefully placed over the specimen, and none of the liquid should be allowed to get on the upper surface of the cover-glass : if any does, don't try to wipe it off-remove the cover-glass and put another on.

We cannot here give instructions in the way of using a microscope: printed instructions, we know from experience, are of practically no value. More can be learned in one hour's lesson from a practical teacher than from many pages of a book. Failing such direct instruction, we must again refer the student to the practical books we have recommended.

Ready prepared histological sections, as well as general sections of such animals as Amphioxus, Lumbricus, and Hydra, can be obtained from the dealers already mentioned and other dealers in microscope-slides.

\section{EmbRYology.}

The student should look out for frog-spawn on ponds in March, and keep some in a glass jar with a little pond-weed 
to supply oxygen. The whole development from ovum to late tadpole-stage can be watched, and a much better notion of the process obtained than from any written account-so far, that is, as external form goes. A tadpole affords good practice in fine dissection.

If the student can gain access to an artificial incubator, he would do well to study the early development of the chick by taking eggs at the end of one, two, three, and four days of incubation (and as many other stages as he pleases), and carefully opening them in a dish of warm water. The egg should be laid on its side, and the part of the shell that lies uppermost-carefully removed.

The making of sections of tadpole or chick is beyond the means of the solitary worker, but if he can get prepared sections from a dealer he should examine them carefully.

For the rabbit, pregnant females can be easily obtained and dissected. The placenta and other extra-embryonic structures can be examined; and if the embryos are welladvanced, they may be hardened in formalin, and sections cut with a sharp scalpel. Fig. 2 was drawn from embryos treated in this way.

\section{Furtier Study.}

Finally there may be among the readers of this volume many who will be anxious to extend their knowledge of Zoology in general, or of the particular types we have taken. For further practical work the London University syllabus for Honours in Intermediate Science, and that for the Pass B.Sc. examination, afford a good selection of types to study. These can be obtained from the dealers already mentioned, who issue price lists.

For additional reading, Parker's Elementary Biology (Macmillan) is an admirable work, covering both animal and vegetable kingdoms. Parker and Haswell's Text-Book of Zoology (Macmillan, 2 vols.) is unquestionably the best and most complete English work on Zoology in general. For those who desire a smaller work of the same kind, no better one can be recommended than 'Thomson's Outlines of Zoology (Pentland). 
For more information on our Vertebrate types the reader may refer to the general works already mentioned or cited below, and to Marshall's Vertebrate Embryology-a most. admirable book. Wilson's The Cell in Development and Interitance (Macmillan) will give him the fullest account of modern views on the ultimate structure of the cell, and the physical basis of heredity; and Halliburton's Kirke's Physiology (Murray), the latest information on Animal Physiology. For Amphioxus he should refer to Willey's Amphioxus and the Ancestry of Vertebrates (Macmillan). For more general accounts of the Vertebrates Wiedersheim and Parker's Comparative Anatomy of Vertebrates (Macmillan) will be useful.

The volumes of the Cambridge Natural History, and those of the Treatise on Zoology edited by Professor Ray Lankester, supply the most recent and most detailed information on the several divisions of the Animal Kingdom. The former are rather more popular and less severely technical, the latter contain the latest suggestions and conclusions in theoretical morphology. Professor Lankester's own essay on recent modifications of theoretical views concerning the cœlum, nephridia and cœlomic ducts is specially interesting and important. The volumes of the Treatise on Zoology which have hitherto appeared include Minchin's account of Sporozoa, Bather's of Echmodermata, Pelseneer's of Mollusca, Fowler's of Hydrozoa, and others.

In relation to the earthworm, Darwin's Vegetable Mould and Earthworms may be mentioned, and Huxley's volume on the crayfish in the International Scientific Series should be read, not merely for the sake of its precise scientific description, but because it exhibits so clearly the importance of zoological study to general culture.

Many of the books mentioned ought to be found on the shelves of any good public library. 


\section{INDEX.}

A

BDOMEN of crayfish, 3!2

Abdominal pores, 173

Absorption, 18

Acarid, 413

Accelerator nerves, 91

Acetabulum, 75, 149

Achromatin, 43

Aciculum, 350

Acoela, 446

Acrania, 209

Acromion, 73

Adlaptation, 127, 232

Adipose tissue, 58

Adradial, 392

Adrenals, 33, 15 \&, 190

Afferent nerve-fibres, 90

Albumelı, 13, 228, 217

Alcyonaria, 435

Alecithal, 210

Alimentary canal, 5, 12, 139, 173, $197,225,269,309,329,338$

Ali-sphenoid, 80, 166

Allantois, 134, 259, 265

Alteruation of generatious, 363

Alveoli, 36, 268

Amnion, 134, 258, 26 t

Amniota, 451

Amoba, 38, 399

Amoboid motion, 8!

Amphibia, 158, 41 ?

Amphicelons, $14 i$

Amphioxus, 192

Ampullae, 113

Amylopsin, 17

Anabolism, 10, 45

Analogy, 11

Anastases, 43

Anastomosis, Jugular, 127

Anatomy, 11
Ancestry of animals, 281, 323

Animal and plant, 10, 40, 381

Animal pole, $22 \mathrm{l}$

Anuelida, 439

Auodouta, 32!

Anopheles, 423

Autennæ, 304

Antennule, 304

Anthers, 291

Anthomedusae, $43 t$

Anthozoa, 435

Auura, 158, 232, 450

Anus, 12, 192, 226, 328, 336

Aorta of mussel, 330

„, "vertebrates, 30, 144, 177, 200

Appendages, 303

Appendicular skeleton, 66

Aptera, 415

Arachnida, 442

Arch, Aortic, 28, 133

(See also Arches, Vascular.)

„, , Branchial (see Arches).

,, , Hyoid (see Arches).

,", Neural, 66

Archenteron, 224, 386

Arches, Cartilaginous visceral, 162, $175,181,236,275$

Archiaunelida, 439

Archinephric duct, 238, 278

Area opaca, pellucida, 219

Areolar tissue, 5l

Arteries and veins, 21, 80

$$
\begin{aligned}
& \text { " " , of crayfish, } 31 \text {. } \\
& \text { " ", , , , dogfish, } 176 \\
& \text { " " , ", frog, 144 } \\
& \text { " " , ", mussel, } 330 \\
& \text { "geo ", ", rabbit, } 29
\end{aligned}
$$


Arthrobranchs, 314

Arthropoda, 322, 440

Arthrostraca, 441

Articulation, 306

Aspidogastera, 437

Assimilation, 40

Association of ideas $9 t$

Astacus, 301

Aster, 44

Astragalus, 77, 150

Atlas, 69, 147

Atriopore, 193

Atrium, 194, 204, 227, 233

Auditory capsules, 159

,, organ, 113, 154, 187, 275, 320,333

" ossicles, 82, 113, 154, 165, 275

Auricles, 27, 142, 175

Autostylic skull, 181

Aves, 452

Axial skeleton, 66

Axis, 69

,, -cylinders, 89

DACTERIA, 17

B Basi-branchial, 181

$$
\begin{aligned}
& \text {, -liyal, 181 } \\
& \text { " -occipital, } 80 \\
& \text { ", podite, 304 } \\
& \text { ", -pterygium, } 183 \\
& \text { "-sphenoid, 80, } 166
\end{aligned}
$$

Bile, $1 \dddot{6},-61$

,, -duct, $61,86,140,174$

Bladder, Urinary, 63, 141, 269

Bladder-worm, 372

Blastoderm, 249

Blastodermic vesicle, 263

Blastogenic, 287

Blastomeres, 371

Blastopore, 223, 231, 252, 263, 315

Blastosphere, 222, 229

Blastostyle, 390, 393, 394

Blind spot, 117, 277

Blood, 57, 225

, of amphioxus, 200

", , crayfish, 314

" ", dogfish, 179

", ,earthworm, 310

„, frog, 157

", rabbit, 20, 57

Blood-vessels of earthworm, 340
Blood-vessels of mussel, 330 (See also Arteries, Capillaries, Veins.)

Blood-vessels, Origin of, 270

", , Structure of, 87

Bojanus, Organ of, 331

Bone, 53, 157, 273

Botallus, Duct of 142,246

Bowman's capsule, 64, 244

Brachyura, 441

Brain, 100, 150, 184

Branchial heart, 331

Branchiocardiac groove, 302

Branchiostegite, 302

Breatling (see Respiration).

Bronchi aud bronchioles, 36, 268

Budding, 383

Bulb, 103, 120, 152, 185

Bulbus asteriosus, 143, 176

Bulla, 80

Byssus, 334

CAECUM, 16, 19

$\cup$ Calcaneum, 77, 150

Calcar, 150

Calciferous glands, 339

Canaliculi, 54

Canines, 14, 80, 81

Capillaries, 18, 21, 31, 63

Capitulum, 66

Carapace, 302

Carboliydrates, 13

Cardiac, 310

Carotid gland, 142

Carpopodite, 304

Carpus, 74, 149

Cartilage, 21, 52, 179, 273

Castration, 290

-bone, 54, 83, 161

Caudal vertebræ, 71

Cell, 42

" -anus, 401

i, -mouth, 401

," -products, 42, 45

, wall, 40

C'ellulose, 13, 19, 40

Cement, 57

Central nervous system, 89, 96

Centrolecithal, 321

Centrosome, 43

Centrosphere, 43

Centrum, $66,179, .73$ 
Cephalochorda, 209, 447

Cephalothorax, 302, 443

Cerato-branchial, 181

Cerato-liyal, 82, 181

Cercaria, 360, 362

Cerebellum, 103, 119, 152, 185

Cerebral hemisplieres, 107, 119, 133, 151,185 vesicles, 101, 13 Ł, 235

Cervical grooves, 302

Cestoda, 437 vertebrae, 68

Chaetae, 351

Chaetigerous sac, 350

Chaetopoda, 439

Chalazæ, 218

Change of function, 207

Cheek-teeth, 14

Chela, 306

Chelicerae, 442

Chelonia, 451

Chick, development of, 247

Chitin, 201, 338

Chitinous hooks, 367

Chloragogenous cells, 431

Chlorophyll, 40, 381

Chloroplastids, 381

Chloroplasts, 408

Chondrin, 52

Chondrocranium, 159

Chordata, 208, 416

Chorion, 265

Choroid, 115

" fissure, 276

Chromatin, 43, 409

Cliromatophores, 381

Chromosomes, 41, 409

Chyle, 18, 27, 146

Chyme, 16

Cilia, 49, 400

Ciliata, 432

Circulation of Amphioxns, 200

$$
\begin{aligned}
& \text { ", crayfish, } 343 \\
& \text { " dogfish, } 175 \\
& \text { ", earthworm, } 310 \\
& \text { " frog, } 142 \\
& \text { " mussel, } 330 \\
& \text { ", rabbit, } 20 \\
& \text { ", vertebrate embryo, } 269
\end{aligned}
$$

Cirri, 193

Claspers, 183, 189

Class, 132

Claviclo, $73,132,148$
Clitellum, 336, 3 t5

Cloaca, 141, 155, 174, 190, 267, 328

Cnidoblasts, 378

Cuidocil, 379

Coccidium, 417

Cochlea, 114, 133

Cocoon, 345

Colenterata, 386, 433

Coclom, 5, 6, 134, 139, 171, 196, 204, $225,241,252,326,337,346,350$, 364,386

Coelomata, 3s6, 434

Coelomic ducts, 317

Coenurus, 439

Coleoptera, 445

Colon, 16

Colonial animals, 386

Columella, 154, 163

Commissures, 104, 342, 352

Condyles, 80

Cones, 116

Conjugation, 402, 420.

Conjunctiva, 116

Connective tissues, 51,225

Consciousness, 94, 318, 333

Contractile vacuole, 39

Contraction, 81, 376

Conus arteriosus, 143, 176

Coracoid, 73, 148

Cornea, 115

Corneal facets, 318

Cornua of hyoid, 82, 163, 181

Corpora bigemina, 152

", quadrigemina, 103, 133

Corpus adiposum, 156

" callosum, 101, 133

Corpuscles, 51

, , Blood-, 20, 157, 179

, , Bone-, 54

", Cartilage-, 52 , , Pacinian, 111

Cortex, 400

Cotyloid, 75 of kidney, 61

Coxopodite, 304

Cranial nerves of dogfish, 186

, , , frog, 152

Craniata,"447 "rabbit, 104

Cranio-facial apparatus, 80

Cranium, 77

Crescents, 424

Crocodilia, 451 
Crop, 339

Crura cerebri, 103, 104

Crustacea, 322, 441

Crystalline Cone, 318

Ctenidia, 326

Cuticle, 400, 417, 428, 410

Cyclostomata, 448

Cysticercus, 372, 438

D) ACTYLOPODITE, 304

Decapoda, 322, 4.41

Deciduate, 206

Decussation, 103

Degeneration, 335

Demibranchs, $\mathbf{1 7 5}$

Dendrons, 89

Dental formula, 81, 132

Dentine, 57, 170

Dentition (see Teeth), 14

Dermal denticles, 170

Dermis, 50, 225

Determinants, 295

Development, 11

Diaphragm, 6, 36, 133

Diaster, 44

Diastole, 29, 312

Diatoms, 39

Differentiation, 44, 45, 286, 382

Digenea, 437

Digestion, 12, 310, 331

Dipnoi, 449

Diptera, 445

Direction, Sense of, 115

Dissection, 52

Distomidae, 355, 3557

Distribution of animals, 11, 42, 331

Dominants, 2ُ18 nerves, 105

Dorsal pores, 338

Drepanidium ranarum, 427

Duct, 60

, of Botallus, 142

"Ductless glands," 32, 154, 190

Ductus arteriosus, 28, 272 venosus, 272

Drodenum, 16, 140, 174

Dyads, 215

TAR (see Auditory organ).

If, , External, 114, 130
Earthworm, $340^{3}$

Ecdysis, 309

Echinococcus, 374

Echiuroidea, 439

Ectoderm, 377

Ectoplasm, 38

Ectoplastic, 46

Efferent nerve fibres, 90

Egg-case, 188

Elasmobranchii, 191, 448

Elastic ligament, 325

Embryo, 213, 257

Emulsification, 17

Enamel, 56, 170

," -organs, 267

Encystment, 41

Endoderm, 377

, lamella, 392

Endolymph-duct, 114, 154, 187, 275

Findomysium, 81

Endopliragmal system, 308

Endoplasm, 38

Endoplastic, 45, 46

Endopodite, 304

Endoskeleton, 382

Endostyle, 198

Endothelium, 21, 48

Energy, 8

Enteron, 381, 386

Entomostraca, 441

Epiblast, 223, 231, 249, 315

Epibranchial, 181

Epidermis, 50, 134, 157, 217

Epididymis, 124, 189, 280

Epiglottis, 15

Epi-hyal, 82

Epimeron, 303

Epiphyses, 55, 67, 133, 273

Epipodite, 304

Episternum, 148

Epithelinm, 47, 48, 49, 59, 111, 225, 382

Epitokous, 351

Equilibration, 115, 119, 152, 186

Ethmoid, 80

Euglena, 405

Einstachian tube, 115

Entheria, 454

Fvolution, 296

Excretions, 8, 17, 46

Excretory organs (see Kidney. Ne. phridium, Renal).

Exhalent, 324 
INDEX.

Ex-occipital, 20, 161, 167

Exopodite, 304

Exoskeleton, 302, 371

Extensor surface, 72

Extra-branchial, 182

Extra-embryonic structures, 251, 261

Eye, 115, 134, 154, 187, 318

", , Pineal, 152

," -spots, 203

F AECES, 9, 19

1 Fallopian tube, 123, 262

Family, 132

Fasciculi, 85

Fasciola hepatica, 355

Fat-body, 156

Fats, 13, 58

Fatty acids, 18

Femur, 76, 150

Fenestra obturator, 75

$$
\text { , ovalis, } 115
$$

Ferments, 13$$
\text { rotunda, } 115
$$

Fertilization, 213, 228, 247, 262, $321,334,343,381,403$

Fibres, Connective-tissue-, 51

$$
\text { ", , Muscle-, 84, } 85
$$

Fibro-cartilage, 52

Fibula, 76, 150

Fin-girdles (see Limb-girdles)

,2 -rays, 180, 183, 201

Fins, 134, 169, 182, 192

Fissorium, 429

Fissures of spinal cord, 96, 274

Flagellata, 431

Flagellum, 213, 381

Flat-fishes, 289

Flexor surface, 72

Flocculi, 103

Flounder, 289

Follicle, 212, 228, 261

Food of amoeba, 38

$$
\begin{aligned}
& \text {, Amphioxus, } 194 \\
& \text { ", crayfish, } 301 \\
& \text {, dogfish, 169 } \\
& \text { ", earthworn, } 337 \\
& \text { ", frog, 139 } \\
& \text { ", hydra, 377 } \\
& \text { ", mussel, 321 } \\
& \text { "paramecium, } 401 \\
& \text { ", rabbit, 9, 12 }
\end{aligned}
$$$$
\text { ", ", tadpole, } 245
$$

Foot, 326

Foramen, Condylar, 110

", Lacerum anterius, 106,

107

, , Magnum, 77

posterius, 108

Foraminifera, 430

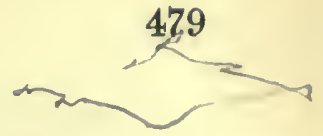

Fornix, 104

Fowl, Ovum of, 247

Frontals, 80

Functions, 11

Function, Change of, 207

VALL-BLADDER, 17, 61, 140, U 174

Gametes, 214, 284, 295, 413

Gametocytes, 285, 413, 424

Ganglia, 97

$$
\begin{aligned}
& \text {, Abdominal, } 317 \\
& \text {, Cerebral, 316, 332, } 312 \\
& \text {, Cervical, } 99 \\
& \text {, Coeliac, } 99 \\
& \text {, Gasserian, } 107 \\
& \text {, Mesenteric, } 99 \\
& \text {, Optic, 319 } \\
& \text {, Origin of, } 274 \\
& \text {, Osphradial, } 333 \\
& \text {, Parieto-splanchnic, } 333 \\
& \text {, Pedal, 333 } \\
& \text {, Spinal, } 97 \\
& \text {, Sub-œesophageal, } 317 \\
& \text {, Supra-œsophageal, 33, } 314 \\
& \text {, Sympathetic, 98 } \\
& \text { Thoracic, } 317
\end{aligned}
$$

Gastric juice, 16

Gastroliths, 311

Gastrula, 222, 229, 250, 315, 385

Gelatin, 13, 51

Gemmarium, 387

Genus, 132

Gephyrea, 439

Germ cells, 214

Germinal area, 247

$\begin{array}{ll}\text { " } & \text { epithelium, 261 } \\ \text { " } & \text { layers, 224, 233 } \\ \text { " } & \text { ridge, 245, 278 } \\ \text { " } & \text { vesicle, 212, } 247\end{array}$


Germ-plasm, 384

Giant-fibres, 342

Gill-rays, 182 ",-slits, 134, 170, 174, 192, 208, $226,240,268$

Gills, 134, 157, 174, 240, 314, 326

Gizzard of crayfish, 209

Gland-cells, 380 earthworm, 339

Glands, 13, 59

, , Cowper's, 123, 125

,, , Digestive, 13, 225

, , Epidermal, 157

", , Green, 316

", Harderian, 119

,, , Infra-orbital, 14

, , Lachrymal, 119

,, , Mammary, 125, 133

,, , Parotid, 14

,, , Perineal, 125

", Prostate, 125

,, , Rectal, 125, 174

, , Salivary, 61

,, , Sebaceous, 50, 125

, , Sublingual, 14

Glochidium, 334 Submaxillary, 14

Glomerulus, 64, 146, 214

Glomus, 200, 204, 239

Glottis, 15, 35, 139

Glycerine, 17

Glycogen, 24, 212

Gnathostomata, 448

Gonads, 121, 155, 188, 193, 206, 225, $245,280,320,335,343,383,395$

Gonoduct, 121

Gonotheca, 390

Green gland, 316

Grey matter, 96, 103

Gymnomyxa, 429

Gymnophiona, 450

\section{HABITS, 94}

H Hæmal arch, 180

Haematochrome, 407

Hæmocyanin, 314

Hæmoglobin, 20, 340

Haemoproteus, 427

Haemosporidia, 423

IIair, 5, 133

Hare, 130

Hatteria, 451, 452
Haversian canals, 53

Head-fold, 258

Hearing, 113

Heart, 6, 27, 134, 142, 175, 212, $237,269,330$

Hemiptera, 445

Hepatic crecum, 199

Heredity, 124, 126, 218, 281

Hermaphrodite, 38 t

Heteronereis, 351

Heterotype division, 217

Hetero-zygote, 220

Hexacanth embryo, 371

Hip-girdle, 75, 149, 183

Histology, 11, 38, 157, 321

Holoblastic, 222

Holocephali, 448

Homology, 11

Homoplasy, 325

Homotype division, 217

Homo-zygote, 220

Hormones, 293

Humerus, 73, 149

Hyaloplasm, 43

Hydra, 376

Hydranth, 389

Hydrocope, $3 \dot{8} 9$

Hydrotheca, 397

Hydrozoa, 434.

Hymenoptera, 444

Hyoid (see also Arches, Visceral), $82,141,163$

Hyomandibular, 181

Hyostylic skull, 181, 191, 282

Hyperpharyngeal groove, 198

Hypoblast, 224, 233, 252, 345

Hypobranchial, 181

Hypodermis, 338

Hypostome, 377

TLEUM, 16, 140, 174

Ilium, 75, 149

Incisors, 14, 80, 81

Incus, 82, 114, 165

Individuality, 397

Infra-branchial chamber, 328

Infundibulum, 103, 152, 185

Infusoria, 432

Inhalent, 324

Inhibited nerves, 91

Insecta, 444

Instinct, 94, 130 
Interradial, 392

Inter-renal body, 190 opening, 332

Interstitial cells, 378

Intervertebral pad, 69

Intestine, 16, 140, 17 $7,199,236,267$, $312,330,339$

Histology of, 86,330

Invertebrata, 322

Iris, 86,116

Ischiopodite, 304

Ischium, 75, 149

Iter, 102

JAWS, 80, 81, 161, 181, 304 Joints (see Articulations). Jugal, 80

\section{KARYOKINESIS, 43, 44}

II Katabolism, 10, 45

Katastases, 26, 31, 43

Keber, Organ of, 332

Keratin, 50

Kidneys of dogfish, 189

$$
\begin{array}{ll}
\text { ", frog, } 155 \\
\text { ", mussel, } 332 \\
\text {,, rabbit, 5, 63 }
\end{array}
$$

\section{T ABIAL cartilages, 182 \\ 1 ,, palps, 329}

Lachrymal, 81

Lacteals, 18, 27, 146

Lacunæ, 54

Lagomorpha, 132

Lamellibranchia, 334

Lancelet, 192

Larva, 212, 245, 334

Laryngo-tracheal chamber, 141

Larynx, 35, 268

Lateral folds, 258

$$
\text { ," line, } 171
$$

" plate, 235,253

Laurer-Stieda canal, 358

Laverania malariae, 42:3

Lens, 116, 276

Lepidoptera, 445

Leporidse, 132

Leptomedusae, 431

Lepus, 131

Limb-girdles, 71, 148, 182
Limbs, 3, 66, 71, 131, 149, 303

Lithobius, 417

Liver, 17, 61, 140, 174, 199, 236, $213,268,311,330$

, -cells, 62

," -fluke, 355

Lobosa, 430

Lobules of liver, 61

Lumbar vertebræ, 70

Lumbricus, 336

Lungs, 6, 34, 134, 141, 243, 268

Lymph, 26, 200

„y -glands, 26, 58

" -hearts, 147

", -sinuses, 139, 146

" -stomata, 26

Lymphatics, 26, 32

MACRURA, 441

1 Malacostraca, 441

Malarial Parasite, 423-427

Malleus, 82, 114, 165

Mammalia, 5, 121, 133, 453

Manatee, 453

Mandible of crayfish, 304 , dog, 81

Mand̈ibulä arch, 163, 236

Mantle, 325

," chamber, 326

Manubrium, 391, 393

Marrow of bones, 57,58

Mastication, 14

Mastigophora, 412

Matrix, 51

Maturation, 213, 228, 262, 391, 403

Maxilla of crayfish, 304

", ", dog, 80
, frog, 161

Maxillipedes, 304

Measly pork, 372

Meckel's cartilage, 161, 181, 236

Medulla, 400

" oblongata, 103

," of kidney, $6 \mathrm{t}$

Medullary sheath, 90

Medusa, 387, 391

Megagamete, 426

Megameres, 371

Meganucleus, 401

Melanin, 423

Members, 11

Membrane-bone, 54, 83, 161, 171 


\section{Mendel, 218}

Meroblastic, 248

Meropodite, 304

Merozoites, 424

Mesencephalon, 107

Mesenteron, 224, 232, 250, 309, 386

Mesentery, 5, 12

Mesoblast, 224, 233, 252, 345

Mesoglœa, 377

Mesonephric duct (sce also Wolffian dinct), 189

Mesonephros, 189, 244, 278

Meso-pterygium, 183

Mesosternum, 148

Metabolism, 10, 45

Metacarpals, 74,149

Metacromion, 73

Metagenesis, 396

Metamerism, 65, 167, 193, 237, 336

Metamorphosis, 245, 288

Metanephros, 189, 278

Metapleural folds, 193, 227

Metapophyses, 70

Meta-pterygium, 183

Metatarsals, 77, 150

Metatheria, 454

Metazoa, 45, 403, 433

Metencephalon, 101

Microgamete, 426

Micromeres, 371

Micronncleus, 401

Milk-teeth, 14, 268

Nineral salts, 13

Miracidium, 360

Molars, 14, 80, 81

Mollnsca, 334

Monocystis, 413

Monogenea, 437

Monotremata, 453

Morphology, 10, 11

Morula, 262

Motor nerves, 91

Month, 12, 137, 139, 170, 193, 226, $239,309,329,336,377$

Mucin, 14

Mucous membrane, 59

Müllerian duct, 244, 280

Muscle, 84, 225

MLuscles, Adductor, 325

,, , Cardiac, 87

,, , Extensor, 307

,, Extrinsic, 311

", Flexor, 307
Muscles, Intercostal, 36

, , Intrinsic, 310

,, , Involnntary, 86

," , Oblique, 118

, , Papillary, 29

, , Rectus, 118

," , Respiratory, 36, 141, 175

,, , Skeletal, 86

, , Sphincter, 16, 197

, , Splanchnic, 86

, , Voluntary, 86

Muscle-tail cells, 378

Mutation, 287

Myocœlomic pouches, 224

Myocyte fibrillae, 413

Myomere, 171, 192, 208, 237, 242

Myotome (see Myomere).

NARES, 37, 77, 137, 139

1 Nasals, 81, 161

Natural selection, 284

Navel, 264

Neck, 3, 137

Nematocysts, 378

Nemertinea, 436

Nephridia, 204, 238, 331, 341, 346, 364,368

Nephrostome, 204, 238, 332, 341

Nereis, 349

Nerve, Abducent, 106

,, , Auditory, 108

,, , Chorda tympani, 108

,, , Depressor, 109

,, , Facial, 107

,, , Glossopharyngeal, 108

, , , Great anricular, 99

," , Gustatory, 107

", Hyomandibular, 153, 186

", , Hypoglossal, 110, 150, 153

", Lateral, 186

, Mandibular, 107

,, , Maxillary, 107

,, , Ocnlomotor, 106

, , Olfactory, 105

,, , Ophthalmic, 107, 186

,, , Optic, 105

, , Palatine, 108, 153, 186

,, , Pathetic, 106

,, , Phrenic, 99

,, , Pneumogastric, 109

,, , Recurrent laryngeal, 109

¿ Spinal accessory, 110 
Nerve, Splanchnic, 99

,, , Superior laryngeal, 109

," , 'Trigeminal, 107

,, , Vagus, 108, 153, 186

", , Vidian, 186

(See also Nerves, Cranial, Spinal.)

Nerve-cells, 89, 157

,, -centres, 104

,, cord, 341

", -fibres, 90, 274

Nerves, 90

roots, $97,184,202$

" , Cranial, 104, 152, 186

," , Spinal, 99, 150, 181, 202

Nervous system, 89, 150, 181, 202, $225,273,316,332,311,379$

Neural arch, 66, 273

", crest, 274

", folds, $233,252,264$

" plate, $180,223,234$

", spine, 180

, tube, 231

Neurenteric canal, 224, 231, 252

Neurilemma, 90, 274

Neuroglia, 89

Neuropodium, 350

Neuroptera, 445

Nictitating eyelid, 119

Notochord, 58, 159, 180, 200, 224, $225,234,252,264,273$

Notopodium, 350

Nucleo-centrosome, 408

Nucleoli, 43

Nucleus, 39

Nutrition, Holophytic, 381

$$
\begin{aligned}
& \text { ", Holozoic, } 351 \\
& \text { ", of amceba, 35 } \\
& \text { ", Amphioxus, } 194 \\
& \text { ", "earthworm, } 337 \\
& \text { ", hydra, 377 } \\
& \text { ", ", mussel, 324 } \\
& \text { ", rabbit, 9 }
\end{aligned}
$$

BELIA, 387

Odontoblasts, 57

Odontoid process, 69

Essophagus, 6, 15, 140, 174, 309, 329,339

Olecranon fossa and process, 73,74

Olfactory capsules, 159,181

" lobes, 101, 101, 184

$" \quad$ sacs, $112,134,154,187,275$
Oligochaeta, 440

Omosternum, 148

Ö̈cyte, 426

Oökinete, 426

Oötype, 369

Opercular fold, 241

Optic cup, 276

, lobes, 103, 133, 152, $18 \tilde{5}$

", stalk, 275

", thalami, 103

", tracts, 106, 152

,, resicle, 275

Orbiculare, 82, 114

Orbito-sphenoid, 80, 166

Order, 132

Organic compounds, 8

Organs, 11

Origin of nerves, 105

Orthoptera, 445

Ossicles, Auditory, 82, 114, 165, 275

Ossification, $\mathbf{5 5}$ Gastric, 309

Osteoblasts, 54

Osteoclasts, 55

Ostia, 312

Otic capsules, 159, 181

Otoliths, 154, 187, 333

Ovals, 424

Ovary, 122, 155, 188, 193, 333, 313, 384 (See also Gonad.)

Oviducal gland, 188

Oviduct, 122, 155, 188, 228, 280, $320,333,344$

Ovum, 122, 123, 155, 185, 201, 212, $228,321,331,345,381$

Oxy-hæmoglobin, 20

PAEDogenesis, 364

Palæontology, 282

Palate, $15,77,81,165$

Palatine, 81, 162

Palato-pterygoid, 161, 181, 236

Palp, 352

Pancreas, 17, 140, 174, 243, 268

Pancreatic juice, 17

Papillæ, Circumrallate, 112

$$
\text { , Foliate, } 112
$$

Parachordals, 159

Paramecium, 399

Parainylum, 408, 411

Parapodia, 349

Parasphenoid, 162 
Parazoa, 433

Parietals, 80

Parieto-frontals, 161

Parthenogenesis, 363

Patella, 77

Pectoral girdle, 72, 148, 183

Pedipalpi, 442

Peduncles of cerebellum, 104

Pelecypoda, 334

Pelvic girdle, 75, 149, 183

Pelvis of kidney, 63

Penis, 125

Pepsin, 16

Peptone, 16

Pericardio-peritoneal pores, 173, 242

Pericardium, 6, 59, 134, 139, 173, 241, 326

Perichondrium, 21, 53

Perilymph, 114

Perimysium, 85

Periosteum, 54

Periotic, 80, 167

Peripheral nervous system, 89

Perisarc, 389

Peristaltic action, 19, 87

Peristomium, 352

Peritoneum, 5, 59

Perradial, 392

Peyer's patches, 19, 58

Phagocytes, 245

Phalanges, 74, 77, 149, 150

Phalangida, 443

Pharyngo-branchial, 181

Pharynx, 15, 174, 195, 236, 339, 352

Phylum, 208

Physiology, 10, 11

Pincers, 304

Pineal, 33, 103, 152

Pimna of ear, 114, 130

Pisces, 448

Pituitary, 33, 103, 152, 185, 267

Placenta, 133, 265

Placoid scales, 170

Plant and animal, 10, 40, 381

Planula, 395

Plasma, 20

Plastic products, 45

Platylelmia, 435

Pleura, 6, 37, 59

Pleurobranchs, 315

Pleuron, 303

Plexus, Brachial, 100, 150 „, Lumbo-sacral, 100
Plexus, Sciatic, 150

, , Solar, 99, 154

Podobranchs, 314

Polar bodies, 218, 228, 217, 262, 384 cells, 214

Poles of ovum, 221

Polychaeta, 3 19, 440

Polycladida, 436

Polymorphism, 398

Polystomum, 437

Pons Varolii, 103, 152

Portal system, 23, 134, 208

Postaxial surface, 72

Præ-oral hood, 193

Preaxial surface, 72

Pre-coracoid, 148

Premaxilla, 80, 161

Premolars, 14, 80, 81

Prephyloric, 310

Pre-sphenoid, 80, 166

Pressure-sense, 171

Primitive characters, 282

", hypoblast, 223,250

, streak, 251, 264

Procœlous, 147

Proctodæum, 226, 236, 269, 309, 345

Proglottides, 366, 438

Pronephros, 237, 278

Prone position, 73

Pronuclei, 213, 228, 384

Pro-otic, 161

Propodite, 304

Pro-pterygium, 183

Prosencephalon, 101

Prostomium, 352

Proteids, 12, 42

Protoplasm, 38, 42, 212, 400

Protopodite, 304

Prototheria, 454

Protovertebræ, 235, 253

Protozoa, 45, 322, 403, 429

Pseudo-navicellae, 414

Psendopodia, 38

Pterocardiac, 310

Pterygoid, 81, 162

Ptyalin, 14

Pubis, 75, 149

Pulse, 22

Pupil, 116

Pylangium, 143, 176

Pyloric, 310

Pyramid, 63

Pyrenoids, 408 
UADRATE, 161

Quadrato-jugal, 161

R ADIAI, CANAL, 392

Radius, 74, 149

Rami communicantes, 97, 153

Rana, 137

Ranvier, Nodes of, 90

Receptaculum ovorum, 3 tt

Recessives, 218

Rectum, 16, 174, 330

Redia, 360, 361

Reduction divisions, 214

Reflex action, 92

Rejuvenation, 402, 403

Renal excretion, 64

Repetition of similar parts, 65

Reproduction, Asexual, 41, 382 of protozoa, 41, 403

$"$ Sexual, 121, 156, 188, $204,212,228,247$, $261,320,333,343,383,403$

Reptilia, 450

Respiration, 34, 141, 174, 207, 240, $259,265,294,314,329,340$

Restiform bodies, 185

Retina, 115, 116, 276

Retinula, 319

Rhabdocoelida, 436

Rhabdom, 319

Rhizopoda, 412, 429

Rhyncocephalia, 451

Ribs, 66, 68, 126, 127, 180

Rods, 116

Rostellum, 367

Rostral, 182

Rostrum, 302

SACCULUS, 114, 154, 187

" $\quad$ rotundus, $16,19,58$

Sacral vertebræ, 70, 75, 148

Saliva, 14

Sarcolemma, 85

Sarcous rods, 86

Sauropsida, 453

Scaplognathite, 305

Scapula, 73, 148

Schizogony, 419, 424

Scleritcs, 302

Sclerotic, 115
Scolex, 373

Scorpionida, 443

Scyllium, 169

Secretion, 13

Secretory nerves, 91

Segmental duct, 238

Segmentation, 214, 220, 228, 248,262 , cavity, 222, 229, 249

Semicircular canals, 113

Seminal vesicle, $156,189,204$

Sense-organs, 91, 111, 154, 187, 202, $225,275,318,333,342$

Sensory tubules, 171,187

Septa, 338

Serous membrane, 259

Sesamoid bones, 77

Setæe, 302, 337

Sexual characters, Secondary, 290

", dimorphism, 290

"selection, 290

Shell, 248, 325

Shoulder-girdle, 72, 148, 183

Sinuses, 178, 314

Sinus venosus, 142,175

Sipunculoidea, 499

Skeleton, 65, 147, 179, 200, 272 $302,325,338,377,400$

Skin, 50, 59, 137, 157, 225

,) -teeth, 170

Skull, 77, 134, 164, 180

Slipper animalcule, 399

Smell, 112

Soaps, 18

Solenocytcs, 347

Soma, 285

Somatic cells, 274

, mesoblast, 226

Somato-plasm, 384

Somites, Mesoblastic, 226, 235, 252, 264,336

Specialized charactcrs, 282

Species, 130

Spermary, 122

Spermatozoa, 122, 124, 213, 320, 344,384

Sperm-blastophor, 345

Spermiduct, 122

Sperm-sacs, 189

Spermoblast, 344

Spermospores, 344

Spermothecæ, 344

Sphenethmoid, 161 
Sphincters, 16, 17, 197

Spiders, 443

Spinal cord, 96, 150, 184, 202, 225, 274

„, nerres, 96, 99, 150, 184, 202, 274

Spindle, Nuclear, 44

,, , Striated, 322

Spiracle, 168, 174

Splanchnic mesoblast, 226

Spleen, 33, muscles,

Splitting of mesoblast, 234, 259, 345

Spongioplasm, 43

Sporocyst, 360

Sporogony, 419,426

Sporozoa, 412, 430

Sporozoite, $414,415,426$

Squamata, 451

Squamosal, 80, 161

Staggers, 375

Stapes, 82, 114, 165

Starch, 13, 14, 381

Statocyst, 392

Steapsin, 17

Sternebrac, 68

Sternum of crayfish, 303

Stigma, 407

", ", frog, 148

Stimuli, 91

Stomach, 15, 140, 174, 329

Stomodxum, 226, 236, 267, 309, 345

Stratum corneum, 50

') mucosum, 50

mucosum, 50
spindle, 319

Stylo-hyal, 82

Sub-germinal cavity, 249

,, -order, 132

Suckers, 239

Suctoria, 432

Sugar, 13

Sun the source of energy, 10

Supine position, 73

Supra-branchial chamber, 328

Supra-occipital, 80

Sweat-glands, 60

Swimmerets, 304

Symbiosis, 382

Symmetry, Bilateral, 65

Sympathetic system, 98, 153

Symphysis, 76

Synangium, 143, 176
Systemic heart, 331

Systole, 29, 312

TADPOLE, 238

Taenia, 439

Taenia serrata, 375

,, solium, 366-375

Taeniarhynchus saginata, 374

Tail, 1, 121, 137, 169, 212

Tail-fold, 258

Tape-worm, 366-375

Tarsus, 77, 150

Taste, 112

Teeth, 14, 56, 80, 81, 139, 170, 265

Teleostomi, 449

Telolecithal, 226

Telson, 303

Tendon of crayfish, 307

," , vertebrata, 52

Tentacles, 376

Tergum, 303

Testis, 122, 156, 188, 320, 333, 313

Tetrads, 214

Thalamencephalon, 101, 152, 185

Thoracic duct, 27

", vertebr:a, 66

Thorax of rabbit, 3

Thymus, 33, 154, 190, 208, 266

Thyro-hyal, 82, 163

Thyroid, 32, 154, 190, 207, 266

Thyrojd gland, 294

Tibia, 76,150

Tissue-complexes, 59

'Tissues, 11, 45, 47

Toads, 158

Tongue, 14, 139, 174

, -bars, 201

Trabecula, 159

Trachea, 35, 266

Transverse process, $66,147,180$

Trematoda, 363, 437

Trichocysts, 400

Tricladida, 436

'Trophozoite, 415, 425

Truncus arteriosus, 142, 175

Trypsin, 17

Tuberculum, 66

Tubules, Uriniferons, 64, 146

Tunicata, 208

Turbellaria, 436

Turbinals, 81, 112

Tympanic bulla, 80

" membrane, 114, 154 
Tympano-Eustachian recess, 153, 163,273

-hyal, 82

Typhlosole, 339

ITLNA, 74, 149

Umbilicus, 264

Urea, 8, 63

Ureter, 63, 86, 156, 189, 332

Urethra, 124, 278

Urinary bladder, 86, 141, 257, 267

Urine, 8 sinus, 190

Urinogenital (see Urogenital)

Urocardiae, 310

Urochorda, 416

Urodela, 158, 230, 450

Urogenital duct, 156

iv sinus, 189

Urostyle, 147

system, 122,276

Uterus, 123, 262

't masculinus, 124

Utriculus, 113, 154, 187

VACUOLE, Contractile, 39, 401

", , Food-, 401 380

agina, 123

Valve, Eustachian, 29

,, , Ileo-colic, 19

", , Longitudino-spiral, 143

," , Mitral, 29

", , Spiral, 174

,, , Tricuspid, 29

Valves in veins, 23

" of shell, 325

Vall Beneden's blastopore, 261

Variability, 127

Variation, 126, 287

Vasa efferentia, 156, 189, 243

Vascular system, 20, 142, 175, 200, $235,267,312,330,310$

Vas deferens, 122, 189, 278, 320, 343

Vasifactive tissue, 339

Vegetative pole, 213

Veins, 21, 22

" and arteries, 21, 30
$" \quad$ of crayfish, 314
$", \quad$, dogfish, 178
", frog, 145

Veins of mussel, 331

", ", rabbit, 23,24

", ", vertebrate embryos, 235, 267

Velum, 197

Ventricles of brain, 96, 101, 102, 151,185

Vermicule, "26 heart, $27,142,175$

Vermiform appendix, 16, 19

Vermis, 103

Vertebral column, 1, 66, 147, 171, 179,271

plate, 233, 251

Vertebrarterial canal, 68

Vertebrata, 1, 133, 208, 447

Vosicles, cerebral, 101, 233, 251

,', optic, 273

Vestibule, 123

Vestiges, 28, 31 Ј

Villi of Chorion, 263 intestine, 18

Visceral arches, 162, 234 mass, 326

Vitelline membrane, 229, 260, 339

Vomer, 81, 162

Vorticella, 432

WALKING-LEGS, 304

Waste-material, 8

Weissman, 284

Whito matter, 96, 103

Wolffian duct, 242,278 tubules, 242

Work, 7, 8, 40, 45

YIPHISTERNUM, 68, 148

X Xiphosura, 412

YOLK, 210, 245

1 ," -cells, 227

", -sac, 255, 262

", -sac placenta, 262

TOANTHARIA, 435

1 Zona radiata, 260

Zooid, 387

Zygapophyses, 67, 147

Zygocardiac, 310

Zygoma, 80

Zygote, 426 



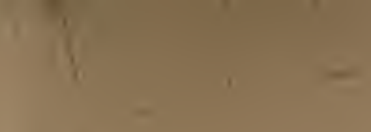




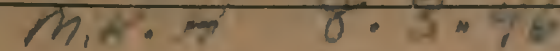
University of Toronto Library

DO NOT

REMOVE

THE

GARD

FROM

THIS

POCKET

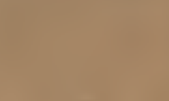
6. H

(1) $\quad 4$

a)

- 8

वे $i$

$\mathrm{H}+\mathrm{H}$

a.

E.

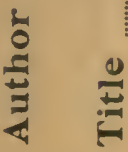

Acme Library Card Pocket Under Pat. "Ref. Index File"

Made by LIBRARY BUREAU 
